Slavistische Beiträge · Band 314

(eBook - Digi20-Retro)

\title{
Bernadette Koecke
}

\section{Diminutive \\ im polnisch-deutschen Übersetzungsvergleich}

\section{Eine Studie zu Divergenzen und Konvergenzen im Gebrauch einer variierenden Bildung}

Verlag Otto Sagner München · Berlin · Washington D.C.

Digitalisiert im Rahmen der Kooperation mit dem DFG-Projekt „Digi20“

der Bayerischen Staatsbibliothek, München. OCR-Bearbeitung und Erstellung des eBooks durch den Verlag Otto Sagner:

http://verlag.kubon-sagner.de

(c) bei Verlag Otto Sagner. Eine Verwertung oder Weitergabe der Texte und Abbildungen, insbesondere durch Vervielfältigung, ist ohne vorherige schriftliche Genehmigung des Verlages unzulässig. 


\title{
SLAVISTISCHE BEITRÄGE
}

\author{
Begründet von \\ Alois Schmaus \\ Herausgegeben von \\ Peter Rehder \\ Be irat:
}

Tilman Berger - Walter Breu - Johanna Renate Döring-Smirnov

Wilfried Fiedler - Walter Koschmal · Milos Sedmidubsky - Klaus Steinke

BAND 314

\section{VERLAG OTTO SAGNER MÜNCHEN 1994}




\section{Bernadette Koecke}

\section{Diminutive im polnisch-deutschen Übersetzungsvergleich}

Eine Studie zu Divergenzen und Konvergenzen im Gebrauch einer variierenden Bildung

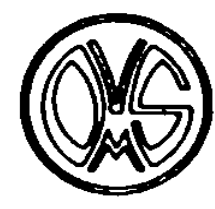

VERLAG OTTO SAGNER

MÜNCHEN 1994 


\title{
Meinen Eltern mit Dank
}

\author{
Bayorlsche \\ Staatsbibliothek \\ München
}

ISBN 3-87690-580-X

(c) Verlag Oto Sagner, München 1994

Abteilung der Firma Kubon \& Sagner

D-80328 München 


\section{VORWORT}

Die vorliegende Untersuchung wurde im Sommersemester 1992 von der Philosophischen Fakultät der Rheinischen Friedrich-Wilhelms-Universität zu Bonn als Dissertation angenommen. Für den Druck ist sie geringfügig überarbeitet worden.

Unterstützt wurde die Arbeit durch ein Stipendium nach dem Graduiertenförderungsgesetz des Landes Nordrhein-Westfalen.

Mehreren Personen und Institutionen gilt mein besonderer Dank:

Herrn Prof. Dr. Helmut Keipert, der mein Interesse am Thema geweckt und die Arbeit stets mit wohlwollender Kritik begleitet hat. Wertvolle Anregungen von germanistischer Seite verdanke ich Herrn Prof. Dr. Dr.h.c. Johannes Erben.

Das Deutsche Polen-Institut in Darmstadt, insbesondere Herr Dr.h.c. Karl Dedecius, sowie die Hörspielredaktion des Westdeutschen Rundfunks Köln haben mir freundlicherweise wichtige Texte zur Auswertung zugänglich gemacht.

Annette Kittlik-Hermanns und Dieter Hermanns danke ich für die hilfreiche Unterstützung bei der Anfertigung des Manuskripts.

Schließlich gilt mein Dank Herrn Prof. Dr. Peter Rehder für die Aufnahme der Arbeit in die Reihe Slavistische Beitrage.

Brüssel, im Juni 1994 


\section{INHALTSVERZEICHNIS}

1. EINLETUNG

1.1. Thematische Zielsetzung und die Methode des Ubersetzungsvergleichs

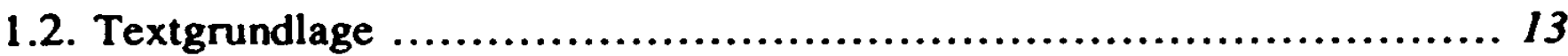

1.3. Die Forschung zum Diminutiv im Überblick ......................... 14

\section{LINGUISTISCHE GRUNDLEGUNG}

2. ZUR THEORIE DES DIMINUTIVS .................................... 32

2.1. Konzepte der Erfassung des Diminutivphänomens ..................... 32

2.2. Die Diminutivbildung im Polnischen .................................. 41

2.3. Die Diminutivbildung im Deutschen ................................. 57

2.4. Der semantisch-pragmatische Aspekt des Diminutivs ................... 70

2.4.1. Zum Begriff der Expressivität .................................. 71

2.4.1.1. Expressive Werte in der Bedeutungsstruktur einer lexikalischen Einheit ....................................................... 73

2.4.1.2. Expressivität und die Formantien ................................ 77

2.4.2. Die Bedeutungskomponenten des Diminutivs ..................... 79

2.4.3. Die inhaltlichen Leistungen des Diminutivs ..................... 82

2.4.3.1. Neutralisierte Formen ....................................... 84

2.4.3.2. Lexikalisierte Formen ....................................... 87

3. ZUR THEORIE DER UBERSETZUNG .................................... 94

3.1. Die Entwicklung übersetzungstheoretischen und -methodischen

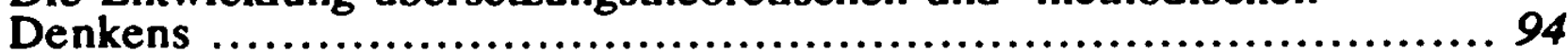

3.2. Ansätze einer Übersetzungskritik ................................. 102

3.3. Die Frage der Relation von Ausgangstext und Zieltext ................ 108

3.3.1. Determinanten des Übersetzungsvorgangs .................... 110

3.3.2. Die Forderung nach einer "Äquivalenz des Sinns" .............. 113

3.4. Äquivalenzebenen der Diminutivübertragung -

Exkurs (124127)

\section{EMPIRISCHE ANALYSE}

4. DIMINUTIVE IM UBERSETZUNGSVERGLEICH ........................... 130

4.1. Wiedergabemuster in der polnisch $\Rightarrow$ deutschen

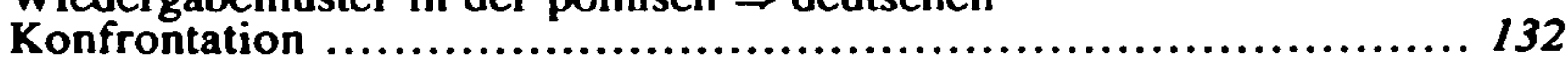

4.1.1. Übertragung synthetischer Diminutive ....................... 135

4.1.2. Übertragung analytischer Diminutive ........................ 180 
4.1.3. Übertragung analytisch-synthetischer Diminutive ............... 194

4.1.4. Diminutivische Übertragung von Nichtdiminutiven .............. 20I

4.2. Statistische Auswertung beider Übersetzungsvergleiche .............. 207

4.2.1. Verteilung der Belege auf die Wiedergabetypen:

Polnisch $\Rightarrow$ Deutsch ........................................... 208

4.2.2. Verteilung der Belege auf die Wiedergabetypen:

Deutsch $\Rightarrow$ Polnisch

4.3. Divergenzen und Konvergenzen im Diminutivgebrauch beider Sprachen .................................................... 231

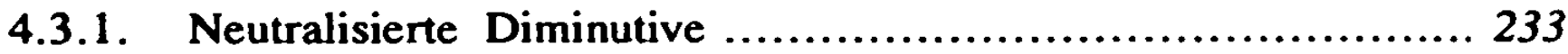

4.3.1.1. Angleichung von Basislexem und Diminutiv ................... 234

4.3.1.2. Funktionale Differenzierung von Basislexem und Diminutiv ....................................................... 239

4.3.2. Formal-semantische Restriktionen ........................... 248

4.3.3. Pragmatisch-stilistische Inkompatibilitäten ..................... 253

4.3.3.1. Konventionalisierte Kommunikationssituationen ................ 255

4.3.3.2. Individuelle Kennzeichnung ............................... 277

4.4. Gegenüberstellung der Übersetzungsvergleiche - Interferenzen beider Sprachen

5. RESÜMEE: Diminutive im Polnischen und Deutschen "vergleichbar und unvergleichlich" 


\subsection{THEMATISCHE ZIELSETZUNG UND DIE METHODE DES ÜBERSETZUNGSVERGLEICHS}

Wer sich mit dem Diminutiv, einem scheinbar marginalen Wortbildungsdetail, beschäftigt, wird bald feststellen, daß es einen reichen und vielgestaltigen Forschungsgegenstand abgibt. Das Phänomen, daß diesen variierenden (modifizierenden) Bildungen eine über die primäre Assoziation statischer Verkleinerung weit hinausgehende Dynamik sprachlicher Differenzierung und Nuancierung zukommt - was ihren Reiz als stilistisches Mittel ausmacht -, ist den verschiedensten Sprachen gemeinsam. Dabei lassen sich aber wesentliche Unterschiede in den Möglichkeiten der formalen Bildeweise und der Verwendung in der Rede feststellen. Man greift nun auf Bekanntes zurück, wenn man voraussetzt, daß Diminutive slavischer Sprachen einem Nichtslaven Schwierigkeiten bereiten, insofern er sich nicht nur einer Vielzahl spezifischer Diminutivsuffixe gegenübersieht, sondern gerade auch Kenntnis der jeder Sprache eigenen Gebrauchsgewohnheiten mitbringen muß.

In welcher Weise und warum bei der Übersetzung des Textes einer Sprache in eine andere Irritationen auftreten können, soll, am Beispiel des Sprachenpaares Polnisch-Deutsch vorgeführt, Gegenstand der Untersuchung sein. Die Unterschiedenheit beider Sprachen in Vielfalt der formalen Mittel und besondere Vorliebe für ihre Verwendung zum einen, sowie in Formenarmut und Sparsamkeit im Gebrauch zum anderen bietet die Grundlage für eine ergiebige Forschungsdiskussion. Wesentliche Vorleistungen wurden bereits von einzelsprachlich orientierten Arbeiten erbracht; mit zunehmender Bedeutung der kontrastiven Sprachwissenschaft ist Diminutiven auch von diesem Ansatz gewisse Aufmerksamkeit zuteil geworden. Auch wenn das Diminutivphänomen in seiner Heterogenität verschiedene Betrachtungsweisen zuläßt, bildet den Schwerpunkt der Studien die Erforschung des Diminutivsystems unter vorwiegend morphologischen Aspekten, in der Regel von den einzelnen Suffixen oder der isolierten lexikalischen Bedeutung der Worteinheit ausgehend. Als belangvolles Forschungsdesiderat ist aber die Beachtung der Differenzierbarkeit einer Sprache mit Coseriu - in System, Norm und Rede anzusehen. Mit ihr nämlich ist bisherigen Unzulänglichkeiten und Versäumnissen entgegenzutreten: Einerseits sind die an sich wertvollen systembezogenen Abhandlungen so von der unzulässigen Verquickung der Ebenen freizuhalten, andererseits können Fragen des Funktio- 
nierens von Diminutiven in Texten präziser gefaßt werden und sich daneben behaupten.

Will man eine zuverlässige Aussage über Diminutive in ihrer Textbedeutung treffen, so wird erst die Einbindung dieser Derivate ins Textganze, in dem sie erst ihre Wirksamkeit entfalten und ihre Bestimmung erfahren können, dieser Aufgabe gerecht. Textorientierte konfrontative Untersuchungen sind nun in der Lage, den normgemäßen Einsatz dieses Mittels für die jeweilige Sprache anzuzeigen und damit einzelsprachlich relevante Teilaspekte sowie Hinweise auf Gemeinsamkeiten und Unterschiede beider Sprachen zu liefern. Ein Handikap dieses Verfahrens liegt aber darin, daß es immer nur auf mehr oder weniger vergleichbare, nie aber auf exakt dieselben aktuellen Situationen verwiesen ist. Beobachtungen unter gleichen situativen Bedingungen kann dagegen allein ein Übersetzungsvergleich erbringen, nur er kommt in der Sprachenkonfrontation der Forderung einer konkreten Texteinbettung des Diminutivs nach:

In der Übersetzung wird die Sprache, in welche übersetzt wird, durch die andere gleichsam gestellt. Die Untersuchung der Lösung, welche die Übersetzung eines bestimmten Sprachmittels der Ausgangssprache bringt - immer wieder bringt -, zwingt den Betrachter zur "Feineinstellung". Auch er sieht sich hier gestellt, und manche von inm mitgebrachte Simplifikation wird dabei als solche enthüllt.'

Der geforderten Textsensibilität wegen verbietet es sich auch, die nun gewählte Methode des Übersetzungsvergleichs auf der Basis von konstruiertem bzw. isoliertem Wortmaterial durchzuführen: Es erscheint in Anbetracht der Manipulationsgefahr weder sinnvoll, auf selbstgebildete und übersetzte Mustersätze bzw. -texte oder selbstdurchgeführte Übersetzungen gegebener Sätze bzw. Texte zurückzugreifen, ${ }^{2}$ noch einen Vergleich "kontextloser verbaler Elemente, seien es nun isolierte Wörter, Syntagmen oder Sätze ${ }^{n 3}$, durchzuführen. Es ist davon auszugehen, daß es sich für die Belange dieser Studie als fruchtbar erweist, außerhalb linguistischer Intentionen entstandene, vorhandene ausgangssprachliche Texte und ihre Übersetzungen heranzuzichen und dabei eine möglichst vielseitige Auswahl des Textkorpus zu treffen. Eine weitere methodische Forderung besteht in der systematischen, nicht bloß selektiven Erfassung und Auswertung des ausgewählten Materials, um Zufälligkeiten auszuschließen und

'GAUGER 1971. S.115.

2 Vgl. dazu die Kritik an bisherigen kontrastiven Studien, insbesondere an Verfahren des Übersetzungsvergleichs, von WANDRUSZKA 1973, bes. S.7f.

${ }^{3}$ REISS 1981 a. S.311; sie spricht in diesem Fall von "Übersetzungsgleichungen", die sie streng vom eigentlichen Übersetzungsvergleich geschieden wissen will. 
dafür ein Spektrum sprachlicher Realisierungsmöglichkeiten vorzustellen. Eine solchermaßen angelegte Dokumentation soll auch insofern einen Beitrag zur Diskussion der Diminutivthematik leisten, als der Problemkreis Diminutiv und Übersetzung bisher in eher "aphoristischer" Weise angegangen worden ist. Da der Untersuchung in der Regel lediglich eine einzige Übersetzungsversion zur Verfügung stand, handelt es sich bei ihr um einen interlingualen Einfach-Vergleich.4

Ist man bestrebt, tatsächlich kommensurable Vergleichsbedingungen für beide Sprachen herzustellen, dann ist die unilaterale Konfrontation in bestimmter Hinsicht unzulänglich: Indem sie einseitig ausgerichtet ist und die eine Sprache immer nur in der ausgangssprachlichen, die andere in der zielsprachlichen Position beläßt, verzerrt sie deren Verhältnis; die eine wird ausschließlich in der vorteilhaften Lage, "aus dem Vollen (...) schöpfen"s zu können, betrachtet, die andere nur in der Beschränkung durch den Zwang, "Entsprechendes leisten" zu müssen, so daß sie "ihre eigensten Möglichkeiten nicht voll entfalten [kann], während ihre Lücken und Schwächen besonders deutlich werden" ${ }^{\text {6 }}$. Diesem Mißstand kann nur durch den bilateralen Übersetzungsvergleich abgeholfen werden, wenn nämlich die involvierten Sprachen jeweils sowohl als Ausgangswie als Zielsprache zu Wort kommen können.

Die gebotenen Übersetzungslösungen sollen aber nicht unkritisch hingenommen werden, sondern hinsichtlich ihrer Angemessenheit und Qualität einer Beurteilung unterzogen werden. Denn erst die Zusammenschau von geglückten und gescheiterten Entscheidungen kann den Charakter einer Sprache in seiner Besonderheit zum Vorschein treten lassen.

Die Methode des Übersetzungsvergleichs ist aber nicht Selbstzweck, vielmehr heuristisches Mittel, um die Vielzahl der Fragen anzugehen, die sich mit dem Hauptanliegen der Untersuchung verbinden, die spezifische Leistung einer Sprache im Gebrauch eines modifizierenden Sprachelements und die Art und Weise von deren Realisierung herauszuarbeiten. Im näheren zeigt dieser deskriptive Sprachvergleich zunächst, wie der Zieltext auf Diminutiveinheiten der Vorlage reagiert und welche Art von Relationen - Übereinstimmungen, partielle

\footnotetext{
4 Vgl. dazu die von REISS 1981a. S.312-316 vorgenommene Unterscheidung von grundsätzlich drei Formen des Übersetzungsvergleichs - intralingualen, interlingualen und multilingualen mit der weiteren Spezifizierung jeweils in Einfach- und Mehrfach-Vergleich.
}

S WANDRUSZKA 1973. S.19.

- WANDRUSZKA 1969. S.7. 
Entsprechungen und Unterschiede - sich ergeben. So ist, anders ausgedrückt, erkennbar, wie ein Text sich mal für die analoge Struktur entscheidet, mal auf diese Entsprechung verzichtet und eventuell kompensatorisch vorgeht, mal sein Potential ohne Vorgabe zum Einsatz bringt. Auf der Grundlage dieser Erörterungen legt der Übersetzungsvergleich Mittel an die Hand, Kriterien und Beweggründe aufzudecken, warum Diminutive gewählt werden oder auf sie verzichtet wird, womit auch die Ursachen für das ungleiche Frequenzverhältnis beider Sprachen aufgezeigt werden können. Des weiteren ist man in die Lage versetzt, im Rekurs auf verschiedene Sprechsituationen die den Sprachen jeweils eigenen Gebrauchsgewohnheiten und -normen, d.h. sprachengebundene Präferenzen zu ergründen und letztlich Vergleichbares und Unvergleichliches ${ }^{7}$ im polnischdeutschen Sprachkontakt, das Miteinander von Sprachen in ihrer Individualität, ersichtlich werden zu lassen. ${ }^{8}$

Zur Bewältigung dieses Frageprogramms bietet sich als Vorgehensweise eine Zweiteilung der Arbeit an: in einen die theoretischen Grundlagen referierenden und einen das Materialkorpus analysierenden Abschnitt. Vorrangiges Erkenntnisziel ist die Beschreibung polnischer nominaler Diminutive, die der deutschen dagegen, in ihrer Funktion als "Folie", bleibt ihr nachgeordnet. Die zunächst vorgenommenen einzelsprachlichen Darstellungen zum Diminutivsystem beider Sprachen, auf der Basis bereits geleisteter Forschungsarbeit zusammengestellt, sind eine notwendige methodische Hinleitung zum vorgegebenen zentralen Fragenkomplex. Der breite Raum, der anschließend übersetzungstheoretischen Belangen, im besonderen der Frage übersetzungsrelevanter Textkriterien und den daraus resultierenden Äquivalenzpostulaten, zugestanden wird, rechtfertigt sich aus der Notwendigkeit, ein Instrumentarium zur Durchführung der nachfolgenden Übersetzungsanalyse und -beurteilung zu schaffen.

Den Ausgangspunkt des zweiten, korpusorientierten Teils bildet nach einer Erörterung des hier anzuwendenden Diminutivterminus die Katalogisierung und

7 Vgl. WANDRUSZKA 1969.

- Daß der methodische Ansatz des Übersetzungsvergleichs nicht nur zur Erforschung der Unterschiede und Gemeinsamkeiten von Sprachen in ihrem gegenwärtigen Zustand fruchtbar gemacht werden kann, sondern auch wertvoll, ja unverzichtbar bei der Untersuchung von frühen und grundlegenden Sprachbeziehungen ist, zeigen Forschungen zum griechisch-slavischen Übersetzungsproblem. So sind Übersetzungsvergleiche u.a. in der Lage, den Nachweis zu erbringen, wie sich in der Entstehungsphase einer Sprache Veränderungen in der Übersetzungstechnik prăgend auf die Entwicklung ihres Wortschatzes auswirken können. Vgl. dazu KEIPERT 1977 (Kap.3, bes. S.84ff), der dies am Beispiel des kirchenslavischen Wortbildungstyps -tełs vo vorfünt; ferner s. auch KEIPERT 1989a und KEIPERT 1989b. 
zahlenmäßige Erfassung von Regularitäten und Mustern der Umsetzung dieser Modifikationsformen. Diese Bestandsaufnahme zeigt formal-strukturelle Parallelen, vornehmlich Disparitäten im interlingualen Austausch, kurz: Übersetzungsprobleme, die im anschließenden, schwerpunktsetzenden Kapitel grundsätzlich - auf dem Wege der kritischen Deutung von Einzelfällen - auf Möglichkeiten und Grenzen der Übersetzbarkeit bzw. des Erzielens von Äquivalenz zurückgeführt werden sollen. Mit dieser Darstellung wird es dann möglich, die entscheidende Frage nach Divergenzen und Konvergenzen beider Sprachen in pragmatisch-stilistischer Hinsicht zu klären, damit auch in einer Ausschnittsbeleuchtung Einblick in das je spezifische Gepräge der thematisierten Sprachen zu gewinnen.

Den Abschluß bildet die bilaterale Auswertung der bisherigen Ergebnisse. Sie soll, auf der Grundlage statistischer Erhebungen, Veränderungen der Nutzung des Diminutivprogramms der Sprachen in Abhängigkeit von ihrer Stellung als Quellen- bzw. Zieltext, d.h. mögliche Beeinflussungen durch den interlingualen Kontakt aufzeigen.

\subsection{TEXTGRUNDLAGE}

Die Auswahl des zugrundegelegten Textkorpus ist anwendungsbestimmten Kriterien gefolgt, indem versucht wurde, Sprachzeugnisse heranzuziehen, die einen lebendigen Diminutivgebrauch erwarten ließen. Zum einen sind dies Dokumentationen nichtartifizieller, nichtkonstruierter, also eher spontaner, kolloquialer Redeweise in Alltagssituationen und unformellem Umgang. Ideal wäre es gewesen, gesprochene Rede auswerten zu können, die Untersuchung sah sich aber darauf verwiesen, ausschlieBlich schriftlich fixierte Texte heranzuziehen. Diesem Mangel ist durch die Aufnahme von literarischen Texten begegnet worden, die zu großen Teilen Elemente der geforderten Kolloquialität, z.B. durch häufige Verwendung direkter Rede, aufweisen können. Im einzelnen kam es dann zur Auswahl u.a. von Erinnerungen, Tagebüchern, Hörspielen und Gesprächsaufzeichnungen.

Ein anderes Selektionskriterium stellte der bevorzugte thematische Gegenstand und seine Auswirkung auf die sprachliche Gestaltung bestimmter literarischer Genres dar. So war zu erwarten, daß Literatur über und für Kinder und Sachbücher, wie populärwissenschaftliche tierkundliche Werke, Koch- und 
handwerkliche Anleitungen, reiches Auswertungsmaterial zur Verfügung stellen würden. Im Bestreben, einen möglichst breiten Textsortenquerschnitt zu bieten, hat auch Belletristik, im näheren Romane und Erzählungen, Eingang gefunden.

Um gleiche Bedingungen zu schaffen, war es geboten, Parität zwischen den sprachlichen Quellen des Polnischen und Deutschen hinsichtlich der genannten Anforderungen zu erzielen. Doch erwies es sich als schwierig, ein allen genannten Kriterien genügendes Textangebot zusammenzustellen. Es fehlte nicht nur an Übersetzungen nichtbelletristischer Texte, sondern es zeigte sich auch, daß, sofern solche der einen Sprache übersetzt waren (z.B. deutsch $\Rightarrow$ polnisch), oft kein Pendant in der anderen Sprache vorzufinden war (z.B. polnisch $\Rightarrow$ deutsch). Dies machte insbesondere einen Mehrfach-Vergleich (Untersuchung mehrerer Übersetzungen eines Textes) unmöglich, und hinsichtlich des einzig wählbaren Verfahrens des Einfach-Vergleichs ergab sich dann auch das Problem, zuweilen nur auf qualitativ unbrauchbare Übersetzungen zurückgreifen zu müssen.

Aufnahme gefunden haben schließlich acht Texte für jede Sprache mit ihren Übersetzungen (die drei polnischen Hörspiele - M.Nurowska, W.Rumel, M.Tonecki - werden als ein Text verstanden), die im einzelnen dem Literaturverzeichnis zu entnehmen sind. Die Erfassung des Textmaterials beschränkte sich auf durchschnittlich 120 Seiten pro Werk, mit Ausnahme der Kinderbücher von J.Korczak und M.Ende, die ganz durchgesehen wurden, sowie der Sachbücher von M.Lemnis/H.Vitry und R.Gööck, die zwar vollständig durchgesehen, deren Diminutive aber nur in Auswahl exzerpiert wurden.

\subsection{DIE FoRSCHUNG ZUM DIMINUTIV IM ÜBERBLICK}

Angesichts des seit Jahrzehnten anhaltenden Interesses an dem in der Sprache existierenden morphologisch komplexen Wortmaterial - sei es in Form theoretischer Beschäftigung mit Verfahren und Gesetzmäßigkeiten der Wortbildung oder praktischer, sprachvergleichender Analyse von deren Produkten scheint es zweckdienlich, bereits geleistete Forschungsarbeit auf dem Gebiet der Diminutivbildung vorzustellen, zumal die folgende Untersuchung unter Einbeziehung der bisherigen Ergebnisse durchgeführt werden soll. Die zu dieser Thematik in großer Zahl verfaßten Arbeiten zwingen jedoch dazu, auf einen vollständigen und detaillierten Literaturbericht zu verzichten und sich auf die 
wesentlichen Beiträge zu beschränken, so daß die Aufarbeitung nur in einer summarischen Wiedergabe der Forschungsschwerpunkte bestehen kann. ${ }^{9} \mathrm{Da}$ auf ein nur chronologisches Vorgehen verzichtet wurde, erwies sich die Anordnung der gesammelten Literatur aufgrund ihrer Heterogenität bezüglich Inhalt, Zielsetzung und Arbeitsmethode als nicht ganz unproblematisch. Es wurde versucht, mit einer thematischen Gliederung eine zweckmäßige Systematik zu erreichen.

Entstehung, Entwicklung und Anwendung der Diminutive bieten ein so reich gefächertes Untersuchungsrnaterial, daß sie innerhalb der polnischen Wortbildungsforschung einen privilegierten Platz eingenommen haben. Als Anhaltspunkt für das Ausmaß des regen Interesses kann z.B. die Spezialbibliographie zur slavischen Wortbildung (bisher sechs Hefte, die die Jahre 1874-1981 umfassen $)^{10}$ herangezogen werden, in der allein 157 Publikationen als solche ausgewiesen werden, die sich mit Problemen der Diminutivbildung des Polnischen beschäftigen. " Stellt man diesen die Veröffentlichungen zum deutschen Diminutiv gegenüber, so zeigt der Forschungsbericht von ETTINGER $1980^{12}$ (berücksichtigter Zeitraum: Ende 19.Jh.-1975), daß sie in quantitativer Sicht mit den obigen nicht konkurrieren können. Diese sind hierin eher mit den romanistischen Arbeiten zur Diminutivmodifikation - von der geäußert wird, sie sei das "enfant chérin $^{\text {13 }}$ der Sprachwissenschaft - vergleichbar.

Der Eindruck, das Thema sei hinlänglich und in vielen Einzelheiten erfaßt, muß insofern relativiert werden, als mit der Akzentuierung der Quantität ein Mangel an Qualität einhergeht: Die unterschiedlichen Betrachtungsweisen, die aus den vielfältigen linguistischen Aspekten des Forschungsobjekts resultieren, bringen zwangsläufig eine breite Streuung der Literatur in verschiedene Teildisziplinen der Sprachwissenschaft mit sich. Dies hat dann wesentlich dazu beigetragen, daß viele Studien, ohne Bezug auf vorangegangene Forschungs-

- Weitere, hier nicht erwăhnte publizierte Literatur ist der Bibliographie dieser Arbeit zu entnehmen.

$10 \mathrm{Vgl}$. MATERIALY 1958-1983.

" Da das Sachregister der einzelnen Hefte nicht einheitlich strukturiert ist, kann die ermittelte Zahl nur als Näherungswert mit Tendenz nach oben verstanden werden. Bei dieser Fülle ist es nicht ganz verständlich, wenn Diminutivstudien eingeleitet werden mit der Behauptung, "das Diminutiv der polnischen Gegenwartssprache frist[e] ein wenig beachtetes Dasein", es sei denn, der Verfasser bezieht dieses Manko auf die westdeutsche Polonistikforschung, was aber seinen weiteren Ausführungen nicht zu entnehmen ist (s. POHL 1980. S.161).

$12 \mathrm{Vgl}$. dort (S.48) die tabellarische Übersicht.

${ }^{13}$ ETTINGER 1980. S.1. 
erkenntnisse zu nehmen, entstanden sind und damit bereits formulierte Fragen häufig nur wiederholt erörtert worden sind. $\mathrm{Zu}$ beanstanden ist darüberhinaus oft auch die Form der Darstellung. Zum einen handelt es sich meist um kleinere, das Problem nur in gedrängter Form darbietende Aufsätze oder solche, die die Diminutive innerhalb eines größeren Rahmens lediglich als Nebenthema berücksichtigen. Dazu zählen besonders dialektologische Untersuchungen, die einen GroBteil der oben genannten Zahl ausmachen. Zum anderen haben Diminutive eine thematisch wohl zu breit angelegte Ausführung erfahren, wenn der Versuch unternommen wird, eine systematische Darstellung zu bieten, indem auf eine Reihe grundlegender Probleme sowohl aus diachronischer als auch synchronischer Sicht eingegangen wird. Mit der parallelen Darstellung von z.B. Herkunft und historischer Entwicklung der Suffixe, Ableitungskriterien und -faktoren, Produktivität, Lexikalisierungsprozessen, Funktion und Stilwert der Diminutive in einer Sprache ist die eigentlich nötige, gezielte Beschäftigung mit Einzelaspekten wiederum ausgeblieben. ${ }^{14}$

Im einzelnen läßt sich die Forschungsarbeit wie folgt nachvollziehen: Außer in Spezialstudien haben Diminutive als Kategorie der Wortbildung, wenn auch in sehr knapper Form, Beachtung in Grammatiken sowie Handbüchern zur Wortbildungslehre gefunden, in denen der morphologisch-phonologische Aspekt der Beschreibung dominiert. Als polnische Grammatiken können genannt werden: die Gramaryka jezyka polskiego von SZOBER $1953^{15}$, der innerhalb des Abschnitts "Nauka o budowie wyrazów" im Kapitel "Formy słowotwórcze poszczegolnych cześci mowy", und zwar beim Substantiv unter dem Titel "Przyrostki tworzace rzeczowniki od rzeczowników", den \$210 "Rzeczowniki zdrobniaje i pieszczotliwe" (S.122) den Diminutiven widmet und sich dabei mit der bloßen Auflistung der an der Bildung beteiligten Suffixe (neun verschiedene Gruppen) und der Nennung entsprechender Beispiele begnügt. Auch im parallel aufgebauten Adjektivkapitel werden, ohne explizite Erwähnung, acht Formantien mit vermindernder Bedeutung oder mit Angabe der Intensität einer Eigenschaft in \$235 "Przyrostki tworzace przymiotniki od przymiotników" (S.132) genannt. In Ulaszyns Beitrag zur Wortbildung in dem Sammelwerk von BENNI et al.

i4 Diesen Mangel an Schwerpunktsetzung beklagt u.a. ETTINGER 1986 bei wissenschaftlichen Erstlingswerken und bezeichnet solche Leistung als "wenig ergiebig für die Entwicklung der theoretischen Grundlagen der Diminutivforschung" (S.439).

is Bei dem hier zugănglichen Exemplar, 3.Aufl. 1953, handelt es sich um die von W.Doroszewski überarbeitete Fassung der 2.Aufl. von 1923; die 1.Aufl. erschien 1914-1916. 
1923 wird versucht, innerhalb der Wortarten die Wortbildungsprodukte hinsichtlich ihrer Bedeutung zu klassifizieren, anders als bei SZOBER 1953, der formale Einteilungskriterien heranzieht. Diminutivische Formen, die nur bei Substantiven Berücksichtigung finden, werden zusammen mit den Augmentativen in einer eigenen Bedeutungsgruppe besprochen, vgl. \$23 "Rzeczowniki zdrobniałe" (S.230-232). Auch hier kommt es nicht über eine Suffixaufzählung hinaus, auch wenn sporadisch und unsystematisch knappe Erläuterungen, wie z.B. über Genese, Produktivität, Bedeutung, gegeben werden. Eine noch globalere Betrachtung erfahren die Diminutive in der - wie auch die vorherigen Grammatiken der diachronischen Sprachwissenschaft verpflichteten - Gramatyka wspótczesnego jezyka polskiego von GAERTNER 1938, der sie im Abschnitt "Slowotwórstwo" (S.234-424) gar nicht als eigene Ableitungsklasse heraushebt, sondern die Gesamtheit aller Derivationsprodukte rein formal, je nach Formansbeteiligung, zusammenstellt. DOROSZEWSKI 1963 (1.Aufl.1952) gliedert auf der Grundlage seiner logisch-syntaktischen Theorie in den Podstawy gramatyki polskiej die "Rzeczowniki zdrobniale" (\$209) wohl als eigene Gruppe innerhalb des Kapitels "Rzeczowniki oparte na temacie rzeczownikowym" (S.309-317) heraus, doch gibt auch er sich mit der Nennung einer recht heterogenen Suffixgruppe infolge der Vermengung hochsprachlicher und dialektaler Formen zufrieden. Andere Wortarten bleiben unberücksichtigt. Schließlich ist noch die sehr umfangreiche Wortbildungsstudie von R.Grzegorczykowa, J.Puzynina, K.Kallas und H.Wróbel im Morphologieband der GRAMATYKA 1984 vom Instytut jezyka polskiego PAN zu nennen. Auf der Basis moderner Wortbildungstheorien wird das Großkapitel "Derywaty odrzeczownikowe" zunächst in zwei Unterkapitel "Derywaty modyfikacyjne" und "Derywaty mutacyjne" aufgeteilt, um dann - die in allen vorangegangenen Grammatiken immer nur in einer Gruppe zusammengefaßten Diminutivbildungen als "Nazwy deminutywne" (S.366-368), "Nazwy ekspresywne" (S.369-371) und "Nazwy istot mlodych" (S.371) mit jeweils eigenen Formantien unter die Modifikationen zu ordnen. In dieser synchronischen Beschreibung findet sowohl der morphologisch-phonologische als auch der semantische Aspekt Erwähnung. Auch diminutivische Adjektive werden differenzierter bedacht, indem unter "Derywaty odprzymiotnikowe" u.a. zum einen "Formacje gradacyjne" (S.437-439), zum anderen "Formacje ekspresywne" (S.441) unterschieden werden.

Ein wenig anders sieht es im Deutschen aus, denn bereits bei GRIMM 1826/1831 wird das deutsche Diminutivsystem einer eingehenden Untersuchung unterzogen. Innerhalb des der Wortbildung gewidmeten 2. und 3.Teils seiner 
Deutschen Grammatik nimmt die "Diminution" außerhalb des umfangreichen Ableitungskapitels einen eigenen größeren Absatz ein (T.3. S.664-707). Neben der Erörterung des Begriffs und Wesens der Diminution beschäftigt sich J. Grimm besonders mit der Beschreibung der formalen Seite dieser Bildungen, mit dem Schwerpunkt der Darstellung der historischen Entwicklung. Außer den substantivischen Diminutiven werden auch die übrigen Wortarten einbezogen, die adjektivischen, adverbialen, pronominalen, verbalen Diminutive sowie die der Eigennamen. Neben dem Thema der Verbreitung, Produktivität und Genusproblematik muß die, wenn auch nur in Andeutungen, vergleichende Gegenüberstellung verwandter Sprachen als Besonderheit herausgestellt werden: betrachtet wird die Diminution des Lateinischen, Griechischen, Sanskrit, Slavischen, Litauischen und Romanischen. Eine ähnliche, doch weniger ausführliche, diachron angelegte Beschreibung findet sich bei WILMANNS 1899 (1.Aufl. 1896), der im zweiten Band seiner Deutschen Grammatik die Wortbildungsprozesse vom Gotischen bis hin zum Neuhochdeutschen verfolgt. Bei Dominanz des formalen Gesichtspunkts werden die Diminutivformen innerhalb des Großabschnitts "Substantivum" im Kapitel "Substantiva mit ableitenden Suffixen", welches wiederum nach in der Sprache vorkommenden Affixen unterteilt ist, behandelt (vgl. I-Suffix: S.270, n-Suffix: S.316-324, d-Suffix: S.362). In der stärkeres Gewicht auf das Neuhochdeutsche legenden Deutschen Grammatik von PAUL 1920 werden die substantivischen Diminutive zusammen als eigene Bedeutungsgruppe innerhalb des Ableitungskapitels aufgeführt (Bd.5. \$42. S.48-52). Besprochen wird vor allem die Gebräuchlichkeit und Konkurrenz der beiden Suffixe -chen und -lein sowie der Bedeutungsaspekt dieser Bildungen. Auch diminutive Formen der Adjektive (S.102f) und Verben (S.120f) werden kurz genannt. Schließlich können noch drei jüngere, um synchronische Darbietung bemühte Werke erwähnt werden: Zunächst Die deutsche Sprache von BRINKMANN 1971 (1.Aufl.1962), wo - ausgehend von einer Beziehung zwischen Form und Inhalt sprachlicher Einheiten - die Diminutive im 2.Kapitel: "Klassen des Substantivs" bei den Überlegungen zur "Verteilung der Ableitungsgruppen auf das grammatische Geschlecht" Berücksichtigung finden (S.17f). Ferner die Deutsche Grammatik von ERBEN 1980 (1.Aufl.1958), der in äußerst komprimierter Weise die Diminutivformen des Substantivs - "Affektische Verkleinerungs-Bildungen" (S.127f) - hauptsächlich aus semantischer Sicht betrachtet. Und dann H.Wellmanns Beitrag in der DUDEN-GRAMMATIK 1984, in dem ebenfalls die Funktionen der Präfix- und Suffixbildungen als Gliederungskriterien herangezogen und dabei die "Diminutivbildungen" als eigener Komplex der semantischen 
Abwandlung (Modifikation) recht knapp besprochen werden (S.459-461). Ebenso finden diminutive Formen der Verben (S.439) und Adjektive (S.489) Erwähnung.

In vergleichbarer Art und Weise wird das Diminutivphänomen in eigenständigen Gesamtdarstellungen zur Wortbildungslehre dargeboten. ${ }^{16} \mathrm{Zu}$ nennen wären für das Polnische die der synchronischen Beschreibungsmethode verpflichteten, erst relativ spät erschienenen Bücher von GRZEGORCZYKOWA 1984 (1.Aufl.1972), vgl. S.53-57; 69-71, und der zunächst erste Band von GRZEGORCZYKOWA/PUZYNINA 1979. Da in letzterem das Substantivmaterial nur formal nach den beteiligten Suffixen gegliedert ist, sei hier bloß auf das 9.Großkapitel mit der Darstellung der -k-Formantien verwiesen, vgl. S.114-215. Im Deutschen ist die Auswahl größer, einen orientierenden Überblick über die hauptsächlich synchronischen Probleme der Diminution geben die bekannten Gesamtdarstellungen von KLUGE 1913 (S.25-29), HENZEN 1957 (1.Aufl.1947) (S.140-152; 203; 223f), FLEISCHER 1974 (1.Aufl.1969) (S.178-182; 200; 272; 321) sowie ERBEN 1983 (1.Aufl.1975) (S.79-82; 95-97). Die umfassendste Bestandsaufnahme für die deutsche Sprache ist von der Forschungsstelle Innsbruck des Instituts für deutsche Sprache geleistet worden. Bisher sind drei Bände von DEUTSCHE WORTBILDUNG 1973-1984 zuzüglich eines Morphem- und Sachregisterbandes erschienen, in denen sich unter Einbeziehung bereits publizierter Fachliteratur die Ableitungsmöglichkeiten deutscher Verben, Substantive und Adjektive systematisch beschrieben finden. Zur Diminutivmodifikation vgl. Bd.1. S.115f; Bd.2. S.27, 34-38, 56f, 123-135; Bd.3. S.412f.

Waren die Diminutive bisher nur als Teilgebiet aller Wortbildungskonstruktionen einbezogen worden, so konnten sie - deren Komplexität sich nur schwer in enge Wortbildungskapitel zwängen läßt - in Aufsätzen bzw. Monographien zum alleinigen Gegenstand der Untersuchung werden. Das Forschungsmaterial zum Phänomen der Diminuierung, im Polnischen in extenso dargeboten, kann und soll hier lediglich in Auswahl der wohl wichtigsten Beiträge besprochen werden. Zu dem am häufigsten behandelten Sachgebiet zählt die Beschreibung der in Frage kommenden Suffixe. Der Darstellung der einzelnen Suffixe in ihrem historischen Entwicklungsprozeß sind die beiden komparativen Arbeiten von

\footnotetext{
${ }^{16}$ Auf eine Kommentierung der Leistung wird an dieser Stelle verzichtet, da Parallelen zu den Grammatiken bestehen und damit sich keine wesentlich anderen Gesichtspunkte bei der Beschreibung ergeben.
} 
BELIĆ 1901/1904 sowie die umfangreiche und ungleich bedeutendere Monographie von WARCHOL 1984, die das Diminutivpotential der slavischen Sprachen inventarisieren, gewidmet. Während die am Anfang der Diminutivforschung stehende Abhandlung von A.Belic nicht mehr als einen Überblick über die Diminutiv- und Augmentativbildung der Substantive und Adjektive im Russischen, Čechischen, Slovenischen und Serbischen zu geben versucht, bemüht sich S. Warchol um systematisches Aufzeigen des Ursprungs und Wandels expressiver Bildungen mit dem suffixalen -k- und -c-Element bis in die Gegenwart, verbunden mit der Betrachtung ihrer strukturell-semantischen Eigenschaften. Neben Substantiven und Adjektiven werden auch Adverbien und Verben im Altkirchenslavischen, Bulgarischen, Makedonischen, Serbokroatischen und Slovenischen, im Russischen, Ukrainischen und Weißrussischen, im Polnischen, Čechischen, Slovakischen, Ober- und Niedersorbischen sowie Polabischen berücksichtigt. Eine Thematisierung ausschlieBlich polnischer Diminutivformen ist den Arbeiten von DOBRZYŃSKI 1974/1988 zu verdanken. Auf der Grundlage in großem Umfang exzerpierten Materials aus gegenwärtigen und historischen Quellen werden in diachronischer Sicht ausgewählte Suffixe vorgestellt. Band 1 befaßt sich mit der Produktivität, Funktion sowie Ausdehnung des Einzelsuffixes $-\boldsymbol{e}$ auf verschiedene semantische Bereiche. Im 2.Band werden appellative Hypokoristika, speziell solche, die durch Anfügen verschiedener Suffixe (60 werden aufgenommen) an ein reduziertes Basismorphem entstanden sind, zusammengestellt. Schließlich ist noch die diachronische Studie von RECZEK 1968 zu nennen, der anhand unterschiedlicher Textsorten besonders die Entwicklung der stilistischen Funktionen von Diminutivbildungen, vom Beginn des Schriftums bis hin zur Gegenwart, allerdings nur andeutungsweise ins Auge faßt.

Bei den vornehmlich synchronisch orientierten Beiträgen können grundsätzlich Beschreibungen, die schwerpunktmäßig der formalen bzw. der semantischen Seite nachgehen, unterschieden werden. Zu den erstgenannten und gewiß wertvollsten dieser Gruppe zählt die morphologisch ausgerichtete Monographie über die expressiven Substantive auf -ik, -k-, -isko und -ina der Gegenwartssprache von KREJA 1969, deren Mittelpunkt das Aufzeigen der Distributionsmechanismen von -ek und -ik bildet. Darüberhinaus beschäftigt sich der Verfasser mit den phonologischen Veränderungen des Basismorphems infolge der -k-Suffigierung, der Frage des Genus dieser Bildungen sowie den Funktionen der expressiven Formantien; das Heranziehen historischen Materials hat lediglich unterstützenden Charakter. Exemplarisch für die Vermengung vielfältiger Aspekte der Diminutivproblematik auf begrenztem Raum ist der Aufsatz von SIATKOWSKA 1967: 
sie unternimmt den Versuch, die aktuellen Diminutive der westslavischen Sprachen in "właściwe" und "emfatyczne" - mit Angabe der beteiligten Suffixe einzuteilen. Ferner ermittelt sie die Gebrauchshäufigkeit einzelner Formantien sowie die allgemeine Frequenz von Diminutiven in den einzelnen Sprachen, greift dabei nur auf Wörterbuch- und Grammatikbeispiele zurück, was zu einem verzerrten Bild des eigentlichen Gesamtbestandes führt. Nicht zuletzt führt die Autorin phonetische und semantische Einschränkungen bei den Ableitungsvorgängen sowie Bedeutung und Stilwert der Diminutive an. Eine morphologische, auf der Basis der strukturellen Semantik angelegte Darstellung der diminutiven, augmentativen und expressiven Derivate des Polnischen und Russischen bietet STANKIEWICZ 1954. Viele der hier nur fragmentarisch angedeuteten sowie weitere Probleme sind auch Gegenstand kleinerer Studien geworden: Produktivität und relative Häufigkeit der Substantivdiminutive in den einzelnen slavischen Sprachen hat MÜLLER-OTT 1970a und MÜLLER-OTT 1970b überprüft, eine morphologische Analyse der maskulinen Diminutivbildungen des Polnischen führt WRÓBEL 1969 durch, morphonologische Besonderheiten der substantivischen und adjektivischen Diminutivformen der Gegenwartssprache zeigt POHL 1980 auf, BURDZANOWSKA 1978 schließlich bemüht sich um eine Beschreibung der Diminutivbildungen auf der Grundlage des generativen Grammatikmodells.

Dem Stellenwert entsprechend überwiegen die Substantivuntersuchungen, nur wenige Publikationen wählen Adjektivdiminutive zum Thema, vgl. KREJA 1957 und JADACKA 1978 - beide behandeln das -awy-Suffix - sowie den modern orientierten Beitrag von JANUS 1986. Etwas zahlreicher sind die Beiträge zur Diminutivderivation der Verben, so z.B. DOROSZEWSKI 1957, OTREBSKI 1968, WARCHOL 1972 und MACZYŃSKI 1982. Letztlich sind auch Namenhypokoristika Mittelpunkt einer Analyse geworden, so bei KURZOWA 1970b, die männliche Kosenamen in Volksliedern untersucht, bei WROBEL 1973, der die morphologischen Mechanismen der Vornamenbildung aufzuzeigen versucht, bzw. bei IWANOWA-PERCZYŃSKA 1978 mit ihrer vergleichenden Aufzeichnung über aktuelle polnische und russische Koseformen.

Neben den vielen Schriften, in denen die formale Seite, der Wortbildungsprozeß des Diminutivs, im Vordergrund steht, sind auch solche zu nennen, die in erster Linie dem semantischen Aspekt, mit Berücksichtigung der Beziehungen zwischen den verschiedenartigen Bedeutungsschattierungen, Rechnung tragen. Unter ihnen ragt die Erörterung zu einer der Primärfragen (nach der bis heute umstrittenen Hierarchie der Bedeutungskomponenten des Diminutivs) von GA- 
WROŃSKI $1928^{17}$ heraus, die den eigentlichen Beginn der polnischen Diminutivforschung markiert und direkt im AnschluB zu einer lebhaften Diskussion anregte: siehe dazu die auf verschiedene sprachliche Mittel zum Ausdruck von Emotionen eingehenden Ausführungen von OBREBSKA 1929, WEDKIEWICZ 1929, DLUSKA 1930, alle dem hypokoristischen Wortschatz gewidmet. Hierzu zählt auch der etwas später erschienene Aufsatz über nomina propria von ZAREBINA 1954, der an die vorangehenden anknüpft. Fruchtbare Ansätze auf diesem Gebiet vermittelt besonders HELTBERG 1964, indem sie die geläufigen semantischen Kriterien nicht nur nennt, sondern mit in die Darstellung der Derivationsvorgänge von Diminutiven und Augmentativen einbezieht. Mit den wichtigen Problemen des Bedeutungswandels, der sog. Neutralisierung und Lexikalisierung befassen sich u.a. die Artikel von BIRKENMAJER 1924, SARNOWSKI 1985, SARNOWSKI 1987 und MACZYŃSKI 1986 sowie die Monographie von SARNOWSKI 1991. Erwähnenswert sind ebenfalls die etwas allgemeiner gehaltenen Abhandlungen von WARCHOL 1974a und GRABIAS 1981, in denen versucht wird, Beziehungen zwischen Expressivität und Wortbildung herauszustellen.

Ein weiteres recht beliebtes Sachgebiet der Forschung ist die Erkundung des Diminutivgebrauchs eines bestimmten Dichters oder einzelner literarischer Gattungen. Die Verfasser bemühen sich - wenn auch mit verschiedenen Intentionen - zum einen um die Registrierung der benutzten Suffixe, teilweise verbunden mit einer formalen Analyse der Diminutivstruktur, zum anderen, auf der Grundlage stilistischer Interpretationen, die möglichen, hauptsächlich konnotativen Bedeutungen auf der Ebene der Rede herauszuarbeiten. Leider beruhen die meisten Gradangaben des sog. emotionalen Wertes auf rein subjektiver Einschätzung, so daß man nachvollziehbare und verbindliche Kriterien vermißt. ZALESKI 1958 beurteilt den Diminutivgebrauch bei Dichtern des 16.Jh.s, REPA 1981 den von Kazimierz Brodziński, NOWOTNA-SZYBISTOWA 1969 von Stanisław Ignacy Witkiewicz, PLUTA 1985 von Rafal Urban usw. Darüberhinaus werden von KACZMAREK 1936, PLUTA 1967 und ADAMISZYN 1977 Volkslieder aus GroBpolen, Schlesien und Kleinpolen behandelt, sowie von KOPEĆ 1966 und POLAŃSKA 1967 Diminutivbildungen in der Kinderliteratur. Mit dem obigen Themenkreis beschäftigt sich auch die maschinenschriftliche Leipziger 17 Die Erstveroffentlichung erschien in: Prace lingwistyczne ofiarowane Janowi Baudouinowi
de Courtenay dla uczczenia jego dziatalnasci naukowej 1868-1921. Krakow 1921. S.241-256. 
Dissertation von RUDOLPH $1983^{18}$. Nach allgemein-theoretischen Erwägungen zu Problemen der Wortbildungslehre stellt sie Derivationsregeln mit Angabe von Regularitäten und Restriktionen der -k-Modifikanten auf, ${ }^{19}$ um dann einen Überblick über potentielle Bedeutungen der Diminutive sowie über ihre Funktionen in der sprachlichen Kommunikation, zum einen im umgangssprachlichen, zum anderen im künstlerischen Stil, zu geben. Instruktiv ist die recht detailliert strukturierte Zusammenstellung der Funktionen aktueller Diminutive (ausgenommen hypokoristische Bildungen) in der Umgangssprache, als weniger ergiebig erweist sich dagegen die etwas oberflächliche Gliederung für Lyrik, Prosa, Kinderliteratur und einige Liedgattungen.

Abgesehen von der mehr oder weniger zufäligen Berücksichtigung einiger landschaftlich geprägter Suffixarten in den genannten Beiträgen zur Hoch- und Schriftsprache sind auch etliche dialektologische Nachforschungen unternommen worden. Unter ihnen ist die u.a. dem Diminutivsystem der lachischen Dialekte gewidmete Monographie von LASKOWSKI 1966 hervorzuheben, die beispielgebend und modellhaft für weitere Studien, nicht nur zur Mundart wirkte. Die meisten Arbeiten konzentrieren sich, wenn auch in knappen Zügen, auf die Erkundung der den Dialekten (dem schlesischen, kleinpolnischen, zentralpolnischen u.a.) jeweils eigenen Suffixformen, vgl. z.B. PLUTA 1980, BASARA 1972, STASZEWSKA 1985 oder KLOBUS 1985.

Die Arbeiten zur Diminution im Deutschen reichen, wie im Polnischen, von beiläufiger Erwähnung über die Untersuchung von Einzelaspekten bis hin zum Versuch einer Definition oder gar zur umfassenden Präsentation des Diminutivsystems, auch wenn sich eine weniger umfangreiche Auswahl, insbesondere der synchronischen Studien, bietet. Da mit ETTINGERs (1980) Überblick über Arbeiten zur Modifikation bereits ein ausführlicher Forschungsbericht zum deutschen Diminutiv in vorteilhaft systematisierter Form vorliegt (vgl. S.47-83), möge an dieser Stelle eine knappe Repetition seiner bis 1975 berücksichtigten Literatur - ergänzt durch Neuerscheinungen - genügen. "Unter diachronischen Gesichtspunkten ist die Diminution im Deutschen nahtlos behandelt worden.

\footnotetext{
18 Liegt auch in publizierter Form vor, jedoch um die Darstellung der formalen Seite verkürzt, vgl. RUDOLPH 1984.

${ }^{19}$ Bei diesen Derivationsregeln, besonders für die Maskulina, fallt auf, daß die Verfasserin sie auf der Grundlage des bereits von KREJA 1969 ausfuhrlich ermittelten Materials zur Distribution der Suffixe -ek und -ik (vgl. S.24-69) beschreibt, diese Quelle aber nicht eigens als ihre Vorlage zu erkennen gibt.
} 
POLZIN [1901 - B.K.] bespricht die Zeit bis zum Jahre 1600, GÜRTLER [1909a - B.K.] behandelt die Diminutive für den Zeitraum 1600-1750, PFENNIG [1904 - B.K.] schließlich geht auf die Zeit ab 1750 ein". ${ }^{20}$ Richtungweisend wirkte die Dissertation von POLZIN 1901. Im Mittelpunkt dieser sowie der sich anschließenden und mit den vorgelegten Thesen sich auseinandersetzenden Publikationen steht die Entwicklungsgeschichte der beiden Diminutivsuffixe -chen und -lein mit Bevorzugung der Frage nach fremdsprachlichem Einfluß und Herkunft sowie nach der primären Bedeutung der Diminutive. Während Polzin sich zum einen für eine Beeinflussung von seiten des Lateinischen ausspricht und zum anderen die Kleinheit als ursprüngliche Bedeutung ansieht, geben WREDE 1908 in seiner großen Untersuchung der Sprachatlas-Diminutive und sein Schüler HASTENPFLUG 1914 - dessen Dissertation zu den ersten Versuchen gehört, anhand von statistischen Auswertungen den Diminutivgebrauch des 12. und 13.Jh.s systematisch zu erfassen - eine neue Diminutivdefinition, indem sie die Personennamen als Ursprung fassen und damit das kosende Element an den Anfang stellen. Die These von der lateinischen Einwirkung auf die Entstehung der deutschen Diminutive lehnen sie ebenfalls ab. Um eine Vermittlung zwischen beiden zugespitzten Positionen bemühte sich ÖHMANN 1946: lateinischen Einfluß will er nur noch für das Althochdeutsche gelten lassen, im Mittelhochdeutschen zeigt er Abhängigkeiten sowohl vom Lateinischen als auch Französischen auf. Die Leistung der Doktorarbeit von GÜRTLER 1909a, die sich des Problems der unterschiedlichen Distribution der beiden deutschen Diminutivformen im Frühneuhochdeutschen annimmt, liegt auf einem anderen Gebiet, die begonnene Diskussion führt er nicht fort. Mit diesen Beiträgen gehören die Diminutive zu den wenigen gut aufgearbeiteten Bereichen des Frühneuhochdeutschen. Nach jahrelangem Forschungsstillstand ist mit dem von W.Besch, W.Lenders und H.Moser geleiteten Bonner Forschungsvorhaben "Grammatik des Frühneuhochdeutschen" ein methodischer Neuansatz versucht worden, indem mittels maschineller Auswertung sprachhistorischer Quellen ein Überblick über Verhältnisse und Entwicklungsprozesse dieses Zeitabschnitts erstellt werden konnte. Im Rahmen dieser EDV-Untersuchung sind auch die Diminutivsuffixe - anhand eines nach Landschaften und Zeiträumen sorgfältig sortierten Korpusmaterials - einer Analyse unterzogen worden. So konnten anhand einer Reihe von Beobachtungen,

\footnotetext{
${ }^{20}$ In dieser komprimierten Form leitet ETTINGER 1980 (S.49 - Hervorhebung B.K.) sein Kapitel "Diachronische Studien" ein.

${ }^{21}$ Komplettiert wird die Abhandlung durch die eigens publizierte Inventarisierung des benutzten Materials, vgl. GÜRTLER $1909 \mathrm{~b}$.
} 
wie z.B. hinsichtlich einer landschaftlichen Bevorzugung, einer zeitlichen Entwicklung (d.h. Zunahme der Diminuierung), einer Verteilung auf Gattungen oder hinsichtlich der konkurrierenden Varianten, Ergebnisse der früheren Arbeiten bestätigt, korrigiert und sinnvoll ergänzt werden, vgl. WEGERA 1982.

Der umfangreichen, aus genetischer Sicht urteilenden Literatur stehen nur vereinzelte, sehr knapp gefaßte Artikel, die sich um die Darstellung der Diminutivproblematik in der gegenwärtigen Hoch- und Schriftsprache bemühen, gegenüber. Ein umfassender Versuch, das moderne deutsche Diminutivsystem in dessen verschiedenen Aspekten zu beschreiben, steht noch immer aus. Zum Frühesten zählen die Aufsätze von SANDERS 1893/1894 und GARTNER 1898, der - obwohl bei ETTINGER 1980 schon zum synchronischen Forschungsteil gezählt - letztlich bei seiner Beschreibung des Wettstreits von -chen und -lein der historischen Betrachtungsweise verpflichtet bleibt, während SANDERS 1893/1894 rein synchronisch das einzelne Suffix, -chen, im System der Sprache untersucht: neben dem Bedeutungsproblem geht er auf Besonderheiten bei der Bildung dieser Derivate ein und liefert damit erste Ansätze einer formalen Beschreibung der Regularitäten und Restriktionen. Wenig Fortkommen, vielmehr nur Paraphrasierung von bereits Bekanntem, bringen die in großen zeitlichen Abständen aufeinander folgenden Beiträge von PREUSLER 1942/43, dessen Untersuchung eher durch reihende Beobachtungen als durch systematische Auswertung gekennzeichnet ist, und ÖHMANN 1972. Hier wird zum wiederholten Male auf die Konkurrenz der beiden Modifikanten um den Vorrang in der neuhochdeutschen Schriftsprache eingegangen. Anders die drei folgenden, sich gegen die historisch-vergleichende Methode wendenden Beiträge, die wertvolle Informationen liefern: SIEBERER $1966^{27}$ und SEIDEL-SLOTTY 1947 bemühen sich um eine eingehende Charakteristik des Diminutivphänomens auf der Grundlage seiner semantischen Struktur. Zur Klärung der vielschichtigen Bedeutungsmöglichkeiten werden meist andere Sprachen als Vergleichsbasis herangezogen. Auch die bei ETTINGER 1980 unerwähnt gebliebene Abhandlung von KRUISINGA 1942 macht sich - hier explizit - die Arbeitsmethode des Sprachvergleichs zunutze, wenn über die semantischen Grundfunktionen der Diminutivund Affektivsuffixe in den germanischen Sprachen, der englischen, der niederländischen und der deutschen Gegenwartssprache, geforscht wird. Gedrängt, aber anregend ist schließlich die semantisch-stilistisch orientierte Studie von LOCKWOOD 1974/75, die besonders Fragen der möglichen Funktionen der Diminutiv-

2 Hierbei handelt es sich um den Nachdruck des bereits 1950-1952 erschienenen Aufsatzes. 
suffixe oder der Lexikalisierung skizziert.

Mit einem anderen Themengebiet, dem Diminutivgebrauch in poetischen Werken, befassen sich die beiden Dissertationen von PFENNIG 1904, der F.Schiller und seine Zeitgenossen heranzieht, und RAMSTEIN 1969 (maschinenschriftlich) - von ETTINGER 1980 nicht berücksichtigt -, die ausgewählte Prosatexte aus der neueren deutschen Literatur der Autoren Th.Mann, H.Hesse, E.T.A.Hoffmann, J.v.Eichendorff und E.Mörike analysiert. Während die frühere Arbeit - teils diachronisch, teils synchronisch - sowohl der Formenbildung als auch der Verwendung des Diminutivs nachgeht, interessiert RAMSTEIN 1969 besonders die stilistische Deutung des Diminutivs. Nach dem summarischen Referieren der Wortbildungslehre ihres Lehrers W.Henzen sowie der Grammatik von H.Paul zum Thema Diminution unternimmt die Verfasserin den Versuch, Wesen und Bedeutung des Diminutivs, speziell seine Leistung als Mittel stilistischer Nuancierung zu erforschen. Die zunächst vorgenommene Zuordnung des exzerpierten Materials (2230 Diminutive von Substantiven und Eigennamen) zu zehn Sachgruppen bringt keine nennenswerten Erkenntnisse. Als effizienter enweist sich die Beobachtung der syntaktischen Verwendungsart, vor allem die Rolle der die Diminutive begleitenden Attribute. Weniger nachhaltigen Eindruck hinterläßt dagegen der letzte Teil dieser Arbeit, in dem die Belege ihrem stilistischen Ausdruckswert nach in zwölf Gruppen gegliedert werden, in denen das Diminutiv als Zeichen der Kleinheit, als Affektträger im laudativen Sinn und als Affektträger in restriktiver, euphemistischer, ironischer und pejorativer Bedeutung auftritt. Letztlich liefert auch das Auffüllen der aus der wissenschaftlichen Literatur bereits bekannten Skala von Bedeutungsschattierungen mit Textbeispielen kaum neue Einsichten, zumal auch nach Auffassung der Autorin eine eindeutige Beurteilung und Rubrizierung kaum zu leisten ist.

Hinsichtlich methodischer Neuansätze ist das, was nach 1975 von germanistischer Seite zu diesem Thema veröffentlicht wurde, kaum erwähnenswert: Mit der in Form eines komprimierten Lexikonartikels abgefaßten Miszelle von THIEL 1978, dem ukrainischen Kurzbeitrag von KURKO 1975 über die semantische Struktur und diesbezüglich mögliche Gliederung der Diminutivbildungen sowie mit SCHEIDWEILERs 1984/85 Studie, die eine begrenzte Meinungsumfrage bezüglich der Konnotation von -chen und -lein auswertet, sind bereits alle für diese Arbeit zugänglichen Beiträge aufgezählt.

Die Mehrzahl der Arbeiten beschränkt sich auf die Wortart der Substantive; Adjektivdiminutive sind nur einmal, in der Dissertation von SCHWARZ 1905, der Entstehung und Entwicklungsgang des Suffixes -lich(t) herausgearbeitet hat, 
Gegenstand einer Untersuchung geworden. Eigenständige Beiträge zur verbalen Diminution fehlen gänzlich.

Auffälig größeres Interesse ist der Diminutivbildung in den deutschen Mundarten geschenkt worden. Da es nicht sinnvoll erscheint, einzelne Schriften hervorzuheben, sei auch hier auf ETTINGER 1980 verwiesen, besonders die tabellarische Übersicht (S.48), der in chronologischer Reihenfolge Verfasser und der jeweils besprochene Dialekt zu entnehmen ist. Es werden die Suffixvarianten zahlreicher Mundarten beschrieben, wobei die Dominanz der niederdeutschen Studien in Kenntnis der These von der Diminutivarmut des norddeutschen Sprachraums etwas überrascht. Auch wenn der Großteil der Beiträge ohne Bezug auf vorhergegangene Ergebnisse verfaßt wurde und sich damit innerhalb eines Dialektgebiets eine recht heterogene Darstellung ergab, kennzeichnet alle die gemeinsame thematische Schwerpunktsetzung. Überlegen erweist sich die Untersuchung der formalen Bildungsweise der Diminutive, während semantischstilistischen Aspekten in sehr geringem Umfang Beachtung geschenkt wird. Ergänzend muß u.a. auf den Aufsatz von LEYS 1970 zum Diminutivsystem einer westfälischen Mundart und die materialreiche Monographie von HOFMANN 1961 über Diminutive im Nordfriesischen hingewiesen werden, die, ausgehend vom nordfriesischen Mundartmaterial des 19.Jh.s, die These von der Diminutivarmut bzw. -losigkeit des Niederdeutschen entscheidend unterminiert hat. ${ }^{23}$ Unter den Neuerscheinungen der letzten Jahre zum Problem der diatopischen Unterschiede kann zum einen SEEBOLDs 1983 überblicksartig zusammenfassender Handbuchartikel herausgegriffen werden, zum anderen der instruktive Beitrag von TIEFENBACH 1987, der - immer mit Bezug auf vorausgegangene Forschungsarbeit - zunächst auf die sprachgeographische Verteilung der verschiedenen Suffixtypen eingeht, dann sein Augenmerk auf deren Übernahme in die überlandschaftliche Schriftsprache richtet, um schließlich den mundartlichen Befund in seiner Genese zu erläutern. Damit knüpft der Autor an die deutsche Tradition, die Frage nach der ursprünglichen Herkunft und Bedeutung der Diminutive, an und führt die zu Beginn dieses Jahrhunderts aufgenommene Diskussion fort, indem er sich mit den in der Forschung geäußerten Positionen auseinandersetzt und neue Lösungen anbietet: so hält er F.Wredes Theorie für

20 Diese bis dahin geltende und in allen Handbüchern verbreitete Lehrmeinung geht auf die Ergebnisse der Diminutivuntersuchungen von WREDE 1908 zurück: "Das (...) mittlere Niederdeutsch ist im allgemeinen diminutivlos oder mindestens diminutivarm" (S.90). 
"wenig wahrscheinlich" und spricht sich für den "umgekehrten Weg" aus. ${ }^{24}$ In Anlehnung an andere Wortbildungsphänomene nimmt er für die Diminutive eher eine Übernahme von appellativischem Sprachgut in das onomastische Inventar an.

Bei allen genannten Bemühungen vermißt man bis jetzt eine systematisch angelegte Monographie sowohl zum polnischen als auch zum deutschen Diminutivsystem, die die bisher ermittelten (nicht immer zugänglichen) wertvollen Forschungsergebnisse der Einzel- und Spezialstudien kritisch beleuchtend zusammenträgt, wie in anderen Sprachen bereits geschehen. Hervorzuheben sind die richtungweisende Arbeit des schwedischen Romanisten HASSELROT 1957 sowie HASSELROT 1972 hauptsächlich für das Französische, GOOCHs 1967 Studie über das Spanische, ferner für die lettische Sprache die umfangreiche Dissertation von RŪKSE-DRAVINAA 1959. Mit gewisser Einschränkung gehören hierhin auch die Abhandlungen von BRATUS 1969 und POLTERAUER 1981 über die russische Sprache.

Arbeiten zur Sprachtheorie im allgemeinen und zur Wortbildungslehre im besonderen greifen in den letzten Jahren immer häufiger auf eine kontrastive Methodik zurück. Die Einbeziehung mehrerer Sprachen läßt linguistische Fakten oftmals deutlicher zutage treten, als dies Jurch Analysen an nur einer Sprache möglich wäre. Im Rahmen dieser allgemeinen Tendenz wuchs auch das Interesse am synchronischen Sprachvergleich des Deutschen und des Polnischen. ${ }^{25} \mathrm{Be}-$ achtenswert ist dabei die - speziell bei der Diminutivforschung - einseitige Verteilung: während der Großteil der Studien von polnischer Seite beigetragen wurde, sind deutsche Wissenschaftler nur in geringem Maß beteiligt. Die Arbeiten aus Polen stammen vorwiegend von polnischen Germanisten, bei denen eine didaktische Zielsetzung, die Bemühung um eine Verbesserung des Deutschunterrichts, im Vordergrund steht. Deutsche Beiträge gehen fast allein auf Untersuchungen ostdeutscher Forscher zurück, die sich vornehmlich unter übersetzungswissenschaftlichen Aspekten der Konfrontationsarbeit gewidmet haben. Auch speziell auf dem Gebiet der Gegenüberstellung deutscher und polnischer

26 TIEFENBACH 1987. S.26.

$25 \mathrm{Vgl}$. dazu den Literaturbericht zur deutsch-polnischen kontrastiven Linguistik für den Zeitraum 1965-1980 von MIEMIETZ 1981, die über zweihundert Positionen - mitunter unveroffentlichte Abhandlungen - vom Bereich der Aussprache bis hin zu dem der Syntax bzw. dem lexikalisch-semantischen Teilbereich zusammengetragen und besprochen hat. 
Diminutivprobleme sind von westdeutschen Slavisten noch keine Beiträge geleistet worden.

Im einzelnen sind folgende sprachvergleichende Diminutivuntersuchungen verfaßt worden: Außer der nur thesenhaft angelegten Miszelle von IPPOLDT 1958 beschäftigen sich mit dieser Thematik in der Konfrontation des Polnischen und Deutschen drei in deutscher Sprache abgefaßte polnische Dissertationen. In der unter sprachdidaktischen Gesichtspunkten angefertigten Arbeit von GRUCZA 1974 bilden die Diminutiv- und Augmentativsuffixe nur einen Teilbereich der behandelten präfixalen und suffixalen Substantivderivation. Im Mittelpunkt steht die Auflistung der Eigen- und Fremdsuffixe beider Sprachen sowie der anschlieBende Vergleich konstitutionell identischer, ähnlicher und verschiedener Bildungen. Am Ende jedes Abschnitts werden dann die Relationen zwischen polnischen und deutschen Suffixformen tabellarisch aufgezeigt, die die quantitative Divergenz der formalen Mittel der Diminuierung in beiden Sprachen verdeutlichen sollen. DRAŻYŃSKA 1974/75 macht die Diminutive zum alleinigen Gegenstand ihrer Analyse. Der Großteil der Arbeit ist der einzelsprachlichen Betrachtung gewidmet, zunächst dem Deutschen, dann dem Polnischen. Die Beschreibung der formalen Seite der polnischen Diminutive, d.h. der Frage, mit welchen Stämmen sich Diminutivsuffixe verbinden sowie welche lautlichen Veränderungen der Basis durch die Derivation verursacht werden können, geschieht auf der Grundlage der Ergebnisse von KREJA 1969, indem diese in Übersichtstabellen zusammengefaßt werden. Dieser Analyse folgt unter Verwendung des generativen Modells die eigentliche strukturelle Konfrontation der Diminutive, wobei anhand des Belegmaterials, dessen Herkunft nicht wie bei GRUCZA 1974 unerwähnt bleibt, vorab ein Inventar der verschiedenen Konstruktionstypen erarbeitet wird, um dann die Binärbeschreibung in Form des binären Stammbaums folgen zu lassen. ${ }^{26}$

Einen multilateralen Sprachvergleich, der neben dem Deutschen und Polnischen das Niederländische mit einbezieht, hat KLIMASZEWSKA 1983 vorgestellt. ${ }^{27}$ Im Gegensatz zu den beiden Darstellungen der formalen Seite gilt ihr Augenmerk besonders dem semantischen Bereich. Getrennt nach Sprachen werden die Diminutive zunächst jeweils auf dem Hintergrund des vorhandenen Schriftums zur Diminutivproblematik vorgestellt. Verdienstvoll und aufschluß-

\footnotetext{
* Während die Dissertation lediglich in maschinenschriftlicher Fassung vorliegt, ist der Konfrontationsteil als Aufsatz zugänglich, vgl. DRAŻY ŃSKA-DEJA 1979.

${ }^{n}$ Diese Publikation beruht auf der auch in deutscher Sprache abgefaßten maschinenschriftlichen Doktorarbeit, vgl. KLIMASZEWSKA 1979.
} 
reich zugleich ist die Realisierung der wiederholt geäußerten, in der traditionellen Modifikationsforschung aber nicht beachteten Forderung nach Differenzierung der sprachlichen Ebenen, indem versucht wird, semantische Bedeutungen der Diminutive im Sprachsystem von semantisch-stilistischen Funktionen in der Sprachverwendung zu trennen, so daß die bis dahin andauernde, aus dieser Vermengung resultierende Kontroverse in der Beurteilung des diminutiven Wertes eine Vermittlung finden könnte. Der einzelsprachlichen Untersuchung folgt die wohl etwas zu knapp bemessene sprachvergleichende Darstellung, in der viele Details der Konvergenz und Divergenz nur angedeutet werden. Dennoch ist es gelungen, den Grad der typologischen Nähe der drei Sprachen hinsichtlich ihrer Gebrauchsfrequenz und expressiven Wertigkeit deutlich zu machen. Bei der Angabe des Materialkorpus für die pragmatische Untersuchung wird leider nur global von exzerpierten schriftlichen (Wörterbücher, Belletristik, Gebrauchsprosa) und mündlichen (vor allem Notizen nach Rundfunksendungen) Belegen gesprochen, bis auf die Nennung der Wörterbücher vermißt man ein Quellenverzeichnis mit genauem Nachweis.

Konfrontierende Arbeiten, die eine andere methodische Möglichkeit, den Übersetzungsvergleich, nutzen, sind bisher, abgesehen von vereinzelten, allgemeinen Hinweisen zur Übersetzungsproblematik bei Diminutiven, nur selten vorgelegt worden. Der Themenkreis Diminutiv und Übersetzung, der vom letztlich nicht immer zufriedenstellenden Nebeneinander der Sprachen abrückt und dafür das Miteinander, den direkten Vergleich, ermöglicht, ist nur für das Deutsche, das dem Französischen, Italienischen und Russischen gegenübergestellt wurde, betreten worden. Wiederum sind es Dissertationen, die sich bemühen, die genannte Problematik in den Kanon wissenschaftlicher Untersuchungen einzubeziehen. Die der romanistischen Forschung verpflichteten Züricher Studien von FISCHER $1962^{28}$ und PELLEGRINI 1977 konnten beide mit dem Exzerpieren von Übersetzungsmöglichkeiten aus Werken der schönen Literatur zunächst Erkenntnisse zur unterschiedlichen Nutzung dieser sprachlichen Mittel im Deutschen sowie im Französischen bzw. Italienischen vermitteln. Daraus konnten dann weitere Beobachtungen über das Funktionieren der Diminutive in verschiedenen Kommunikationssituationen in den jeweiligen Sprachen abgeleitet werden, die die Diminutivforschung um neue Aspekte bereichert haben. Während FISCHER 1962 einen einzigen deutschen Ausgangstext mit unterschiedlichen französischen Übersetzungen konfrontiert, arbeitet PELLEGRINI 1977 mit

2 Die wichtigsten Ergebnisse sind in einem Aufsatz wiederholt worden, vgl. FISCHER 1963. 
vier deutschen und drei italienischen Prosatexten bilateral.

Die Methode des Übersetzungsvergleichs, allerdings ohne systematische Auswertung eines geschlossenen Textkorpus, sondern nur anhand ausgewählter Beispiele, macht sich auch die Jenaer maschinenschriftliche Dissertation von TENNERT $1982^{29}$ zu eigen, die beachtenswerte Ansätze zur Analyse des Diminutivs im Russischen bietet. Hier geht es vornehmlich um die Erarbeitung breit einsetzbarer Wiedergabemuster bei der Schaffung äquivalenter Texte, wobei unilateral - das Russische als Ausgangs-, das Deutsche als Zielsprache - verfahren wird. Als Teilbereich finden die Diminutive auch Berücksichtigung in der Leipziger Doktorarbeit von ANDRJUSCHICHINA 1968 ${ }^{30}$. Im Rahmen der gegenüberstellenden Untersuchung von Personenbezeichnungen (im engeren Sinne nomina agentis) werden Wiedergabemöglichkeiten russischer Diminutivderivate, gebildet mit Suffixen der subjektiven Einschätzung, mit deutschen Sprachmitteln erstellt. Als Materialbasis dienen Beispiele aus Wörterbüchern, aus Film, Funk, Fernsehen und Presse, vor allem aber aus Übersetzungen russischer belletristischer Literatur des 19. und 20.Jh.s ins Deutsche; insgesamt werden über 110 (!) Werke angegeben. Auch wenn keinerlei Angaben zur Art der Auswertung gemacht werden, ist beim Umfang dieses Korpus anzunehmen, daß keine vollständige und systematische Analyse vorgenommen wurde.

Ähnlich konstruktive Beiträge, in denen die polnischen Diminutive thematisiert worden wären, konnten bis auf den knappen Artikel von KRAJCARZ $1981 \mathrm{a}$, der den Übertragungsmöglichkeiten niederländischer Diminutive ins Polnische nachgeht, nicht festgestellt werden.

Vor diesem Hintergrund bietet es sich durchaus an, auch die Diminutivforschung in der Slavistik aus neuer Perspektive, nämlich durch übersetzungsvergleichende Untersuchungen, zu ergänzen. ${ }^{31}$

20 Zusammengefaß̊ zugănglich in TENNERT 1984.

${ }^{30}$ Liegt auszugsweise als Aufsatz vor, vgl. ANDRJUSCHICHINA 1967.

31 Vgl. auch die Forderung des Romanisten ETTINGER 1986 (S.443), der sich für einen Fortgang der Studien auf slavistischer Seite einsetzt. 


\section{LINGUISTISCHE GRUNDLEGUNG}

\section{ZUR TheORIE DES DIMINUTIVS}

Bevor das Material ausgewählter Texte überhaupt erst erfaßt und später untersucht werden kann, ist es geboten, einige Überlegungen zum Begriff des Diminutivs vorauszuschicken. In die folgenden Ausführungen zum Charakter der Diminutive sowie zum gegenwärtigen Diminutivsystem des Polnischen und Deutschen sind neben der Auswertung vorhandener Ansätze auch Erkenntnisse aus der bereits vorgenommenen, aber aus methodischen Gründen erst im Abschnitt "Empirische Analyse" ausgewerteten Textlektüre eingegangen. So stellt das Folgende kein isoliertes, beliebig einsetzbares Textsegment dar, sondern bereitet unmittelbar auf die Auswertung vor, indem es wichtige bei der Textlektüre aufgeworfene Fragen zunächst theoretisch klärt. Dem Verständnis einer synchronisch angelegten Studie entsprechend bleiben Fakten zum Ursprung und historischen Entwicklungsprozeß $B$ der jeweiligen Diminutivformen unberücksichtigt.

\subsection{KONZEPTE DER ERFASSUNG DES DIMINUTIVPHÄNOMENS}

Der traditionelle und etablierte grammatische Fachterminus Deminutiv/ Diminutiv (vgl. lat.deminuere/diminuere: "durch Hinwegnahme eines Teils ein Ganzes vermindern, verringern, schmälern, schwächen" ${ }^{n}$ ) allein gibt keine erschöpfende Charakteristik dieser sprachlichen Erscheinung. Mit der Einengung auf nur den einen Teilaspekt, Diminutiv als Verkleinerungsform, wird weder seine Ambivalenz angedeutet, noch der damit verbundene komplexe Ausdrucksreichtum erfaßt. ${ }^{2}$ Entsprechend gehört es innerhalb der einschlägigen Literatur quasi zur Pflichtübung, diese terminologische Unangemessenheit anzuprangern und inhaltliche Präzisierung zu fordern. Mit der dann folgenden Begriffsbestimmung, die neben dem begrifflichen Element der Kleinheit besonders auf die

' GEORGES 1951. Sp.2027 und 2172. Die von der Forschung uneinheitlich gebrauchte Schreibweise De-Diminutiv ist laut THESAURUS 1909-34 (Sp.484), der die Lemmata de-' diminuere als Synonyme verzeichnet, ohne Unterschied zu verwenden.

2 Trotz seiner begrenzten Aussagekraft wird hier der herkömmliche Ausdruck Diminutiv beibehalten. 
Möglichkeit einer emotionalen Nuancierung durch Diminutive hinweist, ${ }^{3}$ wird der Versuch unternommen, die Unzulänglichkeit der Bezeichnung aufzuheben. Die Mehrdeutigkeit gab und gibt immer noch Anlaß zu Diskussionen, in denen die Frage der Dominanz der einen bzw. anderen Bedeutungskomponente erörtert wird. Ohne auf die im einzelnen geführte Kontroverse eingehen zu wollen, sind lediglich die im Laufe der Zeit herausgebildeten Positionen festzuhalten.

Während die einen hauptsächlich im affektiven Charakter die Wirkung der Diminutive sehen wollen, sprechen sich die anderen für das Merkmal der Kleinheit als Grundbedeutung aus.' Auch wenn Anstrengungen unternommen worden sind, eine Abgrenzung zu schaffen, so z.B. zwischen deminutywa wlaściwe und deminutywa emfatyczne ${ }^{6}$ oder zwischen echten, logischen Diminutiven, die der Ebene des begrifflichen Denkens angehören und übertragenen, psychologischen Diminutiven, die der Sphäre des Affektischen zuzurechnen sind, ${ }^{7}$ zeigt der Sprachgebrauch, daB eine solche Trennung streng theoretischer Art ist und ein exaktes Auseinanderhalten in jedem einzelnen Fall unmöglich er-

${ }^{3}$ Für diese vorherrschende Lehrmeinung kann stellvertretend GAWROŃSKI 1928 angeführt werden, der formuliert: "Jest ono [deminutivum - B.K.] co najmniej w rownej mierze wykladnikiem pierwiastk6w intelektualnych co emocjonalnych" (S.199); und ebenso GRIMM 1831 (S. 664): "Das diminutivum hat nicht nur den ausdruck des kleinen, geringen (weiwotৎ), sondern

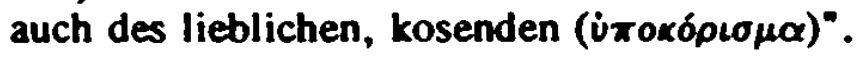

"Vgl. z.B. SIEBERER 1966, der von "Wortformen der Anteilnahme" spricht: "Das wesentliche Merkmal eines Deminutivs ist also seine Gefühlsbetontheit. Oft ist Kleinheit mitvorgestellt, hăufig bleibt sie ganz aus dem Spiel" (S.86) oder die Aussage GAWROŃSKIs 1928: "uczaciowe [elementy - B.K.] dominuja o wiele czesciej i silniej, nizby sie na podstawie naszych gramatyk wydawalo (...), one to sie przeważnie cisna na pierwszy plan" (S.203).

s Zu den Verfechtern dieser Auffassung sind u.a. DOROSZEWSKI 1928 (vgl. S.239) und vor allem die Mehrzahl der Verfasser von Grammatiken zu zăhlen. In enger Verbindung mit den verwickelten Beziehungen zwischen den Bedeutungsschattierungen steht die hăufig diskutierte diachronische Frage nach dem Ursprung der Diminutive und damit der Vorrangigkeit eines der beiden Faktoren in der sprachgeschichtlichen Entwicklung. Zur Auseinandersetzung im Deutschen vgl. den Forschungsbericht weiter oben S.23ff und 27f. Für die polnische Seite mogen BELIC 1901 und auch DOROSZEWSKI 1928 angeführt werden, der primăr die Bedeutung "pochodzenie czegos od czegos" für das Diminutivsuffix ansetzt, das erst nachtrăglich mit expressivem Wert gefült worden sei: "To, co od czegos pochodzi z natury rzeczy musi nie dorownywax 'pierwowzorowi', a wiec musi byc mniejsze, male, to zas co jest male (zwlaszcza dziecko), latwo usposobic może do nastroju pieszczotliwego" (S.239).

- Zu dieser Unterteilung, die hauptsăchlich auf formaler Grundlage basiert, vgl. SIATKOWSKA 1967. S.157.

' Siche dazu die Erorterung bei SEIDEL-SLOTTY 1947. S.25. 
scheint. ${ }^{8}$ Folge dieser engen genetischen Verflechtung ist eine Konfusion der terminologischen Verwendung innerhalb der Fachliteratur. ${ }^{9}$ Die Aufzählung nur weniger Termini mag zur Verdeutlichung definitorischer Uneinheitlichkeit genügen: um die Bezeichnung Diminutive gruppieren sich die Ausdrücke Verkleinerungen, Affektive, Suffixformen der subjektiven Wertung, Expressiva; zdrobnienia, spieszczenia, hipokorystyka, formacje ekspresywne bzw. affektische Verkleinerungsbildungen oder formacje deminutywno-hipokorystyczne als synthetische Begriffe u.v.a.m.

Ungeachtet dessen bleibt eine Interpretation im Rahmen moderner Wortbildungstheorien möglich: Diminution ist hier zu verstehen als formale und semantische Modifikation eines Grundwortes, die durch Anfügen bestimmter Wortbildungsmittel erreicht wird. ${ }^{10}$ Innerhalb der Wortbildung, im speziellen Fall der Derivation, die es ermöglicht, aus bereits existierenden sprachüblichen Einheiten immer neue lexikalische Beziehungen für neue Situationen zu schaffen, kann also mittels "quantifizierend-qualifizierender Suffixe" "l ein vorhandenes Basislexem semantisch abgewandelt werden. Das bedeutet, daß "zum Inhalt des gegebenen Ausgangsbegriffs ein ergänzendes, modifizierendes Merkmal hinzugefügt

\footnotetext{
- Näheres zu dieser besonders das Polnische betreffenden Problematik siehe die Ausfuhrungen im folgenden Kapitel, S.41ff.
}

- Kritisch äußert sich dazu u.a. GRZEGORCZYKOWA 1971 (S.123), die am Beispiel der Monographie von KREJA 1969 das ungeklărte Verhältnis zwischen den drei traditionell unterschiedenen Kategorien: Expressive, Diminutive, Augmentative beklagt, das sich an der Inkonsequenz der Bezeichnungsverwendung sogar zwischen Titel und folgendem Text erneut zeigte.

10 Während in der Polonistik bzw. Germanistik bei der Beschreibung diminutivischer Konstruktionen die Frage ihrer Zugehörigkeit zur Grammatik bzw. Lexik kaum Diskussionsgegenstand war - ihre Zuordnung zur Wortbildung wurde allgemein akzeptiert -, ist dieser Punkt in der russistischen Forschung häufig problematisiert worden. U.a. formale Kriterien, Kongruenz zwischen Substantiv und entsprechender Diminutivform, besonders die Genusparallelität, werden als Argument genutzt, Diminutivsuffixe neben den Flexionsendungen zum Paradigma eines Substantivs zu zählen, ihnen die Qualităt einer grammatischen Kategorie zuzusprechen. Vorwiegend semantische Faktoren (Bedeutung von Grundwort und Diminutiv stimmen nicht vőllig überein) unterstützen demgegenüber die These vom eigenständigen Lexem. Die Standpunkte im einzelnen stellt kurz POLTERAUER 1981 vor, vgl. S.10-14, bes. Anm.1 zur weiterführenden Literatur. Die Positionen früherer Zeit, in russischen Grammatiken des 18. und 19.Jh.s, erläutert u.a. BIEDERMANN 1981 (S.65-81).

"Vgl. dazu WANDRUSZKA 1967. S.161ff bzw. in überarbeiteter Fassung WANDRUSZKA 1969. S.85ff. 
wird", ${ }^{12}$ ohne daß sich an seiner Wortklassenzugehörigkeit etwas ändert. Die Derivate können durch die hinzukommenden spezifizierenden Komponenten zum Teil "('diminutiv') sachlich-begrifflicher", zum Teil "('meliorativ', 'pejorativ', 'expressiv') subjektiv-aspektueller oder emotional-wertender Natur" 13 sein.

Die Zuweisung der Diminutivbildungen zum Modifikationstyp der Ableitung geht auf M.Dokulil zurück, der entsprechend dem unterschiedlichen Charakter der Beziehung von onomasiologischer Basis und onomasiologischem Merkmal drei Grundtypen der Derivation ausgegliedert hat: den Mutationstyp, den Transpositionstyp und eben den Modifikationstyp, welchem außer den Diminutiven die onomasiologischen Kategorien der Augmentative, der Motion, der Tierjungen und der Kollektive zugerechnet werden. ${ }^{14}$ Diese Art der Klassifikation ist insofern gut geeignet, als die Diminution als Wortbildungstypus klar von anderen Verfahren abzugrenzen ist.

Im Gegensatz zu Dokulils formaler Anordnung in Zusammensetzung, Ableitung und gegebenenfalls Präfixbildung entwickelt etwa gleichzeitig (1965/66) E.Coseriu innerhalb seiner Sprachtheorie eine leistungsbezogene Einteilung der Wortbildung auf der Grundlage semantischer und syntaktischer Kriterien, wobei die Wortbildung in den größeren Rahmen der lexematischen Strukturen gestellt wird und innerhalb dieser den sekundären paradigmatischen Strukturen entspricht. Je nach grammatikalischer Determination des primären Begriffs, den sie implizieren, werden drei Typen der sekundären Strukturen unterschieden: Modifizierung bzw. Modifikation, Entwicklung und Derivation (später Komposition). ${ }^{15}$ Modifikation oder "homogene Derivation" wird als "komplementäre

${ }^{12}$ DOKULIL 1968. S.209.

i3 SEIDELMANN 1979. S.166.

14 Vgl. hierzu DOKULILs 1962 theoretische Ausführungen im Rahmen seiner Wortbildungslehre des Čechischen, S.29-49, hes. S.46f.

is Vgl. COSERIU 1973b, vor allem S.85-102. Auch GAUGER 1971 (S.60-134) differenziert in seiner Wortbildungstheorie unter Berücksichtigung inhaltlich-funktionaler Kriterien drei Leistungstypen des sog. "durchsichtigen Wortes": Ausgriff, Verschiebung und Variation (dazu s. unten S.37ff); hierbei ist das Verfahren der Variation mit der Diminutivbildung als dem bedeutsamsten Vertreter mit der oben genannten Modifikation gleichzusetzen. In Anlehnung an Dokulil und von einem funktionalen Wechselverhältnis zwischen Ausdrucks- und Inhaltsseite in der Wortbildung ausgehend, schlăgt auch SEIDELMANN 1979 eine Dreigliederung vor: semantische Wortbildung oder Modifikation, syntaktische Wortbildung oder Transposition und semantosyntaktische Wortbildung oder Mutation, vgl. S.161-172. Bei der Bestandsaufnahme der Substantive der deutschen Gegenwartssprache gliedert H.Wellmann die Ableitung zum einen nach Mustern der semantischen Modifikation (Kap.3) und zum anderen nach Mustern der semantischen Transposition (Kap.4-9), vgl. DEUTSCHE WORTBILDUNG 1975. Auch 
Determination eines ganzen Lexems ${ }^{n 16}$ verstanden, die keine spezifische Satzfunktion des modifizierten primären Begriffs impliziert; vielmehr handelt es sich hierbei um eine Quantifizierung (Diminution, Augmentation), Wiederholung oder Qualifizierung eines primären Wortes. ${ }^{17}$ Richtungweisend für die Diminutivproblematik ist aber seine Forderung, auch im Bereich der Modifikationsforschung die bei einer Untersuchung der funktionellen Sprache wichtige Differenzierung der sprachlichen Ebenen in System, Norm und Rede zu beachten. ${ }^{18}$ Auf der abstrakten Ebene des Systems - das als "Summe von distinktiven Funktionen (Oppositionsstrukturen) ${ }^{n 19}$ betrachtet wird und die Gesamtheit der möglichen Realisierungen enthält - bedeutet Diminution die Veränderung eines Lexems nach der Formel "Lexem - $x^{n},{ }^{20}$ d.h. eine "objektive Minderung des Gemeinten"21. Hier beziehen sich die Diminutivderivate auf - durch die jeweilige Basis genannte - quantitativ reduzierte Objekte der Wirklichkeit, sie erhalten die denotative Bedeutung klein. Im Bereich der Sprachverwendung muß dann zwischen Norm und Rede unterschieden werden: Während die Norm alle vom System vorgegebenen usuellen und traditionellen Realisierungen einer Sprachgemeinschaft umfaßt, repräsentiert die Rede als "konkrete Realisierung der Sprachtechnik"22 den individuellen Sprachgebrauch mit all seinen okkasionellen Varianten, die kontextuell oder auch situationell determiniert sein können. Abweichungen von diesem Prinzip, weitere Differenzierungen wie Meliorative, Pejorative u.a. können erst an dieser Stelle wirksam werden.

die polnische Wortbildungslehre verfährt in ähnlicher Weise, indem entsprechend der Formansfunktion drei verschiedene Derivatklassen aufgestellt werden: derywaty transpozycyjne, derywaty mutacyjne und derywaty modyfikacyjne, vgl. GRZEGORCZYKOWA 1984. S.27f; eine noch weiter differenzierte Typisierung geben R.Grzegorczykowa und J.Puzynina in ihren Wortbildungsbeitrăgen der GRAMATYKA 1984, vgl. S.319-321.

${ }^{16}$ COSERIU 1973a. S.52.

$17 \mathrm{Vgl}$. insbesondere die Erörterungen in COSERIU 1971b, aber auch das Kap.4.2.1. "Modifizierung" in COSERIU 1973b. S.90-92.

is. dazu den Beitrag von COSERIU 1971 a.

19 COSERIU 1973a. S.40.

20 ETTINGER 1974, der in seiner Studie, von Coserius Wortbildungsmodell ausgehend, ein Teilgebiet innerhalb der Modifikation, die Quantifizierung der Substantive, für einige romanische Sprachen beschreibt, hat versucht, das Postulat der Dreiteilung auf den Bereich der Diminutiv- und Augmentativbildungen zu übertragen; vgl. vor allem S.20f.

${ }^{21}$ COSERIU 1970. S.110.

2 COSERIU 1973a. S.38. 
In ähnlicher Weise behandelt auch H.-M.Gauger, der in der Sicht der "bewußtseinseigenen Sprachuntersuchung"23 die Wortdurchsichtigkeit" thematisiert, das Problem einer Einordnung der Diminutivformen. Im Anschluß an Coseriu arbeitet er das Begriffspaar Derivationsprogramm und Norm heraus. ${ }^{25}$ Mit ersterem ist gemeint, daß eine mehr oder minder große Zahl von durchsichtigen Wörtern, die ein identisches Verhältnis zu ihren entsprechenden Grundwörtern aufweisen, eine derivationelle Reihe bilden kann. Die Entstehung eines solchen Affixprogramms wird durch vier Kriterien bestimmt: Form (bei Diminutiven: Suffixe), Inhalt, Wortklasse und eventuell Genus. Dieses derivationelle Programm als bloße "in der synchronisch gesehenen Sprache wirksame und greifbare bewußtseinsmäßige Anlage" ${ }^{m 26}$ ist zu unterscheiden von dem "in der Sprache faktisch Verwirklichten" ${ }^{\text {27}}$; vielfältige Restriktionen formaler und inhaltlicher Art schränken nämlich die Aktivität des Programms ein und lassen lediglich einen Teil dessen zur Durchführung gelangen. Dementsprechend werden auch die verschiedenen Programme der diminutiven Variation durch die Norm in ihrer Realisierung eingeengt.

Auf der Grundlage des oben Gesagten definiert Gauger den Sondertyp der variierenden Derivation wie folgt:

Das Diminutiv (...) mein[t] also zunăchst eine Kleine (...) Variante des vom Grundwort gemeinten Dings. Dies muB, wenn man die so lebhafte Reaktion des Sprachbewußtseins auf diese Bildung als das entscheidende Kriterium in Rechnung stellt, als die Grundintention, der Kern dieser Bildung betrachtet werden (...) So gesehen erscheinen die Verhăltnisse einfach und klar. In der konkreten Außerung jedoch sind sie oft flüchtig und verwickelt. ${ }^{2}$

${ }^{23}$ GAUGER 1971. S.9.

24 In seinem Beitrag zur Wortbildungslehre, exemplifiziert am Französischen und Spanischen, geht er vom Ansatz des durchsichtigen Wortes aus, das im Gegensatz zum selbstăndigen undurchsichrigen Wort durch seine formal-inhaltliche Abhăngigkeit von einem anderen, seinem jeweiligen Grundwort, gekennzeichnet ist. Diese wird durch das Schema z.B. "le pommier $\Leftrightarrow$ la pomme)" verdeutlicht, wobei die Pfeilrichtung, die interpretiert wird als "lehnt sich bewuBtseinsmaßBig an" oder "verweist im Bewußtsein auf" (GAUGER 1976. S.142), den ausschlaggebenden Unterschied zu der üblichen bewußtseinsfremden Darstellung "la pomme $\Rightarrow$ le pommier" ausmacht. Beide existieren im Bewußtsein nebeneinander, sind koprăsent, vgl. GAUGER 1971. S.12-15.

2s Vgl. dazu GAUGER 1971. S.45-59. Mit der Bezeichnung Programm greift er auf die von WANDRUSZKA 1969 konzipierten Betrachtungen zurūck.

\%AUGER 1976. S.148.

${ }^{n}$ GAUGER 1976. S.149.

20 GAUGER 1971. S.106. 
Die Bestimmung der Diminutive als variierende Bildungen spiegelt ihren Charakter trefflich wider, allein schon die terminologische Parallele zur Musik: Thema $=$ Grundwort : Variation = Diminutiv ist überzeugend. ${ }^{29}$ Ihre spezifischen Kennzeichen werden besonders durch die Abgrenzung gegenüber dem ausgreifenden und verschiebenden Leistungstyp offengelegt. ${ }^{30}$ Dreierlei ist hervorzuheben: Von der Verschiebung unterscheidet die Diminutive der fehlende Wortklassenwechsel. Zweitens ist, wie erwähnt, für alle durchsichtigen Formen ihre fundamentale Grundwort-Derivat-Opposition charakteristisch, ${ }^{31}$ doch kommt es im einzelnen auf das jeweilige Verhältnis an. Das durch eine "Konstituentenanalyse $^{\text {32 }}$ zerlegbare Morphemgefüge eines abgeleiteten Wortes weist zwei Glieder auf, das basisfähige Grundmorphem und ein wortbildendes Morphem, ${ }^{33}$ denen ein sog. semantisches Determinationsverhältnis zugrunde liegt. Während den Suffixen grundsätzlich ein allgemeiner, kategorieller Wert zugesprochen wird, d.h., daß sie als Determinatum die begriffliche Grundklasse sowie die grammatische Funktionsklasse der Neubildung festlegen und durch das spezifizierende Zusatzmerkmale einbringende Grundwort determiniert werden, ist die Lage bei diminutivischen Suffixableitungen genau umgekehrt: das Diminutivsuffix als Determinans "richtet sich auf den Inhalt" der Basis, das Determinatum, "es 'qualifiziert' den ihm logisch vorausgehenden Inhalt und ist demnach einem Adjektiv vergleichbar". ${ }^{34}$ Drittens, mit der Diminutive von der ausgreifenden Derivation trennenden eigenen Determinationsrichtung hängt eng eine

20 Vgl. GAUGER 1971. S.102; zur Bevorzugung des Ausdrucks variierend gegenüber modifizierend, quantifizierend-qualifizierend oder appreziativ (Ch.Bally) s. S.125, Anm.267.

× Zu den Erörterungen im einzelnen s. GAUGER 1971. S.118-134.

" Die Bedeutung dieser Beziehung betonte schon SEIDEL-SLOTTY 1947 (S.32): "Das Diminutiv steht in irgendeiner Art von Zugehörigkeit zum Grundwort, ist aber nicht das Grundwort selbst". Auf den Charakter des Nebeneinander weist auch HELTBERG 1964 (S.94) hin: "Sa to jednostki jezykowe, ktorych specjalny charakter polega na tym, ze moga one istnie jedynie jako formacje oboczne przy podstawowej formacji, neutralnej zarówno pod wzgledem rozmiaru. jak i emocjonalno-stylistycznym."

3 S. hierzu die Darlegung bei FLEISCHER 1974. S.49-51.

33 Zur Struktur von Neuwortkomplexen vgl. ERBEN 1983. S.27f.

34 GAUGER 1968. S.94. Am Beispiel zweier homophoner, in ihrer Funktion aber verschiedener, spanischer Suffixe -azo wird hier sehr anschaulich die unterschiedliche Richtung der Determination im abgeleiteten Wort demonstriert. Nebenbei sei darauf hingewiesen, daß die im entscheidenden Schaubild vorgestellten Begriffe Determinans, Determinatum in GAUGER 1971. S.119 irrtümlicherweise vertauscht wurden. Vgl. ferner BALLY 1950 (S.250, \$399), der schon früher auf diese Erscheinung aufmerksam gemacht hat. 
weitere, inhaltliche Beziehung zwischen Derivat und Basiswort zusammen. Während das variierende Wort, obwohl klar vom Grundwort geschieden, doch semantisch in seiner unmittelbaren Nähe bleibt, lediglich in einer besonderen Ausprägung zu seiner Vorlage erscheint, entfernt sich bei aller Ähnlichkeit die ausgreifende Bildung erheblich weiter vom durch das Grundwort Gemeinten, es schafft einen neuen Inhalt. Für dieses "spezifische Miteinander von Verharren und Veränderung" ${ }^{35}$, das teilweise sogar zur Austauschbarkeit von Grundwort und Derivat führen kann, ist der Begriff zentripetales Verhältnis im Gegensatz zu zentrifugal geprägt worden.

Davon ausgehend, daß deutliche Parallelen zwischen dem Bau komplexer lexikalischer Einheiten und synonymischen syntaktischen Konstruktionen bestehen, ${ }^{36}$ können auch Suffixdiminutive in eine semantisch "äquivalente" syntaktische Wortverbindung bzw. -gruppe als explizitere Ausdrucksmöglichkeit umgeformt werden. Der die Suffixe determinierende Charakter läßt diese bei derartiger Transposition "in erster Näherung als Stellvertretung für ein Attribut aus dem Umkreis des Adjektivs kłein beschreiben" ${ }^{37}$. Je nach Sprecherabsicht können auch Adjektive, die den expressiv-emotionalen Aspekt widerspiegeln, an die Stelle von klein treten. Damit stellt sich neben das Register der quantifizierend-qualifizierenden Suffixe das der adjektivischen bzw. adverbialen Determination, d.h. morphologische Einheiten geraten in Wettstreit mit in "äquivalenter" Funktion stehenden lexikalisch-syntaktischen Mitteln. Dieser Auffassung zufolge hat sich vor allem in der romanistischen Fachliteratur ${ }^{38}$ die Vorstellung zweier Diminuierungsverfahren herausgebildet: der synthetischen und analytischen Diminution. ${ }^{39}$ Umstritten bleibt allerdings die Frage der Gleichwertigkeit dieser

3s GAUGER 1971. S.125.

* Zum gegenseitigen Verhăltnis von Wortbildung und Syntax vgl. die Ausführungen von DOKULIL 1964.

${ }^{37}$ DEUTSCHE WORTBILDUNG 1975. S.127.

3* Diese Tatsache laßßt sich wohl mit der Sonderstellung des Französischen hinsichtlich der Lebendigkeit suffixaler Diminutive gegenüber den anderen romanischen Sprachen erklăren: den überreichen produktiven Suffixprogrammen des Spanischen, Italienischen und Portugiesischen steht ein durch die Norm erheblich reduziertes Potential entgegen; einen Teil seiner Aufgaben hat das Syntagma mit petit übernommen.

${ }^{\circ} \mathrm{Zu}$ dieser formalen Aufgliederung vgl. die Angaben in der ausführlichen und wertvollen, hauptsảchlich historisch ausgerichteten Arbeit von HASSELROT 1957 (S.171f). In einer jüngeren slavistischen Arbeit von KLIMASZEWSKA 1983 (S.12) ist auf diesen Ansatz zwar 
beiden formal ungleichen Bildeweisen bezūglich ihrer Leistung. Nicht selten wird den Verfahren gerade infolge der "Verschiedenheit des formalen Instruments" 40 unterschiedlich starke Ausdruckskraft zugesprochen:

Das im Sprachlichen oft zu beobachtende, über den bloß instrumentellen Charakter hinausgehende Eigengewicht der Form, ihre sich im Inhaltlichen auswirkende Eigenschwerknaf, ist auch hier in Rechnung zu stellen."

Inwieweit innerhalb einer Sprache durch Attribuierung mit klein Äquivalenz erreicht werden kann, bleibt hier dahingestellt, ${ }^{42}$ sicher ist lediglich, daß der praktizierte Ersatz suffixaler Formen durch analytische beim Vergleich zweier in ihren Gebrauchsgewohnheiten sich unterschiedlich verhaltenden Sprachen gerechtfertigt erscheint. Ob die beiden Verfahren dasselbe oder nur ähnliches leisten, sei der Beurteilung zu einem späteren Zeitpunkt überlassen.

Mit dieser mehr oder minder detaillierten Einführung in die Problematik dieser Spracherscheinung sind bereits Grundlinien für die folgenden Überlegungen und Fragen vorgezeichnet worden.

\begin{abstract}
verwiesen worden, ohne ihn aber vergleichend auszuarbeiten. Auch ihr Bezug (vgl. S.12) auf HASSELROT 1957 (S.306) betreffs der Einteilung in morphematische und syntagmatische Diminutive bleibt unklar und ist wohl auf eine fehlerhafte Übernahme der Aussagen in GAUGER 1971 (S.114, Anm. 247) zurückzufüren. Bereits SEIDEL-SLOTTY 1947 (S.31) hat wenn auch nicht in dieser expliziten Form - auf verschiedene Techniken der Diminutivbildung aufmerksam gemacht. Am Beispiel von Tasse unterscheidet sie die Modifizierung durch Suffixe: Täßchen, durch adjektivische Umschreibung: kleine Tasse sowie im Deutschen zusătzlich durch ein Kompositum: Mokkatasse; bei letzterem liegt allerdings schon eine semantische Spezialisierung vor.
\end{abstract}

¿ GaUger 1971. S.115.

"GAUGER 1971. S.115; seine Bewertung demonstriert er am Vergleich von span. -ito und franz. petit.

42 Entschieden gegen dieses Konzept wendet sich REITER 1988 (S.659-661). Die Kleinheitshypothese ablehnend sieht er das Prinzip der Diminutive allein in der Perspektivregulierung. Zur Begründung seiner Annahme diskutiert er anhand von fünf Erscheinungen den Unterschied von derivationellen Formen und Attribuierungen durch klein. Gewiß ist dies ein möglicher Ansatz, doch muß darauf hingewiesen werden, daß bei seiner Darstellung der analytischen Bildeweise das Attribut klein nicht die Variabilităt eines Suffixes zugesprochen bekommt. Die Bedeutung des Attributs wird von vornherein lediglich auf die reine Größenangabe reduziert, andere mögliche Bedeutungsnuancen werden außer acht gelassen. 
Es gilt nun, einen Blick auf die formalen, später die inhaltlichen Ausprägungen des Diminutivphänomens zu werfen, um im folgenden dessen vielfältige Leistungen korrekt beurteilen zu können. Die angedeuteten divergenten Verhältnisse in beiden Sprachen verlangen nach einer getrennten Darstellung der Diminuierungsmöglichkeiten.

Die polnische Sprache ist durch ein auffallend reich facettiertes morphologisches System zur Bildung von Diminutiven gekennzeichnet. Dieser wichtigsten Gruppe innerhalb der Modifikation steht ein mannigfaltiges Angebot an Suffixen zur Verfügung. Die terminologische Deutung des Begriffs Diminutiv und die damit verbundene Bestimmung, welche Bildungen, die von bereits in der Sprache existierenden Wörtern entstehen - überwiegend von Substantiven und Adjektiven sowie Personen- und Verwandtschaftsnamen, seltener von Verben, Pronomen und Numeralien -, unter ihn zu fassen sind, gestaltet sich im Polnischen weitaus problematischer als im Deutschen.

Während für einen Teil der Forschung die Beschäftigung mit Diminutivbildungen hauptsächlich in der bloßen Zusammenstellung der potentiellen Suffixe bestand ${ }^{1}$ und die unmittelbar naheliegende Trennung zwischen Hochsprache und Dialekt nicht vorgenommen wurde, ${ }^{2}$ bemühten sich andere Studien, von den zwei Bedeutungsschichten ausgehend, seit jeher um mögliche Differenzierungen und Klassifizierungen innerhalb dieses Formenreichtums. Die Eigenart dieser Suffixe, einerseits in vermindernder, andererseits in kosender Funktion aufzutreten, hat viele polnische Forscher dazu veranlaßt, zwei eigenständige Kategorien, das Diminutiv - verstanden als intellektuelle Kategorie, die über die geringere Größe des Objekts informiert, nicht selten mit zusätzlicher expressiver Bedeutung - und das Hypokoristikum - als emotionale Kategorie, die über positive bzw. negative Einstellungen des Sprechers informiert, meist mit jeweils eigenen

\footnotetext{
' Stellvertretend sei hier die Grammatik von DOROSZEWSKI 1963 (S. 316, \$209) genannt, wo unter dem Stichwort rzeczowniki zdrobniale eine bunte Vielfalt von Suffixen aufgezählt wird: -cio, -as, -s, -as, -is, -isia, -osia, -us, -usia, -un, -unio, -unia, -ul, -ulo, -ula, -ek, -aszek, -iszek, -uszek, -yszek, -ka, -efka, -ycaka, -ulka, -uszka, -ko, -aczko, -atko, eniko, ekko, -icako, -uszko, -ik(-yk), -uk, -cha.

${ }^{2}$ Diesen Mangel beklagt deutlich u.a. URBAŃCZYK 1945 (S.16): "czesto mowi sie o obfitosci polskich sufiksow deminutywnych, jednakze nawet w obszernych gramatykach brak informacji o geograficznym ograniczeniu niektórych sposrod nich".
} 
morphologischen Mitteln -, anzunehmen. ${ }^{3}$ Eine Auswahl der Positionen zum Trennungsversuch ist rasch skizziert: E.Stankiewicz erreicht eine Zweiteilung der Derivate mittels formaler Kriterien. Indem er, die lexikalische Bedeutung des motivierenden Substantivs nicht beachtend, lediglich die Struktur der Formantien heranzieht, unterscheidet er Formen mit quantitativen (einfachen) Suffixen (=Diminutive) und mit qualitativen (zusammengesetzten) Suffixen (=Hypokoristika). Neben diesem allzu schematischen, in der konkreten Umsetzung kaum haltbaren Vorschlag steht J.Kurylowiczs Ansatz, der sich für eine durch die Art der Derivationsbasis bedingte Trennung ausspricht: während Diminutive hauptsächlich durch Suffigierung appellativischer Lexeme entstehen, ist der anthroponymische Wortschatz Grundlage für die Bildung von Hypokoristika. Kuryłowicz unterstreicht dabei, daß beide Gruppen mit denselben Formantien gebildet werden. ${ }^{5}$ Unter Berücksichtigung formal-semantischer Aspekte hat sich auch S.Warchol in Anlehnung an den von J.Zima entworfenen Typ der expresivita inherentnt $t^{6}$ dafür entschieden, auf dieser Ebene formacje o charakterze deminutywnym und formacje o charakterze ekspresywno-hipokorystycznym mit jeweils eigenem Suffixregister voneinander abzugrenzen. ${ }^{7}$ In gleicher Weise hat auch $\mathbf{R}$. Grzegorczykowa nazwy deminutywne und nazwy ekspresywne als zwei Modifikationskategorien angeführt; formacje okreslajace istoty mtode $i$ mate bzw. nazwy istot mtodych werden bei beiden Forschern parallel dazu als eigenständige

3 Manifest wird diese Haltung u.a. in der ENCYKLOPEDIA 1978 (S.400 und 321), wo zdrobnienia und spieszczenia als getrennte Stichworte aufgenommen werden, sowie im SLOWNIK TERM.LIT. 1976 (S.71 und 154) mit der Unterscheidung von deminurivum und hipokoristikon.

- Vgl. dazu STANKIEWICZ 1954, bes. S.468.

'S. hierzu KURYLOWICZ 1956, bes. S.5 und 13.

- ZIMA 1961, der sich mit der Differenzierung expressiven Wortschatzes beschäftigt, arbeitet drei Haupttypen der Expressivität lexikalischer Mittel im Cechischen heraus: expresivita inherentnf, die fester Bestandteil der lexikalischen Bedeutung eines Zeichens ist, sie resultien allein aus der Wortbildungsstruktur, der Basis und dem Suffix (vgl. Kap.2); bei der expresivita adherentní kommt der Einfluß des äußeren Kontexts hinzu (vgl. Kap.3); und die expresivita kontextova verdankt ihre aktuelle Wirkung allein dem Kontext, der Situation, außerhalb dessen sind die Lexeme neutral und unmarkiert (vgl. Kap.4).

'Warchoł gehört zu denjenigen, die sich, durch viele Beiträge bezeugt, am intensivsten mit dieser Problematik befaßt haben; dabei blieb er nicht nur im Rahmen des Polnischen, expressive Bildungen auch anderer slavischer Sprachen wurden vergleichend herangezogen. Speziell zur Trennungsfrage vgl. WARCHOL 1974a, bes. S.75f. 
Gruppen herausgestellt. ${ }^{8}$

Diesen Vorschlägen stehen kritische Meinungen gegenüber, die infolge der wechselseitigen Abhängigkeit des intellektuellen und emotionalen Moments auf Schwierigkeiten einer derartigen formalen und semantischen Abgrenzung hinweisen. ' Sie betonen, daB es sich hierbei eigentlich nur um eine rein theoretisch haltbare Zweiteilung handelt, die in der Praxis angesichts eines lebendigen Prozesses des fortwährenden Sich-Durchdringens aufgehoben wird. Diesem Hinweis folgend ging man in einigen Beiträgen auf Kompromisse ein: neben den "reinen" Diminutiven und "reinen" Hypokoristika nahm man eine dritte, eine Übergangsgruppe mit diminutivisch-hypokoristischen Derivaten an, die beide Komponenten beinhalten soll. ${ }^{10}$ Zum anderen wurde mit der Einsicht in die Unzuverlässigkeit des Bedeutungskriteriums nur eine breite, nicht weiter untergliederte Klasse der sog. formacje deminutywno-hipokorystyczne anempfohlen."

Berücksichtigt man E.Coserius Forderung nach der Dreiteilung in System, Norm und Rede, so bietet sich eine Lösungsmöglichkeit an, die der Problematik wohl am ehesten gerecht wird. Dem Beispiel S.Ettingers folgend ${ }^{12}$ hat Z.Klimaszewska anhand ihrer pragmatischen Analyse folgende Explikation vorgenommen. Sie geht davon aus, daß sich auf der abstrakten Ebene des Sprachsystems zwei deutlich voneinander zu differenzierende Suffixarten bestimmen lassen: zum

- Vgl. daZu GRZEGORCZYKOWA 1984. S.53-57.

- Gegen eine scharfe Grenzziehung sprechen sich vor allem GAWROŃSKI 1928 (S.200 passim), HELTBERG 1964 (S.96), BRODOWSKA-HONOWSKA 1967 (S.13) sowie SIATKOWSKA 1967 (S.157) aus.

10 Vgl. z.B. die Darstellung von HELTBERG 1964, bes. S.95f sowie die von KREJA 1969. S.15-18.

" BARTMIŃSKI 1973 (S.139) prăgte diesen synthetischen Begriff, der u.a. auch von ADAMISZYN 1977 übernommen wurde. Ein weiterer Klărungsversuch kommt von S.Grabias, der im Rahmen seiner Erlăuterungen zum Phănomen der Expressivităt in der Sprache auch auf diminutivische Formen eingeht. Indem er nicht von der lexikalischen Bedeutung ausgeht, sondern der morphologischen Ebene eines Wortbildungsprodukts, das in diesem Falle zum einen durch den Wert des angefügten Suffixes, zum anderen durch den des motivierenden Grundwortes (welches entweder stilistisch neutral oder schon selbst markiert sein kann) konstituiert wird, kommt er zu dem SchluB: "Przeciwstawienie deminutywow hipokorystykom stanowi w gruncie rzeczy opozycje wartosci znaczeniowej wnoszonej przez formanty (deminutywne) do wartosci calych konstrukcji slowotworczych. Emocjonalnost jest zjawiskiem znacznie szerszym niż deminutywnosc." Vgl. dazu GRABIAS 1978, bes. S.92 sowie seine monographische Darstellung GRABIAS 1981, dort das Kap. "Ekspresywnosc formantow", S.41-76; Zitat ebd. S.66.

${ }^{12} \mathrm{Vgl}$. dazu S.36, Anm.20 dieser Arbeit. 
einen Suffixe der Verkleinerung, die eine rein quantitative Verminderung des Objekts anzeigen und die Derivate mit dem semantischen Merkmal klein versehen; sie verleihen diesen zusätzliche denotative Bedeutung, die Diminutive beziehen sich somit auf eine kleinere Variante des durch das Grundwort bezeichneten Denotats. Zum anderen führt sie Suffixe der Expressivität auf, die keine Modifikation im Denotatsbezug hervorrufen, sondern sich auf dasselbe Denotat wie das Basislexem beziehen; ihre Leistung besteht allein darin, die emotionale Haltung des Sprechers zum gegebenen Sachverhalt zum Ausdruck zu bringen. ${ }^{13}$ Diese Gesetzmäßigkeit - ein der polnischen Sprache eigenes Charakteristikum, das innerhalb des deutschen Sprachsystems keine Entsprechung findet - läßt sich jedoch nicht, wie bisher geschehen, mechanisch auf die Ebene der Sprachverwendung (sprachliche Norm, individuelle Rede) übertragen; hier wird die Zweiteilung teilweise egalisiert, und es ergibt sich ein "verwickeltes Bild der Berührungspunkte, Übereinstimmungen oder Überschneidungen der beiden Arten von Suffixen in ihrer semantischen Leistung". ${ }^{14}$ Vor diesem Hintergrund ist dann lediglich von einer Kategorie auszugehen, der Diminuierung, die als kategorialer Oberbegriff in Opposition zur Augmentation zu verstehen ist. Diese verfügt dann über verschiedene formale Ausdrucksmittel und erfüllt auf der Sprachgebrauchsebene unterschiedliche semantisch-stilistische Funktionen, so daß die Bezeichnungen Diminutiv und Hypokoristikum ihm unterzuordnen sind. ${ }^{\text {is }}$

Nun soll ein kurzer Blick auf die zur Disposition stehenden morphologischwortbildenden Mittel geworfen werden. Auf der Grundlage des Dargelegten bietet sich im speziellen folgendes Bild innerhalb des polnischen Diminutivsystems: Zu den wichtigsten Vertretern der Verkleinerungssuffixe sind die Formantien mit -k- zu zählen: -ek/-ik (-yk) (zegarek, Wojtek/ bucik, J6zik, krzyżyk) für Maskulina, -ka (szafka, Tereska) für Feminina, -ko (drzewko) für Neutra. ${ }^{16}$ Zweierlei ist als Besonderheit gegenüber den deutschen Diminutiven hervorzuheben: das genusspezifische Auftreten der Suffixe, d.h., daß sie analog

13 S. hierzu KLIMASZEWSKA 1983. S.60f und S.78f.

14 KLIMASZEWSKA 1983. S.83.

is Vgl. KLIMASZEWSKA 1983, u.a. S.80.

${ }^{16} \mathrm{Vgl}$. GRZEGORCZYKOWA 1984. S.53 und GRAMATYKA 1984. S.367. Zur Genese der verschiedenen Suffixe und der Herausbildung der heute produktiven Mittel kann auf die Aufsătze von BELIĆ 1901/1904 sowie auf die Monographie von WARCHOL 1984 (S.156-195, 316-319), die neben den Substantiven auch die anderen Wortarten berücksichtigen, verwiesen werden. 
zum kongruenten Verhalten der Adjektive sich dem grammatischen Geschlecht des vorangehenden Grundwortstammes angleichen ${ }^{17}$, sowie deren polyfunktionaler Charakter. Sie dienen nicht nur der Bildung weiterer Derivate innerhalb der Modifikation, wie z.B. zur Movierung (student - studentka), große Verwendung finden sie auch bei der Bildung von Mutationsderivaten, bei nomina instrumenti (przykrywka), nomina patientis (wkladka, odcinek), nomina attributiva (Smieszek, zaglówka) u.a., sowie bei der Bildung von Transpositionsderivaten, z.B. nomina actionis (zarobek, przer6bka). ${ }^{18}$

Darüberhinaus machen sich im Gebrauch der -k-Diminutivsuffixe Restriktionen gleichermaßen morphologisch-semantischer wie phonetischer Art geltend. Von den meisten Abstrakta, z.B. von suffigierten Wortformen, (pracowitosk, nauczycielstwo), von Substantiven, die absolute Begriffe bezeichnen, (poczatek, szczescie), von Substantiven, die Negatives zum Inhalt haben, (morderca, paraliż), auch von Eigennamen u.v.a.m., ist eine Diminutivderivation auf der Ebene der Norm nicht möglich, als Okkasionalismen sind solche Wörter aber durchaus bildbar. ${ }^{19}$ Innersprachlich bestimmte Beschränkungen gibt es hauptsächlich für die Bildung maskuliner Diminutive. Die Varianz in der Verwendung von -ek, -ik(-yk) läßt wohl keine eindeutige Distribution erkennen, dennoch sind gewisse Regularitätstendenzen auszumachen. Maßgeblich für die Wahl einer dieser positionellen Varianten ist zumeist die Struktur des Wortstamms des motivierenden Substantivs, genauer das im Auslaut stehende Phonem oder die Phonemfolge. ${ }^{20}$ Im allgemeinen ist ihre Distribution komplementärer Natur, doch besteht gelegentlich die Möglichkeit, daß an ein und dieselbe Basis beide Formantien ek und -ik treten, vgl. kąt - kątek/kącik. Während hier beide Formen gleichberechtigt sind, kann es in anderen Fällen zu semantischen Diffe-

17 S. z.B. HELTBERG 1964. S.100f.

I* WARCHOL 1974a (S.77) schreibt: "Na gruncie wspołczesnego jezyka polskiego taka na ogó uniwersalna funkcje slowotworcza maja formanty $z$ sufiksalnym $-k-"$. Vgl. auch DOROSZEWSKI 1928 (S.9-57 und 58-71), der in seinen Darlegungen den Suffixen -ek und -ka jeweils sieben verschiedene Funktionen zuerkennt. Fortgesetzt worden ist diese Arbeit von SCIEBORA 1983, die den aktuellen Wortschatz anhand des Stownik jezyka polskiego (red. W.Doroszewski) klassifiziert hat.

19 Genaueres dazu in KLIMASZEWSKA 1983. S.73-76.

20 Eine ausführliche Analyse der herrschenden Regularitäten verdankt die Forschung der Untersuchung von KREJA 1969 (vgl. im einzelnen S.24-69 und 71-84). Die Ergebnisse der Distribution der beiden Affixe ek und -ik sind übersichtlich in tabellarischer Form in der Dissertation von DRAŻYŃSKA $1974 / 75$ (S.44-51) zusammengestellt worden. Derivationsregeln für Diminutivbildungen der polnischen Literatursprache für alle drei Genera versucht RUDOLPH 1983 (S.35-53) aufzustellen. 
renzierungen kommen, vgl. st6́ - stolek (lexikalisierte Form, 'Hocker')/stolik (Diminutiv, 'kleiner Tisch') oder przedzial - przedzialek (lex. Form, 'Scheitel')/przedzialik (Dim., 'kleines Abteil'); das Diminutiv wird dann immer durch -ik ausgedrückt. Auch wenn das ek-Suffix zahlenmäßig ${ }^{21}$ in der Gegenwartssprache überwiegt, wird das -ik-Formans durch seine Dominanz hinsichtlich der potentiellen Positionen, in denen es erscheinen kann, als Hauptvertreter angesehen. Generell wird beobachtet, daß das Affix -ik im gegenwärtigen Polnisch immer weitere Verbreitung zuungunsten von ek findet. ${ }^{22}$

Darüberhinaus sind grundsätzlich bei der Bildung von Diminutiven zu beachten: die am Stammende des Grundwortes stattfindenden morphonologischen Alternationen, z.B. dach - daszek, kapelusz - kapelusik; droga - dróżka; okno - okienko, ${ }^{23}$ sowie die Möglichkeit, daß bei einigen Ableitungen die Basis gegenüber dem Stamm des motivierenden Substantivs um ein Affix erweitert bzw. reduziert wird, z.B. bei der sog. desintegrativ-suffixalen Derivation, wie sie u.a. bei den individuellen Bildungen des Typs kamień - kamyk ${ }^{24}$ vorliegt.

21 Vgl. die Angaben bei SIATKOWSKA 1967. S.160.

2 S. dazu KREJAs 1969 (S.69f) Fazit sowie WARCHOLs 1979 und WARCHOLs 1982 ergãnzende Beobachtungen zu dieser Rivalität, der das wachsende Übergewicht auf die Tatsache zurückführt, daß -ik vor allem bei Fremdwörtern mit hartem Stammauslaut gegenüber -ek dominieren kann. Gewisse Bedenken an dem dezidierten Übergewicht von -ik ăußert GRZEGORCZYKOWA 1971 (S.124). Genaue Zahlenangaben zur Produktivităt der übrigen Suffixe finden sich bei MÜLLER-OTT 1970a (für das Polnische vgl. S.575) und MÜLLER-OTT 1970 b (für das Poln. vgl. S.12-15), die ihre Untersuchung - auf die Substantivdiminutive mit dem Element -k- (auch in seinen palatalisierten Formen) beschränkt - für alle slavischen Sprachen durchgeführt hat.

2 Eine Besprechung des Einflusses der Suffixe -ik und der anderen -k- Formantien auf die vorausgehende Basis, bes. der qualitativen Änderung der Vokale und der vorangehenden Konsonanten, bietet KREJA 1969 (S.118-145); die Resultate sind wiederum von DRAZYŃSKA 1974/75 überblicksartig in einer Tabelle zusammengefaßi worden, vgl. S.56-70. In diesem Zusammenhang siehe auch die Aufsätze von BURDZANOWSKA 1978 und POHL 1980, die auf der Grundlage einer generativen Betrachtung versuchen, phonologische und morphologische Regeln für den Prozeß der Diminutivbildung im Polnischen aufzustellen. Schließlich ist noch auf die Arbeit von MICHALEWSKI 1984 hinzuweisen, der im Rahmen seiner Distributionscharakteristik der polnischen Suffixe hauptsächlich die morphonologischen Veränderungen der Derivate behandelt und dabei auch phonetische und morphologische Beschränkungen bei der Verbindungsfähigkeit einzelner Suffixe aufzeigt.

20 Vgl. dazu KREJA 1963. S.134f, er spricht von der sog. derywacja wymienna. Năheres zum Begriff desintegrative Derivation vgl. weiter unten S.50. 
Neben diesen strukturell gesehen einfachen Suffixen verfügt die Hoch- und Schriftsprache über zusammengesetzte bzw. erweiterte Suffixbildungen:2s eczek (noseczek, Janeczek), -iczek/-yczek (stoliczek, Kaziczek/wierszyczek); -eczka (gl6weczka, Aneczka); -eczko (słrweczko) ${ }^{26}$ sowie die eher nur sporadisch gebrauchten und teilweise dialektal gefärbten Formantien -uszek (dzbanuszek, paluszek), -iszek (braciszek), -yszek (kawałyszek), -aszek (mlodzieniaszek) ${ }^{27}$ bei den Maskulina; -uszka (paczuszka), -yczka (wieżyczka), -ulka (rzeczulka) bei den Feminina und -uszko (serduszko) für das Neutrum. ${ }^{28}$

Faßt man den Terminus Verkleinerungssuffix etwas weiter, so kann man auch diejenigen Formantien, die vorwiegend bei der Benennung junger und kleiner Lebewesen herangezogen werden, dazurechnen. ${ }^{29}$ Zunächst ist auf das in dieser Funktion früher äußerst produktive Formans e (krolicze) ${ }^{30}$ hinzuweisen, das im Laufe der Zeit eine semantische Umwertung erfuhr und damit seinen emotional-expressiven Charakter verlor. Diese Funktion übernahm dann das morphologisch sehr aktive, infolge der Kumulation von $-\mathrm{e}$ und -ko entstandene Suffix -ątko (kurczątko, dzieciątko). Seiner Fähigkeit entsprechend, sich an verschiedenartige Basismorpheme anzuknüpfen, blieb es nicht nur auf die Bil-

2s In diesem Sinne unterscheidet z.B. LASKOWSKI 1966 bei seiner Untersuchung der lachischen Dialekte: "a) formanty podstawowe, o prostej budowie (...) oraz b) formanty wtorne (...) zawierajace jako drugi (ostatni) czlon formantu deminutywny sufiks -ek, -ka lub -ko, w zależnosci od rodzaju gramatycznego wyrazu podstawowego" (S.82).

${ }^{20}$ Bei diesen Derivaten gilt es zu berücksichtigen, daß sie bereits sekundäre Bildungen sind, die durch erneute Suffigierung bereits diminuierter Formen entstanden sind, z. B. nos - nos + ek nos + ecz + ek. Es handelt sich hierbei nicht um selbständige, sondern um strukturell geteilte, zweigliedrige Formantien, POLTERAUER 1981 (S.28-33) nennt sie zusammengesetzte Suffixe 2. Grades und unterscheidet sie somit von den - im folgenden angeführten - zusammengesetzten Suffixen 1.Grades; vgl. dazu auch weiter unten S.SIf.

$27 \mathrm{Zu}$ den beiden letztgenannten Suffixen, auch für die beiden anderen Genera, siehe die Ausarbeitungen hinsichtlich Herkunft, Verbreitung und Produktivităt in der Hochsprache von KARPLUK 1968 und KARPLUK 1982.

2al. dazu die Zusammenstellungen bei SIATKOWSKA 1967. S.158, KLIMASZEWSKA 1983. S.60 und in der GRAMATYKA 1984. S.367. Da eine klare Trennung zwischen Suffixen der Hochsprache und der Mundarten nicht vorzunehmen ist, könnte die Anzahl noch erhöht werden, deshalb hier nur eine Auswahl. Siehe auch RUDOLPH 1983 (S.44f, 47f und 49f), die diese Formen unregelmäßige Deminutiva nennt.

20 Als eigenen, von den Diminutiven gesonderten Modifikationstyp ordnen diese Bildungen WARCHOL 1974a (S.75f) und GRZEGORCZYKOWA 1984 (S.57) ein, vgl. oben S.42f.

30 S. dazu HELTBERG 1964 (S.98-100) sowie die Arbeit von ZYCH 1981; ferner die ausführliche Untersuchung von DOBRZYŃSKI 1974, der u.a. zeigt, daß - neben der Bezeichnung von Tierjungen sich auch auf andere semantische Bereiche ausgedehnt hat. 
dung von Bezeichnungen in der semantischen Gruppe junger Lebewesen beschränkt und verselbständigte sich. Zur Steigerung der Ausdruckskraft gibt es dann noch die Möglichkeit, ein zusammengesetztes Suffix 2.Grades zu bilden: -qteczko (dzieciąteczko). ${ }^{31}$ Daneben, als Pendant zum e-Formans, entstand das, zunächst nur auf nordpolnische Dialekte begrenzte, maskuline Suffix -ak (kociak), auch hier mit der Doppelungsoption -aczek (kociaczek). ${ }^{32}$ Diese derivationelle Entwicklung kann dazu führen, daß manchmal eine Variantenreihung des Typs kocie - kociątko - kociak möglich wird. Schließlich bleibt noch das unproduktive Fremdsuffix etka (rozetka) zu erwähnen. ${ }^{33}$

Im Anschluß an die oben geäußerten Überlegungen sind den bisher aufgezählten Formantien nun die sog. Suffixe der Expressivität entgegenzusetzen. Die häufig mit einem palatalisierten Element, -\$-, $-\mathfrak{k -},-\mathbf{n}-$, -1-, gebildeten Derivationsmittel sind meist durch Regionalität und umgangssprachlichen Charakter gekennzeichnet, auch dabei ist zwischen einfachen und zusammengesetzten zu unterscheiden: -us (chlebus, Piotrus), $-\mathbf{s}$ (ptas, Antos), seltener -is/-ys (goląbis, Gabrys), -as (chloptas), ${ }^{34}$ dann eniek (kwiateniek), -ulek (mężulek) sowie -'o (dziadzio, J6zio), -'u (piesiu, Tadziu) ${ }^{35}$ und die kombinierten -cio (syncio, Stefcio), -sio (brzusio), -siu (Stasiu), -unio (ojczunio, Wacunio) für Maskulina; -usia (córusia, Ewusia), -sia (spódnisia, Malgosia), -isia/-ysia (glowisia, Marysia), -cia (żabcia, Jukcia), -’a (nózia, Ania) sowie -ula (ciotula, Basiula), -unia (raczunia, Elżunia), -uchna (dziewuchna, Liduchna), -enka (czapeńka, Zosieńka), -uleńka (babuleńka) und -ina/-yna (chacina/butelczyna), -inka/ -ynka (kobiecinka/drożynka) für die Feminina; -usio (jabłusio), -unio (serdunio), -uchno (pudehuchno), -eńko (miasteńko), -'o (l6zio) und andere weniger

"Zur Genese und Funktionsentwicklung der beiden Suffixe e und -atko vgl. WARCHOL 1974b, bes. S.156-161.

$32 \mathrm{Zu}$-ak siehe den Beitrag von TASZYCKI 1961.

33 Vgl. GRZEGORCZYKOWA/PUZYNINA 1979. S.142.

34 Anders deuten GRZEGORCZYKOWA/PUZYNINA 1979 (S.290) dieses Beispiel: sie rechnen das - $t$ - dem Suffix und nicht dem erweiterten Wortstamm zu.

3s Genetisch gesehen ist es die Endung des Vokativ Sing.mask., die hier in der Funktion des Nominativs steht; recht ausführlich bemüht sich darum ZALESKI 1959. 
produktive für das Neutrum. ${ }^{36}$ Charakteristisch ist für die gesamte Gruppe die Übernahme von Suffixen anderer Genera, so daß z.B. Formantien des Femininums für maskuline Substantive, -'a (Bozia), oder umgekehrt solche des Maskulinums für feminine Substantive, -us, -ulek (corus, Basiulek), eingesetzt werden können. Diese Art des Wechsels des grammatischen Geschlechts wird im allgemeinen als beliebtes Mittel zur Steigerung bzw. Erneuerung verlorengegangener Emotionalität eingesetzt. ${ }^{37}$

Die vom System vorgagebene Art und Weise der Verteilung der oben zusammengestellten Diminutivformantien, der Umstand, daß die Verkleinerungssuffixe hauptsächlich an nomina appellativa, die expressiven an nomina propria, hier vor allem Vornamen ${ }^{38}$ und Verwandtschaftsbezeichnungen, treten, erfährt auf der Ebene der Rede - wie die Beispiele zeigen - oft eine Umkehrung der Positionen. ${ }^{39}$ Wie W.Dobrzyŕski nachzuweisen versucht hat, sind die appellativischen Hypokoristika nicht nur sporadisch vorkommende und obendrein allein mundartlich begrenzte Bildungen, vielmehr erweisen sie sich teilweise als höchst

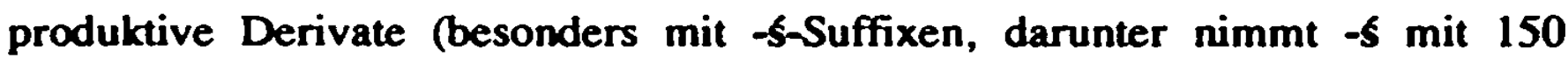
Belegen den führenden Platz ein, gefolgt von -us und -sia; ferner mit -cia sowie die aus dem Südwesten Polens stammenden, durch Kontakt mit ostslavischen Dialekten entstandenen Suffixe mit dem -n-Element, -eniek, eńka, eńko ${ }^{40}$ ), die nicht selten bereits Eingang in den Wortschatz der Standardsprache gefunden

* S. auch die Beispiele bei SIATKOWSKA 1967. S.167, KLIMASZEWSKA 1983. S.60f, DOBRZYŃSKI 1988 (in alphabetischer Reihenfolge) sowie in der GRAMATYKA 1984. S.370, hier werden die diminutivisch-expressiven Affixe zusammen in einer Gruppe mit allen übrigen expressiven Suffixen behandelt. CYRAN 1977 reiht die hier aufgezăhiten - aber auch die adjektivischen Diminutivsuffixe - in sein zweites Kapitel "Tendencje slowotworcze w gwarach rótniace je od jezyka ogólnopolskiego" ein, damit versteht er sie als produktive Suffixe verschiedener Dialekte, vgl. bes. S.49-62.

${ }^{7}$ Mit auf diese Art gebildeten Hypokoristika beschäfigt sich vor allem OBREBSKA 1929; vgl. auch HELTBERG 1964. S.100f.

36 Anhand der populärsten dreizehn weiblichen und männlichen Vornamen der Nachkriegszeit hat GLADYSZ 1968 versucht, Tendenzen bei der suffixalen Bildung von Kosenamen im gegenwărtigen Polnisch aufzuzeigen und ihre Verbreitung innerhalb des Sprachgebiets festzastellen.

30 Über den gegenseitigen Einfluß appellativer und proprialer Lexik berichten SMOCZYŃSKI 1962 und SZYMCZAK 1969.

* Vgl. dazu die Anmerkungen von URBAŃCZYK 1945. S.16f. 
haben. ${ }^{41}$ Natürlich erstreckt sich ihr Verwendungsbereich vornehmlich auf die Umgangssprache, besonders die Sprache im intim-familiären Kreis, im Gespräch mit Kindern und über sie sowie in zunehmendem Maße in der Schüler-, Studenten-, überhaupt der Jugendsprache, z.B. biolcia ('biologia'), francio ('jezyk francuski'), dyrcio ('dyrektor'), interek ('internat'), legitka ('legitymacja'). ${ }^{42}$ Sicherlich haben diese Lexeme nicht die spontane, direkte kosende Wirkung zum Ziel, ihre Expressivität liegt vielmehr im Ausdruck von Humor, Gleichgültigkeit und sogar Geringschätzung gegenüber geltenden Konventionen.

Die formale Seite betreffend gilt es im weiteren auf das uneinheitliche Verhalten der Basis des motivierenden Substantivs im Derivationsprozeß hinzuweisen. Wie schon bei den Verkleinerungssuffixen erwähnt, spielt auch bei der Bildung von Hypokoristika, nur in entschieden größerem Maße, neben der nur selten vorkommenden reinen Suffixableitung die sog. derywacja dezintegralna ${ }^{43}$ eine bedeutende Rolle. Gemeint ist die ursprünglich den Personennamen eigene Derivationsweise, die sich darin äußert, daß die Derivate mittels Suffigierung eines um ein bestimmtes Segment verringerten Stammes des Ausgangswortes geformt werden. ${ }^{4}$ Diese Kürzung geschieht meist mechanisch und kann verschiedene Teile der Basis erfassen. Als häufigstes Verfahren gilt die Reduktion des letzten Elements des vollen Namens oder eines appellativischen Ausdrucks, vgl. Barbara - Ba-sia, kolega - kole-s, bufetowa - buf-cia; seltener des ersten oder mittleren Segments, z.B. Jakub - Kub-us, Anastazja - Nast-eńka, korepetycja - rep-cia; Ausnahmen bilden Formen, die aus verschiedenen Teilen des

"Im Gegensatz zu WARCHOLs 1974a (S.79) Behauptung: "mamy tu do czynienia z tworem użytym doraznie, najczesciej jednorazowo i zapewne w celach o charakterze stylistycznym", konnte DOBRZYŃSKI 1988, der über 1000 hypokoristische Appellativa, mit 60 verschiedenen Formantien gebildet, aus unterschiedlichen Quellen zusammengetragen hat, feststellen, daß über 200 dieser Bildungen bereits Aufnahme im dialektale Bildungen prinzipiell ausschließenden elfbändigen Slownik jezyka polskiego (red. W.Doroszewski) erfahren haben, vgl. S.l15 sowie die Erwăhnungen bei den einzelnen Beispielen.

$42 \mathrm{Zu}$ den Beispielen vgl. DOBRZYŃSKI 1988. S.17, 19, 22, 48.

${ }^{3}$ DOBRZYŃSKI 1988 (S.5) führt in seiner Untersuchung diesen Terminus auf H.Ulaszyn zurück, der im Zusammenhang mit durch Wortstammkürzung entstandenen Ableitungen von pochodniki dezintegralne sprach. Vgl. dazu auch KREJAs 1976 (S.41-45) Betrachtung über die sog. derywacja wsteczna in hypokoristischer Funktion.

4 In KURYLOWICZs 1968 (S.176) auf Personennamen angewandter Terminologie wird dieser Wortbildungsvorgang mit dem Begriff mutylacja (= "niemorfologiczne okaleczenie imienia") belegt. 
Stammes kombiniert werden, vgl. Konstanty - Kost-us, Leokadia - Lodzi-a. ${ }^{45}$ Schließlich ist noch die in der Alltagssprache recht verbreitete Möglichkeit der sog. derywacja alternacyjna ${ }^{46}$ zu nennen, eine Bildeweise, die auf dem Wechsel eines harten zum weichen Konsonanten im Stammauslaut beruht: ${ }^{47}$ R6ża R6́zia, nos - nosio, häufig in Verbindung mit Basisdesintegration, vgl. Henryk - Henio, ciot-ka - ciocia. $^{48}$

Typisch für das Polnische und in gleichem Maße für die anderen slavischen Sprachen ist die große Dynamik, die die Bindung diverser Diminutivformantien an ein Basislexem auszeichnet. Oft können an ein Grundwort kettenartig die verschiedensten Suffixkombinationen angehäuft werden, die in der Regel als Anzeiger unterschiedlicher Stufen von Expressivität dienen. Man spricht von einer Graduierung des diminutivischen Wertes, und zwar von Diminutiven 1. und 2. Grades als den häufigsten Formen.9 ${ }^{49}$ Letztere bzw. solche höheren Grades sind immer Ableitungen, deren motivierendes Substantiv bereits ein Diminutivderivat darstellt; ${ }^{50}$ in vielen Fällen ist eine erneute Suffigierung infolge des Verlusts ihres ursprünglichen diminutivischen Charakters nötig, vgl. skrzynia - skrzyn-ka - skrzyn-ecz-ka, tato - tat-us - tat-us-ek, Maria - Marysia - Mar-ys-ka - Mar-ysi-en-ka u.a. Zu beachten ist dabei, daß allein bei Konkreta zunächst eine reale Verkleinerung, dann expressive Abstufungen erfolgen können, bei Abstrakta lediglich qualitative Nuancen möglich sind, vgl. minuta - minut-ka - minut-ecz-ka. Die über zwei Glieder hinausgehende

as Vgl. die Ausarbeitung von WROBBL 1973, der recht ausführlich auf die verschiedenen Umformungsmöglichkeiten der Anthroponyme eingeht und versucht, gewisse Wortbildungsgesetzmäßigkeiten beim Aufbau der Hypokoristika festzustellen: innerhalb des maskulinen und femininen Paradigmas, in Abhängigkeit des Basismorphems, stellt er jeweils fünf verschiedene Untertypen heraus, s. S.39-46. Mit grundsătzlich möglichen Derivationsverfahren bei Eigennamen beschäftigt sich die Arbeit von KOSYL 1981, vgl. S.69-71.

${ }^{46} \mathrm{Vgl}$. KREJA 1976. S.43f.

${ }^{47}$ Zur Genese der weichen Konsonanten $\$, \varepsilon, \varepsilon$ vgl. den Beitrag von URBAŃCZYK 1945. S. $17 f$.

4 Eine andere Interpretation des Typs nosio - nos-ek, piesio - pies-ek, nózia - nóz-ka, drozia - dróz-ka schlagen KREJA 1976 (S.41t) und DOBRZYŃSKI 1988 (S.54 u.11f) vor; sie werden verstanden als durch Abtrennung von ek bzw. -ka gebildete Ableitungen mit den Suffixen -'o bzw. -'a.

* Den Begriff Diminuierungsgrad, der von S.Karcevskij in die Forschung eingefürt wurde, hat HELTBERG 1964 (S.94) übernommen und weiterentwickelt, vgl. auch LASKOWSKI 1966. S.83, Anm.14.

so Vgl. auch S.47, Anm.26. 
Suffixkumulation ist fast ausschließlich den Namenhypokoristika und den mit ihnen verbundenen Verwandtschaftsnamen, vor allem in vertrauten, familiären Gesprächssituationen, oder dem Volksliedgut ${ }^{51}$ vorbehalten. Sowohl die Vielfalt als auch die lebhafte Dynamik dieser Bildungen im Sprachgebrauch ist dem deutschen System fremd, strukturelle Äquivalenz läßt sich nicht finden.

Zeigten sich quantitatives Überangebot und eben gezeigte Suffixanhäufung als besondere Kennzeichen der polnischen Formantien gegenüber den deutschen, so ergeben generative Strukturanalysen, daß das Polnische über weniger Konstruktionstypen als das Deutsche verfügt: in Frage kommen nur die Wortbildungskonstruktionen $K(D) \Rightarrow S t+$ Suff (wlosek), $K(D) \Rightarrow D+$ Suff (chudzinka), seltener $K(Z) \Rightarrow Z+$ Suff (parowozik) und als Ausnahme $K(Z R B) \Rightarrow W G+$ Suff (niezapominajka), während im Deutschen besonders die reich ausgebauten Zusammensetzungen die Typenzahl vermehren. ${ }^{52}$ Bezüglich des Basisaufbaus weisen die Diminutivbildungen ein recht differenziertes Bild auf, möglich sind Diminutive mit substantivischer Basis (wino - winko), mit adjektivischer (niewinny - niewiniątko), mit verbaler (wiązac - wiazka/wiazzecaka) sowie mit Numeralien (tysiąc - tysiączek) oder mit Verbalabstrakta als Basis (spanie spanko). ${ }^{33}$ Die Distanz zwischen Grundwort und abgeleitetem Wort kann recht groß werden, wenn sich ein von Alternation, Vokalschwund, Basisreduktion und Suffixanhäufung begleitetes komplexes Derivat entwickelt. ${ }^{\text {s4 }}$

$\mathrm{Zu}$ der bedeutendsten Gruppe der Substantivdiminutive treten - gegenüber dem Deutschen in ausgeprägterer Weise - Diminutivformen von Adjektiven, Adverbien und Pronomen, auch wenn dabei der Terminus Diminutiv explizit kaum noch begegnet. Durch Suffigierung, seltener durch Präfigierung, entstehen Modifikationsderivate, die verschiedene, ja sogar diametral entgegengesetzte Intensitätsgrade, zum einen Abschwächung, zum anderen Verstärkung der mit

si S. z.B. die Beispielsammlung von ADAMISZYN 1977. S.51-59 oder PLUTA 1967.

\$2 Vgl. dazu die Ergebnisse der konfrontativen Arbeit von DRAŻYŃSKA 1974/75 (S.109-111) sowie die tabellarische Gegenüberstellung der beiden Sprachen (S.124f), oder besser zugänglich in der Zusammenfassung der Arbeit, DRAŻYŃSKA-DEJA 1976 (S.21-23 und 29-31). Zu den

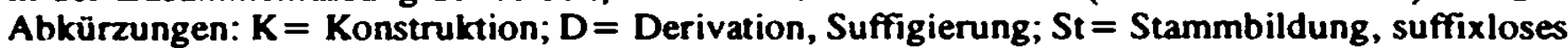
Simplex; Suff = Suffix; $Z=$ Zusammensetzung; $Z R B=$ "unfeste" Zusammenbildung, d.h. mit Wortgruppe als Kernglied; WG = Wortgruppe (vgl. DRAŻYŃSKA 1974/75. S.93 und 97 bzw. DRAZYNSKA-DEJA 1976. S.20 und 23).

33 Vgl. auch hierzu DRAŻYŃSKA 1974/75. S.125f oder DRAŻYŃSKA-DEJA 1976. S.39.

st Vgl. die Angaben S.46, Anm.23. 
dem Grundwort vorgegebenen Eigenschaft zum Ausdruck bringen. Eine parallele semantische Interpretation ist ebenso bei den substantivischen Formen gegeben, möglich ist eine minimalisierende (diminuierende) als auch maximalisierende (verstärkende) Bedeutung. Hypokoristika oder Wendungen wie ostatni kawaleczek, ani słoweczka sind vor allem als Zeichen gesteigerten Ausdrucks zu verstehen. ss

Der unterschiedlichen Funktion entsprechend differenziert man also Adjektivdiminutive und die entsprechenden Adverbien in zwei Gruppen, in eigentliche Diminutive und Intensiva ${ }^{36}$ bzw. in formacje gradacyjne, genauer formacje stopniowania bezwzglednego (im Gegensatz zu den "regulären" Steigerungsformen), und formacje ekspresywne. ${ }^{57}$ Anzeiger jener immer deadjektivisch geprägten Bildungen der ersten Gruppe ist das recht produktive Suffix -awy (czerwonawy, -a, $-\mathbf{e},-0)$ mit den selteneren Nebenformen -kawy (zielonkawy), erlawy (chuderlawy). Daneben werden auch gelegentlich die Suffixe -owaty (glupkowaty), -owity (slabowity) und die weniger produktiven Präfixe przy(przyciasny) und niedo- (niedorozwinięty) eingesetzt. ${ }^{58}$ Es sind Ableitungen, die dazu dienen, den Grad des durch das Basislexem genannten Merkmals zu mindern und mit der Formel: " $A\left(A_{1}\right)$ - 'nie calkiem (dosyc) $A_{1}{ }^{\prime \prime}$, vgl. bylo ciemnawo ('nie calkiem ciemno /troche ciemno'), umschrieben werden können, womit eine "intensywnosć poniżej normy" angegeben wird. ${ }^{39}$ Zusätzlich, durch eine andere Perspektivsetzung, kann auch eine Umschreibung nach dem Schema " $A\left(A_{1}\right)$ - 'zbyt (zanadto) $A_{1}$ '" angenommen werden, vgl. te kartofle sa jeszcze

ss Der Gedanke der Verringerung eines Gegenstandes o.a. zum Zweck der Verstärkung eines Eindrucks findet sich bei NIEDZWIEDZKI 1919: "czesto, chcac spotegowac wrażenie przedmiotu, koncentrujemy niejako ten przedmiot, i wyrażamy także jezykowo w postaci skupionej, zwezonej, w malenkich rozmiarach, czyli w formie zdrobnialej" (S.45).

so Vgl. dazu z.B. KURKOWSKA 1954. S.99 und GRZEGORCZYKOWA 1984. S.69f.

"7 Diese Einteilung findet man in der GRAMATYKA 1984. S.437-439 und 441; KLIMASZEWSKA 1983 (S.66-69) bietet in Anlehnung u.a. an Z.Klemensiewicz die antonymische Paanung Detensiva - Intensiva an.

st Zum Bestand der formalen Mittel s. JANUS 1986. S.100 und GRAMATYKA 1984. S.439. Zur Genese der abschwächenden Funktion des Suffixes -awy vgl. die Analyse von KREJA 1957. Einen Überblick über die Adjektivdiminution aller slavischen Sprachen gibt die frühe Arbeit von BELIĆ 1904.

\$o Vgl. GRAMATYKA 1984. S.439. 
twardawe ('zbyt twarde /troche za twarde'), so daß hier eine "intensywnosć przekraczajaca norme" zur Geltung kommt. ${ }^{\infty}$

Die Existenz dieser Derivate wird hauptsāchlich durch die Semantik ihres Grundwortes bestimmt, unerläßliche Bedingung sind steigerungsfahige Eigenschaften: z.B. Farben (niebieskawy); Geschmacksrichtungen (gorzkawy); weitere Eigenschaften, wie körperliche Ausmaße (chudawy), Alter (starawy), Temperatur (cieplawy); physische Eigenschaften von Gegenständen, wie Ausmaß, Gestalt u.a. (krótkawy, płaskawy, twardawy); psychische Eigenschaften von Menschen (tepawy); physische Eigenschaften von Lebewesen (slabawy); oder solche, die der Beurteilung des Sprechers unterliegen (nudnawy, trudnawy). ${ }^{61}$

In enger Verbindung mit den abschwächenden Bildungen stehen die Intensiva bzw. formacje ekspresywne, die eine Steigerung des durch die Basis genannten Attributs ausdrücken. ${ }^{62}$ Träger dieser Bedeutung sind die einfachen und zusammengesetzten Suffixe (jeweils in der maskulinen Form angegeben): -utki (zdrowiutki, wszyściutko), -uśki (miluśki, takuśki), -usi (drobniusi), -eńki (bladzieńki), -uteńki (cichuteńki, każdziuteńki), -usieńki (malusieńki, samiusieńki), -uchny (bialuchny), -aśny (grubaśny) sowie die Präfixe prze- (przepiękny), arcy- (arcyciekawy), super- (supernowoczesny), ultra- (ultrakatolicki), extra- (extramocny), pra- (pradawny). ${ }^{63}$ Ähnlich wie bei den substantivi-

${ }^{\infty}$ S. nochmals GRAMATYKA 1984. S.439 und auch JANUS 1986. S.105. Mit der traditionellen Interpretation der -awy-Derivate setzt sich auch JADACKA 1978 auseinander und sieht aber die Möglichkeit, geringe Intensität einer Eigenschaft mit Hilfe morphologischer Mittel auszudrücken, nicht gegeben. Sie differenziert die -awy-Bildungen in solche mit objektiver oder subjektiver Funktion, d.h., die modifizierte Basis kann einerseits subjektiven Charakter haben, dann: "mamy do czynienia z eufemicznym okresleniem cechy", oder andererseits objektiven, dann informiert sie "o nieosiagnieciu, niepeinosci cechy podstawowej" (S.158). Ihre Darlegungen lassen wieder ein Nichtbeachten der drei Sprachebenen erkennen.

6l Zu dieser Einordnung vgl. GROCHOWSKA 1975 (S.338-341), die sich vor allem mit den strukturellen und semantischen Restriktionen dieser Derivate beschäftigt. Auch in der chemischen Terminologie sind Derivate auf -awy mit substantivischer Basis möglich, miedziawy, rteciawy, aber dann nur als Opposition zu den Adjektiven miedziowy, rteciowy; vgl. KOWALIK 1977. S.71, Anm.16.

62 RITTER 1976 (S.357) gebraucht den paradox formulierten Begriff Diminutiv der Verstärkung.

63 Vgl. zu dieser Auswahl GRAMATYKA 1984. S.441 und KLIMASZEWSKA 1983. S.67. Über die morphologische Struktur der expressiven und auch -awy-Bildungen sowie mögliche morphonologische Veränderungen des Stammes informiert die Monographie von KOWALIK 1977, vgl. S.110-112, $173 \mathrm{f}$ und 177-180. Auch hier ließe sich die Liste der Suffixe verlăngern, 
schen Diminutiven können durch Suffixkombination ganze Reihen der Verstärkung aufgestellt werden: malutki - maluteńki - malutenieczki, mlody - mlodziutki - mlodziusieńki usw.

Wiederum ist auch hier die Verbindungsfähigkeit der morphologischen Elemente mit ihren Basisformen durch Restriktionen unterschiedlichen Charakters bedingt. Entscheidende Voraussetzung ist, daß diese Formen ausschließlich von qualitativen Adjektiven geformt werden. Faßt man die Beschränkungen innerhalb dieser ganz allgemein zusammen, so zeigt sich, daß sie z.B. nicht von Wörtern buchsprachlichen, gehobenen Stils (milosierny, egoistyczny) gebildet werden können, oder aber, daß ihre Grundlage in erster Linie einfache, nicht komplexe Adjektivstämme stellen. Nach semantischen Gruppen unterschieden ist zu erkennen, daß sich die Adjektivdiminutive auf drei Hauptgruppen verteilen: 1. "przymiotniki parametryczne (oznaczajace wielkosci mierzalne)", vgl. króciutki, szczuplutki, latwiutki; 2."przymiotniki i przysłowki oceniajace", vgl. wesolutki, zdolniutki; 3. "wyrażenia oznaczajace barwy, ksztalty, smaki", vgl. źf́ciutki, okraglutki, slodziutki. ${ }^{64}$ AufschluBreich ist nun die ansatzweise zu erkennende Distribution der Suffixe -utki und -awy innerhalb dieser Gruppen. In der Gruppe der Adjektive, die meßbare Größen bezeichnen, werden die Intensiva von denjenigen Adjektiven gebildet, die auf einer vorgestellten Gradskala das quantitativ oder qualitativ geringere Ende markieren, die Detensiva dagegen von einigen, die am anderen Ende der Skala liegen: z.B. krociutki dlugawy, szczuplutki - tegawy, latwiutki - ciezzkawy. Auch in der Gruppe der bewertenden Adjektive beobachtet man eine Verteilung, bei der die Intensiva von Grundwörtern, die hauptsächlich eine positive Eigenschaft auf der Bewertungsskala anzeigen, entstehen; Detensiva dann umgekehrt von jenen mit negativen Eigenschaften, z.B. wesolutki - smutnawy, zdolniutki - tepawy. Für die Bildungen der dritten Gruppe sind ähnliche Distributionen nicht auszumachen, sie werden von beiden Suffixtypen (ausgenommen Adjektive, die geometrische

berücksichtigte man die dialektalen Varianten, dazu s. KURKOWSKA 1954. S.105 oder das Beispielmaterial in den einschlägigen Arbeiten von ADAMISZYN 1977 S.57-59 oder PLUTA 1967. S.47f.

a Diese Einteilung beruht auf der Zuhilfenahme der Relation Positiv - Komparativ, die JANUS 1986 (S.103-105) auf der Grundlage ihrer semantischen Analyse der Adjektive (vgl. JANUS 1981) vorgenommen hat. In der 1.Gruppe ist die Grundstufe der Diminutive semantisch gesehen als Komparativ, in der 2.Gruppe dann als Positiv zu deuten. 
Formen bezeichnen) fast gleichermaßen genutzt: ź6łciutki - z6łtawy, słodziutki slodkawy u.a. ${ }^{63}$

Nun noch zum Bedeutungsaspekt der Intensiva. Die durch sie mitgeteilte gesteigerte Intensität einer Eigenschaft kann mit dem Zusatz bardzo transformiert werden: " $A\left(A_{1}\right)$ - 'bardzo (lub: bardzo, bardzo) $A_{1}{ }^{\prime \prime} .66$ Doch, wie schon der Terminus Expressiva verrät, bleibt es nicht allein bei der elativisch geprägten Funktion. Vielmehr kommt neben der semantischen Komponente bardzo eine expressive zur Geltung, die sich in Übereinstimmung mit den Substantivdiminutiven als emotionale Einstellung des Sprechers zum Sachverhalt äußert:

[Derywaty - B.K.] maja funkcje ekspresywna, charakteryzuja nie desygnat, ale mówiacego, informujac o jego skJonosci [sic!] do przesady, emocjonalnosci, a czesto sa po prostu znamieniem pewnego stylu. ${ }^{67}$

Jene Derivate aus der Gruppe der sog. "przymiotniki oceniajace" nennen jeweils Eigenschaften, Qualitäten, die vom Sprecher als positive bewertet werden; will man lediglich eine gewisse Intensität anzeigen, ohne positive Reaktion, dann genügt die umschreibende Formulierung mit bardzo, vgl. suchuteńka ziemia wenn man sich auf die Erde setzen will - aber bardzo sucha ziemia - wenn man feststellt, daß die Pflanzen zu gießen sind. ${ }^{68}$

Mit dem oben dargestellten reichhaltigen Suffixprogramm der polnischen Sprache ist die Möglichkeit der synthetischen Diminuierung erfaßt. Der Vollständigkeit halber bleibt zuletzt anzuführen, daß neben dieser auch auf die mit Adjektiven oder Adverbien umschreibende, die analytische Bildeweise zurückgegriffen werden kann, wobei das Grundwort je nach semantischer Funktion durch die entsprechenden Attribute ergänzt wird, z.B. male okno statt okienko bzw. mily kot statt kotek..$^{69}$ Zur Intensivierung des diminutivischen Wertes wird oft die analytische mit der synthetischen Diminution verbunden (male okienko; mily

as Vgl. JANUS 1986. S.105 sowie ihre tabellarische Zusammenstellung auf S.108.

* So die GRAMATYKA 1984. S.441; die Doppelung von bardzo gilt für die zusammengesetzten Suffixe. Für NIEDŹWIEDZKI 1919 stellen diese Bildungen "spotegowane pojecia" dar, die umschrieben werden können mit "bardzo, nader, ze wszystkiem, zupetnie" plus Grundwort, vgl. S.45. Auch JANUS 1986 (S.110, Anm.20) erweitert die Interpretation um das Bedeutungselement zupelnie.

${ }^{67}$ GRZEGORCZYKOWA 1984. S.70.

6* Vgl. hierzu GRZEGORCZYKOWA 1978. S.121f.

${ }^{\infty}$ Zur Begriffsbestimmung und Einschătzung beider Verfahren hinsichtlich ihrer Leistung vgl. S.39f. 
kotek) und dabei sogar - was eine Besonderheit des Polnischen darstellt - das Attribut ebenfalls diminuiert (maleńkie okienko; milutki kotek). Diese Art der Übereinstimmung von diminuierten Adjektiven und Substantiven kann zu der obligatorischen grammatischen Kongruenz beider Formen in Beziehung gesetzt werden. ${ }^{70}$

\subsection{DIE DIMINUTIVBILDUNG IM DEUTSCHEN}

Methodische Gründe zwingen dazu, auch einen Blick auf die Vergleichssprache zu werfen. Ganz im Gegensatz zur polnischen Diminutivbildung, die zu den wichtigsten Abschnitten innerhalb der Wortbildungslehre zählt, spielen die deutschen variierenden Suffixe eine eher periphere Rolle im morphologischen System dieser Sprache. Hierin liegt wohl einer der Hauptgründe für die geringere Beschäftigung mit der Diminutivthematik. Da es an vergleichbaren Einzeluntersuchungen zur gegenwärtigen Schriftsprache unter synchronischem Aspekt mangelt, ist man auf allgemeiner und kürzer gefaßte Wortbildungsdarstellungen angewiesen.

Dessen ungeachtet verfügt auch die deutsche Sprache über ein weithin offenes formales Programm der quantifizierend-qualifizierenden Suffixe, an dem alle drei Hauptwortarten, Substantive, Adjektive/Adverbien und - wenn auch nur vereinzelt - Verben, mit jeweils eigenen Affixen beteiligt sind. Innerhalb der Wortklasse der Substantive hat sich im Laufe der Zeit in der deutschen Schriftsprache ein kohärentes System entwickelt, das die beiden Formative -chen' (Körbchen, Hündchen ${ }^{2}$, Evchen) und -lein ${ }^{3}$ (Blümlein, Tüchlein, Fränzlein)

T Vgl. dazu JANUS 1986. S.115.

' H.Wellmann gliedert die substantivischen -chen Bildungen in zwei Gruppen: -chen' mit der formalen Definition "BS-chen $\Rightarrow$ Attr. (klein) + BS" (=Basissubstantiv) und die kleine Gruppe -chen ${ }^{2}$ (Dummchen, Liebchen, Altchen) mit adjektivischer Basis (=BA), die einem abweichenden syntaktischen Muster angehört, in dem das Suffix anders zu deuten ist: es "verweist auf denjenigen, dem das im BA genannte Merkmal zugesprochen wird": vgl. DEUTSCHE WORTBILDUNG 1975. S.124 und 317, Zitat S.317. Gegen diese Trennung spricht sich KLIMASZEWSKA 1983 (S.42) aus, die die zweite Gruppe als Ableitungen von substantivierten Adjektiven interpretiert.

2 Zur Benennung von Tierjungen mit Hilfe von Diminutivsuffixen vgl. SEIDEL-SLOTTY 1947. S.32-34. 
verwendet. ${ }^{\star}$ Neben diesen beiden gängigsten Diminutivsuffixen sind zum Inventar der Diminutivmorpheme die synchron gesehen kaum mehr produktiven -el und -ling, die im Gegensatz zu jenen in ihrer Leistung polyfunktional sind," sowie das Fremdsuffix ette zu rechnen. Während -el-Bildungen (meistens mit Basisumlaut) wie Bündel, Büschel, Gürtel heute - auch wenn die Verbindung zu Bund, Busch, Gurt noch deutlich ist - ihren diminuierenden Charakter gegenüber dem Grundwort meist verloren haben und zu Simplizia geworden sind, ${ }^{6}$ bewegen sich die semantischen Merkmale der -ling-Derivate im Bereich von ' 'klein' bis 'schlecht', vom Deminutiven (wenn das BS keine Person bezeichnet) bis zum Pejorativen (BS= Personenbez.) ${ }^{n 7}$, so Dichterling, Schreiberling. ${ }^{8}$ Mit geringer Frequenz begegnet auch das aus dem Französischen übernommene -ette-Formans, z.B. Sandalette, Operette, meist bereits lexikalisierte Bildungen.9

Außer den hier aufgelisteten einfachen Affixen sind zwei Möglichkeiten der Suffixerweiterung, der sog. "geminierten diminution" 10 anzuführen: zum einen die vor allem im nord- und mitteldeutschen Sprachgebiet übliche Suffixkombination elchen, die analog zu Bildungen wie Vögelchen (hier wird el zum

${ }^{3}$ Vgl. DEUTSCHE WORTBILDUNG 1975. S.124f.

4 Zur Entstehungsgeschichte dieser beiden Ableitungsmittel vgl. u.a. GRIMM 1831. S.665-673 und $676-680$.

${ }^{3}$ Vgl. dazu Wellmanns Differenzierung der Morpheme nach Ableitungsmustern in DEUTSCHE WORTBILDUNG 1975. S.104 und 86.

${ }^{6}$ Hierzu s. FLEISCHER 1974. S.137 und 180.

7 DEUTSCHE WORTBILDUNG 1975. S.126, vgl. dazu auch Abschnitt 3.3.3. und 3.3.4. dieses Handbuchs.

- Zur Substantivbildung mit -ling vgi. WELLMANN 1969, der die uneinheitliche Bedeutung dieser Derivate zur Darstellung bringt, indem er das Suffix -ling in sieben verschiedene Ableitungsmuster gliedert. HENZENs 1957 Feststellung (S.1660), den meisten Ableitungen mit -ling wohne "irgendwie" diminutivische Bedeutung inne, lảBt sich somit nur für jene Ableitungsmorpheme bestătigen, die für die Attribute aus dem lexikalischen Paradigma schlecht, unbedeutend stehen. Darüberhinaus s. auch MÜLLER 1953 (S.56-75), der im Rahmen seiner Arbeit über pejorative Substantivableitungen zwischen -ling als reinem, pejorativen Modifikationssuffix und -ling als komplexem Ableitungsaffix unterscheidet.

' FLEISCHER 1974 (S.181) zählt darüberhinaus auch -ine-(Violine) und -it-Bildungen (Meteorit) dazu.

10 Vgl. dazu GRIMM 1831. S.681f. 
Stamm gezählt) Derivate des Typs Wägelchen, Löchelchen bildet, " zum anderen die Variation -leinchen, wie z.B. in Fräuleinchen, Kindeleinchen. Diese "pleonastischen"12 Modifikationsformen können entweder zum Ausdruck einer verstärkten Affektivität oder bei Verlust des spezifischen Bedeutungsgehalts der einfachen Form als Anzeiger einer erneuten Diminuierung dienen. ${ }^{13}$ Die Funktion der Wertungsdifferenzierung bleibt allerdings eher ungebräuchlich; verglichen mit dem Polnischen ist eine ähnliche Stufenbildung und damit verbundene Suffixkombinationsfreiheit nicht gegeben.

Will man der Diminution in ihrer Gesamtheit gerecht werden, bleibt es nicht aus, neben den suffixal bestimmten Diminuierungsformen auf eine moderne Bildeweise mit stetig wachsender Verbreitung ergänzend hinzuweisen. Es handelt sich um ein Präfixoid, das Fremdmorphem Mini-, entstanden unter dem Einfluß des Englisch-Amerikanischen. ${ }^{14}$ In Anlehnung an das seit Mitte der sechziger Jahre aufkommende Muster Minirock (engl. mini-skirt) ${ }^{15}$ wird dieser lebendige Bildungstyp "vom Durchschnittssprecher oft ohne stilisierende Absicht (...) zur Prägung von Sachbez. gebraucht ${ }^{n 16}$, vgl. Minipreis, Ministaubsauger. In diesem Falle steht Mini- stellvertretend für die Attribute niedrig, kurz, gering, einfach u.a. Doch zeigt sich, daß "die spielerische Bildung mit stilistischer Nebenabsicht heute weitaus, vor allem in der Zeitungssprache ${ }^{n 17}$ dominiert, vgl. Mini-Aufstand, Mini-Kapitalismus. Die Mehrzahl der Fügungen sind noch Ad-hoc-Bildungen, die den bereits etablierten als Lexikoneintrag noch folgen

"Wellmann führt die Erweiterung der Grundform -chen auf phonetische Gründe zurück: ein auf - $\mathrm{g}-$ bzw. -ch- ausiautender Substantivstamm verlangt ein zusammengesetztes Suffix: Kräg-elchen, Sïch-elchen, vgl. DEUTSCHE WORTBILDUNG 1975. S.27.

12 HENZEN 1957. S.147.

13 Dieses in der polnischen Hochsprache verbreitete Phänomen der sog. "zweiten Diminuierung" ist im Deutschen eher in den Mundarten ausgeprägt, vgl. BRANDSTETTER 1963/64. S.344f und MOSER 1969. S.109f.

i4 Einen kurzen historischen Abriß zu Mini-gibt KANN 1973. S.208f.

is So die Deutung von KÜPPER 1970. S.211f.

16 DEUTSCHE WORTBILDUNG 1975. S.127.

17 DEUTSCHE WORTBILDUNG 1975. S.127; vgl. auch die Miszelle MINI-BETRACHTUNG 1967. 
müssen. ${ }^{18}$ Vergleicht man den durch die Morpheme ausgedrückten stilistischen Wert miteinander, so werden die traditionellen Suffixe als "sanft verniedlichende" interpretiert, das Präfix Mini- als solches, das "dynamisch/aggressiv/rekordanstrebend $^{\text {19 }}$ verkleinert.

Mit jenen teilweise inhaltlich verwandt sind die reihenhaft auftretenden Klein-Bildungen wie Kleinstadt, Kleinwagen, Kleinanzeige. Eine Gegenüberstellung dieser Zusammensetzungen, diesmal hinsichtlich des objektiven Merkmals der Kleinheit, mit entsprechenden -chen bzw. -lein Formen legt ihre eigentlich "klassenstiftende Funktion" offen, ${ }^{20}$ sie werden vor allem genutzt zur Benennung von festen Unterklassen (kleineren Exemplaren) der durch das Basissubstantiv genannten Dinge. Dementsprechend ist Kleinkind nicht synonym mit Kindchen und Minibus nicht mit Büslein zu gebrauchen; -chen und -lein Diminutiven ist diese Funktion primär nicht eigen, möglich wird sie eventuell durch semantische Isolierung wie z.B. bei Mädchen, Fräulein.

In die Reihe der Diminuierungsmittel kann ferner das eigentlich nur expressiv (und nicht zur objektiven Verkleinerung) genutzte Suffix -i gestellt werden. ${ }^{21}$ In Parallele zum Polnischen zeigt damit auch das Deutsche - allerdings mit gänzlich anderer Suffixquantität - die Möglichkeit, als Diminutive im weiteren Sinne verstandene Expressiva mit eigenen morphologischen Formen zu bilden. Die bis in die letzte Zeit gültigen Feststellungen, das Suffix -i werde allein zur Bildung hypokoristischer Derivate herangezogen und bleibe somit in der Anwendung auf Rufnamen und Anredeformen beschränkt, ${ }^{22}$ bedürfen einer Korrektur. Neuere Untersuchungen ${ }^{23}$ zeigen einen Wortbildungstrend der Gegenwartssprache auf, der darin besteht, daß in einigen Sondersprachen, beson-

It S. dazu den an Belegmaterial reichen Aufsatz von KANN 1973.

10 KANN 1973. S.209; er sieht darin den "Zeitgeistwandel" widergespiegelt.

$30 \mathrm{Vgl}$. DEUTSCHE WORTBILDUNG 1975. S.128.

"1 Vgl. dazu DUDEN-GRAMMATIK 1984. \$827, S.460; wie jung diese Entwicklung ist, sieht man daran, daß weder die die Schriftsprache des 20.Jh.s untersuchenden Verfasser der DEUTSCHEN WORTBILDUNG 1975, noch die Studie zur Wortbildung in gesprochener Sprache von GERSBACH/GRAF 1984/1985 -i-Derivate berücksichtigen.

2 So FLEISCHER 1974, der im Kap. "Onomastische Suffixe von Personennamen" (S.200t) davon spricht, daß das appellativische System diese expressiv-kosenden Formen nicht kennt. Zu den Namenkurzformen auf $-i$ vgl. auch FLEISCHER 1968. S.23f.

${ }^{2}$ S. GREULE 1983/84 mit Glossar sowie die Beispiele bei MUTHMANN 1988. 
ders den Sprachvarianten von Schülern, Studenten, dieses aus dem althochdeutschen Diminutivsuffix -i(n) stammende ${ }^{24}$ Formans sich durch vermehrtes Auftreten auf den appellativischen Wortschatz ausgedehnt hat und sich erneut zum produktiven Suffix entwickelt. Neben seiner traditionell hypokoristischen Funktion, die in vertrauten Kommunikationssituationen zum Einsatz kommt - so bei Vornamen, Anna - Anni, Wolfgang - Wolf, Verwandtschaftsnamen, Vater Vati, oder ganz allgemein in der Sprache mit Kindern, Schnuller - Schnulli usw. -, hat das -i besonders zwei dafür bisher nicht typische semantische Bereiche erfaßt: Personenbezeichnungen wie Wessi (Westdeutscher), Sympi (Sympathisant), Bubi (Bub(e)) und auch Instrumentbezeichnungen wie Kuli (Kugelschreiber), Kasi (Kassettenrekorder). ${ }^{25}$ Der Inhalt des abgeleiteten Wortes kann dabei mit einer "liebevollen, teils verkleinernden, teils abschätzigen Nuance $^{\text {m26 }}$ versehen werden. Diese Ausweitung des Verwendungsbereichs wird auf den in jüngster Zeit bedeutenden Einfluß der Lehnwörter auf $-\mathbf{i} /-\mathbf{y}$, besonders jener aus der englischen Sprache wie Baby, Buggy u.a., zurückgeführt. Hinzu kommt die wachsende Beliebtheit von Kurzwörtern des Typs Uni (Universität), Touri (Tourist), die von den obigen Expressiva zu unterscheiden sind. ${ }^{27}$ Besonderes Kennzeichen aller -i Bildungen ist ihre fast obligatorische Zweisilbigkeit; bei mehrsilbigen Basiswörtern geht, parallel zum Polnischen, mit der Affigierung eine Reduzierung des Grundwortes einher, vgl. Bri-gitte - Gitti, Rud-olf Rudi; Am-erikaner - Ami, Pull-over - Pulli, bei Vornamen tritt das -i an meist schon als Koseformen verwendete Namenkurzformen, z.B. Lotte ( $=$ Charlotte) - Lotti. Auch wenn dieser sprachliche Trend der Inhaltsnuancierung vorrangig in der mündlichen Kommunikation dominiert, gelangen erste Bildungen allmählich in die geschriebene Sprache.

In Hinblick auf die Verhältnisse im Polnischen muß schließlich noch auf weitere, besonders für das Deutsche charakteristische Wortbildungsmittel zum Ausdruck von Gefühlswertungen hingewiesen werden, die zum semantischen Umfeld der Diminutive gerechnet werden können. Gemeint sind die systemhaft

24 Zur Herkunftshypothese vgl. GREULE 1983/84. S.208f. Interessant ist, daß $-i(n)$ als selbstăndiges Suffix noch heute Diminutive im Schweizerdeutschen bilden kann.

25 Vgl. dazu GREULE 1983/84. S.208-213.

${ }^{20}$ GREULE 1983/84. S.214.

${ }^{27}$ GREULE 1983/84 hat den Bestand an -i-Wōrtern in acht verschiedene Gruppen zu klassifizieren versucht, vgl. S.208. 
entwickelten expressiven Personenbezeichnungen. ${ }^{28}$ Der erste Wortbestandteil, der sich aus nominalen bzw. verbalen Stämmen zusammensetzen kann, ist Träger der lexikalischen Bedeutung, wie z.B. in Gemüse-fritze, Schlau-peter, Zappelphilipp, als zweite Konstituente treten suffixartig gebrauchte, nach Genus differenzierte Vornamen - oft in verkürzter Form - auf, wie Klatsch-heini ( $\Leftarrow$ Heinrich) - Klatsch-trine (- Katharina), Heul-peter - Heul-suse ( $\leftarrow$ Susanne), Bummel-fritze ( $\Leftarrow$ Friedrich) - Bummel-liese ( $\Leftarrow$ Elisabeth), seltener sind es gängige Familiennamen wie in Schlau-meier, Drücke-berger u.a. ${ }^{29}$ Indem auch diese suffixähnlichen Elemente affektische Zusatzmerkmale in die Wortbedeutung einbringen, lassen sie sich mit den obigen polnischen Suffixen der Expressivität vergleichen: sie "helfen ironische, scherzhafte, zärtliche, abschätzige, freundliche, grobe (...) Schattierungen auszudrücken" ${ }^{\text {30 }}$. Diese emotional gefärbten Bildungen stehen dann den neutral gebrauchten Personenbezeichnungen gegenüber, vgl. Gemüsemann, Klatsche, Bummler usw. Auch für dieses lebendige Wortbildungsmuster gilt, daß es eigentlich aus dem Bereich der Mundarten stammt, aber allmählich über die Umgangssprache Einzug in die Schriftsprache hält.

In Kenntnis dessen, daß die schriftsprachlichen -chen und -lein infolge der Entwicklung einer überregionalen Sprachform Vereinheitlichungen darstellen, kann erst die Beachtung der mannigfachen Sprachvariationen in den Mundarten das Gesamtbild der deutschen Diminutive abrunden. Auch wenn eine eingehende und systematische Beschreibung der Geschichte der Diminutivsuffixe für einzelne Orts- oder Regionalmundarten noch ansteht, ${ }^{31}$ ist herrschende Meinung, daß die Formengeographie des deutschen Diminutivs durch einen schon lang bekannten Nord-Süd-Gegensatz geprägt ist: -k- mit den Varianten -ken, -ke, -kin, -ske, -sche, der palatalisierte Typ -tje u.ä. in den diminutivarmen nieder- und mitteldeutschen Mundarten vs. -I- mit den Varianten -(e)l, -le, -li, -la, -erl(e) u.ä. in

22 Die Berücksichtigung dieser Formen geht auf die Dissertation von ANDRJUSCHICHINA 1968 zurück. In ihrer vergleichenden Studie zu Personenbezeichnungen des Russischen führt sie u.a. als Entsprechungen russischer Diminutivsuffixe deutsche Bildungen des Typs Bummelliese - Bummelfritze an, vgl. S.157-191.

20 Vgl. dazu auch DEUTSCHE WORTBILDUNG 1975. S.364-368 und 390-392.

3 ANDRUUSCHICHINA 1967. S.36.

${ }^{31}$ Wertvoll und maßgebend ist die, wenn auch ăltere Studie von WREDE 1908, darüberhinaus auch der Beitrag von GARTNER 1898. S.169-172. 
den diminutivreichen oberdeutschen Mundarten. ${ }^{32}$ Abweichungen von diesen beiden Haupttypen sind z.B. das mecklenburgisch-pommersche -ing, das konsonantenlose Suffix -i im Berner Oberland. ${ }^{33}$

Etwas ausführlicher muß nun auf die beiden Hauptvertreter der Diminutivformantien eingegangen werden. Wichtigstes formales Charakteristikum dieser Derivate ist die obligatorische Einheitlichkeit des Genus sowie das Fehlen einer flexivischen Pluralmarkierung. Ungeachtet des Grundwortes ziehen die Suffixe zwangsläufig das neutrale Geschlecht nach sich: ${ }^{34}$ Schrank (mask.) - Schränkchen (neutr.), Tasse (fem.) - Täßchen (neutr.), Kleid (neutr.) - Kleidchen (neutr.) oder auch bei Propria Hans (mask.) - Hänschen (neutr.), Brigitte (fem.) - Brigittchen (neutr.). ${ }^{35}$ Somit sind die Diminutive des Deutschen anders als die von Polyfunktionalität gekennzeichneten polnischen Diminutivformative durch Suffix und Genus eindeutig charakterisiert. ${ }^{36}$

Der Gebrauch dieser sprachhistorisch als kombinierte Suffixe geltenden -chen ( $\approx-$ ikin-) und -lein ( $\approx-$ ilin-) Formen ist, auch wenn von ihnen gesagt wird "they are twins" ${ }^{37}$, keineswegs wahllos und ohne gewisse Einschränkungen möglich. Die schriftsprachliche Wahl eines der Suffixe bleibt von mehreren

32 Zur These der Diminutivarmut des Niederdeutschen vgl. weiter oben S.27.

3 Eine knappe Zusammenfassung der bisherigen Forschungsergebnisse über Form, Herkunft und geographische Verbreitung im deutschen Sprachgebiet (mit Karte) mit weiterführender Literatur bietet SEEBOLD 1983.

34 Im Rahmen seiner Erörterungen zum Zusammenhang von Ableitungsgruppen und grammatischem Geschlecht bei der Klasseneinteilung des Substantivs konstatiert BRINKMANN 1971 (S.17): "In der Regel gehoren Wörter gleicher Ableitung auch zu demselben grammatischen Geschlecht. Das ist besonders deutlich bei den Diminutiva, die früher im Genus dem Grundwort folgten, jetzt aber unabhăngig vom Grundwort stets Neutrum sind (...). Die Ableitungsgruppe bestimmt das Genus." Ferner äußert er zur Zusammengehörigkeit von Diminutiven und Kollektiven: "Wăhrend in Gebirge die Ausmaße des Grundwortes gesteigert sind, werden sie in Gr̈rtchen verringert. Beide Bildungen gehören zur Klasse der Neutra, obwohl sie sich in verschiedener Richtung vom Grundwort entfernen. Was das Neutrum jeweils leistet, hăngt davon $a b$, wie es dem Grundwort entgegengesetzt ist. Kollektiva und Diminutiva haben verschiedene Antipoden." (S.18)

$3 s$ Vom Genuswechsel nicht betroffen sind el, vgl. Barbara (fem.) - Bärbel (fem.), -i, vgl. Ursula (fem.) - Uschi (fem.) und auch die Prăfixe Mini-/Klein-, vgl. Rock (mask.) - Minirock (mask.), Bahn (fem.) - Kleinbahn (fem.).

36 Auch wenn MARKEY 1983 (S.55) für das hauptsăchlich bayerische I- Diminutiv eine Multifunktionalităt konstatiert, verteilt sich diese doch auf Nomen und Verben, so daß auch hier die Eindeutigkeit erhalten bleibt.

${ }^{37}$ LOCKWOOD 1974/75. S.307. 
Faktoren abhängig. Zunächst einmal machen sich rein innersprachliche, euphonische Gründe geltend, indem die morphonologische Struktur des Grundwortes, genauer des Auslauts, die Realisierung der Morphemvariante determiniert; z.B. -lein wird nicht bei Basisauslaut auf -J(e) verwendet (Teil-chen), -chen in der Regel nicht bei auf $-\mathbf{g}$, $-\mathbf{c h}$, $-\mathbf{n g}$ endenden Stämmen (Krüg-lein, Bäuch-lein, Züng-lein). ${ }^{38}$ Neben dieser komplementären sind auch Möglichkeiten einer freien Distribution zu verzeichnen. ${ }^{39} \mathrm{Zu}$ beachten ist bei der freien $\mathrm{Wahl}$, daß in einigen Fällen die Ableitungen zur Bedeutungsdifferenzierung genutzt werden, wie bei dem schon klassischen Beispiel Männchen ('männliches Tier') - Männlein (Dim. zu Mann) und Weibchen ('weibliches Tier') - Weiblein (Dim. zu Weib). Neben der skizzierten Bindung an Merkmale der phonetischen Umgebung steht das Auftreten beider Suffixe in Abhängigkeit von diastratischen Kriterien, ${ }^{40}$ währenddessen diatopische Faktoren wohl keinen Einfluß mehr ausüben. Auf der Grundlage umfangreicher Materialsichtung wird konstatiert, daß

der Gebrauch von -chen' und -lein in der Hochsprache nicht mehr durch die Heimat des Autors bestimmt ist wie in den Mundarten, was historisch aus der teilweise führenden Rolle mitteldt. Autoren, Grammatiker und Drucker bei der Ausbildung der nhd. Schriftsprache zu erklären ist. ${ }^{\star 1}$

Schließlich kann die Varianz in der Verwendung sowohl in Verbindung mit bestimmten rhythmischen und metrischen Wirkungen stehen, als auch durch Stillage und Textgattung bedingt sein. Hinsichtlich der Häufigkeitsfrage hat man anhand verschiedener Textsorten- bzw. Wörterbuchauswertungen für das gegenwärtige Deutsch ein Frequenzverhältnis für -chen : -lein wie 4:1 bzw. 3:1 erstellen können. ${ }^{2}$ das die dominierende Stellung von -chen in hochsprachlichen Texten bezeugt, während der Einsatz des Formans -lein im wesentlichen seiner Markierung wegen eingeschränkt bleibt:

\footnotetext{
3* Zu den Kombinationsregularităten beider Suffixe mit dem Grundwort im einzelnen vgl. FLEISCHER 1974. S.179 und DEUTSCHE WORTBILDUNG 1975. S.125.
}

* Siehe dazu DEUTSCHE WORTBILDUNG 1975. S.131.

- Vgl. DEUTSCHE WORTBILDUNG 1975. S.132.

^ DEUTSCHE WORTBILDUNG 1975. S.I31f; FLEISCHER 1974 (S.178f) spricht noch von einer geographischen Differenzierung der beiden Formantien.

22 Vgl. die Angaben in DEUTSCHE WORTBILDUNG 1975. S.131. 
it is at home in elevated, especially poetic and biblical style; it is the hallmark of folksong and common in the traditional fairy tale."

Mit diesem Befund zur heutigen Geltung - zum einen Vorherrschaft von -chen, zum anderen stilistische Spezialisierung bei -lein, welches kaum noch produktiv ist, denn "okkasionelle Bildungen, die auf eine Produktivität des Morphems schließen lassen, bleiben vereinzelt" währenden, von vielerlei Faktoren abhängigen sprachhistorischen Prozesses vor. Um es in aller Kürze zu summieren, war -[nn, später in der Form -lein, sowohl für das südliche als auch nördliche Sprachgebiet in der mittelhochdeutschen Periode das am häufigsten verwendete Diminutivsuffix der deutschen Sprache. Mit dem Aufkommen des nördlichen -chen, zunāchst sporadisch in regionalen Quellen, setzt das Ringen um die Vorrangstellung beider Formative ein. Im 16. Jh. beginnt es sich allmählich in Sachprosa, Urkunden und Geschäftssprache durchzusetzen, während -lein über seine mundartlichen Grenzen hinaus weiterhin in der Belletristik und in religiösem Schriftum gebraucht wird. Erst recht spät, Mitte des 18.Jh.s, hat sich -chen als hochsprachliche Form endgültig etabliert und -lein verdrängt. Mit der Hinwendung zu volkstümlichen Sprachformen innerhalb der romantischen Dichtung erleben die -lein Diminutive dann einen erneuten Aufschwung, deren Gebrauch aber nicht mehr als eine Modeströmung blieb. ${ }^{45}$ Zur Verdeutlichung der Entwicklung seien die prozentualen Verschiebungen in der Diachronie angeführt: ${ }^{46}$

"LOCKWOOD 1974/75. S.312; interessant und dazu passend sind auch die Ergebnisse von drei Testreihen (exemplifiziert an moselfrănkischen Mundartsprechern) von SCHEIDWEILER 1984/85, der von -chen als prosaisch-nüchternen Bildungen spricht und -kin-Diminutive als poetisch-mărchenhaft charakterisiert.

« DEUTSCHE WORTBILDUNG 1975. S.125.

4s Zur Entwicklungsgeschichte der beiden Suffixe sind schon zu Beginn des Jahrhunderts wichtige Forschungsergebnisse von GÜRTLER 1909 sowie PFENNIG 1904 geliefert worden. Vgl. auch ÖHMANN 1972 sowie den neueren Beitrag von TTEFENBACH 1987, der u.a. die Übernahme der verschiedenen Suffixtypen in die überlandschaftliche Schriftsprache problematisiert.

${ }^{40}$ Entnommen ist diese Tabelle der Darstellung in DEUTSCHE WORTBILDUNG 1975. S.135, leider sind die -i-Bildungen hier nicht mitberücksichtigt worden. 


\begin{tabular}{|l|c|c|}
\hline & um 1800 & heute \\
\hline \multirow{2}{*}{$\begin{array}{l}\text {-chen' } \\
\text {-lein }\end{array}$} & $55,2 \%$ & $68,7 \%$ \\
\cline { 2 - 3 } el $^{1}$ & $41,3 \%$ & $16,6 \%$ \\
\cline { 2 - 3 } -lings $_{\text {-ette }}$ & $2,5 \%$ & $2,6 \%$ \\
\cline { 2 - 3 } Mini- & $0,6 \%$ & $2,3 \%$ \\
\cline { 2 - 3 } & $0,4 \%$ & $1,6 \%$ \\
\cline { 2 - 3 } & - & $8,2 \%$ \\
\hline
\end{tabular}

Neben dem Zuwachs der -chen-Derivate zuungunsten der -lein-Formen ist ein deutliches Aufkommen von Mini-Bildungen zu beobachten.

Berücksichtigt man diastratische Gesichtspunkte, so sind die Differenzen bezüglich der sprachlichen Schichtung insofern bemerkenswert, als sich die Zahlenverhältnisse der gesprochenen Sprache geradezu spiegelbildlich zu denjenigen der geschriebenen verhalten. Auswertungen weisen nach, daß das Morphem -chen "eher eine Ausnahme darstellt und stattdessen das weniger hsprl. Muster -lein ganz entschieden bevorzugt wird". 47 Innerhalb der berücksichtigten Lemmata ist -lein (hier als Einheitsform für alle phonetischen Varianten verstanden) sogar das zweithäufigste Suffix im gesamten Korpus. Die Umkehrung wird möglich, da besonders die im schriftsprachlichen Material festgestellten euphonischen Restriktionen bei der Bildeweise von -lein-Diminutiven ihre Gültigkeit zum großen Teil einbüßen. Überraschend ist ferner der Unterschied im Gebrauch des el-Musters, das in der Alltagssprache noch durchaus als produktives Diminutivsuffix auftritt, während in der geschriebenen Sprache fast ausschließlich isolierte Formen begegnen, die bereits den Status von Simplizia erreicht haben. Eine weitere Differenz liegt schließlich in dem zahlenmäßig unterschiedlichen Suffixinventar der Diminutivbildung: lediglich drei Muster der gesprochenen Sprache stehen sechs (rechnet man die -i-Morpheme nicht dazu) Mustern des geschriebenen Typus entgegen. Die Verteilung und fast alleinige Dominanz der -lein-Formative verdeutlicht die folgende Zusammenstellung: ${ }^{48}$

"7 Vl. dazu die Ergebnisse von GERSBACH/GRAF 1984. S.183f, 211f, 216 und 231-234; Zitat S.231.

4 Vgl. GERSBACH/GRAF 1984. S.234. 


\begin{tabular}{|l|c|c|c|c|}
\hline \multirow{2}{*}{ Gruppe } & \multicolumn{2}{|c|}{ Lemmata } & \multicolumn{2}{c|}{ Belege } \\
\cline { 2 - 5 } & abs. & $\%$ & abs. & $\%$ \\
\hline -lein 1 & 261 & 94,57 & 787 & 95,51 \\
\cline { 2 - 5 } chen 1 & 10 & 3,62 & 10 & 1,21 \\
\cline { 2 - 5 } -el 1 & 5 & 1,81 & 27 & 3,28 \\
\cline { 2 - 5 } Gesamt & 276 & 100,00 & 824 & 100,00 \\
\hline
\end{tabular}

Abschließend soll noch auf die Verwendungsmöglichkeiten der Diminutive innerhalb der Hochsprache eingegangen werden. Ihr Gebrauch ist zunächst durch strukturell-morphologische Restriktionen geprägt: eine Affigierung erfolgt fast ausschließlich von Substantivstämmen, ${ }^{49}$ die beinahe immer mit der Umlautung eines dazu fähigen Basisvokals verbunden ist; ${ }^{50}$ nur selten sind andere Wortarten betroffen, in diesen Fällen ist dann mit der Modifikation eine Transposition verbunden. In der Regel unterliegt der Diminuierung sowohl eine Vielzahl von Simplizia als auch von Komposita und präfigierten Derivaten, Beschränkungen gelten aber u.a. für bereits suffigierte Formen - ausgenommen -er-Derivate oder auf en oder em auslautende Substantive. ${ }^{\text {st }}$ Andererseits zeigen generative Strukturanalysen eine relativ große Beweglichkeit der Diminutivsuffixe; so können sie "sowohl die Basis als auch das Satellitenglied (Bestimmungswort) komplexer Konstruktionen konstituieren" ${ }^{52}$, ein Charakteristikum, das dem Polnischen fremd ist, vgl. Schönheitsgrübchen, Fähnchenständer u.a.

Bestimmt wird der Verwendungsbereich der Diminutive in der Gebrauchsnorm des Deutschen schlieBlich durch semantische Restriktionen. Bieten bei den nomina propria hauptsächlich die Vornamen Möglichkeit zur Diminution, so lassen sich bei den nomina appellativa in neutralem Sprachgebrauch keine

^9 DRAŻYŃSKA $1974 / 75$ (S.126f; oder DRAŻYŃSKA-DEJA 1976. S.38f) teilt nach der von ihr durchgeführten Strukturanalyse die möglichen Stămme der Diminutive neben den rein substantivischen (Lämpchen) weiter ein in: a) numerale Stämme (Milliönchen), b) Stämme mit substantivierten Adjektiven (Dickerchen), c) mit substantivierten Präpositional-Adverbien (Hinterchen) und d) mit substantivierten Verbalabstrakta (Schrittchen).

so Vgl. dazu Wellmanns Alternationsübersicht, DEUTSCHE WORTBILDUNG 1975. S.36-38. Zur weiteren Formverănderung der Basis vgl. ebd. S.34f sowie die ältere Arbeit von SANDERS 1894. S.93-100 und 130-136, der nur die -chen-Bildungen betrachtet.

s1 Über diese Art von Restriktionen im einzelnen vgl. WILMANNS 1899. S.322-324.

52 DRAŻYŃSKA 1974/75. S.92; zu den im Deutschen möglichen Konstruktionstypen vgl. S.95-97 sowie oben S.52, Anm.52. 
Ableitungen von Stoffnamen und Abstrakta bilden, da sie keine objektive Verminderung des Denotats zulassen; möglich sind Derivate eigentlich nur als Okkasionalismen bzw. nur in Fügungen auf der Ebene der Rede: Lüftchen, Bierchen, Pöstchen, Wissenschäftchen. Der so entstandene ausschließlich expressive Charakter dieser Bildungen wird in der Literatursprache als stilistisches Mittel eingesetzt. Außer den genannten werden ganz selten Bezeichnungen für Instrumente, Maschinen und nicht pluralfähige Sammelwörter diminuiert. ${ }^{53}$

Nicht nur Bezeichnungen von Lebewesen und Gegenständen, auch solche von Eigenschaften und Handlungen können in diesem Sinne modifiziert werden, denn (im Falle der Adjektive bzw. Adverbien)

mit der Grundleistung des Beiworts, zu charakterisieren, abstufend-wertend zu kennzeichnen, hängt seine potentielle Graduierbarkeit zusammen. ${ }^{\text {st }}$

Auch wenn die semantische Abwandlung von Adjektiven im Vergleich zu der substantivischen Modifikation als viel weniger ausgebildet erscheint, existiert eine reihenhaft erfolgende modifizierende Umwandlung mit dem Suffix -lich,"ss der "wohl etwas diminutives"s6 anhaftet. Diese Derivate drücken eine Abschwächung aus, "das Objekt, auf das sie sich beziehen, hat die angegebene Eigenschaft nicht im vollen $\mathrm{Maße}^{\mathrm{n} 57}$; die Varianz besteht also in der unzureichenden Annäherung an die Bedeutung des Grundwortes: Der Charakter dieser Bildungen wird umschrieben mit der Formel "BA + -lich $=$ 'etwas BA'" .

$\$ 3$ S. dazu DEUTSCHE WORTBILDUNG 1975. S.130f; natürlich handelt es sich hierbei nicht
um eine systematische Zusammenstellung aller Restriktionen, sondern um eher zufälig zu-
sammengetragene, die noch einer genaueren Untersuchung bedürfen. Die im Bereich der
nomina appellativa in Frage kommenden Bildungen konnen in mehrere semantische Gruppen
zusammengefaßt werden; vgl. dazu die Einteilung bei KLIMASZEWSKA 1983. S.46f, die sich
großtenteils an Wellmanns Aufzählung anlehnt (DEUTSCHE WORTBILDUNG 1975. S.130).
Um eine Einteilung ihrer Belege in Sachgruppen hat sich auch RAMSTEIN 1969 (S.37-59) bei
ihrer Auswertung des Diminutivgebrauchs in Prosawerken des 19. und 20.Jh.s bemüht.

st ERBEN 1980. S.170.

ss Vgl. zu diesem Bildungstyp die Darstellung von SCHWARZ 1905. S.7-24 sowie die Studie von SPYCHER 1955-1957, bes. 1956. S.452 und 1957. S.420.

\$6 GRIMM 1831. S.687.

${ }^{57}$ FLEISCHER 1974. S.272; SEIDEL-SLOTTY 1947 (S.48) spricht hier von einer "Milderung der Eigenschaften".

s Vgl. DEUTSCHE WORTBILDUNG 1978. S.412 (BA= Basisadjektiv); ERBEN 1983 (S.95f) ordnet diese deadjektivischen Derivate mit dem Inhaltsmerkmal '(nur) annähernd, nicht in vollem Maße' der Gradation zu. Es fallt auf, daß von einer Adjektivdiminution expressis verbis nicht mehr gesprochen wird, der Terminus Diminutiv bleibt sowohl im Polnischen als 
Der nur schwach vertretene Typus der Restriktivbildungen im Vergleich zu den produktiven -lich-Konstruktionen in verschiedenen anderen Funktionen wird hauptsächlich von Farbbezeichnungen (bläulich, rötlich) und Bezeichnungen einer sonstigen Eigenschaft, besonders von viel gebrauchten Personenmerkmalen (süßlich, ärmlich, kränklich), fast regelmäßig mit Basisumlaut, gebildet. ${ }^{39}$

Neben den -lich-Ableitungen, die den polnischen Formen auf -awy, den Detensiva, gleichgesetzt werden können, beginnen immer mehr Bildungen mit den Formantien -oid (anarchoid) und quasi- (quasiofriziell) in Gebrauch zu kommen, deren Wertindikator mit 'beinahe BA' zu umschreiben ist. ${ }^{60}$

Ganz zu fehlen scheinen in der deutschen Sprache die den polnischen Intensiva entsprechenden Suffixderivate. Der Steigerung und Verstärkung signalisierende Wert dieser Formative hat sich auf andere sprachliche Mittel verlagert; er wird im Deutschen mittels der Präfixe, z.B. ur-gemütlich, und präfixartig gebrauchter Kompositionsglieder, z.B. erz-dumm, blut-jung, stock-dunkel, schnee-weiß, wiedergegeben. ${ }^{61}$

Von der Wortbedeutung her gesehen gibt es auch im Deutschen nicht nur Diminutive, die mit Hilfe des obigen Registers der quantifizierend-qualifizierenden Suffixe gebildet werden, sondern auch solche, die durch semantisch "äquivalente" lexikosyntaktische Syntagmen zustande kommen. Wie bei dem ersten, dem sog. synthetischen Diminutionstyp, so kann auch der analytische entsprechend dem gegebenen adjektivischen Modifikationswert des Suffixes sowohl zur objektiven Größen- bzw. Umfangsangabe als auch zum Ausdruck der Gefühle und Affekte genutzt werden: kleine Tür statt Türchen oder niedliches Tier statt Tierchen. Besondere Ausdrucksintensivierung kann durch Kombination beider, des synthetischen und analytischen Verfahrens, erreicht werden: kleines Türchen oder niedliches Tierchen.

Soweit zur Konfrontation der vornehmlich formalen Verhältnisse der Diminutivbildung in beiden Sprachen. Die nun folgenden grundsätzlichen Überlegun-

auch im Deutschen auf die Suffixderivate der Substantive beschrănkt.

so $\mathrm{Vgl}$. dazu die Angaben in DEUTSCHE WORTBILDUNG 1978. S.412. Kontrastiert man diese Ergebnisse der geschriebenen mit denen der gesprochenen Sprache, so wird die Seltenheit dieses Musters auch hier bestätigt, s. GERSBACH/GRAF 1985. S.558f und 598.

${ }^{\infty}$ Hierzu vgl. DEUTSCHE WORTBILDUNG 1978. S.412 und ERBEN 1983. S.96.

61 Zum Ausdruck eines höheren Grades, der Intensivierung einer Eigenschaft vgl. ERBEN 1980. S.168, Anm.688 sowie SEIDEL-SLOTTY 1947. S.48f. 
gen zur semantischen und pragmatischen Seite können für beide Sprachen gemeinsam angestellt werden.

\subsection{DER SEMANTISCH-PRAGMATISCHE ASPEKT DES DIMINUTIVS}

Es war bereits im Zusammenhang mit der Forschungskontroverse zur Bedeutungs- und Funktionsentwicklung der Diminutive im Laufe der Sprachgeschichte die Rede von der engen Verflechtung der beiden Hauptfunktionslinien der Modifikationsderivate, einerseits der Angabe einer Umfangsminderung, andererseits des Ausdrucks einer emotional-expressiven Wertung. Unter der Maßgabe, daß Sprache nicht nur dem Zweck des nüchternen Informationsaustausches dient, vielmehr auch Mittel persönlichen Ausdrucks ist, ${ }^{1}$ bietet jene Seite der affektischen Qualifizierung dem Sprecher Möglichkeiten, sein individuelles Verhältnis zum Gegenstand bzw. zur Situation anzuzeigen und/oder den Leser/ Hörer emotional zu beeinflussen. Diese Art der Einflußnahme bildet schon ein Grundthema der antiken Rhetorik, die drei Grade der Persuasion kennt: das docere, verstanden als der intellektuelle Weg, sowie delectare und movere als den affektischen Aspekt der Redeintention. ${ }^{2}$ Während das delectare lediglich auf die sanften Affektstufen zurückgreifend "Sympathie des Publikums mit dem Redegegenstand (im Parteisinne des Redners) und mit dem Redner selbst ${ }^{n 3}$ erreichen soll, erzeugt das movere eine "seelische Erschütterung des Publikums"4. Als gedanklich-sprachliches Mittel der Pathoserregung wird die Form der amplificatio empfohlen, die "eine im Parteiinteresse vorgenommene gradmäBige Steigerung des von Natur aus Gegebenen durch die Mittel der Kunst"s beabsichtigt. Dieser Begriff gibt einen Rahmen ab, der auch für das Phänomen der Diminution brauchbar gemacht werden kann, insofern mit ihm eine Hervorhebung der Aussage erzielt werden soll, die nicht nur mittels Steigerung

' Genaueres zum Thema Sprachfunktion siehe im folgenden übersetzungstheoretischen Teil.

2 Zu den folgenden Erlăuterungen vgl. die Darstellung von LAUSBERG 1973. \$\$256-259.

${ }^{3}$ LAUSBERG 1973. \$257, S.141.

- LAUSBERG 1973. \$257, S.143.

3 LAUSBERG 1973. \$259. 


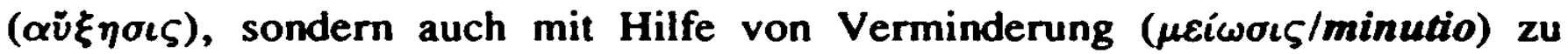
erreichen ist. ${ }^{6}$ Gemeint ist damit, daß durch die Verminderung dem Zuhörer die beabsichtigte Aussage nachdrücklicher, als wenn das Gesagte im Normalzustand (=Grundwort) belassen würde, emotional nahegebracht und in seiner Bedeutsamkeit für ihn intensiviert werden kann.

\subsubsection{Zum Begriff der Expressivitat}

Die konstitutive Emotionalität/Affektivität der Diminutive bedingt, daß sie dem sog. expressiven Wortschatz zugerechnet werden' und damit spezifische Funktion als stilistische Mittel erlangen können. ${ }^{8}$ Vorab Erläuterndes zu den Begriffen Expressivität/Emotionalität: Das Phänomen der sprachlichen Expressivität, das im psychologisch-stilistischen Grenzbereich angesiedelt wird, gehört hinsichtlich seiner Stellung, die es in sprachwissenschaftlichen Betrachtungen einzunehmen hat, zu den wohl umstrittensten Problemen dieser Disziplin. Während Untersuchungen zur Expressivität, die aus dem Gebrauch bestimmter sprachlicher Mittel resultiert, seit jeher literaturwissenschaftliche Theorien beschäftigen, beginnt die theoretische Erfassung seitens der Sprachwissenschaft erst mit F.de Saussures Schüler Charles Bally. Mit seinem bis heute gültigen, fundamentalen Werk machte er u.a. auf jene affektischen Elemente aufmerksam, die bereits im Sprachsystem verankert sind. Damit war ein wichtiger Schritt auf dem Weg der Trennung expressiver Zeichen vom expressiven Gebrauch der Zeichen getan. 9 Seitdem haben sich in Abhängigkeit vom Ansatz verschiedene

\footnotetext{
- LAUSBERG 1973 bezieht sich dabei u.a. auf QUINTILIANUS 1975, bei der Steigerung auf Buch VIII. Kap.4.3, bei der Abschwăchung auf Buch VIII. Kap.4.28.

'In diesem Sinne außer sich u.a. SIEBERER 1950-52 (S.86): "Wir konnen (...) ein von Sprache zu Sprache verschieden großes Bedürfnis voraussetzen, einen größeren Teil des Wortschatzes gleichsam in zwei Auflagen vorrătig zu haben: neben einer nüchternen eine intim, anheimelnd oder kosend gefärbte Wortschatzgarnitur" (Hervorhebungen weggelassen B.K.).
}

So z.B. HELTBERG 1964 (S.94), die konstatiert: "Mamy tu do czynienia z jednym z najważniejszych Srodków stylistycznych".

- Bally zählt zu den Begründern der Stilistik als neuer sprachwissenschaftlicher Disziplin, die verstanden wird als Erforschung sprachlicher Ausdrucksmittel einer Sprachgemeinschaft unter dem Gesichtspunkt ihres affektischen bzw. emotionalen Gehalts, vgl. z.B. BALLY o.J. Zur theoretischen Erfassung sprachlicher Expressivităt siehe BALLY 1926. speziell Kap. "Mécanisme de l'expressivité linguistique" (S.139-181). Eine Zusammenstellung seiner Forschungs- 
Konzeptionen der Betrachtung sprachlicher Expressivität herausgebildet. Jüngere Arbeiten, die die systemimmanente Vorgehensweise ablehnen, betonen die Notwendigkeit der Berücksichtigung außersprachlicher Erscheinungen beim Erfassen sprachlichen Gefühlsausdrucks, indem sie fordern:

Choc teoria ekspresji jezykowej ma ujmowac sposoby przejawiania sie ekspresywnosci $w$ jezyku $i$ w zwiazku $z$ tym sposoby powstawania $i$ funkcjonowania jezykowych znakow ekspresywnych, to jej formulowanie powinno się odbywać w kontekscie analizy peinego aktu komunikacyjnego. ${ }^{10}$

Was die inhaltliche Bestimmung der Termini angeht, herrscht auch hier offenkundige Unsicherheit. Vereinfacht dargestellt, lassen sich zwei Auffassungen, eine engere und eine weitere, herausarbeiten. Zum einen wird Expressivität lediglich mit dem Ausdruck von Emotion - als gefühlsmäßige Beurteilung der Erscheinungen verstanden - gleichgesetzt, wobei Emotionalität/Affektivität in Kontrast zum Intellektuellen auf der einen und Voluntativen auf der anderen Seite gesetzt wird." Etwas weiter gefaßt wird dieses Phänomen in Entwürfen, die den Willensproze $B$ miteinbeziehen, so daß Expressivität für sie das Resultat einer gefühls- und willensmäßigen Haltung des Sprechenden bedeutet. ${ }^{12}$ Dem steht die Überzeugung entgegen, daß Expressivität mit dem Ausdruck aller inneren (psychischen) Vorgänge des Senders, seiner Persönlichkeit, in Verbindung zu bringen ist. ${ }^{13}$ Am weitesten gehen die der Pragmatik verpflichteten Interpretationen, die jegliche individuellen Eigenschaften des Sprechers, innere und äußere, wie Alter, Geschlecht, Bildung etc. in Betracht ziehen; Expressivität ist dann die Vergegenwärtigung der Person des Senders im sprachlichen Zeichen. ${ }^{14}$

ergebnisse liegt auch in polnischer Übersetzung vor, vgl. BALLY 1966.

${ }^{10}$ GRABIAS 1981. S.17; er betrachtet das Phänomen der Expressivität im Bereich der Wortbildung.

"In dieser Form z.B. in den Arbeiten von S.Ullmann vertreten, vgl. ULLMANN 1972a. S.90ff.

${ }^{12}$ So in der klassischen Theorie von Ch.Bally verstanden, vgl. BALLY 1926. Kap.III, die auch später Grundlage für die Expressivităts-Studie lexikalischer Erscheinungen von ZIMA 1961 war.

${ }^{13}$ Expressivität in diesem Sinne hat ihre Entsprechung in BÜHLERs 1934 (vgl. S.28 passim) Terminus Ausdruck. In dieser Deutung wird dieser später auch in den Beiträgen von WIERZBICKA 1969 (vgl. bes. das Kap. "Problemy ekspresji i ich miejsce w teorii semantycznej") oder SKUBALANKA 1973a genutzt.

${ }^{14}$ Genaueres zu den angegebenen Standpunkten vgl. auch bei GRABIAS 1981. S. 19-22. 
Einen Klärungs- und Abgrenzungsversuch hat auch S.Grabias unternommen, indem er im Anschluß an T.Skubalanka, zwischen Prozeß und Eigenschaft unterscheidend, folgenden Zusammenhang sehen will:

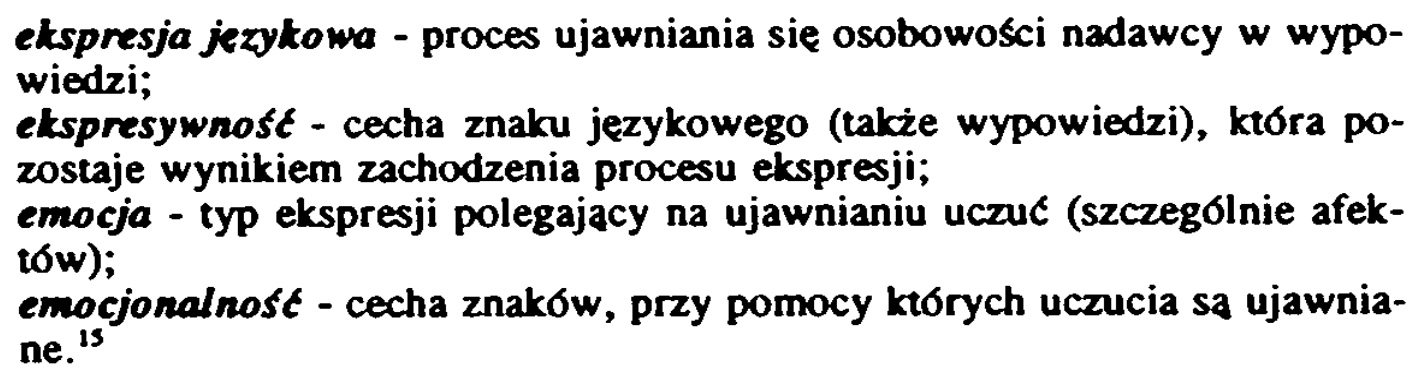

Mit dieser Festlegung sieht er die Aufgabe einer Theorie der sprachlichen Expressivität darin festzustellen, auf welche Weise die Person des Senders, im speziellen seine Empfindungen und Wertungen, in sprachlichen Aussagen zu Tage treten. ${ }^{16}$ Berücksichtigt man bestimmte Faktoren, so z.B., ob die zum Vorschein kommende Emotion Folge eines unbewußten oder bewußten und gezielten Prozesses ist, ferner, ob jene in der Bedeutung der Aussage enthalten ist oder außerhalb dieser zustande kommt, lassen sich laut Grabias theoretisch mindestens drei Typen von ekspresja unterscheiden: komunikowanie, wyrazanie und przejawianie sie osobowosci nadawcy w jezyku. ${ }^{17}$

\subsubsection{Expressive Werte in der Bedeutungsstruktur einer lexikalischen Einheit}

Jede dieser Möglichkeiten, Emotionen anzuzeigen, kann mittels einer großen Anzahl von sprachlichen Mitteln unterschiedlichster Art realisiert werden. Dem Vorhaben der folgenden Untersuchung entsprechend interessieren in erster Linie Wörter und Wortbildungsformantien, die als sprachliche Einheiten mit der beschriebenen Expressivität in Verbindung zu bringen sind. Wichtigste und umstrittenste Frage zugleich ist die nach dem Status dieser expressiven Werte innerhalb der Bedeutungsstruktur einer lexikalischen Einheit. ${ }^{18}$ Für gewöhnlich

is GRABIAS 1980. S.479.

16 Diesem Verstăndnis von Expressivităt will sich auch diese Arbeit anschließen.

17 Vgl. dazu die Erläuterungen in GRABIAS 1981. S.26-28.

18 Der andauernden Diskussion um die unterschiedlichen Auffassungen vom Wesen der Wortbedeutung bewußt, soll hier nur ein für diese Untersuchung zweckmāßiger Bedeutungsbegriff als Arbeitsgrundlage übernommen werden. 
wird davon ausgegangen, daß sich die Bedeutung von sprachlichen Ausdrücken als Einheiten des Lexikons aus einem begrifflichen Kern und verschiedenen "Mit- und Nebenbedeutungen", die häufig auch unter dem Stichwort Konnotationen $^{19}$ zusammengefaßt werden, konstituiert. Kontrovers aber und bis heute uneinheitlich interpretiert ist das Problem dieser "zusätzlichen", nichtbegrifflichen Komponenten. Ohne auf das breite Spektrum der Positionen im einzelnen eingehen zu können, soll es genügen zu erwähnen, daß die Bandbreite vom völligen Ausschluß der nichtbegrifflichen Elemente von den am Aufbau der Bedeutung eines Wortes beteiligten Größen - unter ihrer Deutung als Erscheinung des Gebrauchs - bis hin zu einer sehr weit angelegten Bedeutungsinterpretation reicht, deren Vertreter jegliche Informationen außerhalb des begrifflichen Inhalts zur lexikalischen Bedeutung zählen.

Fruchtbare Anknüpfungspunkte lassen sich in K.O.Erdmanns Entwurf finden, der neben dem "begrifflichen Inhalt" die Ausdrücke Nebensinn und Gefiihlswert (bzw. Stimmungsgehall) geprägt hat. ${ }^{20}$ Nebensinn, mit welchem "Resultate unterschiedlicher begrifflicher Verarbeitung der Wirklichkeit" erfaßt werden, und Gefühlswert, gedeutet als Ergebnis "unterschiedlicher emotionaler Stellungnahmen ${ }^{21}$, werden nicht erst durch den Redekontext erzeugt, vielmehr bringt sie das Wort selbst neben dem begrifflichen Inhalt als Vorgabe in die Gebrauchskontexte ein.

In diesem Sinne kann auch Th.Schippans Verständnis vom Strukturaufbau der lexikalischen Einheit gedeutet werden, wenn sie mit den Begriffen Wertungspotenz und Gefühlswert wertende und emotionale Merkmale als semantische

\footnotetext{
${ }^{10}$ Um diesen so vieldeutig verwendeten Ausdruck hat sich eingehend RÖSSLER 1979 bemüht, indem sie zunächst seiner verschiedenartigen Verwendungsweise in der Sprachphilosophie, Literaturtheorie und Sprachwissenschaft nachgegangen ist, um dann eine Konnotationssystematik zu entwerfen. Auch DIECKMANN 1979, der den gegenwärtigen Diskussionsstand als "chaotisch" bezeichnet, hat einen Versuch unternommen, den unter dem Stichwort Konnotation zusammengetragenen komplexen Sachverhalt, die - wie er selbst sagt - entstandene "linguistische Rumpelkammer", wenigstens teilweise zu ordnen, vgl. bes. S.18-48.
}

$20 \mathrm{Vgl}$. die Studie von ERDMANN 1910 (S.103-126), die erstmals schon 1900 veröffentlicht wurde und für beinahe alle nachfolgenden Forscher, die sich mit der Bedeutungslehre befaßt haben, hinsichtlich der Bedeutungsdifferenzierung zum Vorbild wurde. Er definiert wie folgt: "ich verstehe unter dem Nebensinn alle Begleit- und Nebenvorstellungen, die ein Wort gewohnheitsgemäß und unwillkürlich in uns auslost; unter dem Gefühiswert oder Stimmungsgehalt alle reaktiven Gefühle und Stimmungen, die es erzeugt" (S.107).

21 DIECKMANN 1979. S.8; im Rahmen der Konnotationserforschung hat sich dieser erneut mit den Thesen Erdmanns und seiner Rezeption auseinandergesetzt und dabei festgestellt, daß die Wiedergabe der Erdmannschen Dreiteilung in begrifflicher Inhalt, Nebensinn, Gefühlswert häufig falsch oder mißverständlich erfolgt ist. 
Gegebenheiten festsetzt. ${ }^{22}$ In diesem Konzept, das auf der Erkenntnis der unterschiedlichen Determiniertheit der einzelnen Wortinhalte beruht, sind die genannten Bestandteile von weiteren, über diese hinausgehenden informativen Elementen, die mit dem Lexem verbunden sind, zu trennen. Denn zum einen sind die Wortinhalte "Abbilder des widergespiegelten Objekts, des Bezeichneten, und zwar Abbilder in ihrer Einheit von Rationalem und Emotionalem "23 - was der signifikativen Bedeutung ${ }^{24}$ entspricht -, zum anderen aber sind sie "Abbilder der Wortverwendung"2s. Diese "Mitinformationen", die in der Literatur unter verschiedenen Begriffen subsumiert werden: stilistische Bedeutung, pragmatische Bedeutung, kommunikative Bedeutung, Konnotationen etc. ${ }^{26}$, besitzen gegenüber den ersten eine andere Qualität und werden als "gesellschaftlich invariante Abbildelemente verschiedener Faktoren kommunikativer Ereignisse, kommunikativen Handelns ${ }^{\text {"27 }}$ verstanden.

So umstritten und unzulänglich die Beschreibung der stilistischen Bedeutung als Markierung des Verwendungsbereichs und der Verwendungsrestriktionen im einzelnen auch sein mag, kann doch als Anhaltspunkt die von E.Riesel und E.Schendels vorgenommene Dreiteilung der "absoluten stilistischen Bedeutung" genutzt werden:

- die funktionale Komponente der Stilfarbung markiert die Zugehörigkeit eines Wortes zu einer bestimmten kommunikativen Sphäre

- die normative Komponente der Stilfärbung gibt den Punkt über (gewăhlt, geschwollen) bzw. unter (lit.-umgangssprachlich, salopp-umgangssprachlich, vulgär) der Grundnorm (normalsprachlich $=$ "Stilfärbung, die von der bildungstragenden Schicht in allen Stilen

2 Sie definiert diese wie folgt: "Dabei soll unter Werrungspotenz die Eigenschaft verstanden werden, daß in das verallgemeinerte ideelle Abbild, das sich auf ein Formativ bezieht, gesellschaftliche Wertungen eingeschlossen sind, die aus gesellschaftlichen Erfahrungen mit dem Bezeichneten gewonnen werden und als verkürzte 'eingefrorene' Werturteile charakterisiert werden kőnnen" und weiter "wir meinen mit Gefühlswert eines Lexems seine Potenz, emotionale Einstellungen, die die Gesellschaft oder Gruppen zum Bezeichneten haben, auszudrücken", SCHIPPAN 1979. S.681.

2 SCHIPPAN 1979. S.683.

24 Zur Opposition denotative vs. signifikative Bedeutung vgl. APRESJAN 1963. S.106.

is SCHIPPAN 1979. S.683.

${ }^{26}$ Im weiteren Verlauf der Arbeit wird auf den Ausdruck stilistische Bedeutung zurückgegriffen.

n7 SCHIPPAN 1984. S.156f.

2 Gemeint sind damit die sog. Funktionalstile, Genaueres dazu siehe unten S.124ff. 
als Nullfärbung, als neutrale Basis empfunden wird") auf der Hőhenskala der Ausdrucksschattierungen an;

- die expressive Komponente der Srilfärbung wird lediglich als Opposition expressives vs. nichtexpressives Ausdrucksmittel verstanden, ohne an dieser Stelle schon die Art der Expressivităt angeben zu kőnnen. ${ }^{20}$

Eine etwas stärker untergliederte Auffassung von der stilistischen Bedeutung, die die kommunikativen Bedingungen der Wortverwendung angibt, zeigt sich in Schippans Ansatz. Folgende Informationen sieht sie hier zusammengefaßt: ${ }^{30}$

- die emotionalen Bedingungen des Wortgebrauchs" "bilden sowohl die emotionale Einstellung des Senders zum Denotat ab als auch die 'kommunikative Hohenlage', die Beziehungen zwischen den Kommunikationspartnern" ${ }^{\text {"32. }}$. Anders als Riesel/Schendels ${ }^{33}$ sieht sie bereits im kontextunabhängigen Lexem differenzierte Bewertungskomponenten enthalten; es sind über die positive/negative Haltung hinausgehende Spezifizierungen wie scherzhaf, ironisch, zärtlich, abwertend u.a.;

- die kommunikative Ebene des Wortgebrauchs, die die Kommunikationssituation kennzeichnet, vgl. ungezwungen, salopp, formlich, offiziell u.a.;

- die Funktionsbereiche des Wortgebrauchs, wie z.B. administrativ, fachsprachlich usw.; - die soziale Geltung des Wortgebrauchs, womit die Zugehörigkeit zur Lexik einer bestimmten sozialen Gruppe angezeigt wird;

- die regionale Bindung des Wortgebrauchs;

- die zeilliche Gebundenheit des Wortgebrauchs;

- die politisch-weltanschauliche Bindung des Wortgebrauchs;

- die Modalitäten der Kommunikativen Handlungen und der Wortgebrauch.

Erst eine Zusammenschau aller oben aufgezählten Merkmale kann eine Gesamtcharakterisierung eines kontext- und situationsunabhängigen sprachlichen Zeichens gewährleisten. Die in den beschriebenen Ansätzen vorgenommene Zweiteilung der Bedeutungselemente hat eher theoretischen Charakter. Sie ist im

$29 \mathrm{Vgl}$. RIESEL/SCHENDELS 1975 (S.29-34), die diese Einteilung im Rahmen ihrer Erstellung einer deutschen Funktionalstilistik vornehmen. In diesem Sinne kann man auch DIECKMANN 1979 (S.39f) verstehen, wenn er sagt: "Die stilistischen Wirkungen beruhen auf dem Wissen der Sprachbenutzer von dieser Heterogenităt und den Geltungsbereichen der Wörter in den verschiedenen Dimensionen der Variabilităt (geographisch, sozial, funktional etc.)" und fortfährt "Gefühlswerte o.a (...) kommen erst dann ins Blickfeld, wenn man berücksichtigt, daß die Sprecher nicht nur ein Wissen über die Zugehörigkeit bestimmter Sprachmittel zu bestimmten Kommunikationsbereichen haben, sondern diese Kommunikationsbereiche bzw. die Sprechergruppen auch bewerten."

30 $\mathrm{Zu}$ den folgenden Ausführungen der - in ihrer Terminologie - Konnotationen vgl. SCHIPPAN 1984. S.157-160.

31 Diese Markierung ist zu unterscheiden von der oben angeführten emotional-wertenden Bedeutung.

${ }^{2}$ SCHIPPAN 1984. S.158.

3 Auch GRABIAS 1981 glaubt bei der nichtaktualisierten Wortschatzeinheit, die Haltung des Sprechers "jedynie w ogolnych kategoriach aprobaty lub dezaprobaty, tj. uczuc dodatnich albo ujemnych" (S.38) angeben zu können. 
Einzelfall nicht so strikt durchzuführen, ist lediglich von heuristischem Interesse, denn jene zusätzlichen Inhaltskomponenten (= stilistische Bedeutung) sind keineswegs additiv mit der signifikativen Bedeutung verbunden, vielmehr beeinflussen und bedingen beide Seiten einander. Zu unterstreichen ist schließlich, daß alle genannten Bedeutungselemente, die expressiven Größen in Grabias' Sinne eingeschlossen, als Inhaltskomponenten des Langue-Wortes (lexikalische Bedeutung) lediglich potentielle Eigenschaften sind, die erst im Sprachgebrauch individuell realisiert werden, so daß in immer neuen Kombinationen eine aktualisierte Variante (aktuelle Bedeutung) möglich wird. ${ }^{34}$ Entscheidend ist dabei die Betonung des "dynamischen" Charakters der Bedeutungsstruktur; in diesem Sinne äußert sich auch R.Stolze:

Die Wortbedeutung [wird - B.K.] nicht als fixierter Schnittpunkt einer bestimmten Menge von Bedeutungsmerkmalen verstanden, sondern als ein elastischer Gesamtkomplex aus semantischen und pragmatischen Merkmalsdimensionen, deren wandelbare Konstellationen jeweils auf die Textebene projiziert werden. ${ }^{35}$

\subsubsection{Expressivität und die Formantien}

Soweit zum Themenkreis Expressivität auf lexikalischer Ebene, nun noch einiges zur Expressivität auf morphologischer Ebene. Bedenkt man, daß es im Bereich expressiver Wörter unterschiedliche Mittel zur Erzeugung von Expressivität gibt, dann kann man mit Grabias grundsätzlich unterscheiden zwischen: wyrazy o ekspresywności implicytnej und wyrazy o ekspresywności eksplicytnej. ${ }^{36}$ Zur letzteren Gruppe zählt u.a. der hier interessierende formal motivierte Typ, jener mit expressiv markierten Morphemen bzw. Phonemen. Auch wenn die Existenz expressiver Wortbildungsmittel in der Forschung anerkannt wird (die Gegenposition ist ebenso vertreten), bleibt deren Begriffsbestimmung unscharf. Um eine Präzisierung dieses expressiven Wertes hat sich Grabias bemüht, indem er Typen von durch Formantien ${ }^{37}$ getragener Expressivität zusammen-

\footnotetext{
*3 $\mathrm{Zu}$ der Unterscheidung lexikalische vs. aktwelle Bedeutung vgl. SCHMIDT 1963.

35 STOLZE 1982. S.104.

$36 \mathrm{Vgl}$. GRABIAS 1981. S.39f.

37 GRABIAS 1981 betrachtet auch Formantien als eigenständige semiotische Zeichen, die sich in syntaktischen, semantischen und pragmatischen Kategorien beschreiben lassen; dementsprechend unterscheidet er für den Bereich der substantivischen Wortbildungsmittel struktural-
} 
zustellen versucht hat. Eliminiert man jeglichen Einfluß des äußeren und inneren Kontexts $^{38}$, um so lediglich die alleinige expressive Beschaffenheit der Formantien zu berücksichtigen, dann lassen sich gegeneinander abgrenzen: formanty melioratywne, formanty pejoratywne und die Übergangsgruppe formanty melioratywno-pejoratywne.

Zur Klasse der meliorativen Formantien werden Suffixe gezählt, die Hypokoristika bilden. Berücksichtigt man das Verhältnis, welches zwischen dem emotionalen Wert und anderen Bedeutungskomponenten besteht, die die Formantien einbringen, dann lassen sich zwei Unterklassen bilden: 1. Jene Suffixe, die ausschließlich expressive Qualitäten in das unverändert gebliebene Grundwort einbringen; sie dienen allein der Pragmatik, semantische Funktionen erfüllen sie nicht: dazu gehören u.a. -cia (mamcia), -uńcio (taturício), -unia (czekoladunia), -us (chlebus); ${ }^{39}$ 2. Suffixe, die neben dem expressiven Wert auch intellektuelle, insbesondere diminutivische (Element der Kleinheit) Faktoren enthalten, dazu zählen die polyfunktionalen Formantien -ek, -ik, -ka, -ko in diminutivischer Funktion (mit Ausnahme derjenigen, die nur als Anzeiger der reinen Verminderung dienen), ferner Morpheme, die zusammengesetzte Diminutive bilden, z.B. eczka, -ulko, und letztlich das Suffix -ątko. ${ }^{20}$ SchlieBlich ist dieser Klasse auch die Gruppe derjenigen Formantien, deren expressive Beschaffenheit zwischen Meliorativität und Pejorativität pendelt, zugeordnet worden, vgl. -ina, -ula, -ys, -ak u.a. ${ }^{41}$

Natürlich ist die Beachtung expressiver Markierung von Suffixen für die emotionale Aussage eines Derivats unerläßlich, doch in gleichem Maße zu beachten (in vielen Untersuchungen vernachlässigt) ist der Einfluß der sich mit

ne, semantyczne und pragmatyczne funkcje formantu, letztere mit den Komponenten: modal$n y$, emocjonalny ("wskazuje na aprobate (melioratywnosc), dezaprobate (pejoratywnosc) lub postawe emocjonal nie ambiwalentna", S.46); vgl. S.46-53.

3* Innerer Kontext ist als Wortbildungskontext $2 \mathrm{u}$ verstehen, d.h. als das Verhältnis, das zwischen Basis und Formans entsteht.

39 Aus dem 134 Formantien umfassenden Inventar, das 72 meliorative und 62 pejorative Einheiten enthält, sind hier nur diejenigen, die zur Bildung von Modifikationsderivaten dienen und zum Themenbereich Diminutive gehören, aufgezăhlt; die vollständige Zusammenstellung vgl. in GRABIAS 1981. S.71-74.

* Seine Formansbeobachtungen hat GRABIAS 1981 gleichzeitig genutzt, um Stellung zum ewigen Streitthema Diminutiv vs. Hypokoristikum zu nehmen, vgl. oben S.43, Anm. 11 .

¿Zur Klasse der hier nicht interessierenden pejorativen Formantien vgl. GRABIAS 1981. S.66-69. 
dem Formans verbindenden Basis. Je nach Kombination von Grundwort und Wortbildungsmorphem können bei der Modifikation verschiedene Schattierungen der Expressivität realisiert werden. Eine positive Wertung der Erscheinungen wird erreicht durch: 1 . Verbindung emotional neutraler Wörter mit meliorativen Formantien, 2. Zusammentreffen positiv markierter Grundwörter mit meliorativen Formantien. Beim letztgenannten Fall handelt es sich um eine Art Intensivierung des Gesagten. Für den ProzeB der negativen Wertung gelten die gleichen Bedingungen mit umgekehrter Wertungsrichtung. Erscheinungen der Ironie werden möglich durch eine paradoxe Konstellation, zum einen negativ markierte Basislexeme mit meliorativen Formantien, zum anderen positiv markierte $\mathbf{A b}$ leitungswörter mit pejorativen Suffixen. Und schließlich die äußerst komplizierte, weil vor allem im Metasprachlichen angesiedelte Möglichkeit, Komik und ähnliche expressive Nuancen auszudrücken. ${ }^{42}$

\subsubsection{Die Bedeutungskomponenten des Diminutivs}

Faßt man jene Ausführungen der vorangegangenen Abschnitte zusammen, dann zeigt sich, daß die Bedeutungsstruktur eines Diminutivs auf Sprachsystemebene aus der Kombination der jeweiligen Bedeutungskomponenten des Grundwortes und derjenigen des Suffixes resultiert. Mit der Zugehörigkeit des Diminutivs zum Wortbildungstyp der Modifikation hängt zusammen, daß der begriffiche Kern der motivierenden Basis, der das Denotat widerspiegelt, ohne Veränderung auch die begriffliche Grundlage des Diminutivs bildet: Grundwort und Derivat unterscheiden sich nicht hinsichtlich dessen, was bezeichnet wird, vielmehr wie, in welcher subjektiven Form, es vom Sprecher dargestellt wird. Demzufolge sind neben dem begrifflichen Inhalt folgende Bedeutungsbestandteile maßgeblich am Aufbau beteiligt: ${ }^{43}$

Signifikante Stellung kommt dem Merkmal klein zu. Der spezifische Charakter dieser sprachlichen Einheit erlaubt es, sie in die Nähe der wertenden Komponente zu rücken. Es ist anzunehmen, daß damit, ohne das Anzeigen eines objektiven Kleinseins völlig auszuschließen, vordergründig ein auf subjektiver Einschätzung beruhende Kleinheit ausgedrückt werden soll.

\footnotetext{
2 Im einzelnen dazu GRABIAS 1981. S.171-175.

* Zum folgenden vgl. auch TENNERTs 1982 (S.61-77) semantische Analyse des Diminutivs.
} 
Eine ebenso tragende Säule der Diminutivbedeutung stellt die wertende Komponente dar. Durch sie kann eine spezifische, aus der Sicht eines bestimmten Sprechers in einer bestimmten Situation vorgenommene, sei es positive, sei es negative Bewertung der genannten Person, der Dinge bzw. Situationen erfolgen. Häufig bestimmen diese Wertung nicht unbedingt die tatsächlichen Eigenschaften des zu bewertenden Objekts, vielmehr beruht sie auch auf bestimmten Bewußtseinszuständen des Sprechenden, Emotionen unterschiedlichster Art, von Liebe über Mitgefühl und Neid bis zur Verachtung. Damit ist auch schon der nächste, mit dem wertenden auf das engste verknüpfte Faktor, die emotionale Komponente, erwähnt. Doch ist wohl nicht davon auszugehen, daß das Diminutiv bereits auf Langue-Ebene über eine exakte emotionale Differenzierung verfügt. Sinnvoller ist es, lediglich eine Richtungsangabe, meliorativ oder pejorativ, anzusetzen. Durch das emotional-wertende Bedeutungselement erfährt man also eher etwas über die Gefühlshaltung des Sprechers als über besondere Charakteristika des bezeichneten Objekts. Diesem nicht unwesentlichen Umstand ist vor allem in Hinblick auf den Übersetzungsvergleich Rechnung zu tragen. Der Bezug, bei dem sich die Wertung auf das Denotat richtet, erweist sich als nur eine von mehreren Möglichkeiten. In vielen Fällen zeigt die jeweilige Realisierung im Text, daß das durch das Diminutiv bezeichnete Objekt lediglich Stellvertreterfunktion für die eigentliche, aus dem Kontext erst zu erschließende Bewertung eines ganz anderen Gegenstandes, einer Situation übernimmt.

$\mathrm{Zu}$ den festen Bedeutungsbestandteilen zählt schließlich die stilistische Komponente. Auch wenn sie mit den beiden letztgenannten Elementen in einem starken Wechsel- und Abhängigkeitsverhältnis steht, ja sogar auf ihnen beruht, ist sie aufgrund ihres breiteren Situationsbezugs gesondert zu berücksichtigen. Um den Vorschlag von Th.Schippan hier aufzugreifen, liefert diese Größe insbesondere Informationen über die "emotionalen Bedingungen", die "kommunikative Ebene" und die "Funktionsbereiche" des Wortgebrauchs der sprachlichen Einheit. ${ }^{44}$ Damit wären die wichtigsten Bedeutungskomponenten eines Diminutivs skizziert. ${ }^{\text {ss }}$ Selbstverständlich sind sie nur als potentielle

4 Vgl. weiter oben S.76.

4s Im Unterschied hierzu vgl. REITERs 1988 (S.662f) Einschätzung, der sowohl Kleinheit als auch subjektive Einschätzung und Emotionalität nicht als semantische Inhalte eines sprachlichen Zeichens anerkennen will. Die beiden letztgenannten faßt er vielmehr - im Gegensatz zur traditionellen Diminutivforschung - als Komponenten der Redestrategie auf, des "Prinzips, nach dem die Auswahl der Zeichen für die Mitteilung gesteuen wird" (S.662). Mit dem Faktor subjektive Einschätzung ist die Vermittlung der jeweils individuellen Sicht des Sprechers gemeint, die gleichzeitig stets hörerorientiert ist. Emotionalität in den Varianten Zärtlichkeit. 
Möglichkeiten aufzufassen, die, jeweils den Bedingungen des Textes unterstellt, unterschiedlich aktualisiert werden können.

Diese Andeutungen weisen bereits darauf hin, daß die lexikalische Bedeutung eines sprachlichen Zeichens zwar ein notwendiges, nicht aber hinreichendes Kriterium für seine inhaltliche Leistung auf der Ebene der Sprachverwendung darstellt. Insbesondere in Hinblick auf den durchzuführenden Übersetzungsvergleich erscheint es unabdingbar, vom Text und nicht nur der isolierten sprachlichen Einheit als primär interessierender Erscheinung auszugehen. Dementsprechend wird eine Beurteilung des diminutivischen Wertes an gegebener Stelle nur im Zusammenhang des das Diminutiv umgebenden Textes, im Redeakt, dort, wo Sprache sich manifestiert, möglich sein. Erst mit dieser Einbettung ist die Voraussetzung für das Verständnis der getroffenen Aussage geschaffen. ${ }^{46} \mathrm{Mit}$ dieser dialektischen Interdependenz von sprachlichen Zeichen und Text ergibt sich eine vielfältige Determiniertheit der sprachlichen Äußerung. Wenn man nun Text/Textsegmente als "Beziehungsgeflecht aus Information, Intention und Situation ${ }^{\text {47 }}$ betrachtet, dann erscheint es zweckmäßig, für das Entschlüsseln der Leistung eines Diminutivs den von H.Schmidt geprägten Begriff der stilistischen Information einzuführen. ${ }^{48}$ Darunter sind jene vom Text signalisierten Informationen zu verstehen, die Aufschluß über die Kommunikationsbedingungen geben, im einzelnen über den Kommunikationssituationsbezug und das Kommunikationsziel an der jeweils gegebenen Textstelle. ${ }^{49}$ Als Kategorie der Sprachverwendung beruht die stilistische Information zunächst auf den oben zusammengestellten Komponenten der lexikalischen Bedeutung, besonders der stilistischen Komponente. Doch erst die Ergänzung dieser Ebene, bedingt durch die Wech-

Ironie usw. versteht er dann als "Mutmaßungen des Hơrers über die Mitteilungsabsicht des Sprechers" (S.663).

* So ist auch bei GAUGER 1971 zu lesen: bei der Diminution ist, "wie vielleicht bei keiner anderen Erscheinung, der Kontext, die Situation von größter Wichtigkeit, denn hier wird sehr oft die Beziehung zwischen Sprecher und Horer und die Beziehung beider zur beredten Sache bestimmend", sie kann deshalb "nur innerhalb konkreter Äußerungen als das, was sie eigentlich ist und leistet, beurteilt werden" (S.108).

47 STOLZE 1982. S.23.

48 Vl. dazu die Darlegungen in SCHMIDT 1979. S.65-78. Etwas ungünstig ist die Terminuswahl, stilistische Information darf nicht verwechselt werden mit dem oben festgelegten Bedeutungselement des Langue-Wortes, der stilistischen Komponente.

*o Genauer zu diesen Begriffen vgl. unten S.122ff. 
selwirkung zwischen Textelement und einrahmendem Kontext, sprachlicher wie außersprachlicher Art, macht die Dimension der stilistischen Information aus.

\subsubsection{Die inhaltlichen Leistungen des Diminutivs}

Zuletzt ist noch ein Blick auf die in den morphologischen Mitteln angelegte Vielfalt des Ausdrucks zu werfen. Vorab kann festgehalten werden, daß die beiden Funktionsbereiche des Diminutivs als Grenzmarkierungen auf einer Bedeutungsskala vorzustellen sind, Kleinheit etwa als Anfangspunkt, subjektive Wertung als Endpunkt. Dazwischen können weitere, aus Mischformen dieser beiden sich ergebende Funktionsmöglichkeiten angesiedelt werden. Die Übergänge sind jeweils fließend, nicht statisch zu verstehen. ${ }^{30}$ Bei der Anzeige von Kleinheit ist zu unterscheiden, ob mit der Darstellung der Verminderung eines Sachverhalts, meist eines Gegenstands oder Lebewesens in seinem Umfang, Dauer u.a. ein absolutes und normiertes $\mathrm{Ma} ß$ innerhalb eines homogen herausgebildeten Größensystems zum Tragen kommen soll oder die Kleinheit eher als subjektive, sprecher- und situationsabhängige Darstellung gemeint ist. Der erste Fall, bei dem die Möglichkeit einer subjektiven Einschätzung ausgeschlossen ist, muß somit allen übrigen Funktionsfeldern gegenübergestellt werden, hier ist die klein-Komponente verbindliches und konstitutives Merkmal der Wortbedeutung. ${ }^{\text {s1 }}$ Beim engegengesetzten Funktionsbereich, der subjektiven Wertung, fehlt das Kleinheitselement völlig, er trifft für alle nichtverkleinerungsfähigen Lexeme, besonders Abstrakta, zu. Hier, wo eine subjektive Einschätzung und

\footnotetext{
so $\mathrm{Vgl}$. hierzu die schematische Darstellung bei TENNERT 1982. S.38f.

si In diesen Zusammenhang paßt die Diminutivinterpretation von REITER 1988, der die vielfach angenommene Kleinheitshypothese verwirft. Er sieht das Prinzip der Diminuierung nicht in der Angabe einer meßbaren absoluten Größe als vielmehr in der "Regulierung der Perspektive", d.h. der Positionierung eines darzustellenden Objekts hinsichtlich seiner Bedeutsamkeit in Relation zu seinem Bezugsbereich. Im Diminutiv sieht er eine Erweiterung der Perspektive, im Pendant, dem Augmentativ, eine Verengung. Ausgangspunkt dieses Perspektivbzw. Anteiligkeitskonzepts war der von ihm in Märchen beobachtete Wechselgebrauch von DIMINUTIVUM, AUGMENTATIVUM und NORMATIVUM in ein und derselben Textpassage für ein Objekt.
} 
persönliche Anteilnahme am Gesagten, eine Sprecherperspektive indiziert werden soll, können die mannigfaltigsten Gemütsbewegungen signalisiert werden. ${ }^{52}$

Beim Sichten der zunächst nach rein formalen Kriterien exzerpierten Beispiele zeigt sich, daß sich eine Vielzahl der Bildungen nicht ohne Schwierigkeit den obigen Funktionsfeldern zuordnen läßt. Dazu zählen besonders jene Lexeme, die zwar mit typisch diminutivischen Formantien versehen sind, jedoch keine spezifisch modifizierenden Eigenschaften mehr besitzen, und infolgedessen für sie in der Fachliteratur der Terminus deminutywa formalne ${ }^{53}$, im Gegensatz zu den formacje aktualnie deminutywno-ekspresywnest geprägt worden ist. Jene Bildungen haben teilweise oder gänzlich ihren diminutivischen Bezug zum ursprünglich motivierenden Substantiv eingebüßt, so daß sie am Ende dieses Prozesses als eigenständige, vom Grundwort unabhängige Wörter, die dann auch eine andere Realität als dieses bezeichnen, dastehen können. Die hier stattfindende Bedeutungsveränderung hat man sich nicht als unvermittelt oder übergangslos vorzustellen, vielmehr ist sie Ergebnis unterschiedlicher, u.a. durch politische, wirtschaftliche oder kulturelle Veränderung bedingter, sprachhistorischer Entwicklungen. Die Lebendigkeit dieses Prozesses bringt mit sich, daß die große Anzahl derjenigen Lexeme, die sich semantisch mehr oder weniger weit von ihrer Basis entfernt haben, nicht auf ein und demselben Niveau anzusiedeln sind. Vereinfacht gesagt, unterscheidet man zwei Stadien des Bedeutungswandels, die mit den Stichworten Neutralisierung und Lexikalisierung belegt werden."

\footnotetext{
52 S. dazu die Ergebnisse der pragmatischen Untersuchung von KLIMASZEWSKA 1983. S.5459 und 91-101; Anhaltspunkte für die Vielfalt der Ausdruckswerte des Diminutivs liefert auch die Studie von RAMSTEIN 1969, vgl. S.88-145. Zu den semantisch-stilistischen Funktionen der Diminutive vgl. auch das VII.Kap. (S.120-166) der umfangreichen Dissertation über lettische Diminutive von RÜKE-DRA VIṆA 1959. Hinweise über den Einsatz von Diminutiven als stilistisches Mittel geben auch Handbücher der Stilistik, vgl. z.B. SCHNEIDER 1959. S.15-19, FAULSEIT/KÜHN 1972. S.135f und 168, RIESEL/SCHENDELS 1975. S.174-177 sowie KURKOWSKA/SKORUPKA 1959. S.44-52. Vgl. auch die Ausführungen in Kap. 2.1. dieser Arbeit.

53 Zu dieser Bezeichnung siehe besonders BASARA 1972, der formale Diminutive an einer Mundart kurz demonstriert und von ihnen sagt: "Ich formalnie deminutywny charakter sygnalizuja jedynie formanty, pelniace tu funkcje wylacznie strukturalna" (S.59). Andere Forscher betonen den Aspekt der Entstehung und sprechen von deminutywa historyczne, vgl. z.B. WARCHOL 1974a. Im Deutschen begegnen die Begriffe: feste Diminutive, vgl. BRANDSTETTER 1963/64 (S.338ff) oder bildungsneutrale Diminutive, vgl. FÖRSTER 1982. S.319.
}

* So STASZEWSKA 1985. S.242 oder KLIMASZEWSKA 1983, die von lebendigen Diminutiva spricht, s. S.49 und 78.

ss Dazu vgl. die Erlăuterungen bei LASKOWSKI 1966. S.77-81. 


\subsubsection{Neutralisierte Formen}

Zum Typ der neutralisierten Bildungen können jene Derivate gerechnet werden, die semantisch gesehen ihre enge Bindung zur Basis beibehalten, alle Wirkungsmöglichkeiten eines Diminutivs aber abgegeben haben. Indem eines der wichtigsten Pfeiler der Diminution, die Opposition zum Grundwort, nicht mehr gegeben ist, wird auch der modifizierende Charakter der Diminutive aufgehoben, so daß sie nur noch als neutrale, nicht markierte Lexeme empfunden werden können. Innerhalb dieser lassen sich mehrere Formen der Neutralisierung aussondern: Zu den am klarsten zu umgrenzenden Fällen, weil in ihrer Entwicklung zunächst abgeschlossen, zählen jene Einheiten, bei denen mit dem Verlust des diminutivischen Wertes eine neue funktionale Polarisierung zwischen Grundwort und Derivat eingetreten ist, indem letzteres die Position des unmarkierten Basislexems eingenommen hat und gleichzeitig das ursprünglich motivierende Substantiv in Richtung Augmentativ verschoben hat. Damit hat das Basislexem seine Neutralität verloren und eine neue semantische Markierung erhalten, vgl. ksiązka - księga, czapka - czapa, worek - wór, córka - córa. Die erstgenannten Wörter sind zu Normalbezeichnungen für 'Buch', 'Mütze', 'Sack', 'Tochter' geworden. Diesen Oppositionswechsel, der eine Umdisponierung der ganzen Modifikationsreihe bewirkt, faßt man unter den Begriff Transversion, ${ }^{56} \mathrm{vgl}$. ksiega (Grundwort $\Rightarrow$ Augmentativ) - książka (Dim. 1.Grades $\Rightarrow$ Grundwort) książeczka (Dim. 2.Grades $\Rightarrow$ Dim. 1.Grades). Im Gegensatz zur Diminutivbildung, die zur sog. "derywacja dodatnia" (Grundwort wird um ein Formans erweitert) gerechnet wird, gehört dieser Wortbildungsvorgang der sog. "derywacja ujemna (wsteczna)" an, die in der Kürzung - hier Abwerfen des -k- Suffixes und evtl. Alternation des auslautenden Stammkonsonanten - besteht. ${ }^{37}$

In diesen Kreis gehören auch die isolierten Diminutivbildungen, die heute kein bzw. ein bedeutungsverschiedenes Basislexem neben sich haben. Primär als Verkleinerungsbildungen verstanden, haben sich Derivate wie matka $(\Leftarrow \operatorname{mac})$, scieżka ( $\leftarrow$ sçdza); Märchen ( $\leftarrow$ Mär), Kaninchen ( $\leftarrow$ Kanin), Veilchen ( $\leftarrow$ Veil) im Laufe der sprachgeschichtlichen Entwicklung zu bedeutungsneutralen

\footnotetext{
so Vgl. dazu KREJA 1974 (S.171), der formuliert: "to przeksztalcenie funkcji zneutralizowanego elementu jezykowego (neutralizatu), czyli negatywnego czlonu opozycji, można by nazwac transwersja".

57 Leider erweist sich eine Festlegung der Markierungsrichtung in vielen Fällen als problematisch, denn die Genese eines Basis-Derivat-Paares läßt sich nicht immer sicher und zuverlässig angeben. Eingehend beschäftigt sich damit - dabei verschiedene Typen der "derywacja wsteczna" herausstellend - der Aufsatz von KREJA 1970.
} 
Diminutiven gewandelt. Eine Reihe isolierter Formen existiert lediglich in idiomatischen Wendungen, wie jemandem ein Schnippchen schlagen ( $\Leftarrow$ Schnipp 'schnelle Bewegung mit der Schere'), mach doch keine Mätzchen! (wohl = Matz 'dummer, törichter Kerl'). Schließlich begegnen auch syntagmaintern motivierte Diminutivmodifikationen mit gängiger Grundform, vgl. biec z górki na pazurki ('sehr schnell herunterlaufen'), mówič słodkie sł́wka ('Süßholz raspeln'); vom Hölzchen aufs Stöckchen kommen, nur ein Rädchen im Getriebe sein u.v.a.m., die meist nur als semantische Stütze der idiomatischen Aussage dienen. ${ }^{\text {s8 }}$

Recht deutlich zu bestimmen sind auch jene bildungsmäßig fest gewordenen Diminutive, die das zu ihnen gehörende Primärwort nicht verdrängt haben, sondern in gleicher Bedeutung neben ihm auftreten, so daß man von QuasiSynonymen sprechen kann, z.B. bei lódź - lódka, wies - wioska, nic - nitka, skarpeta - skarpetka. Durch die häufigere Anwendung der modifizierten Formen kommen die motivierenden Substantive immer mehr aus dem Gebrauch und wirken zuweilen veraltet.

In ähnlicher Weise sind auch Personen- und Verwandtschaftsnamen vom Neutralisierungsprozeß betroffen; besonders im anthroponymischen Bereich führt der ständige Gebrauch modifizierter Formen zum Verlust diminutivischer Dimensionen. Namen wie Mirek ( $\Leftarrow$ Miroslaw), Maciek ( $\Leftarrow$ Maciej), Joasia ( $\Leftarrow$ Joanna), Wladka ( $\leftarrow$ Whadyslawa) konnten sich in der gesprochenen Sprache zu durchaus gängigen, neutralen Ausdrucksmitteln entwickeln. Die Überbeanspruchung dieser ursprünglich hypokoristisch gedachten Bildungen im täglichen Umgang hat sie aus ihrer Stellung als stilistische, individuell einzusetzende Auswahlmöglichkeit verdrängt. Noch mehr: ihre Unmarkiertheit macht es möglich, sie bereits bei offiziellen Situationen zu verwenden und läßt mittlerweile einige von ihnen sogar zu vollständigen, amtlichen Namensformen werden. ${ }^{39}$ Auch suffigierte Verwandtschaftsnamen, wie babcia, dziadek, ciocia, wujek u.a., sog. Höflichkeitsdiminutive, ${ }^{60}$ deren Anwendung primär auf die Anrede der betreffenden Personen als Ausdruck von besonderer Verehrung beschränkt blieb, haben ihre Bedeutung erweitert. Ihre subjektive Einschätzung wurde nivelliert, die Grundformen baba, dziad ungebräuchlich, ciotka, wuj seltener und die

\footnotetext{
se Für das Deutsche vgl. auch LOCKWOOD 1974/75. S.308f.

so Vgl. IWANOWA-PERCZYŃSKA 1978 (S.22), die sich zu "zdrobnienia neutralne od imion wlasnych osobowych" außert.
}

${ }^{\infty}$ Dazu und zum semantischen Wechsel s. RITTER 1976. S.359-361. 
suffigierten Formen auf eine andere Ebene, allein zur Bezeichnung der gemeinten Person, übertragen. N.Reiter faßt die geschilderte Erscheinung der Diminutivverfestigung prägnant zusammen: ${ }^{61}$

DIM. [Diminutive - B.K.] und AUG. [Augmentative - B.K.] signalisieren Perspektiven, die vom Mehrheitlichen, dem Normalen abweichen, es sind Ausnahmen. Wird, was Ausnahme ist, überstrapaziert, so wird es mehrheitlich, bildet keine Ausnahme mehr, sondern fügt sich ins Normale ein.

A.Brandstetter bringt für diesen Prozeß der Einbuße den Begriff exdiminutivische Tendenz ein. In Anlehnung an Extendenzen bei Metaphern, Hyperbeln und Euphemismen glaubt er auch hier einen der "Bedeutungsentleerung nahe verwandten Vorgang ${ }^{n 2}$ zu sehen. Hinzuzufügen bleibt, daß mit der Entleerung einer fest umrissenen Bedeutung, hier des diminutivischen Gehalts, immer eine Erweiterung des Bedeutungsumfangs einhergeht. ${ }^{63}$

Schwieriger ist die Deutung derjenigen Bildungen, die sich dem neutralen Status des Grundwortes angenähert haben, allerdings ohne sich mit ihm zu decken. Eine solche quasi doppelt besetzte Stelle des Basislexems begegnet vorzugsweise bei zum Alltag des Menschen gehörenden Gebrauchsgegenständen derselben Art, aber unterschiedlicher Ausmaße, vgl. pudlo - pudelko, zegar zegarek; Tüte - Tütchen, Kanne - Kännchen. Ohne Zweifel zielt die suffigierte Form auf die Bezeichnung der jeweils kleineren Variante des Objekts ab, doch kommt es letztlich vielmehr darauf an, daß sie funktional anders determiniert ist. Nicht die Markierung des Größenunterschieds ist vorrangig, sondern die Tatsache, daß Grundwort und Diminutiv zu anderen Zwecken genutzt werden. ${ }^{4}$ Folgende Gegenüberstellungen verdeutlichen dies: pudlo wird z.B. in Verbindung mit pudlo na kapelusze, pudlo na tort gebraucht, pudelko dagegen mit pudelko do soli, pudelko zapalek; zegar bezieht sich auf zegar ścienny, zegar stojący, zegarek dagegen auf zegarek na ręke, zegarek kieszonkowy; Tüte steht im Zusammenhang mit Tüte Obst, Tütchen dagegen mit Gewürtüt-

\footnotetext{
o1 REITER 1988. S.675.

62 BRANDSTETTER 1963/64. S.345.
}

a Über die Wechselwirkung von Extension und Intension vgl. ULLMANN 1973. S.289.

* GAUGER 1971 (S.131) formuliert dazu: "Die Vorstellung klein bleibt in diesen Wörtern durchaus lebendig, nur meinen sie nicht mehr generell ein kleines Exemplar des durchs Grundwort Gemeinten, sondern etwas in bestimmter Weise qualitativ anderes". 
chen; Kanne wird im Sinne von Kaffeekanne, Kännchen von Mikchkännchen ${ }^{65}$ gebraucht. Die Reihe der morphologisch als Diminutive ausgewiesenen Einheiten ließe sich mit Paaren wie ogrod 'Garten' - ogrodek 'Vorgarten', ulica 'Straße' - ulicaka 'Gasse', torba 'Tasche' - torebka 'Handtasche'; Tisch 'EBtisch' - Tischchen 'Cafétisch', Decke 'Tischdecke' - Deckchen 'Zierdecke' u.a. beliebig erweitern, wobei der Grad der Bedeutungsspezifizierung von Fall zu Fall unterschiedlich stark ausfallen kann.

Es steht außer Frage, daß die angeführten Beispiele bezüglich des Kriteriums der inhaltlichen Nähe zum Grundwort den Neutralisierungen zuzurechnen sind; bedenkt man die offenkundige Bedeutungsdifferenzierung, die Tatsache, daß eine Einheit "sui generis" Lexikalisierungen zu sprechen. Gauger sieht in jenen Grenzfallen im Rahmen seiner drei Leistungstypen des durchsichtigen Wortes eine "charakteristische Symbiose von ausgreifender und variierender Funktion (...) Es sind Ausgriffe, die sich - in einer unbestimmten Weise - auf die Variation stützen" ${ }^{67}$ So scheint es angebracht, die Vielzahl - besonders im Polnischen - derartiger Bildungen als Übergangsgruppe zwischen neutralisierten und lexikalisierten Formen zu verstehen. Während Einheiten der beiden Pole für einen Übersetzer fast durchgängig leicht auszumachen sind, erfordern die Derivate der Schnittmenge genaue Kenntnis des Sachverhalts.

\subsubsection{Lexikalisierte Formen}

Unter Lexikalisierungen werden Modifikationsderivate gefaßt, die ihren Grundwörtern gegenüber die größte Selbständigkeit erlangt haben, so daß sie im Gegensatz zu den neutralisierten Derivaten in Wörterbüchern generell mit einem eigenen Lemma angeführ werden. ${ }^{68}$ Die mit der Suffigierung erfolgte semantische Verschiebung ist nicht lediglich eine spezifische Variation des vom motivie-

\footnotetext{
as In der găngigen Wendung ein Kännchen Kaftee, so z.B. bei einer Bestellung im Café, wird die modifizierte Form nicht als Bedeutungsspezifizierung, sondern im Sinne kleine Kanne gebraucht.
}

¿ GAUGER 1971. S.131.

${ }^{67}$ GAUGER 1971. S.132.

al Vgl. SIEBERER 1966 (S.102): "Deminutiva werden Bestandteil des Lexikons, (...) wenn sie sich in einer vom Primărwort abliegenden Sonderbedeutung fixieren." 
renden Substantiv Gemeinten, sie ist mehr, sie stellt eine deutliche Änderung im Denotatsbezug dar - eine neue Benennung entsteht. Bei diesem Prozeß der semantischen Weiterentwicklung werden die bestehenden Bindungen zwischen morphologischer Struktur des Wortes und seiner Semantik verwischt bzw. gelöst:

Wyrazista formacja slowotworrcza przeksztalca sie w wyraz bedacy jednolitym znakiem pełniacym swoja funkcje oznaczania, niezależnie od pierwotnych funkcji jego skladnikow slowotwórczych.

Jene deminutywa pozorne ${ }^{70}$ bzw. quasi-deminutiwa" versteht man "jako tworzywo jezykowe do celow tzw. nominacji wtórnej" ${ }^{n}$. Mit der Lexikalisierung der Diminutive wird die Möglichkeit genutzt, neue Inhalte zu benennen. So steht bei einer nicht unwesentlichen Zahl von Spracheinheiten neben dem Grundwort einerseits die Diminutivform mit aktivem Suffix, andererseits die Diminutivform in neuer symbolischer Funktion. Mit der Bedeutungsveränderung der letzteren ist in keinem der Fälle Ersetzbarkeit durch die dazugehörige Basis mehr gegeben. Viel weniger das Moment der Kleinheit als eine Bandbreite verschiedener Assoziationen anderer Art, die das Verhältnis zum Grundwort kennzeichnen, geben Anlaß zur neuen Formbildung. Basara konstatiert:

Te nowe znaczenia maja różne zródła pochodzenia. W gre tu wchodza najrozmaitsze skojarzenia postrzezeniowe, powodujace zmiane znaczen. Od momentów zewnetrznych, podobienstwa ksztaltów (...) poprzez podobienstwo funkcji (...) do momentow uczuciowych, emocjonalnych. ${ }^{n}$

Wollte man die Lexikalisierungsmechanismen innerhalb der großen heterogenen Gruppe der Diminutivbildungen charakterisieren, so lassen sich im wesentlichen vier Übergangsmodi beschreiben. Zu der gängigsten Art der Bedeutungsveränderung zählt die Metaphorisierung. ${ }^{74}$ Hierbei findet auf der Grundlage ähnlicher Bedeutungsmerkmale die Übertragung eines Wortes aus seinem eigent-

"SARNOWSKI 1991. S.11.

' VgI. SARNOW'SKI 1987. S.60.

"Vgl. SARNOWSKI 1991.

n SARNOWSKI 1987. S.61.

${ }^{n}$ BASARA 1972 . S.60.

${ }^{4} \mathrm{Zu}$ den folgenden Ausführungen vgl. SARNOWSKIs 1991 Lexikalisierungsmodelle, die er für das Russische (S.18-34) und das Polnische (S.61-70) vorschlägt und mit zahlreichen Beispielen belegt. Siehe auch RITTER 1976, die skizzenhaft metaphorische Bildungen des Russischen zusammengestellt hat. 
lichen Bedeutungszusammenhang in einen anderen, fremden, statt, die sich auf zahlreiche semantische Bereiche erstrecken kann, vgl.:

oczko (pierścionek z oczkiem) 'Edel- bzw. Schmuckstein eines Ringes' zu oko 'Auge',

ząbek (czosnku) 'Knoblauchzehe' zu zqbb 'Zahn', daszek (na lampe) 'Lampenschirm' zu dach 'Dach', gołąbki 'Krautrouladen' zu goląb 'Taube', paluszki 'Salzstangen' zu palec/paluch 'Finger', mieczyk 'Schwertlilie' zu miecz 'Schwert', bratek 'Stiefmütterchen' zu brat 'Bruder', szafirek 'Träubelchen bzw. Träubelhyazinthe' zu szafir 'Saphir"7s; Sternchen 'Markierungszeichen in Texten' zu Stern, Grübchen 'kleine Vertiefung am Kinn' zu Grube, Hörnchen 'Gebäckstück aus Blätter- oder Hefeteig' zu Horn, Früchtchen 'Kind oder Jugendlicher, den man für ungeraten hält' zu Frucht ${ }^{76}$, Bäuerchen 'bei Säuglingen: Aufstoßen' zu Bauer,

Pfaffenhütchen 'Pflanze mit vierkantigen karminroten Früchten' zu Pfaffenhut 'Kopfbedeckung eines Geistlichen',

Kätzchen 'Blütenstand der Birke, Erle u.a.' zu Katze,

Landkärtchen 'zur Familie der Fleckenfalter zählendes Insekt' zu Landkarte.

Eng mit jenen Bildungen verbunden ist der Typ der Generalisierung. Ihm liegen nämlich meist metaphorische Diminutive zugrunde, die mehrfach, d.h. für eine ganze Gruppe von Gegenständen, gebraucht werden können. Sie beruht auf einer Ausdehnung des Anwendungsbereichs:

Zbieżnosc pewnych cech przedmiotów, leżaca u podstaw metafory, daje sie zaobserwowac nie miedzy pojedynczymi przedmiotami, lecz miedzy znacznie wieksza ich liczba. ${ }^{n}$

Folgende Beispiele lassen sich anführen:

glówka 'oberer Teil eines Gegenstandes in abgerundeter bzw. kugelförmiger Gestalt' zu glowa 'Kopf' - glowka szpilki 'Stecknadelkopf, glowka zapalki

\footnotetext{
's An diesem Beispiel ist interessant zu beobachten, wie unterschiedlich einzelne Sprachen bei der Namengebung verfahren können. Ist im Polnischen der Aspekt der Farbe (saphirfarben) herausgegriffen worden, bietet im Deutschen die Form der Blüte (traubenăhnlich) die Vorlage zur Benennung.
}

* Vgl. dazu MÜLLER 1953. S.25.

${ }^{n}$ SARNOWSKI 1987. S.65. 
'Streichholzkopf, glowka gwoździa 'Nagelkopf, głwka skrzypiec 'Geigenschnecke';

nózka 'Gegenstand, der an die Form und Funktion eines Beines erinnert' zu noga 'Fuß' - nóżka kieliszka 'Fuß eines Glases', nózka grzyba 'Stiel eines

Pilzes' nóżka rosliny 'Pflanzenstengel', nóżka cyrkla 'Zirkelschenkel'; mostek 'etwas in Form einer Brücke' zu most 'Brücke' - mostek dentystyczny 'Zahnbrücke', mostek skrzypcowy 'Geigensteg', mostek nawigacyjny 'Kommandobrücke'78, mostek 'gymnastische Übung';

maszynka 'Gerät bzw. Vorrichtung, die eine bestimmte Tätigkeit mechanisiert' zu maszyna 'Maschine' - maszynka do golenia 'Rasierapparat', maszynka do kawy 'Kaffeemaschine', maszynka do mielenia mięsa 'Fleischwolf', maszynka do krojenia chleba 'Brotmaschine';

proszek 'fein zerkleinerter, zerriebener Stoff' zu proch 'Pulver' - proszek do pieczenia 'Backpulver', proszek do prania 'Waschpulver', mleko w proszku 'Trockenmilch', proszek scierny 'Schleifstaub'.

Die dritte Gruppe umfaßt die sog. Metonymisierung. Auch wenn die Grenzen zwischen Metapher und Metonymie fließend verlaufen, soll letztere, verstanden als "Ersetzung eines Ausdrucks durch einen anderen Ausdruck, der zu ihm in einer realen, d.h. kausalen, räumlichen oder zeitlichen Beziehung "79, nicht aber in einem semantisch-begrifflichen Zusammenhang steht, mit ein paar markanten Fällen eigens hervorgehoben werden, vgl.:

gwiazdka 'Weihnachten' zu gwiazda 'Stern',

okienko 'Schalter, bes. in Bahnhöfen, Banken, Postämtern' zu okno 'Fenster', gl6wka 'Kopfball' zu glowa 'Kopf',

raczka 'Griff, Henkel, Hebel u.a.' zu reka 'Hand', marcinki 'spätherbstliche Birnensorte' zu Marcin 'Martin';

Kreisel 'Kinderspielzeug' zu (wahrsch.) Krause 'Krug, Topf', Köpfchen 'pfiffiger Verstand, Ideenreichtum' zu Kopf.

Als letztes läßt sich das Lexikalisierungsmodell der Detaillierung (Konkretisierung) ausgliedern. Es umfaßt neben individualisierenden bzw. singularisierenden Formen. die im Gegensatz zu der durch die Basis vorgegebenen Gesamtheit oder Masse ein individuelles, selbständig existierendes Teil davon

\footnotetext{
nit diesem Beispiel ist nur eins von vielen genannt. Das Heranziehen von Diminutivsuffixen zur Neubildung technischer Termini gehör zum gängigen Verfahren in der polnischen Wortbildung, Näheres dazu s. MÜLLER-OTT 1972 und MATUSCHEK 1977.
}

"LEWANDOWSKI 1979. S.492, Artikel "Metonymie". 
benennen, auch konkretisierende Bildungen, mit denen das verarbeitete Produkt aus einem bestimmten Rohstoff bezeichnet wird, ${ }^{\infty} \mathrm{vgl}$.:

bożek 'Götze' zu Bóg 'Gott',

Śnieżynka 'Schneeflocke' zu Śnieg 'Schnee',

trawka 'Grashalm' zu trawa 'Gras',

ciastko 'Keks, Gebäck' zu ciasto 'Teig',

choinka 'Weihnachtsbaum' zu choina 'Nadelbaum, bes. Kiefer',

wątrobka 'Lebergericht' zu wątroba 'Leber',

salatka 'mit Marinaden zubereitete kalte Speise in diverser Zusammensetzung'

zu salata 'Blattsalat';

Stäubchen 'Staubkorn' zu Staub,

Knöchel 'Fuß-, Fingerknöchel' zu Knochen,

Lüftchen 'Brise, leichte Luftbewegung' zu Luft,

Teilchen 'Korpuskel (Terminus der Kernphysik)' zu Teil,

Männchen/Weibchen 'Tier männlichen/weiblichen Geschlechts' zu Mann/ Weib.

Auf die morphologischen Mittel der Diminution als Quelle für lexikalische Neubildungen (Formen, die einen Sonderstatus innerhalb der vierten Gruppe einnehmen) greift ein weiterer nicht zu vernachlässigender Bereich zurück. Diminutivsuffixe werden hier zum Aufbau einer systematischen wissenschaftlichen Terminologie genutzt. Im Unterschied zu den oben erwähnten, auf metaphorischen Übertragungen beruhenden, botanischen und zoologischen Populärnamen ist die Basis des neu entstandenen Ausdrucks bereits selbst ein Terminus. Sarnowski erläutert diesen Tatbestand wie folgt:

Oba terminy (niedeminutywny i deminutywny) musza funkcjonowac obok siebie w ramach tej samej dyscypliny naukowej. W sferze desygnatow powyższe oznacza sytuacje, w której dwa desygnaty (jeden oznaczony przez deminutywum, drugi przez niedeminutywum) znajduja się w okreslonej reainej zaleźnosci."

In Abhängigkeit vom Fachgebiet können mit der formalen Basis-Derivat-Opposition mindestens drei Relationsarten angezeigt werden: ${ }^{82}$ Abkunft (pochodnoś) in der Chemie, z.B. tlen 'Sauerstoff' - tlenek 'Oxyd (Verbindung eines chemi-

\footnotetext{
* Genaueres zu diesen lexikalisierten Formen findet sich am Beginn der Monographie von KREJA 1969. S.18-23; speziell zu singulativen Bildungen vgl. KREJA 1972 und LOCKWOOD 1974/75. S.313f. Darüberhinaus zur singularisierenden und konkretisierenden Funktion vgl. ZWOLIŃSKI 1968.
}

" SARNOWSKI 1987. S.65.

" Vgl. SARNOWSKI 1985. S.34-39. 
schen Grundstoffs mit Sauerstoff)', jod 'Jod' - jodek 'Jodid (Verbindung des Jodes mit Metallen und Nichtmetallen)', arsen 'Arsen' - arsenek 'Arsenid (Arsen-Metall-Verbindung)'. Die Grundwort-Diminutiv-Paarung spiegelt hier das Verhältnis eines Elements zu dessen Verbindungen mit anderen chemischen Stoffen. In der botanischen und zoologischen, besonders ornithologischen Terminologie ist es die Hyperonymie (zawieralnosc), z.B. paproc 'Farn (Filix)' - paprotka 'Tüpfelfarn (Polypodium)', bob 'Puffbohne (Vicia faba maior)' - bobik 'Pferdebohne (Vicia faba minor)', bąk 'Rohrdommel (Botaurus stellaris)' baczek 'Zwergdommel (Ixobrychus minutus)', drozd 'Drossel (Turdus)' droździk 'Weindrossel (Turdus musicus)'. Die Gegenüberstellung von Nichtdiminutiv- und Diminutivform wird hier dazu genutzt, ein Unterordnungsverhältnis innerhalb der Pflanzen- oder Tiersystematik zu kennzeichnen. In erster Linie handelt es sich um die Unterscheidung von Gattung (=Grundwort) und Art (=Diminutiv) im Linneschen Sinne. Schließlich kann man in der Botanik eine dritte Relation, die Kohyponymie (równoleglość), beobachten. Dabei dient die morphologische Opposition nicht zur Charakterisierung eines Unterordnungs-, sondern eines Nebenordnungsverhältnisses, so z.B. zweier Gattungen innerhalb einer Familie: groch 'Erbsenpflanze (Pisum)' - groszek 'Platterbse (Latyrus)', die der Familie der Schmetterlingsblütler angehören. ${ }^{83}$ Grundsätzlich läßt sich zur Spezialisierung von Diminutiven sagen:

Jeżeli system terminologiczny okreslonej dyscypliny naukowej widziec jako twor przestrzenny, to można powiedziec, iż formacje deminutywne użyte tu jako terminy rozbudowuja go najczesciej " $w$ d 61 ", ewentualnie " $w$ glab", nigdy zas "wzwyż"."

Soweit zum derivationellen Material aus morphematischer Sicht, der ein recht breit angelegter Diminutivbegriff zugrundeliegt. Nicht unberechtigte Kritik an dieser bisher üblichen Interpretationsmethode, die, um Elemente einer Wortbildungskonstruktion herauszuarbeiten, ihren Schwerpunkt auf die Form und innere Struktur legt, wird von Vertretern einer semantisch orientierten Beschreibungsart geäußert. ${ }^{\text {s }}$ Der Vorteil dieses Verfahrens liegt in der ganzheitlichen

* Eine ähnliche Nutzung diminutiver Formen ließ sich für das Deutsche auf Anhieb nicht nachweisen, auch wenn eine Reihe botanischer (Maiglöckchen, Steinröschen, Gänseblümchen u.a.) und zoologischer (Flughörnchen, Geißeltierchen, Johanniswürmchen, Rotkehlchen, Seepferdchen u.a.) Termini auf der Grundlage von Diminutiven entstanden sind; dazu $s$. LOCKWOOD 1974/75. S.310.

"SARNOWSKI 1985. S.40.

2s Vgl. dazu die Überlegungen von MACZYŃSKI 1986. S.279f. 
Betrachtung einer Spracheinheit begründet, d.h., es berücksichtigt nicht allein strukturelle, sondern vielmehr inhaltliche Funktionen einer gegebenen Wortbildung; es wird von ihrer aktuellen, vom konkreten sprachlichen Kontext abhängigen Bedeutung ausgegangen. Unter Voraussetzung dieser Prämisse ist der Gebrauch des Terminus deminutywa formalne unzutreffend. ${ }^{86}$ In den meisten Fällen bezieht er sich nämlich auf Derivate, deren Suffixe eine andere als die für Diminutive charakteristische Modifikationsfunktion erfüllen; den Morphemen kommt vornehmlich Mutationsfunktion zu. Unzureichend erscheint die Begriffswahl zudem auch im Hinblick darauf, daß ein bestimmtes Wortbildungsformans (hier -k-) als Anzeiger unterschiedlicher Wortbildungsprozesse dienen kann, also mit Blick auf das gerade im Polnischen bekannte Phänomen der Polyfunktionalität von Affixen. Eine treffende Summierung des Problems bietet Maczyński:

Gdy idzie o polskie formanty afiksalne[,] ich cecha charakterystyczna jest brak specjalizacji funkcionalnej, stad wiazanie stalej dominanty tresciowej $z$ okreslonymi wykladnikami formalnymi jest nieuzasadnione, sugeruje bowiem, ze np. deminutywa sa klasa formalna, tj. wyodrebniona na podstawie cech formalnych, podczas gdy w rzeczywistosci plaszczyzna ich wyodrebnienia jest wspolna im wszystkim semantyczna tresc kategoriaina ujeta wtornie w odpowiednie schematy formalne."

Befolgt man diese Interpretation, so engt sich das Verständnis des Terminus Diminutiv wesentlich ein.

* Dazu und zu den entsprechenden Argumenten s. MACZYŃSKI 1986. S.282f.

"MACZYŃSKI 1986. S.284. 


\subsection{DIE ENTWICKLUNG UBERSETZUNGSTHEORETISCHEN UND -METHODISCHEN DENKENS}

Grundlage für den durchzuführenden übersetzungsvergleich ist nun die Skizzierung wichtiger übersetzungstheoretischer Entwicklungen sowie eine $\mathrm{Be}$ leuchtung einschlägiger Aspekte methodischer Art. Die Diversität der Sprachen und die damit verbundenen Verständigungsprobleme, die bereits im biblischen Mythos vom Turmbau zu Babel ihren Ausdruck fanden, forderten immer schon nicht nur praktische Vermittlungsleistungen, sie waren ebenso Auslöser theoretischer Überlegungen zum Thema Übersetzen. Die entstandene Auseinandersetzung, in der wiederholt - u.a. von theologischer, philosophischer, ästhetischer, kommunikationswissenschaftlicher Seite sowie von verschiedenen Disziplinen der Linguistik - dem Phānomen des Übersetzens und der Übersetzung nachgegangen worden ist, hat sich

vereinfacht formuliert, in zwei komplexen, aufeinander bezogenen Problembereichen konkretisiert:

1. in der übersetzungstheoretisch bestimmten Frage nach der interlingualen Übersetzbarkeit bzw. Nichtübersetzbarkeit;

2. in der übersetzungsmethodisch bestimmten Frage nach den jeweiligen

Äquivalenzmaßstäben und den daraus ableitbaren Übersetzungsstrategien, Übersetzungsmethoden und Übersetzungstechniken'.

Vorwiegend waren es die Übersetzer selbst, die sich, sei es in Vor-oder Nachworten zu ihrem Werk, sei es in eigenständigen Abhandlungen nicht nur mit ihren speziellen Problemen auseinandersetzten, sondern sich auch um prinzipielle Äußerungen zur Verfahrensweise bemühten. ${ }^{2}$ Ungeachtet des breiten Spektrums dieser übersetzungstheoretischen Ansätze mangelt es der traditionellen Übersetzungstheorie an Kontinuität und systematischer Erfassung.

Während Überlegungen übersetzungsmethodischer Art schon aus früherer Zeit bekannt sind, wurden Reflexionen zum Übersetzbarkeitsproblem erst mit der Romantik, die für die Geschichte der Übersetzungstheorie unabweisbar eine Zäsur bedeutet, aktuell. Neben F.Schleiermacher war es im deutschsprachigen

' WILSS 1977. S.29 (Hervorhebungen B.K.).

${ }^{2}$ Die wichtigsten übersetzungstheoretischen Beitrăge der Vergangenheit u.a. von Hieronymus, M.Luther, J.W.von Goethe, F.Schleiermacher, W.von Humboldt sind in der Anthologie von STÖRIG 1963 nachzulesen. Mit dieser Auswahl ist den Hauptströmungen der europăischen Übersetzungsgeschichte Rechnung getragen worden, zu denen die Übersetzung der Bibel, der Werke des klassischen Altertums, danach der europäischen Nationalliteraturen in andere europăische Sprachen und schließlich die Übersetzungen strukturferner Sprachen gehören. 
Raum besonders W.von Humboldt, mit dessen Erkenntnis von der "wesenhaften Ungleichheit der Einzelsprachen" die Frage nach Grenzen und Unzulänglichkeiten des Übersetzens zum Zentrum übersetzungswissenschaftlicher Erörterungen wurde. An die Stelle der bisher geltenden These von der uneingeschränkten Austauschbarkeit sprachlicher Zeichensysteme trat die Zurückhaltung gegenüber dieser Vorstellung einer immer möglichen adäquaten Übersetzbarkeit von Texten. Diese Position ist dann im 20.Jh. von der Sprachinhaltsforschung (L.Weisgerber) aufgenommen und ausgebaut worden, auch die Sapir-Whorf-Hypothese ("linguistisches Relativitätsprinzip") steht - allerdings ohne Explikation - im Einklang mit Humboldts Sprachauffassung. ${ }^{3}$ In neuerer Zeit wurde ihr wiederum die frühere Extremhaltung von den Vertretern der Leipziger Übersetzungswissenschaftlichen Schule entgegengesetzt. ${ }^{4}$ Die proklamierte These von der prinzipiellen Möglichkeit des Übersetzens - "Wenn wir davon ausgehen, daß in jeder Sprache alles ausdrückbar ist, so können wir daraus folgern, daß (...) alles aus jeder gegebenen in jede gegebene Sprache übersetzbar ist"s - resultiert weitgehend aus Untersuchungen von pragmatischen (sachbezogenen) Texten. Mit dem aus methodischen Gründen vorgenommenen Verzicht auf literarische Texte und damit auf formbetonte Sprachelemente sollen nur "objektivierbare" Äquivalenzbeziehungen von quellen- und zielsprachlichen Texten berücksichtigt werden. Demzufolge gilt die Vorstellung von der uneingeschränkten Übersetzbarkeit für Texte mit vorwiegend "denotativem" Charakter. ${ }^{6}$

Ganz anders stand es um das Interesse an übersetzungsmethodischen Fragestellungen, bereits Cicero gebraucht die antithetische Formulierung ut interpres ut orator, zwei übersetzungsmethodische Grundprinzipien, die über Horaz, Quintilian, Hieronymus, Luther, Schleiermacher' bis in das 19.Jh. hinein die

\footnotetext{
${ }^{3}$ Über die historische Entwicklung und Auseinandersetzung beider konträrer Prinzipien von der Übersetzbarkeit und Unübersetzbarkeit orientieren ausführlich KOLLER 1987. Kap.6 und WILSS 1977. Kap.3.
}

4 Vgl. dazu u.a. die Ausführungen von KADE 1968 und JÄGER 1975.

S KADE 1968. S.68.

- Eine Korrektur dieser gängigen Kontrastierung der beiden Positionen hat KOSTA 1986 versucht, er sieht jene Auffassungen nur in einem "scheinbaren Widerspruch", vgl. seine Erläuterungen auf S.59-63.

' Entsprechende frühe Übersetzungskonzeptionen sind in Polen von P.D.Huet, Dawid Pilchowski und F.N.Golanski formuliert worden, vgl. ZIETARSKA 1973. S.315f sowie die wertvolle Anthologie von BALCERZAN 1977, der polnische übersetzungstheoretische Positionen von 1440-1974 zusammengetragen hat. Für das russische Schrifttum dagegen fehlt eine 
Fachdiskussion geprägt haben. Gemeint sind damit die beiden theoretischen Extreme der wortgetreuen bzw. der freien Wiedergabe oder, anders gesehen, die Frage, ob sich die Übersetzung dem Original (ausgangssprachenzugewandt) anzupassen oder umgekehrt das Original sich der Übersetzung (zielsprachenzugewandt) unterzuordnen hat; Positionen, die die Problematik der traditionellen philologisch orientierten Übersetzungstheorie deutlich charakterisieren.

Einen bemerkenswerten Aufschwung erfuhr die Beschäftigung mit der Übersetzungsproblematik nach 1945, begünstigt durch drei Faktoren: ${ }^{8}$ zum einen das Aufkommen der Maschinenübersetzung, zum anderen eine bis dahin nie dagewesene Zunahme übersetzter Schriften' - neben literarischen Werken besonders naturwissenschaftliche Literatur -, die in dem vielzitierten Ausspruch "nous sommes à l'áge de la traduction" 10 auf einen Punkt gebracht wurde; schließlich durch die neu erwachten Bemühungen um die Sprachkomparatistik. Mit dem Bestreben, maschinelle Übersetzungen zu ermöglichen, geriet die Übersetzungsdiskussion vermehrt in das Blickfeld der Linguistik." Seitdem begann sich die

vergleichbar repräsentative Zusammenstellung, vgl. die Erörterungen bei KEIPERT 1981.

- Weitere Gründe für die Belebung des Interesses führt WILSS 1977 (S.40-42) an, vgl. auch die Überlegungen von ZIETARSKA 1973. S.309.

- Ein Blick in die laufenden Veroffentlichungen des Index translationum. Repertoire international des traductions. Paris 1(1932)ff führt dies unmittelbar vor Augen.

10 Im Vorwort des ersten Heftes der Zeitschrift Babel (1955. S.3) vom Präsidenten der Fédération Internationale des Traducteurs, P.-F.Caille, eingebracht. Vgl. z.B. REISS 1970, die diesen Slogan an den Anfang ihrer Überlegungen zum Thema Übersetzen stellt.

"Hinsichtlich dieser Ausweitung muß zur Forschungslage in Polen angeführt werden, daß sich u.a. angesichts der Bevorzugung literarischer Texte bei der Erörterung von Übersetzungsfragen - insbesondere des Problems der przektadalnosḱ/nieprzektadalnosḱ - eine Disziplin entwickelt hat, die vornehmlich Domäne der Literaturwissenschaft blieb. Abgesehen von einzelnen kleineren linguistisch konzipierten Abhandlungen und auch Beiträgen zur maschinellen Übersetzung hat sich eine systematisch angelegte, linguistisch ausgerichtete Übersetzungswissenschaft mit modernem theoretisch-methodischen Schriftum, wie sie z.B. von sovetischen, DDRund westlichen Wissenschaftlern vorgestellt wurde, nicht entwickelt. Als äußeres Kennzeichen dieser Tendenz mag es genügen zu erwähnen, daß der Terminus przektad/tlumaczenie bis heute nicht in sprachwissenschaftlichen Wörterbüchern oder Bibliographien aufgenommen wurde, sondern lediglich in entsprechenden literaturwissenschaftlichen Nachschlagewerken. Bei dieser Lage bietet es sich in einer polnisch-deutschen sprachvergleichenden Studie gleichsam an, die neuere übersetzungswissenschaftliche Forschungssituation in Deutschland vorzustellen. 
moderne linguistisch orientierte Übersetzungswissenschaft ${ }^{12}$ mit eigenständigem Objektbereich und eigener Zielsetzung als ein Zweig der Sprachwissenschaft ${ }^{13}$ allmählich zu konstituieren.

Mit der Etablierung dieser jungen Fachwissenschaft ging eine perspektivreiche Methodendiskussion einher. Begünstigt u.a. durch die nur schwer überbrückbare Dichotomie von literarischem Übersetzen und Fachübersetzen sowie die zweifache Ausrichtung des Terminus Übersetzung, zum einen als Prozeß, zum anderen als Resultat verstanden, konnten vielfältige, sehr unterschiedliche, wenn nicht widersprüchliche theoretisch-methodische Ansätze Eingang finden. Neben den rein linguistischen bzw. linguistisch-kommunikationswissenschaftlichen ${ }^{14}$

${ }_{12}$ Nach WILSS 1975 (S.520) geht der Terminus Übersetzungswissenschaft auf Schleiermacher zurück, in der modernen Verwendung wurde er zum ersten Mal von A.V.Fedorov genutzt, im deutschen Sprachraum wohl zunächst von O.Kade.

${ }^{13}$ Auch wenn bereits mehrfach Aufgaben und Arbeitsziele formuliert wurden, ist die "Legitimationskrise, in der sich die Übersetzungswissenschaft sowohl im Blick auf die Übersetzungspraxis als auch im Blick auf andere Wissenschaftszweige (vergleichende/kontrastive Sprachwissenschaft/Stilistik, vergleichende Literaturwissenschaft, Textwissenschaft, Kommunikationswissenschaft, linguistische Datenverarbeitung, automatische Sprachanalyse) befindet, (...) (noch) nicht überwunden" (KOLLER 1987. S.10). So wollen die einen Übersetzungswissenschaft als Teilgebiet der vergleichenden Sprachwissenschafi (vgl. JÄGER 1975. S.192-202 oder JÄGER 1968) bzw. des synchron deskriptiven Sprachvergleichs (vgl. z.B. WILSS 1977. S.69f) verstanden wissen, die anderen sie den Disziplinen der angewandten Linguistik (vgl. REISS/VERMEER 1984. S.1f) zuordnen. Für die polnische Seite sei der Vorschlag von LEBIEDZIŃSKI 1981 (S.15) zitiert: "Przekładoznawstwo ogólne wyodrebnia sie w autonomiczny dzial filologii w trojkacie: jezykoznawstwo - literaturoznawstwo - przekładoznawstwo. Natomiast przekladoznawstwo szczególowe tworza dwie galezie: jedna galaź wywodzi sie z jezykoznawstwa, druga z przekladoznawstwa ogólnego". Jüngste Publikationen verlangen nach einer Neuorientierung, nicht nur nach Emanzipation von den Nachbardisziplinen, sondern auch nach immer noch ausstehender Integration von Theorie und Praxis. Mit der Ablehnung der Übersetzungswissenschaft als "Teilbereich einer Teildisziplin" wird die Forderung erhoben, Übersetzungswissenschaft als "interdisziplinäre, multiperspektivische Einheit zu verstehen, die von der komplexen Realität des Übersetzens und nicht von den axiomatischen Modellen der Linguistik ausgeht" (SNELL-HORNBY 1986. S.12).

14 $\mathrm{Zu}$ den wichtigsten Vertretern dieser Richtung zählen außer A.V.Fedorov, J.C.Catford und R.Jakobson vor allem die Mitglieder der Leipziger Übersetzungswissenschaftlichen Schule, O.Kade, A.Neubert, G.Jäger, die mit ihrer Translationslinguistik, mittels linguistisch-kommunikationswissenschaftlicher Modelle, die Mechanismen des Übersetzungsprozesses nachzuvollziehen versuchen. Thre vorwiegend auf der langue-Ebene operierende Ubersetzungstheorie beschränkt sich, wie vorher erwähnt, allein auf naturwissenschaftlich-technische Textgattungen, bei welchen die "Invarianz des Inhalts" sprachlicher Äußerungen als Grundproblem im Mittelpunkt steht. Die verschiedenen Stellungnahmen polnischer Wissenschaftler zur Übersetzungsproblematik wurden in den beiden Sammelbänden RUSINEK 1955 und POLLAK 1975 vorgestellt. Daraus können die Beiträge von KLEMENSIEWICZ 1955 (besser zugänglich in: Jezyk Polski. 34(1954). S.65-76) und INGARDEN 1955 besonders hervorgehoben werden, wo zum ersten Mal linguistische Überlegungen bei der Betrachtung des Übersetzungsprozesses eine Rolle spielen. Wertvolle Anregungen aus sprachwissenschaftlicher Sicht haben auch ansatzweise WOJTASIEWICZ 1957 und LEBIEDZINSSKI 1981 geliefert. Vgl. auch die referierende Arbeit 
sowie literaturwissenschaftlichen ${ }^{15}$ Zugangsmöglichkeiten haben besonders die textlinguistische, psycholinguistische, soziolinguistische, anthropologische und didaktische Betrachtungsweise eine berechtigte Position bei der Untersuchung von Übersetzungsprozeduren eingenommen. ${ }^{16}$ Der Versuch, die zahlreichen Methoden zu klassifizieren, ${ }^{17}$ führte zu einer globalen Zweiteilung, ${ }^{18}$ indem der theoretische, sprachenpaarübergreifende Ansatz und der sprachenpaarbezogene Ansatz, der wiederum in einen deskriptiven und einen angewandten Teil zerfält, unterschieden wird. Während ersterer faktorenanalytische Modelle des Übersetzungsvorgangs erarbeitet, beschäftigt sich der deskriptive Zweig mit der Beobachtung konkreter Translationsakte, der Entwicklung einer übersetzungswissenschaftlich relevanten Texttypologie sowie mit der Ermittlung von objektiven Äquivalenzkriterien; der anwendungsorientierte Ansatz schließlich ist auf Untersuchungen im Bereich des Übersetzungsunterrichts, der Übersetzungstechnik und der Übersetzungskritik ausgerichtet. ${ }^{19}$

Da sich der Großteil der bisherigen Abhandlungen allein um die übersetzungsprozessuale Theorieforschung bemühte und der anwendungsorientierten Übersetzungswissenschaft kaum Aufmerksamkeit geschenkt wurde, was zu einem regelrechten Theorieüberschuß führte, stellten sich in letzterer Zeit verstärkt Appelle nach einer Auflösung dieses Mißverhältnisses von Theorie und Praxis, nach der "Sichtbarmachung der theoretischen Ansätze durch konkrete Anwen-

von ZIETARSKA 1973 mit einem Überblick über die polnischen Studien in den Jahren 19501973.

is Für die Schaffung einer eigenen Theorie der literarischen Übersetzung, die sich vornehmlich an der Theorie der Dichtkunst und Hermeneutik orientieren müsse, sprachen sich KLOEPFER 1967 und LEVÝ 1969 aus. Vgl. auch die Angaben in BALCERZAN 1985.

${ }^{16}$ Deutlich wird die diverse Aufsplitterung z.B. bei KÖNIGS 1986, der seinen Forschungsbericht zur Übersetzungswissenschaft den Arbeitsschwerpunkten der einzelnen Wissenschafter entsprechend nach den unterschiedlichen Perspektiven systematisiert.

$17 \mathrm{Vgl.} \mathrm{dazu}$ in WILSS 1977 den Abschnitt "Methodologie der Übersetzungswissenschaft", S.93-100.

18 Dieser Vorschlag stammt von KADE 1968 (vgl. S.94f), der seinerseits auf FEDOROV 1968 zurückgreift. Siehe ferner LEBIEDZIŃSKI 1981, der sich ebenso für die Einteilung in przektadoznawstwo ogolne und przektadoznawstwo szczegolowe ausspricht, vgl. S.9 passim.

19 Genauere Ausführungen zu diesen Basisformen s. bei WILSS 1975. S.520-535. Vgl. auch KOLLER 1987 (S.95-101), der den Aufgabenbereich der Übersetzungswissenschaft in acht Hauptkomplexe gliedert, dabei den von "Übersetzungstexten ausgehende[n] Sprachvergleich (...) mit dem Ziel der Herausarbeitung von potentiellen Übersetzungsäquivalenzen " (S.98) - wie er im folgenden vorgenommen wird - explizit als Teilaufgabe der linguistisch-sprachenpaarbezogenen Übersetzungswissenschaft nennt. 
dung ${ }^{20}$, ein. ${ }^{21}$ In diesem Sinne ist dann auch die vorliegende Studie mit ihrer materialausgerichteten Detailuntersuchung zu verstehen.

Von besonderer Bedeutung für die moderne Übersetzungswissenschaft waren und sind immer noch Bemühungen um eine textlinguistische Fundierung ihres Objektbereichs. Sie nutzte dabei die Forschungsergebnisse der noch relativ.jungen Textlinguistik, die vor allem die bisherige Beschränkung auf den Satz als oberste sprachliche Einheit zugunsten einer Erweiterung des Betrachtungsfeldes zum Text hin aufgab. Da gerade Sprache als Rede in Texten vorkommt, ${ }^{22}$ hat es besonders der Übersetzer "nicht mit der Quellensprache und der Zielsprache als abstrakten Zeichensystemen zu tun, sondern mit konkreten Äußerungen" ${ }^{23}$. Es liegt hier die Beobachtung zugrunde, daß
Ausdrücke, die in isolierter Lexikoneintragung polysem sind (...), im Kontext monosemiert werden, d.h. die ihnen inhärente Mehrdeutigkeit wird durch den konkreten sprachlichen und/oder situativen Kontext einge- schränkt und zur funktionellen Eindeutigkeit reduziert. ${ }^{24}$

Mit der Erkenntnis, daß Übersetzung als eine spezifische Form der Sprachverwendung anzusehen ist, wurde die Forderung laut, daß eine wissenschaftliche Übersetzungstheorie nicht auf der Grundlage einer vergleichenden Analyse zweier Sprachsysteme, sondern nur auf der Basis von Texten zweier Sprachen entwickelt werden, und damit dem Bereich einer linguistique de la parole angehören müsse.

Die Tatsache, daß die teilweise programmatisch formulierten Thesen letztlich doch nicht selbstverständlich umgesetzt worden sind, somit der Paradigmenwechsel von der lexematischen bzw. Satzebene hin zur Textebene von der modernen Theorie der Übersetzung nicht in vollem Maße vollzogen wurde,

20 SNELL-HORNBY 1986. S.20.

21 Die seit Ende der fünfziger Jahre erschienenen wichtigsten unselbständigen Forschungsbeiträge - nach den drei genannten Teildisziplinen systematisiert - sind von WILSS 1981 als Fortsetzung der Sammelausgabe von STÖRIG 1963 zusammengetragen worden. Vgl. auch die Anthologie von SNELL-HORNBY 1986, die unter der Prämisse einer Neubestimmung des Theorie-Praxis-Bezugs der modernen Übersetzungswissenschaft die maßgeblichen Aufsätze der ersten Hälfte der achtziger Jahre vorstellt.

2 Auf die "Texthaftigkeit" von Sprache hat u.a. HARTMANN 1971, einer der deutschen Begründer der Textlinguistik, hingewiesen.

23 BARCHUDAROV 1977. S.7. Er gehört innerhalb der sovetischen Forschung zu den Initiatoren der Textlinguistik im übersetzungstheoretischen Aufgabenbereich. Innerhalb der Leipziger Schule ist besonders NEUBERT 1983 hervorzuheben.

24 KOSTA 1986. S.41. 
veranlaßte E.Coseriu, die aus dieser Perspektive falschen und richtigen Fragestellungen in der Übersetzungstheorie als wiederholten Appell zusammenzutragen. Als die vier entscheidenden Fehlansätze stellt er heraus:

1) Die Problematik der Uebersetzung und des Uebersetzens wird als eine die Einzelsprachen (die "langues") betreffende Problematik angegangen.

2) Es wird von der Uebersetzung (bzw. von der "idealen", aber theoretisch schon "unmöglichen" Uebersetzung) wenigstens implizite verlangt, dass sie alles in den Originaltexten Gemeinte und durch diese Texte als gemeint Verstandene mit den Mitteln der Zielsprache wiedergibt; sie könne dies aber nicht, und deshalb sei sie schon ihrem Wesen nach "unvollkommen", wenn auch praktisch notwendig.

3) Die Uebersetzung als rein einzelsprachlich bezogene Technik ("Uebertragung") wird dem Uebersetzen (d.h. der Tätigkeit der Uebersetzer) gleichgesetzt. Dies führt u.a. zu dem Paradoxon, dass die Uebersetzung zwar theoretisch unmöglich, empirisch jedoch eine Realität sei.

4) Es wird eine abstrakte optimale Invarianz für die Uebersetzung überhaupt angenommen. ${ }^{23}$

Bei der Kritik, die Übersetzungsschwierigkeiten würden eher langue- als parolespezifisch behandelt, führt Coseriu am Beispiel der Bedeutungsproblematik aus, daß Ziel einer jeden Übersetzung - die Unterscheidung der drei Hauptarten des sprachlichen Inhalts: Bezeichnung, Bedeutung, Sinn vorausgesetzt - nicht die Wiedergabe der gleichen Bedeutung, sondern der gleichen Bezeichnung und des gleichen Sinns zu sein hat. ${ }^{26}$ Ferner sieht er eine Auflösung des geltenden Widerspruchs der eigentlich unmöglichen, in der Praxis aber existierenden Übersetzung in der unabdingbaren Unterscheidung von Übertragung, verstanden als reine Technik der Herstellung zwischensprachlicher Äquivalenzen, und Übersetzen, verstanden als die tatsächliche komplexe Tätigkeit der Übersetzer, die nicht an rationale, sondern nur empirische Grenzen stößt. Damit verbunden ist schließlich das Problem der abstrakten optimalen Invarianzforderung. Da es sich beim Übersetzen um eine finalistisch und historisch bedingte Tätigkeit handelt, verlangt dieses nach textthematisch, -funktional und -pragmatisch differenzierten Äquivalenzen. ${ }^{27}$

Um textuelle Aspekte des Übersetzungsprozesses haben sich besonders F.Paepcke, Ph.Forget und R.Stolze bemüht. Mit ihrer hermeneutischen Orientierung, bei der der Übersetzer mit seinem spezifischen Vorwissen, seinem ausgangssprachlich gebundenen Sprachgefühl und letztlich seiner zielsprachlich gerichteten Formulierungskompetenz in das Zentrum des Übersetzungsgesche-

2S COSERIU 1978. S.18.

${ }^{20}$ Genaueres dazu s. Kap.3.3.2. dieser Arbeit.

${ }^{27}$ Eine ausführliche Kommentierung der Aussagen ist nachzulesen bei COSERIU 1978. 
hens rückt, ist neben der ausschließlich linguistisch ausgerichteten Übersetzungstheorie eine weitere Extremposition geschaffen worden. Da der Sinn von Texten nicht als statische Größe betrachtet wird, werden jegliche Schematisierungs- und Normierungsversuche abgelehnt und stattdessen die Tätigkeit des "intuitiven Textverstehens" und des "zielsprachlichen Gestaltens" als wesentliche Faktoren des Übersetzungsvorgangs hingestellt. Neben der Basisforderung, daß der "Text der Aktionsraum des Übersetzens" zu sein hat, liegen diesem Ansatz hauptsächlich die beiden Prinzipien der "Individualität" und "Übersummativität" von Texten, die eine Übersetzung zum "Nicht-Anderen" werden lassen, zugrunde. ${ }^{28}$

Kennzeichen übersetzungstheoretischer Bemühungen in jüngster Zeit sind zum einen die Skopostheorie (Theorie vom "Zweckprimat") von H.J.Vermeer $^{29}$, zum anderen J.Holz-Mänttäris Theorie des translatorischen Handelns. ${ }^{30}$ Von einer funktionalen Perspektive ${ }^{31}$ ausgehend, liegt beiden Konzepten zugrunde, daß sie sich von den überkommenen Vorstellungen des Übersetzens als Übertragung von Wörtern bzw. Texten aus einer Sprache in eine andere abwenden. Vielmehr rückt auf der Basis eines handlungsorientierten Textbegriffs die Anschauung in den Vordergrund, die Übersetzung eines Textes sei als spezifische Form von Handeln aufzufassen, durch welches "in einer neuen, anderen Situation ein neuer, anderer Text ebenfalls 'funktionieren', d.h. eine bestimmte, von anderen Kommunikationspartnern intendierte und verwirklichte Funktion erfüllen kann" ${ }^{\text {32 }}$. Damit wird die Situation, in der der Text als

Vgl. dazu PAEPCKE/FORGET 1981 und STOLZE 1982, bes. Kap.2.

20 Vgl. REISS/VERMEER 1984. Die Verfasser sind bestrebt, eine umfassende Translationstheorie zu konzipieren, die auf kulturwissenschaftlicher Grundlage aufbaut und sowohl textlinguistische als auch hermeneutische Aspekte in ihre Überlegungen einbezieht.

* Vgl. die Überlegungen von HOLZ-MÄNTTÄRI 1984. In diesen Zusammenhang gehört auch die Arbeit von NORD 1988. In Anlehnung an die beiden wichtigsten Bausteine der modernen Übersetzungswissenschaft entwickelt sie ein übersetzungsrelevantes Textanalysemodell, das in gleichem Maße für Wissenschaft, Ausbildung und Praxis nutzbar zu machen sein soll.

${ }^{31}$ Die Mőglichkeit einer funktionalen Ausrichtung ist innerhalb der übersetzungswissenschaftlichen Literatur bereits 1971 von REISS 1986 (S.93ff) in die Diskussion eingebracht worden. Doch wird hier die Orientierung eines Zieltexts am gegebenen Auftrag als Sonderfall betrachtet, erst später setzt sich allmählich die grundsätzliche Forderung nach einer Bevorzugung der Zieltextfunktion gegenüber der des Ausgangstextes bei der Wahl der Übersetzungsmethode durch; vgl. dazu NORD 1988. S.283, Anm.1.

${ }^{32}$ NORD 1988. S.276. 
"Botschaftsträger" zu verstehen ist, zum zentralen Element erhoben. Vermeer äußert dazu:

Eine Translation ist also immer auch ein transkultureller Transfer, die möglichste Lösung eines Phänomens aus seinen alten kulturellen Verknüpfungen und seine Einpflanzung in zielkulturelle Verknüpfungen. ${ }^{3}$

Mit der Einbettung der Übersetzung in den zielsprachlichen kommunikativen Zusammenhang wird offensichtlich, daß der Zweck des Zieltextes ein primärer Maßstab für die Bestimmung von Übersetzungsverfahren zu sein hat. Diese Orientierung an der Funktion des Zieltextes (Translatskopos) zieht gleichzeitig eine Schwächung der Gewichtung des Ausgangstextes nach sich.

Nach diesem Überblick zur Forschungssituation gilt es, das Augenmerk speziellen, für die folgende empirische Analyse relevanten Einzelfragen zuzuwenden. Im Mittelpunkt der Ausführungen soll die Diskussion des Äquivalenzbegriffs stehen. Das den Übersetzungsprozeß bestimmende Auffinden äquivalenter Ausdrucksmittel in der Zielsprache nimmt auch bei der Beurteilung von Übersetzungen eine zentrale Stelle ein.

\subsection{ANSÄTZE EINER ÜBERSETZUNGSKRITIK}

Angesichts des erwähnten traditionellen Forschungsschwerpunkts rückte die Ergebnisuntersuchung erst allmählich ins Blickfeld. Der übersetzungskritische Vergleich von Vorlage und Übersetzung, der Gegenstand der folgenden Erörterungen sein soll, ist wohl erstmals von Katharina Reiss thematisiert worden.' Mit dem Ziel, "objektive Kriterien und sachgerechte Kategorien für die Beurteilung von Übersetzungen aller $\mathrm{Art}^{\mathrm{m2}}$ zu erstellen, sollte der bisherigen unzulänglichen, meist nur auf intuitionsgelenkten Teilaussagen fußenden Kritik ein methodisch stringenter Bezugsrahmen gegeben werden. War jene mehr oder

33 VERMEER 1986. S.34.

' Vgl. dazu REISS 1968 und REISS 1986. Ihrem methodologischen Modell folgten Arbeiten von POPOVIĆ 1973, WILSS 1974, HOUSE 1977 u.a., die sicherlich zu den bedeutendsten Studien zur Begründung der Übersetzungskritik gezählt werden kőnnen. Die Ansätze von Reiss, Popovic und Wilss hat KOLLER 1987 (S.196-206) gegenübergestellt und diskutier.

${ }^{2}$ REISS 1986. S.7. 
minder fundierte, vornehmlich bei literarischen Texten praktizierte Übersetzungskritik weitgehend eine nicht an die ausgangssprachliche Version gebundene Translatkritik ${ }^{3}$, spricht sich Reiss für die ausgangstextabhängige Form der Kritik aus. Das Charakteristikum dieser Translationskritik besteht nämlich in einer vergleichenden Betrachtung und Analyse von Ausgangs- und Zieltext." Erst mit dieser Gegenüberstellung wird es möglich, z.B. Kenntnis über Strukturähnlichkeiten und -unterschiede von Ausgangs- und Zielsprache oder Rückschlüsse über die vom Übersetzer vorgenommene Strategie zu erlangen. Unter der vorläufigen, noch nicht sonderlich nuancierten Vorgabe, jeder ÜbersetzungsprozeB sei ein "bipolarer Vorgang, der sich in der Gestaltung eines zielsprachlichen Textes unter ständiger Rückbindung an einen ausgangssprachlichen Text erfüllt" ${ }^{\text {"6 }}$, und dabei habe das Auffinden "optimaler Äquivalenzen" als oberstes Postulat zu gelten, hat Katharina Reiss in Anlehnung an textlinguistische Bestrebungen als Grundlage ihres Modells eine übersetzungsrelevante Texttypologie entworfen.

So sollte der erste Schritt der ausgangssprachlichen Textanalyse für den Übersetzer und Kritiker gleichermaßen in der Festlegung des Texttyps bestehen, um daraus bereits entscheidende Instruktionen für die Übersetzungsmethode ableiten zu können. Im Anschluß an die drei Sprachzeichenfunktionen des Bühlerschen Organon-Modells ${ }^{7}$ - Darstellung, Ausdruck, Appell - ${ }^{8}$ legte Reiss drei Grundfunktionen von Texten fest, "die jeweils von der Kommunikationsintention des Textautors bestimmt werden"9, und definierte demnach folgende Textypen: den inhaltsbetonten ( = informativen), den formbetonten ( $=$ expressiven), den appellbetonten (= operativen), denen sie schließlich noch einen

${ }^{3} \mathrm{Vgl}$. auch KOLLER 1987 (S.206-209), der von ZS-Textkritik (ZS = Zielsprache) spricht.

- Zu den Termini Translation (= Bezeichnung für den Übersetzungsvorgang) und Translal (= Produkt einer Translation) vgl. die im Anschluß an die Leipziger Schule vorgenommenen terminologischen Unterscheidungen von REISS/VERMEER 1984. S.6.

S Vgl. dazu REISS 1986. S.17-24.

${ }^{\circ}$ REISS 1986. S.11.

7 Auch die osteuropäische linguistische Übersetzungstheorie hat Versuche unternommen, den Bühlerschen Ansatz aufzugreifen, allerdings ohne Rezeption der westlichen Entwürfe, vgl. dazu KOSTA 1986. S.50.

- Vgl. BÜHLER 1934. S.28ff.

- REISS/VERMEER 1984. S.205. 
vierten, den audiomedialen Texttyp ${ }^{10}$ hinzufügte. " Damit überwand sie die traditionelle Dichotomie von literarischen vs. pragmatischen Texten. ${ }^{12}$ Mit dem Wissen um Mischformen innerhalb eines Textes wird bei der Klassifikation der Texttyp je nach Dominanz einer der vertretenen Funktionen bestimmt. Steht die Vermittlung von Inhalten im Vordergrund, so handelt es sich um einen informativen, bei dessen Übersetzung "Invarianz auf der Inhaltsebene" 13 angestrebt wird; beabsichtigt der Autor sein Informationsangebot auch noch künstlerisch zu gestalten, dann spricht man vom expressiven Texttyp, bei dessen Übertragung die "Analogie der Form"14 zu fordern ist. Beim operativen Texttyp schließlich geht es neben der Inhaltsvermittlung besonders um persuasive Gestaltung, so daß es auf die "Erhaltung des textimmanenten Appells"1s ankommt.

Davon ausgehend, daß trotz erheblicher Variabilität konkreter Texte Regularitäten der Sprachverwendung festzustellen sind, konnten weitere Differenzierungen vorgenommen werden. Den Texttypen als übergeordneten Einheiten wurden verschiedene Textsorten zugeordnet. ${ }^{16}$ Zieht man in die Überlegungen sogar Textsortenkonventionen ${ }^{17}$ mit hinein, so ist die Textsortenklassifizierung durch die Unterscheidung von Textsortenklassen, z.B. Roman bzw. Vertrag, Textsorten, z.B. Kriminal-, Zukunfts-, historischer Roman bzw. Miet-, Kauf-,

10 Darunter werden Texte zusammengefaßt, die "zwar schriftlich fixiert, aber mit Hilfe eines nicht-sprachlichen Mediums in gesprochener (oder gesungener) Form an das Ohr des Empfängers gelangen", vgl. REISS 1986. S.34.

"Zum Texttyp als Kategorie der Übersetzungskritik siehe REISS 1986. S.24-53. Die in Klammern gesetzten Texttypenbenennungen sind infolge kommunikationstheoretischer Überlegungen Modifizierungen ihres ersten Ansatzes, vgl. REISS 1976, bes. S.11-15.

12 Daneben ist auch NEUBERT 1968 zu nennen, der - wenn auch in anderem Zusammenhang von einer Vierfachdifferenzierung der Textarten ausgeht.

${ }^{13}$ REISS 1986. S.37.

14 REISS 1986. S.38.

is REISS 1986. S.47.

16 Unterschieden wird ein: a) informativer Textyp mit den Textsorten Sachbuch, Bericht, Aufsatz, Urkunde, Gebrauchsanweisung, Kommentar u.a.; b) expressiver Texttyp mit den Textsorten Lyrik, Novelle, Roman, Biographie, Lehrgedicht, Komödie, Schauspiel u.a.; c) operativer Textyp mit den Textsorten Satire, Predigt, Propaganda, Reklame, Demagogie, Pamphlet u.a. Vgl. dazu die graphische Darstellung bei REISS 1976. S.19 bzw. REISS 1986. S.34, 42, 45 .

17 Verstanden werden darunter "charakteristische Sprachverwendungs- und Textgestaltungsmuster" (REISS/VERMEER 1984. S.177), die sich "durch ihre Rekurrenz (...) zu sprachlichen Verhaltensregularitäten in bestimmten Kommunikationssituationen" (S.183) verfestigen. 
Handelsvertrag und Textsortenvarianten, z.B. zu Kriminalroman der Detektivroman oder Thriller bzw. zum Mietvertrag der Einheitsmietvertrag, um ein weiteres $\mathrm{zu}$ nuancieren. ${ }^{18}$

Bei den vorangegangenen Darlegungen ist immer implizit Funktionskonstanz zwischen ausgangs- und zielsprachlichem Text vorausgesetzt worden. Die Erhaltung der kommunikativen Funktion gilt hier als Normalfall, während Funktionswechsel entsprechend der "Verschiedenheit der translatorischen Zwecksetzungen" ', die wiederum andere Übersetzungsstrategien nach sich ziehen, als Sonderfall besondere Maßstäbe an die Übersetzungskritik stellen würde.

Die genannten Texttypen werden auf dem Hintergrund innersprachlicher Instruktionen und außersprachlicher Determinanten, die den Übersetzungsprozeß, d.h. das Auffinden "optimaler Äquivalente" bestimmen, beschrieben. Während zur sprachlichen Kategorie der Übersetzungskritik semantische, lexikalische, grammatische und stilistische Instruktionen gezählt werden, die je nach Textsorte in unterschiedlicher Rangfolge zu berücksichtigen sind, umfaßt die pragmatische Kategorie die folgenden Faktoren: den engeren Situationsbezug, den Zeit-, den Orts- und Empfängerbezug, die Sprecherabhängigkeit und affektive Implikationen, die die innersprachliche Gestaltung wesentlich unterstützen. ${ }^{20}$

Neben berechtigter Anerkennung blieb dieser texttypologische Ansatz in der Forschung nicht unumstritten. Nur exemplarisch sollen die wohl gravierendsten Unzulänglichkeiten aufgezeigt werden: W.Koller kritisiert einerseits die willkürliche Beschränkung auf nur drei Funktionen, bei einem wohl größeren Potential an Sprachfunktionen, ${ }^{21}$ andererseits die Schwierigkeit einer immer zweifelsfreien Zuordnung von Texten zu diesem Typologieschema. ${ }^{22}$ Allerdings kann auch er keine überzeugende Alternative bieten, sein Vorschlag zur "Methodik einer wissenschaftlichen Übersetzungskritik" bleibt sowohl hinsichtlich der Präzi-

18 Zur Textsortentheorie vgl. die Betrachtungen in Kap.11 bei REISS/VERMEER 1984.

19 REISS/VERMEER 1984. S.134.

20 Näheres dazu bei REISS 1986. Kap.3 und 4.

${ }^{21}$ Eine Erweiterung der Bühlerschen Dreiteilung hat JAKOBSON 1960 (S.355-357) vorgenommen, indem er die poetic, phatic und metalingual function hinzugenommen hat.

2 S. KOLLERs 1987 Darlegung S.199-206. 
sion der Ausformulierung als auch der Anwendbarkeit deutlich hinter dem Reissschen Ansatz zurück. ${ }^{23}$

In ähnlicher Weise äußert sich auch J.House, die an der reibungslosen Umsetzung, der Praktikabilität der Reissschen Theorie Zweifel hegt:

Her model of translation quality assessment is programmatic only, she gives no indication as to the precise method of establishing textual function and textual type. ${ }^{24}$

Auch R.Stolze sieht in Übereinstimmung mit Wilss das Klassifikationssystem von Reiss als Versuch einer "idealisierenden Modellbildung" an, in dem der für jeden Einzeltext charakteristische Aspekt der "Multiperspektivität" und "Polyfunktionalität" außer acht gelassen wird; ${ }^{25}$ so können z.B. künstlerische Texte alle drei Funktionen abdecken. Die Abkehr von derartigen Generalisierungstendenzen verdichtet sich schlieBlich in der Forderung:

An die Stelle der traditionellen Typologien sollten hier Prototypologien treten, die nicht auf Trennung, sondern auf Fokussierung hinzielen und dabei "verschwommene Ränder" und Mischtypen zulassen. ${ }^{20}$

Stolzes Hauptkritik aber richtet sich gegen die Parallelsetzung von Sprach- und Textfunktion. Während Bühler in seinem Modell von Funktionen von Zeichen in der Sprache ausgeht, übertrage Reiss diese unzulässigerweise auf konkrete Texte und nennt sie Textfunktionen. ${ }^{27}$

Neue Anregungen bot der übersetzungskritischen Diskussion J.House. Im Unterschied zu Reiss, die vor allem den Text selbst und damit die Sprache zum Ausgangspunkt ihrer Betrachtung macht, unternimmt jene den Versuch, ein pragmatisch-funktionales Analysemodell zu entwickeln, in dessen Mittelpunkt Sprachbenutzer- und Sprachgebrauchsdimensionen stehen: ${ }^{28}$

${ }^{2} \mathrm{Vgl}$. dazu KOLLER 1987. S.210-216.

24 HOUSE 1977. S.23f.

${ }^{2 s}$ Hierzu s. STOLZE 1982. S.113.

${ }^{30}$ SNELL-HORNBY 1986. S.16. Vgl. auch ihre graphische Darstellung auf S.17.

${ }^{27}$ Genaueres dazu in STOLZE 1982. S.114-116; vgl. auch weiter unten S.117, Anm.53. Zu nennen ist auch KOSTA 1986 (bes. S.46-52), der versucht hat, "die Spezifität der literarischen Texte am Beispiel einiger bekannter Texttypologien zu beleuchten und die Insuffizienz in der Gleichsetzung von Sprach- und Textfunktion zu erweisen". In diesem Zusammenhang macht STOLZE 1982 (vgl. S.116, Anm.125) ferner darauf aufmerksam, daß bei der Beschreibung des expressiven Texttyps Reiss eher Anregungen von JAKOBSON 1960 hinsichtlich des von ihm definierten Begriffs der poetischen Funktion der Sprache aufgreift.

3 Vgl. HOUSE 1977. S.42. 
A. Dimensions of Language User:

B. Dimensions of Language Use:
1. GEOGRAPHICAL ORIGIN

2. SOCIAL CLASS

3. TIME

1. MEDIUM [SIMPLE/COMPLEX]

2. PARTICIPATION [SIMPLE/COMPLEX]

3. SOCIAL ROLE RELATIONSHIP

4. SOCIAL ATTITUDE

5. PROVINCE

Die in Teil A. genannten Sprachbenutzerdimensionen stimmen im großen und ganzen mit der bekannten Coseriuschen Differenzierung in diatopisch, diastratisch, diaphasisch überein. ${ }^{29}$ Mit dem unter B. aufgeführten Kriterium Medium (simple/complex) soll der Gegensatz von geschriebenen und gesprochenen Texten bedacht werden, mit Participation (simple/complex) ist die monologische bzw. dialogische Form der Rede gemeint, wobei jeweils mit complex verschiedene Mischformen erfaßt werden. Die interpersonalen Komponenten, zum einen Social Role Relationship, die in "symmetrical" (Relation zwischen zwei auf gleicher sozialer Rangstufe Stehenden) und "asymmetrical" (Relation zwischen zwei nicht Gleichgestellten) gegliedert ist, und zum anderen Social Attitude, die den Grad an persönlicher Nähe oder Distanz - von "intimate" bis "frozen" anzeigt, sollen für die Beziehung Leser vs. Autor verantwortlich sein. Mit dem letzten Begriff ihres Rasters, Province, sollen die Textsorten und Funktionalstile erfaßt werden. ${ }^{30}$

Dieses Bewertungsschema mit acht Subkategorien, den "situational dimensions", für die linguistische Korrelate aufgezeigt werden müssen, ist zunächst an den Ausgangstext anzulegen. So entsteht ein die Textfunktion charakterisierendes "textual profile", das zur Norm für den Zieltext gemacht wird und dann eine Beurteilung über dessen Leistungsfähigkeit erlaubt. ${ }^{31}$

Sicherlich reicht der an pragmatischen und situationellen Bedingungen der Sprache ausgerichtete Ansatz von House in Hinblick auf eine Objektivierung von Äquivalenzkriterien weit über den ihrer Vorgänger hinaus, die jenem Aspekt geringere Bedeutung beigemessen haben, doch zeigt die Praxis, daß auch dieses Modell Einstufungsschwierigkeiten bereitet, hauptsächlich bei den medialen und

\footnotetext{
20 Zu dieser Terminologie vgl. COSERIU 1973a. S.32f.

30 Ausfürlicher hierzu vgl. HOUSE 1977. S.37-50.

31 S. HOUSE 1977, bes. S.49f.
} 
interpersonalen Faktoren. ${ }^{32}$ Problematisch ist ferner die allzu gewichtige Dominanz der Pragmatik, was sich auch aus äquivalenztheoretischer Sicht - mit der Festlegung, daß die pragmatische Äquivalenz der semantischen vorzuziehen sei $-{ }^{33}$ bemerkbar macht.

Angesichts dessen, daß unterschiedliche Sprachstrukturen, unterschiedliche situationsgebundene Sprachverwendungsgewohnheiten und unterschiedliche soziokulturelle Gegebenheiten zweier Sprachgemeinschaften bestehen und diese sich wesentlich auf die Qualität einer Übersetzung auswirken können, werden die Bemühungen um geeignete Maßstäbe für die Beurteilung von Übersetzungen weiterhin aktuelles Thema der Übersetzungswissenschaft bleiben. Bei der Suche nach einer zufriedenstellenden Antwort auf die so häufig gestellte Frage: Wann ist eine Übersetzung gut? hat sich der Terminus Äquivalenz zum Schlüsselbegriff herauskristallisiert. Es erweist sich daher im folgenden als notwendig, ihn zu präzisieren, um eventuelle Bewertungskriterien an die Hand zu bekommen.

\subsection{DIE FRAGE DER RELATION VON AUSGANGSTEXT UND ZIELTEXT}

Daß Übersetzen "keine Kommunikationsleistung als Gebrauch eines Kodes zur Übertragung von Nachrichten"1 ist, bei der der Übersetzer lediglich als "passive Schaltstelle"2 auftritt, vielmehr ein "Textverarbeitungs- und Textverbalisierungsprozeß, der von einem ausgangssprachlichen Text zu einem möglichst äquivalenten zielsprachlichen Text hinüberführt" ${ }^{33}$, und dabei die trans-

\footnotetext{
$\therefore$ Vgl. dazu KÖNIGS 1979 (S.37 und Anm.27 auf S.141), der über eine - von einer Studentengruppe vorgenommenen - Erprobung dieses Schemas informiert. Infolge unterschiedlicher Ergebnisse konnte dem Modell eine nur "subjektive Funktionalität" zuerkannt werden.

$30 \mathrm{Vgl}$. HOUSE 1977. S.28.

1 PAEPCKE 1979a. S.320, seine Kritik richtet sich gegen den einseitigen kommunikationstheoretischen Ansatz.

2 SNELL-HORNBY 1986. S.13.

'WILSS 1977. S.72 (Hervorhebung B.K.). 
latorische Kompetenz ${ }^{4}$ als wesentlicher Faktor angesehen wird, gehört mittlerweile zu den verbindlichen Auffassungen linguistischer Übersetzungstheorie. Infolge der fortwährenden Modifizierung dieser Disziplin erfuhr auch eines der Kernprobleme des Übersetzungsvorgangs, die Herstellung von Äquivalenz/ Adäquatheit ${ }^{5}$ bzw. die Frage nach der Invarianz ${ }^{6}$, mannigfaltige und nicht selten Verwirrung stiftende Interpretationen. Von einem Konsens in der Begriffsbestimmung, deren Versuch doch in kaum einer westlichen bzw. osteuropäischen Publikation fehlt, kann in der Tat keine Rede sein. Gemeinsam ist allen lediglich die grundlegende Feststellung, daß mit dem aus der Fachterminologie der Logik entliehenen Begriff der Äquivalenz', der gleichermaßen im Mittelpunkt der Kontrastiven Linguistik steht, ${ }^{8}$ eine spezifische Relation zwischen einem Ausgangstext (bzw. -elementen) und einem Zieltext (bzw. -elementen) gemeint ist.

Zum Äquivalenzbegriff läßt sich eine Reihe von Definitionsansätzen zusammentragen, in denen sich ein jeweils anderes Verständnis von der Art dieser Beziehung widerspiegelt: ${ }^{9}$ beginnend mit Jakobsons Begriff der equivalence in difference, mit dem nichts anderes gemeint ist als das Ziel der Herstellung von Äquivalenz trotz existierender Strukturverschiedenheiten der Sprachen, ${ }^{10}$ über Nidas formal equivalence und dynamic equivalence," Catfords textual equiva-

\footnotetext{
4 Diese wird nicht als "rein sprachliche Fähigkeit, sondern als eine über die Beherrschung von grammatikalischen und lexikalischen Regeln von AS und ZS hinausgehende linguistische und extralinguistische Fähigkeit" verstanden, so KOSTA 1986. S.83.
}

s Zu diesem Begriffspaar vgl. z.B. REISS 1984. In Abhängigkeit vom Zweck (Skopos), der "Dominante aller Translation", schlägt sie eine klare Differenzierung beider Termini vor, so daß, verkürzt gesagt, unter Adäquatheit eine "Angemessenheit" in bezug auf den gewählten Zweck verstanden wird, Äquivalenz (= Gleichwertigkeit) dagegen nur bei gleicher kommunikativer Funktion von Ausgangs- und Zieltext gefordert wird. Vgl. auch die Definition von KLEMENSIEWICZ 1955 (S.93), der im Anschluß an sowjetische Arbeiten den Terminus adekwatnose in die polnische Forschung einbrachte.

- Năheres zum Terminus Invarianz s. JÄGER 1975. S.87ff.

7 REISS/VERMEER 1984 (S.128f) sprechen sich demgegenüber für eine Herkunft aus dem Bereich der Elektrotechnik aus.

- Vgl. dazu KOLLERs 1987 Ausführungen auf S.176-186.

- Die Bemühungen um immer neuere Definitionsversuche hat SNELL-HORNBY 1986, die bisher 58 unterschiedliche Äquivalenztypen feststellen konnte, heftig kritisiert, sie spricht von einem "Wildwuchs von Äquivalenztypen" (S.15), vgl. S.13-16.

${ }^{10} \mathrm{Vgl}$. JAKOBSON 1971. S.262.

"Vgl. NIDA 1964. S.159. 
lence, ${ }^{12}$ Kades Formulierung der Invarianz auf der Inhaltsebene, ${ }^{13}$ House' Forderung der equivalence of pragmatic meaning, ${ }^{14}$ Jägers kommunikative $\ddot{A q u i v a l e n z}$ und funktionelle Äquivalenz, ${ }^{15}$ den von Reiss geprägten Begriff der funktionalen Äquivalenz einer "kommunikativen Übersetzung"16 sowie Neuberts text-bound equivalence ${ }^{17}$ bis hin zu Paepckes Zielbegriff des glücklichen $\ddot{U}$ bersetzens, ${ }^{18}$ der das Definitionschaos keineswegs aufhebt.

\subsubsection{Determinanten des Übersetzungsvorgangs}

Will man Äquivalenz in ihrer Gesamtheit erfassen, so ist Wilss' Forderung zuzustimmen, "ein hinlänglich detailliertes Faktoreninventar für die MeBbarkeit der Äquivalenz"19 zu schaffen. Grundlegend für eine derartige Angabe von Gesetzmäßigkeiten ist sicherlich die Kenntnis der wesentlichen den ÜbersetzungsprozeB konstituierenden textinternen und textexternen Kommunikationsgrößen.

In diesem Sinne hat Katharina Reiss für die Kommunikative Übersetzung ( = Übersetzungstyp, bei dem Funktionskonstanz von Ausgangs- und Zieltext vorausgesetzt wird) folgende Rahmenbedingungen für einen Übersetzungsvor-

\footnotetext{
12 Vgl. CATFORD 1965. S.49.

13 Vgl. KADE 1968. S.63.

14 Vgl. HOUSE 1977. S.28.

is Vgl. JÄGER 1975. S.37 und 107-109. Zur Kritik an Jägers und Nidas Terminologieauffassungen s. KOSTA 1986. S.86-92.
}

$16 \mathrm{Vgl}$. REISS 1983. S.200 und 208 bzw. REISS 1984. S.87. Bereits KLEMENSIEWICZ 1955 (S.93-95) betont den funktionalen Zugang: "Żadanie przekladu polega nie na kopiowaniu, nie na odtwarzaniu, tym mniej na przetwarzaniu elementow i struktur oryginalu (...), ale na uchwyceniu ich funkcji i wprowadzeniu za elementy i struktury oryginału takich elementow i struktur wlasnego jezyka, które by byly w miare możnosci substytutami i ekwiwalentami o takiej samej funkcionalnej wydolnosci, zdatnosci i skutecznosci, w czym wlasnie okazuje sie ich adekwatnosc, wspołmiernosc, wspolstosownosc, rownowartosciowosc" (S.93, Hervorhebung B.K.).

17 Vgl. NEUBERT 1984. S.68 bzw. NEUBERT 1986. S.87.

18 Vgl. PAEPCKE 1986. S.112.

19 WILSS 1977. S.157. 
gang zusammengetragen: ${ }^{20}$ Als erstes ist der Produzent des Ausgangstextes zu nennen, der "mit seinem Text ein Informationsangebot an den Ausgangstextrezi-

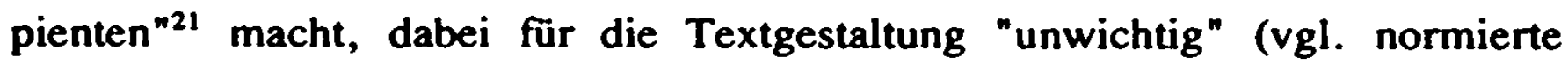
Texte wie Formulare, Gesetzestexte u.a.) bis "ausschlaggebend" 22 (z.B. literarische Texte) sein kann.

Dann ist die Rolle der Ausgangssprache zu beachten: "sie hat vom System (SAUSSURE's 'langue') her wie in den Verwendungsbedingungen (COSERIU's 'Norm') ihre ganz spezifischen Charakteristika (= Strukturen) ${ }^{n 23}$, so daß es beim kommunikativen Übersetzen nicht auf das bloße Substituieren der sprachlichen Segmente ankommt, sondern auf die funktionsgerechte Wiedergabe mit den der Zielsprache eigenen Mitteln. U.a. ist hier der - im Coseriuschen Sinne diatopischen, diastratischen und diaphasischen Gestaltung der jeweiligen Sprache Rechnung zu tragen.

Schließlich ist der Text der Ausgangssprache als sprachliches Gebilde zu berücksichtigen. Jedem Text werden drei sog. "Seinsweisen" zuerkannt: zum einen ist er - abgesehen von völlig standardisierten Texten - ein "Individuum", da sich in ihm die spezielle, d.h. "individuelle Sprachzeichenauswahl des Produzenten zur Verbalisierung seines Informationsangebots niederschlägt" ${ }^{24}$, zum anderen ist er ebenso Realisation einer bestimmten Textsorte und eines übersetzungsrelevanten Texttyps. Diesen Text gilt es nun in allen drei Dimensionen in der Zielsprache zu erhalten, dabei muß er sowohl seine Individualität bewahren, als auch durch zielsprachenanaloge Konventionen sowie zielsprachenkonforme Typenauswahl ersetzt werden.

Die Textkonstitution sowie Textrezeption beeinflussen darüberhinaus die beiden Variablen des situationellen Kontextes und der sozio-kulturellen Einbettung des jeweiligen Textes. Sprache wird nämlich nicht in einem Vakuum verwirklicht, sondern "ergibt sich aus einer bestimmten Situation innerhalb eines

\footnotetext{
${ }^{30}$ Ausführlicher zu diesem Faktorenmodell s. REISS 1983. S.200-202 sowie REISS/VERMEER 1984. S.148-153.

${ }^{21}$ REISS/VERMEER 1984. S.149.

2 REISS 1983. S.201.

22 REISS 1983. S.201.

थ REISS/VERMEER 1984. S.149.
} 
kulturellen Rahmens" 25 , der von Sprachgemeinschaft zu Sprachgemeinschaft divergieren kann. ${ }^{26}$

Zu den aufgezählten Komponenten kommt zuletzt die bedeutende, ambivalent ausgerichtete Stellung des Übersetzers hinzu, der sowohl in der Person des Rezipienten des Ausgangstextes als auch des Produzenten des Zieltextes auftritt und mit seiner Sprach-, Sach- und Übersetzungskompetenz versucht, die Vorlage zu verstehen, d.h. die inner- und außertextlichen Zusammenhänge zu erkennen und zu interpretieren, um die Mitteilungen des ausgangssprachlichen Textes in die Strukturen der Zielsprache einzubringen. ${ }^{27}$ Mit dem Verstehen einer ausgangssprachlichen Version ist immer verbunden, daß "jede Übersetzung auf einer Rezeption des AS-Textes beruht ${ }^{\text {"28; }}$; geht man von einer "Multiperspektivität und Übersummativităt von Texten" ${ }^{m 9}$ aus, so kann es nicht nur eine einzig mögliche und richtige Übersetzung geben.

Der Vollständigkeit halber sei noch der durch bestimmte Erwartungsnormen geprägte Empfänger(kreis) einer Übersetzung angeführt, der seinerseits das ihm Dargebotene erkennen, verstehen und deuten wird.

Eine weitere Spezifizierung der den Übersetzungsprozeß steuernden sprachlichen und nichtsprachlichen Faktoren unternimmt auch Ch.Nord mit ihrem senderabhängigen Modellvorschlag einer übersetzungsrelevanten Textanalyse, der dem Übersetzer eine verläßliche Basis für jede einzelne übersetzerische Entscheidung liefern soll. Sind in den bisherigen Konzepten ${ }^{30}$ textexterne, situative Kriterien auch schon - wenn auch mit unterschiedlicher Akzentuierung - berücksichtigt worden, so rücken sie in ihrem Analysemodell, das auf einem handlungsorientierten Textbegriff und einem funktionalistischen Übersetzungskonzept

25 SNELL-HORNBY 1986. S.13.

${ }^{20}$ Diesen Faktor hebt auch in Anlehnung an WOJTASIEWICZ 1957 (S.105) SZYMCZAK 1973 (S.26) als Hauptproblem des Übersetzens hervor, wenn er sagt: "glowna trudnose przekladu polega nie na istnieniu różnic w strukturze jezyka $A$ i jezyka $B$, lecz na istnieniu odrebnosci środowiskowo-kulturowych, ktorych odbiciem sa fakty leksykalno-semantyczne i stylistyczne".

27 Vgl. z.B. PAEPCKE 1968 und PAEPCKE 1979b mit seinem hermeneutischen Konzept, das im Verstehen und Deuten eines Textes die obersten Gebote für jedes Formulieren, d.h. Übersetzen sieht.

3 REISS 1983. S.202.

29 STOLZE 1982. S.100.

${ }^{30} \mathrm{Vgl}$. dazu NORD 1988. S.42f. 
aufbaut, in den Mittelpunkt des Interesses; die "semantisch-syntaktischen Textualitätsmerkmale" (textinterne Kriterien) bleiben zunächst sekundären Charakters. ${ }^{31}$ Doch die Trennung der beiden Komponentenfelder ist lediglich begrifflicher Art, denn deren praktische Anwendung verdeutlicht ihre wechselseitige Abhängigkeit.

Mit dem Wissen um die am Übersetzungsprozeß beteiligten Determinanten lassen sich erste richtungweisende Rückschlüsse auf den Charakter der geforderten Äquivalenz ziehen: sie ist nicht als statische Größe zu verstehen, vielmehr zeigt sich, daß Äquivalenz ein "variabilitätsorientierter Begriff ist, der sich in einem ständigen Oszillieren zwischen Zugabe und Defizit im Vergleich zum Original ausdrückt ${ }^{\text {"32 }}$, so daß sich, wie häufig gefordert, kein generalisierbares Beschreibungsmodell mit übereinzeltextlich gültigen Kriterien aufstellen läßt. Die Suche nach zielsprachlichen Entsprechungen ist immer für jeden Text neu und individuell aufzunehmen. Reiss faßt treffend zusammen:

Damit erweist sich Äquivalenz als ein flexibler Begriff für eine Relation zwischen AT und einem ZT, die bei jedem Text (und jedem seiner Elemente in seinem Beitrag zwm Sinn dieses Textes) eine Neuordnung der Relevanz aller jener Faktoren erfordert, die den ÜbersetzungsprozeB beeinflussen. Selektion und Hierarchisierung dieser relevanten Faktoren erfolgen durch den Übersetzer. ${ }^{33}$

\subsubsection{Die Forderung nach einer "Äquivalenz des Sinns"}

Wesentliche Impulse zur weiteren Präzisierung der Äquivalenzdefinition und damit auch Lösungsvorschläge zum Übersetzbarkeits- vs. UnübersetzbarkeitsProblemkreis kamen von Eugenio Coseriu. Für ihn ist die individuelle Sprachgestaltung der jeweiligen Einzelsprachen, die sich darin äußert, daß

die Inhalte zweier verschiedener Sprachen (...) oft nicht nur nicht in einem Verhăltnis von $1 \mathrm{zu} 1$, und nicht nur nicht in einem "rationalen" Verhältnis vom Typ 1 zu 2 (bzw. 1 zu 3, 1 zu 4 usw.) (...), sondern einfach in einem

31 Die Zusammensetzung aller Faktoren wird mittels des auf der sog. LASSWELL-Formel basierenden "W-Fragen"-Schemas veranschaulicht; zum Fragenkomplex S. NORD 1988 (S.41), zu den Ausführungen des Modells S.40-165.

3 STOLZE 1982. S.196 (Hervorhebung B.K.).

30 REISS 1984. S.88 (Hervorhebung B.K.). 
"irrationalen" Verhältnis zueinander stehen, (...) so dass sehr viele Inhalte zweier Sprachen "inkommensurabel" sind"

nicht, wie häufig beklagt, "das Problem par excellence der Uebersetzung, sondern vielmehr ihre Voraussetzung, die Bedingung ihrer Existenz ${ }^{\text {"3s. }}$.

Von der Forderung einer "Autonomie der Textebene" im Rahmen seiner textlinguistischen Erörterungen ausgehend, grenzt Coseriu drei verschiedene Ebenen des Sprachlichen gegeneinander ab: die Ebene des Sprechens im allgemeinen, die Ebene der Einzelsprachen und die Ebene des Textes. Besonders die vorgenommene Differenzierung von historischer Einzelsprache und Text konnte nutzbringend in die übersetzungstheoretische Diskussion übernommen werden, indem sich - wie bereits oben angedeutet - allmählich eine Abkehr von der Betrachtung isolierter, kleinerer Einheiten der lexematischen Ebene und eine Hinwendung zu den komplexeren sprachlichen Einheiten, den Texten, vollziehen konnte. In einem nächsten Schritt wurden den drei Ebenen der Sprache "jeweils spezifische Funktionen" und damit "unterschiedliche allgemeine Werte des Inhalts ${ }^{\text {"36 }}$ zugeteilt: $:^{37}$

$$
\begin{aligned}
& \text { "Welt" - Bezeichnungsfunktion - Bezeichnung } \\
& \text { Einzelsprache - einzelsprachliche F. - Bedeutung } \\
& \text { Text - Textfunktion - Sinn }
\end{aligned}
$$

Die Anwendung dieser Dreiteilung des sprachlichen Inhalts proponierte er nun zur Klärung noch ausstehender übersetzungstheoretischer Fragen, ${ }^{38}$ denn bei der geforderten Wiedergabe von Textinhalten kommt es ihm zufolge nicht auf die Übertragung der gleichen Bedeutung - womit die jeweils einzelsprachlich gebundenen Denkinhalte gemeint sind -, sondern der gleichen Bezeichnung worunter die von der Einzelsprache trennbaren Inhalte, der "Bezug auf das aussersprachlich Gemeinte ${ }^{m 9}$ verstanden wird - und die Übertragung des gleichen Sinns - als "der besondere Inhalt eines Textes oder einer Texteinheit ${ }^{\text {40 }}$ -

* COSERIU 1978. S.19.

3s COSERIU 1978. S.24.

* COSERIU 1980. S.43; Genaueres zu seinen textlinguistischen Darlegungen vgl. S.35-50.

37 Zum Schema und seiner Erläuterung s. COSERIU 1980. S.47.

3 Zu den folgenden Ausführungen vgl. den Beitrag COSERIU 1978, bes. S.18-26.

${ }^{30}$ COSERIU 1978. S.20.

- COSERIU 1978. S.21. 
an, und zwar mittels der Bedeutungen der jeweiligen Zielsprache. ${ }^{41}$ Mit der Herausstellung der Dichotomie Bezeichnung - Bedeutung ergibt sich also, daß zunächst nur Äquivalenz in der Bezeichnung gefordert werden kann; die Beziehung zwischen den Bedeutungen, den Instrumenten zur Mitteilung des Inhalts der Ausgangs- und Zielsprache, ist hierbei lediglich eine indirekte, sie "besteht nur insoweit, als analoge oder verschiedene Bedeutungen dieser Sprachen sich einander in der Bezeichnung 'entsprechen' ". ${ }^{2}$ Der Weg dieser Bedeutungsfindung im Übersetzungsprozeß kann in zwei Phasen gegliedert werden, zuerst die semasiologische, in der der Übersetzer die Bezeichnung des Originals verstehen muB, dann die onomasiologische, in der es die Entsprechung der Zielsprache festzulegen gilt.

Parallel zu den obigen Überlegungen hat auch H.Lebiedzinski - von der Überzeugung: "przekladamy nie slowa lecz tresc $c^{\text {n3 }}$ ausgehend - Kritik an der Vermengung und Mißachtung der Begriffsopposition desygnat - oznaczenie in der linguistisch orientierten übersetzungstheoretischen Forschung geäußert. ${ }^{44}$ Sein Verständnis von desygnat, definiert als "element rzeczywistosci wyrażony w mowie", und oznaczenie als "jezykowy wyraz desygnatu"45 kommt der Coseriuschen Auffassung von Bezeichnung und Bedeutung recht nahe: ${ }^{46}$

Sedno przekładu stanowi wlasciwie nie "tlumaczenie", lecz zastepowanie oznaczen oryginalu oznaczeniami przekladu, który by w miare możnosci wyrażaly zespót desygnatów jesli nie tożsamy, to maksymalnie bliski desygnatom wyrażonym oznaczeniami oryginalu' ${ }^{47}$;

und weiter bei der Formulierung der Aufgabe des Übersetzers:

${ }^{41}$ Eine Anwendung der Coseriuschen Textlinguistik des Sinns auf den Bereich der Übersetzung literarischer Texte hat bereits KOSTA 1986 vorgenommen, vgl. im speziellen S.52-58.

42 COSERIU 1978. S.23. Das schon ofters bemühte Beispiel von G.Frege "Morgenstern Abendstern" zur Illiustrierung der Identităt des Bezeichneten und Diversităt der Bedeutung zeigt, daß unterschiedliche Bedeutungen dasselbe bezeichnen kőnnen; vgl. COSERIU 1970. S.105f.

๑ LEBIEDZIŃSKI 1981. S.20.

“ Vgl. LEBIEDZIŃSKI 1981. S. 14f; zu seinen Betrachtungen zum genannten Kontrastpaar, die er im Rahmen seiner auf der Basis der sog. metoda tresciowa vorgenommenen Erörterungen zur allgemeinen Übersetzungswissenschaft anstellt, vgl. vor allem Kap.III.3.

4s LEBIEDZIŃSKI 1981. S.23.

${ }^{46}$ Wie unterschiedlich und teilweise verwirrend die Terminologie auf diesem Gebiet ist, hat KOSTA 1986 im AnschluB an ALBRECHT 1973 (S.21) durch Erweiterung um neuere Arbeiten gezeigt, vgl. Tabelle I: Zeichentheoretische Konzeptionen, S.54.

${ }^{47}$ LEBIEDZIŃSKI 1981. S.20. 
Elementarna czynnosc tlumacza jest taka: zaznajomienie sie z oznaczeniem $w$ oryginale (oze $O$ ), uswiadomienie sobie jego desygnatu (ds), znalezienie oznaczenia w przekladzie (oze P) do tego desygnatu, co można ując w formule: oze $\mathrm{O} \Rightarrow \mathrm{ds} \Rightarrow$ oze $P .{ }^{4}$

Was hier als Formel dargestellt wird, faßt Coseriu in folgendem Schema zusammen:49

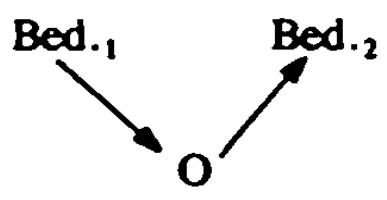

Bezeichnung

Die Erkenntnis, daß an der Herstellung von Texten außer sprachlichen in unterschiedlichem Maße auch außersprachliche Mittel beteiligt sind, zieht nach sich, daß es beim Übersetzen neben der erwähnten Äquivalenz in der Bezeichnung auch um Äquivalenzen in bestimmten Situationen und Kontexten geht. ${ }^{\text {so }}$ Damit wird die dritte Art des sprachlichen Inhalts, der Sinn, aufgegriffen. Die Beziehung zwischen Bezeichnung, Bedeutung und Sinn stellt sich wie folgt dar:

Sprachliche Zeichen haben Bedeutungen, mittels derer sie etwas Außersprachliches bezeichnen. Dieser komplexe Sachverhait stellt auf einer höheren semiotischen Ebene wieder den Ausdruck für eine Inhaltseinheit höherer Art dar, den Sinn. ${ }^{31}$

Das, was in einem Text gemeint, bezeichnet wird, ist also seinerseits Zeichenträger, im übertragenen Sinne signifiant (in Saussurescher Terminologie), für einen bestimmten Sinn, das signifie der Textzeichen.

Bei der Frage nach Entstehung und Aufbau des Sinns geht Coseriu von einem durch ihn modifizierten Schema des Organon-Modells von K. Bühler sowie dessen Erweiterung durch R.Jakobson ${ }^{32}$ aus: Neben den drei Grundrelationen des sprachlichen Zeichens in Texten als konkreten Redeakten zu ihrer Umgebung, nämlich zum Sprecher (Ausdrucksfunktion), zum Hörer (Appellfunktion)

\footnotetext{
4EBIEDZIŃSKI 1981. S.24 (Hervorhebung B.K.).

^ COSERIU 1978. S.22.

so VgI. COSERIU 1978. S.23.

s1 COSERIU 1980. S.49; Genaueres dazu s. S.48-50.

52 Vgl. weiter oben S.105, Anm.21.
} 
und zur außersprachlichen Wirklichkeit (Berichtfunktion) ${ }^{53}$ leitet sich der Sinn darüberhinaus aus noch anderen Arten der Relation her:

Zu nennen sind hier "Relationen mit anderen Zeichen", die nochmals aufgeschlüsselt werden in "Relationen mit einzelnen Zeichen in materieller und inhaltlicher Hinsicht" - hier handelt es sich einerseits um Erscheinungen wie Reim, Assonanz, Alliteration u.ä., andererseits um die sog. "bildungsdurchsichtigen" Wörter; in "Relationen mit Gruppen bzw. Kategorien von Zeichen" dazu zählt z.B. die Beziehung eines suffigierten Diminutivs zu der Gruppe der Diminutive; und in "Relationen mit ganzen Zeichensystemen". Dieses Verhältnis betrifft die Konnotation (im Hjelmslevschen Sinne), die sprachlichen Zeichen aufgrund ihrer Zugehörigkeit zu einer historischen Sprache eigen ist. Historische Sprachen gelten in funktionaler Hinsicht als nie völlig homogen; regionale, sozial und sprechersituationsbedingte Unterschiede führen dazu, daß ein Zeichen jeweils das Subsystem konnotiert bzw. evoziert, dem es angehört. ${ }^{54}$ Darüberhinaus können Zeichen in "Relationen mit Zeichen in anderen Texten" stehen gemeint sind damit Beziehungen zu Texten, die zur sprachlichen und kulturellen Tradition einer Sprachgemeinschaft gehören, wie z.B. die Aufnahme von Redewendungen, Sprichwörtern, Zitaten. ${ }^{\text {ss }}$ Schließlich sind zwei außertextliche Relationen zu berücksichtigen: die "Relationen zwischen Zeichen und 'Sachen' ", d.h. die "ikastische Funktion" des Zeichens als direkte Nachahmung der bezeichneten Sache durch das signifiant des Zeichens ${ }^{56}$, und als letzter Typ die "Relationen zwischen Zeichen und 'Kenntnis der Sachen' ". Anders formuliert: Zeichen funktionieren im Text auch durch die "Relationen, die zwischen den Zeichen und unserem Wissen von den bezeichneten Sachen bestehen, durch die Relationen zwischen den Zeichen und den Kenntnissen, die die Zeichenbenutzer

53 Die Notwendigkeit einer Korrektur des Modells ergab sich aus der Tatsache, daß Bühler keine Unterscheidung zwischen den Funktionen des Zeichens in der Sprache und denen im Text vornahm: während Ausdruck und Appell Funktionen des Zeichens im konkreten Redeakt sind, bezieht sich die Darstellungsfunktion auf das virtuelle Zeichen. Für die Darstellungsleistung des Zeichens in seiner Verwendung ist deshalb der Terminus Bericht eingefüht worden, der damit auf derselben Stufe wie Ausdruck und Appell fungiert, vgl. COSERIU 1980. S.53-67. Zur Auseinandersetzung mit den Unzulänglichkeiten des modifizierten Bühlerschen Modells vgl. auch LALEWICZ 1973.

so Im einzelnen vgl. COSERIU 1980. S.68-80.

ss COSERIU 1980. S.80-82.

So Vgl. COSERIU 1980. S.82-88. 
vom Bezeichneten haben".57 Diese Beziehungen können verschiedener Natur sein, gedacht wird dabei an zwei Faktoren, den Kontext und die Situation, die den Redeakt determinieren können. Coseriu faßt diese beiden üblichen - seiner Meinung nach unzureichenden - Größen zunächst unter dem Oberbegriff $\boldsymbol{U} \boldsymbol{m}$ felder zusammen, um danach weitere notwendige Differenzierungen zu unternehmen: Situation - unmittelbar/mittelbar, Region - Zone, Bereich, Umgebung, Kontext - einzelsprachlicher Kontext, Rede-Kontext, Außer-Rede-Kontext und Redeuniversum. .8 $^{\text {s8 }}$

Die genannten Relationstypen, deren Funktion die Evokation ${ }^{59}$ ist, können vom Sprecher/Autor individuell genutzt werden und so zum Sinn eines Textes beitragen. Damit ist zunächst zu sagen, daß sich der Sinn eines Textes aus der Kontamination der verschiedenen Arten der Evokation mit den Bühlerschen Zeichenfunktionen Ausdruck, Appell und Darstellung konstituiert.

Nicht als Kontrast, vielmehr als Ergänzung zur Coseriuschen Sinn-Konzeption ist das Sinn-Verständnis der kommunikationstheoretisch ausgerichteten Beiträge der Vertreter der Leipziger Schule zu bedenken. Während beim erstgenannten den Sinn-Begriff textuelle Einzelaspekte dominieren, wird er hier als außersprachliche Kategorie aufgefaßt. ${ }^{60}$ In seiner adressatenorientierten Betrachtung definiert G.Jäger den kommunikativen Wert eines Textes - dessen Auffassung er in seinen zahlreichen Studien mehrmals modifiziert hat - als die

Gesamtheit der in einem Verstehensprozeß der phonetischen oder graphischen Reprăsentation eines Textes auf der Grundlage vorhandener Verstehensvoraussetzungen (...) vom Adressaten zugeordneten BewuBtseinsinhalte. $^{\circ 1}$

Drei grundlegende Komponenten, die es zu ermitteln gilt, konstituieren die Invariante der Übersetzung: Neben den aktuellen sprachlichen Bedeutungen, "die der dem Text (bzw. der Äußerung) als sprachliches Substrat unterliegenden

"T COSERIU 1980. S.89.

s* Genauere Ausführungen dazu vgl. COSERIU 1980. S.88-101.

\$ Vgl. COSERIU 1980. S.102.

“ KADE 1980 (S.94) 2.B. bestimmt den Sinn als eine "außersprachliche, weil nur in Verbindung mit einem konkreten Situationsbezug existierende GroßBe und zugleich eine übersprachliche Größe; denn er ist (...) nicht an eine bestimmte Einzelsprache gebunden".

61 JÄGER 1986. S.10f. 
Satzfolge (bzw. dem Satz) zukommen" ${ }^{\text {"2 }}$, zu denen sowohl die semantischen als auch pragmatischen Bedeutungen gehören ${ }^{63}$, sowie dem aktualisierten assoziierten Sachwissen - beide zusammen als Äußerungsbedeutung zusammengefaßt ist der kommunikative Sinn einer Äußerung bzw. eines Textes zu nennen, der seinerseits "verfasserseitig als der mit der sprachlichen Handlung verbundene Zweck verstanden" ${ }^{\text {"64 }}$ wird.

Dieses oben aufgezeigte Ineinander von sprachlichem Kotext und außersprachlichem Kontext, den "übersummativen Inhalt", 65 gilt es bei der Textanalyse zu entschlüsseln. Nach hermeneutischer Auffassung - um sie abschließend noch einmal anzuführen - entwickelt sich der Sinn eines Textes erst mit seiner Rezeption durch den Leser bzw. Übersetzer. Weil dieser Verstehensprozeß an Individuen geknüpft ist, wird er "nicht nur vom thematischen Textaufbau, den Zeichenrelationen und der Textfunktion bestimmt, sondern auch von der hermeneutischen Grundhaltung des Textinterpreten selbst" ${ }^{\text {66 }}$. Textverstehen ist danach keine systematisch angelegte Analysearbeit, eher eine individuelle, personale Tătigkeit ${ }^{67}$ im Medium der Sprache,

in der der Übersetzer das Verstandene nicht einfach kritisch auflöst oder unkritisch reproduziert, sondern es mit seinen eigenen Formulierungen in seinem eigenen Horizont auslegt und neu zur Sprache bringt ${ }^{\text {st }}$.

Auch wenn der vorgetragene Sinn-Entwurf in Hinblick auf komplexe Texteinheiten konzipiert wurde, ist es möglich - wie in dieser Studie -, ihn auf weniger umfangreiche Textpassagen und weniger komplexe Textarten zu übertragen. ${ }^{69}$ Im Anschluß an das Dargelegte und in Übereinstimmung mit P.Kosta,

Q2 JÄGER 1983. S.55.

$63 \mathrm{Vgl}$. zu diesen Invarianten die Angaben in JÄGER 1975. S.87-107.

* JÄGER 1983. S.57; Erläuterungen zu den einzelnen Komponenten S. JÄGER 1986. S.10-29.

as STOLZE 1982. S.104.

\$ STOLZE 1982. S. 102.

${ }^{67}$ Grundsätzliches zum hermeneutischen Verstehensbegriff vgl. STOLZE 1982. S.33-44.

* STOLZE 1982. S.42.

की In seinen Betrachtungen betont COSERIU 1980 ausdrücklich, daß Sinngebung nicht ausschließlich literarischen Texten vorbehalten ist: "Alle Texte haben Sinn, natürlich auch die Texte, die im alltäglichen Leben verwendet werden. Denn auch dieses Sich-einfach-auf-eine- 
Sinn als Ensemble einer "sprachlichen und einer logischen (erkenntnismäßigen) Kategorie $^{n 70}$ zu begreifen, soll für diese Untersuchung von der Prämisse ausgegangen werden, daß die Äquivalenz des Sinns als oberstes Postulat jeden Übersetzens anzusetzen ist.

\section{4. ÄQUIVALENZEBENEN DER DIMINUTIVUBERTRAGUNG}

Mit der Erkenntnis, daß die Schaffung totaler bzw. idealer bilingualer Identität auf allen Ebenen des sprachlichen Zeichens sowie innerhalb der gleichen linguistischen (strukturellen) Bereiche eines Textes ein in der Praxis unerreichbares theoretisches Konstrukt bleibt, müssen die Bemühungen des Übersetzers dahin gehen, ein Optimum an funktioneller Äquivalenz ${ }^{1}$ herzustellen, d.h. die zielsprachliche Rekonstruktion der höchstrangigen Invariante der Übersetzung, des aktuell gemeinten Sinns von Aussagen der Textvorlage zu sichern und dabei die real notwendigen Verluste so gering wie möglich zu halten. Die so verstandene Forderung nach funktioneller Äquivalenz ist dann erfüllt, wenn "zwei Texte [bzw. Textpassagen, Textsegmente - B.K.] den gleichen Sinn bei Wahrung der Hierarchie der diesen konstituierenden Bezugsgrößen haben"2.

Da angesichts der bekannten Strukturverschiedenheit sowie der divergierenden Erfahrungskomponenten zweier Sprachen - hier Polnisch und Deutsch - auch oder gerade einzelne Einheiten wie die Diminutive nicht immer auf allen Ebenen zugleich invariant gehalten werden können, müssen Möglichkeiten gefunden werden, die relevanten Informationen im Text zu kompensieren. Dabei bestimmen zwei Prinzipien, das Prinzip der Selektion und das Prinzip der Hierarchisierung ${ }^{3}$ die Auswahl einer zielsprachlichen Entsprechung. Als diffizil erweist sich jedoch die Festlegung möglicher Bezugspunkte (eines tertium comparatio-

empirische-Wirklichkeit-Beziehen, dieses Keinen-darüber-hinausgehenden-fiktiven-Sinn-Haben, ist eine Art von Sinn" (S.49).

TOSTA 1986. S.279.

$'$ Genaueres dazu s. oben in Kap.3.3.1.

${ }^{2}$ KOSTA 1986. S.283.

3 Vgl. die Erorterungen in REISS 1984. S.86 
nis). Diverse Ansätze, üblicherweise im Anschluß an die Erkenntnisse der Semiotiktheorie von der Triade der syntaktischen, semantischen und pragmatischen Dimensionierung eines Zeichens, bieten unterschiedliche Beschreibungsmodelle an.

Der schon häufiger diskutierte Vorschlag von W.Koller geht z.B. von fünf Bezugsrahmen (Invarianten) aus: ${ }^{4}$

- dem außersprachlichen Sachverhalt (= denotative Äquivalenz);

- den im Text durch die Art der Verbalisierung vermittelten Konnotationen

(= konnotative Äquivalenz);

- den Text- und Sprachnormen (Gebrauchsnormen) (= textnormative Äquivalenz);

- dem Empfänger (= pragmatische Äquivalenz);

- bestimmten formal-ästhetischen Eigenschaften des Ausgangstextes (= formale Äquivalenz).

Diese Anregungen aufgreifend haben Stolze und Kosta die Anwendbarkeit der einzelnen Größen erörtert. Während R.Stolze die Aufteilung beibehält und lediglich terminologische Änderungen vornimmt, um den allzu strapazierten Äquivalenz-Terminus durch nuancierte Wendungen zu entlasten - sie spricht von inhaltlicher Invarianz und konnotativer Analogie, die den Aspekt der Genauigkeit ausmachen, von gebrauchsnormativer Adäquatheit sowie von pragmatischer Wirkungsgleichheit und expressiver Entsprechung, die zum Aspekt der Gleichwertigkeit gezählt werden,- s strafft P.Kosta den Gliederungsansatz, indem er den zweiten, dritten und vierten Äquivalenzaspekt des Kollerschen Modells zusammenfaßt. Im Rahmen seiner Betrachtung literarischer Texte setzt er vier "obligatorische Translationsinformationen"6 fest: die formal-ästhetische, die semantische (denotative), die pragmatisch-stilistische, worunter die Entsprechung bezüglich der stilistischen Höhenlage sowie der Stilfärbung verstanden wird, und schließlich die textfunktionale Information sollen als Bezugspunkte für die Beurteilung seiner Texte gelten. ${ }^{7}$

4 Vgl. KOLLER 1987. S.187.

s S. die Angaben in STOLZE 1982. S.178-191.

- KOSTA 1986. S.282.

${ }^{7} \mathrm{Vgl}$. dazu KOSTAs 1986 detaillierte Ausführungen in Kap.1.2.5.6 - 1.2.5.10. 
Für die Zwecke der folgenden Untersuchung, Diminutive als Textkonstituenten genau zu erfassen, ist es nötig, nach "Inhalt" und Textmeinung sowie "Wirkung" und Redestrategie dieser Einheiten zu fragen. Auch wenn in Anlehnung an die obige Terminologie die Herstellung semantischer Äquivalenz als einstweilige Grundbedingung funktional äquivalenten Übersetzens anzusehen ist, gilt es im speziellen Fall der Diminutive, die Priorität pragmatisch-stilistischer Äquivalenz zu gewährleisten. Weniger wichtig erscheint dagegen der Erhalt der formalen Struktur der ausgangssprachlichen Elemente, da die hier zu beschreibenden Diminutive nicht in den Bereich der sog. "formbetonten Elemente" gehören. Ihre Form (signifiant) ist - mit Coseriu gesprochen - lediglich als "Instrument zweiten Grades" zu verstehen (sie ist zunächst Instrument für die Bedeutung und diese ist wiederum Instrument für die Bezeichnung), nicht aber als Faktor, der direkt zur Konstitution des Textsinns beiträgt. ${ }^{8}$

Befolgt man die Darlegungen zu den die Diminutive determinierenden Bedeutungskomponenten, ${ }^{9}$ so ist es für die Rekonstruktion des diminutiven Wertes an aktueller Textstelle angebracht, auf jene Spezifizierungen zurückzugreifen und sie als relevante Größen anzusetzen: Informationen, die auf der begrifflichen Komponente des Diminutivs beruhen - vereinfachend semantische Information genannt; zum anderen Informationen, deren Grundlage die wertende und emotionale, teilweise auch die stilistische Bedeutungskomponente sein kann emotional-wertende Information; und schließlich die übergeordnete stilistische Information, die sich aus der Kontamination der beiden vorangehenden Bedeutungen mit denen anderer Textelemente und -erscheinungen ergibt - stilistische Information. ${ }^{10}$ Diese drei Informationen machen somit diejenigen Größen aus, die als Bezugsebenen (tertia comparationis) des Übersetzungsvergleichs von Diminutiven angesetzt werden sollen und zugleich als Kriterien für die Beurteilung des erzielten Äquivalenzgrades hilfreich sein können. Genauer muß nun auf die Dimension der stilistischen Information eingegangen werden.

Von der Prämisse ausgehend, daß Stal zu verstehen ist als die Art und Weise der Formulierung eines Textes, d.h. als die Wahl bestimmter sprachlicher Mittel, die sich einerseits an festgelegten Normen bzw. Konventionen einer Sprachgemeinschaft ausrichtet, andererseits die Intention des Senders dokumentiert, womit

- Vgl. COSERIU 1978. S.29f.

- Vgl. dazu oben Kap.2.4.2.

${ }^{10} \mathrm{Vgl}$. dazu auch TENNERT 1982. S.176. 
dann Auskunft über den Sprecher und seine Einstellung zum Gegenstand bzw. Sachverhalt gegeben wird," erscheint es besonders bei der Diminutivbetrachtung unumgänglich, Stileigenschaften vorrangig in die Beschreibung interlingualer Äquivalenzbeziehungen einzubeziehen.

Das Erreichen pragmatisch-stilistischer Äquivalenz, ${ }^{12}$ die in der ZeichenSprachbenutzer-Sprachgebrauchs-Dimension begründet ist, erweist sich insofern als nicht unproblematisch, als "die die Pragmatik reflektierenden stilistischen Selektionsregeln sprachgebunden sind, also nicht in interlinearer Weise von der QS in die ZS übertragen werden können" ${ }^{\prime 13}$. Demzufolge soll zum gemeinsamen Bezugspunkt die bereits als stilistische Information definierte Information der Textelemente über die Kommunikationsbedingungen herangezogen werden. ${ }^{14}$

Versteht man sprachliche Kommunikation als spezifische Form menschlichen bzw. sozialen Handelns, bedeutet dies, allgemein formuliert, daß sie stets unter bestimmten Umständen, unter bestimmten Bedingungen oder eben in einer Situation stattfindet. Um ein Erfassen der vielfältigen, einen Text determinierenden Kommunikationsbedingungen, insbesondere der Kommunikationssituation, hat sich z.B. Heide Schmidt mit ihrem Modell bemūht, in das sie nicht nur den "psychischen Zustand" des Senders (Sprecher/Autor), sondern darüberhinaus die "Gesamtheit der Beziehungen, in die er bei der Kommunikation eintritt, seine 'Situation' im Kommunikationsereignis", is einzubeziehen versucht hat.

Dabei werden drei Faktoren der Kommunikationssituation als wesentlich hervorgehoben: die Art der kommunikativen Tätigkeit (Kommunikationssphäre), der die führende Rolle innerhalb aller Komponenten zukommt, dann die Bedingungen für die Ausführung der kommunikativen Tätigkeit (Übermittlungs-

"Vgl. dazu PÜSCHEL 1983, bes. S.105.

${ }^{12}$ Die Art, in der hier und spăter dieser Terminus verstanden wird, ist nicht zu verwechseln mit Kostas Definition; hier wird er vielmehr im Sinne von Übereinstimmung bezüglich der stilistischen Information verwendet.

${ }^{13}$ SCHMIDT 1979. S.78. Auch KOSTA 1986 (S.105) sieht die Schwierigkeit darin begründet, daß "im Bereich der langue die Nichtübereinstimmung bezüglich der Stratifizierung (Schichtung) von AS und ZS die Regel ist". Ebenso kommt BUTTLER 1982 (S.21) beim Vergleich von hovorovy jazyk - razgovormaja rez' - jezyk potoczny zu der Überzeugung: "stratyfikacja każdego jezzyka slowianskiego ma charakter swoisty, idiomatyczny, (...) wlasciwa jednemu jezykowi hierarchia odmian nie powtarza sie $w$ pozostalych. Wynika to z różnego uksztaltowania systuacji historycznej, spolecznej i komunikatywnej mowiacych nimi wspolnot narodowych".

14 S. dazu weiter oben, S.81f.

is SCHMIDT 1979. S.51. 
bedingungen) und die Charakteristika der diese Tätigkeit ausübenden Personen. ${ }^{16}$ Für die Zwecke dieser Arbeit soll es genügen, vorzugsweise das Kriterium der Kommunikationssphäre zu berücksichtigen. Anknüpfungspunkte für eine Diskussion der verschiedenen "Arten der kommunikativen Tätigkeit" lassen sich in den Konzeptionen der Funktionalstilistik finden.

EXKuRS: Die Heterogenität natürlicher Sprachen spiegelt sich in dem Klassifizienungsversuch in Hochsprache, Umgangssprache, Dialekt, Regiolekt, Soziolekt, Slang, Jargon, Technolekt u.a. wider. Die Ausprägung dieser "inneren Mehrsprachigkeit"17 resultiert aus dem Umstand, daß es sprachliche Charakteristika - von der phonologischen über die morphologische, syntaktische bis hin zur semantischen Ebene - gibt, die für Sprecher bestimmter Regionen, für bestimmte Gruppen oder Gesprächssituationen typisch sind. Mit Coseriu definiert man diese "sprachlichen Varietäten"18 einer Sprachgemeinschaft in einem bestimmten Sprachstadium als diatopisch, diastratisch, diaphasisch bedingte Diasysteme und unterscheidet dementsprechend Dialekt, Gruppensprache und Sprachstil. ${ }^{19}$

Andere - für die Übersetzungsproblematik geeignetere - Gliederungskriterien, die der Differenzierung des sprachlichen Gebildes Rechnung tragen, bietet der methodische Ansatz der Funktionalstilistik, der in der Tradition vor allem der Prager Schule steht. ${ }^{20}$ Die Grundidee der funktionalen Spezialisierung von Texten beruht auf der Erkenntnis der Bedeutung der Sprachverwendungsarten in den unterschiedlichen Bereichen gesellschaftlicher Tätigkeit sowie der kommunikativen Sprachfunktionen. Während in den Arbeiten von B.Havránek - dem eigentlichen Begründer der Theorie von der funktionalen Schichtung -, entsprechend den Grundfunktionen der Sprache, die Alltags- bzw. Gesprächs- oder Konversationssprache (einfache mitteilende Funktion), Sach- und Fachsprache (fachlich mitteilende Funktion) und Dichtersprache (poetisch-

$16 \mathrm{Vgl}$. dazu das Schema und die dazugehörigen Ausführungen bei SCHMIDT 1979. S.52-55. Siehe ebenso KADE 1980 (S.96-149), der die Kommunikationssituation als "Bezugsgrundlage für einen dynamischen Äquivalenzbegriff" (S.96) diskutiert.

17 WANDRUSZKA 1975. S.333.

$18 \mathrm{Vgl}$. NABRINGS 1981 (S.17), die Konzeptionen zur Erforschung innersprachlicher Heterogeniät diskutiert.

$19 \mathrm{Vgl}$. COSERIU 1988. S.280-292.

20 Vgl. dazu die Beiträge des Sammelbandes SCHARNHORST/ISING 1976/1982. 
ästhetische Funktion) unterschieden werden, ${ }^{21}$ wird in späteren Entwürfen diese traditionelle Gliederung durch stärkere soziolinguistische Orientierung korrigiert. ${ }^{22}$ Neben der Verknüpfung von Kommunikationssituation und bestimmter sprachlicher Ausprägung eines Funktionalstils wird als weitere Komponente die der gesellschaftlichen Konventionen hervorgehoben, man spricht vom "normierten Charakter" ${ }^{23}$ der Funktionalstile.

Die Tatsache, daß "eine menschliche Sprache (...) ein einzigartig (...) dynamisches Polysystem, ein Konglomerat von Sprachen"24, von Varietäten ist, Funktionalstile dagegen in jeder Sprache anzutreffen sind, hat für übersetzungswissenschaftlich orientierte Untersuchungen insofern Bedeutung, als "Funktionalstile prinzipiell leichter übertragbar" sind "als die Varietäten ('Existenzformen') einer Sprache" . ${ }^{25}$ Kosta konstatiert dazu:

\begin{abstract}
Während nämlich "Existenzformen" die Architektur der langue einer bestimmten Sprache spiegein, die sich (...) als eine Summe von Mikrosprachsystemen einer bestimmten historischen Einzelsprache bezeichnen läßt und somit ein einzelsprachlich spezifisches Inventar an unvergleichbaren grammatisch-lexikalischen Ausdrucksmitteln herausbildet, sind "Funktionalstile" rekurrente Verwendungsweisen, die von kommunikativen und daher einzelsprachlich unspezifischen Faktoren wie z.B. "Kommunikationssphäre" - "Kommunikationssituation" - "Stilsphäre" abhängig sind. ${ }^{20}$
\end{abstract}

Obwohl das Prinzip der funktionalstilistischen Einteilung wohl unbestritten ist, ist der "Begriff des Funktionalstils noch weitgehend eine Arbeitshypothese"27, die sowohl in der Germanistik als auch Polonistik erst noch einer eingehenden Beschäftigung bedarf, ganz zu schweigen von einer bilingualen konfrontativen Untersuchung auf diesem Gebiet. Es gibt einige mehr oder weniger vergleichbare Versuche, die Funktionalstile zu klassifizieren, wobei die Anzahl, die Benennungen, teilweise die weiteren

${ }^{21}$ Mit der Bezeichnung funktionale Sprache sind die heute als funktionale Stile ausgegliederten Kategorien gemeint; funktionaler Stil gebraucht Havránek zur Benennung von Erscheinungen anderer Art.

2 S. hierzu die Publikation von BENES/VACHEK 1971 sowie SCHARNHORST 1981 und JEDLIČKA 1981.

${ }^{23}$ FLEISCHER/MICHEL 1979. S.243.

${ }^{24}$ WANDRUSZKA 1979. S.39 (Hervorhebung B.K.).

${ }^{25}$ KOSTA 1986. S.135.

26 KOSTA 1986. S.135.

${ }^{27}$ FLEISCHER/MICHEL 1979. S.24. 
Untergliederungen divergieren. ${ }^{28}$ Man kann von einer sich allmählich etablierenden Differenzierung von fünf Hauptkategorien ausgehen.

Für die deutsche Gegenwartsprache ist die von Riesel vorgeschlagene Unterteilung in: Stil der bffentlichen Rede, Stil der Wissenschaft, Stil der Presse und Publizistik, Stil der Alltagsrede, Stil der schönen Literatur anzuführen; ${ }^{29}$ für die polnische Sprache Skubalankas Gliederung in: styl potoczny, styl przemówień, styl urzedowo-kancelaryjny - die beiden letztgenannten stellt sie als zwei selbständige Typen heraus -, styl naukowy, styl publicystyczny $i$ styl dziennikarski und styl artystyczny. ${ }^{30}$ Zweifellos stellt diese Systematisierung mit der Beschränkung auf diese wenigen Kommunikationsbereiche lediglich ein Grundmodell dar, dessen allzu grobes Raster sicherlich nicht allen stilistisch relevanten Textformen gerecht werden kann.

In Anlehnung an Riesel, die versucht hat, die sprachlich-stilistischen Merkmale, die sog. Stilzüge, als Kennzeichen jeder Verwendungsweise darzulegen, ${ }^{31}$ soll noch kurz auf die Charakteristik des für die Diminutivbetrachtung besonders interessierenden Stiltyps der Alleagsrede eingegangen werden. ${ }^{32}$ Entsprechend seiner Verwendungssphäre kann er weiter subklassifiziert werden in: 1. Alltagsstil im Familien- und Freundeskreis; 2. Alltagsstil im Beruf; 3. Alltagsstil im Verkehr mit Freunden, ohne jegliche offizielle Bindung, ${ }^{33}$ wobei allgemein die mündliche Form die übliche ist, jedoch die schriftliche Variante, wie z.B. Privatkorrespondenz und Tagebücher, auch zu berücksichtigen ist. Abgesehen von der Mannigfaltigkeit der Thematik gilt ebenso die Vielfalt der sprachlichen Basis, bedingt durch die jeweilige soziale, berufliche, territoriale Zugehörigkeit des Sprechers, als Besonderheit der Alltagssprache, so daß

28 VI. dazu RIESELS 1975 kurzen Überblick auf S.50-53.

29 Vgl. RIESEL 1959. S.13f sowie RIESEL/SCHENDELS 1975. S.18-28.

* Vgl. KURKOWSKA/SKORUPKA 1959. S.231. In spăteren Arbeiten, vgl. z.B. SKUBALANKA 1976 (S.261), BUTTLER 1982 (S.27), WILKON 1987 (S.57), wird ein modifiziertes Schema vorgestellt, in dem die Funktionalstile dem hierarchisch übergeordneten Gegensatzpaar jezyk pisany - jezyk mowiony zugeordnet werden.

" Vgl. RIESEL 1959 (Teil V), die auch die Realisierung jedes Stilzugs durch die entsprechenden lexikalisch-phraseologischen, grammatischen und phonetischen Mittel kurz beschreibt. S. auch die Besprechung der Grundkategorien in KURKOWSKA/SKORUPKA 1959. S.231-351 und WILKON 1987. Kap.IV.

"Vgl. bes. RIESEL 1970; zum Aspektreichtum von Umgangssprache vgl. BICHELs 1973 umfangreiche Studie. In der Polonistik ist auf die Arbeiten von BUTTLER 1982 und WILKOŃ 1987 sowie LUBAŚ 1979 zu verweisen.

3 VgI. RIESEL 1970. S.74. 
bald hochsprachliche, bald dialektale, bald Argot- und Jargon-Elemente Eingang finden können.

Als ihr grundlegender Stilzug wird die ungezwungen-lockere und entspannte Gesamthaltung der Sprecher im privaten Umgang miteinander genannt. Für den Alltagsstil spezifisch wird daneben, von der Forschung übereinstimmend, der mit dem ersten eng verflochtene Zug der Emotionalität und subjelativen Bewertung herausgestellt. $^{34}$ Beide spielen bei der Diminutivbewertung eine entscheidende Rolle. Darüberhinaus sind "Ausdrucksökonomie einerseits und Ausdrucksfülle (behagliche Umständlichkeit) andererseits", ferner "Anschaulichkeit und Dynamik" sowie "Humor und Satire" ${ }^{\text {35 }}$ weitere nennenswerte Kennzeichen des Alltagsstils. SchlieBlich üben Automatismen, der Gebrauch vorgefertigter Muster, eine bedeutende Funktion aus. ENDE DES EXKURSES

Die der Funktionalstilistik zu entnehmende Klassifizierung der Kommunikationssphären soll für die Diminutivuntersuchung lediglich als Anhaltspunkt verstanden werden, der je nach Bedarf im analysierenden Teil erweitert werden kann. Es bleibt festzuhalten: die Kommunikationssituation, mit der Komponente Kommunikationssphäre, sowie das Kommunikationsziel sind die invariant zu haltenden Vergleichsgrößen, an denen sich die stilistische Umsetzung eines Textes/Textsegments aus der einen in eine andere Kommunikationsgemeinschaft zu orientieren hat. Jene Informationen bestimmen nämlich die

Auswahl und Anordnung der sprachlichen Elemente in verschiedensprachigen Texten, d.h. die in verschiedenen Kommunikationsgemeinschaften für eine bestimmte Art der Kommunikationssituation typischen sprachlichen Mittel in ihren typischen Kombinationen sowie mit ihren typischen Frequenzcharakteristika. ${ }^{*}$

Mit der Einsicht, daß bei der "Sprachverwendung auf allen Ebenen ein unerläßliches $\mathrm{Maß}$ an Konsens und eine Fülle von Konventionen innerhalb einer Sprachgemeinschaft wirksam" ${ }^{37}$ werden, gehört für den Übersetzer, der situa-

\footnotetext{
34 Vgl. RIESEL 1970. S.83f. In demselben Sinne formulient BUTTLER 1982 (S.28): "Jego [stylu potocznego - B.K.] zasadnicze wyznaczniki stanowia spontanicznosc, ekspresywnosc, charakter wartosciujacy, orientacja familiarna". Zum Expressivitătskriterium s. SKUBALANKA $1973 b$.
}

3s RIESEL 1970. S.84.

SCHMIDT 1977. S.111.

37 REISS/VERMEER 1984. S.183 (Hervorhebung B.K.); Konventionen werden hier im Rahmen der Textsortendiskussion besprochen. Grundsătzliches zu diesem Begriff ist nachzulesen u.a. bei WUNDERLICH 1972. 
tionsadäquate Formulierungen zu finden hat, zur Sprachverwendungsbeherrschung besonders die Kenntnis solcher meist nur einzelsprachlich gültiger Textaufbaunormen, die sich im Verlauf der Sprachgeschichte herausgebildet haben. Ziel des folgenden Übersetzungsvergleichs wird es sein, zu prüfen, inwieweit bei der Wiedergabe von Diminutiven derartige charakteristische sprachliche "Verhaltensregularitäten in bestimmten Kommunikationssituationen " 38 der Vorlage bei der zielsprachlichen Version berücksichtigt wurden.

Daß die infolge des Sinnerschließungsprozesses realisierten Entscheidungen über die zielsprachlichen Formulierungen dem Diktum der funktionellen Äquivalenz Rechnung zu tragen haben, ist bereits deutlich herausgestellt worden. Doch darüberhinaus gilt es zu bedenken, daß je nach Distanz oder Nähe zu diesem Postulat verschiedene Grade bzw. Stufen der Entsprechung ${ }^{39}$ möglich sind, wobei - um es nochmal zu betonen - die Zuordnung zu ihnen bei aller gegebener Objektivität letztlich meist nur Ergebnis der subjektiven Einschätzung des gerade Urteilenden sein kann. Ist Übereinstimmung auf allen Bezugsebenen, einschließlich der lexikalisch-strukturellen, festzustellen, dann kann man von totaler Äquivalenz sprechen. Optimale/maximale Äquivalenz ${ }^{* 0}$ wird erreicht, wenn trotz bestehender struktureller Unterschiede größtmögliche Parallelität von Ausgangs- und Zieltext mit Hilfe kompensatorischer Mittel erzielt werden kann. Reicht eine Übertragung nur annähernd an das Ideal der funktionellen Äquivalenz heran, dann besteht nur approximative Äquivalenz. Bleibt der Übersetzungsvorschlag hinter diesen Forderungen zurück, so hat man es mit einer fehlerhaften, einer Nicht-Äquivalenz zu tun. ${ }^{41}$

Wesentliche Voraussetzung für die Verwirklichung einer funktionell äquivalenten Übersetzung ist neben dem Aufspüren der maßgeblichen Invariante die

3* REISS/VERMEER 1984. S.183.

* In ähnlichem Sinne äußert sich u.a. BARCHUDAROV 1975 (S.189): "Pravil'nee budet govorit' o raznoj stepeni èkvivalentnosti perevoda, o bol'sem ili men'కem ego približenii $k$ 'polnost'ju èkvivalentnomu'".

^ Vgl. dazu BARCHUDAROVs 1975 (S.233) Begriff maksimal'naja ekvivalentnost' und JÄGERs 1975 (S.145f) Terminus maximale Äquivalenz.

" Zu verweisen ist auf KADE 1968 (S.79-81), der im Rahmen seines Modells zur Darstellung interlingualer Äquivalenzbeziehungen vier Äquivalenztypen zusammenstellt: totale, approximative, fakultative, Null-Äquivalenz: vgl. dazu auch KOSTA 1986. S.63-70. Diese Typen sind hier in dem oben beschriebenen Sinne modifiziert. 
Wahl der geeigneten linguistischen Ebene. ${ }^{42}$ In Abhängigkeit von der Beschaffenheit der Zielsprache muß nämlich häufig ein Wechsel dieser Ebene veranlaßt werden. Neubert konstatiert in diesem Zusammenhang:

Es ist eine alte übersetzerische Erfahrung, daß Unübersetzbarkeit auf einer niederen strukturellen und semantischen Ebene durch Expandierung der gewählten Übersetzungseinheit, also durch Erweiterung des Rahmens des QS-Zeichens und dann mögliche semantisch-pragmatische Kompensierung auf höherer struktureller Ebene ausgeglichen werden kann."

Als Beispiele derartiger Kompensation können genannt werden: "Phrase für Wort, Satz für Phrase, Satzfolge für Satz etc.; Lexik für Grammatik, Stil für Lexik, Pragmatik für Semantik, aber auch Lexik für Lautung und auch umgekehrt komplexe Grammatik für einfache Lexik etc. " ${ }^{\text {/4 }}$. Von welchen Möglichkeiten im konkreten Fall der Diminutive Gebrauch gemacht wird, muß die kommende Gegenüberstellung zeigen.

Auf dem Hintergrund der oben vorgenommenen theoretischen Grundlegung zum Problem des Übersetzens und der damit verbundenen Äquivalenzfrage soll nun am konkreten Textmaterial gezeigt werden, in welcher Weise und unter welchen Bedingungen Diminutive in Texten beider Sprachen zum Einsatz kommen.

${ }^{42}$ Zu den linguistischen Ebenen bzw. Übersetzungseinheiten vgl. KOMISSAROV 1973. S.185190 und BARCHUDAROV 1975. Kap.4.

$₫$ NEUBERT 1983. S.100.

4NEUBERT 1983. S. 100. 


\section{EMPIRISCHE ANALYSE}

\section{Diminutive IM ÜBersetzungSVergleich}

Die Gegenüberstellung der nominalen Diminutivsysteme des Polnischen und Deutschen in ihren Möglichkeiten und Restriktionen hat neben gewissen Gemeinsamkeiten erhebliche Unterschiede ausmachen können. Auch wenn beide Sprachen über ein lebendiges Modifikationsprogramm verfügen, besteht frappierende Disproportion in der Quantität der morphologischen Mittel. Während das variantenreiche polnische Suffixsystem, erweitert durch die dem Deutschen prinzipiell fremde Möglichkeit der Stufen- bzw. Reihenbildung (erneute Diminuierung einer diminutivischen Form), in der Lage ist, neben der Art und Weise auch den Intensitätsgrad z.B. der jeweiligen Empfindung zu signalisieren, bewirkt die zumeist festgelegte stilistische Markierung von -lein, daß im Deutschen die komplexen Aufgaben der Diminution zunächst allein auf dem Suffix chen ruhen.

Bei einer nach strukturellen Gesichtspunkten orientierten Parallelisierung der synthetischen Bildungen lassen sich drei Entsprechungsgruppen angeben:' 1.einem Diminutiv der Ausgangssprache steht kein formal entsprechendes Element in der Zielsprache gegenüber (1:0); 2. umgekehrt, der Ausgangssprache fehlt eine formale Entsprechung zur Zielsprache (0:1); 3. Ausgangs- und Zielsprache verfügen über gleiche formale Mittel $(1: 1)$, hierbei ist aber im konkreten Fall darauf zu achten, in welchem Maß sich die Diminutivbildung der intendierten Sprache als "vollwertiges Äquivalent", "Teiläquivalent" oder vielleicht nur "Scheinäquivalent" ${ }^{2}$ der Vorgabe herausstellt und damit ein real anderes Äquivalenzverhältnis geschaffen wird.

Mit diesem Wissen um die divergierenden Diminuierungsmöglichkeiten in beiden Sprachen auf Langue-Ebene gilt es zu beobachten, wie Diminutive in direkter Konfrontation, in konkreten Texten, zum Einsatz kommen. Der Vergleich von Original und Übersetzung, das Schaffen einer gemeinsamen Grundlage, soll nun einen Nachweis über den tatsächlichen Gebrauch von Diminutiveinheiten erbringen, ihr Funktionieren an derselben Stelle, d.h. in derselben Situation, erhellen.

\footnotetext{
' Zu dieser Einteilung vgl. die Beobachtungen von TENNERT 1982. S.89-93, besser zugănglich in TENNERT 1984. S.674f.

2 TENNERT 1984. S.674.
} 
Die erste bestimmende Maßnahme, die Auswahl der Diminutiveinheiten im Textkorpus, soll sich auf der Basis der oben geschaffenen Begriffsdarlegung vollziehen. So wird ein Textelement als Diminutiv verstanden, wenn es drei Bedingungen erfüllt: wenn es a) durch die genannten Modifikationssuffixe formal gekennzeichnet ist, b) damit zugleich in semantischer Opposition zum entsprechenden Grundwort auftritt und c) in der Regel derselben Wortart wie das Ausgangswort angehört. Berücksichtigung sollen vornehmlich nomina appellativa finden, daneben Adjektive, Adverbien und Pronomen sowie nomina propria, im einzelnen Vor- und Verwandtschaftsnamen, vorausgesetzt, sie alle werden noch als aktuelle Bildungen empfunden. Dazu gehören dann auch jene auf dem Wege des Neutralisierungsprozesses befindliche Derivate, während bildungsmäßig festgewordene diminutivische Wortformen nicht einbezogen werden (sog. "historische Diminutive"). Ausgeschlossen bleiben ebenfalls unmißverständlich lexikalisierte Formen, lediglich jene aus dem nur schwer abzugrenzenden Übergangsbereich sollen aus methodischen Gründen Eingang finden. Nicht aufgenommen werden schließlich an idiomatische Wendungen gebundene Diminutivformen, ihre Beschreibung stellt ein eigenes Problem dar und gehört eher zum Themenkreis der Phraseologie.

Die Kenntnis der erarbeiteten generellen Angaben zur Modifikationsform des Diminutivs bildet eine unverzichtbare Voraussetzung für die Beschäftigung mit ihnen. Denn wollte man sich auf einschlägige einsprachige Wörterbücher verlassen bzw. sie insbesondere bei strittigen Formen hinsichtlich ihrer Abgrenzung zum formalen Diminutiv zu Rate ziehen, wird man bald feststellen müssen, wie unzulänglich ihre lexikographische Erfassung ausfältt. Sowohl die Auswahlkriterien eines Diminutivderivats als auch die das Lemma erläuternden Vermerke scheinen eher nach dem Prinzip der Zufälligkeit als nach dem der konsequenten Strukturierung geprägt zu sein und bleiben damit für den Wörterbuchbenutzer unergiebig und kaum brauchbar. ${ }^{3}$

Mit der Annahme der Existenz zweier Diminutionsverfahren bietet es sich an, neben den Affixdiminutiven, den synthetischen Bildungen, auch analytische Diminutive in den Vergleich einzubeziehen. Als problematisch erweist sich dabei das Herausgreifen der das Basiswort begleitenden Epitheta. Die Leistungsfülle eines formal eindeutig markierten Suffixdiminutivs macht es unmöglich, parallel alle das Grundwort erläuternden Worteinheiten zu erfassen; die ungleiche Bilde-

\footnotetext{
3 Über diesen "desolaten Zustand" (S.66) der lexikographischen Darstellung der Modifikation, am Beispiel von vier romanischen Sprachen, hat ETTINGER 1984 geklagt und einige Verbesserungsvorschlăge geboten.
} 
weise zwingt zur Eingrenzung. Es mag daher genügen, nur diejenigen analytischen Bildungen des ausgangssprachlichen Textes aufzunehmen, deren nähere Bestimmung das Element der Kleinheit - in quantitativer wie qualitativer Hinsicht - beinhaltet. All diejenigen Attribute, die nur in meliorativer bzw. pejorativer Funktion genutzt werden (vgl. mily kanarek, ladny dom, biedne dziecko, niedliches Mädchen u.a.) bleiben als analytische Fügungen in der Vorlage unberücksichtigt.

\subsection{WIEDERGABEMUSTER IN DER POLNISCH $\Rightarrow$ DEUTSCHEN KONFRONTATION}

Will man untersuchen, wie beschriebene Diskrepanzen oder auch Konvergenzen auf Textebene realisiert sind, ist es bei der Kontrastierung angebracht, vorab Regelmäßigkeiten unter strukturellem Aspekt nachzugehen, mögliche Übersetzungsprinzipien aufzudecken und sie zu systematisieren. Dabei bietet es sich an, auf Anregungen bereits erfolgreich durchgeführter Übersetzungsvergleiche zurückzugreifen. Gedanken zum Übersetzungsproblem polnischer Diminutive im Deutschen hat sich D.Müller-Ott gemacht, die von grundsätzlich drei Möglichkeiten der Wiedergabe ausgeht:

1. das ausgangssprachliche Suffixdiminutiv wird auch im Deutschen mit einem Diminutiv übersetzt;

2. es wird versucht, zumindest einen Teil der im polnischen Diminutiv beinhalteten Semantik im Deutschen mit anderen Mitteln wiederzugeben;

3. die beste Übersetzung des suffixalen Diminutivs ist der Verzicht auf dessen Wiedergabe. ${ }^{4}$

Mit dem ersten Vorschlag erfaßt sie also alle Übertragungen des Typs Suffixdiminutiv $\Rightarrow$ Suffixdiminutiv: chmurka $\Rightarrow$ Wölkchen, braciszek $\Rightarrow$ Brüderchen. Unter der zweiten Gruppe werden insbesondere Umschreibungen verstanden, die aus dem substantivischen Grundwort und einem dieses präzisierenden Attribut bestehen, dabei werden neben klein, vgl. spacerek $\Rightarrow$ kleiner Spaziergang, auch weitere Ergänzungen berücksichtigt, vgl. herbatka $\Rightarrow$ guter Tee, biurokratyczny folklorek $\Rightarrow$ miese bürokratische Folklore u.a. Diese Übertragung kann

• Vgl. die Ausführungen von MÜLLER-OTT 1983. S.108-112. 
mit dem Umsetzungsmuster Suffixdiminutiv $\Rightarrow$ analytisches Diminutiv formalisiert werden. Der ersatzlose Verzicht, den die Autorin vor allem bei Hypokoristika und Diminutivformen, die im Zusammenhang mit Kindern gebraucht werden, vorschlägt, wäre dann als Realisierungstyp Suffixdiminutiv $\Rightarrow$ Nichtdiminutiv zu fassen. Besonders der Umgang mit Koseformen ist ein geeigneter Gradmesser für die Qualität einer Übersetzung; so ist ein "deutscher Text mit vielen Väterchen, Mütterchen und Täubchen unschwer als schlechte Übersetzung "s zu identifizieren.

Daß das vorgestellte Grundmodell lediglich einen Ausschnitt potentieller Übertragungstechniken vorführt, zeigen S.Ettingers Erörterungen, die ein wesentlich nuancierteres Inventar an Übersetzungsmöglichkeiten anbieten. In Anlehnung an die Postulate M.Wandruszkas sieht er nämlich in der "Notwendigkeit einer möglichst genauen Einteilung der sprachlichen Fakten sowohl in der Ausgangssprache als auch in der Zielsprache", d.h. in der Entwicklung eines "feinen Instrumentariums", 6 eine der Grundforderungen des gelungenen Übersetzungsvergleichs. Der Parallelität der Probleme wegen will die folgende Untersuchung Ettinger folgen, der, in kritischer Auseinandersetzung mit bisherigen Übersetzungsanalysen romanischer Sprachen und des Deutschen, von einem Modell mit theoretisch achtzehn möglichen Übersetzungstypen ausgeht.' Soll dieses Paradigma für das Sprachenpaar Polnisch-Deutsch fruchtbar gemacht werden, so ist eine speziell für diese Sprachenkonstellation geltende Modifizierung bestimmter Wiedergabemuster geboten.

Nach Durchsicht des gesamten Textmaterials ließen sich die diminutivischen Formen zunächst in - berücksichtigt man auch die diminutivische Übertragung von Nichtdiminutiven des Ausgangstextes - vier strukturell verschiedene Gruppen gliedern, die je nach Übertragungsmodus weiter zu unterteilen sind:

${ }^{3}$ MÜLLER-OTT 1983. S.106.

- ETTINGER 1980. S.166.

7 Damit die Eigenart seiner Klassifizierung deutlich wird, lohnt es, die Übertragungsmöglichkeiten kurz vorzustellen, vgl. ETTINGER 1980. S.166f:
DS $\Rightarrow$ DS
$P+S \Rightarrow D S$
$P+D S \Rightarrow D S$
$S \Rightarrow$ DS
$D S \Rightarrow P+S$
$P+S \Rightarrow P+S$
$P+D S \Rightarrow P+S$
$S \Rightarrow P+S$
$D S \Rightarrow P+D S$
$P+S \Rightarrow P+D S$
$P+D S \Rightarrow P+D S$
$S \Rightarrow P+D S$
DS $\Rightarrow S$
$P+S \Rightarrow S$
$P+D S \Rightarrow S$
DS $\Rightarrow$ fr.W.
$P+S \Rightarrow$ fr. $W$.
$P+D S \Rightarrow$ fr.W.
(DS $=$ Wort mit Diminutivsuffix; $P=$ petit; $S=$ Substantiv; fr.W. = freie Wiedergabe). 

A. Synthetisches Diminutiv $\Rightarrow$
2. Analytisches Diminutiv
3. Analyt.-synthet. Dim.
4. Nichtdiminutiv
5. freie Wiedergabe

1. Synthetisches Diminutiv

(SD)

(AD)

(ASD)

(ND)

(frW)

1. Analytisches Diminutiv

2. Analyt.-synthet. Dim.

B. Analytisches Diminutiv $\Rightarrow$

C. Analyt.-synthet. Dim. $\Rightarrow$
3. Synthetisches Diminutiv

4. Nichtdiminutiv

5. freie Wiedergabe

1. Analyt.-synthet. Dim.

2. Analytisches Diminutiv

3. Synthetisches Diminutiv

4. Nichtdiminutiv

5. freie Wiedergabe

1. Synthetisches Diminutiv

2. Analytisches Diminutiv

3. Analyt.-synthet. Dim.
(AD)

(ASD)

(SD)

(ND)

(frW)

(ASD)

(AD)

(SD)

(ND)

(frW)

(SD)

(AD)

Verglichen mit Ettingers Systematik erweist es sich als zweckmäßiger, alle analytischen Wendungen in einer Gruppe zusammenzufassen (=AD) und nicht das Syntagma "maly/klein + Substantiv" von anderen sowohl quantitativ als auch qualitativ gebrauchten Wortfolgen "Epitheton + Substantiv" (bei Ettinger der Umsetzung "freie Wiedergabe" zugeordnet) zu trennen. Eine Hervorhebung des Wiedergabetyps "P $+S^{n}$ rechtfertigt sich wegen der durch sprachhistorische Entwicklungen bedingten spezifischen Funktion des epithetischen Adjektivs petit speziell für das Französische. ${ }^{8}$ Sinnvoll scheint auch eine weitere Umschichtung, indem die Zusammensetzung von " $S$ " eine Erweiterung erfahren soll. Neben dem entsprechenden nichtsuffigierten Grundwort soll der ND-Typ auch alle jene Substantive enthalten, die mit dem diminutivischen Wert des Primärtextes in gewisser Weise korrespondieren; unter den frW-Typ soll dann lediglich das subsumiert werden, was sich vom Original entfernt hat, formal und semantisch nicht mehr direkt an es gebunden ist."

\footnotetext{
- Um Angaben über den Gebrauch und die Leistung von petit hat sich FISCHER 1962 (S.23ff, bes. S.61ff) in ihrem deutsch-franzosischen Übersetzungsvergleich bemüht; vgl. auch WANDRUSZKA 1969. S.94; darüberhinaus weiter oben S.39, Anm.38.

- Ettingers Sihema hat sich bereits die niederländisch $\Rightarrow$ polnische Vergleichsstudie von KRAJCARZ 1981 a zunutze gemacht, der, ohne es für seine Zwecke zu modifizieren - bis auf das Weglassen diminutivischer Wiedergaben von Nichtdiminutiva des Originals -, die Struktur der Übersetzungstypen beibehalten und sie entsprechend ihrer Produktivität aufgenommen hat (vgl. S.67).
} 


\subsection{1. Übertragung synthetischer Diminutive}

Dem obigen Schema möglicher Umsetzungsmodi folgend soll nun die Analyse der interlingualen Beziehungen von Diminutiveinheiten des Polnischen als Ausgangssprache und des Deutschen als Zielsprache vorgenommen werden. Sowohl hierfür als auch für den weiteren Verlauf gilt es, aus dem zusammengetragenen umfangreichen Wortmaterial lediglich eine möglichst repräsentative Auswahl der für den jeweiligen Wiedergabetyp charakteristischen Beispiele anzuführen. ${ }^{10}$ Dabei bietet es sich an, auf die verschiedenen inhaltlichen Aspekte des Diminutivs einzugehen.

$S D \Rightarrow S D:$

Mit dieser ersten Übersetzungstechnik werden Fälle formaler Substitution erfaßt, die also auf ein Suffixdiminutiv ebenfalls mit einem diminutivischen Derivat reagiert haben."

Na pókach stalo nieprzebrane mnóstwo sloi i słoikow podlug hierarchii dat, każdy $z$ odpowiednim napisem i notatka. (IWA: 64)

W pobliżu miejsca, gdzie mieszkal ojciec, byl niewielki las, borek, niedaleko miasteczka Biskupice. (FOR: 37)

...i wydostala $z$ dużej torby książeczkę. Byla to malenka, zlożona z kilkunastu kartek ksiażeczka... (FOR: 17)

* 1 jasnego, lekkiego piwa zagotowujemy $z$ 1/2 l wody, 2-3 goździkami i kawaleczkiem kory cynamowej. (LEM:

26)

In den Regalen standen unzählig viele Gläser und Gläschen, nach Daten geordnet, jedes mit Aufschrift und Notiz. (IWA: 52)

Nicht weit von Vaters Haus, in der Nähe des Städtchens Biskupice, befand sich ein Wäldchen... (FOR: 37)

Dann holte sie aus der großen Tasche ein Büchlein hervor. Es war klein und hatte nur wenige Seiten... (FOR: 23)

* 1 helles, leichtes Bier mit $1 / 21$ Wasser, 2-3 Nelken und einem Stückchen Zimt aufkochen. (LEM: 29)

Diese Beispiele führen den Gebrauch eines Diminutivs in reiner, nicht nur subjektiver Verkleinerungsfunktion vor: gemeint sind male sloje, maly bor, mala ksiqzika, mały kawal. Betont wird die Größenangabe eines Objekts in

10 Grundbedingung für die Aufnahme der Belege in diesen Abschnitt der Analyse ist ihre zielsprachengerechte Wiedergabe, d.h., nur gelungene Übersetzungsentscheidungen, nicht aber übersetzerische Fehlinterpretationen haben Eingang gefunden.

"Zur Auflösung der folgenden Kürzel (z.B. IWA: 64) vgl. den Beginn des Literaturverzeichnisses; die dem Kürzel folgende Zahl gibt die betreffende Seite des Werkes an. 
einem Fall durch die Gegenüberstellung von Grundwort und Derivat, vgl. sloje sloiki, im andern durch die vorangehende oder folgende attributive Ergänzung, niewielki las oder malenka ksig̨zeczka; beim letzteren ist wohl eher an eine Umfangs- als eine Größenminderung gedacht.

Eine Variante der Verkleinerungsfunktion bieten die beiden folgenden Belege:

I zawsze w pewnej chwili jest $z$ pagórka widok na niebieska tafle jeziora $z$ biala, ledwo dostrzegalna plamka perkoza, ze sznurem kaczek ciagnacych nad trzcinami. (MIL: T)

Jej stopy sa najpierw tuż nad trawa i nie ruszajac nimi unosi sie powoli coraz wyżej,...aż jest już tylko punkcikiem miedzy oblokami... (MIL: 39)
Und immer, irgendwann, ist vom Hügel die Aussicht auf die blaue Fläche eines Sees mit dem weißen, kaum zu erspähenden Fleckchen des Haubentauchers, mit der über dem Schilf dahinziehenden Entenschnur. (MIL: 5)

Ihre Füße sind zuerst dicht über dem Gras, und ohne sie $z u$ bewegen, schwebt sie allmählich empor,...bis sie schon, nur ein Pünktchen, zwischen Wolken ist... (MIL: 50)

Hier ergibt sich die Kleinheit des Objekts aus der Perspektive des Betrachters. Bei gleichbleibender absoluter Größe minimalisiert allein die sich vergrößernde räumliche Entfernung das Gemeinte. Unterstützt wird der Eindruck des Kleinseins durch die Beifügungen ledwo dostrzegalna und juz tylko.

Diminutive im Dienste der Verminderung eines Objekts begegnen schließlich auch an folgenden Textstellen:

Mial cere dziecka...i oczy niebieskie z czerwonymi zylkami. (MIL: 13)

Wkladala zloty lańcuszek z ziarenkami jak lebki od szpilek, a medalion...chowala w kieszonce za pasem. (MIL: 17)

Przez ciemnosc zielonymi kropelkami fosforu blyszczala tarcza zegarka. (NOW: 126)

Jola...jadla drugie śniadanie, skladajace sie $z$ chleba, masla i dlugich zielonych ogórkow, które zrecznie obierala srebrnym nożykiem... (IWA: 85)

To mniej wiecej opowiadal doktorowi, gdy siedzieli pod kasztanem, przed domkiem kolonijnym... (IWA: 26)
Seine Hautfarbe war die eines Kindes.... und die Augen waren blau mit roten Adderchen. (MIL: 13)

Sie legte ein goldenes Kettchen mit Körnchen, groß wie Stecknadelköpfchen, an und das Medaillon...steckte sie in ein Gürteltäschchen. (MIL: 19)

In der Dunkelheit glänzte das Zifferblatt der Uhr mit grünen Phosphorpinktchen. (NOW: 79)

Jola... [verzehrte] ihr zweites Frühstück. Es bestand aus Brot, Butter und langen grünen Gurken, die sie mit einem silbernen Messerchen geschickt schälte... (IWA: 75)

Dies ungefähr erzählte er dem Doktor, während sie unter der Kastanie vor dem Sommerlager-Häuschen saßen... (IWA: 10) 
Dwa bukjeciki fiokkow, wrzuce je do jeziora, zapach maja przywiedly, ich pora dawno minela, sa drobne jakies $i$ niewydarzone. (FIN: 84)

...Marynia wziela mnie w swoje obroty...i ani sie spostrzeglam, a już bylam w spódniczce, fartuszku i kaftaniku z bialego płótna... (FOR: 61)

Zasmażke rozprowadzamy dokladnie, by nie dopuscić do powstania grudek... (LEM: 51)

To znow wyniesie Macius klatke $z$ kanarkiem, zawiesi na galezi, otworzy drzwiczki klatki i patrzy, i slucha. (KOR: 62)

Rozdano [czarnym] dzieciom choragiewki bialych narodów. (KOR: 110)
Zwei Veilchensträußchen, ich werfe sie in den See, sie duften welk, ihre Zeit ist lange vorbei, sie sind irgendwie klein und mißraten. (FIN: 101)

...Marynia nahm sich meiner an..., und in kurzer Zeit schon stand ich fertig in Rock, Schürzchen und weißem Leinenmieder da. (FOR: 58)

Die Schwitze gründlich...verrühren, damit keine Klümpchen entstehen... (LEM: 57)

Ein andermal nimmt Hänschen das Bauer mit dem Kanarienvogel und hängt es an einen Zweig. Dann öffnet er das Türchen und schaut $\mathrm{zu}$ und horcht. (KOR: 86)

Fähnchen der weißen Völker wurden an die [schwarzen] Kinder verteilt. (KOR: 150)

Der Gebrauch der diminutivischen Wortform ist Ergebnis der nicht selten anzutreffenden sprachlichen Erscheinung, insbesondere des Polnischen (im Deutschen ist ihr nur ausnahmsweise zu begegnen), Objekte derselben Art, aber anderen, kleineren Ausmaßes morphologisch zu kennzeichnen: So sind die das Auge durchziehenden Adern dünner und feiner als die den ganzen Körper durchlaufenden Blutbahnen; die Tasche hinter dem Gürtel bietet nicht soviel Einsteckplatz wie eine übliche Hosentasche; Blumen einer kurzstieligen und zarteren Sorte, wie es Veilchen sind, lassen sich z.B. im Vergleich zu dick- und langstieligen Rosen lediglich zu einem kleineren Strauß binden. Ein Ferienhaus wird sich in seiner Größe und Ausstattung von einem herkömmlichen Wohnhaus meist unterscheiden. Die Tür eines Käfigs ist in ihren Ausmaßen deutlich kleiner als die eines Hauses, und schließlich hat die zumeist papierene Fahne, die z.B. auf Kundgebungen geschwenkt wird, eine andere Größe als die, die an einem Mast angebracht ist. Häufig geht mit dieser Diminuierung auch eine Spezialisierung einher. Während die Kette als Sicherungs- oder Befestigungsmittel dient, wird ein Kettchen als Halsschmuck genutzt. So ist auch das Messerchen als feines Schälmesser für Obst bzw. Gemüse zu verstehen, oder das Schürzchen nicht in der Funktion eines Kleidungsschutzes, sondern als Bestandteil einer festlichen Volkstracht gedacht.

Die Nennung der Kleinheit eines Gegenstandes wird auch für eine Aussage im übertragenen Sinne genutzt: 
Czul wyraźnie bolesny cieżar uwierajacego go w okolicy serca kamyczka. Taka bowiem postac przybralo niefrasobliwe, lekkie jak bańka mydlana, ziarenko euforii...Kamyczek $\delta w . . . r o z-$ rastal sie i powiekszal i w krótkim czasie przemienil sie z kamyczka w kamien... (FIN: 32)

Milczalam. Wiedizalam, że niezależnie od tego, czy i co powiem, ona bedzie mówila. Niepytana, nieproszona, opowie wszystko mnie, obcej, pierwszy raz spotkanej. Kamyczek zlecial - czekalam lawiny. (FIN: 98)

Po wojnie mówiono mi o niej i o jej rodzicach, mialam wiec przed soba jak na dloni bieg ich losów, najpierw spokojny i gladki, nieco leniwy jak nie zmacona fala tafla naszej rzeczki, potem nagle zalamujacy sie, porwany przez wir, gwaltownie spadajacy $w$ otchlan. (FIN: 95)
Deutlich spürte er die schmerzende Last des Steinchens, das ihn in der Herzgegend drückte. Diese Gestalt hatte das sorglose, gleich einer Seifenblase leichte Kömchen Euphorie angenommen...Dieses Steinchen [wuchs]...und vergrößerte sich so, daß es sich binnen kurzer Zeit aus einem Steinchen in einen Stein verwandelte... (FIN: 95f)

Ich schwieg, weil ich wußte, was immer ich sagte, sie würde sprechen. Ungefragt, ungebeten würde sie mir, der Fremden, der zum ersten Mal Angetroffenen, alles erzählen. Ein Steinchen war herabgerollt, ich wartete auf die Lawine. (FIN: 82)

Nach dem Krieg haben mir die Leute von ihr und ihren Eltern erzählt. Ganz übersichtlich lag nun ihr weiterer Lebenslauf vor mir. Erst ruhig und glatt, ein wenig träge wie die von keiner Welle getrübte Oberfläche unseres Fiüßchens, dann plötzlich aufgewühlt, fortgerissen von einem Strudel und mit dem Wasserfall in den Abgrund stürzend. (FIN: 133)

Mit den Diminutiven wird der beabsichtigte Gegensatz stārker herausgestellt: der Geringfügigkeit und Bedeutungslosigkeit (kamyczek) bzw. der Harmlosigkeit und Vertrautheit (nasza rzeczka) steht das Schmerzliche, das Unwägbare, die Bedrohlichkeit gegenüber. In ähnlicher Funktion begegnet das Diminutiv auch im wörtlichen Sinne:

Przez mokre dróżi po deszczu przeprawialy sie [ślimaki] $z$ jednego trawnika na drugi... Wziete $w$ reke, wciagaly sie w skorupke, ale zaraz wysuwaly sie znowu... (MIL: 18)

...gdy wtem uslyszelismy walenie $w$ drzwi, domek zatrzasl sie, a obraz pejzaż nadmorski $z$ mewa $i$ wydmami spadl ze sciany...,krótka cisza i znów walenie $w$ drzwi... (FIN: 84)
Über die feuchten kleinen Wege nach dem Regen setzten [die Schnecken] von einem Rasen zum anderen über...In die Hand genommen, verzogen sie sich ins Häuschen, doch kamen sie gleich wieder hervor... (MIL: 20)

...da donnerte jemand gegen die $\operatorname{Tür}[$, das Häuschen bebte und die Küstenlandschaft mit den Möwen und den Dünen fiel von der Wand[,]...eine kurze Stille und wieder das Donnern an der Tür... (FIN: 100f)

In beiden Fällen handelt es sich um Kleinheit angesichts der Gefahr, zum einen der möglichen Bedrohung des Tieres durch den Menschen (worauf es im Schnekkenhaus Schutz findet), zum anderen der Angst und des schutzlosen Ausgeliefertseins gegenüber der SS, die das Haus erstürmt. 
Doch ebenso sind in dieser Gruppe Diminutive als Ausdrucksmittel einer positiven Stimmung zu finden:

Caly piekny ogródek zalożyla Magdalena i wystarczylo już tylko dbac żeby go nie zapuscic. (MIL: 99)

...zblizala sie linia lasu i za pierwszymi jego drzewami otwieral sie zupelnie inny swiat: $z$ pagóreczka w dolinke, tu borek, tu rojścik, kręcace sie dróżki... (MIL: 87)

...patrzyl na rodzinny krajobraz jak na przyjaciela spotkanego po latach...Jechali wsród plaskich rownin, monotonnych, stepowych...A kiedy wreszcie w kotlinie, pod zamkowym wzgórzem ukazalo sie miasteczko i ono zdumialo go... (FIN: 32f)
Das ganze schöne Gärtchen hatte Magdalena angelegt, und es genügte etwas Pflege schon, um es zu erhalten. (MIL: 135)

Die Linie des Waldes nahte, und hinter den ersten Bäumen öffnete sich eine ganz andere Welt: Hügelchen und Tälchen, hier ein Wäldchen, dort etwas Sumpf, gewundene kleine Pfade... (MIL: 119)

...er musterte die heimatliche Landschaft wie einen nach Jahren wieder getroffenen...Freund. Sie fuhren durch eine flache, monotone Steppenebene... Und als sich endlich im Kessel unter dem Burghügel das Städtchen zeigte, machte auch dieses ihn...staunen. (FIN: 96)

Hier soll zunächst Wertschätzung, Gefallen an der Schönheit des Gartens angezeigt werden; im zweiten Beleg ist es die Entdeckungsfreude, die Sehnsucht nach etwas Neuem, die im Originaltext durch eine Aneinanderreihung mehrerer diminutivischer Bildungen noch unterstrichen wird. Im letzten Beispiel wird dann die Rührung und Freude bei der Ankunft in der Heimatstadt signalisiert.

Die nun folgenden Exempel:

...myslalam sobie, że moge godzinke odpoczać i pospac, o godzinke opóźnić trudne spotkanie z Siniawka... (FIN: 70)

[M:] Można jeszcze kieliszeczek? - [S:] W karafce dno widac, jak pan dojedzie do domu? (NUR: 18)
Ich überlegte...,daß ich ein Stündchen ausruhen und schlafen, die schwierige Begegnung mit Siniawka aufschieben dürfte... (FIN: 114)

[M:] "Noch ein Gläschen? - [S:] Die Karaffe ist fast leer...wie wollen sie jetzt noch nach Hause kommen? (NUR: 22)

bieten einerseits die Möglichkeit einer euphemistischen, d.h. einer verhüllenden und beschönigenden Wendung, andererseits die des Ausdrucks von Understatement, der Abmilderung, der Verharmlosung eines unangenehmen Tatbestandes.

Im Dienste einer qualitativen Verminderung stehen nun jene Ausschnitte:

Podjechal pod bude platforma na gumowych kolach. Obiad zjadl spiesznie i podcial chude boki kudlatych koników. (NOW: 107)
Er fuhr an die Bude heran in einem Gefährt auf Gummirädern. AB eilig sein Mittagbrot und klatschte auf die mageren Flanken der zottigen Pferdchen. (NOW: 61) 
Byla to mala miescina: ubogie żydowskie sklepiki, chaty na wpół porozwalane... (FOR: 114)

Ja jestem bosa i w samodzialowej koszulinie. Prawdopodobnie jest ostra zima... (FOR: 11)
[Das Städtchen] war sehr klein: ärmliche jüdische Lädchen, halbzerfallene Hütten. (FOR: 107f)

Ich war barfuB, in einem handgewebten Hemdchen. Es war wohl ein harter Winter... (FOR: 17)

Armseligkeit, Bedürftigkeit und Armut sind die Stichworte, die den Inhalt des Diminutivs hier bestimmen.

In der Funktion von Herabsetzung und Geringschätzung treten die folgenden Derivate auf:

Wampir...zaczal śpiewać. Zrazu cicho, potem mocno i ochryple. "Wróce $\mathrm{i}$ ucaluje twoje recen"..."Wróce, polożyć musze kres twej mece...", ktos podchwycil, przylaczajac sie zdartym tenorkiem. (NOW: 127)

Boleslaw podszedl do klawiatury i jednym palcem wystukal melodie piosenki, jaka spiewal kiedys w wojsku w mlodosci. (IWA: 117)
Vampir...begann zu singen. Erst leise, dann kräfig und heiser. "Ich kehre zurück und küsse deine Hände"... "Ich kehre zurück und mach deiner Qual ein Ende..." hakte jemand mit verzerrtem Tenorstimmchen ein. (NOW: 80)

Boleslaw trat an die Tastatur und klimperte mit einem Finger die Melodie eines Liedchens, das er einst in jungen Jahren beim Militär gesungen hatte. (IWA: 110)

Es wird sowohl abschätzig über die Qualität der Männersingstimme - ergänzt durch zdartym - als auch über ein bestimmtes, wohl schlichtes Lied gesprochen, auf dessen Niveau auch der Kontext - in der übersetzten Fassung vielleicht noch deutlicher durch die umgangssprachlich-abwertende Formulierung klimperte hinweist.

Exemplarisch für ironischen Gebrauch eines Diminutivs ist diese Stelle zu nennen:

Osobliwoscia doliny Issy jest wieksza niż gdzie indziej ilošc diablow...Ci, co je widzieli, mówia, że diabel...nosi zielony fraczek, żabot, wlosy splecione w harcap, biale ponczochy i przy pomocy pantofli na wysokich obcasach stara sie ukryé kopyta, których sie wstydzi. (MIL: 9)
Die Besonderheit des Issatals liegt in der Zahl seiner Teufel...Diejenigen, die sie gesehen haben, sagen, ein Teufel...trage ein grünes Fräcklein, ein Jabot, die Haare zum Zopf geflochten, weiße Strümpfe und bemühe sich, durch Schuhe mit hohen Absätzen die Hufe, deren er sich schämt, zu verstecken. (MIL: 7f)

Sie unterstreicht die bereits kontextuell ausgedrückte Ironie angesichts des herrschenden Aberglaubens. Mit einer Abmilderung von Ironie hat man es indes im nächsten Musterbeispiel zu tun:

Ścisnal ja mocno za lokiex. "Odczep sie", warknela. Poruszyla sie niecierpliwie..."Madralinko", syknal zlowrogo, "nie podskakuj". (NOW: 38)
[Er] drückte sie mächtig am Ellbogen. "Hau ab!" fauchte sie. Machte eine ungeduldige Handbewegung..." Schlauköpfchen", zischte er unheilverkündend, "hab dich nicht so". (NOW: 21) 
Eine weitere Variante diminutivischen Wertes findet sich in den Belegen:

Niebo bez chmurki, slonce wysoko, poludnie. (MIL: 65)

"Na mapach rysuje sie tylko duże wyspy, boby sie wszystkie nie zmiescily. Jeżeli jest na mapie chocby tylko kropeczka, to wyspa jest duża." (KOR: 6)

"Tylko pamietaj: gdybys komu choc stowko powiedzial, to już wiesz, co bedzie. To jest nasz najtajniejszy zwiazek." (KOR: 32)
Der Himmel ohne ein Wölkchen, die Sonne hoch, Mittag. (MIL: 87)

"Auf den Landkarten sind immer nur die großen Inseln eingezeichnet, denn für alle ist kein Platz. Wenn auf der Landkarte nur ein Pünktchen verzeichnet ist, dann ist es schon eine große Insel." (KOR: 7)

"Aber wenn du jemandem auch nur ein Sterbenswörtchen sagst, dann weißt du ja, was dir geschieht. Das ist unser allergeheimster Bund." (KOR: 43f)

Die Betonung der Geringfügigkeit, äußerster Intensität im negativen wie positiven Sinne (hier unterstützt durch das begleitende chocby/choc) dient jedesmal der Verstärkung, Intensivierung der Aussage, was im letzten Beispiel im Zieltext durch die Verwendung eines Kompositums (zusammengesetzt aus sterbendes 'schwaches, kaum hörbares' - Wörtchen) zusätzlich verdeutlicht wird.

Ein besonders beliebter Bereich, in dem Diminutivformen zum Einsatz kommen, ist der Themenkreis Kind und Tier. Hier vermischt sich die Komponente der Kleinheit mit der der affektischen zu einer Einheit, um in der Regel diverse Gefühlsschattierungen, wie z.B. Vertrautheit, Sympathie, Niedlichkeit, Zierlichkeit oder auch Sorge, Anteilnahme und Mitleid anzuzeigen, vgl.:

Ulożyl Oleńke $w$ ramionach jak w kolysce, i, wolnym krokiem idac ku domowi, kolysal ja w te i owa strone. To nóżki, to glowka dziewczynki potracaly o liście, o krzaki... (IWA: 130)

...dziecko leżalo cichutko i bylo okragle i duze, zaczerwienione od snu jak jabhuszko. (FIN: 41)

Chlopiec prowadzi za reke mala dziewczynke, która ladnie, predziutko przebiera nózkami i ma jasne wloski. (KOR: 72)

A tu mówi: "Teraz Ala wytrze nosek, żeby byl suchy". (KOR: 80)

A kiedy wszystko rozda, otwiera raczki i wola radośnie: "Nie ma, nie ma nic!" (KOR: 81)
Dann legte er Ola in seinen Armen wie in einer Wiege zurecht, ging mit langsamen Schritten nach Hause und wiegte sie dabei hin und her. Köpfchen und Füße des Kindes stießen abwechselnd gegen Blätter und Büsche... (IWA: 125)

Das Kind lag ganz ruhig, es war rund und groß und rosig im Schlaf wie ein Äpreichen. (FIN: 60)

Der Junge hielt ein kleines Mädchen an der Hand, das niedlich und schnell seine Füßchen setzte und blondes Haar hatte. (KOR: 97)

Und hier sagt er: "Jetzt putzt sich Ala das Näschen, damit es trocken ist". (KOR: 109)

Und wenn sie alles verteilt hatte, dann offnete sie die Händchen und rief fröhlich: "Alles weg, alles weg!" (KOR: 110) 
[F:] Dzieci moje nieszczesne, piskleta moje biedne, otwieraja dzjobki, jeśc wolaja, a ten lajdak nic sobie $z$ tego nie robi... (TON: 3)

Odwrócilem sie - biegl za mna. "Do widzenia piesku", powiedzialem i po raz pierwszy dotknalem jego sierści, która byla ciepła i przyjemna w dotyku. (FIN: 68)

...a tam przejeżdżal kupiec, wiozacy na wozie owce. Bylo im ciasno i jedna sie zadusila. Kupiec sie strasznie rozzloscil, zrzucil ja $z$ wozu, kopnal noga $i$ odjechal, a Frania wlasnym oddechem owieczke ocuciła, nakarmila chlebem i mlekiem. (FOR: 18)

Zbliżyl sie Macius do klatki - ptaszek umilkl na chwile przestraszony, ale po chwili jeszcze glośniej i piekniej zaspiewal. (KOR: 15)

Szczurek musial być napadniety $w$ drodze, bo mial jedna lapkę odgryziona i kulal. (KOR: 99)
[F:] Meine unglücklichen Kinder, die armen kleinen Küken, sperren die Schnäbelchen auf, hungrig sind sie und dieser Schuft macht sich gar nichts draus... (TON: 3 )

Ich drehte mich um, er folgte mir. "Auf Wiedersehen, Hündchen", sagte ich und berührte zum ersten Mal sein Fell, das warm und angenehm war. (FIN: 111f)

Da kam ein Händler vorbei, der hatte Schafe auf seinem Wagen. Die Schafe waren so zusammengepfercht, daß ein Lämmchen erstickte. Der Händler wurde schrecklich wütend, er warf es vom Wagen, gab ihm einen Tritt und fuhr davon. Frania aber erweckte das Lämmchen mit ihrem Atem wieder zum Leben und gab ihm Brot und Milch. (FOR: 24)

Hänschen näherte sich dem Bauer - das Vögelchen verstummte für einen Augenblick ganz erschreckt, schmetterte aber ein Weilchen später um so lauter und schöner los. (KOR: 20)

Die Ratte mußte unterwegs angefallen worden sein, denn ein Prötchen war verletzt und sie hinkte. (KOR: 133)

Was allen oben angeführten Beispielpaaren bereits zu entnehmen ist, gilt ebenso für die übrigen $S D \Rightarrow$ SD-Belege: bis auf wenige Ausnahmen ist im Zieltext immer mit einem der Vorlage entsprechenden Lexem geantwortet worden. Mit ausgetauschtem Grundwortelement sind z.B. zu nennen:

Na [babci] wezwania na spacerze, żeby wzial ja za reke nie odzywal sie zaraz, chowal sie za pniem drzewa $i$ w ogole tak sie urzadzal, zeby wyrwać z tej okraglej, różowej kulki lekliwe skargi: "oje, oje". (MIL: 51)

Tomasz, czesto podrapany i potluczony do krwi, wiedzial, że najlepszym srodkiem sa liście babki, przykladal taki liść i przewiazywal rane kawalkiem płótna. (MIL: 84)

Naczelny lekarz przychodzil czesto. Stanawszy przy lózku, drwil: "Wyjmie-
Während der Spaziergänge antwortete er nicht sofort auf die Hilferufe [der Großmutter], versteckte sich hinter dem Baum und richtete es meistens so ein, $\mathrm{da} \beta$ er aus dem kugligen, rosa Persönchen die angstvollen Klagen: "O je, $O$ je" herauslocken konnte. (MIL: 67)

Thomas, der oft zerkratzt und blutig gestoßen war, wußte, daß die Salbeiblätter das beste Mittel sind; so ein Blatt legte er auf und verband die Wunde mit einem Leinenläppchen. (MIL: 114)

Der Oberarzt kam häufig. Er stand am Bett und spottete: "Wir nehmen dir das 
my żelastwo, panie bratku, i znów...", czynil wymowny gest, "fiut, pod cele". (NOW: 149)
Eisen raus, Bürschchen, und dann...", er machte eine sprechende Geste, "zack, in die Zelle". (NOW: 102)

Neben den genannten nominalen sind auch adjektivische Modifikationsderivate der Vorlage in diminutivische Formen übertragen worden. Ausnahmslos sind sie den sog. Detensiva, jenen Bildungen in abschwächender Funktion, zuzuordnen:

We wnetrzu mnóstwo ludzi i geste, sinawe od dymu powietrze. (NOW: 1260)

Światlo lamalo sie $w$ zielonkawym szkle butelki. (NOW: 36)

...zastanawial sie nad tym, jak malo jest skupione ich zycie, jak bardzo uciekaja od czarniawego domku leśniczówki... (IWA: 131)

...przedwieczorna pora, kiedy drzewa rzucaja dlugie cienie, a powietrze nasycone jest lekka niebieskawa mgielka... (FIN: 54)

[Sos] powinien byc slodkawo-kwaskowaty, korzenny, lecz z dominujacym smakiem pieczeniowym. (LEM: 67)

Artur Dilbin, wówczas juź nie pierwszej mlodosci a nawet starszawy, uchodzil za czlowieka statecznego... (MIL: 51)

Calowal matke...i z pieszczota w swojej rece wyczuwal jej palce. Grubawe i lagodne. (IWA: 165)

...ghupkowata Kasia, duplikat takiej samej Walerci sprzed lat pietnastu, zaniosla przed nim lampe do zimnego, dusznego pokoju. (IWA: 41)

Drinnen waren eine Masse Leute und dicker, bläulicher Mief. (NOW: 80)

Das Licht brach sich im grünlichen Glas der Flasche. (NOW: 19)

...[er] überlegte: Wie wenig ist unser Leben aufeinander bezogen, wie oft fliehen wir das schwärzliche Haus der Försterei... (IWA: 126)

...wenn die Bäume lange Schatten werfen und die Luft durchzogen ist von einem leichten bläulichen Dunst... (FIN: 44)

[Die Soße] soll süsslichsauer und würzig sein, jedoch mit charakteristischem Bratengeschmack. (LEM: 72f)

Artur Dilbin, damals schon nicht von erster Jugend, ja sogar ältlich, war als ein ernsthafter Mann angesehen... (MIL: 67)

Er küßte seine Mutter...und spürte voller Zärtlichkeit ihre Finger in seiner Hand. Dickliche, sanfte Finger. (IWA: 163)

Die dümmliche Kasia, das Duplikat jener Walercia vor fünfzehn Jahren, trug die Lampe vor ihm her in sein kaltes, stickiges Zimmer. (IWA: 27)

In allen Fällen drücken die Adjektive eine Unvollständigkeit der jeweiligen Eigenschaft aus, bei Farben sind es gewisse Farbabstufungen - beim vierten Beleg verdeutlicht durch den Zusatz lekka -, bei der Angabe der Geschmacksrichtung und des Alters sowie der Anzeige körperlicher Ausmaße und physischer Verfassung handelt es sich um eine Abschwächung im Sinne von nieco/prawie/ zbliżony do. 
Abweichend davon ist noch ein beinahe idiomatisiertes Indefinitpronomen in verstärkender Funktion zu erwähnen, das im Deutschen substantivisch übersetzt wurde:

[J:] Pozwole sobie zauważyé, że dzisiaj wasy...jakby troszke, odrobinke krótsze...może o pare milimetrów. (TON: 14f)
[J:] Ich erlaube mir, zu bemerken, daß der Schnurrbart...heute morgen ein Spurchen, ein klein wenig kürzer zu sein scheint...vielleicht paar Millimeter. (TON: 27f)

Schließlich gehören in die SD $\Rightarrow$ SD-Gruppe Diminutive des anthroponymischen Wortschatzes (Verwandtschaftsnamen inbegriffen). Dabei gilt es, grundsätzlich zu unterscheiden zwischen ausgangssprachlichen Bildungen, auf die im Zieltext mit deutscher Entsprechung reagiert wurde und solchen, die in ihrer polnischen Lautung übernommen wurden:

Ale Macius mial byc wyslany na bezludna wyspe. (KOR: 5)

[Macius] posadzi kanarka na palcu i opowiada, pyta sie, czy pamięta królowe i króla,...Stasia, Helcię... (KOR: 80)

"A pamietasz, jakes Antkowi zeby wybila?" (KOR: 105)

"Tatus codziennie chodzi $z$ rana albo pod wieczor, a $w$ niedziele to sie tu modli ze mna. Czyta z tej książki, co mamusia miala." (IWA: 102)
Hänschen aber sollte auf eine einsame Insel verbannt werden. (KOR: 5)

[Hänschen] setzt ihn sich auf den Finger und erzählt ihm etwas. Er fragt ihn, ob er sich auf die Königin und den König,...Peter, Leni...noch besinnen könne. (KOR: 109)

Und weißt du noch, wie du dem Toni die Zähne ausgeschlagen hast?" (KOR: 142)

"Vati kommt jeden Tag hierher, morgens oder gegen Abend, und am Sonntag betet er mit mir. Er liest mir aus dem Buch vor, das Mutti gehörte." (IWA: 95)

Während die Vornamen Helcia und Antek mit den deutschen hypokoristischen Namenäquivalenten - bei beiden eine mittels Mutilation entstandene Kurzform wiedergegeben werden, ist bei der Übersetzung von Macius das Suffixelement wohl beibehalten, der Name selbst aber durch einen für ein deutsches Kinderbuch gängigen Vornamen ersetzt worden (Maciej (dt. Matthias) $\Rightarrow$ Hans). Bei den Elternbenennungen geht das Polnische von den bereits kosend gebrauchten Kurzformen mama/tato aus, die deutsche Version dagegen modifiziert die Grundformen Mutter/Vater.

Für die zweite, beliebtere Übersetzungsvariante lassen sich folgende Belege anführen:

Od babki Michaliny czyli Misi Tomasz nigdy nie dostal żadnego prezentu... (MIL: 15)
Von Großmutter Michalina oder Misia hatte Thomas nie ein Geschenk bekommen... (MIL: 17) 
Przed ogniskiem w sadzie Domcio [= Dominik] snul mysli teologiczne... (MIL: 63)

Przystaneli koło kiosku piegowatej Jadzi [ $=$ Jadwiga]. (NOW: 63)

W drzwiach stal poslugacz Kubus [ $\leftarrow$ Jakub] Stopkarz. (NOW: 151)

...jedne wakacje spedzil calkiem jako korepetytor Zosi [ $=$ Zofia]. (IWA: 28)

Frania [ $\leftarrow$ Franciszka] siedziala pod chata blisko drogi $i$ jadla chleb $z$ mlekiem... (FOR: 18)
Vor dem Feuer im Obstgarten spann Domcio theologische Gedanken... (MIL: 85)

Sie blieben beim Kiosk der sommersprossigen Jadzia stehen. (NOW: 39)

An der Tür stand der Pfleger Kubus Stopkarz. (NOW: 104)

...[er] hatte sogar einmal die gesamten Ferien dort verbracht, als Nachhilfelehrer für Zosia. (IWA: 13)

Frania saß vor dem Haus am Wege und aB Brot mit Milch. (FOR: 24)

Eine Variation der Übertragungsweise, die polnischen Namensformen in den deutschen Text zu integrieren, bieten die Beispiele:

Stach usmiechal sie nimi, naokolo ust w usmiechu tworzylo sie wiele zmarszczek... (IWA: 97)

Przy pomocy Janka, Żyda ze Slawska, Edzia i Olka...wyladowal fortepian... (IWA: 111)
Stas lächelte mit ihnen, rund um seinen Mund bildeten sich bei diesem Lächeln viele Runzeln... (IWA: 89)

Janek, der Jude aus Slawsk, Edek, Olek...halfen Stas, den Flügel abzuladen... (IWA: 104)

In beiden Fällen greift der Übersetzer auf die eigentlich im Originaltext vorherrschende Namensform, Stas und Edek, beides auch schon Modifikationsderivate ( $\Leftarrow$ Stanislaw und $\Leftarrow$ Edward), zurück.

Fast alle der oben zusammengetragenen Beispiele treten zwar in diminutivischer Form, jedoch ohne aktuell gemeinten diminutivischen Wert auf, was allein schon ihr durchgängiger Gebrauch in den Texten vermuten läßt. In gezielt eingesetzter Weise dagegen - die in direkter Umgebung verwendeten Grundformen mögen das verdeutlichen - wird in diesen Mustern vorgegangen:

W drugim roku takiego smutnego życia urodzilam syna...Kochalam mego synka niezmiernie, ale bylam bardzo mloda, nie wiedzialam, jak wychowywac dziecko... (FOR: 70)

"A to filut z Tyldzi! Nie wiedzialem, że Żydów chowa"... Okreslenie, jakiego użyl mówiac o bratanicy, bylo...niestosowne...Wszyscy, którzy chociażby raz jeden widzieli Marylde, emerytowana nauczycielke,...żachneliby sie wraz ze mna. (FIN: 66)
Im zweiten Jahre dieses traurigen Lebens gebar ich einen Jungen...Ich liebte mein Söhnchen sehr, war aber noch sehr jung und wußte nicht, wie man Kinder erzieht. (FOR: 65f)

"Ein Filou ist das Tildchen. Ich habe gar nicht gewußt, daß sie Juden versteckt hält!"...Der Ausdruck, den er benutzte, als er über seine Nichte sprach, war...unangebracht...Alle, die Fräulein Marilda, die pensionierte Lehrerin,....auch nur ein einziges mal gesehen hatten, wären zusammen mit mir empört gewesen. (FIN: 108f) 
Nasz pies nazywal sie Czing...Pies byl smutny, nie ulegalo watpliwosci... Wodzil wzrokiem za mucha, spacerujaca po oknie, a na pieszczotliwe "no co Czingusiu, cos taki smutny?" zawodzil cichutko, tkliwie glosem klametu. (FIN: 34)

Najglośniej rozprawial wśród nich Wladek zwany Wampirem..."Wampirek", jak powiadala Zoskka, ostatnia jego kochanica. (NOW: 127)

Baltazarowi wielu zazdroscilo i slusznie. Zostajac leśnikiem nie mial nic, a teraz gospodarstwo, krowy, konie i nie chata a dom...Surkont niczego nie odmawial czegokolwiek poprosil "Baltazarek" i tu już można bylo pokazywać palcem na czolo. (MIL: 28)
Unser Hund hieß Tsching...Der Hund war traurig, das stand außer Zweifel...Sein Blick folgte einer über die Fensterscheibe spazierenden Fliege, und wenn man ihn zärtlich anredete: "Na, Tschingchen, warum bist du so traurig?", winselte er leise und fein mit der Stimme einer Klarinette. (FIN: 32f)

Am lautesten redete Wladek, genannt Vampir..." Vampirchen", wie Zoska, seine letzte Geliebte sagte. (NOW: 80)

Viele beneideten Baltazar, und mit Recht. Als er Förster geworden war, hatte er nichts, und jetzt Landwirtschaft, Kühe, Pferde, und nicht eine Hütte, sondern ein Haus...Surkont schlug nichts ab, worum auch immer das Baltasarchen bat - mit dem Finger auf die Stim hätte man tippen kōnnen! (MIL: 34)

Ähnlich wie beim appellativischen Wortmaterial werden auch die Namenhypokoristika zum Ausdruck der meliorativen bzw. pejorativen Einstellung des Sprechers genutzt, hier ist es die Anzeige von Zuneigung, spöttischer Geringschätzung, Mitleid, Verharmlosung und Ironie. Eine weitere Verwendung der Koseformen, die vornehmlich in der Anrede zu finden sind, zeigen die nächsten Fälle an:

[M:] Fajgele, zlotko moje, czegos ty sie na mnie zawziela? (TON: 2)
[M:] Fajgele, mein Goldchen, was hast's denn so auf mich abgesehen? (TON: 2)

Hier ist es Schmeichelei, ein Werben um die Gunst der angesprochenen Person.

Jeszcze raz zawolala: "Pan nie wie, co oni...oni wczoraj..." Ale i tym razem nie dal jej dokonczyc: "Wiem", powiedzial," "wiem...ja wszystko wiem, dziecinko". (FIN: 31)
Noch einmal rief sie: "Sie wissen ja nicht, was die da gestern, gestern..." Aber auch diesmal ließ er sie nicht ausreden. "Ich weiß", sagte er, "ich weiB alles, Kindchen". (FIN: 95)

Es ist kein Kind, sondern eine junge Frau gemeint, die angesichts der schrecklichen Umstände, die sie erlebt hat, beruhigt und besänftigt werden soll.

W pare dni potem kupowal kwiaty. "Ladne", powiedzial do ogrodnika, "i dużo". Ogrodnik wybaluszyl oczy. "Karolek!!... kwiaty!?"... Nacial pek krwistych peonii. (NOW: 66f)
Ein paar Tage danach kaufte er Blumen. "Schöne", sagte er zum Gärtner, "und viel." Der Gärtner bekam Telleraugen. "Karolek!! ... Blumen?!" ... Schnitt einen Haufen blutigroter Päonien ab. (NOW: 43)

Erstaunen über das ungewöhnliche Verhalten von Karol, Blumen zu verschenken, soll signalisiert werden. 
Boleslaw wstrzasnal sie: "Ta muzyka przerazliwie mnie denerwuje". - "Mnie takze", powiedzial Stas..." Sluchaj, Bolu", powiedzial nagle poważnie... (IWA: 112)
Boleslaw schüttelte sich. "Diese Musik geht mir entsetzlich auf die Nerven." "Mir auch", sagte Stas..."Hör mal, Bolek", sagte er plötzlich ganz ernst... (IWA: 105)

Hier wird ein Diminutiv der Vertrautheit gewählt, um anzuklagen. Zu bemerken ist auch die Wahl des üblicheren hypokoristischen Suffixes -ek in der Übersetzung.

$S D \Rightarrow A D$

Anders als in der vorangegangenen Gruppe hat der Übersetzer im folgenden auf die synthetische Form der Wiedergabe verzichtet. Die Suffixdiminutive wurden aufgelöst in das Syntagma "attributives Adjektiv + Basislexem", wobei klein als Äquivalent des Derivationssuffixes dominiert. Doch beschränkt sich die Semantik von klein keineswegs auf die reine Größenangabe gegebener Objekte, auch die von den Formantien der Vorlage getragenen positiven wie negativen Wertungen - dabei immer im Kontext eingebettet - können vom entsprechenden Attribut angezeigt werden. Folgende Textausschnitte sind anzuführen:

Domcio tymczasem stal na brzegu rzeki i...puszczal $w$ wode kamyki...Falki goniace jedna druga w coraz szerszych kregach kolysaly rozpostartymi liścmi... (MIL: 65)

Zjedli to wszystko i Jola poszla spać do swojej "alkowy", jak nazywala salonik, a Wiktor szedl po schodach do siebie. (IWA: 38)

Szlo sie tam zboczem, potem troche $w$ dó, gdzie stala krynica z żurawiem, $z$ czterema srebrnymi bialodrzewkami, a potem byla brama. (IWA: 54)

Lubil te strony, bylo tu faliscie, zboże rozkladalo sie dużymi lanami $i$ stare wyjeżdzone drogi tworzyly rodzaj wawozikow otoczonych $z$ obu stron bujna trawa... (IWA: 89)

Mogila ogrodzona byla bialym brzozowym piotkiem, bardzo zwyczajnej struktury; po prostu powtykano na krzyż patyki. (IWA: 101)
Indessen stand Dominik am Flußufer und ließ...Steinchen übers Wasser gleiten...Kleine Wellen, eine nach der anderen in immer größeren Kreisen verfolgend, schaukelten die ausgebreiteten Blă̈tter... (MIL: 87)

Dann ging Jola in ihren "Alkoven" schlafen, wie sie den kleinen Salon nannte, und Wiktor stieg die Treppe zu seinem Zimmer hinauf. (IWA: 23)

Man ging den Hang entlang, dann etwas hinunter zu dem Ziehbrunnen mit den vier kleinen Silberpappeln, dort lag der Eingang. (IWA: 41)

Er liebte die Gegend, sie war leicht gewellt. Das Getreide stand in großen Schlägen, und die alten, ausgefahrenen Wege bildeten kleine Schluchten, die zu beiden Seiten von üppigem Gras ...eingerahmt waren. (IWA: 79)

[Der Grabhügel] war von einem kleinen Birkenholzzaun ganz gewöhnlicher Konstruktion umgeben, man hatte ein- 
Po drodze, starannie dobierajac plaskie kamyki puszczalam kaczki na wode, a na mostku, za którym otwieral sie widok na miasto, usiadlam na chwile, spuscilam nogi i dyndajac nimi przygladalam sie swemu odbiciu w wodzie... (FIN: 9)

Aż doszedl do jeziora i spotkal dzikich mieszkariców wyspy. Bo na srodku jeziora byla wysepka, a trzech dzikich czerpalo wode lupina kokosowego orzecha. (KOR: 100)

Pokoik wydawal sie teraz nawet duży, gdy w nim nie bylo ani fortepianu, ani wujowego ciala i Oli zrobilo sie troche nieprzyjemnie. (IWA: 169)

Jedno chuchro jest mężczyzna, drugie kobieta...Kobieta drobila kroczki, pochylona do przodu, jak gdyby za chwile miala upaśc. (FIN: 60)

O swojej Śmierci dowiedzial sie z gazety w miasteczku, przez które przechodzil. (KOR: 124) fach Pflöcke über Kreuz in den Boden gesteckt. (IWA: 94)

Unterwegs sammelte ich flache Kiesel und warf Hüpfsteine ins Wasser, auf der Kleinen Brücke aber, hinter der sich der Blick über die Stadt öffnete, setzte ich mich für eine Weile und lieB die Füße baumeln, während ich mein Spiegelbild im Wasser betrachtete... (FIN: 13)

Endlich kam er an einen See und fand die wilden Bewohner der Insel. Mitten im See lag nämlich eine kleine Insel, und drei Wilde schöpften gerade mit einer Kokosnußschale Wasser. (KOR: 135)

Das relativ kleine Zimmer kam ihr jetzt groß vor, da der Flügel und der Körper ihres Onkels fehlten, und Ola wurde es ein bißchen unheimlich. (IWA: 168)

Das eine Klappergestell ist ein Mann, das andere eine Frau... Die Frau machte ganz kleine Schritte, vornübergeneigt, als würde sie gleich hinfallen. (FIN: 118)

Von seinem eigenen Tod erfuhr er aus der Zeitung, als er durch eine Kleinstadt kam. (KOR: 168)

Man kann die obigen Beispiele durchweg als Diminutive der Verkleinerung ansehen, für die im polnischen die synthetische, im deutschen Text die analytische Bildeweise vorgezogen wurde. Es geht in erster Linie um die Einstufung der Ausmaße hinsichtlich der Kategorie groß-klein. Wiederum läßt sich dabei die Tendenz des Polnischen beobachten, bei Objekten derselben Art, aber verschiedener Größe das kleinere Exemplar, in Opposition zu dem durch das Grundwort benannten, mit dem Diminutiv zu belegen. So bilden die beim Steinchenwerfen entstehenden Wasserbewegungen kleinere, sanftere Wellen als z.B. die durch den Wind aufgeworfenen Meereswellen. Ein schmaler Pfad, der durch ein hohes Getreidefeld führt, ist natürlich in seiner Größenordnung von einer Bergschlucht zu unterscheiden. Ebenso kann die Umzäunung eines Grabes immer nur niedriger als der Zaun am Haus oder Garten sein, oder die Brücke über einen nicht sehr breiten Fluß. (wenige Zeilen weiter heißt es: "marna rzeczulka, osiem metrów szerokosci") immer nur kleiner als die über einen größeren; so wird 
auch eine auf einem See gelegene Insel kein besonders großes Areal bilden usw. Erwähnenswert sind dann noch die Wiedergabe von pokoik, wo die Wortfolge kleines Zimmer, durch das Adverb relativ ergänzt, eine interessante Übersetzungsvariante bietet, sowie die quasi zweifache analytische Diminuierung im vorletzten Beispiel, in dem auch das Adjektiv klein durch das Attribut ganz ergänzt wird, um der ausgangssprachlichen Formulierung, wo mit Verb drobic ('stawiać drobne kroki') die diminutivische Wirkung verstärkt werden soll, gerecht zu werden. Schließlich sei auf die präfixoide Bildung Kleinstadt für miastecako verwiesen, der im Unterschied zum Syntagma bereits eine klassenstiftende Funktion zukommt und damit der oben angesprochenen Eigenart des Polnischen zuzurechnen ist.

Auch im weiteren begegnen in dieser Gruppe Verminderungen, diesmal in bezug auf das Alter (im zweiten Beispiel mit dem Epitheton jung deutlich gemacht):

Dormesko byl spokojny, mama Maciusia spokojna, ten chlopczyk, którego widzial podczas wojny $w$ chacie, spokojny. (KOR: 100)

Tomasz...bral swoj leszczynowy kij - i na polowanie. Sztuka polegala na tym, żeby zbliżyc sie cicho i żeby, sploszone, nie skoczyly za predko w brzezinke. (MIL: 92)
Dormesko war ruhig gewesen, Hänschens Mutter war ruhig, der kleine Junge, den er im Krieg in der Hütte gesehen hatte, war ruhig. (KOR: 136)

Thomas...nahm seinen Haselnußstock und ging auf die Jagd. Die Kunst beruhte darauf, leise heranzukommen, um zu vermeiden, daß die Vipern, aufgescheucht, zu schnell in die jungen Birken entwischten. (MIL: 125)

in bezug auf die Intensität einer Erscheinung:

...miedzy drzewami wilgoc poczela parowac. Mialo byc sloneczne popoludnie. "To tylko taki majowy deszczyk", Ola ciagnela pomalutku watek swych mysli. (IWA: 101)

[M:] Dojechać dojade, żeby tylko było czym. Póńno sie zrobilo. - [S:] Póńno. - [M:]. Trzeba sie zbierac. Ona nie lubi jak sie pónno wraca. Wszystko uslyszy, każdy szmerek...a potem na drugi dzien gadanie... (NUR: 18)

oder in bezug auf Länge bzw. Umfang:

Malwina powtórzyla pierwsza strofke dwa razy, urwala gwaltownie i potem dlugo milczala. (IWA: 165) ...die Feuchtigkeit begann zwischen den Bäumen zu verdunsten. Ein sonniger Nachmittag kündigte sich an. "Es war nur so ein leichter Mairegen." Ola spann langsam den Gedankenfaden fort. (IWA: 93f)

[M:] Das wäre kein Problem...fragt sich nur, womit? - [S:] Ja...es ist schon ziemlich spät geworden. - [M:] Ich muB mich jetzt aufraffen. Sie hat nämlich was gegen spätes Nachhausekommen. Und sie hört alles...das leiseste Geräusch... und dann kommen die Vorhaltungen...am nächsten Tag... (NUR: 22)

Malwina wiederholte die erste kurze Strophe zweimal, brach ab und schwieg lange. (IWA: 163f) 
Entsprechend der Bedeutung des Grundwortes wird das Suffix nicht in kkein, sondern in das jeweils passende Attribut umgewandelt.

Darüberhinaus lassen sich auch bei diesem Wiedergabetyp Belege finden, bei denen das Diminutiv zum Ausdruck meliorativer bzw. pejorativer Einstellungen genutzt wird. Fast durchweg erfüllt klein die jeweiligen Funktionen; klein im Sinne von 'bescheiden', 'ärmlich', 'armselig' steht in den Fällen:

Biureczko pod oknem, pekaty tom Larousse'a, zlocona oprawa "Listów z mojego miyna"...I zapach stechlizny mrocznego pokoju. (FIN: 59)

Podwórko bylo male, czyste, ale smutne. Konie i krowy miescily sie w czerwonym budynku na parterze, na pietrze bylo mieszkanko, w którym odbywala sie operacja. (IWA: 105)

Bracia wybrali $z$ gniazd kurzych kilka jaj. Jeden $z$ nich poszedl do Lejbki (sklepik w sasiednim domu), dostal za nie dwa sledzie... (FOR: 16)
Der kleine Schreibtisch am Fenster, der dicke Band Larousse, die Goldprägung auf den "Lettres de mon moulin". Und der Modergeruch des halbdunklen Zimmers. (FIN: 116)

Das Gehöft klein, sauber, aber traurig. Die Pferde und Kühe standen in einem roten Gebäude im Erdgeschoß, im Oberstock lag die kleine Wohnung, in der die Behandlung durchgeführt wurde... (IWA: 97)

Die Brüder holten ein paar Hühnereier aus den Nestern, einer von ihnen ging zu Lejbka (das war ein kleiner Laden im Nachbarhaus) und tauschte die Eier gegen zwei Heringe ein. (FOR: 22)

In den nächsten Beispielen ist das Epitheton klein gleichzusetzen mit 'unbedeutend', 'nebensächlich', 'unmaßgeblich':

[P:] Znowu mijamy jakas stacyjke. Tutaj też zupelnie pusto. Jakies male, wymarle miasteczko. (RUM: 9)

Dwadzieścia wiorst w kilka godzin to naprawde byl dla niej spacerek, moglaby zrobic chyba sto, tym swoim lekkim chlopskim chodem. (MIL: 81)

Kiedy ludzie nie mieli aeroplanów tylko ptaki mogly fruwac. To dziwne: muszka mogła fruwac, a czlowiek nie. (KOR: 61)

lle tego mieszkalo w jednej prostej, hawajskiej melodyjce, która wydobywal ze starego fortepianu nieskomplikowanymi ruchami palców. (IWA: 147)
[P:] Da ist wieder eine kleine Station. Liegt völlig verlassen da. Muß irgendeine Kleinstadt sein... wie ausgestorben . (RUM: 13)

Zwanzig Werst in einigen Stunden, das war für sie wirklich ein kleiner Spaziergang, sie hätte auch hundert machen können mit dem ihr eigenen leichten Bauernschritt. (MIL: 110)

Als die Menschen noch keine Flugzeuge hatten, da konnten nur die Vögel fliegen. Seltsam: Eine kleine Fliege konnte fliegen, ein Mensch nicht. (KOR: 83)

Was steckte nur alles in einer einzigen, kleinen Hawaiimelodie, wie er sie mit schlichten Fingergriffen aus dem alten Flügel hervorlockte! (IWA: 143) oder mit 'unwichtig' zu umschreiben, weil eine Routineangelegenheit vorliegt:

"Może już gada ze sledczym", rozmyslal Kaczor. "W tym pokoju jest
"Vielleicht spricht er schon mit dem Untersuchungsrichter", überlegte Ka- 
biurko, w kacie zielona szafa. Protokolik. Sledczy zapisuje skwapliwie." (NOW: 11) czor. "In dem Zimmer ist ein Schreibtisch, in der Ecke ein grüner Schrank. Ein kleines Protokoll. Der Untersuchungsrichter schreibt genau mit." (NOW: 13)

oder als 'unwichtig' zu verstehen, weil das Bezeichnete in einer verlassenen, einsamen Gegend gelegen ist:

...przed czterema godzinami odlożylam sluchawke telefonu $w$ malej oberży wysoko w górach, trzeciorzedna miejscowosc $z$ trzeciorzednym lodowcem okrytym zazwyczaj mglami,...jest tu jedna tylko oberża, hotelik pusty... (FIN: 83)
Vor vier Stunden habe ich den Telefonhörer aufgelegt, oben in dem kleinen Berggasthof. Eine Ortschaft dritter Klasse, und ein Gletscher dritter Klasse, gewöhnlich von Nebelschwaden verdeckt,...es gibt hier nur einen Gasthof; das kleine Hotel ist leer... (FIN: 98)

Desweiteren ist der Gebrauch von klein zum Ausdruck von Sympathie bzw. Mitleid zu vermerken:

"Jest niedawno, ale znam ja [dziewczynke] na wskros; jej duszyczka nie ma przede mna tajemnic. Każda jej mysl czytam jak na dloni." (KOR: 33)

"Wstydzilbyś sie, chamie, taki ciężki worek na dzieciaka ladowac." (KOR: 27)
"Sie [das Mādchen] ist noch nicht lange da, aber ich kenne sie schon durch und durch; ihre kleine Seele birgt für mich kein Geheimnis. Ich kann jeden ihrer Gedanken lesen, als hätte ich ihn in der Hand." (KOR: 45)

"Du solltest dich doch schämen, du dummer Kerl, so einem kleinen Jungen diesen schweren Sack aufzuladen." (KOR: 36)

In wertender Funktion begegnen neben klein auch Epitheta, die die Aussage der suffixalen Konstruktion explizit angeben:

[I:] Przestań pan pieprzyć! - [Dy:] Tak nie można...Szkoda mi tego kumpla ze stoczni... - [I:] Pewnie biedaczek nie ma co teraz...żrec. (RUM: 32f)

[I:] Musze powiedziex, że nawet zaimponowala mi pani. - [Dz:] Oo! - [I:] Tak, odwaga $z$ jaka pani wypowiada swoja filozonjkę życiowa. Nie każdego na to stac. - [Dz:] Nie mam sie czego wstydzić. Nie kradne, nie robie żadnym szwindli, uczciwie pracuje... (RUM: 20)
[I:] Hören Sie doch auf mit dem Gefasel! - [D:] Sie sind ungerecht....Mir tut der Kollege von der Werft wirklich leid... - [I:] Der arme Teufel... hat jetzt bestimmt... nichts zu fressen. (RUM: 39)

[I:] Ich muß gestehen, Ihre Haltung hat mich ziemlich beeindruckt. - [J:] Hört, hört! - [I:] Ja, der Mut, mit dem sie Ihre bescheidene Lebensphilosophie verkünden. Das macht Ihnen so bald keiner nach. - [J:] Ich brauch mich wirklich nicht zu schämen. Ich stehle nicht, ich drehe keine krummen Dinger, ich gehe ehrlich meiner Arbeit nach. (RUM: 24f)

Dies kann auch in Verbindung mit einem Hypokoristikum geschehen:

"Nie, ciociu, nich ciocia porzuci wszelkie nadzieje. Ja sie już w Wilku nie ożenie..." (IWA: 63)
"Nein, liebe Tante, gib alle Hoffnung auf. Ich werde niemanden aus Wilko heiraten..." (IWA: 50) 
Analytische Wiedergabe erfuhren auch Adjektive und Adverbien. Lediglich zwei Detensiva sind als Entsprechung zu verzeichnen, vgl.:

Wiewiórka ma zima futerko szarawe, ale nie calkowicie szare. (MIL: 8)

Gdy przyszedl do leśniczówki, było już pónawo. Brat gdzies poszedl. (IWA: 146)
Das Eichhörnchen hat im Winter ein Fellchen, das fast, aber nicht ganz, grau ist. (MIL: 6)

Als er in der Försterei anlangte, war es schon recht spät, sein Bruder fortgegangen. (IWA: 142)

Dem stehen aber mehrere Intensiva mit den beiden attributivischen Wendungen "ganz + Grundwort" bzw. "sehr + Grundwort" gegenüber:

"Leżalam sobie cichutko, czasem uszczknelam korzonek, czasem kes kartofla... woda popilam..." (FIN: 93)

Zbudzilo ja cieniutkie drżenie szyb. Usiadla gwaltownie, calkiem rozbudzona, swiadoma wszystkiego. (FIN: 7)

Leżal bezbronny jak dziecko i jak dziecko nieswiadomy zla, które sie rozpetalo.... Leciutko przesunela dionia po jego wlosach. (FIN: 7)

"Potem podbiegl drugi, jasniutki na wlosach, krzyknal cos po ichniemu, odtracil tamtego i wystrzelil sam." (FIN: 24)

Matka Stefanii, drobniutka kobieta, zapalila wlasnie lampe naftowa - Swiatla w chacie nie bylo... (FIN: 55)

... wlosy dość ubogie, splotla mi jedna z dziewczat $w$ warkoczyk, a ze byl króciutki, to wplotla dluga wstazkke... (FOR: 56)

Macius mieszkal wtedy przez dwa tygodnie w chacie, gdzie byl chlopczyk taki sam jak Ala, maly, i podobnie mówil, ale byl cichutki, siedzial cale dnie kolo pieca i patrzyl, i bardzo rzadko cos powiedzial. (KOR: 81)
"Ich lag da ganz still, manchmal knabberte ich an einer Wurzel, manchmal einen Happen Kartoffel, dazu einen Schluck Wasser..." (FIN: 137)

Ein ganz feines Beben der Fensterscheiben weckte sie. Sie setzte sich sofort auf, war völlig wach und wuBte alles. (FIN: 10)

Er lag da, wehrlos wie ein Kind und wie ein Kind das Böse nicht ahnend, das sich entfesselt hatte...Ganz leicht streichelte ihre Hand Piotr's Haar. (FIN: 10)

"Dann lief ein anderer hin, ganz helles Haar, der rief was in seiner Sprache, schob den ersten zur Seite und scho $B$ selbst." (FIN: 29)

...Stefanias Mutter, eine sehr zarte Frau, zündete gerade die Petroleumlampe an - Strom gab es dort nicht... (FIN: 45)

Ein Mädchen flocht aus meinem recht dünnen Haar einen Zopf, doch weil er sehr kurz wurde, flocht sie ein langes Band hinein. (FOR: 53)

Hänschen wohnte damals zwei Wochen lang in einer Hütte, in der ein ebenso kleiner Junge wie Ala lebte. Er sprach auch ähnlich, aber er war sehr still, saß den ganzen Tag am Ofen und schaute, und sehr selten sagte er etwas. (KOR: 110) 
In statistischer Hinsicht bleibt dieser Wiedergabetyp ohne Relevanz. Denn unter Äquivalenzgesichtspunkten erweist sich die Überbetonung in der deutschen Fassung, in der der Übersetzer den "einfachen" diminutivischen Wert der Vorlage durch den Zusatz eines Quantitätsadjektivs zum Suffixdiminutiv akzentuiert, als redundant, vgl.:

Niestety, zaczynaly sie na mnie walic kamienie: suna powoli ze wszystkich stron, takie ogromne glazy jak ten, który popekal, a jestem tak mala jak ziarenko maku... (FOR: 98)

Ja mam już osiem lat i niańcze dwoje mlodszych - braciszek ma rok i jeszcze nie chodzi... (FOR: 19)

[N:] Zapisana zostala [historia] w starej ksiq̨żeczce, po której już dawno śladu nie zostalo - poprostu sie spalila. (TON: 2)

Królowie powiesili na scianach mapy wszystkich cześci swiata i na wyspach, które im sie podobaly, wkluwali szpilki $z$ choragiewkami. (KOR: 5)
Dann fielen Steine auf mich, von allen Seiten rollten riesengroße Felsbrocken auf mich $\mathrm{zu}$, die unserem geborstenen Mühlstein glichen. Ich war wie ein winziges Mohnkörnchen. (FOR: 93f)

Ich war acht Jahre alt und muBte auf meine beiden jüngeren Geschwister aufpassen. Mein kleines Brïderchen war ein Jahr alt und konnte noch nicht laufen... (FOR: 25)

[E:] Eingeschrieben wurde [die Geschichte] in ein kleines altes Büchlein, von dem schon seit langem jede Spur fehlt - es ist einfach verbrannt. (TON: 1)

Die Könige hingen Landkarten aller Erdteile an die Wände, und auf die Inseln, die ihnen gefielen, steckten sie Nadeln mit kleinen Fähnchen. (KOR: 5f)

$S D \Rightarrow N D:$

Nach der diminutivischen, synthetischen bzw. analytischen, Wiedergabe soll mit vorliegender Formel jene Übersetzungsmöglichkeit erfaßt werden, die auf ein ausgangssprachliches Diminutivderivat mit einem nichtmodifizierten, suffixlosen Grundwort reagiert. Die Fülle der exzerpierten Beispiele macht es erforderlich, das Kürzel ND näher zu bestimmen. Eine Spezifikation zeigt, daß die abgeleitete Einheit in der zielsprachlichen Version häufig nicht lediglich auf das zugrundeliegende Basislexem reduziert wird, sondern andere, nichtdiminutivische Mitel zur Auflōsung der ausgangssprachlichen Vorgabe herangezogen werden. Dementsprechend lassen sich mindestens fünf Umsetzungsweisen feststellen: 


\section{1. $S D \Rightarrow$ Basislexem:}

Die am stärksten vertretene Gruppe bilden die deutschen Wiedergaben mit dem entsprechenden Basislexem. Auch hierzu gehören vorab die Fälle, in denen Suffixdiminutive in nicht emotional-wertender Funktion auftreten. Dazu zählen zum einen Derivate, deren Markierung lediglich formaler Art ist und dementsprechend in der Übertragung weder formal noch semantisch nachzuvollziehen ist, vgl.:

Z podwórka dolecial hałas. Pisk. Smiech. (NOW: 11)

Na placyku przed sklepikiem dzieci obsypywaly sie piachem... (NOW: 131)

Nisia siedziala przy oknie wychodzacym na puste, kamienne podwórze, na jej kolanach leżala czysta sciereczka, na sciereczce stała patelnia. (FIN: 64)

Wioska byla polożona nad rzeczulka, chaty byly $z$ malymi wyjatkami kume... (FOR: 44)

[N:] ... [dziedziczka] zapalala Swieczki przed niezliczonymi świetymi obrazami i znów kleczala, wzdychała i modila sie. (TON: 3 )

...usuwamy slonine, aby skorka bazanta pieknie, lecz niezbyt silnie sie przyrumienila. (LEM: 72)

Po zagotowaniu zupe przecieramy przez sitko $\mathrm{i}$ rozcieńczamy $1 / 2$ I wrzacej wody. (LEM: 25)

Klóca sie i już do bicia sie porwali. A dozorca zapisuje na tabliczce, ile slów powiedzieli...I zapisal Maciusia, choć on nic nie mówil. (KOR: 113)
Vom Hof kam Lärm. Quietschen. Lachen. (NOW: 13)

Auf dem Platz vor dem Lädchen schmissen die Kinder sich mit Sand... (NOW: 84)

Nisia saß am Fenster, das zum leeren, gepflasterten Hof hinausblickte, auf ihren Knien lag ein sauberer Lappen, auf dem Lappen stand eine Pfanne. (FIN: 66)

Das Dorf lag an einem kleinen Fluß, die Häuser waren, von ein paar Ausnahmen abgesehen, elende Hütten... (FOR: 45)

[N:] ... [die Gutsbesitzerin] zündete Kerzen an vor unendlich vielen Heiligenbildern und kniete nieder, seufzte und betete. (TON: 5 )

...den Speck abnehmen, damit die Haut des Fasans schön, jedoch nicht zu stark bräunen kann. (LEM: 76)

Danach die Suppe durch ein Sieb streichen und mit $1 / 2$ I kochendem Wasser verdünnen. (LEM: 28)

Sie stritten sich und wollten schon aufeinander einschlagen. Der Aufseher aber schrieb auf die Tafel, wieviel Wörter jeder gesagt hatte...Und auch Hänschen schrieb er auf, obwohl der gar nichts gesagt hatte. (KOR: 155)

Zum anderen und hauptsächlich aber lassen sich Belege anführen, die der oben schon angesprochenen Einordnung hinsichtlich einer Größeneinstufung, vornehmlich im räumlichen, aber auch wertenden Sinne, dienen:

Miedzy debami, na zboczu, jest cmentarz, a na nim, w kwadracie lańcuchów
Zwischen Eichen, am Hang, liegt der Friedhof, und dort, inmitten von Qua- 
laczacych kamienne shupki, leża przodkowie Tomasza... (MIL: 11)

Na stole dziadka leżalo wiele ksiażek a na obrazkach w nich ogladalo sie korzenie, liscie i kwiaty. (MIL: 15)

Z okienka splynal blady świt. Jeszcze spali. (NOW: 9)

Pogrzebal w kieszeniach. Wyciagnal plaski zloty zegarek. (NOW: 34 )

Czeska biżuteria blyszczala w pudelku, wabiac wzrok. (NOW: 49)

Trzyczwartak pogladzil [wasiki] $z$ namyslem, przysunal do geby lusterko, zadarl nos, wydal usta - obejrzal dokladnie. (NOW: 124)

...poszedl razno, pogwizdujac i machajac teczka, w której dźwigal dwie koszule i szszoteczke do zebów. (IWA: 29)

...nalala mu kieliszek wơdki, odniosla lampke w glab pokoju... (IWA: 129)

Józef wdrapal sie po drabince, przycupnal na walizce $i$ do chwili, gdy szofer zapuścil motor, nie wierzyl, że uda mu sie wyjechac z miasta. (FIN: 27)

...siegnela reka po poduszeczke najeżona szpilkami, zachowana tu $z$ dawnych, krawieckich czasow... (FIN: 65)

Aromat espresso, krzyk neonow, widelczykiem dlubiac ciastko....smiech. (FIN: 84)

Zdyszana stoje przy zielonym plotku, miejskiej zagrodzie, ktora zamyka dziesiex maleńkich stolików, nikt przy nich nie siedzi, zbyt zimno, by kawe popijac na dworze. (FIN: 85) draten, die steinerne mit Ketten verbundene Pfeiler abstecken, liegen Thomas' Ahnen... (MIL: 10)

Auf Großvaters Tisch lagen viele Bücher, und auf ihren Bildern sah man Wurzeln, Blätter und Blumen. (MIL: 16)

Aus dem Fenster floß bleicher Dämmer. Sie schliefen noch. (NOW: 10)

Wühlte in den Taschen. Zog eine flache goldene Uhr heraus. (NOW: 17)

Der böhmische Schmuck glänzte in der Schachtel und lockte den Blick an. (NOW: 25)

Dreiviertel streichelte [den Schnurrbart] nachdenklich, führte den Spiegel an die Schnauze, hob die Nase, blies die Mundpartie auf - er besah sich genau. (NOW: 78)

Er schritt munter aus, pfiff vor sich hin und schwenkte die Tasche, in der er zwei Hemden und seine Zahnbürste trug. (IWA: 14)

Sie schenkte ihm ein Gläschen ein, sie trug die Lampe hinten ins Zimmer... (IWA: 124)

Józef stieg die Leiter hoch und hockte sich auf seinen Koffer; bis zu dem Augenblick, da der Fahrer den Motor anließ, glaubte er nicht, daß es ihm gelingen würde, die Stadt zu verlassen. (FIN: 87)

Sie...griff nach dem mit Nadeln gespickten Kissen, das sich aus früheren Schneiderzeiten erhalten hatte... (FIN: 67)

Das Aroma des Espresso, der Schrei der Neonröhren, ich stochere mit der Gabel im Kuchen herum...Lachen. (FIN: 99)

Außer Atem stehe ich an dem grünen Zaun, der Einfriedung, die zehn Tischchen umschlieBt. Niemand sitzt dort, es ist zu kalt, um draußen Kaffee zu trinken. (FIN: 101) 
Na miejscu komina jest kuchnia, tak zwana angielska. Pewnego razu przywiózl ojciec z miasta majstra, blachy, drzwiczki żelazne...i zaczeto budować. (FOR: 27)

Caly mazurek smarujemy (przy pomocy piorka lub miekkiego pedzelka) surowym íottkiem... (LEM: 232)

Szybko uplywaly Maciusiowi godziny...Zbieral zoledzie, patyki, robil ogródek kolo muru, niby parkan, niby domki z piasku. (KÓR: 18)

Meżczyzna, któremu Józef placil za przejazd - potomek znanej w Z. rodzinie zlodziejaszków i zawadiakow obrzucil go bacznym spojrzeniem... (FIN: 26f)
Dort, wo der alte Herd gestanden hatte, befand sich jetzt ein sogenannter englischer Herd. Vater war eines Tages mit einem Ofensetzer aus der Stadt gekommen, hatte Blech, Ofentüren...gebracht...und mit der Arbeit begonnen. (FOR: 30)

Den ganzen Mazurek mit Hilfe einer Feder oder eines weichen Plattpinsels mit rohem Eigelb bestreichen... (LEM: 237)

Die Zeit verging jetzt schnell...Er sammelte Eicheln und kleine Stöcke, legte sich an der Mauer einen Garten an, errichtete einen Zaun, baute Häuschen aus Sand. (KOR: 24)

Der Mann, bei dem er für die Fahrt bezahlte, Nachkomme einer in $Z$. bekannten Diebes- und Schlägerfamilie, blickte Jozef forschend an... (FIN: 87)

Hier zeigt sich erneut, daß der polnische Text bei von der sog. Normgröße abweichenden Objekten derselben Art zur Unterscheidung diminutivische Bildungen einsetzt. So z.B. zur Kennzeichnung der geringeren Ausmaße eines Grabpfeilers, eines Zellenfensters, eines Handspiegels, einer tragbaren Lampe, einer Stufenleiter am LKW, eines Nadelkissens, einer Umzäunung eines Straßencafés im Unterschied zu einer Säule oder einem Mast, einem Wohnungsfenster, einem Hängespiegel, einer Steh-bzw. Hängelampe usw. Auch im letzten Beispiel soll mit der diminuierten Form ein Straftäter kleinerer Delikte angezeigt werden.

Als zweites sind die Übertragungsbelege solcher Objekte zu nennen, die bereits in einem wertenden Textzusammenhang stehen. Dazu gehört in erster Linie alles, was mit Kindern und Tieren in Verbindung gebracht werden kann, z.B.:

Bor pozbawiony byl $w$ tej stronie poszycia i Stas...widzial, jak bledziutka sukienka Oli migala pomiedzy pniami. (IWA: 98)

Serduszko dziewczynki bilo pod jego palcami, czul je reka i obawial sie, ie jego bije tak samo. (IWA: 129)

Nagle [dzieci] przestaly plakac i naj-
Da der Nadelwald hier frei war von Unterholz, konnte Stas....sehen, wie Olas blaßfarbenes Kleid zwischen den Stämmen hin- und herhuschte. (IWA: 90)

Das Herz des Kindes schlug unter seinen Fingern. Er spürte es mit der Hand und fürchtete, sein eigenes schlüge ebenso. (IWA: 124)

Plötzlich hörten [die Kinder] auf zu weinen, und die Jüngste - drei Jahre! - 
mlodsza - trzy latka! - zawolala: "Tato, tato, chodź do nas!" (FIN: 20)

Rozpoznal nawet dzieciaki gospodarzy, którzy go ukrywali. (FIN: 58)

I Czing szczekal cichutko, ledwo, ledwo merdal ogonkiem, po czym lizal nas $w$ policzki. (FIN: 35 )

...matka przechylajac glowe na ramie także byla podobna do figlamego ptaszka o tuczonej szyjce. (FIN: 61) rief: "Papa! Papa, komm zu uns!" (FIN: 38)

Er erkannte sogar die Kinder der Bauersleute, die ihn versteckt hielten. (FIN: 70f)

Dann bellte Tsching leise, wedelte ganz sachte mit dem Schwanz und leckte uns die Backen. (FIN: 33)

....seine Mutter, die den Kopf zur Seite legte, sah selbst aus wie ein neckischer Vogel mit rundem Hals. (FIN: 119)

Darüberhinaus lassen sich aber auch Muster aus beliebig anderen Bereichen anführen, deren wertenden Charakter bereits der weiter umgebende Kontext vorzeichnet:

Ciala stawaly sie wilgotne od potu. W glowach dudnila pustka. "Piwo jasne, zofte", tesknil szeptem Seta, "z piankq..." (NOW: 95)

"Przez las można isce i isć...Czlowiek sie nie zmeczy. Gdzies pod krzaczkiem odsapnie i dalej..." (NOW: 102)

"Wiedzialem", rzekl ochryple, "wiedzialem, że wrócisz." $\ldots$. Z leżacego na podlodze plaszcza wyciagnal póllitrówke...Napelnili szklaneczki. (NOW: 134)

I nagle, gdy tak rysowal, zachcialo mu sie zobaczyc Julcie, Jole czy Tunie, i wprost od kreslunku wyruszyl znajoma drożyna..., i po dwudziestu minutach wchodzil na ganek dworu wilkowskiego. (IWA: 78f)

[K:] Tyle mojego co tu [na cmentarz] sobie do meża przyjde, na laweczce posiedziec na sloneczku... (NUR: 2)

"Niech nam lord nie zwraca glowy, bierzemy Maciusia na okret handlowy i zjemy razem pożegnalne sniadanko." (KOR: 58)
Die Leiber wurden feucht vor SchweiB. In den Köpfen trommelte die Leere. "Ein helles, goldgelbes Bier", jieperte Seta flüsternd. "Mit Schaum." (NOW: 50)

"Durch einen Wald kann man gehen und gehen...Man wird nicht müde. Irgendwo unter einem Busch verschnauft man, und dann weiter..." (NOW: 56)

"Ich habs gewußt", sagte er heiser. "Ich habs gewuBt, daß du kommst."...Aus dem Mantel, der am Boden lag, holte er eine Halbliterflasche...Sie füllten die Gläser. (NOW: 87f)

Plötzlich, als er so zeichnete, hatte er große Lust, Julcia oder Jola oder Tunia zu sehen, machte sich direkt auf den vertrauten Weg und betrat nach zwanzig Minuten die Vorlaube des Gutshauses von Wilko. (IWA: 68)

[F:] Das einzige, was ich noch vom Leben habe, ist hier auf den Friedhof zu kommen, zu meinem Mann, das bißchen auf der Bank sitzen in der Sonne. (NUR: 2)

"Lord, lassen Sie uns in Ruhe, wir nehmen jetzt Hänschen mit auf das Handelsschiff und essen dort gemeinsam zum Abschied ein Frühstück." (KOR: 79) 
Auch Adjektive bzw. Adverbien und Indefinitpronomen lassen sich in dieser Gruppe finden. Neben vereinzelten Detensiva, z.B.:

...ciemnosć na zboczu gestniala...Rzeka pod nimi przeblyskiwala bladawo. (MIL: 86)

Patrzyl uważnie i w oczach mial ciepły, zlotawy blask. (FIN: 73)

treten besonders Intensiva auf, deren formale Markierung kaum eine semantische Entsprechung hat, vgl.:

Powolutku wsuneli sie $w$ gestwine, w połmrok. (MIL: 93)

$Z$ rozchylonymi ustami wsluchiwal sie w stabiutkie cykanie [zegarka]. (NOW: 34)

"To mamusia tutaj pochowana? Dlaczego nie na cmentarzu?" - "Taki świat", odpowiedziala Ola, "a tutaj bliziutko." (IWA: 102)

O ile drażnily go piosenki hawajskie, które tak cichutko wygrywal Staś,... (IWA: 114)

$Z$ pokoju Boleslawa padlo światlo na zadżdżony las - w jego promieniu widac bylo cieniutkie nitki przelatujacych kropelek... (IWA: 123)

Kiedy pani zawolala, aby sie wynosila za drzwi, dziewczyna uczula sie bardzo szcześliwa, zebrala prędziutko szatki w wezelek... (FOR: 40)

Teraz dodajemy 1 spory peczek drobniutko posiekanego kopru i maly peczek drobno posiekanego szczypiorku. (LEM: 57)

Mase jajeczna natychmiast wylewamy na patelnie $z$ silnie rozgrzanym, leciutko rumianym maslem. (LEM: 105)

Wstaje Maciuś raniutko, ściele lożko, czyści buty, ubranie, ogień zapali, wode zagotuje... (KOR: 141)

Ale lubi staruszke, bo tak wolniutko idzie,...takim dobrym wzrokiem patrzy na Maciusia... (KOR: 141) ...die Dunkelheit auf dem Hang wurde dichter...Bleich glänzte unter ihnen der FluB auf. (MIL: 118)

Seine Augen hatten einen warmen, goldenen Glanz. (FIN: 150)

Langsam schoben sie sich ins Dikkicht, ins Halbdunkel. (MIL: 128)

Mit offenem Mund lauschte er dem schwachen Ticken [der Uhr]. (NOW: 18)

Hier ist deine Mutti begraben? Warum nicht auf dem Friedhof? - "Der ist so weit weg", antwortete Ola, "hier aber haben wir's nahe." (IWA: 94)

So sehr ihn die Hawailieder reizten, die Staś leise spielte,... (IWA: 107)

Aus Boleslaws Zimmer fiel Licht in den verregneten Wald, in seinem Umkreis sah man die feinen Fäden der fallenden Tropfen... (IWA: 117)

Als die junge Frau diese Worte vernahm, war sie glücklich. Sie packte eilig ihre Sachen... (FOR: 40)

Nun ein Bund feingehackten Dill und ein kleines Bund feingehackten Schnittlauch zugeben. (LEM: 63)

Die Eimasse sofort in die stark erhitzte Pfanne mit der leicht gebräunten Butter giessen. (LEM: 107)

Hänschen steht früh auf, macht sein Bett, putzt die Schuhe, bürstet seinen Anzug aus, macht Feuer, setzt Wasser auf... (KOR: 192)

Aber er liebt die Alte, weil sie so langsam geht,...Hänschen freundlich anschaut... (KOR: 193) 
Gdyby mlody król powiedzial wszystko, co mysli, i gdybym ja powiedzial wszyściutko, co myśle - moze nie bylibysmy wrogami. (KOR: 93)
Wenn der junge König alles sagen würde, was er denkt, und wenn auch ich alles sagen würde, was ich denke, vielleicht wären wir dann keine Feinde. (KOR: 127)

Erwähnenswert sind dann zwei weitere Textstellen, in denen die diminutivische Kennzeichnung des Adjektivs in kompensatorischer Weise auf das dazugehörige Substantiv übertragen wurde:

Przymknal oczy okolone promieniami malutkich i ostrych zmarszczek. (NOW: 54f)

Dzieciak spal. Twarz mial maleńkq i czerwona... (NOW: 109)
Er kniff die Augen zu. Strahlen kleiner und scharfer Faltchen umrandeten sie. (NOW: 31)

Der Säugling schlief. Hatte ein kleines, rotes Gesichichen... (NOW: 63).

Eine Wiedergabe mit dem vorgegebenen Grundwort haben auch Anthroponyme erfahren, sowohl einige Vornamen:

Napisala Irenka, ze lalka do sufitu stlukła sie. (KOR: 111)

Aż raz Macius znalazl...taki list: "Kochany królu,... Wiec ci dziekuje. Zosia." (KOR: 141f)

als auch Verwandtschaftsbezeichnungen:

Na poczatku zimy przyjechala z Dorpatu w Estonii babka Dilbinowa,...stanowila w tym przeciwiensto babci Misi, ze troszczyla sie o wszystko... (MIL: 48)

Chlopak podszedl do wujka. (NOW: 136)

W milczeniu [mala Ola] dygnela przed stryjaszkiem. (IWA: 98)

Wiktor siedzial $w$ powozie z Julcia, jej córeczkami i Tunia. (IWA: 74)

Mam dwoje dzieci: synek ma pieć lat... (FOR: 72)
Irene schrieb, die Puppe, die bis zur Decke reichte, sei ihr entzwei gegangen. (KOR: 151)

Bis Hänschen eines Tages...einen Brief fand: "Lieber König!...Darum danke ich dir. Sophie." (KOR: 193)

Wintersanfang kam aus Dorpat in Estland die GroBmutter Dilbin angefahren...und kümmerte sich, im Gegensatz zu Großmutter Misia, um alles... (MIL: 63)

Der Bursche trat an den Onkel heran. (NOW: 89)

Schweigend knickste [die kleine Ola] vor ihrem Onkel. (IWA: 90)

Wiktor saß in dem Wagen mit Julcia, ihren Töchtern und Tunia. (IWA: 62)

Ich hatte zwei Kinder. Der Sohn war fünf... (FOR: 67)

Dasselbe gilt auch für diese Fälle in der Anrede:

...spojrzal [doktor] Wiktorowi w oczy:

"Wiktorku", powiedzial (doktor do wszystkich mowil "ty")... (IWA: 26)

...zaprotestowalam cicho: Agasiu, nie moge tego sluchac...jestem chora... (FIN: 22) ...blickte [der Doktor] Wiktor in die Augen. "Wiktor", sagte er (der Doktor sprach alle mit Vormamen an)... (IWA: 10)

...als ich...leise protestierte: "Agaria, ich kann das nicht hören. Ich bin krank." (FIN: 27) 
"Cicho, Ludeczku, jak możesz. Nie badź taki niewychowany." (FIN: 60)

Ale ona powiedziala: Paulinko, musisz uciekac. U nas bedzie rewizja. (FIN: 98)

[K:] Nie ma co sie unosic, pani Sabinko. Tyle jej, co tu sobie kwiatki posadzi. (NUR: 3)

Za każdym pytaniem wuja Wiktor patrzyl na niego przez chwile..." A tak, tak, prosze wujaszka...", mówil... (IWA: 44)

"Nie zostawie ciebie, mateczko, w wiezieniu; razem uciekniemy." (KOR: 15)
"Still, Ludek, wie kannst du nur. Sei nicht ungezogen." (FIN: 119)

Sie aber sagte: "Paulina, du muBt fliehen. Bei uns gibt es Durchsuchung." (FIN: 83)

[F:] Regen Sie sich nich auf, Frau Sabine. Die hat ja auch nicht mehr davon als BlumengieBen und aus. (NUR: 3)

Nach jeder Frage schaute Wiktor seinen Onkel eine Weile...an..."Ach so, Onkel", sagte er... (IWA: 30 )

"Aber ich lasse dich nicht im Gefängnis, Mutter, wir flüchten zusammen." (KOR: 20)

Der deutsche Zieltext ignoriert die hypokoristische Vorgabe und greift stattdessen vornehmlich auf die Namensform in polnischer Lautung zurück.

\section{2. $S D \Rightarrow$ Basislexem + Kontextaussage:}

Die Fallbeispiele der vorherigen Gruppe können (mit wenigen Ausnahmen) auch so gedeutet werden, daß der diminutivische Inhalt des jeweiligen Derivats aus dem umgebenden Textzusammenhang bereits implizit zu erschließen ist. Dies gilt für Diminutive sowohl in quantitativer Hinsicht, wenn z.B. vom Fenster einer Gefängniszelle, das immer relativ klein sein wird, die Rede ist, oder in qualitativer Hinsicht, am deutlichsten bei Wendungen in den Bereichen Kind bzw. Tier, wo die positive Wertung schon der Textumgebung zu entnehmen ist. Diese mitenthaltenen Informationen scheinen dem deutschen Text zu genügen, so daß auf eine Diminuierung verzichtet werden kann.

Im Gegensatz dazu zeichnen sich die in der nun folgenden Gruppe zusammengefaßten Belege dadurch aus, daß die diminutivische Aussage im Ausgangstext auch explizit Ausdruck findet. Hierbei gibt sich die deutsche Version erst recht mit der ausdrücklichen, beschreibenden Formulierung zufrieden, ohne den Inhalt nochmals in komprimierter Form (diminutivisch) anzugeben. Häufig läßt sich beobachten, daß bei Substantivdiminutiven des Polnischen attributivische Ergänzungen im unmittelbaren bzw. mittelbaren Kontext die explizite Information tragen:

Galus potarl policzki. Zachrześcil zarost....Kaczor spojrzal naŕ podejrzliwie
Galus rieb die Wangen. Der Bartwuchs knirschte...Kaczor blickte ihn argwöh- 
i też potarl policzki. Mial zarost nikty puszek prawie. (NOW: 10)

Piach żólty i sypki, prawie mazowiecki, zsypal sie z kó [wozu] z leciutkim szmerem, bylo dosć goraco i pot splywal po ciele i karku Stanislawa...; patrzyl bezmyslnie przed siebie i czul jak gdyby szmerek piasku w swoim ciele. (IWA: 109)

Stas wiedzial jedno tylko, że nad stajnia jest okienko, które powinno byc teraz oswietlone, okienko male, prawie kwadratowe, z czterema dużymi szybami. (IWA: 127)

Wtedy sie jeszcze nie balam i moja siostra też nie - zapomnialam dodać, że szla ze mna moja mlodsza siostra i ona też puszczala kaczki na wode i dyndala nogami nad rzeka,...marna rzeczulka, osiem metrów szerokości - ..., dopiero kiedy poszlyśmy dalej ulica za mostkiem..., dopiero wtedy stanelyśmy nagle... (FIN: 9)

Nie bylo to pierwsze jego schronienie... Lato trwalo przepiekne, laskawe dla żniw, upalne. Na stryszku bylo duszno, w kilku skapych metrach kwadratowych unosil sie kurz suchej slomy i czlowiek zrobil sobie niewielka szpare w zewnetrznej ścianie stodoly. Przez te szpare mógl jednym okjem ogarnac skrawek swiata: lake przed zagroda chlopska i pasemko szosy. (FIN: 57)

Niech pani tu przyjedzie, powinnam byla powiedziex, siadziemy na lawce... przy wielkim, nieksztaltnym chlopskim stole..."Nie lepiej w kawiarni, na miescie...i to w jakiejs matej, na boku." "Co to znaczy na boku?" spytala. "Żeby bylo cicho i malo ludzi." - "No, dobrze, w kawiarence Bel nad jeziorem, tam o tej porze roku jest pusto..." (FIN: 83)

A potem nie pamietal Macius, który jest grób kanarka, wiec wszedzie krzy- nisch an und rieb sich auch die Wangen. Er hatte geringen Bartwuchs - fast Flaum. (NOW: 12)

Der gelbe lockere, beinahe masowische Sand fiel mit einem leichten Gerausch von den Rädern [des Wagens], es war heiB, der SchweiB rann Stanislaw vom Nacken den Rücken hinunter...Er blickte gedankenlos vor sich hin und empfand das Geräusch des Sandes gewissermaßen im eigenen Leib. (IWA: 102)

Stas wuBte nur, daß es über dem Stall das Fenster gab, das jetzt erleuchtet sein muBte, das kleine, fast quadratische Fenster mit den vier Scheiben. (IWA: 122)

Damals hatte ich noch keine Angst, und auch meine Schwester nicht, ich habe vergessen zu sagen, daB meine jüngere Schwester mit mir gegangen war und ebenfalls Hüpfsteine ins Wasser geworfen und mit den Beinen über dem FluB gebaumelt hatte - ...ein kammerliches Flaßchen, acht Meter breit -....erst als wir die Straße jenseits der Brücke entlangliefen..., erst dann blieben wir stehen... (FIN: 13)

Es war nicht sein erstes [Versteck]... Der Sommer blieb schön, der Emte gnädig, heiß. Auf dem Scheunenboden war es stickig, über den karglichen Quadratmetern wirbelte trockener Strohstaub. Der Mann hatte sich eine schmale Ritze in die Außenwand gemacht; durch sie konnte er mit einem Auge ein Stück Welt erfassen: die Wiese vor dem Bauernhof und einen Streifen Chaussee. (FIN: 69)

Kommen Sie her, hätte ich sagen sollen, wir können auf der Bank...an dem großen, ungeschlachten Bauerntisch sitzen..." Nein, besser in einem Café, in der Stadt...und zwar in einem kleinen, irgendwo abseits." - "Was heiBt abseits?" fragte sie. - "Still und mit wenig Menschen." - "Na gut, dann im Café 'Bel' am See, dort ist es um diese Jahreszeit leer." (FIN: 98f)

Später wußte Hänschen nicht mehr, welches Grab dem Kanarienvogel ge- 
zyki male wkopal...Malenkie byly mogilki jak dla czterech ptaszkow, ale wszystko, jak patrzec z daleka, wydaje sie male. (KOR: 84) hörte, darum grub er überall kleine Kreuze ein...Klein waren die Gräber, so wie für vier Vögel, aber von weitem sieht alles klein aus... (KOR: 115)

Während die genannten Auszüge auf die Kleinheit abheben, zeigen die folgenden eine im Kontext explizierte Intensivierung der Aussage, sei es in positiver oder negativer Form, an:

Już teraz nie bedzie sie nudzil...Bedzie mial strasznie dużo pracy. Musi dokładnie obejrzex ogród więzienny, kazdy zalamek muru, każde przy murze drzewko. (KOR: 15)

Zerknal na butelke...Podali mu butelke. Podziekowal. "Od rana nic", wyjaśnil chuchajac w garsc, "nawet kropelki." (NOW: 118f)

Fele polożono opodal w kacie, przy zbiegu dwoch murow... Nad grobem stal krzyż brzozowy otoczony sztachetami, ale mogilka byla zaniedbana. Wiktor popatrzył na ten wzgórek, na którym nie bylo ani jednego kwiatka... (IWA: 54)
Und nun wird er sich auch nicht mehr langweilen... Nun hat er schrecklich viel Arbeit. Er muß sich den Gefängnisgarten ganz genau ansehen, jeden Mauerwinkel, jeden Baum an der Mauer. (KOR: 19)

Er linste auf die Flasche...Sie gaben ihm die Flasche. Er dankte. "Seit heute früh noch nichts", erklärte er und blies sich in die Hände. "Nicht mal einen Tropfen." (NOW: 72)

Fela hatte man unweit bestattet, in der Ecke, die hier zwei...Mauern bildeten. Zu Häupten des Grabes erhob sich ein mit Staketen umgebenes Birkenkreuz, aber der Hügel selbst war vernachlassigt. Wiktor betrachtete diesen Hügel, auf dem es keine einzige Blume gab... (IWA: 41)

Schließlich begegnet neben dem in der ausgangssprachlichen Version diminutivisch ausgedrückten Segment auch eine zusätzliche kontextuelle qualitative Angabe des Gemeinten, vgl.:

"Gdy wszystko skonczy sie dobrze ...wyjade ze starym, na pewno wyjade daleko $z$ miasta. To ma być mała wies, gleboko w lasach, koniecznie sosnowych..." Lasy sosnowe to lepsze niż wszystko dla takich jak jego stary. Beda chodzic po tym lesie co dzień. Wynajda sobie zaciszna polanke. I tam wyciagna sie w zielonej trawie na dlugie godziny. (NOW: 100)

Wtedy przyszla Jola, jeszcze nie ubrana, w bialym stroju porannym, pelnym zapachów i koronek. Byla śliczna i uśmiechnieta, zupelnie swobodna i nawet zaraz zaczela mówic Wiktorowi o dniu wczorajszym...Ale Wiktor potraktowal od razu jej przeprosiny naj-
"Wenn alles gut ausgeht...fahre ich mit dem Alten weg, ganz bestimmt weit weg von der Stadt. Ein kleines Dorf muß es sein, tief in den Wäldern versteckt, Kiefernwälder jedenfalls..." Kiefernwälder sind das allerbeste für so Leute wie seinen Alten. Sie werden täglich durch diesen Wald gehen. Werden eine stille Lichtung finden. Und sich dort im grünen Gras für lange Stunden ausstrecken. (NOW: 55)

Da kam Jola, noch nicht fertig angekleidet, in weißem Spitzenneglige und in einer Duftwolke. Sie war hübsch und heiter und ganz ungezwungen, sie fing sogleich an, vom vorigen Tag zu reden...Wiktor aber quittierte ihre Abbitte mit einem schroffen "Nicht der Rede 
bardziej radykalnym "szkoda gadac"... Zobaczyl, iż delikatna i ladna kobiecina przeploszyla sie... (IWA: 85)

Spostrzeglszy porcelanowa lede z labedziem osobnik $w$ mundurze... reka... wskazal porcelanowy kicz. Jozef podal go uslużnie, po czym otworzyl szuflade i wyjal z niej kilka podobnych kiczow z porcelany i brazu. Byly to nedzne resz$k i$ sklepiku zmarlej ciotki i jedynie przez pamiex o niej Jozef nie wyrzucil ich na smietnik. (FIN: 28)

[M:] Wspaniale grzybki. Już nie pamietam, kiedy takie kosztowalem... (NUR: 18)

Lubi Macius lowic ryby. A tu nagle tal mu sie zrobilo zlapanej rybki. Niechby siedziala w morzu. Dlaczego ja krzywdzi? A gdyby Maciusia zlapala ryba na haczyk i wciagnela do morza? (KOR: 65)

"O, teraz kanarkowi zmieni sie wode, nasypie piasku swieżego, bedzie znów czysto. Teraz da sie kanarkowi swieza salatke." (KOR: 80 )

Mala Ola przyszla z glebi domu... Dźwigała w reku lalke, dosć oskubana ..."Boże! Jakaż ogromna!", zawolal Stanislaw..."Ale lalke ma straszna! Widzialem takie sliczne lalki za granica. Zapomnialem jej przywiezt. To doprawdy nieczuly ze mnie stryjcio!" (IWA: 98)
wert"...Er sah, wie die feinfuhlige habsche Frau erschrak... (IWA: 75)

Als der Uniformierte eine Leda mit Schwan erblickte,...wies [er] mit der Hand...auf den Kitsch aus Porzellan. Józef reichte ihm das Stück bereitwillig, offnete dann eine Schublade und holte ähnlichen Kitsch aus Porzellan und Bronze hervor. Es waren die kammerlichen Reste des Ladens seiner verstorbenen Tante. Nur im Gedenken an sie hatte Jozef das Zeug nicht auf den Müllhaufen geworfen. (FIN: 89)

[M:] Phantastisch, diese Pilze! Kann mich nicht mehr erinnern, wann ich zum letzten Mal solche Pilze gegessen habe... (NUR: 22)

Hänschen geht gem angeln. Und dann tut ihm plötzlich wieder der gefangene Fisch leid. Wäre er doch lieber in der See geblieben. Warum hat er ihm weh getan? Und wenn nun so ein Fisch käme, Hänschen auf den Angelhaken spießte und ins Meer zöge? (KOR: 89)

" $\mathrm{O}$, jetzt bekommt mein Kanarienvogel frisches Wasser, sauberen Sand. Gleich ist alles wieder sauber. Und jetzt bekommt er frischen Salat." (KOR: 109)

Aus dem Inneren des Hauses kam die kleine Ola...In der Hand trug sie eine arg zerrupfte Puppe..." Gott, was ist sie gro $B$ geworden! "rief Stanislaw..." Aber die Puppe ist schrecklich! Ich habe im Ausland so hübsche Puppen gesehen, leider aber vergessen, ihr eine mitzubringen. Ja, ich bin wirklich kein liebewoller Onkel!" (IWA: 90)

In allen Fällen ist sowohl den die Diminutive begleitenden Attributen als auch der näheren Textumgebung die Einstellung und Intention des Sprechers zu entnehmen, so daß die deutsche Fassung die Sehnsucht nach der Freiheit, das Begehren einer Frau, die Schilderung eines ärmlichen und bescheidenen Geschäftes, das Lob der Speise, das Mitleid mit dem Tier und die Sorge um es sowie die Selbstkritik mit dem Grundwort allein ausreichend zum Ausdruck bringen kann.

Für die Weglassung diminutivischer Adjektive bzw. Adverbien gilt nun, daß der Modifikationsgehalt entweder von den ihnen zugeordneten Substantiven 
(meist bei Vergleichen) oder seltener von anderen, die Diminutive erläuternden adjektivischen Bildungen getragen wird:

Stojac już w nocy, w mokrym zapachu lasu, podniósi ja na rece, leciutka byla jak ptaszek, przycisnal do siebie... (IWA: 130)

"Wyszlam wiec powoli na szose, te tutaj..., prosciutka, biala jak wyprasowana wstatka." (FIN: 99)

Wyszukal Macius ladne, równiutkie miejsce pod drzewem na samym szczycie gory... (KOR: 84)
Draußen in der Nacht, im feuchten Waldgeruch hob er sie auf den Arm sie war leicht wie ein Vogelchen -, drückte sie an sich... (IWA: 125)

"Langsam ging ich hinaus auf die Landstraße, auf die da[,]...dieses gerade, weiße, wie gebugelte Band." (FIN: 84)

Hänschen suchte sich eine häbsche, ebene Stelle unter einem Baum ganz oben auf dem Gipfel des Berges... (KOR: 114).

\section{3. $S D \Rightarrow$ Synonymlexem:}

Eine weitere Möglichkeit, ein diminutivisches Element des Ausgangstextes zu kompensieren, besteht in der Wahl eines synonymen Lexems, das bereits in seiner nichtmodifizierten Form den diminutivischen Inhalt in sich trägt:

Ten dom z kolei na górce, z ganku widok na nieduże jezioro... (MIL: 88)

W malym brzozowym gaiku przy drózce do strumienia drzewa rosly gesto i ziemie zascielala warstwa suchych liści... (MIL: 91)

Na sasiednim polu pasl konie syn naszego sasiada...Zaczelam wyrywac zielsko z pszenicy, kiedy chlopak podszedl do drózki oddzielajacej nasze pole... (FOR: 69)

Owszem, rozmawiali o potocznych rzeczach, jezdzili nawet razem na spacery, zachodzil do niej po ksiażki, znajdowali sie bardzo czesto sami w osobnych pokojach, w lesie, na l6dce... (IWA: 39)

Po drodze, starannie dobierajac plaskie kamyki puszczalam kaczki na wode... (FIN: 9)

"Wiec w ostatnim stadium getto zweżone zostalo do jednej uliczki?" (FIN: 100)
Das Haus auf einem Hügel schaut zu einem kleinen See hinunter... (MIL: 120)

In einem kleinen Birkenwäldchen am Pfad, der zum Bache führte, wuchsen die Bäume dicht, und die Erde war mit einer Schicht dürrer Blätter bedeckt... (MIL: 123)

Auf dem Nebenfeld hūtete der Sohn unseres Nachbarn die Pferde...Als ich das Unkraut aus dem Weizen zu ziehen begann, kam er bis an den Feldrain, der unsere Felder trennte... (FOR: 64)

O ja, sie unterhielten sich über Alltägliches, sie ritten zusammen aus, er kam zu ihr, um Bücher zu holen, sie befanden sich oft allein in einem Zimmer, im Wald, im Kahn... (IWA: 25)

Unterwegs sammelte ich flache Kiesel und warf Hüpfsteine ins Wasser... (FIN: 13)

"Also war das Ghetto im letzten Stadium auf diese eine Gasse beschränkt?" (FIN: 105) 
W bramie, na murze, wisiala tablica, na której byly wypisane nazwiska mieszkancow kamienicy; u spodu widniala karteczka z napisem: "Sutereny i różne mieszkania do wynajecia". (FOR: 109)

Przyslano lalki bez glów, harmonijki, które nie graly, zapisane kajety, polamane szczotki do zebów,...skórzane paski do lyżew...; bylo z tym wiele klopotu, ale dzieci sie cieszyly. (KOR: 110)

Leży Macius w szpitalu...Jest bardzo slaby. Znow zasnal - znow sie przebudzil: "Prosze przyniesc moje pamiatki." Pedzi samochód do izdebki Maciusia. (KOR: 146)

...na pietrze bylo mieszkanko...Dwa pokoje strzelca i pokoik chlopakow. (IWA: 105)
Im Torweg hing an der Wand eine Tafel, auf der die Namen der Bewohner standen. Darunter klebte ein Zettel mit der Aufschrift: "Wohnungen zu vermieten, auch Kellerwohnungen". (FOR: 103)

Es kamen Puppen ohne Köpfe, Mundharmonikas, auf denen man nicht mehr spielen konnte, vollgeschriebene Hefte, zerbrochene Zahnbürsten,... Lederriemen für Schlittschuhe...; man hatte viel Scherereien mit diesem Kram, aber die Kinder freuten sich doch. (KOR: 149)

Hänschen liegt im Krankenhaus....Er ist sehr schwach, schläft ein, erwacht wieder und bittet: "Bitte bringen Sie mir meine Andenken." Ein Auto rast zu Hänschens Mansarde. (KOR: 198)

...im Oberstock lag die kleine Wohnung....mit den beiden Räumen des Waldhüters und dem Zimmer der Jungen. (IWA: 97)

Die deutschen Entsprechungen führen trefflich die Bandbreite des semantischen Gehalts der Wortbildungskonstruktion "Basislexem + Diminutivsuffix" vor Augen. Die neu gewählten Lexeme, die Simplizia, bringen direkt eine genauere Spezifizierung mit sich, die bei den Derivaten aber erst dechiffriert werden muß: so meint z.B. Hügel (=górka) eine kleinere, aber eben auch sanft ansteigende Bodenerhebung; Pfad bzw. Feldrain (=dróżka) einen schmalen Weg, einen Wegstreifen; Kahn (=l6dka) ein kleines und flaches Ruderboot; Kiesel (=kamyk) einen kleinen abgerundeten Stein oder Mansarde (=izdebka) ein engeres Dachgeschoßzimmer. Besonders deutlich wird das unterschiedliche Vorgehen beider Sprachen im letzten Textbeispiel, während das Polnische den Größenunterschied mit Hilfe morphologischer Mittel anzeigt (pokбj - pokoik), bemüht das Deutsche für diese Aussage zwei bedeutungsähnliche Lexeme (Raum Zimmer).

Auch Diminutive in qualitativer Funktion erfahren im Zieltext eine Wiedergabe durch Synonyme:

...dopóty krecil sie, aż jeden $z$ bylych żolnierzy pozwolil mu używać od czasu do czasu swego karabinu. Zreszta stanowilo to zaplate za milczenie o pewnych sprawkach. (MIL: 62) ...er war so lange herumgerutscht, bis einer der früheren Soldaten ihm erlaubte, von Zeit zu Zeit seinen Karabiner zu benutzen. Übrigens machte das den Lohn für sein Schweigen über verschiedene Afraren aus. (MIL: 83) 
Nie przestawal nucic ani na chwile modnych piosenek europejskich, wiatr zawial inny od tych melodyj. (IWA: 99)

...za rowem ciagnely sie malutkie poletka, za poletkami w gaszczu wisniowych drzew stala zapadnieta chatka starej Maryjki... (IWA: 115)
Er summte ununterbrochen modeme europäische Schlager, und aus diesen Melodien wehte ein anderer Wind. (IWA: 91)

...hinter dem [Graben erstreckten] sich kleine Felder..., und hinter den Feldern stand im Laubwerk der Kirschbäume die Kate der alten Maryjka... (IWA: 108)

Mit Affäre soll hier ein kleinerer, unangenehmer Vorfall ausgedrückt werden, mit Schlager ein leicht eingängiges, nicht anspruchsvolles Lied und mit Kate ein kleines, ärmliches Holzhaus. Bei der Angabe von Zuneigung und Mitleid hat sich der Zieltext für folgende Lexeme entschieden:

[S:] Dziecko jeszcze male, dwóch lat nie ma. Raz nawet jej przypilnowalam malutkiej, jak miala egzamin. To potem zapraszala, żebym zachodzila. I że dziewuszka mnie polubila... (NUR: 1)

"Daj mu teraz spokój", powiada żona. "Widzisz przecie: dzieciak ledwo zipie. Niech sie wyspi. Masz czas jutro przepytac." (KOR: 26)

Rzeczywiście nasze źrebieta przeistaczaly sie w konie...Mysl, aby jednego zostawic do pracy u siebie, dawno zarzucilismy - bedzie mąz dalej odrabiac za obróbke ziemi, a nasze koniki pojda na dlug. (FOR: 86)
[S:] Das Kind ist noch ganz klein. Einmal hab ich sogar Babysitter bei der gespielt, als sie zur Prüfung mußte. Sie hat mich wieder zu sich eingeladen, ich sollte ruhig mal vorbeischauen, die Kleine hätte mich auch liebgewonnen... (NUR: 1)

"Laß ihn jetzt in Frieden", sagt die Wursthändlerin, "du siehst doch, der Kleine kann ja kaum noch piepsen. Soll er sich erst mal ausschlafen. Morgen kannst du ihn dann ausfragen." (KOR: 36)

Und wirklich, unsere beiden Fohlen wuchsen zu prächtigen Pferden heran...Den Gedanken, eines der Pferde für uns zu behalten, hatten wir schon lange verworfen. Mein Mann mußte eben weiterhin arbeiten gehen....Bei dem Gedanken, daß die beiden Fohlen unsere Schulden tilgen würden... (FOR: 81)

Einen interessanten Ausschnitt dieser Gruppe bilden die Beispiele für die Übertragung des Derivats kawalek, das kontextlos, als lexikalische Einheit, recht undifferenziert, dementsprechend beliebig einsetzbar ist und vom deutschen Text wie folgt spezifiziert werden kann:

...rozleg! sie w sypialni krzyk i Monkiewicz ukazal sie na progu $w$ dlugiej nocnej koszuli rozdartej w kilku miejscach, tak, ze plótno zwisalo kawalkami. (MIL: 44)

[F:] Motel? Co zrobimy bez ciebie? Z powodu kawalka wasa morduje sie ojca ...[es] ertönte im Schlafzimmer ein Schrei, und Monkiewicz erschien auf der Schwelle in einem langen Nachtgewand, das an mehreren Stellen so zerrissen war, daß das Leinen in Fetzen herunterhing. (MIL: 57)

[F:] Motel? Was sollen wir ohne dich tun? Wegen einer Schnurrbartspitze 
dzieciom? Morderca! Przeklinam cie! (TON: 11)

"Rekinów kolo wyspy nie ma. Wiec phynelismy pod woda, tylko kawalek trzciny wystawal. A przez dziurke w trzcinie oddychalismy." (KOR: 104) mordet man den Vater den Kindern weg? Mörder!!! Ich verfluche dich! (TON: 20)

"Haie gab es bei der Insel nicht, so sind wir denn unter Wasser geschwommen, nur ein Ende Schilfrohr ragte heraus. Durch das Loch im Rohr haben wir geatmet." (KOR: 141)

Nennenswert sind auch die Textstellen, in denen das Deutsche mit einem Verbalabstraktum reagiert, vgl.:

A ile bylo bójek po szkolach, trudno zliczyc. (KOR: 27)

I bojki ich byly dziwne. Pobija sie, pokrwawia, a nie ma w nich zlosci... (KOR: 116)

Na luki wybieral leszczyny... Wpelzal w cień pod krzakami, gdzie nie ma trawy tylko splatane poklady suszu...Leszczyna pnie sie tam do góry żeby wyjrzec na swiatlo i wtody, zupelnie prosta, bez gałzek, nadaje sie najlepiej. (MIL: 47)
Und wie oft es in den Schulen zu Prügeleien kam, das ließ sich kaum zählen. (KOR: 37)

Auch ihre Schlägereien waren seltsam. Sie schlugen sich gegenseitig blutig, und dabei waren sie gar nicht zornig... (KOR: 158)

Zum Bogen suchte er sich Haselnußzweige aus...Er kroch in den Schatten unter die Sträucher, wo es kein Gras, nur Schichten trockener Blätter gab... Die Haselnußzweige, die von dort zum Licht streben, sind dann ganz stark, ohne Verästelungen, und so eignen sie sich am besten. (MIL: 61)

Zum einen kann das Suffix -(er)ei, zum anderen -ung mit der morphologischen Basis sich verästeln ('sich in viele immer dünner werdende Zweige teilen') die Aufgabe übernehmen, die diminutivische Aussage zu kennzeichnen.

Belege lassen sich ebenso für expressive Personenbezeichnungen anführen:

"Leca lata", powiedzial ze zloscia Tapeta, "cholemie leca...Kiedys też bylem kozak...a ty szczawik, synek jeszcze." (NOW: 54f)

...[Kwadratowy] znalazl sie za brama wiezienia... Wpuszczajacy straznik przymrużyl wyblakle oko i mruknal: "Wpadles, bratku." Każdemu to mowil. (NOW: 140)
"Die Jahre fliegen", sagte Tapete böse. "Ganz verdammt fliegen sie...Früher war ich auch mal ein Dragoner...Und du ein Dachs, noch ein Hosenmatz." (NOW: 31)

...[Quadratisch] war...nun hinter dem Gefängnistor...Der Wachthabende zwinkerte mit ausgebleichtem Blick und brummte: "Geschnappt, Bursche." Das sagte er jedem. (NOW: 93)

Bei Adjektiven begegnen lediglich vereinzelt Wendungen mit ausgetauschtem Grundwort. Bei den folgenden Beispielen ist auch interessant zu beobachten, wie das Deutsche auf die drei unterschiedlichen Suffixe des Adjektivs maly immer nur mit ein und demselben Lexem reagiert: 
...malenkie pojazdy wlokly za soba kurz po drugiej stronie doliny. (MIL: 46)

Szli powoli...Spocil sie, choc bylo chlodnawo. (NOW: 69)

...okno [suterenki] - byly to cztery malutkie szybki, które tylko polowa wychodzily nad ziemie podworka. (FOR: 110)

Na mrowisko tak samo przyjemnie patrzec jak na morze. Rzuci Maciuśs okruszek listka albo kory, patrzy, jak mrowki ciagna - takie malusienkie. (KOR: 62)

[M:] Ale ma pani mieszkanko, pierwsza klasa. Urzadzone. Czysciutko, cieplutko. Mebelki jak trzeba. (NUR: 8) ...auf der anderen Seite des Tals, wo winzige Gefährte den Staub nach sich zogen. (MIL: 60)

Sie gingen langsam...Er geriet in Schweik, obwohl es kühl war. (NOW: 45)

Das Fenster bestand aus vier winzigen Scheiben, von denen nur die oberen über der Erde lagen. (FOR: 104)

Ein Ameisenhaufen ist ebenso interessant wie das Meer. Hänschen wirft ein Stück Blatt oder Rinde hin und sieht zu, wie die Ameisen sich damit abschleppen - diese winzigen Tiere. (KOR: 85f)

[M:] Die Wohnung...ich muß schon sagen...alle Achtung. Und prima eingerichtet. Gemütlich, warm. Genau die richtigen Möbel. (NUR: 10)

\section{4. $S D \Rightarrow$ Basislexem in Komposita:}

Bei einer nicht unbeträchtlichen Zahl von Belegen fällt die Wiedergabe ausgangssprachlicher Diminutive durch ein Determinativkompositum im Zieltext auf. Am stärksten vertreten ist die Beispielklasse, in der sich das deutsche Kompositum aus dem motivierenden Substantiv als zweitem und dem das Grundwort inhaltlich näher bestimmenden Substantiv als erstem Glied zusammensetzt. Die reichhaltige Auswahl mag die Häufigkeit verdeutlichen:

Na toku (pyl gryzl w oczy i ledwo co majaczylo) biale chustki kobiet i spocone twarze. (MIL: 68)

Zakaszlal charkotliwie, splunal w chusteczke. "Kiepsko pluje", powiedzial... (NOW: 32)

Grzebala w torebce...Spuscila oczy. Przypudrowala sie w lusterku. (NOW: 38)

Szofer zerknal w lusterko i zagadal wesolo... (NOW: 116)
Oben in der Scheuer (der Staub biß in die Augen, und es war kaum etwas zu sehen) weiße Kopftücher der Frauen und schwitzende Gesichter. (MIL: 91f)

Hustete röhrend, spuckte ins Taschentuch. "Üble Spuckerei", sagte er... (NOW: 16)

Wühlte in ihrem Täschchen...Sie senkte die Augen. Puderte sich vorm Taschenspiegel. (NOW: 22)

Der Chauffeur guckte in den Rückspiegel und quatschte fröhlich... (NOW: 70) 
Kaczor pochyliı sie nad wózkiem. Dzieciak spal. (NOW: 109)

Najmniejszym palcem rozluznil pasek [zegarka]. Poszlo bez oporu. (NOW: 116)

Zauważyl ladna wiśniowa lufke na stoliku obok lóżka. (NOW: 153)

Nagle ktos puknal w okno, Wiktor ujrzal biala parasolke, potem bialy kapelusz. (IWA: 57)

Ciocia czym predzej poszla do kuchni przygotowac podwieczorek. Na pewno zaraz wystapi czekolada z pianka jak dla dzieci... (IWA: 58)

Za wielkimi kratami z drutu, które stanowily tylko "przedmurze" specjalnych, ogrzewanych domk6w, siedzialo mnóstwo bialego drobiu, bialych kur i pantarek. (IWA: 61)

Kazia...przysunela sobie wielki klosz szklany, niebieski. Poczela na nim ukladać w desen suche konfitury, wyjmujac je szczypczykami $z$ metalowych puszek. (IWA: 65)

Ujrzal nagle z bliska wielki, cebulasty zegarek, uslyszal cichy brzek zlotej pokrywy. (FIN: 32)

...jeden $z$ nich, najmlodszy, wciąz biegal na drugi koniec rynku, stawal pod daszkiem nad wejsciem do apteki i wypatrywal. (FIN: 45)

Schowala dokumenty do torebki, meziczyzna otworzyl drzwi, poklepal ja po ramieniu. (FIN: 53)

[I:] Jest mloda. Nic sie jej nie stanie, jak troche postoi na korytarzu [pociagu]...Naprawde prosze sie nie przejmowac. Na korytarzu jest pusto, ciepło, sa krzeselka... (RUM: 4)
Kaczor beugte sich über den Kinderwagen. Der Säugling schlief. (NOW: 63)

Mit dem kleinen Finger lockerte er das Armband. Es ging ohne Widerstand. (NOW: 70)

Er bemerkte eine schöne rote Zigarettenspitze auf dem Nachttisch. (NOW: 106)

Plötzlich klopfte jemand ans Fenster. Wiktor sah einen weißen Sonnenschirm und dann einen weißen Hut. (IWA: 44f)

Die Tante eilte in die Küche, um die Vesper zu richten. Sicher würde es gleich Schokolade mit Milchschaum geben wie für Kinder. (IWA: 45)

Hinter großmaschigen Drahtgittern, die nur einen Vorhof für die geheizten Hühnerhäuser bildeten, saßen weiße Leghorn und bunte Perlhühner... (IWA: 49)

Kazia...zog eine große blaue Glasschale zu sich heran. Dort hinein legte sie, zu einem Muster geordnet, die kandierten Früchte, die sie mit einer Kuchenzange aus mehreren Blechbüchsen nahm. (IWA: 53)

Plötzlich erblickte er von nahem die große, zwiebelförmige Taschenuhr und vernahm das leise Klick des goldenen Deckels. (FIN: 96)

...einer von ihnen, der Jüngste, lief immer wieder ans andere Ende des Marktplatzes, stellte sich unter das Vordach über dem Apothekeneingang und hielt Ausschau. (FIN: 54)

Sie steckte die Dokumente in die Handtasche, der Mann öffnete die Tür und klopfte ihr auf die Schulter. (FIN: 52)

[I:] Die Dame ist noch jung. Der schadet es bestimmt nicht, wenn sie sich ein bißchen im Gang die Füße vertritt... Aber ich bitt' Sie, stören Sie sich doch nicht daran. Der Gang ist leer, geheizt, außerdem stehen die Klappsitze zur Verfügung. (RUM: 4f) 
Do emaliowanego rondla wlewamy 11 jasnego, lekkiego piwa..., dodajac lyżke swieżego masła, 1/s tyżeczki kminku, szczypte soli... (LEM: 25)

Zjadl kolacje, rozebral sie, polożyl bardzo wczesnie: glowa go boli. Zostawil otwarty lufcik: bo mu goraco. (KOR: 19)

W glebi ogrodu dom; a tu furtka otwarta; a z boku domek parterowy, a za nim krzaki. (KOR: 31)

Z poczatku gniewalo Maciusia, że o byle glupstwo musial pisac podanie...Ale sie przyzwyczail...Zapisuje w ksiazeczce, co potrzebuje. Potem pisze podanie... (KOR: 83)
In einem Emaillekochtopf 11 helles, leichtes Bier,...einen Löffel Butter, $1 / 3$ Teelöffel Kümmel, eine Prise Salz...zusammen aufkochen. (LEM: 28)

Er aB sein Abendbrot, zog sich aus und legte sich früh ins Bett, angeblich hatte er Kopfschmerzen. Das Oberfenster ließ er offen, ihm sei so heiB. (KOR: 25)

In der Tiefe des Gartens stand ein Haus, die Gartentür war offen, und an der Seite noch ein kleines Häuschen, dahinter Gebüsch. (KOR: 43)

Anfangs hatte sich Hänschen geärgert, daß er um jeder Kleinigkeit willen eine Eingabe an den Rat...richten mußte...Aber dann hatte er sich daran gewöhnt und fand es sogar besser so. In seinem Notizbuch trug er ein, was er brauchte. Dann schrieb er die Eingabe... (KOR: 112)

Beim polnisch-deutschen Textpaar stehen sich also jeweils gegenüber: ein Derivat mit polyfunktionalem Suffix und ein Kompositum mit konkretisierendem Nomen, um, wie an anderer Stelle schon gezeigt, meist von der "Normvorstellung" abweichende, "kleinere" Objekte zu benennen. Eine Variation dieses Musters begegnet in:

Przez okienko wpadl stlumiony stukot. Spacer. Stukot zgrzytal jak piach w zebach. (NOW: 95)

Zeskoczyl ze stolka, odstawil go pod szanke. (NOW: 104)
Durch das Guckloch fiel ersticktes Gepolter ein. Spaziergang. Das Gepolter knirschte wie Sand zwischen Zähnen. (NOW: 50)

Er sprang von dem Tischchen herunter und stellte es wieder unter den Hängespind. (NOW: 59)

Hier kommt hinzu, daß das Basislexem der Vorlage im Zieltext durch ein Synonym ausgetauscht wurde und damit das Gemeinte noch exakter getroffen wird. Eine weitere Besonderheit stellen die folgenden Beispiele dar:

Równocześnie myślal: wiec $z$ nimi podziele ostatnia czastke mego życia, ich krzyk bedzie ostatnim glosem, który zamknie swiat. (FIN: 47)

[P:] Moim zdaniem - jestem co do tego zupelnie przekonany - wolnosci nie można dzielić, tak jak nie można dzielić prawdy na kawalki. (RUM: 6)
Zugleich dachte er: Also mit denen werde ich das letzte Bruchstück meines Lebens teilen, ihr Schrei wird die letzte Stimme sein, die mir die Welt zuschlieBt. (FIN: 75)

[P:] Meiner Ansicht nach, und ich bin mir dessen vollkommen sicher, kann man die Freiheit nicht auseinander dividieren, ebensowenig wie sich die 
Wahrheit in Einzelteile zerlegen läßt. (RUM: 7)

[K:] Ziec doktorat robi. Calymi dniami tylko w tych ksiażkach siedzi. Na paluszkach trzeba, a tu dziecko sie drze...a córka znowuż, nie najlepiej sie czuje. (NUR: 1)
[F:] Der Schwiegersohn promoviert. Er sitzt wochenlang über seinen Büchern, nichts wie lesen und lesen. Man muß die ganze Zeit auf Zehenspitzen gehen, und das Kind brüllt ununterbrochen... Der Tochter, der geht's gesundheitlich auch nicht immer gut. (NUR: 1f)

Hierbei dient die hinzugefügte Worteinheit (Bruch-, Einzel-, -spitzen) weniger der konkreten Spezifizierung als der Intensivierung.

Von den obigen Diminutivübersetzungen sind jene zu unterscheiden, in denen die Kompositionsglieder genau umgekehrt angeordnet sind, d.h., die spezifizierte Wiedergabe des Suffixes folgt als zweites Glied der des vorgegebenen Grundwortes:

Widocznie dotyk do krótkiej sierści [losiowego dywanika] przywolywal wspomnienia wszystkich jego [puchacza] przodków, którzy rozszarpywali sarenki i zajace. (MIL: 73)

Koslawy Karol zapala papierosa. Ruchliwy ognik pokazuje jego twarz z dziwacznymi cieniami, pelzajacymi po policzkach. (NOW: 58)

[M:] Raczki caluje, kochanej pani. Prosze przyjąc kwiatki ode mnie. Zaraz, tylko $z$ papieru wyjme, żeby bylo eleganciej. O, prosze bardzo. (NUR: 7)

Zgodzil sie Macius za pastuszka. Jest teraz Marcinkiem - dwie krowy pasie. (KOR: 125)
Die Berührung mit dem kurzen Fell [des Elchteppichs] rief [im Uhu] augenscheinlich die Erinnerung aller Ahnen zurück, die die Rehkitzen und die Hasen zerrissen haben. (MIL: 98)

Der krummbeinige Karol steckt sich eine Zigarette an. Der bewegliche Feuerpunkt zeigt sein Gesicht mit kauzigen Schatten, die seine Wangen entlangkriechen. (NOW: 34f)

[M:] KüB' die Hand, Frau Sabine. Ich habe mir erlaubt, diesen Blumenstrauß...Augenblick, ich pack ihn aus, so ist's eleganter. $\mathrm{Da}$, bitte sehr. (NUR: 9)

Hänschen verdingte sich als Hirtenknabe. Er hieß nun Heinz und hütete zwei Kühe. (KOR: 169)

Ähnlich wie bei den Substantiven werden auch bei der Adjektivübertragung Determinativzusammensetzungen genutzt. In struktureller Hinsicht trifft man einerseits auf Komposita mit substantivischem Bestimmungswort:

Kokietowala go raczej Jola, mlodziutka wówczas, szesnastoletnia i prześliczna panna. (IWA: 36)

...brat umieral. Czuli to wszyscy widac, bo i mala Ola siedziala cichutka, rozszerzonymi bialymi oczami spogladajac na stryja. (IWA: 161f)
Schöne Augen machte ihm Jola, damals ein blutjunges, sechzehnjähriges und wunderhübsches Fräulein. (IWA: 21)

... sein Bruder starb. Das spürten offenbar alle, denn selbst die kleine Ola saß mucksmäuschenstill und betrachtete ihren Onkel mit weit aufgerissenen, hellen Augen. (IWA: 159f) 
"Stala tam golusieńka na pustym wygonie, cala w sloncu i czekala, żeby ja zabili." (FIN: 23)

"Ty tak z litości, co?" zaśmialam sie, "biedna ofiara wojny, rodziców stracila w powstaniu, samiutka jak palec..." "Przestan, to wstretne i sama wiesz, ze nieprawda." (FIN: 74)
"Sie stand dort splitterfasernackt auf dem leeren Anger, im Sonnenschein, und wartete, daß man sie erschoB." (FIN: 29)

"Du redest so aus Mitleid, was?" Ich lachte auf. "Ein armes Kriegsopfer, hat die Eltern im Aufstand verloren, ist mutterseelenallein..." - "Hōr auf, das ist ekelhaft, und du weiBt, es stimmt nicht." (FIN: 153)

Andererseits bilden Adjektive mit bereits präfixoidem Charakter das Primärsegment der Konstruktion:

...Stas widzial, jak las przemienia sie w niebotyczne drewniane konstrukcje, po których on skacze z lekkoscia wiewiórki, a Malina idzie, malutka, malutka, jak w górach, samym dołem... IWA: 166)

W powrotnej drodze myslal Macius o Ali. Malutka jest, paluszki ma malutkie... (KOR: 74)

Koniec wizyty witala $\mathrm{z}$ ulga, odchodzila $z$ rodzicami czyściutka i pachnaca. patrzyliśmy za nimi uwieszeni plotu. (FIN: 94)
...Stas sah, wie der Wald sich in eine himmelhohe Holzkonstruktion verwandelte, auf der er - leicht wie ein Eichhörnchen - umhersprang, während Malina - winzigklein wie im Gebirge zu FüBen entlangging... (IWA: 164f)

Auf dem Heimweg dachte Hänschen über Ala nach. Klitzeklein war sie, hatte ganz kleine Fingerchen... (KOR: 100ก)

Sie begrüßte das Ende des Besuchs erleichtert und ging mit ihren Elterm davon, pieksauber und duftend. Wir schauten ihnen, am Zaun hängend, nach. (FIN: 132)

In allen Fällen dient der Zusatz der Ausdrucksverstärkung im Sinne von 'sehr' (blutjung, pieksauber) bzw. 'ganz' (splitterfasernackt, mutterseelenallein).

5. $S D \Rightarrow$ Basislexem + Ergänzungen:

Gelegenheitsbildungen stellen jene Wiedergaben dar, in denen neben dem vorgegebenen Grundwort auf vielfältige und nicht einheitlich systematisierbare lexikalische bzw. syntaktische Ergänzungen zurückgegriffen wird. Mal finden sich Possessivpronomen:

"Niech pani usiądzie, dziecinko, za moim przykladem na mchu i zwróci uwage na niezwyklosć powszednich zjawisk, takich, na przyklad, jak spiew ptaków, szum wiatru..." (FIN: 31)

Staral sie teraz Macius najmniej siedzieć w pokoju...Albo na gorze przy-

"Setzen Sie sich wie ich ins Moos, mein Kind, und beachten Sie das Ungewöhnliche der alltäglichen Erscheinungen wie zum Beispiel den Gesang der Vögel, das Rauschen des Windes..." (FIN: 94)

Hänschen bemühte sich, so wenig wie möglich im Zimmer zu sitzen... Entweder schmückte er auf dem Berg seinen 
strajal cmentarzyk kwiatami, albo popłynie do latarni... (KOR: 89)

mal Indefinitpronomen als Zusatz:

...otwieral sie zupelnie inny Swiat: $z$ pagóreczka w dolinke, tu borek, tu rojścik, krecace sie dróżki... (MIL: 87)

\section{Mal sind es restriktive Adverbien:}

[Pies] rwal przed siebie, uciekal...Nikl mi w oczach, coraz mniejszy, ale wciaż widoczny, to czarny punkcik, to czama kreska skaczaca po szosie. (FIN: 70)

A odbywać narady i jesć rybki, szczególnie po tylu cieżkich przejsciach - to nie dla królow interes. (KOR: 56)

mal werden Adjektive genutzt:

Noc byla ciepla, wietrzna...W domach ani swiatelka, gesta czern, sen. (FIN: 59)

Im weiteren begegnen, besonders bei Stoffbezeichnungen, Ergänzungen mit Stück:

Okno bez kraty...Na dole wisi mocny, skórzany pas...W górze okna też jakaś skórka. (KOR: 22)

Nareszcie. Z poparzonych rak Maciusia odebral klocek rozpalony - kaplan ludożerców. (KOR: 50)
Friedhof mit Blumen, oder er fuhr zum Leuchtturm hinüber... (KOR: 121)

...[es] öffnete sich eine ganz andere Welt: Hügelchen und Tälchen, hier ein Wäldchen, dort et was Sumpf, gewundene kleine Pfade... (MIL: 119)

[Der Hund] hetzte davon, er floh...,da verschwand er mir fast aus den Augen und wurde immer kleiner, nur noch ein schwarzer Punkt, ein schwarzer Strich auf der Chaussee. (FIN: 114)

Und Beratungen abhalten, wenn man nur Fische im Magen hat, das ist, besonders nach so vielen schweren Erlebnissen, nun einmal nichts für Könige. (KOR: 76f)

Die Nacht war warm und windig...In den Häusem brannte kein einziges Licht, dichtes Schwarz, Schlaf. (FIN: 116)
[Das Fenster] war unvergittert...Unten hängt ein starker Ledergurt... Oben am Fenster ist auch ein Stück Leder. (KOR: 29)

Endlich! Der Priester der Menschenfresser nahm Hänschen das glühende Stück Holz aus den verbrannten Händen. (KOR: 69)

\section{oder Ergänzungen in Form von Höflichkeitswendungen:}

"Jeszcze chwilke", blagala Kampanella. "zapomnialam dodac..." (KOR: 16)
"Einen Augenblick, bitte", flehte Campanella, "ich wollte noch hinzufügen..." (KOR: 21)

Neben diesen lexikalischen Gestaltungsmöglichkeiten sind nun mit syntaktischen Mitteln vorgehende Kompensationen des diminutivischen Gehalts des Ausgangstextes anzuführen:

"Zaraz, chwileczke nie moge ich znaležt, nie wiem, gdzie je mamusia polożyla..." (FIN: 15)
"Gleich, gleich, einen Augenblick, ich kann die Schlüssel nicht finden, ich weiß nicht, wo Mama sie hingelegt hat..." (FIN: 23) 
Hier wird die diminutivische Aussage durch die Verdopplung des nebengeordneten Lexems erreicht. Als ein ebenso probates Mittel erweist sich die Nachdruck erzeugende Reihung von Synonymen:

[M:] Co ja mam robić? Siedziec w tym swoim pokoju i myslec jak tam mój sad niszczeje. Nikt nie wie co jemu potrzeba. Ja tam każde drzewko znam. Whasnymi rekami sadzilem... (NUR: 16)
[M:] Was soll ich denn anfangen? Soll ich vielleicht ruhig in meinem Zimmer hocken und daran denken, wie mein Garten jetzt zu Grunde geht. Keiner weiß, wie man den zu pflegen hat. Ich kenne dort jeden Strauch und jeden Baum. Ich habe jeden mit eigenen Händen gepflanzt. (NUR: 19)

Die verstärkende Komponente des Diminutivs im nun folgenden Beispiel wird durch den Kunstgriff, einen neuen, eigenen Kurzsatz zu bilden, erzielt:

"Nic sie nie bój...to Żydów strzelaja. Zlaź z wozu i do lasu",....kiedy usiadlam w trawie na skraju lasu, widac już bylo wszystko, wszyściutenko... (FIN: 22)

[M:] Ale ma pani mieszkanko, pierwsza klasa. (NUR: 8)
"Keine Angst,... die schießen auf Juden. Steig ab und geh schnell in den Wald" $\ldots$, als ich mich ins Gras setzte, sah man bereits alles. Alles. (FIN: 27)

Im letzten Beispiel gelingt die Wiedergabe eines Diminutivs in laudativer Funktion mit Hilfe einer Satzerweiterung.

$S D \Rightarrow f r W:$

Sind die unter der Formel ND gefaßten Wiedergaben in der Regel als Nichtdiminutive in lediglich formaler Sicht zu verstehen, weil sie in semantischpragmatischer Hinsicht meist auf verschiedene Weise kompensiert werden, so stellen die mit dem Kürzel frW bedachten Übertragungen die eigentlichen formal-semantischen Nichtdiminutive dar. Drei Verfahren lassen sich bei der Umsetzung eines Diminutivs des Ausgangstextes beobachten:

\section{Substitution durch andere Lexeme:}

Hierbei findet ein punktueller Austausch statt, d.h., das Suffixdiminutiv wird durch ein semantisch anders als das Grundwort des Diminutivs besetztes Substantiv ersetzt; dabei kann zum einen Wortklassenidentität gewahrt werden:

W sobote po wyplacie spotkali sie nad Pociecha...W wydeptanej wśród trzcin kotlince rozlożyli marynarki. (NOW: 62f)
Am Samstag nach der Auszahlung trafen sie sich an der Pociecha...An einer heruntergetretenen Stelle im 
Schilf breiteten sie die Jacken aus. (NOW: 39)

Nie znaczy to, aby wysluchal z rozpacza wiadomosci, która mu zakomunikowala wlaśnie nad jeziorkiem, ze Michal chce sie $z$ nia żenić w paździemiku. (IWA: 168)

Stanal przy mogile i oparl sie reka o pochylony pien, którego gladkie i chropawe czqsteczki odczuwal palcami. (IWA: 131)

Zabralam predko dzieci do chaty, maż pobiegl po pastuszka i krowe ... (FOR: 80)
Das heißt nicht, daß er verzweifelt gewesen wäre, als sie ihm an dieser Stelle mitteilte, Michal wolle sie im Oktober heiraten. (IWA: 167)

Am Grab blieb er stehen und stützte sich mit der Hand an einen schrägen Stamm, dessen teils glatte, teils rauhe Oberfläche er mit den Fingern spürte. (IWA: 126)

Ich schaffte die Kinder nach Hause, mein Mann lief auf die Wiese, holte unseren Großen und die Kuh. (FOR: 74)

Auszumachen sind ebenso hypokoristische Personennamen, für die der Übersetzer entweder die Grund- oder Kurzform anderer Namen stellvertretend herangezogen hat:

...pyta sie [kanarka], czy pamieta...Stasia, Helcie, Klu-Klu. (KOR: 80)

Rozumiem, dlaczego Franek nie chcial z nimi zaczynac. (KOR: 130)

Wspominać o Bum-Drumie, Felku, Klu-Klu, smutnym królu nie pozwolili. (KOR: 14)

Die andere Gruppe bilden nun jene Wiedergaben, die auf einem Wortartwechsel beruhen. Dazu zählen zunächst Adjektive:

Niebo zalegal szafir bez chmurki od samego rana... (IWA: 146)

Ale oto robi sie rumor, wbiega gromadka moich wczorajszych druhen $z$ okrzykiem... (FOR: 61)

Tak sobie skupilam grupke przyjació... (FOR: 74)

Tam ukryly sie resztki dawnych mieszkańców, ucieklszy przed zaraza. (KOR: 64)

...a pozostala garstka ukryla sie w lesie za rzeka... (KOR: 78)
Er fragt [den Kanarienvogel], ob er sich auf...Peter, Leni, Klu-Klu noch besinnen könne. (KOR: 109)

Nun begreife ich, warum Stefan auf der Insel mit innen nicht anbinden wollte. (KOR: 176)

Es war ihr nicht gestattet, über BumDrum, Fritz, Klu-Klu oder den traurigen König zu sprechen. (KOR: 17)
Wolkenloses Blau überzog seit dem frühen Morgen den Himmel... (IWA: 142)

Da stürzten einige Brautjungfern ins Zimmer und kreischten... (FOR: 58)

Und ich fand einige Freunde... (FOR: 69)

Da halten sich die letzten Urbewohner versteckt, nachdem sie vor der Epidemie geflüchtet sind. (KOR: 87)

...[als] die wenigen Überlebenden sich 
im Wald jenseits des Flusses versteckten,... (KOR: 106)

Auch Adjektivableitungen aus Scheinpartizipien auf substantivischer Basis sind zu finden:

...palenisko [fajki] zamykalo sie na metalowe wieczko z dziurkami. (MIL: 15)

Antonia wysuwala naprzod brzuch w fartuchach w prazikj. (MIL: 14)

Koniec nosa $z$ fioletowymi żylkami dziadek lubil brac w dwa palce... (MIL: 75)

Ferner werden Adverbien (Pronominal- bzw. Lokaladverbien) herangezogen:

"Po co sie tyle tego robi?" zapytal Kazi. - "Aby sie niektóre $z$ tych sloik6w dostaly wybranym." (IWA: 68)

Przez parkan i brame przychodzili na placyk coraz to inni chlopcy $z$ miasta. (NOW: 119)

Na tej uliczce znajdowal sie wielki, czteropietrowy dom. (FOR: 108) ...der Pfeifenkopf war mit einem gelochten Metalldeckelchen zugemacht. (MIL: 16)

Antonia schob den Bauch in der gestreiften Schürze vor. (MIL: 15)

Die violett geäderte Nasenspitze pflegte der Grobvater in zwei Finger zu nehmen... (MIL: 101)
"Warum wird davon soviel hergestellt?" fragte er Kazia. - "Damit man einige davon auserwählten Freunden vorsetzen kann." (IWA: 56)

Über den Zaun und durch das Tor kamen immer andere Burschen aus der Stadt hierher. (NOW: 73)

Hier stand ein großes, vierstöckiges Haus. (FOR: 103)

Als besonders beliebt erweist sich die Pronominalsubstitution, das gängige Prinzip der Wiederaufnahme durch Pronomen, häufig unter Zuhilfenahme von Personalpronomen:

Zachlysnela ona stojacego wciaż nieruchomo, niby grudka lodu. Grudka ta topniala w gardle,... (IWA: 139)

...okno - byly to cztery ... szybki... Okienko było zabezpieczone żelazna krata... (FOR: 110)

[S:] Mial pan mieć gazete. - [M:] A jest gazetka, jest. Prosze bardzo. (NUR: 5)

"Wujaszku", powiedziala, "slyszy wujaszek? Tam graja." (IWA: 128)

und Indefinitpronomen:

"Jak dyrektor mówi, żes dziewczynka, tos dziewczynka, i koniec." (KOR: 34) 
$\mathrm{Z}$ rozwalkowanego cienko ciasta wycinamy kieliszkiem do wina kółka, na kótka kładziemy kupke... (LEM: 55)

Oddzielnie podamy zielona salate, przyprawiona jedynie cytryna, oliwa (olejem), szczypta soli i odrobinka cukru. (LEM: 106)

Aber auch Relativpronomen:

Kampanella ... polożyla nieznacznie pudelko czekoladek, pudelko to miala ukryte w kieszeni królewskiego płaszcza... (KOR: 11)
Aus dem dünn ausgerollten Teig mit einem Weinglas Scheiben ausstechen, etwas Füllung daraufgeben... (LEM: 61)

Dazu Salat reichen, der nur mit Zitrone, Öl, einer Prise Salz und etwas Zucker angemacht ist. (LEM: 107)

Campanella legte unmerklich...eine Schachtel Pralinen hin, die sie in der Tasche ihres königlichen Mantels verborgen hatte... (KOR: 15)

sowie Demonstrativ- und Possessivpronomen werden als Stellvertreter für das diminutivische Substantiv genutzt:

Obciać butelke można sznurkiem umaczanym w nafcie: jeżeli ja owinać $i$ podpalic sznurek, szkło peknie naokolo dokladnie w tym miejscu. (MIL: 26)

"Ja pisalem cześciej." - "Ale tatuś nie lubi listów stryjcia." (IWA: 102)
Die Flasche durchschneidet man folgendermaßen: Man umwickelt sie mit einer in Petroleum getauchten Schnur, zündet diese an, dann platzt das Glas rundherum genau an der gewünschten Stelle. (MIL: 31)

"Ich habe häufiger geschrieben." "Aber Vati mag deine Briefe nicht." (IWA: 95)

\section{Paraphrasierung:}

Im Gegensatz zum obigen, auf nur ein Lexem begrenzten Verfahren des Ersetzens bedeutet Paraphrasierung, daß eine ausgangssprachliche Phrase, die sich aus dem Diminutiv und in der Regel einem Verb zusammensetzt, im Zieltext in eine verbale bzw. adjektivische Worteinheit meist ohne Diminutivcharakter komprimiert wird. Zur Umschreibung mit Verben lassen sich folgende Belege zitieren:

Tomasz przewracal kartki jego ksiażek teraz już domagajac sie objaśnień. (MIL: 69)

Alfons odchodzit truchcikiem, potem gadal na calym oddziale: "Spokój..." (NOW: 93)

"Drzwi", rzekla urywanym póiglosem, "drzwi zamknij na haczyk..." (NOW: 122)
Thomas blätterte in seinen Büchern, jetzt schon nach Erklärungen verlangend. (MIL: 93)

Alfons trabte fort und verkündete der ganzen Abteilung: "Ruhe im Saal..." (NOW: 47)

"Die Tür", sagte sie abgerissen und mit halblauter Stimme, "verriegle die Tür ..." (NOW: 76) 
Wymylam rece, wlotylam swiateczne szatki... (FOR: 56)

Woźnica dat koniom po wiaderku wody. (FOR: 115)

Rozlegt sie dzwonek. (KOR: 32)
Ich wusch mir die Hände und kleidete mich festlich... (FOR: 53)

Der Kutscher tränkte die Pferde. (FOR: 108)

Es klingelte. (KOR: 44)

Die Komprimierung durch Adjektive gestaltet sich wie folgt:

Kiedy zjawil sie we dworze, w swojej bluzie sciagnietej paskiem, w granatowej czapce na bakier,... (MIL: 27)

Patrzyli, zbiwszy sie $w$ gromadke, na bialy blok naprzeciw. (NOW: 142)

Zwlaszcza skóra nad sztywnym kodnierzykiem wojskowym oficera...byla niezmiernie biala, podbita thuszczykiem... (IWA: 50)

Kazia mu przyniosla duża paczke, starannie zwiqzana sznurkami... (IWA: 92)

Jest niska i krepa,...oczy male i nieforemne, brazowe, zawsze poknyte lzawa mgielka... (FIN: 21)

[S:] Po co mi trzymac pieniadze na ksiązeczce. Nie ma dla kogo... (NUR: 3)

Do goracego sosu wsypujemy $25 \mathrm{dkg}$ ziemniaków pokrajanych $w$ kosteczke. (LEM: 95)

Eine Sonderstellung nimmt die Übertragung der nur spärlich vertretenen Adjektivdiminutive ein, bei deren Umschreibung - im Unterschied zu den substantivischen Derivaten - erweiternd vorgegangen wird:

Nikogo nie bylo $w$ calym domu ani na podwórzu, wszyscy sie gdzies porozchodzili i bylo cichutko. (IWA: 164)

[S:] Mieszkanie sobie wysprzatam. Nikt mi nie brudzi...Lubie, jak czyściutko jest. Podloga wypastowana, Iśni sie. (NUR: 2)
Wenn er im Herrenhaus erschien mit enggegirteter Bluse, dunkelblauer, schräg aufgesetzter Mütze,... (MIL: 34)

Zusammengedrängt sahen sie auf den weißen Block gegenüber. (NOW: 95)

Besonders die Haut über dem steifen Militärkragen des Offiziers...war unendlich weiß, fettgepolstert... (IWA: 37)

Kazia gab ihm ein großes, sorgfaltig verschnürtes Päckchen... (IWA: 82)

Sie ist stämmig und untersetzt, hat... kleine, unförmige, stets von Tränen verschleierte braune Augen... (FIN: 25)

[S:] Was soll ich mit dem gesparten Geld? Für wen? (NUR: 4)

In die heisse Sauce 250 g kleingewürfelte Kartoffeln geben. (LEM: 98)
Niemand war im Haus oder auf dem Hof, alle hatten sich entfernt, es herrschte tiefe Stille. (IWA: 162)

[S:] Ich habe eine gepflegte Wohnung. Und keiner ist mehr da, der alles durcheinanderbrächte. Ich bin nun mal für Sauberkeit....ich habe Freude daran, wenn alles glänxt. Der Fußboden bei mir ist immer frisch gebohnert...funkelt richtig. (NUR: 2) 
Zum einen wird das polnische Adjektiv in eine attributivische Fügung, zum anderen durch den Zusatz eines erläuternden Satzes aufgelöst, um damit die beabsichtigte intensivierende Wirkung im deutschen Text nachzugestalten.

Von der verbalen bzw. adjektivischen ist schließlich die sog. freie Paraphrase zu unterscheiden, bei der das umschriebene Diminutiv, nicht mehr punktuell faßbar, in einer neuen syntaktischen Konstruktion aufgeht:

Rybak...garnał wioslem, które mialo dwie lopatki... (MIL: 19)

One też czasem nosza chusteczkę od slońca. (FOR: 68)

Malo bylo tego, bo choc jeszcze ze wsi byly jakies graty,...to do takiej suterenowej izdebki nic by sie $z$ tego nie pomiescilo. (FOR: 112)

Tam czasem ktos z domowników spojrzal na [maleristwa], tu musialy samiutkie zostawać po kilka godzin. (FOR: 72)

Deski byly poukladane daleko jedna od drugiej. Ja bylam tak ghupiutka, że kiedy weszlam na te deski, nie pomyślalam, że spódnica moja zwiesza sie pomiedzy nimi - i jak tylko weszli,... wydala mnie moja spódnica. (FOR: 65)

Kolejarz Wróbel, chmurny żoładkowiec, przerywa im zwykle i wtraca swoje o wedkach lub rybach. Jednego razu powiada, że dobrze biora, nastepnego, że źle. I tak w kótko. (NOW: 57)
Der Fischer...schaufelte mit dem Doppelruder... (MIL: 21)

So schützen sie auch manchmal ihren Kopf vor der Sonne. (FOR: 64)

Wir besaßen nur wenig, und einiges, was wir noch von unserer Wirtschaft hatten, mußten wir dalassen... (FOR: 106)

Früher hatte manchmal einer nach [den Kleinen] geschaut, hier aber muBten sie einige Stunden im Haws bleiben... (FOR: 67)

Ich kletterte hoch, hatte aber in meiner Aufregung nicht beachtet, daß die Bretter ziemlich weit voneinander entfernt lagen, so daß mein Rock zwischen ihnen herunterhing und sogleich zu sehen war. (FOR: 61)

Eisenbahner Wróbel, ein düsterer Magenkranker, unterbricht sie gewöhnlich und fängt von seinen Angeln oder den Fischen an. Einmal sagt er, daß sie gut beißen, das nächste $M a l$, daß sie schlecht beißen. Und so geht es rund. (NOW: 33)

\section{Eliminierung:}

Darunter sind schließlich all jene Textstellen zu fassen, bei denen das Diminutiv, ohne in irgendeiner Form ersetzt zu werden, im Zieltext wegfällt:

Tor dzieci ukladaly $z$ darni,...wylożony kawalkami blachy dla rozpedu. (MIL: 24)

W bramach jarzyly sie ogniki papierosów. Ktos gral na harmonii. (NOW: 66)
Die Kinder fertigten aus Rasenstücken die Bahn,...und das Anlaufstück war mit Blech ausgelegt. (MIL: 29)

In den Portalen glühten Zigaretten. Einer spielte Harmonika. (NOW: 42) 
...ostatnim jego lacznikiem z życiem byl fortepian wynajety w miasteczku od jego "siostry w chorobie". (IWA: 119)

...wiatr wzdymal rókowy zagiel jej sukienki odslaniajac $w$ calej okazalości czerwone shupki nóg. (FIN: 95)

Mial tuż przed soba worek jej twarzy, w którym Izawily dwa jeziorka oczu. (FIN: 62)

...podniósl dziecko i pogladzil kepke jasnych wlosów. (FIN: 44)

A tu dzieci z domu wybiegly do ogrodka. (KOR: 33)
Sein letztes Bindeglied zum Leben stellte der Flügel dar, den er bei einer "Schwester in der Schwindsucht" gemietet hatte. (IWA: 114)

...der Wind blähte ihr Kleid auf wie ein rosa Segel und entblößte ihre roten Beine. (FIN: 133)

Ihr Gesicht, unförmig wie ein Sack, war dicht vor ihm, die Augen tränten. (FIN: 121)

...nahm das Kind auf und streichelte ihm über das helle Haar. (FIN: 65)

Und nun kamen die Kinder aus dem Haus gelaufen. (KOR: 44)

Eine noch gravierendere Abweichung vom Quellentext stellen die Beispiele dar, bei denen mit dem substantivischen oder adjektivischen Diminutiv auch das dieses direkt umgebende Textsegment ausgelassen wird:

.... wtedy miód wylewal sie $\mathrm{z}$ plastrów wstawianych tam w ramkach. (MIL: 80)

W miasteczku, o którym mowa, na poczcie, z której ubodzy mieszczanie byli wielce dumni, u naczelnika slużyla ...dziewczyna... (FOR: 38)

Potem nalewano w dzbanki piwo i w glinianych kubkach rozdawano... (FOR: 67) ...dann ergoß sich der Honig aus den Waben. (MIL: 109)

Beim Vorsteher dieser Post, auf die die ärmlichen Bürger so stolz waren, diente das Mädchen... (FOR: 38)

Bier wurde in Tonbechern gereicht. (FOR: 63)

\subsection{2. Übertragung analytischer Diminutive}

In Kenntnis der Kontroverse um die Anerkennung eines neben der derivationellen Diminuierung zweiten, formal verschiedenen Diminuierungsverfahrens ${ }^{12}$ sollen nun die vorgefundenen Übertragungsmuster analytischer Diminutive vorgestellt werden, um später Rückschlüsse über ihre inhaltliche Leistung ziehen zu können. Bei der Auswahl ist das Epitheton klein des Syntagmas "klein + Grundwort" nicht allein als Dimensionsadjektiv verstanden, sondern auch, je nach Anwendungsbereich, in seinen verschiedenen semantischen Variationen

12 Vgl. die Überlegungen im theoretischen Teil S.39f. 
beachtet worden, so daß klein in der Folge nicht in seinem engsten wörtlichen, vielmehr im weitesten Sinne, gleichsam als Formel aufzufassen ist.

Was nun die Reihenfolge der Wiedergabe betriff, soll der formalen Parallelität wegen die analytische Übertragung vorgezogen werden.

$A D \Rightarrow A D:$

Mit diesem Realisierungsmuster ist gleich die umfangreichste Gruppe angesprochen, die einer weiteren Untergliederung bedarf. Semantisch gesehen bietet sich eine Scheidung in quantitative und qualitative Aussagen der attributivischen Fügung an. Innerhalb dieser lassen sich dann der Attributwahl entsprechend im Zieltext verschiedene Übertragungsweisen feststellen.

Zunächst zur Quantitätsangabe, die eine Verringerung in räumlichem und zeitlichem Maße sowie eine Mengenreduzierung umfaßt. Wiederum sollen typische Beispiele den Charakter der folgenden Subklassen offensichtlich machen:

\section{Wahl des entsprechenden Attributs:}

Als erstes sind Diminutivpaare zu nennen, in denen Adjektive, die eine räumliche Ausdehnung, ein geringeres Ausmaß, ohne genauer zu spezifizieren, anzeigen, durch das entsprechende Adjektiv im Deutschen wiedergegeben werden (maly/drobny $\Rightarrow$ klein), vgl.:

...taki ukazal sie Tomaszowi pan Romuald, w malej granatowej czapce $z$ daszkiem... (MIL: 87)

Male okno spiżarni mialo kraty i prócz zapachu byl tu chlód i cien. (MIL: 12)

Mały okuń najlepiej nadaje sie na przynete... (MIL: 19)

Z uwaga, która wydala sie zazdrościa, patrzyl na jego mała, okragla postać. (NOW: 99)

Samo pole boże też bylo zasadzone gesto drzewami i szczelnie wypelnione małymi zacierajacymi sie wzgórkami... (IWA: 54) ...das war für Thomas Herr Romuald mit der kleinen dunkelblauen Schirmmütze... (MIL: 118)

Das kleine Speisekammerfenster hatte Gitter, und außer dem Duft war hier Kühle und Schatten. (MIL: 12)

Ein kleiner Barsch eignet sich am besten als Köder... (MIL: 22)

Aufmerksam, sah wie Neid aus, musterte er seine kleine, rundliche Gestalt. (NOW: 55)

Auch der Gottesacker selbst war dicht mit Bäumen bestanden und mit kleinen, eingesunkenen Hügeln gefüllt... (IWA: 41) 
...w malej oberizy wysoko w górach, trzeciorzedna miejscowosc $z$ trzeciorzednym lodowcem... (FIN: 83)

$\mathrm{Na}$ jednej stronie wielka izba $\mathrm{z}$ alkierzem, gdzie sie miesci rodzina, na drugiej stronie komora i mala izba, gdzie przechowuje sie zboże. (FOR: 12)

...i znowu prosba do kogos o mala pożyczke na poczatek. $Z$ tych malych dhugow utworzyl sie dlug duży... (FOR: 107)

[I:] Co sie stalo, panie profesorze? [P:] W prawej kieszeni...Mala czerwona pastylka... Nitrogliceryna...Nie mam sily... (RUM: 1)

I pokazuje na glowie, na rekach, na nogach duze i male blizny. (KOR: 134)

Juz w pierwszych latach malżenstwa upodobniala sie do swej matki i bylaby zapewnie wiernie powtórzyla jej los dostatni dom w małym miescie, ladne dzieci, ladne suknie... (FIN: 95) ...oben in einem kleinen Berggasthof. Eine Ortschaft dritter Klasse, und ein Gletscher dritter Klasse... (FIN: 98)

Auf der einen Seite lag die große Stube mit dem Alkoven. Dort wohnte die ganze Familie. Auf der anderen Seite befanden sich eine Kammer und eine kleine Stube, in der das Korn aufbewahrt wurde. (FOR: 18)

....und wieder mußten wir Geld borgen. Unsere kleinen Schulden wuchsen zu einer großen Schuld an... (FOR: 101)

[I:] Was ist passiert, Herr Professor? [P:] In der rechten Tasche...Die kleine rote Pille...Nitroglyzerin...Ich schaffe es nicht allein... (RUM: 1)

Und an Kopf, Hand und Bein zeigt er große und kleine Narben. (KOR: 181)

Schon in den ersten Ehejahren wurde sie ihrer Mutter ähnlich. Sie hätte deren Los gewiB treulich wiederholt - ein wohlhabendes Haus in der Kleinstadt, hübsche Kinder, schöne Kleider... (FIN: 133)

Die deutsche Übersetzung im letzten Beispiel ist ein Sonderfall, klein tritt hier in präfixoider Stellung auf. Nun noch einige Belege mit dem Attribut drobny:

...Tomasza przyciagaly szczegolnie fioletowe, czerwone i czarne kwadraciki szkaplerzy i wiazki różaŕcow - i barwa i mnogosć drobnych przedmiot6w. (MIL:23)

Z okresu...przed swoim osiemnastym rokiem życia przechowywala w kufrze kajety zapisane drobnym pismem. (MIL: 51)

Wstal, odsuwajac gwaltownie krzeslo. Poczal chodzic wzdluż szafy drobnymi krokami. (NOW: 133)

Nie widzial, zreszta, ani źd́bel, na które patrzyl, ani drobnych owadów... (IWA: 93) ...doch Thomas Aufmerksamkeit wurde vor allem von den violetten, roten und schwarzen Skapulieren und den Bündeln von Rosenkränzen gefesselt sowie von den Farben und der Fülle der kleinen Dinge. (MIL: 28)

...aus der Zeit vor ihrem achtzehnten Lebensjahr bewahrte sie im Koffer mit kleiner Schrift beschriebene Hefte. (MIL: 67)

Er stand auf und schob heftig den Stuhl weg. Begann mit kleinen Schritten den Schrank entlang auf und ab zu gehen. (NOW: 86)

Doch er sah weder die Halme, auf die sein Blick fiel, noch die kleinen Insekten... (IWA: 83) 
Dziewczyna dluga chwile patrzyla na Józefa, drobne zmarszczki pofalowaly jej czolo, glowe wtulila w ramiona. (FIN: 30)
Das Mädchen dagegen schaute Józef eine ganze Weile an; kleine Runzeln kräuselten ihre Stim, den Kopf zog sie in die Schultern. (FIN: 92)

Davon sind Fälle zu unterscheiden, in denen im Ausgangs- und Zieltext Angaben über Ausmaße mit Spezifikation gemacht werden; im einzelnen sind es Adjektive, die eine Dimension wie Breite, Höhe, Länge, sowie Stärke und Dauer des durch das dazugehörige Substantiv ausgedrückten Objekts hervorheben:

Oto jego portret: duze czolc, waska twarz, w spojzeniu gwaltownosc i duma... (MIL: 52)

Paser zacisnal usta w waska i prosta linię. (NOW: 36)

Stal w sieni i swiecil latarka, a ja wspinalem sie po waskiej, zbutwialej drabinie. (FIN: 67)

Dlugo ida, coraz glebiej, glebiej - aż do waskiego korytarza. Potem korytarzem tak niskim, że podróżny aż sie pochylil. (KOR: 78)

Zatrzymal sie przed niskim o skrzywionym dachu drewniakiem. (NOW: 131)

Widocznie dotyk do krotkiej sienści [losiowego dywanika] przywolywal wspomnienia... (MIL: 73)

Patrzyl na płomienie, wydobywajace sie z suchych, krótkich drewienek, które Katarzyna podkladala pod płyte. (IWA: 127)

Stasiek, z odstajacymi uszami i cienka szyja, nie pociagal Tomasza... (MIL: 27)

Objal ja bez slowa i chudym ramieniem wyczul jej szerokie plecy... (IWA: 132)

Potem potarl policzki, które pokrywal radki i brudny zarost. (NOW: 54)
Hier ist sein Porträt: hohe Stime, schmales Gesicht, im Blick Heftigkeit und Stolz... (MIL: 68)

Der Hehler preßte den Mund zu einer schmalen, geraden Linie. (NOW: 19)

Er stand im Heu [sic!] und leuchtete mir mit einer Laterne, ich kletterte die schmale, morsche Leiter hinauf. (FIN: 109ก)

Lange gehen sie so, immer tiefer und tiefer hinab - bis zu einem engen Korridor. Dann durch einen so niedrigen Korridor, daB sich der Wanderer bücken muB. (KOR: 105)

Vor dem niedrigen Holzhaus mit dem windschiefen Dach blieb er stehen. (NOW: 84)

Die Berührung mit dem kurzen Fell [des Elchteppichs] rief in ihm augenscheinlich die Erinnerung...zurück... (MIL: 98)

Er betrachtete die Flammen, sie schlugen aus den trockenen, kurzen Scheiten, die Katarzyna unter die Herdplatte gelegt hatte. (IWA: 121)

Stasiek mit seinen abstehenden Ohren und seinem dünnen Hals übte auf Thomas keine Anziehung aus... (MIL: 33)

Wortios umfaßte er sie, seine dünnen Arme spürten ihren breiten Rücken... (IWA: 127)

Dann rieb er sich die Wangen, die ein spärlicher und schmutziger Bartwuchs bedeckte. (NOW: 30) 
Mietek Tapeta popatrzyl na Mele, potem musnal Zabka krókim spojrzeniem. Podszedl do kuchni. (NOW: 53)

Trzyczwartak zmarszczyl twarz i wykonal za jej plecami krótki, dziwaczny ruch reka. (NOW: 110)

Szedl powoli, pociagajac nogami, serce mu sie uspokoilo i krótki oddech móg! stać sie glebszym. (IWA: 131)
Tapetenmietek sah auf Mela, dann streifte er Zähnchen mit einem kurzen Blick. Ging zum Herd. (NOW: 29f)

Dreiviertel legte das Gesicht in Falten und machte hinter ihrem Rücken eine kurze, sonderbare Handbewegung. (NOW: 64)

Er ging langsam und schlurfend, sein Herz beruhigte sich, der kurze Atem wurde mit der Zeit länger. (IWA: 126)

Nur selten begegnen in dieser Gruppe Epitheta, die als Negation des Antonyms eines verkleinernden Adjektivs auftreten (häufig in prädikativer Stellung) und vom Übersetzer mit denselben Mitteln seiner Sprache wiedergegeben worden:

Kazanie w dzień Matki Boskiej Zielnej dal niedhugie i równym, spokojnym glosem. (MIL: 39)

Osobliwoscia doliny Issy jest wieksza niż gdzie indziej iloš diablow. Ci, co je widzieli, mówia, że diabel jest nieduży, wzrostu dziwiecioletniego dziecka... (MIL: 9)

Nisia...nie szyla już od dawna, tylko pracowala lopata przy budowie wielkiego mostu na niewielkiej rzere... (FIN: 64)

...w upale skradal sie za nimi, szedl w niedalekjej odleglości, bowiem oni, pewni siebie..., nie ogladali sie wcale. (IWA: 153)
Die Predigt am Himmelfahrtstag war nicht lang, er hielt sie mit gleichmäßiger, ruhiger Stimme. (MIL: 50)

Die Besonderheit des Issatals liegt in der Zahl seiner Teufel. Sie ist dort größer als sonstwo. Diejenigen, die sie gesehen haben, sagen, ein Teufel sei nicht groß, von dem Wuchs eines neunjährigen Kindes... (MIL: 7f)

Sie nähte schon lange nicht mehr, sondern arbeitete mit der Schaufel beim Bau der großen Brücke über den nicht sehr großen Fluß. (FIN: 66)

In der Hitze war er ihnen nachgeschlichen, in nicht sehr großer Entfernung, weil sie, sicher...geworden, sich überhaupt nicht umblickten. (IWA: 151)

Eine Besonderheit der analytischen Bildeweise liegt darin, daß das attributivische Adjektiv der Vorlage außer in seiner Grundform ebenso in diminuierter Form gebraucht werden kann, vgl.:

Dziewczyna polożyla mu dlon na ramieniu. Pielegnowana, malutka dlon, kwitnaca różowo malowanymi paznokciami. (FIN: 80 )

Sosny nie zmienily sie jesiennie, wiecej tylko latalo cieniutkich płatkow czerwonawej kory, opadajacej $z$ góry pni. (IWA: 172)
Das Mädchen legte ihm die Hand auf die Schulter; eine gepflegte kleine Hand, an der die lackierten Fingernägel rosa blühten. (FIN: 139)

Die Kiefern hatten sich noch nicht herbstlich verändert, es flogen allerdings dünne Flocken umher, die rote Rinde an den Stämmen blätterte ab. (IWA: 171) 
Każda $z$ dziewczat przynosi malutka wiazanke ruty - jest to roslina drobniutka, zima i latem zielona... (FOR: 56)
Jedes Mädchen brachte ein Sträußchen Raute mit - das ist eine zarte Planze, die im Sommer wie Winter grünt... (FOR: 53)

Hier reagiert das Deutsche lediglich mit dem nichtmodifizierten Adjektiv; im folgenden Beispiel bemüht sich der Übersetzer, mit einer synonymischen Worteinheit auszugleichen:

Malutkim czótnem pożyczonym od rybaka jechali dlugo przez szuwary... (IWA: 71)

Mit einem winzigen, vom Fischer entliehenen Kahn fuhren sie lange durch Schilf... (IWA: 59)

Darüberhinaus kann das Adjektiv auch in komparativischer oder superlativischer Form begegnen:

Na trawniku trzy agawy, wielka posrodku, dwie mniejsze po bokach, rozsadzaly donice... (MIL: 12)

"Zjedz sobie truskawek, $z$ tych mniejszych", powiedziala Kazia... (IWA: 64)

...ale zadna, najmniejsza nawet okruszyna prochu nie przetrwala... (MIL: 98)
Auf dem Rasen drei Agaven, die große in der Mitte, zwei kleinere an den Seiten, sprengten fast die Kübel... (MIL: 12)

"IB ein paar Erdbeeren, die kleineren", sagte Kazia... (IWA: 52)

...aber nicht einmal die kleinste Aschenspur ist...übriggeblieben... (MIL: 134)

\section{Wahl eines verkleinernden Attributs fur das negierte Antonym:}

Waren die unter Punkt 1. zusammengestellten Exempel in ihren Variationen zahlreich belegbar, sind die beiden jetzt anzuführenden Fallgruppen ungleich seltener. In ihnen wird in der Vorlage nicht auf das einfache, nichtzusammengesetzte Adjektiv, sondern auf die negierte Bildung seines Pendants zurückgegriffen, die vom Übersetzer mit der einfachen Adjektivform erwidert wird:

...z ganku widok na nieduże jezioro... (MIL: 88)

Ubral sie leniwie i wlóczyl po ogrodzie niewielkim i olbrzymim podwórzu... (IWA: 43)

Fela...rozczesywala niedługi warkocz ... (IWA: 53)

Stary doliniarz byl zgarbiony i niewysoki. (NOW: 53)

...nazajutrz rankiem...zrobili brzozowe sztachetki i krzyż brzozowy, gruby i niewysoki. (IWA: 169) ...schaut zu einem kleinen See hinunter... (MIL: 120)

Träge zog er sich an und schleppte sich durch den kleinen Garten in den groBen...Hof. (IWA: 29)

Fela...kämmte sich mit einem gelben Kamm ihren kurzen Zopf. (IWA: 39)

Der alte Taschendieb war gebückt und klein. (NOW: 30)

...[sie] setzten am nächsten Morgen ...einen Birkenzaun und errichteten ein dickes, niedriges Birkenkreuz. (IWA: 168) 
3. Wahl eines verkleinernden Synonyms für eine Negationsbildung:

Hier stehen sich wiederum gegenüber: ein negiertes Adjektiv im quellensprachlichen Text und ein einfaches, jetzt aber synonymisches Attribut im Zieltext, vgl.:

Dziobus wyobrazal sobie tego starego. Chlop niewielki, charakterny, zawsze uśmiechniety. (NOW: 103)

...czlowiek zrobil sobie niewielka szpare w zewnetrznej scianie stodoly. (FIN: 57)

...oswojony potem z ciemnoscia wylowil zarysy desek biegnacych wzdluż ścian i niewielkq przestrzeń dzielaca je od drzwi. (FIN: 46)

...postawil karawan, ulożyl na nim trumne $i$ ciagnie ten nieduży ciężar dla rak, a wielki cięzar dla serca. (KOR: 84)
Dziobus versuchte sich den Alten vorzustellen. Ein mittelgroßer, charaktervoller Kerl, immer lächelnd. (NOW: 57)

Der Mann hatte sich eine schmale Ritze in die Außenwand gemacht... (FIN: 69)

Später, als seine Augen sich an die Dunkelheit gewōhnt hatten, nahm er die Umrisse der Bretter an den Wänden wahr und den geringen Abstand, der sie von der Tür trennte. (FIN: 73)

...[er] stellte den Leichenwagen zu Boden, legte den Sarg darauf und zog diese für die Hände geringe, für das Herz aber schwere Last hinter sich her. (KOR: 114)

Vergleichbar häufig belegt ist das attributivische Syntagma in der zweiten Hauptgruppe der $A D \Rightarrow A D$-Übertragungsweise, in der es in qualitativem Sinne gebraucht wird. Wiederum nach inhaltlichen Kriterien unterschieden, lassen sich zunächst Fälle mit Angabe der Intensität einer visuell, akustisch u.ä. wahrnehmbaren Erscheinung aussondern. Dabei werden die diversen Qualitätsadjektive durchgängig mit der analogen deutschen Bildung wiedergegeben:

Paser zapalit lampke. Rzucala skapy blask na stól. (NOW: 35)

Na pótce nad stolem widać ustawione równo lyżki. Rozjaśnil je nikły promień $z$ trudem wlażacy z brudnego nieba. (NOW: 147)

...okno oswietlone bylo mala lampka; widac bylo nikle i migotliwe cienie, jakie padaly na sciany. (IWA: 127)
Der Hehler steckte das Lämpchen an. Es warf einen sparsamen Schein auf den Tisch. (NOW: 18)

Auf dem Spind überm Tisch waren die gleichmäßig aufgereihten Löffel zu sehen. Ein schwacher Strahl, der mühsam durch den schmutzigen Himmel drang, beleuchtete sie. (NOW: 100)

Das Fenster wurde von einer kleinen Lampe erhellt. Man sah schwache und nüchtige Schatten, die auf die Wände fielen. (IWA: 122) 
...gotujemy na małym ogniu tak dlugo, aż kasza wchlonie wode. (LEM: 32)

Nagle, daleko, uslyszeli cienki psi glos... (MIL: 96)

Wtedy poslyszeli cichy, lecz wyraźny szept $\mathrm{z}$ betonu. (NOW: 12)

Gdzies slychac staby zgrzyt klucza. Otwieraja krate. (NOW: 147)

...bo tylko kiedy scichla rozmowa kropel, ucho lowilo stabe dźwieki, dochodzace od podwórza. (IWA: 122)

...wlaściwie chcial posluchac, jak mysti i obrazy przesuwaja sie przez jego wnetrze, zostawiajac lekki zapach odurzenia. (IWA: 170)

Można też, zamiast wody, zalać kasze lekkim wywarem z grzybów (suszonych). (LEM: 31)

...mlex trzeba bardzo pomalu przy bardzo stabym wietrze, bo to nie jest wiatrak, a ruina. (FOR: 94) ...auf kleiner Flamme so lange kochen, bis die Grütze das Wasser aufgesaugt hat. (LEM: 35)

Plötzlich hörten sie ferne eine dünne Hundestimme... (MIL: 131)

Da hörten sie ein leises, aber deutliches Flüstern vom Beton. (NOW: 14)

Irgendwo hörte man schwaches Schlüsselknirschen. Das Gitter wurde geöffnet. (NOW: 100)

...nur wenn das Gespräch der Tropfen verstummte, fing das Ohr die schwachen Klänge auf, die vom Hof herüberdrangen. (IWA: 116)

...eigentlich aber wollte er zuhören, wie die Gedanken und Bilder ihn durchzogen und den leichten Duft des Berauschtseins hinterließen. (IWA: 168)

Anstatt mit Wasser kann die Grütze auch mit einem leichten Sud aus (getrockneten) Pilzen übergossen werden. (LEM: 34)

Ihr dürft nur sehr langsam und nur bei schwachem Wind mahlen, denn das ist keine Windmühle mehr, sondern eine Ruine. (FOR: 89)

Neben Substantiv- treten auch Adjektivdiminutive in analytischer Form auf, mit Gradadverbien als Attributen zur Verstärkung bzw. Einschränkung der Aussage:

"A tak, tak, prosze wujaszka...", mówil i bardzo krótko odpowiadal. (IWA: 44)

Mial...jasne wassiki, oczy male, bardzo niebieskie. (IWA: 138)

...to szla kobieta. Niska, bardzo szczupla, w starszym wieku. (FIN: 97)

Dużo sie śmiala, w uśmiechu pokazywała bardzo male i bardzo biale zeby. (FIN: 54)

Pieczeń krajemy na niezbyt cienkie
"Ach so, Onkel", sagte er und antwortete sehr knapp. (IWA: 30)

Er hatte...einen hellen Schnurrbart sowie kleine, sehr blaue Augen. (IWA: 134)

...ich sah eine Frau, klein, sehr schlank, in fortgeschrittenem Alter. (FIN: 81)

Sie lachte viel und zeigte beim Lächeln ihre sehr kleinen, sehr weißen Zāhne. (FIN: 44)

Den Braten in nicht zu dünne Scheiben schneiden (ist er als kalter Aufschnitt 
plastry (zimna na bardzo cienkie!)... (LEM: 80)

Kiedy byl zupetnie maly sadzano go na niedźwiedzim futrze... (MIL: 71)

"Masz 17 lat, ale jestes calkiem mala dziewczynka, której brak opieki i czulosci." (FIN: 75)

Zaraz za podjazdem zaczynal sie równy, dose waski kanal i ciagnal sie $w$ alei starych grabow... (IWA: 51) gedacht, allerdings in sehr dünne!)... (LEM: 84)

Als er ganz klein war, setzte man ihn auf ein Bärenfell... (MIL: 96)

"Du bist 17 Jahre alt, aber ein ganz kleines Mädchen, dem Fürsorge und Zärtlichkeit fehlen." (FIN: 154)

Hinter der Zufahrt begann ein gleichmäBiger, ziemlich schmaler Kanal, er zog sich...durch eine Allee alter Weißbuchen. (IWA: 38)

Außer der Intensitätsanzeige lassen sich analytische Diminutive (teilweise mit diminuiertem oder superlativischem Adjektiv) finden, mit denen eine Einschätzung hinsichtlich der Bedeutsamkeit einer Erscheinung oder eines Objekts geleistet wird:

Tego wieczoru w aresztach panowalo przepelnienie. Zawieziono ich autem na peryferie miasta, do malutkiego komisariatu. (NOW: 5)

Nigdy jeszcze tak wyraźnie nie pomyślal Boleslaw o malej wartosci swojego istnienia. (IWA: 116)

Dwór...skladal sie...z najbliższych zausznikow czy ministrow i zwyczajnych pochlebców, używanych do podrzednych ushug... (MIL: 62)

Tomasz nie niósl strzelby, uczestniczyl jako praktykant niskiego stopnia. (MIL: 96)

$Z$ leciutka pogarda patrzyl na biale pochylone brzozy... (IWA: 119)

Nasze zrebieta rosly bardzo ladnie, ale do roboty byly za male. Jeszcze rok, a do lekkiej pracy bedzie można zaprzegac... (FOR: 82)

[D:] Czasem prawda jest bardziej szkodliwa niż drobne klamstwo. (RUM: 6)
Den Abend herrschte in den Arrestlokalen Überfüllung. Sie wurden im Auto an den Stadtrand gefahren, in ein kleines Kommissariat. (NOW: 7)

Noch nie hatte Boleslaw so klar über den geringen Wert seiner Existenz nachgedacht. (IWA: 110)

Die Hofhaltung...bestand...aus den nächsten Vertrauten oder Ministem und aus persönlichen Schranzen, die zu untergeordneten Diensten gebraucht wurden... (MIL: 82f)

Thomas trug kein Gewehr, er nahm als Gehilfe niedrigen Grades teil. (MIL: 131)

Mit leichter Verachtung betrachtete er die weißen, geneigten Birken... (IWA: 113)

Unsere beiden Fohlen gediehen prächtig, doch zum Arbeiten konnte man sie noch nicht verwenden. In einem Jahr würden wir sie zu leichten Arbeiten einspannen können. (FOR: 76)

[D:] Die Wahrheit kann manchmal mehr Schaden anrichten als eine kleine Liige. (RUM: 7) 
Ale czul, że nie żarty, że jedna drobna nieostrożnost może caly swiat pozbawic bialych królów. (KOR: 50)

Felek wypoczywa, a Macius na niego i na siebie pracuje. Skapy zarobek nie bardzo wystarcza. (KOR: 143)

...okazuje sie, że Konstanty nie chce sie uczyc. Nuży go najmniejszy wysilek. (MIL: 52)

Powtarzaly mu najdrobniejsze jego slowa, czyny... (IWA: 42)

Troche razilo to Wiktora, że nie zdradzala najmniejszego zażenowania, że sie nawet nie specjalnie wdzieczyla do niego... (IWA: 86)
Aber er fühlte, daß dies kein Spaß war und daß eine kleine Unvorsichtigkeit die ganze Welt ihrer weißen Könige berauben könnte. (KOR: 69)

Fritz ruht sich aus, und Hänschen arbeitet für inn und für sich. Sein knapper Lohn reicht nicht hin und nicht her. (KOR: 194)

....[es] zeigt...sich, daß Konstantin nicht lemen will. Jede kleinste Anstrengung ermüdet ihn. (MIL: 69)

Sie wiederholten jedes seiner geringsten Worte, seine Taten... (IWA: 28)

Es traf Wiktor ein bißchen, daß sie nicht die geringste Befangenheit verriet und sich nicht einmal bei ihm einschmeichelte... (IWA: 75)

Gaben die vorangehenden Textstellen bei der quantitativen und qualitativen Einschätzung meist keine Wertung vor, d.h. drückten sie lediglich Geringfügigkeit, Nichtbedeutsamkeit aus, bringen die nun gesammelten Auszüge eine offensichtlich emotional-wertende Haltung des Sprechers zur Geltung. Sie dienen in pejorativer Bedeutung zum Ausdruck von Nichtigkeit, Lächerlichkeit, Verächtlichkeit, Armseligkeit u.ä., in meliorativem Sinne zur Anzeige von Bescheidenheit, Mitleid, Teilnahme, Wertschätzung u.ä.:

Niebo zalegal szafir bez chmurki od samego rana, cienie nikle tworzyly rzadka siatke pomiedzy drzewami. (IWA: 146)

Myslal, że jest jakims bezpiciowym, niemadrym stworzeniem, które nie może sie zdobyć nawet na szczuple uczucia. (IWA: 122)

Obojętnost jej wobec jego malej smierci wstrzasnela nim. (IWA: 147)

Przychodzila z rodzicami, w różowej sukience, blyszczacych lakierkach,... dzieci szly do ogrodu i tam rozpoczynalo sie znecanie nad małym, strojnym gosciem. (FIN: 94)
Wolkenloses Blau überzog seit dem frühen Morgen den Himmel, die kümmerlichen Schatten bildeten ein schütteres Netz zwischen den Bäumen. (IWA: 142)

Er dachte, er sei ein geschlechtsloses, unvernünftiges Wesen, das sich nicht einmal zu kärglichen Gefühlen aufraffen könne. (IWA: 116 )

Diese Gleichgültigkeit seinem eigenen kleinen Tod gegenüber erschütterte inn. (IWA: 144)

Sie besuchte uns mit ihren Eltern, im rosa Kleid und mit glänzenden Lackschuhen..., wir Kinder gingen in den Garten, und dort fingen wir an, den kleinen, aufgeputzten Gast zu quälen. (FIN: 131) 
Na stryszku bylo duszno, w kilku skapych metrach kwadratowych unosil sie kurz suchej slomy... (FIN: 57)

Wstapil do karczmy, zjadl skromny posilek. (KOR: 124)

...folwarczek byl malutki, ale rzadny $i$ madrze utrzymany. Dom tez niewielki, skromny, w posilkach przebijajacy sie wlasnymi srodkami... (IWA: 41)

...pito zdrowie mamy, której fiolkowa suknia nic nie zmienila skromnej miny i potulnej postaci. (IWA: 63)

"Kiedy naprawde jestes moim dzieckiem", zasmial sie, "moim kochanym, matym drieckiem..." (FIN: 5)

Matka Stefanii, drobniutka kobieta, zapalila wlasnie lampe naftowa..., pilyśmy herbate $z$ platków ogrodowych róz, wonna i blada... (FIN: 55)

Kiedy to wszystko wspominam, trudno mi uwierzyc, ze takie male, trzynastoletnie dziecko $w$ takich ciezkich warunkach życiowych pracowalo i rozwijalo sie, choc slabo roslo... (FOR: 89)
Auf dem Scheunenboden war es stickig, über den kärglichen Quadratmetern wirbelte trockener Strohstaub. (FIN: 69)

Er betrat einen Dorfkrug und $a B$ eine bescheidene Mahlzeit. (KOR: 168)

Das Gut war klein, aber in Ordnung und klug geleitet. Auch das Haus war klein, bescheiden und in puncto Ernāhrung auf die eigenen Erzeugnisse angewiesen... (IWA: 27)

Man trank auf die Gesundheit der 'Mama', deren violettes Kleid an ihrer bescheidenen Miene und demütigen Haltung nichts änderte. (IWA: 51)

"Du bist doch wirklich mein Kind", lachte er auf, "mein geliebtes, kleines Kind..." (FIN: 7)

Wir tranken duftenden und blassen Rosenblättertee..., und Stefanias Mutter, eine sehr zarte Frau, zündete gerade die Petroleumlampe an... (FIN: 45)

Wenn ich an all das zurückdenke, fällt es mir schwer zu glauben, daß̉ ein schwächliches dreizehnjähriges Kind unter solch schweren Lebensbedingungen arbeiten und sich entwickeln konnte. (FOR: 83)

Auch in diesen Fällen werden für die einzelnen Attribute die deutschen Entsprechungen gewählt.

$\boldsymbol{A D} \Rightarrow \boldsymbol{A S D}$

Die Übertragung eines analytischen Diminutivs durch ein analytisch-synthetisches ist aus Gründen der Komplettierung noch zu nennen. Der Gebrauch eines diminuierten Substantivs innerhalb der Wendung läßt sich in den verschwindend wenigen Beispielen durch kompensatorische Bestrebungen erklären: der Übersetzer reagiert nämlich auf das diminuierte Adjektiv der Vorlage, dessen Modifizierung er auf das dazugehörige Substantiv überträgt, vgl.:

I jak odróżnic istoty, które zjawily sie tutaj $z$ nastaniem chrzescijanstwa od innych, dawnych tubylcow:...od ma-
Und wie soll man die Wesen, die hier mit dem Anbruch des Christentums erschienen sind, von den anderen alt- 
lenikich ludzi, wychodzacych noca ze swoich palacow, pod korzeniami czarnego bzu? (MIL: 9f)

Przymknal oczy okolone promieniami malutkich i ostrych zmarszczek. (NOW: 54f)

Dzieciak spal. Twarz mial maleńką i czerwona, w kaciku ust sciskal gumowy smoczek. (NOW: 109)

Nikt inny nie mial prawa przyjechac. Co innego Kampanella, a co innego jakis tam nieważny krolik malego kraju. (KOR: 10) eingesessenen unterscheiden:...von dem kleinen Völkchen, das nachts aus seinen Palästen unter den Wurzeln des Holunders hervorkommt? (MIL: 8)

Er kniff die Augen zu. Strahlen kleiner und scharfer Fältchen umrandeten sie. (NOW: 31)

Der Säugling schlief. Hatte ein kleines, rotes Gesichtchen, im Mundwinkel steckte ihm ein Gummischnuller. (NOW: 63)

Kein anderer hatte das Recht, zu ihm zu kommen. Campanella bildete eine Ausnahme, aber irgend so ein unwichtiger König eines Kleinen Ländchens? (KOR: 12)

$A D \Rightarrow S D:$

Auch dieses Wiedergabemuster ist selten anzutreffen. Die vorgefundenen Textbeispiele muß man wohl auf subjektive Entscheidungen des Übersetzers zurückführen:

Matka moja przywiozla male zawiniątko $z$ odzieża... (FOR: 41 )

Każda z dziewczat przynosi malutka wiazankę ruty... (FOR: 56)

Cukier, topiac sie pod wplywem wysokiej temperatury, utworzy na powierzchni combra lekko brunatnq...powloke. (LEM: 69)
Sie brachte ein Bündelchen mit Wäsche und Kleidung mit. (FOR: 40)

Jedes Mädchen brachte ein Sträußchen Raute mit... (FOR: 53)

Der in der Hitze schmelzende Zucker bildet auf dem Fleisch eine bräunliche...Schicht. (LEM: 74)

$A D \Rightarrow N D:$

Nur unwesentlich zahlreicher als die der beiden vorangegangenen Umsetzungsmuster sind die Belege für eine nichtdiminutivische Übersetzung. Auch wenn es fast nur Einzelfalle sind, wiederholen sich hier die Übertragungsweisen des Musters SD $\Rightarrow$ ND. Am häufigsten ist das ausgangssprachliche Syntagma, um das erläuternde Attribut reduziert, mit dem substantivischen Grundwort (bei Adjektiven entsprechend mit dem adjektivischen) wiedergegeben worden: 
Tomasz z poczatku nie mógl sie oswoic $z$ mnóstwem drobnych meszek czy zuczkow, ktore lazily po nim i lechtaly. (MIL: 91)

Nabywca na nasze male gospodarst wo znalazl sie predko... (FOR: 92)

Postanowilismy z meżem kupić male Erebie, wyhodować na sprzedaż i za to oddac czesć dlugu. (FOR: 79)

[M:] Mala kawa raz, koniaczek dwa... taki wie pani, wiekszy... (NUR: 6)

Trzeba bylo dużej sily woli, aby wciaż jeszcze smiac sie i żartowac, trzymajac mala bratanice za reke. (IWA: 107)

...jasne bylo, że chce ja o cos spytac, ale nie smiala widac, bo zaraz odeszla podśpiewujac sobie bardzo cicho i wciąz spogladajac na zegarek. (FIN: 13)
Thomas konnte sich anfangs nicht an die unzāhligen Fliegen oder Käferchen gewöhnen, die auf ihm herumkrochen und kitzelten. (MIL: 125)

Einen Käufer für unsere Wirtschan fanden wir schnell. (FOR: 87)

Wir beschlossen, ein Fohlen zu kaufen, es groß zu füttern und dann zu verkaufen, um mit dem Geld die Hālfte der Schulden zu tilgen. (FOR: 72f)

[M:] Einmal Kaffee und einen Kognac ...einen großen. (NUR: 7 )

Es bedurfte großer Willenskraft, um immer weiter $\mathrm{zu}$ lachen und $\mathrm{zu}$ scherzen, während er die Nichte an der Hand hielt. (IWA: 99)

...uns war klar, da 3 sie etwas fragen wollte, sich aber anscheinend nicht getraute, denn sie ging sogleich weiter, wāhrend sie leise vor sich hinsang und ständig nach der Uhr schaute. (FIN: 20)

Seltener ist die analytische Diminutivform in ein synonymes Nomen aufgelöst oder mit Hilfe eines Kompositums ausgedrückt worden:

...najmlodsza siostra nie mogla isc $z$ nami z powodu malego dziecka. (FOR: 76)

...totez ani ja, ani moje siostry nie mialyśmy możliwosci czesto odwiedzać rodziców, bo na przeszkodzie byla praca na swoim i male dzieci. (FOR: 76)

[1:] Przydzialy dzialek budowlanych po śmiesznie malych cenach, wycieczki zagraniczne...- wszystko za pól darmo. (RUM: 12)

Mial bardzo biale wlosy $i$ jasne wasiki... (IWA: 138)
Die jüngste Schwester konnte nicht mitkommen, da sie einen Săugling hatte. (FOR: 71)

...so konnten weder ich noch meine Schwestern die Eltern öfter sehen. Wir hatten alle viel Arbeit in der eigenen Wirtschaft, und die Kleinen konnte man auch nicht so lange allein lassen. (FOR: 71)

[I:] Die Zuteilung von Bauplätzen zu Spottpreisen, die Auslandsreisen..., alles halb geschenkt. (RUM: 15)

Er hatte weißblondes Haar und einen hellen Schnurrbart... (IWA: 134) 
Beim schwach besetzten Typ der freien Wiedergabe lassen sich ebenso Übertragungsparallelen zum synthetischen Verfahren feststellen. Entweder wird das analytische Diminutiv substituiert - hier durch ein indefinites Zahladjektiv bzw. ein Indefinitpronomen -, vgl.:

Oddzielnie, w niewielkiej ilosci wody, gotujemy do miekkosci 5-8 dkg suszonych grzybow. (LEM: 198)

Postanowil isce pieszo. Niewielka suma pieniedzy wystarczy na droge. (KOR: 124)

oder paraphrasiert, d.h. in andere syntaktische Fügungen umgewandelt:

Bic dzieci nie trzeba, bo to bardziej zlosci, glodzic dzieci - jeszcze wiekssze świnstwo, bo moga zachorowac, wyrosna na słabych ludzi. (KOR: 48)

Najwiecej cierpialam nad tym, że mowiac o mnie nie zwracali na mnie najmniejszej uwagi, jakby mnie wcale nie bylo. (FOR: 51)

Przecież tyle ludzi żyje na tak małych gospodarstwach i wcale sie nie martwia. (FOR: 72)

Pólmisek ustawiamy na pare godzin na parterze lodowki, by sos nabral konsystencji delikatnej galaretki. (LEM: 195)

oder ersatzlos eliminiert:

Widziałam go w chlopskiej chacie, stojacej na przedmieściu malego miasta, w ogrodzie... (FIN: 55)
Gesondert in wenig Wasser 50-80 g getrocknete Pilze aufs Feuer stellen. (LEM: 203)

Er hatte beschlossen, zu Fuß zu gehen. Dann reicht das bißchen Geld für den Weg. (KOR: 168)
Man soll Kinder nicht schlagen, denn davon werden sie noch schlimmer, sie zur Strafe hungern zu lassen, ist eine noch größere Gemeinheit, weil sie krank und schwach werden. (KOR: 66)

Am meisten schmerzte es mich, daß sie mich gar nicht beachteten, so, als wäre ich überhaupt nicht da. (FOR: 48)

So viele Menschen lebten wie wir und machten sich nicht solche Gedanken. (FOR: 67)

Die Platte für mehrere Stunden in das unterste Kühlschrankfach stellen, damit die Sauce leicht gelien. (LEM: 200)

Ich habe ihn in der Bauernkate gesehen...; die Kate stand in der Vorstadt in einem Garten... (FIN: 45) 


\subsection{3. Übertragung analytisch-synthetischer Diminutive}

Geht man von zwei formal verschiedenen Verfahren der Diminuierung aus, dann ist auch die dritte Möglichkeit, die Kombination beider, in die Darstellung einzubeziehen. Sie kann als Variation der synthetischen Bildeweise verstanden werden, insofern als im Gegensatz zu den isoliert stehenden Suffixdiminutiven hier solche Einheiten im Quellentext erfaßt werden, die zusätzlich von - je nach Aussage - analogen Adjektiven begleitet werden.

\section{$A S D \Rightarrow A S D:$}

Nicht nur sporadisch folgt der deutsche Zieltext der im Polnischen gern genutzten sog. doppelten formal-semantischen Modifikation. Was den Gebrauch der adjektivischen Attribute angeht, so wiederholen sich die in quantitativer und qualitativer Hinsicht verringernden Epitheta der AD-Gruppe. Auch deren Realisierungsmöglichkeiten decken sich weitgehend. Es dominiert die zielsprachliche Wahl eines entsprechenden Attributs + entsprechendes Basislexem:

Rodzice matki byli urodzeni w małym miasteczku. (FOR: 41)

W małym brzozowym gaiku przy dróżce do strumienia drzewa rosly gesto... (MIL: 91)

Nagle zapalila sie przy ziemi mala, zielona lampka $i$ zobaczyl Maciuś żelazna klape. (KOR: 78 )

...25 dkg chudego wedzonego boczku (który po ugotowaniu pokrajać w drobma kosteczke i dodać do zupy). (LEM: 34)

W rondlu dusimy $w$ malej ilosci wody $z$ dodatkiem sporej lyżki masla pokrajane na drobne shupki jarzyny... (LEM: 51)

Do tego byl krótki żakiecik $z$ zielonego aksamitu, caly naszywany $w$ zlote prazki. (IWA: 162)

$\mathrm{Z}$ uwaga obserwowal tego siódmego.
Die Eltern meiner Mutter kamen in einem kleinen Städtchen zur Welt. (FOR: 41)

In einem kleinen Birkenwäldchen am Pfad, der zum Bache führte, wuchsen die Bäume dicht... (MIL: 123)

Plötzlich leuchtete am Boden ein kleines grünes Lämpchen auf, und Hänschen sah eine eiserne Falltür. (KOR: 105)

..250 g mageren geräucherten Speck (der nach dem Kochen in kleine Stückchen geschnitten und wieder zur Suppe gegeben wird)... (LEM: 37)

In einer Pfanne in wenig Wasser und einem reichlichen Löffel Butter in kleine Stäbchen geschnittenes Gemüse dünsten... (LEM: 57)

Dazu hatte sie ein kurzes, grünes Samtjäckchen mit lauter goldenen Streifen getragen. (IWA: 160)

Aufmerksam beobachtete er den Sieb- 
Byl to chudy chlopina. Kustykal... (NOW: 40)

...krótka cisza i znów walenie $w$ drzwi, dygotanie lichego domku. (FIN: 84)

...dyndala nogami nad rzeka, która nazywa sie Gniezna, marna rzeczulka, osiem metrów szerokosci... (FIN: 9)

Bez ustanku przychodzily obrazy tego co musialo sie dokonac... Jakies sutenerskie spelunki wielkich miast, mezczyźni grajacy w karty... wsród tego on, jej maly Kostus. (MIL: 54)

A przy tym Wiktor czul, ze mu sie ta mala Zosia, wówczas bardzo nieznosna, a która teraz...ogromnie podobala. (IWA: 49) ten. War ein mageres Kerlchen. Hinkte... (NOW: 23)

...eine kurze Stille und wieder das Donnern an der Tür und das Beben des kümmerlichen Häuschens... (FIN: 101)

...[sie hatte] mit den Beinen über dem Fluß gebaumelt...- er heißt Gniezna, ein kümmerliches Flüßchen, acht Meter breit... (FIN: 13)

Unablässig sah sie vor sich, was kommen mußte:...Spelunken der Großstädte, Kartenspielen der Männer,... dazwischen er, ihr kleiner Kostus. (MIL: 71)

Dabei merkte Wiktor, daB ihm diese kleine Zosia, damals ein so unleidliches Mādchen, heute...ungeheuer gefiel. (IWA: 36)

Seltener ist die Wahl von "klein" für das negierte Antonym + entsprechendes

\section{Basislexem:}

Mike'a poznalam w parku niewielkiego, pieknego miasteczka na pograniczu Alzacji. (FIN: 72)

Miasteczko znajduje sie pod Warszawa, jest nieduze, $z$ brukowanym nierowno rynkiem. (NOW: 139)

Wygrzebal kilka polamanych i skruszonych papierosow... Zapalil o niewielki kawalek siarczanej trzaski... (NOW: 5)
Mike habe ich im Park eines kleinen schönen Städtchens an der Grenze des Elsaß kennengelemt. (FIN: 149)

Das Städtchen liegt bei Warschau, ist klein, mit unregelmäßig gepflastertem Marktplatz. (NOW: 92)

Er fischte ein paar geknickte und zerkrümelte Zigaretten heraus...Zündete sie an einem Kleinen Stückchen Schwefelholz an... (NOW: 7)

Die Vorliebe des Polnischen, Kongruenz zwischen Substantiv und Adjektiv in bezug auf ihre diminutivische Form zu erzeugen, läßt sich hier wiederfinden. Die Übersetzer reagieren auf die diminuierten Adjektive entweder mit dem Basislexem oder unter Zuhilfenahme von Gradadverbien, vgl.:

...w kaszy moga sie czasem znajdować malutkje kamyczki i inne zanieczyszczenia... (LEM: 31)

Ola chudziutkie raczyny owinela naokolo jego szyi i przytulila swoj policzek do policzka stryja... (IWA: 130) ...in der Grütze können sich kleine Steinchen und andere Verunreinigungen befinden... (LEM: 34)

Ola legte ihm ihre dünnen Ärmchen um den Hals und preßte ihre Backe an die des Onkels. (IWA: 125) 
Ola wstala bez slowa i wziela go za reke. Poczul raczke chudziutka i zimna. (IWA: 101)

Malutka jest, paluszki ma malutkie i tak strasznie malo rozumie. (KOR: 74 )
Ola erhob sich wortlos und ergriff seine Hand. Er spürte ein sehr mageres und kaltes Händchen. (IWA: 93)

Klitzeklein war sie, hatte ganz kleine Fingerchen und begriff so schrecklich wenig. (KOR: 100f)

$A S D \Rightarrow A D:$

Die analytische Wiedergabe zählt innerhalb der analytisch-synthetischen Gruppe zu dem wichtigsten Muster interlingualen Austausches. Steht in der Vorlage ein durch Attribute ergänztes Suffixdiminutiv, begnügt sich der Zieltext häufig mit der einfachen Diminuierung, indem er auf die analytische Bildeweise zurückgreift und das diminuierte Nomen vernachlässigt. Dies bedeutet in der Mehrzahl der Fälle, daß das Derivat in das entsprechende Basislexem zurückgeführt und durch das zielsprachlich analoge Epitheton ergänzt wird. So können Aussagen in quantitativer Funktion (auch Angaben zum Alter) vorliegen, vgl.: Ambicja gospodyń jest maly ogrodek przy wejsciu. (MIL: 8)

Wbrew przepisom znaleźli sie razem. Celka byla mala i ciemna. (NOW: 5 )

Mieszka w malym drewniaczku naprzeciw pekinu. (NOW: 58)

...otoczeni tatarakiem wjeżdzali w male zatoczki pomiedzy trzcinami, przekradali sie z sykiem i szelestem. (IWA: 71)

Wiktor od trzech lat byl zarzadzajacym malego folwarczku, zapisanego towarzystwu ociemnialych... (IWA: 25)

...Jola miala tam swoja toalete, ale sypiala w malym saloniku za dużym salonem, na kanapie... (IWA: 36f)

...jest to roslina drobniutka..., nie wydajaca nigdy kwiecia, listeczki malutkie maja forme serca. (FOR: 56)
Der Ehrgeiz der Hausfrauen ist ein kleiner Garten vor dem Hauseingang. (MIL: 7)

Entgegen den Vorschriften blieben sie zusammen. Die Zelle war klein und finster. (NOW: 7)

Wohnt in einem kleinen Holzhaus gegenüber vom "Peking". (NOW: 34)

...von Kalmus umgeben, gelangten sie in kleine Binsenbuchten und glitten raschelnd und plätschernd dahin. (IWA: 59)

Wiktor war seit drei Jahren Verwalter eines kleinen, dem Blindenwerk überschriebenen Gutes... (IWA: 9)

Jola hatte dort ihren Toilettentisch, schlief aber auf dem Kanapee im kleinen Salon hinter dem großen Salon... (IWA: 22)

...das ist eine zarte Pflanze, die...niemals blüht und kleine herzförmige Blätter hat. (FOR: 53) 
...uczulam ogromna radosc, że w tej malenkiej ksiażeczce miesci sie taka wielka rzecz, ze te malenkie literki mówia o Frani, o owieczce. (FOR: 18)

A potem nie pamietal Macius, który jest grob kanarka, wiec wszedzie krzyzyki male wkopal. (KOR: 84)

...wskazanym zas [dodatkiem jest] peczek pokrajanej na cieniutkie plasterki rzodkiewki. (LEM: 57)

W chacie nieraz nocowali Niemcy, slyszelismy ich kroki, schodzili do piwnicy, byli tuż, za cienka scianka przesypana kartoflami. (FIN: 90)

Do ich wsi byl kawal drogi od stacji. Wpierw szlo sie mlodym laskiem. (NOW: 101)

[P:] Ciekawe...ciekawe...co też ta mloda ośbka kiedyś napisze o dzisiejszej...wigilii?... (RUM: 25)

Macius mieszkal wtedy...w chacie, gdzie byl chlopczyk taki sam jak Ala, mały... (KOR: 81)
Ich empfand eine ungeheure Freude darüber, daß in diesem kleinen Buch etwas so Großes verborgen war, daß diese kleinen Buchstaben von Frania und dem Lämmchen erzählten. (FOR: 24t)

Später wußte Hänschen nicht mehr, welches Grab dem Kanarienvogel gehörte, darum grub er überall kleine Kreuze ein. (KOR: 115)

Empfehlenswert ist auch ein Bund in dïnne Scheiben geschnittener Radieschen. (LEM: 63)

Manchmal übernachteten Deutsche in der Kate; wir hörten ihre Schritte, sie stiegen in den Keller und waren dicht hinter der dünnen Wand und dem Haufen Kartoffeln. (FIN: 128)

Bis zu ihrem Dorf war es ein ganz schöner Weg von der Bahnstation. Erst ging man durch einen jungen Wald. (NOW: 56)

[P:] Ich bin gespannt...ja...sehr gespannt... was diese junge Person...später einmal...über den heutigen Heiligabend...schreiben wird... (RUM: 30 )

Hänschen wohnte damals...in einer Hütte, in der ein ebenso kleiner Junge wie Ala lebte. (KOR: 110)

Zum anderen begegnen diminutivische Wendungen in qualitativer Sicht, z.B. als Anzeiger ärmlicher Verhältnisse, unbedeutender Objekte:

W przeciwienstwie do Wilka, Rożki byl to domek bardzo skromny $\mathrm{i}$ skromnie prowadzony. (IWA:41)

...na pietrze bylo mieszkanko...Okienka byly male i swiatla bylo w nich niewiele. (IWA: 105)

Rozstawil je na malenkiej, zgrzybialej i brzydkiej toaketce, która stala w rogu. (IWA: 101)

Stodo'ka i oborka byly to male, ledwie sklecone budyneczki... (FOR: 81)
Im Gegensatz zu Wilko war Rożki ein sehr bescheidenes und bescheiden geführtes Haus. (IWA: 27)

...im Oberstock lag die kleine Wohnung....Kleine Fenster, die wenig Licht hereinließen. (IWA: 97)

Er stellte sie auf den kleinen, uralten und häBlichen Toilettentisch, der in der Ecke stand. (IWA: 93)

Stall und Scheune waren zwei kleine, leicht gezimmerte Gebäude... (FOR: 75) 
[D:] Znowu jakas mala stacyjka... Nic nie widac. Ciemno, powygaszane latarnie. (RUM: 16)

Trudno zadac, moi panowie, żeby prosty kmiotek byl wychowawca... (KOR: 34)
[D:] Wieder eine kleine Station...Alles dunkel...Die Beleuchtung nicht eingeschaltet. (RUM: 19)

Sie können kaum verlangen, meine Herren, daß ein einfacher Bauer Erzieher ist... (KOR: 47)

Besonders beliebt ist die Indikation von Liebkosung, Mitleid u.a.:

Wosy jej jasne sterczaly jak straczki wokolo malej gl6wki... (IWA: 157)

Dziecko ciązylo mu coraz bardziej w ramionach, ale nie byl to cieżar wagi malego cialka. (FIN: 43)

...na furmance dzieci. Siedzialy na slomie, jedno przy drugim. Wygladaly jak male, szare myszki. (FIN: 101)

...a tu...siedziala srednich lat, wystrojona pani, w blekitnym, miekkim fotelu, trzymajac na kolanach malutkiego, bialego pieska. (FOR: 109)

Ale szczurek pocztowy go znalazl... Takie male stworzonko, a madrzejszy ma nos niż wszystkie oczy ludzi. (KOR: 99)

Möglich, wenn auch nur vereinzelt, ist die Kompensation eines ausgangssprachlichen Suffixdiminutivs durch ein Kompositum bzw. eine Präfixbildung: Wspinali sie, ciemnosc na zboczu gestniala, lekki wiaterek kolowal $w$ trawach. (MIL: 86)

Dom w Wilku przecinal z przedpokoju idacy ogromny korytarz. Na koncu tego korytarza byl maly ganeczek... (IWA: 64)
Ihr blondes Haar stand in Büscheln von dem kleinen Kopf ab... (IWA: 155)

Das Kind auf seinem Arm wurde immer schwerer, aber das war nicht die Last des kleinen Körpers. (FIN: 63f)

...auf dem Wagen die Kinder. Sie saßen im Stroh, eines neben dem anderen. Wie kleine graue Mäuse sahen sie aus. (FIN: 106)

...und hier...saß eine aufgeputzte Dame mittleren Alters in einem blauen, weichen Sessel und hielt einen kleinen weißen Hund auf dem SchoB. (FOR: 104)

Doch die Briefratte fand ihn...So ein kleines Geschöpf, und seine Nase ist klüger als alle Augen der Menschen. (KOR: 133)
Sie kletterten, die Dunkelheit auf dem Hang wurde dichter, leichter Windhauch kreiste in den Gräsern. (MIL: 118)

Ein breiter, vom Vorflur ausgehender Korridor teilte das Haus. An seinem Ende gab es eine kleine Vorlaube... (IWA: 52)

oder mittels eines synonymen Lexems, vgl.:

Zwrocil wzrok ku oknu. Uliczka byla waska, wciśnieta miedzy drewniaki. (NOW: 133)

Jola 2 Wiktorem postanowili popłynac mala Iodeczka wilkowska do wujostwa na kolacje. (IWA: 37)

Er wandte den Blick zum Fenster. Die Gasse war schmal, quetschte sich zwischen Holzhütten durch. (NOW: 86)

Wiktor und Jola hatten beschlossen, mit dem kleinen Wilkoer Kahn zum Abendessen zu seinem Onkel zu fahren. (IWA: 22) 
Izba od strony pieca jest przegrodzona niewysoka sciana i tworzy mala izdebke, która nazywamy alkierzem. (FOR: 15)
Durch eine niedrige Wand, die den Ofen von einem Teil der Stube trennte, war eine kleine Kammer entstanden, die wir Alkoven nannten. (FOR: 21)

Nur wenige Beispiele sind aus der adjektivischen Diminution anzuführen. Bei den Detensiva entgegnet das Deutsche mal mit dem Grundwort, mal mit einem Kompositum für den zweiten Bestandteil der attributivischen Wortfolge:

Obudzilo go w ciemnosci, ledwo szarawej, stukanie w szybe. (MIL: 95)
In der kaum grau werdenden Dunkelheit wurde er von einem Klopfen ans Fenster wach. (MIL: 129f)

Gesondert eine helle, leicht goldgelbe Mehlschwitze...zubereiten. (LEM: 159)

Bei den Intensiva wird auf das Derivat lediglich mit dem analogen Basislexem reagiert:

"Moja wysepka ma tylko trzy kilometry, jest wiec bardzo malenka." (KOR: 6)

"Pamietam ciebie zupetnie malutka, mialas zawsze przestraszone oczy..." (IWA: 52)

"Cicho, kochanie", odezwala sie kobieta, "leż cichutko, calkiem cichutko..." (FIN: 42)
"Mein Inselchen ist nur drei Kilometer lang, also sehr klein." (KOR: 7)

"Ich weiß noch, als du ganz klein warst, hattest du immer so erschreckte Augen..." (IWA: 38)

"Still, Liebling", sagte die Frau, "sei still, ganz still..." (FIN: 62)

\section{$A S D \Rightarrow S D:$}

So gängig die analytische Übertragungsweise einer ASD-Einheit war, so unüblich ist die durch ein synthetisches Diminutiv. In nur ganz wenigen Fällen verzichtet der deutsche Text auf das attributivische Adjektiv:

Kaczor na mete wracal sam. Od stacji isc musial niedlugi kawalek. (NOW: 122)

Zdyszana stoje przy zielonym plotku, miejskiej zagrodzie, która zamyka dziesiec maleńkich stolików... (FIN: 85)

A on mówil dużo trzymajac w reku te małą kolorowa ksiazzeczkę. (FOR: 17)
Kaczor kam allein nach Haus. Von der Station mußte er ein Stückchen laufen. (NOW: 75)

Außer Atem stehe ich an dem grunen Zaun, der Einfriedung, die zehn Tischchen umschlieBt. (FIN: 101)

Und nun sprach er so lange und hielt das Büchlein in der Hand. (FOR: 23)

Als Typ für die Wiedergabe analytisch-synthetischer Diminutive von vergleichbar marginaler Bedeutung sind auch die beiden letzten Muster, da mit der 
doppelten Diminuierung eine zweifache Markierung gesetzt wird, der der Zieltext eigentlich kaum ausweichen kann:

$A S D \Rightarrow N D:$

Folgende Belege sind zu nennen: Ale mala stracila swoja ufnosć do stryja i bardzo chlodno przyjela jego zainteresowania maka lalka... (IWA: 127)

...wymyla twarz, zobaczyla sie w mahym husterku... (FOR: 40)

...mieso, gotowane w kapuśniaku lub barszczu, kraja na male kawaleczki. Podaje sie je w małych miseczkach. (FOR: 67)

Doch hatte die Kleine das Vertrauen zu ihrem Onkel verloren. Sie reagierte sehr kühl auf sein Interesse an inrer...Puppe.... (IWA:122)

Sie... wusch sich das Gesicht und besah sich im Spiegel. (FOR: 40)

Das in der Suppe gekochte Fleisch wurde in kleine Stücke geschnitten und ebenfalls in Schüsseln gereicht. (FOR: 63)

Die ausgangssprachliche Fügung wird lediglich in das zugrundeliegende Basislexem komprimiert.

$A S D \Rightarrow f r W:$

Dieser Typ ist mit folgenden Textauszügen vorzustellen:

A wiec siadam kolo swej palki i krece male wrzecionko. (FOR: 17)

Waza potlukla sie na drobne kawalki, Macius zakrwawil sobie reke. (KOR: 11)

Plakala cicho, bezglosnie, lzy splywaly waska strużka po policzkach... (FIN: 88)

...na kókka kladziemy kupke i formujemy make, zgrabne pierożki, silnie je brzegami zlepiajac... (LEM: 55)
Ich setzte mich neben meinen "Spinnstock" und spann. (FOR: 23)

Die Vase zersplitterte, Hänschen blutete die Hand... (KOR: 13)

Sie weinte leise, lautlos, die Tränen liefen ihr über die Backen... (FIN: 125)

...etwas Füllung daraufgeben und die Ränder fest zusammendrücken...(LEM: 61)

Wiederum umgeht der deutsche Text die diminutivische Einheit, einmal durch Paraphrasierung, ein anderes Mal schlichtweg durch Eliminierung. 


\subsubsection{Diminutivische Übertragung von Nichtdiminutiven}

Nicht zu vernachlässigen ist schließlich die letzte Großgruppe, in der sich die Verhältnisse umgekehrt haben. Die vorgefundenen diminuierten Formen sind hier als Antworten auf nichtdiminutivische Textstellen des Originals zu verstehen.

$N D \Rightarrow S D:$

In der Hauptsache begegnen in diesem Muster Fälle, in denen ein modifiziertes Substantiv als Reaktion auf das entsprechende Grundwort gewählt wurde:

$W$ jelniaku tuż koło domu znajdowalo sie we mchach tyle rydzów, że starczalo na cale kosze. (MIL: 93)

Dopiero po poludniu zwlókl sie $z$ twardego stolka, nie dotknal obiadu zostawionego na stole $\mathrm{i}$ poszedl na karczowisko. (IWA: 153)

"Jak mialam w puszce wode, sterte korzonkow, kartofli, to bylam spokojna." (FIN: 93)

Puderniczka w reku Henryka, pochylenie glowy ku lusterku, musniecie puszkiem nosa. (FIN: 86)

Kiedy ja spotrzegl[!], siedziala już wsparta o szoferke i miala zamkniete oczy. (FIN: 27)

Zjadl Macius chleb, dwa serdelki, siedzi w krzakach i mysli, co robić. (KOR: 32)

[K:] Prosze wode...- [M:] Po takiej wodzie, to humoru nie ma. Tylko babelków w nos najdzie. (NUR: 6)
Im Tannenwäldchen nah am Haus gab es so viele Reizker, daß man Körbe füllen könnte. (MIL: 127)

Erst am Nachmittag erhob er sich von dem harten Hocker, rührte das auf dem Tischchen bereitgestellte Mittagessen nicht an und begab sich zur Rodung. (IWA: 150)

"Das Wasser in der Büchse, ein Häufchen Wurzeln und Kartoffeln, das beruhigte mich." (FIN: 137)

Das Puderdöschen in Henryks Hand, der Kopf über das Spiegelchen gebeugt, die Quaste streift die Nase. (FIN: 103)

Als er sie entdeckte, saß sie bereits an das Fahrerhäuschen gelehnt und hatte die Augen geschlossen. (FIN: 87)

Hänschen $\mathrm{a} B$ das Brot und zwei Würstchen, saß im Gebüsch und dachte nach, was er tun sollte. (KOR: 44)

[K:] Bitte, Ihr Wasser...- [M:] Wenn man nur so ein Wässerchen trinkt, ist's kein Wunder, wenn man zu nichts mehr Lust hat..., im Grunde kitzeln einen nur die Blasen in der Nase. (NUR: 7f)

Besonders beliebt ist diese Entsprechung bei der Bezeichnung von etwas an sich schon Kleinem oder kurz Dauerndem:

Do korica świata nie odkryje sie po nim nic, prócz imiena na skrawku pergaminu... (MIL: 98)
Bis an das Ende der Welt wird man nichts von ihm entdecken außer dem 
Namen auf einem Stückchen Pergament... (MIL: 134)

Spostrzegla, ze na nia patrzy, uśmiechnela sie i strzepnela dlonia kruszyny cukru ze stolu. (IWA: 68)

"Dlaczego?" zainteresowal sie bezmyslnie ten w kracie, wytracony $z$ drzemki. (NOW: 7 )

Przez chwile kaszke gotujemy na malym ogniu... (LEM: 37)

Auch bei den folgenden ausgangssprachlichen Simplizia ist wohl der ihnen anhaftende verkleinernde bzw. kosende Charakter dafür ausschlaggebend gewesen, daß der Zieltext mit einem passenden suffigierten Lexem entgegnet hat, vgl.:

Wiele czasu zużyl, kiedy już chodzil, na zwiedzanie izb i zakamarkow. (MlL: 12)

Oprócz niego lubily ten zakątek duże rude mrówki... (MIL: 46)

Podloga...zrobiona byla $z$ blachy, pod nia palono ogien a niedźwiedzie puszczano w drewnianych chodakach. Grala muzyka, blacha parzyla i biedne misie stawaly, bo przednie lapy zostawiano bose. (MIL: 72)

Trzyczwartaka w domu nie bylo, Cyganeczka spala, a bachor w lóżku w ogóle sie nie liczyl. (NOW: 125)
Sie bemerkte, daß er sie ansah, lächelte und wischte mit der Hand ein Krümelchen Zucker vom Tisch. (IWA: 56)

"Wieso?" interessierte sich sinnlos der im Karo, aus einem Nickerchen gerissen. (NOW: 8)

Auf kleiner Flamme ein Weilchen kochen... (LEM: 40) 
Wprawdzie to moze nie tak zaraz nastapi, dzis czuje sie doskonale. (IWA: 162)

...każdy nie rozbierajac sie kladl sie byle jak, jak stal, gdziekolwiek, aby odpoczac. (FOR: 63)
Zwar könne das ein Weilchen dauern, heute gehe es ihm ausgezeichnet. (IWA: 160)

Jeder suchte sich ein Plätzchen und legte sich gleich angekleidet hin, um ein wenig auszuruhen. (FOR: 60)

Von dieser Wiedergabeart betroffen sind auch Adjektive bzw. Adverbien. Einerseits lassen sich Derivate für ein Basislexem der Vorlage finden, das eine Farbe oder andere Eigenschaften bezeichnet. Die Abweichung in den ersten drei Beispielen dürfte ihren Grund darin haben, daß der Deutsche aus seinem Weltverständnis gelbe Schlagsahne seltsam findet; auch weiße Wasserflächen und grüne Himmelsflecken sind zumindest von fragwürdiger Akzeptabilität, vgl:

...a chociaż niebo jaśnialo, niebieskie stawy i wszystkie wody, jakie spotykal po drodze, byly biale. (IWA: 46)

Ale $w$ tej chwili przyszla ciotka z taca: rzeczywiscie, jechala czekolada $z$ źótym kremem. (IWA: 60)

Trzeciego dnia przed wieczorem ukazaly sie na zachodzie niebieskie i zielone platy nieba i deszcz przestal padac. (IWA: 127)

Z uwaga...patrzyl na jego mala, okragla postac. (NOW: 99)

...a że przy tym miala w reku wysoka trzcinowa laseczke, nadawalo jej to wyrazu nieoczekiwanie starczego. (IWA: 50)

...a kiedy urodzilam synka i trzeba bylo go ochrzcic, to nie mialam go $w$ co zawinac: byly tylko nedzne laszki. (FOR: 95)

Powiedzial mu to ktos zupelnie bezstronny, którys z kolegów. (IWA: 45) ...und trotz des blauen Himmels glänzten Teiche und alle Wasserflächen, an denen er vorbeikam, weißlich. (IWA: 32)

Doch in diesem Moment kam die Tante mit einem Tablett herein; wie erwartet, brachte sie Schokolade mit gelblicher Schlagsahne. (IWA: 48)

Am dritten Tag zeigten sich im Westen bläuliche und grüliche Himmelsflekken, und der Regen lieB nach. (IWA: 121f)

Aufmerksam...musterte er seine kleine, rundliche Gestalt. (NOW: 54)

Weil sie zudem in der Hand einen langen Rohrstock trug, wirkte sie unerwartet ältlich. (IWA: 37)

Und als ich einem Söhnchen das Leben schenkte und wir ihn taufen lassen wollten, wußte ich nicht, worin ich ihn einwickeln sollte. Ich hatte nur ärmliche Fetzen. (FOR: 90)

Gesagt wurde ihm das von einem gänzlich Unparteiischen, einem seiner Kollegen... (IWA: 31)

Andererseits antwortet der Zieltext diminutivisch hauptsächlich auf Bezeichnungen für Zwischenfarbtöne der Ausgangssprache, die mit einem eigenen Lexem belegt sind: 
Wal tutaj brunatny od opadlych igiel zniżal sie lagodnie w zielona kotlinke... (MIL: 96)

Karol byl rozczochrany, nos mial siny bardziej niż zwykle. (NOW: 62)

Tak to rozmyslajac stanalem nad rowem oddzielajacym ruda, jesienna trawe od wyboistej, jezdnej drogi. (FIN: 69)
Die Böschung, an dieser Stelle bräunlich von abgefallenen Nadeln, senkte sich sanft in eine grünes Tālchen... (MIL: 131)

Karol war ungekämmt, seine Nase bläulicher als gewöhnlich. (NOW: 38)

Dies überlegend, blieb ich an dem Graben stehen, der das rötliche, herbstliche Gras von der mit Schlaglöchern übersăten Landstraße trennte. (FIN: 112)

$N D \Rightarrow A D:$

Die Entscheidung für ein attributivisches Syntagma ist vornehmlich dadurch bestimmt, daß ein Lexem des Quellentextes Kleinheit im weitesten Sinne implizit enthält, z.B.:

...póńniej spostrzeżono, że zboże... przynosi duze zyski i ze oplaca sie $z$ lasu wycinać poletka. (MIL: 13)

Dolem biegla ciemna, pusta ulica, drzewa skweru przypominaly czame, rozczochrane lby. (FIN: 5)

Zbieral żoledzie, patyki, robil ogródek kolo muru... (KOR: 18 )

Palce coraz wiecej bolaly, bo paznokcie od skrobania sie poscieraly, sporo bylo zadrapan na calej rece. (KOR: 18)

Zgodnie z życzeniem Waszej Królewskiej Mosci przywiozlem dziesieciu wyrostk6w dla osobistej ochrony... (KOR: 86)

...später wurde man gewahr, daß das ...Korn große Gewinne bringe und daß es sich rentierte, aus dem Walde kleine Flächen herauszuschlagen. (MIL: 13)

Unter ihnen zog sich die dunkle, leere Straße hin, die Bäume des kleinen Platzes erinnerten an schwarze, zerzauste Köpfe. (FIN: 7)

Er sammelte Eichein und kleine Stökke, legte sich an der Mauer einen Garten an... (KOR: 24)

Die Finger schmerzten immer mehr, denn die Nägel waren von dem vielen Kratzen schon ganz abgewetzt, er hatte lauter kleine Wunden auf der ganzen Hand. (KOR: 24)

Auf den Wunsch Eurer Majestät habe ich zehn junge Burschen hergebracht zum persönlichen Schutz... (KOR: 117)

Aber auch umschreibende Wendungen sind Anlaß für eine analytische Wiedergabe:

Najpierw napomykali mu niesmialo, ogólnie, krażac dokola sedna, używajac porównan i przypowiesci. (MIL: 42)
Zunächst machten sie ihm gegenüber nur Andeutungen, kurze, schüchterne, ganz allgemein gehaltene, sie gingen 
...każda cos doradzala, cos wpinala i ani sie spostrzegtam, a już bylam w spódniczce, fartuszku i kaftaniku z bialego płótna... (FOR: 61)

"No, po podróży trzeba cos zjeśc", powiada. I zaprosil Maciusia do swego dziwnego w latarni mieszkania. (KOR: 73)

$N D \Rightarrow A S D:$ um den Kern herum, führten Vergleiche und Anekdoten an. (MIL: 54)

Jede riet mir etwas, heftete etwas fest, und in kurzer Zeit schon stand ich fertig in Rock, Schürzchen und weißem Leinenmieder da. (FOR: 58)

"Na, nach der Reise kann ein kleiner Imbiß nichts schaden", sagte er. Und dann bat er Hänschen in seine seltsame Wohnung im Leuchtturm. (KOR: 99)

Ohne Relevanz ist das letzte Übertragungsmuster mit lediglich einem Beispiel:

[M:] Czego ty ode mnie chcesz? Czy nie moglabys zostawic mnie choc na chwile w spokoju? (TON: 2)
[M:] Was willst du von mir? Kannst du mir nicht wenigstens für ein kleines Weilchen mei Ruh lassen? (TON: 2)

Wie die Systematisierung des in Form von Wiedergabetypen gesammelten Diminutivmaterials gezeigt hat, kann die Realisierung eines sprachlichen Elements der einen Sprache mit unterschiedlichen Mitteln der anderen Sprache erreicht werden; sie kann von der formalen Analogie bis hin zu kompensatorischen Verfahren verschiedener Art reichen. Faßt man die beobachteten interlingualen Übersetzungsprozeduren zusammen, dann gehört es zur Ausnahme, wenn Deckungsgleichheit auf allen linguistischen Ebenen erzielt werden kann. Will der Übersetzer nicht gegen das Postulat der funktionellen Äquivalenz verstoßen, dann ist es obligatorisch, Veränderungen im Bereich der Grammatik, Lexik und des Stils, weniger der Semantik im Zieltext vorzunehmen. Im Anschluß an das Modell der sog. Transpositionskategorien lassen sich für die Übertragung von Diminutiven die grammatischen Transpositionstypen der Substitution, Kompression und Expansion angeben. ${ }^{13}$ Während mit der Substitution eine formale Umstrukturierung des Zieltextes vollzogen wird, d.h. ein

13 Zur klassifikatorischen Grundlage vgl. BAUSCH 1968, der die bis dahin gültigen Definitionen der herkömmlichen vergleichenden Stilistik (sog. "stilistique comparée" mit J.P.Vinay und J.Darbelnet als den wichtigsten Vertretern) überprüft und die Transpositionskategorie neu zu bestimmen versucht hat. Einen nuancierten Klassifizierungsvorschlag der Transpositionstypen und -arten, der für alle potentiellen lexikalischen Einheiten angelegt ist, unterbreitet KOSTA 1986; zu seiner Beschreibung, Klassifizierung und Modellierung der Übersetzungsverfahren vgl. dort Kap.II, bes. S.289-359. 
Wechsel der "grammatischen und/oder syntaktischen Kategorie/Struktur" ${ }^{4}$, wird mit den Verfahren der Kompression und Expansion zum einen die (implizierende) Reduktion, zum anderen die (explizierende) Erweiterung eines Strukturelements in formaler und inhaltlicher Hinsicht herbeigeführt.

14 KOSTA 1986. S.312. 
Der Katalogisierung der Umsetzungstypen, die nur mit ausgesuchten Textbeispielen belegt wurde, soll nun eine vollständige zahlenmäßige Erfassung und Auswertung des empirisch gegebenen Diminutivmaterials in tabellarischer Form folgen. Diese statistischen Beobachtungen können dazu dienen, Gesetzmäßigkeiten bei Verwendung und Übertragung dieser sprachlichen Erscheinung zu formulieren, was im singulären, punktuellen Hinblick darauf nicht zu leisten ist.

Zunächst gilt es, einen Verteilungsschlüssel für alle 18 vorgestellten Übersetzungstypen zu ermitteln, auf der Grundlage von Textstellenpaaren, in denen mindestens ein Diminutiv zu verzeichnen ist, genauer, der Ausgangssprache ein Diminutiv zugrunde liegt und/oder die Zielsprache diminutivisch reagiert.

In die Statistik sollen zum einen Substantive, zum anderen Adjektive/Adverbien/Pronomen Eingang finden; Personen- und Verwandtschaftsnamen werden nicht berücksichtigt. Ohne Zweifel stellen sie für die Erörterung des Diminutivphänomens wertvolles Anschauungsmaterial und gewähren Einblick in die Gebrauchsgewohnheiten beider Sprachen, doch ihre uneinheitliche, oft arbiträre Übertragungsweise weicht charakteristisch von der des appellativischen Wortschatzes ab und läßt daher keine brauchbaren statistisch-systematischen Aussagen $z{ }^{15}$ Begleitet wird dies durch den Umstand, daß Namen naturgemäß in Texten ständig wiederholt werden müssen und mit ihrer Aufnahme der Gesamteindruck der Statistik einseitig verzerrt würde.

Die Erfassung der vorgegebenen Beobachtungsdaten erfolgt in absoluten und prozentualen Angaben. Grundlage dieses Zahlenmaterials bilden alle gezählten Belege, mit anderen Worten, es wird nicht die einmalige Nennung, sondern das mehrfache Auftreten einer gleichen Bildung registriert.

is Genaueres dazu vgl. weiter unten S.237ff. 
4.2.1. Verteilung der Belege auf die Wiedergabetypen:

Polnisch $\Rightarrow$ Deutsch

An den Anfang der statistischen Analyse soll die aus den zugrundegelegten acht polnischen Werken zusammengetragene Gesamttabelle gestellt werden, ${ }^{16}$ die für die dann folgenden Einzeltabellen Richtwerte geben kann, anhand derer die jeweiligen Abweichungen vom Mittelwert festgestellt und beurteilt werden können.

Tab.1: Gesamtdarstellung polnischer Werke und ihrer deutschen Übersetzungen (total: 2928 Diminutive, davon 2662 Substantive, 266 Adjektive/Adverbien/Pronomen) ${ }^{17}$

\begin{tabular}{|l|l|r|r|r|r|}
\hline \multicolumn{2}{|c|}{$\begin{array}{c}\text { WIEDERGABE- } \\
\text { TYP }\end{array}$} & \multicolumn{2}{c|}{ SUBSTANTIVE } & \multicolumn{2}{c|}{ ADJEKTIVE ETC. } \\
\cline { 3 - 7 } I. & SD $\Rightarrow$ SD & 447 & 16,79 & 26 & 9,77 \\
\cline { 3 - 7 } & SD $\Rightarrow$ AD & 93 & 3,49 & 21 & 7,9 \\
\cline { 3 - 7 } & SD $\Rightarrow$ ASD & 9 & 0,34 & - & - \\
\cline { 3 - 7 } & SD $\Rightarrow$ ND & 1307 & 49,1 & 148 & 55,64 \\
\cline { 3 - 7 } & SD $\Rightarrow$ frW & 135 & 5,07 & 7 & 2,63 \\
\cline { 3 - 7 } & & 1991 & 74,79 & 202 & 75,94 \\
\hline \multirow{2}{*}{ II. } & AD $\Rightarrow$ AD & 284 & 10,67 & 11 & 4,14 \\
\cline { 3 - 7 } & AD $\Rightarrow$ ASD & 5 & 0,19 & 2 & 0,75 \\
\cline { 3 - 7 } & AD $\Rightarrow$ SD & 3 & 0,11 & 1 & 0,38 \\
\cline { 3 - 7 } & AD $\Rightarrow$ ND & 14 & 0,53 & 2 & 0,75 \\
\cline { 3 - 7 } & AD $\Rightarrow$ frW & 23 & 0,86 & - & - \\
\cline { 3 - 7 } & & 329 & 12,36 & 16 & 6,02 \\
\hline
\end{tabular}

${ }^{16}$ Genaueres zum Umfang des exzerpierten Textmaterials vgl. weiter oben S.14.

17 Wegen notwendiger Auf- bzw. Abrundungen können die Summen der Prozentwerte gelegentlich nicht exakt 100\% betragen. 


\begin{tabular}{|c|c|c|c|c|c|}
\hline \multirow{2}{*}{\multicolumn{2}{|c|}{$\begin{array}{c}\text { WIEDERGABE- } \\
\text { TYP } \\
\end{array}$}} & \multicolumn{2}{|c|}{ SUBSTANTIVE } & \multicolumn{2}{|c|}{ ADJEKTIVE ETC. } \\
\hline & & Belegzahl & $\%$ & Belegzahl & $\%$ \\
\hline \multirow[t]{6}{*}{ III. } & \multirow{6}{*}{$\begin{array}{l}\text { ASD } \Rightarrow \text { ASD } \\
\text { ASD } \Rightarrow \text { AD } \\
\text { ASD } \Rightarrow \text { SD } \\
\text { ASD } \Rightarrow \text { ND } \\
\text { ASD } \Rightarrow \text { frW }\end{array}$} & 44 & 1,65 & - & - \\
\hline & & 123 & 4,62 & 6 & 2,26 \\
\hline & & 9 & 0,34 & - & - \\
\hline & & 10 & 0,38 & 1 & 0,38 \\
\hline & & 5 & 0,19 & - & - \\
\hline & & 191 & 7,18 & 7 & 2,64 \\
\hline \multirow[t]{4}{*}{ IV. } & \multirow{4}{*}{$\begin{array}{l}N D \Rightarrow S D \\
N D \Rightarrow A D \\
N D \Rightarrow A S D\end{array}$} & 127 & 4,77 & 41 & 15,41 \\
\hline & & 23 & 0,86 & - & - \\
\hline & & 1 & 0,04 & - & - \\
\hline & & 151 & 5,67 & 41 & 15,41 \\
\hline
\end{tabular}

Die Gesamtbestandsaufnahme bestätigt schon auf den ersten Blick die grundlegenden Erwartungen. Bei Berücksichtigung der drei möglichen Diminutionsverfahren ergab sich eine unterschiedliche Verteilung auf die achtzehn möglichen Wiedergabetypen. Dabei lassen sich drei Frequenzstufen erkennen:

1. Mit Werten um $50 \%$ dominiert in der Substantiv- und Adjektivgruppe die Übertragung eines Suffixdiminutivs durch ein Nichtdiminutiv (SD $\Rightarrow N D$ ). In großem Abstand folgt dann die Übersetzung eines Suffixdiminutivs mit dem entsprechenden Wortbildungsformans ( $S D \Rightarrow S D$ ). Als dritthäufigster Typ ist das Muster $A D \Rightarrow A D$ bei den Substantiven und ND $\Rightarrow$ SD bei Adjektiven etc. noch zur Hauptfrequenzstufe zu zählen.

2. Zur mittleren Stufe gehören die Wiedergabetechniken mit erheblich geringerer Häufigkeit, wie: $S D \Rightarrow$ frW, $N D \Rightarrow S D, A S D \Rightarrow A D, S D \Rightarrow A D$ bei den Substantiven sowie $S D \Rightarrow A D, A D \Rightarrow A D, S D \Rightarrow f r W, A S D \Rightarrow A D$ bei den Adjektiven etc.

3. Zuletzt sind die verbliebenen elf Übersetzungstypen zu nennen, deren Frequenz in der Regel unter $1 \%$ liegt oder die erst gar nicht auftreten.

Zieht man die Zwischensummen heran, werden die Erwartungen hinsichtlich der Grundtendenzen im gegebenen Vergleich am offensichtlichsten belegt. Es zeigt sich, daß der polnisch-deutsche Diminutivaustausch zum überragenden Teil (74,79\%) durch die Übersetzung eines polnischen Suffixdiminutivs geprägt ist, während der am wenigsten wahrscheinliche Fall $(5,67 \%)$ das Einbringen eines 
deutschen Diminutivs für einen nichtdiminutivischen Ausdruck des Polnischen darstellt.

Zwei zunächst nicht zu erwartende Auffälligkeiten sind noch herauszustellen: zum einen überrascht der geringe Anteil des Typs $S D \Rightarrow A D$, wo doch angesichts der Fülle von Modifikationsderivaten des Polnischen im Deutschen auf umschreibend zusammengesetzte Bildungen hätte zurückgegriffen werden können. Zum anderen erstaunt die besonders bei Adjektiven relativ hohe Belegzahl des ND $\Rightarrow$ SD-Musters angesichts der oben genannten klaren Unterrepräsentiertheit diminutivischer Reaktion im Deutschen.

Die Einzeltabellen bieten folgendes Bild:

Tab.2: MILOSZ, "Dolina Issy" (total: 547 Dim., davon 520 Subst., 27 Adj.)

\begin{tabular}{|c|c|c|c|c|c|}
\hline \multirow{2}{*}{\multicolumn{2}{|c|}{$\begin{array}{c}\text { WIEDERGABE- } \\
\text { TYP }\end{array}$}} & \multicolumn{2}{|c|}{ SUBSTANTIVE } & \multicolumn{2}{|c|}{ ADJEKTIVE ETC. } \\
\hline & & Belegzahl & $\%$ & Belegzahl & $\%$ \\
\hline \multirow[t]{6}{*}{ I. } & \multirow{6}{*}{$\begin{array}{l}S D \Rightarrow S D \\
S D \Rightarrow A D \\
S D \Rightarrow A S D \\
S D \Rightarrow N D \\
S D \Rightarrow \text { frW }\end{array}$} & 159 & 30,58 & 6 & 22,22 \\
\hline & & 23 & 4,42 & 2 & 7,41 \\
\hline & & 3 & 0,58 & - & - \\
\hline & & 192 & 36,92 & 9 & 33,33 \\
\hline & & 18 & 3,46 & 1 & 3,7 \\
\hline & & 395 & 75,96 & 18 & 66,66 \\
\hline \multirow[t]{6}{*}{ II. } & \multirow{6}{*}{$\begin{array}{l}A D \Rightarrow A D \\
A D \Rightarrow A S D \\
A D \Rightarrow S D \\
A D \Rightarrow N D \\
A D \Rightarrow f r W\end{array}$} & 55 & 10,58 & 2 & 7,41 \\
\hline & & 1 & 0,19 & - & - \\
\hline & & 1 & 0,19 & - & - \\
\hline & & 1 & 0,19 & - & - \\
\hline & & 1 & 0,19 & - & - \\
\hline & & 59 & 11,34 & 2 & 7,41 \\
\hline \multirow[t]{6}{*}{ III. } & \multirow{6}{*}{$\begin{array}{l}A S D \Rightarrow A S D \\
A S D \Rightarrow A D \\
A S D \Rightarrow S D \\
A S D \Rightarrow N D \\
A S D \Rightarrow \text { frW }\end{array}$} & 8 & 1,54 & - & - \\
\hline & & 8 & 1,54 & 1 & 3,7 \\
\hline & & 1 & 0,19 & - & - \\
\hline & & - & - & - & - \\
\hline & & - & - & - & - \\
\hline & & 17 & 3,27 & 1 & 3,7 \\
\hline
\end{tabular}




\begin{tabular}{|l|l|r|r|r|r|}
\hline \multicolumn{2}{|c|}{$\begin{array}{c}\text { WIEDERGABE- } \\
\text { TYP }\end{array}$} & \multicolumn{2}{c|}{ SUBSTANTIVE } & \multicolumn{2}{c|}{ ADJEKTIVE ETC. } \\
\cline { 3 - 6 } & Belegzahl & $\%$ & Belegzahl & $\%$ \\
\hline IV. & ND $\Rightarrow$ SD & 44 & 8,46 & 6 & 22,22 \\
\cline { 3 - 6 } & ND $\Rightarrow$ AD & 5 & 0,96 & - & - \\
\cline { 3 - 6 } & ND $\Rightarrow$ ASD & - & - & - & - \\
\cline { 3 - 6 } & & 49 & 9,42 & 6 & 22,22 \\
\hline
\end{tabular}

Gleich die erste zu behandelnde Einzeltabelle bietet auch die stärksten Abweichungen von den durch die Gesamttabelle vorgegebenen Positionsparametern. ${ }^{18}$ Auch wenn der Typ SD $\Rightarrow$ ND wie dort die erste Position einnimmt, so ist sein Anteil mit 36,92\% doch erheblich geringer, was auf die spektakuläre Verschiebung des Gewichts zugunsten des Musters $\mathrm{SD} \Rightarrow \mathrm{SD}$ zurückzuführen ist, das sich gegenüber dem Durchschnittswert sogar verdoppelt zeigt. Die überdurchschnittliche Neigung, Suffixdiminutive im Zieltext zu gebrauchen, beweist sich erneut darin, daß sich auch der ND $\Rightarrow$ SD-Typ in seiner Quantität fast dupliziert. Da der vorliegende Quellentext eine überproportional hohe Anzahl von Diminutiven aufweist, hat er einen nicht unerheblichen Einfluß auf die Gesamtstatistik. Doch verändert er nicht die Grundtendenzen, er verzerrt sie lediglich, so daß, bezieht man ihn in die Statistik nicht ein, sich die entscheidenden Parameter wie folgt verändern:

$$
\begin{aligned}
& \text { SD } \Rightarrow \text { SD: } 16,79 \% \text { sinkt auf } 13,45 \% \\
& \text { SD } \Rightarrow \text { ND: } 49,10 \% \text { steigt auf } 52,05 \% \\
& \text { ND } \Rightarrow \text { SD: } \quad 4,77 \% \text { sinkt auf } \quad 3,87 \%
\end{aligned}
$$

18 Die folgenden Beobachtungen zu den Einzeltabellen beziehen sich zunächst immer auf die Substantive; Adjektive sollen aus methodischen Gründen an späterer Stelle in einem besprochen werden. 
Tab.3: NOWAKOWSKI, "Ten stary ztodziej" (total: 407 Dim., dawon 367 Subst., 40 Adj.)

\begin{tabular}{|c|c|c|c|c|c|}
\hline \multirow{2}{*}{\multicolumn{2}{|c|}{$\begin{array}{l}\text { WIEDERGABE- } \\
\text { TYP }\end{array}$}} & \multicolumn{2}{|c|}{ SUBSTANTIVE } & \multicolumn{2}{|c|}{ ADJEKTIVE ETC. } \\
\hline & & \multirow{2}{*}{$\begin{array}{r}\text { Belegzahl } \\
45 \\
\end{array}$} & \multirow{2}{*}{$\frac{\%}{12,26}$} & \multirow{2}{*}{$\begin{array}{r}\text { Belegzahl } \\
11 \\
\end{array}$} & \multirow{2}{*}{$\%$} \\
\hline I. & $\mathbf{S D} \Rightarrow S D$ & & & & \\
\hline & $S D \Rightarrow A D$ & 5 & 1,36 & - & - \\
\hline & SD $\Rightarrow$ ASD & - & - & - & - \\
\hline & $S D \Rightarrow N D$ & 208 & 56,68 & 26 & 65,0 \\
\hline & $S D \Rightarrow f r W$ & 12 & 3,27 & - & - \\
\hline & & 270 & 73,57 & 37 & 92,5 \\
\hline \multirow[t]{6}{*}{ II. } & \multirow{6}{*}{$\begin{array}{l}A D \Rightarrow A D \\
A D \Rightarrow A S D \\
A D \Rightarrow S D \\
A D \Rightarrow N D \\
A D \Rightarrow \text { frW }\end{array}$} & 49 & 13,35 & - & - \\
\hline & & 3 & 0,82 & - & - \\
\hline & & - & - & - & - \\
\hline & & - & - & - & - \\
\hline & & 3 & 0,82 & - & - \\
\hline & & 55 & 14,99 & - & - \\
\hline \multirow[t]{6}{*}{ III. } & \multirow{6}{*}{$\begin{array}{l}\text { ASD } \Rightarrow \text { ASD } \\
\text { ASD } \Rightarrow \text { AD } \\
\text { ASD } \Rightarrow \text { SD } \\
\text { ASD } \Rightarrow \mathrm{ND} \\
\text { ASD } \Rightarrow \mathrm{frW}\end{array}$} & 7 & 1,91 & - & - \\
\hline & & 14 & 3,81 & - & - \\
\hline & & 2 & 0,54 & - & - \\
\hline & & - & - & - & - \\
\hline & & - & - & - & - \\
\hline & & 23 & 6,26 & - & - \\
\hline \multirow[t]{4}{*}{ IV. } & \multirow{4}{*}{$\begin{array}{l}N D \Rightarrow S D \\
N D \Rightarrow A D \\
N D \Rightarrow A S D\end{array}$} & 19 & 5,18 & 3 & 7,5 \\
\hline & & - & - & - & - \\
\hline & & - & - & - & - \\
\hline & & 19 & 5,18 & 3 & 7,5 \\
\hline
\end{tabular}

Vergleicht man diese Ergebnisse besonders mit den soeben korrigierten Prozentangaben der Gesamtstatistik, wird offensichtlich, daß sie im Durchschnittsbereich liegen. 
Tab.4: IWASZKJEWICZ, "Panny z Wilka"/"Brzezina" (total: 333 Dim, davon 274 Subst., 59 Adj.)

\begin{tabular}{|c|c|c|c|c|c|}
\hline \multirow{2}{*}{\multicolumn{2}{|c|}{$\begin{array}{l}\text { WIEDERGABE- } \\
\text { TYP }\end{array}$}} & \multicolumn{2}{|c|}{ SUBSTANTIVE } & \multicolumn{2}{|c|}{ ADJEKTIVE ETC. } \\
\hline & & Belegzahl & $\%$ & Belegzahl & $\%$ \\
\hline \multirow[t]{6}{*}{ I. } & \multirow{6}{*}{$\begin{array}{l}\mathrm{SD} \Rightarrow \mathrm{SD} \\
\mathrm{SD} \Rightarrow \mathrm{AD} \\
\mathrm{SD} \Rightarrow \mathrm{ASD} \\
\mathrm{SD} \Rightarrow \mathrm{ND} \\
\mathrm{SD} \Rightarrow \mathrm{frW}\end{array}$} & 29 & 10,58 & 5 & 8,47 \\
\hline & & 14 & 5,11 & 5 & 8,47 \\
\hline & & - & - & - & - \\
\hline & & 138 & 50,36 & 24 & 40,68 \\
\hline & & 11 & 4,01 & 2 & 3,39 \\
\hline & & 192 & 70,06 & 36 & 61,01 \\
\hline \multirow[t]{6}{*}{ II. } & \multirow{6}{*}{$\begin{array}{l}A D \Rightarrow A D \\
A D \Rightarrow A S D \\
A D \Rightarrow S D \\
A D \Rightarrow N D \\
A D \Rightarrow \text { frW }\end{array}$} & 40 & 14,6 & 4 & 6,78 \\
\hline & & - & - & 2 & 3,39 \\
\hline & & - & - & - & - \\
\hline & & 1 & 0,36 & 1 & 1,69 \\
\hline & & - & - & - & - \\
\hline & & 41 & 14,96 & 7 & 11,86 \\
\hline \multirow[t]{6}{*}{ III. } & \multirow{6}{*}{$\begin{array}{l}\text { ASD } \Rightarrow \text { ASD } \\
\text { ASD } \Rightarrow \text { AD } \\
\text { ASD } \Rightarrow \text { SD } \\
\text { ASD } \Rightarrow \text { ND } \\
\text { ASD } \Rightarrow \mathrm{frW}\end{array}$} & 4 & 1,46 & - & - \\
\hline & & 28 & 10,22 & 1 & 1,69 \\
\hline & & - & - & - & - \\
\hline & & 1 & 0,36 & - & - \\
\hline & & - & - & - & - \\
\hline & & 33 & 12,04 & 1 & 1,69 \\
\hline \multirow[t]{4}{*}{ IV. } & \multirow{4}{*}{$\begin{array}{l}\text { ND } \Rightarrow \text { SD } \\
\text { ND } \Rightarrow \text { AD } \\
\text { ND } \Rightarrow \text { ASD }\end{array}$} & 7 & 2,55 & 15 & 25,42 \\
\hline & & 1 & 0,36 & - & - \\
\hline & & - & - & - & - \\
\hline & & 8 & 2,91 & 15 & 25,42 \\
\hline
\end{tabular}

Ungewöhnlich erscheint hier lediglich die recht hohe Frequenz des Typs ASD $\Rightarrow$ AD. Sie könnte auf die Vorliebe des polnischen Autors für die analytisch-synthetische Diminution zurückzuführen sein. 
Tab.5: KORCZAK, "Krol Macius na wyspie bezludnej" (total: 591 Dim., dawn 565 Subst., 26 Adj.)

\begin{tabular}{|c|c|c|c|c|c|}
\hline \multirow{2}{*}{\multicolumn{2}{|c|}{$\begin{array}{c}\text { WIEDERGABE- } \\
\text { TYP }\end{array}$}} & \multicolumn{2}{|c|}{ SUBSTANTIVE } & \multicolumn{2}{|c|}{ ADJEKTIVE ETC. } \\
\hline & & \multirow{2}{*}{$\begin{array}{r}\text { Belegzahl } \\
94\end{array}$} & \multirow{2}{*}{16,64} & \multirow{2}{*}{$\begin{array}{r}\text { Belegzahl } \\
-\end{array}$} & \multirow{2}{*}{$\%$} \\
\hline I. & $S D \Rightarrow S D$ & & & & \\
\hline & \multirow{5}{*}{$\begin{array}{l}S D \Rightarrow A D \\
S D \Rightarrow A S D \\
S D \Rightarrow N D \\
S D \Rightarrow \text { frW }\end{array}$} & 16 & 2,83 & 6 & 23,08 \\
\hline & & 2 & 0,35 & - & - \\
\hline & & 308 & 54,51 & 16 & 61,54 \\
\hline & & 22 & 3,89 & 1 & 3,85 \\
\hline & & 442 & 78,22 & 23 & 88,47 \\
\hline \multirow[t]{6}{*}{ II. } & \multirow{6}{*}{$\begin{array}{l}\mathrm{AD} \Rightarrow \mathrm{AD} \\
\mathrm{AD} \Rightarrow \mathrm{ASD} \\
\mathrm{AD} \Rightarrow \mathrm{SD} \\
\mathrm{AD} \Rightarrow \mathrm{ND} \\
\mathrm{AD} \Rightarrow \mathrm{frW}\end{array}$} & 53 & 9,38 & - & - \\
\hline & & 1 & 0,18 & - & - \\
\hline & & - & - & - & - \\
\hline & & 1 & 0,18 & - & - \\
\hline & & 3 & 0,53 & - & - \\
\hline & & 58 & 10,27 & - & - \\
\hline \multirow[t]{6}{*}{ III. } & \multirow{6}{*}{$\begin{aligned} & A S D \Rightarrow A S D \\
& A S D \Rightarrow A D \\
& A S D \Rightarrow S D \\
& A S D \Rightarrow \mathrm{ND} \\
& \text { ASD } \Rightarrow \mathrm{frW}\end{aligned}$} & 11 & 1,95 & - & - \\
\hline & & 19 & 3,36 & 2 & 7,69 \\
\hline & & 3 & 0,53 & - & - \\
\hline & & 2 & 0,35 & - & - \\
\hline & & 1 & 0,18 & - & - \\
\hline & & 36 & 6,37 & 2 & 7,69 \\
\hline \multirow[t]{4}{*}{ IV. } & \multirow{4}{*}{$\begin{array}{l}\text { ND } \Rightarrow \mathrm{SD} \\
\mathrm{ND} \Rightarrow \mathrm{AD} \\
\mathrm{ND} \Rightarrow \mathrm{ASD}\end{array}$} & 20 & 3,54 & 1 & 3,85 \\
\hline & & 9 & 1,59 & - & - \\
\hline & & - & - & - & - \\
\hline & & 29 & 5,13 & 1 & 3,85 \\
\hline
\end{tabular}

Auch der dieser Tabelle zugrundeliegende Text weist dem Durchschnitt entsprechende Daten auf. 
Tab.6: FINK, "Skrawek czasu" (total: 308 Dim., dawon 270 Subst., 38 Adj.)

\begin{tabular}{|c|c|c|c|c|c|}
\hline \multirow{2}{*}{\multicolumn{2}{|c|}{$\begin{array}{c}\text { WIEDERGABE- } \\
\text { TYP }\end{array}$}} & \multicolumn{2}{|c|}{ SUBSTANTIVE } & \multicolumn{2}{|c|}{ ADJEKTIVE ETC. } \\
\hline & & Belegzahl & $\%$ & Belegzahl & $\%$ \\
\hline \multirow[t]{6}{*}{ I. } & \multirow{6}{*}{$\begin{array}{l}\mathrm{SD} \Rightarrow \mathrm{SD} \\
\mathrm{SD} \Rightarrow \mathrm{AD} \\
\mathrm{SD} \Rightarrow \mathrm{ASD} \\
\mathrm{SD} \Rightarrow \mathrm{ND} \\
\mathrm{SD} \Rightarrow \mathrm{frW}\end{array}$} & 46 & 17,04 & 1 & 2,63 \\
\hline & & 15 & 5,55 & 7 & 18,42 \\
\hline & & - & - & - & - \\
\hline & & 133 & 49,26 & 19 & 50,0 \\
\hline & & 5 & 1,85 & - & - \\
\hline & & 199 & 73,7 & 27 & 71,05 \\
\hline \multirow[t]{6}{*}{ II. } & \multirow{6}{*}{$\begin{array}{l}A D \Rightarrow A D \\
A D \Rightarrow A S D \\
A D \Rightarrow S D \\
A D \Rightarrow N D \\
A D \Rightarrow \text { frW }\end{array}$} & 32 & 11,85 & 3 & 7,89 \\
\hline & & - & - & - & - \\
\hline & & - & - & - & - \\
\hline & & - & - & 1 & 2,63 \\
\hline & & 1 & 0,37 & - & - \\
\hline & & 33 & 12,22 & 4 & 10,52 \\
\hline \multirow[t]{6}{*}{ III. } & \multirow{6}{*}{$\begin{array}{l}\mathbf{A S D} \Rightarrow \mathbf{A S D} \\
\mathrm{ASD} \Rightarrow \mathrm{AD} \\
\mathrm{ASD} \Rightarrow \mathrm{SD} \\
\mathrm{ASD} \Rightarrow \mathrm{ND} \\
\mathrm{ASD} \Rightarrow \mathrm{frW}\end{array}$} & 5 & 1,85 & - & - \\
\hline & & 16 & 5,92 & 1 & 2,63 \\
\hline & & 1 & 0,37 & - & - \\
\hline & & - & - & - & - \\
\hline & & 2 & 0,74 & - & - \\
\hline & & 24. & 8,88 & 1 & 2,63 \\
\hline \multirow[t]{4}{*}{ IV. } & \multirow{4}{*}{$\begin{array}{l}\mathbf{N D} \Rightarrow \mathbf{S D} \\
\mathbf{N D} \Rightarrow \mathbf{A D} \\
\mathbf{N D} \Rightarrow \mathbf{A S D}\end{array}$} & 12 & 4,44 & 6 & 15,79 \\
\hline & & 2 & 0,74 & - & - \\
\hline & & - & - & - & - \\
\hline & & 14 & 5,18 & 6 & 15,79 \\
\hline
\end{tabular}

Auch diese Zusammenstellung zeigt keine Auffalligkeiten. 
Tab.7: FORNALSKA, "Pamietnik matki" (total: 387 Dim., davon 346 Subst., 41 Adj.)

\begin{tabular}{|c|c|c|c|c|c|}
\hline \multirow{2}{*}{\multicolumn{2}{|c|}{$\begin{array}{l}\text { WIEDERGABE- } \\
\text { TYP }\end{array}$}} & \multicolumn{2}{|c|}{ SUBSTANTIVE } & \multicolumn{2}{|c|}{ ADJEKTIVE ETC. } \\
\hline & & \multirow{2}{*}{$\begin{array}{r}\text { Belegzahl } \\
43\end{array}$} & \multirow{2}{*}{$\%$} & \multirow{2}{*}{$\begin{array}{r}\text { Belegzahl } \\
-\end{array}$} & \multirow{2}{*}{$\%$} \\
\hline I. & $S D \Rightarrow S D$ & & & & \\
\hline & \multirow{5}{*}{$\begin{array}{l}S D \Rightarrow A D \\
S D \Rightarrow A S D \\
S D \Rightarrow N D \\
S D \Rightarrow \text { frW }\end{array}$} & 8 & 2,31 & 1 & 2,44 \\
\hline & & 3 & 0,87 & - & - \\
\hline & & 133 & 38,44 & 28 & 68,29 \\
\hline & & 50 & 14,45 & 2 & 4,88 \\
\hline & & 237 & 68,5 & 31 & 75,61 \\
\hline \multirow[t]{6}{*}{ II. } & \multirow{6}{*}{$\begin{array}{l}\mathrm{AD} \Rightarrow \mathrm{AD} \\
\mathrm{AD} \Rightarrow \mathrm{ASD} \\
\mathrm{AD} \Rightarrow \mathrm{SD} \\
\mathrm{AD} \Rightarrow \mathrm{ND} \\
\mathrm{AD} \Rightarrow \mathrm{frW}\end{array}$} & 35 & 10,12 & - & - \\
\hline & & - & - & - & - \\
\hline & & 2 & 0,58 & - & - \\
\hline & & 9 & 2,6 & - & - \\
\hline & & 10 & 2,89 & - & - \\
\hline & & 56 & 16,19 & - & - \\
\hline \multirow[t]{6}{*}{ III. } & \multirow{6}{*}{$\begin{array}{l}\mathrm{ASD} \Rightarrow \mathrm{ASD} \\
\mathrm{ASD} \Rightarrow \mathrm{AD} \\
\mathrm{ASD} \Rightarrow \mathrm{SD} \\
\mathrm{ASD} \Rightarrow \mathrm{ND} \\
\mathrm{ASD} \Rightarrow \mathrm{frW}\end{array}$} & 1 & 0,29 & - & - \\
\hline & & 21 & 6,07 & - & - \\
\hline & & 2 & 0,58 & - & - \\
\hline & & 7 & 2,02 & 1 & 2,44 \\
\hline & & 2 & 0,58 & - & - \\
\hline & & 33 & 9,54 & 1 & 2,44 \\
\hline \multirow[t]{4}{*}{ IV. } & \multirow{4}{*}{$\begin{array}{l}\mathrm{ND} \Rightarrow \mathrm{SD} \\
\mathrm{ND} \Rightarrow \mathrm{AD} \\
\mathrm{ND} \Rightarrow \mathrm{ASD}\end{array}$} & 14 & 4,05 & 9 & 21,95 \\
\hline & & 6 & 1,73 & - & - \\
\hline & & - & - & - & - \\
\hline & & 20 & 5,78 & 9 & 21,95 \\
\hline
\end{tabular}

Hervorzuheben ist die Verlagerung der Belege von der nichtdiminutivischen zur freien Wiedergabe von Suffixdiminutiven. Obwohl es in seinen Möglichkeiten steht, äquivalent zu übertragen, weicht der Übersetzer auf die ungebundene, freie Umsetzung aus. 
Tab.8: NUROWSKA, "Oferta": RUMEL, "Oddychaj ponad czasem"; TONECKI, "Wosy pana dziedzica" (total: 128 Dim., dawn 119 Subst., 9 Adj.)

\begin{tabular}{|c|c|c|c|c|c|}
\hline \multirow{2}{*}{\multicolumn{2}{|c|}{$\begin{array}{l}\text { WIEDERGABE- } \\
\text { TYP }\end{array}$}} & \multicolumn{2}{|c|}{ SUBSTANTIVE } & \multicolumn{2}{|c|}{ ADJEKTIVE ETC. } \\
\hline & & Belegzahl & $\%$ & Belegzahl & $\%$ \\
\hline \multirow[t]{6}{*}{ I. } & \multirow{6}{*}{$\begin{array}{l}S D \Rightarrow S D \\
S D \Rightarrow A D \\
S D \Rightarrow A S D \\
S D \Rightarrow N D \\
S D \Rightarrow \text { frW }\end{array}$} & 19 & 15,97 & 2 & 22,22 \\
\hline & & 12 & 10,08 & - & - \\
\hline & & 1 & 0,84 & - & - \\
\hline & & 65 & 54,62 & 6 & 66,66 \\
\hline & & 3 & 2,52 & 1 & 11,11 \\
\hline & & 100 & 84,03 & 9 & 99,99 \\
\hline \multirow[t]{6}{*}{ II. } & \multirow{6}{*}{$\begin{array}{l}A D \Rightarrow A D \\
A D \Rightarrow A S D \\
A D \Rightarrow S D \\
A D \Rightarrow N D \\
A D \Rightarrow f r W\end{array}$} & 6 & 5,04 & - & - \\
\hline & & - & - & - & - \\
\hline & & - & - & - & - \\
\hline & & 2 & 1,68 & - & - \\
\hline & & - & - & - & - \\
\hline & & 8 & 6,72 & - & - \\
\hline \multirow[t]{6}{*}{ III. } & \multirow{6}{*}{$\begin{array}{l}\text { ASD } \Rightarrow \text { ASD } \\
\text { ASD } \Rightarrow \text { AD } \\
\text { ASD } \Rightarrow \text { SD } \\
\text { ASD } \Rightarrow \text { ND } \\
\text { ASD } \Rightarrow \text { frW }\end{array}$} & 2 & 1,68 & - & - \\
\hline & & 3 & 2,52 & - & - \\
\hline & & - & - & - & - \\
\hline & & - & - & - & - \\
\hline & & - & - & - & - \\
\hline & & 5 & 4,2 & - & - \\
\hline \multirow[t]{4}{*}{ IV. } & \multirow{4}{*}{$\begin{array}{l}\mathrm{ND} \Rightarrow \mathrm{SD} \\
\mathrm{ND} \Rightarrow \mathrm{AD} \\
\mathrm{ND} \Rightarrow \mathrm{ASD}\end{array}$} & 5 & 4,2 & - & - \\
\hline & & - & - & - & - \\
\hline & & 1 & 0,84 & - & - \\
\hline & & 6 & 5,04 & . & - \\
\hline
\end{tabular}

Entgegen den bisherigen Zahlenverhältnissen findet eine überproportional häufige Wiedergabe eines Derivats durch eine analytische Fügung statt, die dem oben bemerkten auffallend niedrigen Nachweis des SD $\Rightarrow$ AD-Typs widerspricht. Demgegenüber unterrepräsentiert ist das $\mathrm{AD} \Rightarrow \mathrm{AD}$-Muster. 
Tab.9: LEMNISNITRY, "W staropolskiej kuchni" (total: 227 Dim., davon 201 Subst., 26 Adj.)

\begin{tabular}{|c|c|c|c|c|c|}
\hline \multirow{2}{*}{\multicolumn{2}{|c|}{$\begin{array}{c}\text { WIEDERGABE- } \\
\text { TYP }\end{array}$}} & \multicolumn{2}{|c|}{ SUBSTANTIVE } & \multicolumn{2}{|c|}{ ADJEKTIVE ETC. } \\
\hline & & Belegzahl & $\mathscr{\%}$ & Belegzahl & $\%$ \\
\hline \multirow[t]{6}{*}{ I. } & \multirow{6}{*}{$\begin{array}{l}S D \Rightarrow S D \\
S D \Rightarrow A D \\
S D \Rightarrow A S D \\
S D \Rightarrow N D \\
S D \Rightarrow \text { frW }\end{array}$} & 12 & 5,97 & 1 & 3,85 \\
\hline & & - & - & - & - \\
\hline & & - & - & - & - \\
\hline & & 130 & 64,68 & 20 & 76,92 \\
\hline & & 14 & 6,97 & - & - \\
\hline & & 156 & 77,62 & 21 & 80,77 \\
\hline \multirow[t]{6}{*}{ II. } & \multirow{6}{*}{$\begin{array}{l}A D \Rightarrow A D \\
A D \Rightarrow A S D \\
A D \Rightarrow S D \\
A D \Rightarrow N D \\
A D \Rightarrow \text { frW }\end{array}$} & 14 & 6,97 & 2 & 7,69 \\
\hline & & - & - & - & - \\
\hline & & - & - & 1 & 3,85 \\
\hline & & - & - & - & - \\
\hline & & 5 & 2,49 & - & - \\
\hline & & 19 & 9,46 & 3 & 11,54 \\
\hline \multirow[t]{6}{*}{ III. } & \multirow{6}{*}{$\begin{array}{l}A S D \Rightarrow A S D \\
A S D \Rightarrow A D \\
A S D \Rightarrow S D \\
A S D \Rightarrow N D \\
A S D \Rightarrow \text { frW }\end{array}$} & 6 & 2,99 & - & - \\
\hline & & 14 & 6,97 & 1 & 3,85 \\
\hline & & - & - & - & - \\
\hline & & - & - & - & - \\
\hline & & - & - & - & - \\
\hline & & 20 & 9,96 & 1 & 3,85 \\
\hline \multirow[t]{4}{*}{ IV. } & \multirow{4}{*}{$\begin{array}{l}\text { ND } \Rightarrow \text { SD } \\
\text { ND } \Rightarrow A D \\
\text { ND } \Rightarrow \text { ASD }\end{array}$} & 6 & 2,99 & 1 & 3,85 \\
\hline & & - & - & - & - \\
\hline & & - & - & - & - \\
\hline & & 6 & 2,99 & 1 & 3,85 \\
\hline
\end{tabular}

Die hervorstechendsten Veränderungen zeigen sich in der I.Gruppe. Dem geringen Anteil an Übertragungen durch Suffixdiminutive und der völligen Abwesenheit des SD $\Rightarrow$ AD-Typs steht die gewichtige statistische Präsenz der Übersetzung durch Nichtdiminutive gegenüber. 
Faßt man die Adjektivspalten der Einzeltabellen in einem Überblick zusammen, zeigt sich, daß allein der Typ SD $\Rightarrow$ ND konstant hohe Werte aufweist, ansonsten aber die Tabellen durch starke Heterogenität gekennzeichnet sind und damit keinesfalls solch eindeutige Rückschlüsse zulassen wie bei der Analyse der Substantivkolumnen. Eine Erklärung dieses Umstandes kann darin gesucht werden, daß die absolute Zahl der Belege recht niedrig ist, damit jede einzelne Stelle relativ stark ins Gewicht fallt und die Statistik nachhaltig beeinflussen kann. Einen weiteren Anhaltspunkt bietet die den Adjektivderivaten zugrundeliegende Differenzierung in Intensiva und Detensiva, Sprachmittel, die keineswegs beiden Sprachen im gleichen Maße zur Verfügung stehen. Somit stehen die Parameter in unmittelbarer Abhängigkeit von der jeweiligen sprachlichen Realisierung dieser Einheiten im Ausgangstext.

\subsubsection{Verteilung der Belege auf die Wiedergabetypen: Deutsch $\Rightarrow$ Polnisch}

Auf eine erneute Vorstellung der Wiedergabetypen mit Hilfe repräsentativer Beispiele ist an dieser Stelle verzichtet worden, da sich erwartungsgemäß bei der Spiegelung des Sprachenpaares bekannte Übersetzungsmuster wieder einstellen. Ein gravierender Unterschied besteht jedoch in der quantitativen Konstellation, weswegen eine statistische Beschreibung in ausreichendem Maße Aufschluß über die Umsetzungsverhältnisse geben kann. Die Aufstellung der Ergebnisse soll nun analog zur obigen Vorgehensweise erfolgen: 
Tab.10: Gesamidarstellung deutscher Werke und ihrer polnischen Ubersetzungen (total: 1873 Diminutive, davon 1755 Substantive, 118 Adjektive/Adverbien/Pronomen)

\begin{tabular}{|c|c|c|c|c|c|}
\hline \multirow{2}{*}{\multicolumn{2}{|c|}{$\begin{array}{c}\text { WIEDERGABE- } \\
\text { TYP }\end{array}$}} & \multicolumn{2}{|c|}{ SUBSTANTIVE } & \multicolumn{2}{|c|}{ ADJEKTIVE ETC. } \\
\hline & & \multirow{2}{*}{$\begin{array}{r}\text { Belegzahl } \\
162\end{array}$} & \multirow{2}{*}{$\frac{\%}{9,23}$} & \multirow{2}{*}{$\begin{array}{r}\text { Belegzahl } \\
21\end{array}$} & \multirow{2}{*}{$\frac{\%}{17,8}$} \\
\hline I. & $S D \Rightarrow S D$ & & & & \\
\hline & \multirow{5}{*}{$\begin{array}{l}S D \Rightarrow A D \\
S D \Rightarrow A S D \\
S D \Rightarrow N D \\
S D \Rightarrow \text { frW }\end{array}$} & 5 & 0,29 & - & - \\
\hline & & 5 & 0,29 & - & - \\
\hline & & 52 & 2,96 & 13 & 11,02 \\
\hline & & 17 & 0,97 & 1 & 0,85 \\
\hline & & 241 & 13,74 & 35 & 29,67 \\
\hline \multirow[t]{6}{*}{ II. } & \multirow{6}{*}{$\begin{array}{l}A D \Rightarrow A D \\
A D \Rightarrow A S D \\
A D \Rightarrow S D \\
A D \Rightarrow N D \\
A D \Rightarrow \text { frW }\end{array}$} & 340 & 19,37 & 10 & 8,48 \\
\hline & & 98 & 5,58 & 4 & 3,39 \\
\hline & & 40 & 2,28 & 10 & 8,48 \\
\hline & & 37 & 2,11 & 4 & 3,39 \\
\hline & & 24 & 1,37 & - & - \\
\hline & & 539 & 30,71 & 28 & 23,74 \\
\hline \multirow[t]{6}{*}{ III. } & \multirow{6}{*}{$\begin{array}{l}A S D \Rightarrow A S D \\
A S D \Rightarrow A D \\
A S D \Rightarrow S D \\
A S D \Rightarrow N D \\
A S D \Rightarrow f r W\end{array}$} & 32 & 1,82 & - & - \\
\hline & & 6 & 1,34 & - & - \\
\hline & & 11 & 0,63 & - & - \\
\hline & & 2 & 0,11 & 2 & 1,7 \\
\hline & & - & - & - & - \\
\hline & & 51 & 2,9 & 2 & 1,7 \\
\hline \multirow[t]{4}{*}{ IV. } & \multirow{4}{*}{$\begin{array}{l}\mathrm{ND} \Rightarrow \mathrm{SD} \\
\mathrm{ND} \Rightarrow \mathrm{AD} \\
\mathrm{ND} \Rightarrow \mathrm{ASD}\end{array}$} & 886 & 50,48 & 51 & 43,22 \\
\hline & & 33 & 1,88 & 2 & 1,7 \\
\hline & & 5 & 0,29 & - & - \\
\hline & & 924 & 52,65 & 53 & 44,92 \\
\hline
\end{tabular}

Die Bestandsaufnahme bietet auf einer Grundlage von diesmal 17 Mustern (bei den Substantiven) drei auffällige Werte: Es dominiert mit ca. der Hälfte aller Belege (Substantive 50,48\%, Adjektive etc. 43,22\%) die suffixale Umsetzung eines Basislexems der Ausgangssprache (ND $\Rightarrow$ SD). Bei den Substantiven folgt dann mit Abstand die analytische Diminuierung in beiden Sprachen (AD $\Rightarrow A D$ ) 
und schließlich die synthetische (SD $\Rightarrow$ SD). Mit Ausnahme des Typs AD $\Rightarrow$ ASD sind alle übrigen Wiedergabetechniken statistisch zu vernachlässigen. Die weitere Verteilung bei den Adjektiven weicht davon ab, indem als zweithäufigster Typ $S D \Rightarrow S D$, gefolgt von $S D \Rightarrow N D$, begegnet.

Richtet man den Blick auf die Zwischensummen und zieht zum Vergleich die Gesamttabelle des polnisch-deutschen Austausches (Tab.1) heran, so zeigt sich erwartungsgemäß die Umkehrung der Gewichtung, wenn auch nicht so kraß: mit $52,65 \%$ ist die Gruppe IV nicht so prägnant wie Gruppe I in Tab.1, mit 13,74\% fallt umgekehrt Gruppe I nicht so stark ab wie Gruppe IV in Tab.1. Die Abwesenheit der ausschlieBlichen Vorherrschaft einer Übersetzungstypgruppe geht einher mit einer nicht unerheblichen Nutzung der analytischen Bildeweise in der Ausgangssprache (Gruppe II: 30,71\% im Vergleich zu 12,36\% in Tab.1). Ferner wird die Feststellung des geringen Gebrauchs suffixaler Diminutive im Original durch die niedrige Frequenz der Gruppe III $(2,9 \%)$ bekräftigt.

Tab.11: FRISCH, "Stiller" (total: 199 Dim., davon 180 Subst., 19 Adj.)

\begin{tabular}{|c|c|c|c|c|c|}
\hline \multirow{2}{*}{\multicolumn{2}{|c|}{$\begin{array}{l}\text { WIEDERGABE- } \\
\text { TYP }\end{array}$}} & \multicolumn{2}{|c|}{ SUBSTANTIVE } & \multicolumn{2}{|c|}{ ADJEKTIVE ETC. } \\
\hline & & Belegzahl & $\%$ & Belegzahl & $\%$ \\
\hline \multirow[t]{6}{*}{ I. } & \multirow{6}{*}{$\begin{array}{l}\mathrm{SD} \Rightarrow \mathrm{SD} \\
\mathrm{SD} \Rightarrow \mathrm{AD} \\
\mathrm{SD} \Rightarrow \mathrm{ASD} \\
\mathrm{SD} \Rightarrow \mathrm{ND} \\
\mathrm{SD} \Rightarrow \mathrm{frW}\end{array}$} & 38 & 21,11 & 11 & 57,89 \\
\hline & & 2 & 1,11 & - & - \\
\hline & & - & - & - & - \\
\hline & & 4 & 2,22 & 5 & 26,32 \\
\hline & & 2 & 1,11 & - & - \\
\hline & & 46 & 25,55 & 16 & 84,21 \\
\hline \multirow[t]{6}{*}{ II. } & \multirow{6}{*}{$\begin{array}{l}A D \Rightarrow A D \\
A D \Rightarrow A S D \\
A D \Rightarrow S D \\
A D \Rightarrow N D \\
A D \Rightarrow \text { frW }\end{array}$} & 43 & 23,89 & 2 & 10,53 \\
\hline & & 5 & 2,77 & - & - \\
\hline & & 2 & 1,11 & - & - \\
\hline & & 7 & 3,89 & - & $=$ \\
\hline & & 2 & 1,11 & - & - \\
\hline & & 59 & 32,77 & 2 & 10,53 \\
\hline
\end{tabular}




\begin{tabular}{|c|c|c|c|c|c|}
\hline \multirow{2}{*}{\multicolumn{2}{|c|}{$\begin{array}{c}\text { WIEDERGABE- } \\
\text { TYP }\end{array}$}} & \multicolumn{2}{|c|}{ SUBSTANTIVE } & \multicolumn{2}{|c|}{ ADJEKTIVE ETC. } \\
\hline & & \multirow{2}{*}{$\begin{array}{r}\text { Belegzahl } \\
1 \\
\end{array}$} & \multirow{2}{*}{$\%$} & \multirow{2}{*}{ Belegzahl } & \multirow{2}{*}{$\%$} \\
\hline III. & $\mathrm{ASD} \Rightarrow \mathrm{ASD}$ & & & & \\
\hline & \multirow{5}{*}{$\begin{array}{l}\mathrm{ASD} \Rightarrow \mathrm{AD} \\
\mathrm{ASD} \Rightarrow \mathrm{SD} \\
\mathrm{ASD} \Rightarrow \mathrm{ND} \\
\mathrm{ASD} \Rightarrow \mathrm{fr} W\end{array}$} & - & - & - & - \\
\hline & & - & - & - & - \\
\hline & & - & - & - & - \\
\hline & & - & - & - & - \\
\hline & & 1 & 0,56 & - & - \\
\hline \multirow[t]{4}{*}{ IV. } & \multirow{4}{*}{$\begin{array}{l}\mathrm{ND} \Rightarrow \mathrm{SD} \\
\mathrm{ND} \Rightarrow \mathrm{AD} \\
\mathrm{ND} \Rightarrow \mathrm{ASD}\end{array}$} & 67 & 37,22 & 1 & 5,26 \\
\hline & & 7 & 3,89 & $=$ & - \\
\hline & & - & - & - & - \\
\hline & & 74 & 41,11 & 1 & 5,26 \\
\hline
\end{tabular}

Hier wie im folgenden soll Gewicht auf die Zahlenverhältnisse bei den Substantiven gelegt werden. Der Text von Max Frisch und seine Übersetzung weichen von der vorangestellten Übersicht an zwei charakteristischen Stellen ab. Die starke Präsenz des SD $\Rightarrow$ SD-Typs läßt die angesprochene Diskrepanz zum ND $\Rightarrow$ SD-Typ beinahe verschwinden. Ein Grund mag in dem Herkunftsidiom des Autors liegen, der durch die häufige Verwendung von Suffixderivaten der ansonsten diminutivschöpferischen Disposition der Zielsprache vorwegkommt.

Tab.12: HEY, "Engelmacher \& Co." (total: 200 Dim., dawn 192 Subst., 8 Adj.)

\begin{tabular}{|l|l|r|r|r|r|}
\hline \multicolumn{2}{|c|}{$\begin{array}{c}\text { WIEDERGABE- } \\
\text { TYP }\end{array}$} & \multicolumn{2}{c|}{ SUBSTANTIVE } & \multicolumn{2}{c|}{ ADJEKTIVE ETC. } \\
\cline { 3 - 6 } & Belegzahl & $\%$ & Belegzahl & $\%$ \\
\hline I. & SD $\Rightarrow$ SD & 7 & 3,65 & 3 & 37,5 \\
\cline { 3 - 6 } & SD $\Rightarrow$ AD & - & - & - & - \\
\cline { 3 - 6 } & SD $\Rightarrow$ ASD & - & - & - & - \\
\cline { 3 - 6 } & SD $\Rightarrow$ ND & 3 & 1,56 & 2 & 25,0 \\
\cline { 3 - 6 } & SD $\Rightarrow$ frW & - & - & - & - \\
\cline { 3 - 6 } & & 10 & 5,21 & 5 & 62,5 \\
\hline
\end{tabular}




\begin{tabular}{|c|c|c|c|c|c|}
\hline \multirow{2}{*}{\multicolumn{2}{|c|}{$\begin{array}{c}\text { WIEDERGABE- } \\
\text { TYP } \\
\end{array}$}} & \multicolumn{2}{|c|}{ SUBSTANTIVE } & \multicolumn{2}{|c|}{ ADJEKTIVE ETC. } \\
\hline & & \multirow{2}{*}{$\begin{array}{r}\text { Belegzahl } \\
42 \\
\end{array}$} & \multirow{2}{*}{$\%$} & \multirow{2}{*}{$\begin{array}{r}\text { Belegzahl } \\
1 \\
\end{array}$} & \multirow{2}{*}{90} \\
\hline II. & $\mathbf{A D} \Rightarrow \mathrm{AD}$ & & & & \\
\hline & \multirow{5}{*}{$\begin{array}{l}A D \Rightarrow A S D \\
A D \Rightarrow S D \\
A D \Rightarrow N D \\
A D \Rightarrow \text { frW }\end{array}$} & 10 & 5,21 & - & - \\
\hline & & - & - & - & - \\
\hline & & 2 & 1,04 & - & - \\
\hline & & 1 & 0,52 & - & - \\
\hline & & 55 & 28,65 & 1 & 12,5 \\
\hline \multirow[t]{6}{*}{ III. } & \multirow{6}{*}{$\begin{array}{l}\text { ASD } \Rightarrow \text { ASD } \\
\text { ASD } \Rightarrow \text { AD } \\
\text { ASD } \Rightarrow \text { SD } \\
\text { ASD } \Rightarrow \text { ND } \\
\text { ASD } \Rightarrow \text { frW }\end{array}$} & - & - & - & - \\
\hline & & - & - & - & - \\
\hline & & - & - & - & - \\
\hline & & - & - & - & - \\
\hline & & - & - & - & - \\
\hline & & - & - & - & - \\
\hline \multirow[t]{4}{*}{ IV. } & \multirow{4}{*}{$\begin{array}{l}\mathbf{N D} \Rightarrow \mathrm{SD} \\
\mathbf{N D} \Rightarrow \mathrm{AD} \\
\mathbf{N D} \Rightarrow \mathrm{ASD}\end{array}$} & 123 & 64,06 & 2 & 25,0 \\
\hline & & 4 & 2,08 & - & - \\
\hline & & - & - & - & - \\
\hline & & 127 & 66,14 & 2 & 25,0 \\
\hline
\end{tabular}

Ein gänzlich anderes Bild wird dagegen hier geboten, insofern durch die hohe Frequenz der suffixalen Reaktion auf nichtmarkierte Basislexeme bzw. geringe Quantität des SD $\Rightarrow$ SD-Musters die genannte Polarităt noch offensichtlicher wird. Der Textsortencharakter (moderner Kriminalroman - nüchterne Sprache) scheint hier nachhaltigen Einfluß ausgeübt zu haben. 
Tab.13: TUCHOLSKY, "Schloß Gripsholm" (total: 235 Dim., davon 215 Subst., 20 Adj.)

\begin{tabular}{|c|c|c|c|c|c|}
\hline \multirow{2}{*}{\multicolumn{2}{|c|}{$\begin{array}{c}\text { WIEDERGABE- } \\
\text { TYP }\end{array}$}} & \multicolumn{2}{|c|}{ SUBSTANTIVE } & \multicolumn{2}{|c|}{ ADJEKTIVE ETC. } \\
\hline & & Belegzahl & $\%$ & Belegzahl & $\%$ \\
\hline \multirow[t]{6}{*}{1.} & \multirow{6}{*}{$\begin{array}{l}S D \Rightarrow S D \\
S D \Rightarrow A D \\
S D \Rightarrow A S D \\
S D \Rightarrow N D \\
S D \Rightarrow \text { frW }\end{array}$} & 24 & 11,16 & 4 & 20,0 \\
\hline & & - & - & - & - \\
\hline & & 2 & 0,93 & - & - \\
\hline & & 9 & 4,19 & 5 & 25,0 \\
\hline & & 6 & 2,79 & - & - \\
\hline & & 41 & 19,07 & 9 & 45,0 \\
\hline \multirow[t]{6}{*}{ II. } & \multirow{6}{*}{$\begin{array}{l}A D \Rightarrow A D \\
A D \Rightarrow A S D \\
A D \Rightarrow S D \\
A D \Rightarrow N D \\
A D \Rightarrow \text { frW }\end{array}$} & 59 & 27,44 & 3 & 15,0 \\
\hline & & 20 & 9,3 & - & - \\
\hline & & 12 & 5,58 & 2 & 10,0 \\
\hline & & 7 & 3,26 & 2 & 10,0 \\
\hline & & - & - & - & - \\
\hline & & 98 & 45,58 & 7 & 35,0 \\
\hline \multirow[t]{6}{*}{ III. } & \multirow{6}{*}{$\begin{array}{l}\mathrm{ASD} \Rightarrow \mathrm{ASD} \\
\mathrm{ASD} \Rightarrow \mathrm{AD} \\
\mathrm{ASD} \Rightarrow \mathrm{SD} \\
\mathrm{ASD} \Rightarrow \mathrm{ND} \\
\mathrm{ASD} \Rightarrow \mathrm{frW}\end{array}$} & 5 & 2,33 & - & - \\
\hline & & 2 & 0,93 & - & - \\
\hline & & 2 & 0,93 & - & - \\
\hline & & 1 & 0,46 & 1 & 5,0 \\
\hline & & - & $=$ & - & - \\
\hline & & 10 & 4,65 & 1 & 5,0 \\
\hline \multirow[t]{4}{*}{ IV. } & \multirow{4}{*}{$\begin{array}{l}N D \Rightarrow S D \\
N D \Rightarrow A D \\
N D \Rightarrow A S D\end{array}$} & 65 & 30,23 & 1 & 5,0 \\
\hline & & - & - & 2 & 10,0 \\
\hline & & 1 & 0,46 & - & - \\
\hline & & 66 & 30,69 & 3 & 15,0 \\
\hline
\end{tabular}

Hier fällt das Überangebot an Übersetzungen analytischer Diminutive auf, das die Gesamtsumme der Gruppe II (45,58\%), an der jede einzelne Position teilhat, sogar höher ausfallen läßt als die der Gruppe IV (30,69\%). 
Tab.14: ENDE, "Momo" (total: 633 Dim., davon 602 Subst., 31 Adj.)

\begin{tabular}{|c|c|c|c|c|c|}
\hline \multirow{2}{*}{\multicolumn{2}{|c|}{$\begin{array}{l}\text { WIEDERGABE- } \\
\text { TYP }\end{array}$}} & \multicolumn{2}{|c|}{ SUBSTANTIVE } & \multicolumn{2}{|c|}{ ADJEKTIVE ETC. } \\
\hline & & Belegzahl & $\%$ & Belegzahl & $\%$ \\
\hline \multirow[t]{6}{*}{ I. } & \multirow{6}{*}{$\begin{array}{l}\mathrm{SD} \Rightarrow \mathrm{SD} \\
\mathrm{SD} \Rightarrow \mathrm{AD} \\
\mathrm{SD} \Rightarrow \mathrm{ASD} \\
\mathrm{SD} \Rightarrow \mathrm{ND} \\
\mathrm{SD} \Rightarrow \mathrm{frW}\end{array}$} & 48 & 7,97 & 1 & 3,23 \\
\hline & & 1 & 0,17 & - & - \\
\hline & & 1 & 0,17 & -1 & - \\
\hline & & 15 & 2,49 & - & - \\
\hline & & 5 & 0,83 & 1 & 3,23 \\
\hline & & 70 & 11,63 & 2 & 6,46 \\
\hline \multirow[t]{6}{*}{ II. } & \multirow{6}{*}{$\begin{array}{l}A D \Rightarrow A D \\
A D \Rightarrow A S D \\
A D \Rightarrow S D \\
A D \Rightarrow N D \\
A D \Rightarrow \text { frW }\end{array}$} & 86 & 14,29 & - & - \\
\hline & & 39 & 6,45 & 1 & 3,23 \\
\hline & & 15 & 2,49 & 2 & 6,45 \\
\hline & & 6 & 1,0 & - & - \\
\hline & & 8 & 1,33 & - & - \\
\hline & & 154 & 25,56 & 3 & 9,68 \\
\hline \multirow[t]{6}{*}{ III. } & \multirow{6}{*}{$\begin{array}{l}\mathrm{ASD} \Rightarrow \mathrm{ASD} \\
\mathrm{ASD} \Rightarrow \mathrm{AD} \\
\mathrm{ASD} \Rightarrow \mathrm{SD} \\
\mathrm{ASD} \Rightarrow \mathrm{ND} \\
\mathrm{ASD} \Rightarrow \mathrm{frW}\end{array}$} & 18 & 2,99 & - & - \\
\hline & & 2 & 0,33 & - & - \\
\hline & & 8 & 1,33 & - & - \\
\hline & & 1 & 0,17 & 1 & 3,23 \\
\hline & & - & - & - & - \\
\hline & & 29 & 4,82 & 1 & 3,23 \\
\hline \multirow[t]{4}{*}{ IV. } & \multirow{4}{*}{$\begin{array}{l}\mathrm{ND} \Rightarrow \mathrm{SD} \\
\mathrm{ND} \Rightarrow \mathrm{AD} \\
\mathrm{ND} \Rightarrow \mathrm{ASD}\end{array}$} & 337 & 55,98 & 25 & 80,65 \\
\hline & & 11 & 1,83 & - & - \\
\hline & & 1 & 0,17 & - & - \\
\hline & & 349 & 57,98 & 25 & 80,65 \\
\hline
\end{tabular}

Die Durchschnittstendenz der Gesamtauswertung wird in diesem Text, der die absolut gesehen meisten Belege aufweist, bestätigt, es ist lediglich eine leichte Verschiebung der Werte zugunsten der charakteristischen Gruppenkonstellation zu vermerken. 
Tab.15: FRANK, "Das Tagebuch der Anne Frank" (total: 204 Dim., davon 192 Subst., 12 Adj.)

\begin{tabular}{|c|c|c|c|c|c|}
\hline \multirow{2}{*}{\multicolumn{2}{|c|}{$\begin{array}{l}\text { WIEDERGABE- } \\
\text { TYP }\end{array}$}} & \multicolumn{2}{|c|}{ SUBSTANTIVE } & \multicolumn{2}{|c|}{ ADJEKTIVE ETC. } \\
\hline & & Belegzahl & $\%$ & Belegzahl & $\%$ \\
\hline \multirow[t]{6}{*}{ I. } & \multirow{6}{*}{$\begin{array}{l}\mathrm{SD} \Rightarrow \mathrm{SD} \\
\mathrm{SD} \Rightarrow \mathrm{AD} \\
\mathrm{SD} \Rightarrow \mathrm{ASD} \\
\mathrm{SD} \Rightarrow \mathrm{ND} \\
\mathrm{SD} \Rightarrow \mathrm{frW}\end{array}$} & 15 & 7,81 & - & - \\
\hline & & 1 & 0,52 & - & - \\
\hline & & - & $=$ & - & - \\
\hline & & 10 & 5,21 & - & - \\
\hline & & 3 & 1,56 & - & - \\
\hline & & 29 & 15,1 & - & - \\
\hline \multirow[t]{6}{*}{ II. } & \multirow{6}{*}{$\begin{array}{l}A D \Rightarrow A D \\
A D \Rightarrow A S D \\
A D \Rightarrow S D \\
A D \Rightarrow N D \\
A D \Rightarrow \mathrm{frW}\end{array}$} & 34 & 17,71 & 1 & 8,33 \\
\hline & & 5 & 2,6 & - & - \\
\hline & & 4 & 2,08 & 5 & 41,67 \\
\hline & & 5 & 2,6 & - & - \\
\hline & & 9 & 4,69 & - & - \\
\hline & & 57 & 29,68 & 6 & 50,0 \\
\hline \multirow[t]{6}{*}{ III. } & \multirow{6}{*}{$\begin{array}{l}\text { ASD } \Rightarrow \text { ASD } \\
\text { ASD } \Rightarrow A D \\
\text { ASD } \Rightarrow \text { SD } \\
\text { ASD } \Rightarrow \text { ND } \\
\text { ASD } \Rightarrow \text { frW }\end{array}$} & 2 & 1,04 & - & - \\
\hline & & 1 & 0,52 & - & - \\
\hline & & 1 & 0,52 & - & - \\
\hline & & - & - & - & - \\
\hline & & - & - & - & - \\
\hline & & 4 & 2,08 & - & - \\
\hline \multirow[t]{4}{*}{ IV. } & \multirow{4}{*}{$\begin{array}{l}N D \Rightarrow S D \\
N D \Rightarrow A D \\
N D \Rightarrow A S D\end{array}$} & 97 & 50,52 & 6 & 50,0 \\
\hline & & 3 & 1,56 & - & - \\
\hline & & 2 & 1,04 & $=$ & - \\
\hline & & 102 & 53,12 & 6 & 50,0 \\
\hline
\end{tabular}

Ohne Auffäligkeiten, entspricht die Zahlenverteilung dieses Werks noch am ehesten den Mittelwerten von Tab.10. 
Tab.16: RUNGE, "Bottroper Protokolle" (total: 152 Dim., davon I4I Subst., II Adj.)

\begin{tabular}{|c|c|c|c|c|c|}
\hline \multirow{2}{*}{\multicolumn{2}{|c|}{$\begin{array}{c}\text { WIEDERGABE- } \\
\text { TYP }\end{array}$}} & \multicolumn{2}{|c|}{ SUBSTANTIVE } & \multicolumn{2}{|c|}{ ADJEKTIVE ETC. } \\
\hline & & Belegzahl & $\%$ & Belegzahl & $\%$ \\
\hline \multirow[t]{6}{*}{ I. } & \multirow{6}{*}{$\begin{array}{l}S D \Rightarrow S D \\
S D \Rightarrow A D \\
S D \Rightarrow A S D \\
S D \Rightarrow N D \\
S D \Rightarrow f r W\end{array}$} & 14 & 9,93 & - & - \\
\hline & & - & - & - & - \\
\hline & & - & - & - & - \\
\hline & & 1 & 0,71 & - & - \\
\hline & & - & $=$ & - & - \\
\hline & & 15 & 10,64 & - & - \\
\hline \multirow[t]{6}{*}{ II. } & \multirow{6}{*}{$\begin{array}{l}A D \Rightarrow A D \\
A D \Rightarrow A S D \\
A D \Rightarrow S D \\
A D \Rightarrow N D \\
A D \Rightarrow f r W\end{array}$} & 14 & 9,93 & 2 & 18,18 \\
\hline & & 5 & 3,55 & 3 & 27,27 \\
\hline & & 3 & 2,13 & - & - \\
\hline & & 3 & 2,13 & - & - \\
\hline & & 2 & 1,42 & - & - \\
\hline & & 27 & 19,16 & 5 & 45,45 \\
\hline \multirow[t]{6}{*}{ III. } & \multirow{6}{*}{$\begin{array}{l}\text { ASD } \Rightarrow \text { ASD } \\
\text { ASD } \Rightarrow \text { AD } \\
\text { ASD } \Rightarrow \text { SD } \\
\text { ASD } \Rightarrow \mathbf{N D} \\
\text { ASD } \Rightarrow \text { frW }\end{array}$} & 3 & 2,13 & - & - \\
\hline & & 1 & 0,71 & - & - \\
\hline & & - & - & - & - \\
\hline & & - & - & - & - \\
\hline & & - & - & - & - \\
\hline & & 4 & 2,84 & - & - \\
\hline \multirow[t]{4}{*}{ IV. } & \multirow{4}{*}{$\begin{array}{l}\mathrm{ND} \Rightarrow \mathrm{SD} \\
\mathrm{ND} \Rightarrow \mathrm{AD} \\
\mathrm{ND} \Rightarrow \mathrm{ASD}\end{array}$} & 92 & 65,25 & 6 & 54,55 \\
\hline & & 3 & 2,13 & - & - \\
\hline & & - & - & - & - \\
\hline & & 95 & 67,38 & 6 & 54,55 \\
\hline
\end{tabular}

Der eindeutige Schwerpunkt dieser Übersicht liegt beim ND $\Rightarrow$ SD-Typ, dessen Überrepräsentiertheit bei konstanten Werten für die I.Gruppe auffallig zu Lasten der Übertragung analytischer Bildeweisen geht. 
Tab.17: GRZIMEK, "Vierfabige Australier" (total: 189 Dim., dawon 173 Subst., 16 Adj.)

\begin{tabular}{|c|c|c|c|c|c|}
\hline \multirow{2}{*}{\multicolumn{2}{|c|}{$\begin{array}{c}\text { WIEDERGABE- } \\
\text { TYP }\end{array}$}} & \multicolumn{2}{|c|}{ SUBSTANTIVE } & \multicolumn{2}{|c|}{ ADJEKTIVE ETC. } \\
\hline & & Belegzahl & $\%$ & Belegzahl & $\%$ \\
\hline \multirow[t]{6}{*}{ I. } & \multirow{6}{*}{$\begin{array}{l}\mathrm{SD} \Rightarrow \mathrm{SD} \\
\mathrm{SD} \Rightarrow \mathrm{AD} \\
\mathrm{SD} \Rightarrow \mathrm{ASD} \\
\mathrm{SD} \Rightarrow \mathrm{ND} \\
\mathrm{SD} \Rightarrow \mathrm{frW}\end{array}$} & 11 & 6,36 & 2 & 12,5 \\
\hline & & 1 & 0,58 & - & - \\
\hline & & 1 & 0,58 & - & - \\
\hline & & 8 & 4,62 & 1 & 6,25 \\
\hline & & 1 & 0,58 & - & - \\
\hline & & 22 & 12,72 & 3 & 18,75 \\
\hline \multirow[t]{6}{*}{ II. } & \multirow{6}{*}{$\begin{array}{l}A D \Rightarrow A D \\
A D \Rightarrow A S D \\
A D \Rightarrow S D \\
A D \Rightarrow N D \\
A D \Rightarrow f r W\end{array}$} & 51 & 29,48 & - & - \\
\hline & & 10 & 5,78 & - & - \\
\hline & & 3 & 1,73 & 1 & 6,25 \\
\hline & & 7 & 4,05 & 2 & 12,5 \\
\hline & & 2 & 1,16 & - & - \\
\hline & & 73 & 42,2 & 3 & 18,75 \\
\hline \multirow[t]{6}{*}{ III. } & \multirow{6}{*}{$\begin{array}{l}A S D \Rightarrow A S D \\
A S D \Rightarrow A D \\
A S D \Rightarrow S D \\
A S D \Rightarrow N D \\
A S D \Rightarrow \text { frW }\end{array}$} & - & - & - & - \\
\hline & & - & - & - & - \\
\hline & & - & - & - & - \\
\hline & & - & - & - & - \\
\hline & & - & - & - & - \\
\hline & & & - & - & - \\
\hline \multirow[t]{4}{*}{ IV. } & \multirow{4}{*}{$\begin{array}{l}\mathbf{N D} \Rightarrow \mathbf{S D} \\
\mathbf{N D} \Rightarrow \mathbf{A D} \\
\mathbf{N D} \Rightarrow \mathbf{A S D}\end{array}$} & 73 & 42,2 & 10 & 62,5 \\
\hline & & 4 & 2,31 & - & - \\
\hline & & 1 & 0,58 & - & - \\
\hline & & 78 & 45,09 & 10 & 62,5 \\
\hline
\end{tabular}

Mit Blick auf die Zwischensummen läßt sich konstatieren, daß sich die Positionen mit analytischen Diminutiven in der Ausgangssprache den Häufigkeitsvorrang mit den nichtdiminutivischen Vorlagebelegen teilen (Gruppe II: 42,2\%, Gruppe IV: $45,09 \%$ ). Es ist vielleicht nicht unstatthaft zu vermuten, daß sich die dem Autor eigene emotionale Haltung zu seinem Gegenstand in analytischen Bildungen realisiert hat. 
Tab.18: GOOOCCK, "Selbermachen" (total: 61 Dim., davon 60 Subst., I Adj.)

\begin{tabular}{|c|c|c|c|c|c|}
\hline \multirow{2}{*}{\multicolumn{2}{|c|}{$\begin{array}{c}\text { WIEDERGABE- } \\
\text { TYP }\end{array}$}} & \multicolumn{2}{|c|}{ SUBSTANTIVE } & \multicolumn{2}{|c|}{ ADJEKTIVE ETC. } \\
\hline & & Belegzahl & $\%$ & Belegzahl & $\%$ \\
\hline \multirow[t]{6}{*}{ I. } & \multirow{6}{*}{$\begin{array}{l}S D \Rightarrow S D \\
S D \Rightarrow A D \\
S D \Rightarrow A S D \\
S D \Rightarrow N D \\
S D \Rightarrow \text { frW }\end{array}$} & 5 & 8,33 & - & - \\
\hline & & - & - & - & - \\
\hline & & 1 & 1,67 & - & - \\
\hline & & 2 & 3,33 & - & - \\
\hline & & - & - & - & - \\
\hline & & 8 & 13,33 & - & - \\
\hline \multirow[t]{6}{*}{ II. } & \multirow{6}{*}{$\begin{array}{l}A D \Rightarrow A D \\
A D \Rightarrow A S D \\
A D \Rightarrow S D \\
A D \Rightarrow N D \\
A D \Rightarrow \text { frW }\end{array}$} & 11 & 18,33 & 1 & 100,0 \\
\hline & & 4 & 6,67 & - & - \\
\hline & & 1 & 1,67 & - & - \\
\hline & & - & - & - & - \\
\hline & & - & - & - & - \\
\hline & & 16 & 26,67 & 1 & 100,0 \\
\hline \multirow[t]{6}{*}{ III. } & \multirow{6}{*}{$\begin{array}{l}\mathbf{A S D} \Rightarrow \mathbf{A S D} \\
\mathbf{A S D} \Rightarrow \mathbf{A D} \\
\mathbf{A S D} \Rightarrow \mathbf{S D} \\
\mathbf{A S D} \Rightarrow \mathbf{N D} \\
\mathbf{A S D} \Rightarrow \mathbf{f r W}\end{array}$} & 3 & 5,0 & - & - \\
\hline & & - & - & - & - \\
\hline & & - & - & - & - \\
\hline & & - & - & - & - \\
\hline & & - & - & - & - \\
\hline & & 3 & 5,0 & - & - \\
\hline \multirow[t]{4}{*}{ IV. } & \multirow{4}{*}{$\begin{array}{l}\text { ND } \Rightarrow \text { SD } \\
\text { ND } \Rightarrow A D \\
\text { ND } \Rightarrow \text { ASD }\end{array}$} & 32 & 53,33 & - & - \\
\hline & & 1 & 1,67 & - & - \\
\hline & & - & - & - & - \\
\hline & & 33 & 55,0 & - & - \\
\hline
\end{tabular}

Bis auf die überdurchschnittliche Vorherrschaft des ASD $\Rightarrow$ AD-Typs lassen sich keine Besonderheiten aufzählen, doch beruht sie auf der absoluten Zahl von drei Belegen und kann daher nicht als repräsentativ angesehen werden.

Ein Überblick über die Frequenzverhältnisse bei den Adjektiven etc. belegt erneut die schon in der polnisch $\Rightarrow$ deutschen Konfrontation gemachte Beobach- 
tung starker Inkonsistenz und Irregularität; die beiden oben geäußerten Anhaltspunkte sind auch hier zu berücksichtigen. ${ }^{19}$

${ }^{19}$ Siehe dazu oben S.219. 


\subsection{DIVERGENZEN UND KONVERGENZEN IM DIMINUTIVGEBRAUCH BEIDER SPRACHEN}

Mit der Übersicht der Wiedergabetypen und ihrer statistischen Beschreibung konnte bisher lediglich eine formal-deskriptive Vorleistung für das Verständnis interlingualer Beziehungen von Diminutiveinheiten erbracht werden. Der Konstatierung nicht gewichteter Übersetzungsregularitäten und der allein quantitativen Analyse ihrer Realisierung muß nun der Versuch einer darauf Bezug nehmenden systematischen Begründung folgen.

Die Darstellung der Übersetzungstypen in ihrem Gestaltreichtum machte Koinzidenzen und Diskrepanzen, die Diversifikation beider Sprachen beim Einsatz von Diminutiven offensichtlich, so daß im weiteren zu fragen ist, welche Ursachen dieser Inhomogenität und welche Motive der Wahl eines zielsprachlichen Äquivalents zugrunde liegen. Dies zu beantworten, erfordert eine ausgangstextorientierte Übersetzungskritik, ${ }^{20}$ die an ausgewählten, exemplarischen Textpassagen die Bewältigung von Übersetzungsschwierigkeiten in Augenschein nimmt.

Es ist sinnvoll, nur auffällige Phänomene als Ansatz eines Begründungsversuches zu wählen. Die vorangegangenen tabellarischen Gesamtauswertungen (Tab.1 und Tab.10) stellten ausschließlich zwei aufeinander verwiesene Übertragungsweisen durch ihre Frequenzdominanz in den Mittelpunkt des Interesses: die Übersetzung eines polnischen Suffixdiminutivs mit einer nichtdiminutivischen Einheit im deutschen Zieltext bzw., als Spiegelung dessen, die diminutivische Reaktion des Polnischen auf ein Nichtdiminutiv in der deutschen Vorlage. Das Übergewicht dieser Übersetzungsreziprozität berechtigt zum Entwurf einer für jede Sprache charakteristischen Ausgangslage bei der Diminutivübertragung: Dominiert in der polnisch $\Rightarrow$ deutschen Kontrastierung der SD $\Rightarrow$ ND-Typ, so muß davon ausgegangen werden, daß es für die deutsche Übersetzung signifikant ist, auf eine Vielzahl formal markierter Einheiten zu treffen, um diese dann in der Gestaltung des Zieltextes selektiv zu begrenzen. Umgekehrt sieht sich ein polnischer Übersetzer mit hoher Wahrscheinlichkeit einem nicht durch zahlreiche Modifikationsderivate gekennzeichneten Text gegenüber, den er den Anforderungen seiner Sprache entsprechend diminutivisch aufzubereiten hat. Mit anderen Worten: das Deutsche muß augenscheinlich eine Fülle leeren, das Polnische eine

so Năheres dazu vgl. oben S. $102 f$. 
Leere füllen; Überangebot und Unterangebot werden zu den eigentlichen Problemen des zwischensprachlichen Ausgleichs.

Fragt man nun weiter nach den Gründen dieser Disparität, so bietet sich auf den ersten Blick eine einfache Erklärung an: Die Disposition der deutschen Sprache ist gekennzeichnet durch Seltenheit der Bildung im Sprachsystem und/ oder durch häufiges Unangebrachtsein in der Sprachverwendung; für das Polnische gilt dann das Gegenteil. In einem zweiten Schritt aber ist der Aspekt der möglichen unterschiedlichen Gewichtung der diminutivischen Aussage in Rechnung zu ziehen. In diesem Lichte ist dann zu behaupten, daß der häufige $\mathrm{Ge}$ brauch im Polnischen mit einem Schwund des expressiven Wertes des Diminutivs einhergeht, und umgekehrt, daß der spärliche Einsatz im Deutschen gerade mit einer expressiven Belastung dieser Bildung zu erklären ist.

Die hier zusammengetragenen Stichworte Gewichtung, Existenz, Zulässigkeit sollen im folgenden auf dem Wege des Nachvollzugs eines standardisierten Übersetzungsvorgangs in einen Zusammenhang gebracht werden. Nimmt man die deutsche Übersetzung als Paradigma, ergibt sich im einzelnen folgende Sequenz von Fragen, die sich vor der Entscheidung für eine bestimmte Übersetzung stellen:

1. Handelt es sich im vorliegenden Falle überhaupt um eine inhaltlich markierte (Gegensatz: nur formal markierte) Diminutiveinheit? (Kap. 4.3.1.)

2. Wenn ja, steht der Zielsprache dafür eine formal analoge Bildung zur Verfügung? (Kap.4.3.2.)

3. Wenn ja, ist es an dieser Textstelle, in dieser Situation auch angezeigt, auf diese zurückzugreifen? (Kap.4.3.3.)

Damit sind die wesentlichen Problemfelder umrissen; näheren AufschluB über die sich einfachen Regeln entziehende Vielfalt der interlingualen Beziehungen kann allein eine Diskussion exemplarischer Beispiele ${ }^{21}$ im Hinblick auf das Erreichen bzw. Verfehlen funktioneller Äquivalenz ${ }^{22}$ leisten.

${ }^{21}$ In der Regel werden im folgenden neue Textbelege angeführt, in einigen Fällen wird auf Beispiele zurückgegriffen, die sich schon in anderem Zusammenhang als prägnant erwiesen haben.

2 Genaueres dazu vgl. oben S.120. 


\subsubsection{Neutralisierte Diminutive}

Synthetische Diminutivformen, die ihren modifizierenden Charakter eingebüßt und sich mehr oder weniger vom Grundwort entfernt haben, ${ }^{23}$ stellen für den Übersetzer im Übersetzungsprozeß einen ersten gewichtigen Prüfstein dar. Er läuft ständig Gefahr, dieser neutralisierten Bildung eine markierte entgegenzusetzen und so keine Deckungsgleichheit erzielen zu können. Faktisch stellen dabei die Neutralisierungen des Polnischen, sowohl im Ausgangs-, als auch im Zieltext, das Gros des ohnehin schon quantitativ dominierenden Übersetzungsmusters SD $\Rightarrow \mathrm{ND}$ (PI. $\Rightarrow \mathrm{Dt}$.) und ND $\Rightarrow \mathrm{SD}$ (Dt. $\Rightarrow$ Pl.). Neutralisierte Diminutive des Deutschen bilden eher eine Randerscheinung und stellen meist auch kein Problem für den polnischen Übersetzer dar; die Schwierigkeiten liegen also hier ganz auf der Seite der deutschen Übertragung.

Eine Steigerung der Neutralisierung stellen lexikalisierte Bildungen ${ }^{24}$ dar, die eigentlich keine Aufnahme gefunden haben, aber durch diminutivische Übersetzung doch im Typ ND $\Rightarrow$ SD in das Beispielkorpus gelangten. Bei den zu nennenden Fällen liegt durchweg übersetzerische Fehlinterpretation aus mangelnder Sach-bzw. Verwendungskenntnis vor:

$Z$ tej przedzy gospodynie tkaly sukna na domowych warsztatach, zazdrośnie strzegac sekretów wzoru: w jodelke, w siatke, taki kolor na osnowe, taki na odetke. (MIL: 8)
Aus den Spinnfäden webten die Frauen Tuche auf Hauswebstühlen, eifersüchtig die Geheimnisse des Musters hütend: als Tännlein, als Gitter, die Farbe für die Kette und die für den Schuß. (MIL: 6)

Mit jodelka ist hier nicht die semantisch markierte Wortform, sondern eine metaphorisierte Lexikalisierung gegeben, die ihre Entsprechung in dem an einem anderen Bild orientierten Lexem Fischgrätenmuster hat.

Na trawniku pojawialy sie na wiosne kwiaty nazywane kluczyki sw.Piotra. (MIL: 18)

Wkladala zloty laricuszek z ziarenkami jak lebki od szpilek... (MIL: 17)

...[ministranci] chwiali kadzielnicami, nie bojac sie odpowiadali ksiedzu, podawali mu dzbanki z dziobkami podobnymi do księżyców na nowiu. (MIL: 23)

${ }^{2} \mathrm{Vgl}$. oben Kap. 2.4.3.1.

${ }^{24} \mathrm{Zu}$ diesem Begriff s. oben S.87f.
Auf dem Rasen erschienen im Frühling Blumen, Himmelsschlïsselchen genannt. (MIL: 20)

Sie legte ein goldenes Kettchen mit Körnchen, groß wie Stecknadelköpfchen, an... (MIL: 19)

...[die Ministranten] antworteten dem Priester ohne Angst, sie überreichten ihm Krüglein, deren Schnäbelchen der Mondsichel ähnelten. (MIL: 28) 
In diesen Beispielen ist zwar im Gegensatz zur obigen Nicht-Äquivalenz ${ }^{25}$ semantische Äquivalenz ${ }^{26}$ erreicht, doch verkennt die Übersetzung die auf Metaphorisierung beruhende neue lexematische Verwendung als Terminus der botanischen Nomenklatur oder als Benennung des Teils eines Gebrauchsgegenstandes. Eine eventuell die diminutivische Übertragung rechtfertigende expressive Komponente ist dem Text nicht zu entnehmen.

Keinen Anlaß für eine diminutivische Reaktion bieten ebenso die Textauszüge, die den Übersetzer wohl durch die einem Diminutivsuffix ähnelnde Lexemendung dazu verleitet haben:

$Z$ rozpietej koszuli, już bardzo brudnej i wymietej, sterczala sucha i pocieta zmarszczkami szyja. (NOW: 54)

...drewniane okiennice zamykaly sie od zewnatrz i tylko do okiennicy babci Dilbinowej wygrzebano teraz klodke... (MIL: 56)

Aus dem offenen, schon sehr dreckigen und zerknüllten Hemd ragte der trockene und von Fältchen durchschnittene Hals. (NOW: 30)

...die Holzläden schlossen von außen, und nur für Großmutter Dilbins Fensterladen kramte man jetzt ein Vorhängeschlößchen heraus... (MIL: 74)

Es ist nicht zu erkennen, weshalb der Bedeutungsbestandteil klein in den Text eingebracht wurde.

Schwierigkeiten mit Lexikalisierungen bilden aber im Übersetzungsprozeß eher eine Ausnahme. Weitaus größere Probleme bereiten dem Übersetzer neutralisierte Ausdrucksmittel. Unter ihnen fallen besonders zwei Gruppen auf.

\subsubsection{Angleichung von Basislexem und Diminutiv}

Eine Reihe von Textbeispielen zeigt eine enge inhaltliche Anbindung von Grundwort und Derivat. Treten sie in sachlich-informativer Redefunktion auf, dann sind sie gekennzeichnet durch Beliebigkeit in der Verwendung, d.h. Austauschbarkeit selbst in unmittelbarer Nachbarschaft ohne erkennbaren Perspektivwechsel. Der Verlust der Merkmalhaftigkeit verlangt vom Zieltext, will er funktionelle Äquivalenz erreichen, den notwendigen Verzicht auf das variierende Suffix. Er darf sich nicht auf die zu beobachtende Neigung des Polnischen, ${ }^{27}$

${ }^{25} \mathrm{Zu}$ den potentiellen Entsprechungsstufen vgl. oben S.128f.

*o Dazu s. oben S. 122.

27 Auf die immer weitere Verbreitung findende Manier der Diminuierung weist MAŃCZAK 1980 kritisch hin: "Ostatnio zapanowala u nas istna moda na zdrobnienia (...) Jednak mylilby sie ktos. kto by sadził, że moda na zdrobnienia panuje tylko w sklepach i lokalach gastrono- 
ein Diminutiv seiner unmarkierten Form vorzuziehen, einlassen und muß von seinem Textverständnis ausgehend die Modifikationen als redundant einstufen.

Ein Nebeneinander beider Bildungen in engster Umgebung, ohne erkennbare Verwendungsabsicht, begegnet in folgenden Beispielen:

Widza żolnierze, że maly wiezien zupelnie sie uspokoil..., wiec mniej go teraz pilnuja. Dawniej na podwórku musieli chodzic za nim krok w krok, bo okno kancelarii wychodzilo na podworze i naczelnik wiezienia patrzal na żodnierzy... (KOR: 17)

Klu-Klu...wsiadla $z$ Maciusiem na lodke i pojechali. Na morzu zaskoczyła ich taka sobie burza. Ale nawet mala morska burza jest niebezpieczna dla zwyczajnej lodzi. (KOR: 46)

W grudniowe, ponure popoludnie Adela postanowila przerobic swoja sukienke, wlożyla wiec buciki ojca i pobiegla do kolezanki Nisi-krawcowej. (FIN: 64) Kiedy [Nisia] uspokoila sie, skrócila suknie Adeli szybkimi, zrecznymi ruchami... (FIN: 65)

Adela zamknela za soba drzwi...i przysiadla na zydelku bedacym niegdyś wlasnościa ojca Nisi, szewca. (FIN: 64) - Czwarte [lóżko] slużylo Nisi, która, nie przerywajac obiadu, sluchala szybkiego mowienia siedzacej na ojcowskim zydlu Adeli. (FIN: 64)

...ja zas przyrzeklam zaplate $w$ naturze, to jest co tydzien dostarczac pewna ilose masla i jaj. Bylo to dosc trudno, bo jedna krowa w zimie dawala malo mleka...i o jajka też w zimie bylo trudno... (FOR: 85)
Als die Soldaten bemerkten, daß sich der kleine Gefangene völlig beruhigt hatte..., da achteten sie nicht mehr so streng auf ihn. Früher auf dem Hof hatten sie Schritt für Schritt hinter ihm hergehen müssen, denn das Fenster der Kanzlei ging auf den Hof hinaus, und der Gefangnisdirektor konnte die Soldaten beobachten... (KOR: 22)

Klu-Klu...setzte...sich mit Hänschen in das Boot, und los ging die Reise. Auf hoher See überraschte sie ein Gewitter, das war zwar nicht sehr schlimm, aber selbst ein kleiner Sturm auf See ist für ein gewöhnliches Boot gefăhrlich. (KOR: 63)

An einem trūben Dezembernachmittag beschloB Adela, inr Kleid umarbeiten zu lassen, zog also die Schuhe ihres Vaters an und lief zu ihrer Kollegin Nisia, der Schneiderin. (FIN: 66) - Als [Nisia] sich beruhigt hatte, machte sie Adelas Kleid mit schnellen, geschickten Bewegungen...kürzer... (FIN: 68)

Adela schloß die Tür hinter sich... und setzte sich auf den Schemel, der einst Nisias Vater, dem Schuster, gehört hatte. (FIN: 66) - Das vierte [Bett] diente Nisia, die, ohne ihre Mahlzeit zu unterbrechen, Adelas hastigen Worten zuhörte. (FIN: 66)

Ich versprach, ihn dafür mit Lebensmitteln zu entlohnen. Jede Woche lieferte ich eine bestimmte Menge Butter und Eier bei ihm ab. Das fiel uns nicht leicht, denn die eine Kuh gab im Winter nicht viel Milch, und die Hühner...legten auch schlecht. (FOR: 79)

micznych. W rzeczywistosci szerzy sie ona wszedzie. (...) Nie ma żadnego racjonalnego uzasadnienia fakt, ze na naszych oczach prastary wyraz indoeuropejski wies. ktory w postaci niezdrobnialej istnieje od kilku tysiecy lat, jest coraz bardziej wypierany przez wioska" (S.70). 
[N:] A po powrocie z kosciola...dziedziczka poscila i wzdychala, snula sie jak cien po komnatach, zapalala swieczki przed niezliczonymi swietymi obrazami i...modlila sie. (TON: 3) - [N:] Pewnej nocy po zapaleniu niezliczonych swiec przed swietymi obrazami, po odmówieniu niezliczonych modlitw,... (TON: 5)
[E:] Und nach der Rückkehr aus der Kirche fastete sie und seufzte herum, schwebte wie ein Schatten durch die Gemächer des Palastes, zündete Kerzen an vor unendlich vielen Heiligenbildern und...betete. (TON: 5) - [E:] In einer gewissen Nacht, nachdem sie ungezählte Lichter vor heiligen Bildem angezunndet hatte, ungezählte Gebete gesprochen hatte,... (TON: 8)

Von den exemplarisch aufgezählten sind jene Derivate zu unterscheiden, denen zwar noch theoretisch Austauschbarkeit attestiert werden kann, die faktisch aber die fast durchgängig benutzte Form darstellen:

Wiesc o tych zdarzeniach szybko rozeszla sie po sasiednich wioskach... (MIL: 40)

Przez to go psula, bo dotychczas uwazal za zwyczajne, ze sam przyszywal sobie guziki nitka i igla Antoniny, a teraz zwracal sie o kazdy drobiazg... (MIL: 50)

Przede wszystkim Boleslaw zauważyl szafirowy kolor skarpetek. Spod przykrótkich i luźnych spodni kolor ten wydobywal sie dobitnym akcentem. (IWA: 97)

...wyciagnal z [bryczki] dosc lekki kuferek, postawil go na werandzie, zrzucil elegancki gumowy plaszcz, rękawiczki... (IWA: 97f)
Die Kunde von diesen Vorgängen verbreitete sich schnell zu den Nachbardörfern... (MIL: 52)

Also verwöhnte sie ihn; hatte er bislang für selbstverständlich gefunden, seine Knöpfe mit Antoninas Nadel und Faden anzunähen, so kam er jetzt mit jeder Kleinigkeit an... (MIL: 66)

Vor allem bemerkte Boleslaw die saphirblaue Farbe seiner Socken. Unter der etwas zu kurzen und locker sitzenden Hose kam diese Farbe sehr deutlich zum Vorschein. (IWA: 89)

Er...zog einen kleinen Koffer heraus, stellte ihn auf die Veranda, warf den eleganten Regenmantel ab, die Handschuhe... (IWA: 98)

Die Basislexeme wies, nic, skarpety, rękawice sind beinahe gänzlich aus den Texten verdrängt. Interessant ist dagegen zu beobachten, daß im ältesten Sprachdokument wies gleichberechtigt neben wioska anzutreffen ist, was die erwähnte These der Entwicklung zu einer sprachlichen Verengung zugunsten des Diminutivs bestätigen kann, vgl.:

We wsi, gdzie mieszkal mój ojciec, przeszlo piecdziesieciu chlopów odrabialo pariszczyzne tak zwana sprzeżajna...; reszta we wsi pracowala indywidualnie, to jest posiadali jedna krowe ...W liczbie Srednich $w$ wiosce byl moj ojciec, posiadal dwie krowy i konia... (FOR: 43f)
Im Dorf, wo mein Vater wohnte, hatten über fünfzig Bauern einen sogenannten Spanndienst geleistet...Die übrigen besaßen nur eine Kuh...Mein Vater gehörte zu den mittleren Bauern, er hatte zwei Kühe und ein Pferd... (FOR: 43f)

Nimmt man eine Umkehrung des Übersetzungsvergleichs vor (Dt. $\Rightarrow$ Pl.), bestätigt sich der neutralisierte Status der angeführten Diminutive, insofern sie 
als optimale Äquivalente für die entsprechende nichtmarkierte deutsche Einheit gelten können:

Ich meldete es im Dorf... Nach wenigen Tagen und Nächten, das Dorf lebte unter stetem Alarm, war es bereits ein nicht unbeträchtlicher Berg...Das Dorf konnte weder arbeiten noch schlafen... (FRI: 47)

Aber es tut mir nicht leid, Erinnerungen sind mir mehr wert als Kleider. (FRA: 18)

Mein Kleid, mein Rock, alles zu kurz ... (FRA: 67)

Aber Momo konnte sich nicht erinnern, diese Puppe bei einem der Kinder gesehen zu haben. Und sie wäre ihr bestimmt aufgefallen, denn es war eine ganz besondere Puppe. (END: 87)

Die übrigen Hilfsmittel für den Werkzeugkasten:...ein Lineal, möglichst mit Metallkante; ein scharfes Messer; eine Schere... (GÖK: 18)
Zameldowalem o tym $w$ wiosce...Po wielu dniach i nocach - wioska żyla w stanie niustannego alarmu - utwożyła sie spora góra...W wiosce nikt nie pracowal i nie spal. (FRI: 60)

Ale nie żaluje tego, bo wspomnienia wiecej dla mnie znacza niż sukienkj. (FRA: 18)

Suknia, spodniczka, wyrosłam z wszystkiego! (FRA: 85)

Ale Momo nie przypominala sobie, zeby widziala te lalke u któregos dziecka. A na pewno zwrócilaby na nia uwage, bo byla to zupelnie niezwykla lalka... (END: 70)

W kompletnej skrzynce narzedziowej powinny jeszcze znalezc sie:...linijka (najlepiej $z$ metalowym kantem), ostry nóż, nớyczki... (GOK: 15)

Wiederum treten einige Bildungen im Wechsel auf (suknia - sukienka, spodnica - spodniczka), andere haben generell das Grundwort (wies, lala, nożyce) ersetzt.

In den Zusammenhang der semantischen Annäherung von Grundwort und modifizierter Bildung gehört auch das Phänomen der polnischen Hypokoristika, deren Übersetzung einen eigenen Problemkreis bildet. Grundsätzliches zum Thema Namen und Übersetzung sei jedoch vorausgeschickt: Überschaut man die gesammelten Paarungen, dann fallt eine in der Regel recht heterogene und inkonsequente Handhabung bei der Wiedergabe von Personen- und Verwandtschaftsnamen auf, die sich von der bei nomina appellativa unterscheidet. Wie willkürlich und sorglos beim Umgang mit Anthroponymen verfahren wird, äußert sich u.a. darin, daß zuweilen, sogar innerhalb eines Werkes, die Namen sei es in der Grundform, sei es in der modifizierten Form - mal in der polnischen Diktion übernommen werden, vgl. Misia $\Rightarrow$ Misia (MIL:56/73; die erste Ziffer gibt die Seitenzahl des Originals, die zweite die der Übersetzung an), Barbarka $\Rightarrow$ Barbarka(MIL:84/115), Kubus $\Rightarrow$ Kubus(NOW:151/104), Ala $\Rightarrow$ Ala (KOR:72/97), mal mit der deutschen Entsprechung oder sogar einem anderen deutschen Namen übersetzt werden, vgl. Tomasz $\Rightarrow$ Thomas (MIL:10/10), 
Sabinka $\Rightarrow$ Sabine (NUR:3/3), Zosia $\Rightarrow$ Sophie (KOR:142/193) bzw. Janek $\Rightarrow$ Heinz (KOR:26/35). Noch gravierender und für den Leser schwer nachvollziehbar ist es, wenn diese Sprünge innerhalb eines Werkes einem Namen gelten, vgl. Konstantyn $\Rightarrow$ Konstantin (MIL:52/68), und seine hypokoristische Variante Kostus $\Rightarrow$ Kostus (MIL:54/71) ${ }^{28}$

Die Entscheidung des Übersetzers für das Verfahren der sog. phonologischen Kalkierung ${ }^{29}$, d.h. die Nachbildung der Grundform oder auch der hypokoristischen Variante, ist so lange als äquivalente Lösung zu akzeptieren, wie die Benennung einer Person in einem ausgangssprachlichen Text einheitlich erfolgt. Problematisch wird es, wenn sich mehrere Formen eines Namens für eine Person im Text abwechseln, zum einen Grundform und Hypokoristikon (z.B. Dominik Domcio, MIL:60; Ola - Oleńka, IWA:130), zum anderen mehrere hypokoristische Bildungen (z.B. Edek - Edzio [ $=$ Edward], IWA:105 und 111; Stas Stach [ = Stanislaw], IWA:97; Irus - Irka [=Irena], NOW:108), die das Deutsche gar nicht erst morphologisch nachvollziehen kann. Im Normalfall ist kaum davon auszugehen, daß ein deutscher Leser mit den Regularitäten der polnischen Namengebung und ihrer Funktion vertraut ist und damit die alternative Verwendung in ihrer Bedeutung erkennen wird.

Erschwerend kommt hinzu, daß es Eigenart der polnischen Sprache ist, markierte Namensformen als usuelle, ihrer Merkmalhaftigkeit entleerte Bildungen zu nutzen. Mit Ausnahme des formell-offiziellen Sprachgebrauchs sind die hypokoristischen Formen hinsichtlich ihrer Funktion neben die Namensgrundform, ja sogar stellvertretend für sie, getreten..$^{30}$ Diesen unterschiedlichen Gebrauchsbedingungen im Polnischen und Deutschen ${ }^{31}$ muß dann auch eine Übersetzung Rechnung tragen. Wenn der deutsche Übersetzer der alternierenden Personenbezeichnung nicht folgt, wie z.B. bei Agasia $\Rightarrow$ Agafia (FIN:22/27) oder Paulinka $\Rightarrow$ Paulina (FIN:98/83), die Grundform beibehält, ist erkannt

2 Vgl. auch die Erlăuterungen weiter oben S.144ff.

* Vgl. dazu JÄGER 1980. S.62, der am Beispiel der homographischen Wiedergabeart die Probleme der Übersetzung polnischer Namenhypokoristika ins Deutsche skizziert hat.

30 $\mathrm{Zu}$ Hypokoristika besonders in der Anrede konstatiert KLIMASZEWSKA 1983 (S.84, Hervorhebung B.K.): "Vom Sprachsystem her sind diese Bildungen als expressive Derivate aufzufassen, in der Norm der polnischen Sprache haben sie jedoch in vielen Fälen ihre Expressivităt wesentlich eingebüßt und sind oft zu einem konventionellen Ausdrucksmittel geworden... Die nichthypokoristische Anrede dagegen wird als ungewöhnlich und als Zeichen besonderer Fremdheit gewertet, sie wird als sehr offiziell empfunden."

31 Vgl. dazu die konfrontative Studie über das Anredesystem der polnischen und deutschen Sprache der Gegenwart von TOMICZEK 1983, bes. Kap.4.9. (S. 181-193). 
worden, daß jene für den polnischen Text charakteristisch und damit relevant, für den deutschen Text aber redundant ist.

Als regelrecht fehlerhaft ist dann folgende Übertragung einzustufen, die, diesmal die deutsche Namensentsprechung wählend, die unterschiedlichen Gebrauchsnormen verkannt hat:

Wjechali w dziedziniec - Tomasz siedzial wtedy obok Grzegorzunia, który za stary żeby pracować, zajmowal sie pleceniem koszyków. (MIL: 25)

Sie kamen in den Hof geritten - Thomas saß neben Gregorlein, der, zu alt für die Arbeit, sich mit Korbflechten beschäftigte. (MIL: 30 )

Mit dem hypokoristischen Namen wird im deutschen ein falsches Signal ausgesandt, sein Gebrauch kann in diesem Zusammenhang (alter Mann) nur befremden.

\subsubsection{Funktionale Differenzierung von Basislexem und Diminutiv}

Die am stärksten vertretene Gruppe innerhalb der Neutralisierung bilden jene Wortformen, die zwar auch die Komponente klein aufweisen, deren semantisches Gewicht aber auf der Angabe der daraus resultierenden Funktion und Verwendungsart des Objekts liegt ${ }^{32}$ und denen darüberhinaus keinerlei emotional-wertende Information zukommt. Sie sind Resultat der Tendenz der polnischen Sprache, die Angabe unterschiedlicher Größen-, Bedeutungs- und Wertangaben vermittels der Opposition Grundwort - Derivat zu leisten und so die Differenzierung eines gegebenen Begriffsfeldes durchzuführen. Da diesen Diminutivbildungen nicht ein modifizierender, sondern ein determinierender Charakter zukommt, ist beim Übersetzen zu beachten, daß sie mit dem Basislexem nicht beliebig austauschbar sind, andernfalls Einbußen auf der pragmatischen bis hin zur semantischen Ebene die Folge wären. ${ }^{33}$

Aufgrund der unterschiedlichen Dispositionen beider Sprachen hat der deutsche Text in den folgenden Belegen wiederum mit der Vernachlässigung des wortbildenden Formans eine korrekte zielsprachliche Gestaltung vorgenommen. Es lassen sich im weiteren verschiedene (bei weitem nicht alle) Kriterien der funktionalen Differenzierung herauslesen:

2 Vgl. die Ausfürungen von REITER 1988. S.676.

W VI. die schon bei der Vorstellung der Wiedergabetypen gemachten Beobachtungen. 
Kaczor rozsiadl sie wygodnie. Czul zmeczenie. Szofer zerknal w lusterko... (NOW: 116)

...a jeden $z$ nich, najmlodszy, wciaż biegal na drugi koniec rynku, stawal pod daszkiem nad wejsciem do apteki i wypatrywal. (FIN: 45)

Schowala dokumenty do torebki... (FIN: 53)

Nikt prawie tedy nie jechal, a jeśli, to wsiadaly... wiejskie kobiety $w$ bialych chustkach... (FIN: 88)

"Po wojnie wozili mnie po okolicznych wsiach, gdzie mieszkali niegdys Żydzi, i zawieźli nawet do miasteczka. Pokazywali mnie jak w cyrku". (FIN: 93)

[I:] Na korytarzu [pociagu] jest pusto, cieplo, sa krzeselka... (RUM: 4)

[S:] Pożycze panu parasolke. - [M:] Z damska parasolka? Ja? Chce pani ze mnie pawiana zrobic? (NUR: 19)
Kaczor setzte sich bequem. Fühlte sich müde. Der Chauffeur guckte in den Rückspiegel... (NOW: 70)

...und einer von ihnen, der Jüngste, lief immer wieder ans andere Ende des Marktplatzes, stellte sich unter das Vordach über dem Apothekeneingang und hielt Ausschau. (FIN: 54)

Sie steckte die Dokumente in die Handtasche... (FIN: 52)

Fast niemand fuhr auf dieser Strecke, und wenn, dann stiegen Bauersfrauen mit weißen Kopftuichern ein... (FIN: 124)

"Nach dem Krieg haben sie mich durch die Dörfer der Gegend gefahren, wo einst Juden wohnten, ja sogar in die Kleinstadt. Wie im Zirkus haben sie mich herumgezeigt" ${ }^{\prime \prime}$ (FIN: 138)

[I:] Der Gang [des Zuges] ist leer, geheizt, außerdem stehen die Klappsitze zur Verfügung. (RUM: 5)

[S:] Ich geb Ihnen meinen Regenschirm. - [M:] Was, mit einem Damenschirm? Wollen sie mich lächerlich machen? (NUR: 23)

Mit dem Basislexem werden dagegen belegt: z.B. ein Wandspiegel, ein Hausdach, eine Einkaufstasche, ein Schultertuch, eine normale (größere) Stadt, ein Eßzimmerstuhl, ein Herrenschirm. Folgende Zusammenstellungen führen die Differenzierung besonders deutlich vor Augen:

Trzyczwartak poczal rozbierac sie, rozpial kohnierzyk koszuli, bety rozrzucal dookola krzesla... (NOW: 123) Wialo, zacinal deszcz...Ludzie przesuwali sie spiesznie $z$ podniesionymi kolnierzami. (NOW: 126)

...stary Grzegorzunio zasiadal na swojej lawce i cos strugal - jego nożyk, tak starty od użycia, że ostrze zweizalo sie prawie w szydlo, przecinal gałake jednym zamachem -... (MIL: 60) Wielu par koni potrzeba, żeby ruszyc
Dreiviertel begann sich auszuziehen, knöpfte den Hemdkragen ab, schmiß die Klamotten neben den Stuhl... (NOW: 77) - Es wehte, der Regen peitschte...Die Leute gingen eilig mit hochgeschlagenen Kragen vorbei. (NOW: 80)

...das alte Gregorlein setzte sich auf seiner Bank zurecht und schnitzte etwas - mit seinem von Arbeit so abgenutzten Messerchen, daß seine Spitze fast zu einer Ahle geworden war, schlug er den Zweig mit einem Hieb ab... (MIL: 79) 
lokomobile czy mlocamie z miejsca. Niekiedy, choc rzadko, wieziono ja w sasiedztwo, $z$ nawolywaniem, klaskaniem batów i podkladaniem gakzi pod kola. (MIL: 68)

Rozmowa toczyla sie $w$ gabinecie, który stal teraz zakurzony i nieużyteczny, chyba ze jakis pacjent zjawil sie wieczorem pod oslona ciemnosci $z$ razowcem-zaplata w koszyku. (FIN: 17) - Kazde jablko trzeba najpierw ostrożnie-delikatnie zdjac z galazki,... potem ostrożnie-delikatnie wlożyć do wiklinowego kosza... (FIN: 17)

...pod bieżaca zimna woda umyte $\mathrm{i}$ dokladnie osuszone kuropatwy (przepiorki) solimy umiarkowanie wewnatrz i zewnatrz, po czym każdego ptaszka oddzielnie owijamy... (LEM: 73) - Po tym czasie ptaka oskubujemy na sucho, patroszymy... (LEM: 71)

Die Grundwort-Derivat-Opposition wird genutzt, um zu unterscheiden: Hemdbzw. Blusenkragen gegenüber Jacken-bzw. Mantelkragen; dünner Zweig gegenüber kräftigem Ast; Handkorb im Gegensatz zu Erntekorb; Kleingeflügel gegenüber größerem Geflügel wie Fasan, Ente, Pute usw.

b. Eine seltenere Differenzierung ist die in alt -jung:

Zgodzil sie Macius za pastuszka. (KOR: 125)

...otarl z miloscia twarz Maliny, przy czym poczul znowu pod palcami doskonalosć powiek, podobnych do listków wiosennych. (IWA: 132)
- Es bedarf vieler Pferdepaare, um eine Lokomobile von der Stelle zu rühren. Manchmal, doch selten, fuhr man sie in die Nachbarschaft hinüber mit Rufen, Peitschengeknall und Unterlegen von Ästen hinter die Räder. (MIL: 92)

Das Gespräch fand im Praxisraum statt, der jetzt verstaubt war und nicht gebraucht wurde, es sei denn, ein Patient erschien abends im Schutz der Dunkelheit mit einem Schwarzbrot im Korb als Bezahlung. (FIN: 57) - Jeden Apfel mußte man zunächst vorsichtig und zart vom $Z$ weig lösen,...dann vorsichtig und zart in einen Weidenkorb legen... (FIN: 56)

Die...unter fließendem Wasser gewaschenen und gut abgetrockneten Rebhühner (Wachteln) innen und außen mäBig salzen und jeden Vogel gesondert...umwickeln... (LEM: 77f) - Dann den Vogel rupfen, ausnehmen... (LEM: 76) Lider empfand. (IWA: 128)
Die jungen und zarten, gerade aufgegangenen Blätter des Frühlings stehen im Kontrast zu den fallenden, modernden Blättern des Herbstes, vgl.:

Zapachy jesieni: skad pochodza, jakie tworza mieszaniny, nie da sie tego okreslic, butwiejace liscie i igliwie... (MIL: 95)
Hänschen verdingte sich als Hirtenknabe. (KOR: 169)

...[er] wischte zärtlich Malinas Gesicht $a b$, wobei er von neuem mit den Fingerspitzen die Vollkommenheit ihrer den Fruhlingsblättern so ähnlichen

Herbstgerüche: woher sie kommen, welche Mischungen sie bilden, das läßt sich nicht beschreiben; modernde Blätter und Nadeln... (MIL: 130) 
c. Die Opposition grob - fein begegnet in:

Kaszagryczana...stanowi...niezastapiony dodatek (zamiast ziemniaków) do wszelkiego rodzaju odmian polskich zrazów, pieczeni i sosów pieczeniowych. (LEM: 30) - Kaszka krakowska. Jest to bardzo delikatna, drobna, wrecz wykwintna kaszka... (LEM: 35)

W emaliowanym rondlu zagotowujemy * 1 jasnego piwa, a gdy sie raz zagotuje, dodajemy $1 / 1$ gestej, niezbyt kwasnej śmietany. (LEM: 27) - Bezposrednio przed podaniem zupe podprawiamy 1\% 1 slodkiej śmietanki. (LEM: 28)
$\mathrm{Zu}$ allen Arten von Rinder- und Schweinebraten sowie Bratensaucen bildet [die Buchweizengrütze] - anstelle von Kartoffeln - eine unersetzbare Beigabe. (LEM: 33) - Krakauer Gruitze. Eine sehr zarte, feinkörnige, geradezu erlesene Grütze... (LEM: 38)

In einem Emaillekochtopf $* 1$ helles Bier zum Kochen bringen. Einmal kurz aufwallen lassen und $1 / 41$ dicke, nicht zu saure Sahne zufügen. (LEM: 30) Unmittelbar vor dem Anrichten die Suppe mit $1 / \% 1$ süsser Sahne verfeinern. (LEM: 31)

Die feingemahlene, qualitativ bessere Grütze wird von der grobkörnigen, gewöhnlichen unterschieden, so auch die süße, für delikatere Speisen gebrauchte von der dickflüssigen, sauren Sahne.

\section{d. Schließlich ist das Kriterium roh - verarbeitet zu nennen:}

$1 \mathrm{~kg}$ umytych, ugotowanych i obranych burakow szatkujemy na tarce o grubszych "oczkach". (LEM: 157) - Przed podaniem buraczki zakwaszamy umiarkowanie sokiem cytrynowym. (LEM: 158)

Wszedlszy na schodki i nacisnawszy klamke, stapa sie po posadzce z wytartych cegielek, ulożonych ukosnie w jodelke, a swiatlo pada na nia przez zielone, czerwone $i$ żóte szybki... (MIL: 11)
$1 \mathrm{~kg}$ gewaschene, gekochte und geschälte rote Rüben auf einer groben Reibe reiben. (LEM: 159) - Vor dem Anrichten das [Roterübengemüse] mit etwas Zitronensaft leicht ansäuern. (LEM: 159f)

Nachdem man das Treppchen hinaufgegangen ist und die Türklinke gedrückt hat, gelangt man auf einen ausgetretenen Ziegelboden; die Ziegel stoßen schräg in der Form von Tännchen [sic!] aneinander, das Licht fallt darauf durch grüne, rote und gelbe Scheibchen... (MIL: 10)

Der zubereiteten Speise steht der Rohstoff gegenüber, dem Ziegel für dekorative Zwecke der Baustein für das Mauerwerk.

In allen Fällen konnte der Übersetzer dem Problem der Neutralisierung wirksam begegnen, indem er, um funktionell äquivalent zu übertragen, auf das formal analoge Element verzichtet und sich anderer morphologisch-lexikalischer Mittel bedient hat. Um das generell "kleinere" Exemplar des durch das Basislexem Gemeinten, aber vor allem das qualitativ andere im Deutschen korrekt zu 
erfassen, ist auf die Kompositabildung als häufigstes Kompensationsmuster zurückgegriffen worden. ${ }^{34}$

Daß hier nicht immer optimale Äquivalenz, d.h. weitestgehende Übereinstimmung in Absehung von strukturellen Unterschieden erreicht wird, mögen die nachfolgenden Beispiele demonstrieren:

Ognie w wiosce już pogasly i byla tylko czamośc i ten szum. Kiedy już. wszyscy nadeszli na placyk przed kosciolem, skierowali sie gromada na miejsce... (MIL: 44)

Uciekali stamtad przed glodem. Niesli przywiazane na plecach worki i wezelki, a czesto ciagneli $w$ drewnianych wózkach male dzieci. (MIL: 27)

Dom i podwórze pana Romualda odslanialy sie nagle $w$ dole za jeiniakiem maly dworek $z$ drewnianymi kolumienkami ganku, obrosniety czarnym bzem. (MIL: 87)

Nie tylko musial jeździć po lesie, dogladać to tej, to owej roboty,....ale także jeździć nawet do miasteczka. (IWA: 105f)

Ubrany w kurtke $z$ samodzialu, która go klula przez koszule, Tomasz śledzil ruchy ministrantów w komezkach. (MIL: 23)

Z okjenka przybiegl babski glos... (NOW: 10)

Za kumietynia, koło krzyża z blaszanym daszkiem, zwrócil sie na prawo ku wiosce Pogiry... (MIL: 57)

W Wielkim Tygodniu, w kosciele, gdzie obrazy przeslanialy zaslony $z$ kiru, a kolatki stukaly sucho zamiast dzwonków, odwiedzalo sie grób Pana Jezusa. (MIL: 23f)
Die Lichter im Dorf waren erloschen, und es war nur Dunkelheit und dieses Raschein. Als alle auf das Plätzchen vor der Kirche gekommen waren, gingen sie geschlossen zu der Stelle hin... (MIL: 57)

Sie flohen vor dem Hunger. Sie trugen, auf den Rücken gebunden, Säcke und Bündel, und oft zogen sie kleine Kinder in Holzwägelchen mit. (MIL: 33)

Herm Romualds Haus und Hof wurden plötzlich unter jungen Kiefern sichtbar ein kleines Landhaus mit den Holzsäulchen der Vorgalerie, mit Holunder bewachsen... (MIL: 119)

Er mußte nicht nur in den Wald fahren, um diese oder jene Arbeit zu beaufsichtigen,....sondern auch in das Städtchen. (IWA: 98)

Thomas, in einem handgewebten Rock, der ihn durchs Hemd stach, verfolgte die Bewegungen der Ministranten in ihren Chorhemdchen. (MIL: 28)

Durch das Fensterchen kam eine Weiberstimme... (NOW: 11)

Hinter der Kommende neben dem Kreuz mit einem Dächlein aus Blech bog er nach rechts zum Dorf Pogiry ein.... (MIL: 75)

Während der Karwoche suchte man in der Kirche, wo die Heiligenbilder mit Trauerschleiern verhängt waren und anstatt der Glöcklein die Klappern trocken klopften, das Grab des Herm Jesu auf. (MIL: 28)

\footnotetext{
34 Weitere markante Beispiele zur funktionalen Differenzierung und zu kompensatorischen Methoden ihrer Wiedergabe vgl. die Darstellung der Wiedergabetypen, S.154ff, 164ff. $168 \mathrm{ff}$.
} 
Die Bildungen der polnischen Vorlage stehen ausnahmslos in reiner Bezeichnungsfunktion, bieten die sachliche Angabe einer Gegenstandsklasse und zeigen nicht die markierte Ausnahme der Verwendung, sondern die lexikalische Norm an. Im deutschen Zieltext werden mit dem formalen Nachvollzug andere, expressive Konnotationen geweckt und ein vom Ausgangstext nicht beabsichtigter emotional-wertender Zusammenhang hergestellt, was bei dem gegebenen Kontext einen Verstoß gegen die pragmatisch-stilistische Norm darstellt.

Es ist angebracht, placyk und wózek mit Kirchplatz und Handwagen wiederzugeben, da durch die Spezifizierung im ersten Glied des Kompositums die relevante Information ausgedrückt ist. Will man dem Deutschen gerecht werden, so genügt es, für kolumienki das Grundwort Säulen zu setzen, denn nicht eine besonders kleine, zierliche oder dekorative Stütze ist gemeint, sondern eine an Vorbauten oder Veranden von Wohnhäusern übliche, im Gegensatz zu Säulen an meist großen, architektonisch bedeutenden Bauwerken (kolumny). In ähnlich klassenstiftender Funktion ist auch das Kontrastpaar miasto - miasteczko zu verstehen. Während mit miasto in der Regel eine Großstadt bezeichnet wird, bezieht sich die markierte Variante nicht allein auf besonders kleine Städte, sondern generell auf Nicht-Großstädte, so daß Kleinstadt bzw. nur Stadt eine adäquatere Lösung als die im neutralen Sprachgebrauch kaum gebräuchliche Diminutivbildung bieten. Als Unterscheidungskriterium bei komża - komeżka könnte man Alter und hierarchische Stellung anführen: komża als Priestergewand (vgl. "ksiadz...ubral sie w komże" - MIL: 37), das dazugehörige Derivat als Kleidungsstück der Ministranten. Besonders deutlich äußert sich der zielsprachliche Fehlgriff bei Fensterchen (okienko = Zellenfenster), insofern auch der unmittelbare Kontext, die derbe Sprechweise, mißachtet wurde und somit im Deutschen ein von der Vorlage nicht intendierter paradoxer Effekt geschaffen worden ist. Nicht minder verfehlt muten die eigentlich mit anderen Assoziationsbereichen verbundenen Übersetzungen von daszek und dzwonki an. Zielsprachenkonforme Ausdrücke wären eher in den verwandten lexikalischen Einheiten Giebel und Schellen zu finden.

Waren obige Fälle als Verstöße in pragmatisch-stilistischer Hinsicht zu deuten, ist im folgenden sogar die semantische Ebene verfehlt worden:

Zadziera sie glowe $\mathrm{i}$ wtedy ukazuje sie obrosly mchem Chrystus z drzewa. Siedzi w rodzaju kapliczki, jedna reke trzyma na kolanach, a na drugiej wspiera podbródek, bo jest smutny. (MIL: 11)
Man wirft den Kopf in den Nacken, und dann erscheint ein moosbewachsener Christus aus Holz. Er sitzt in einer Art Kapellchen, die eine Hand liegt auf dem Knie, auf die andere hat er das Unterkinn gestützt, denn er ist traurig. (MIL: 11) 
Wazelina usiadl na niskim stolku pod sciana. (NOW: 39)
Wazelina setzte sich auf das niedrige

Tischchen an der Wand. (NOW: 23)

Es ist verkannt worden, daß der Ausgangstext mit dem Derivat zum einen nicht eine Kapelle ('kleineres Gotteshaus'), vielmehr einen Bildstock meint, zum anderen nicht einen kleineren Tisch - dann müßte stolik stehen -, sondern einen Hocker, auch wenn jener eine ähnliche Gestalt hat.

Während in den bisherigen Belegen die Übersetzer sich offenbar vom Prinzip der formalen Analogie haben leiten lassen (SD $\Rightarrow S D$ ), ist in einigen Beispielen der ausgangssprachlichen Wortform in analytischer Weise begegnet worden:

Co pewien czas zlazil, sprawdzal manometr, otwieral drzwiczki i do buchajacej ogniem czelusci miotal debowe polana... (MIL: 67)

[S:] O tu, widzi pani, co sie z tym murkiem zrobilo. Chyba każe to rozwalić i też marmur dam. (NUR: 3)

Butelka wódki stala na stole. (IWA: 128)

Nasza gosposia wziela sie do pracy. (FOR: 100)
Von Zeit zu Zeit erhob er sich, prüfte den Manometer, öffnete die kleine Tür und warf in den feuerspeienden Rachen Eichenklötze... (MIL: 91)

[S:] Sehen Sie sich doch die kleine Mauer an, die bröckelt von allen Seiten ab. Ich werde sie einreißen lassen und auch Marmor aufstellen. (NUR: 3)

Auf dem Tisch stand eine kleine Flasche Wodka. (IWA: 123)

Die kleine Hausfrau ging an die Arbeit. (FOR: 95)

Nichts deutet hier auf eine bewußte Verringerung hin, es handelt sich wiederum um Lexeme, die zwar Objekte kleinen Ausmaßes benennen, gleichzeitig aber auch eine semantische Veränderung erfahren haben. Mit dem gezielten, spezifizierenden Sytagma "klein + Basislexem" mißversteht der Zieltext das Gemeinte, denn im einzelnen werden lediglich die Kesseltür einer Dampfmaschine, die Einfassung eines Grabes, eine übliche Wodkaflasche und schließlich - der krasseste Fehler, weil semantischer Art - eine Haushälterin bezeichnet.

Kehrt man die zu kontrastierenden Sprachen um (Dt. $\Rightarrow$ Pl.), findet man bei den entsprechenden Diminutiven den Verlust ihres allein modifizierenden Charakters bestätigt. Denn die Mehrzahl des ND $\Rightarrow$ SD-Typs bilden neutralisierte polnische Diminutivformen, die für eine nichtdiminutivische Einheit gesetzt wurden. Einige der schon genannten oder vergleichbare Derivate lassen sich auch für diese Übersetzungsrichtung angeben, so daß eine direkte Gegenprobe möglich wird:

"Solche VW-Busse gibt's viele. Wie erkennen Sie den richtigen? Habt ihr

"Takich mikrobusów jest dużo. Jak pani pozna wlasciwy? Nic nie uzgodnilys- 
nicht verabredet?" - "O doch. Die Frau winkt aus dem Fenster." (HEY: 71)

"Wo warst du solange, Alte? Läßt einen da unten schmachten!"..."Ich..." sagte die Prinzessin und steckte den Spiegel wieder ein... (TUC: 46)

Momo stellte sich in die Mitte des freien runden Platzes. (END: 266)

Jetzt ōffnete der Mann die Wagentür, stieg aus und kam auf Momo zu. (END: 90)

Man stochert in der Asche eines verbrannten Hauses, in den Trümmern eines Autowracks, untersucht Blut- und Geschoßspuren, Marmeladegläser... (HEY: 80)

"Das AdreBbuch", sagte Doris, "enthielt einundsechzig Anschriften, fünfundfünfzig davon mit Telefonnummern." (HEY: 33)

Im Vorgarten sortierten die Kollegen gerade die Hausbesetzer, im Licht der Suchscheinwerfer, die gegen die Villa gerichtet waren. (HEY: 41)

Gerfried blickte auf seine dicke, schimmernde Armbanduhr... (HEY: 55)

Er klappte die Uhr wieder zu und steckte sie in die Westentasche. (END: 147)

Kleidung mittel, nicht modisch, nicht unmodern: Hosen, Pulli, Trenchcoat, Kopftuch. (HEY: 10)

Katharina kramte gerade in der Umhängetasche nach ihrem Paß... (HEY: 5)

Der PIad verlief wie in einem Irrgarten kreuz und quer und mündete schlieblich in einem kleinen Raum... (END: 148)

Früher, vor vierzehn Jahren, war er Gemüsehändler, hatte einen Karren und ein Pferd... (FRI: 24) cie?" - "O tak. Kobieta bedzie kiwala przez okienko." (HEY: 77)

"Gdzie bylas tak dlugo, stara? Kazalas mi tam w dole usychac $z$ tesknoty!"... "Ja...", powiedziala Ksieżniczka, chowajac Justerko... (TUC: 57)

Momo stanela posrodku okraglego placyku. (END: 213)

Szary pan otworzyl teraz drzwiczki samochodu, wysiadl i podszedl do Momo. (END: 72)

Grzebie sie w popiołach spalonego domu, w resztkach wraku samochodu, bada slady krwi i kul, sloiki z marmolada... (HEY: 87)

"Ksiqzieczka adresowa", powiedziala Doris, "zawierala szesćdziesiąt jeden adresów, $z$ tego piecdziesiat piec $z$ numerami telefonow." (HEY: 36)

W ogrodku przed domem, w swietle reflektorów skierowanych na wille, koledzy wygarneli wlasnie grupe zajmujaca dom. (HEY: 45)

Gerfried patrzyl na swoj gruby, poiyskujacy zegarek... (HEY: 60)

Zamknal wieczko zegarka i schowal go $z$ powrotem do kieszonki kamizelki. (END: 122)

Odzienie takie sobie, ani modne, ani niemodne: spodnie, pulower, trencz, chustka na glowie. (HEY: 11)

Katarzyna gmerala wlaśnie w torebce, szukajac paszportu... (HEY: 5)

Drózka wila sie jak w labiryncie, wreszcie jednak doprowadzila ich do niewielkiego pokoiku... (END: 123)

Dawniej, czternaście lat temu, handlowal warzywami, mial wozek i konia... (FRI: 28)

Besonders augenfälig wird die funktionale Differenzierung dort, wo Grundwort und Derivat in unmittelbarer Nähe auftreten: 
Ein Speicher und Dachspeicher ist auch noch da, wie im Vorderhaus. (FRA: 21)

...und dann sangen wir alte Volkslieder und dann amerikanische Lieder, und dann sangen wir ein Reiterlied... (TUC: 52)

Ein etwaiger offener Kamin [im Wohnraum -B.K.] muß in Verbindung mit dem Schornstein gebaut und mit einem soliden Fundament ausgerüstet werden. (GÖK: 391)
Na tym samym pietrze od frontu znajduje sie strych, a poza tym jest jeszcze stryszek nad oficyna. (FRA: 23)

...a potem spiewaliśmy stare piosenki ludowe, piosenki amerykariskie, wreszcie pieśn rycerska... (TUC: 64)

Ewentualny otwarty kominek [ $w$ mieszkaniu -B.K.] musi być zbudowany w polaczeniu $z$ kominem i oparty na fundamencie. (GÖK: 347)

Es ist darauf hinzuweisen, daß viele der hier als neutralisiert vorgestellten Bildungen in anderen Kontexten durchaus modifizierende Funktion annehmen können. Dieses ambivalenten Status wegen ist eine eindeutige lexikographische Zuordnung dieser Wortformen nicht möglich, Funktionalität (determinierender Charakter) und Expressivität (modifizierender Charakter) müßten bei jedem Lemma Erwähnung finden.

Den Beispielen für das Deutsche als Ausgangs- und Zielsprache ist zu entnehmen gewesen, daß es sich bei der Wortneubildung im wesentlichen nicht des derivationellen Verfahrens, eher der Komposition bedient. In nur wenigen Fällen hat die Modifizierungsinformation in eine semantisch-kategorielle Information gewechselt; sie seien, obwohl für den Übersetzer meist kein Problem, noch genannt:

...damit die Nachbarn seines Eigenheims...nicht in Aufregung gerieten über seine immerhin ungewohnte Tracht; selbstverständlich trug er auch einen Gürtel mit Revolver. (FRI: 42)

Man könnte denken, es donnert oben, wenn man nicht wüßte, daß nun das Bett von Frau v.Daan ans Fenster geschoben wird. "Ihre Hoheit" im rosa Bettjäckchen muß aus nächster Nähe den frischen Ozon durch ihr schönes, kleines Näschen atmen! (FRA: 76)

Sie klettern die Küste bis fünfzig Meter hoch empor und bauen ihre Nester unter Grasbüischel oder in Felslöcher. (GRZ: 25)

Im Londoner Zoo pflegte ein tasmanisches Rattenkänguruh ein Bündel Stroh ...aby sasiedzi...nie wpadli $z$ powodu jego niezwyklego stroju w zdenerwowanie: oczywiście mial również pas i rewolwer. (FRI: 54)

Można by pomyśler, że na górze grzmi, gdyby sie nie wiedzialo, że to tylko Iożko pani van Daan jedzie pod okno. "Jej wysokosc" w różowej lizesce musi z calkiem bliska wdychać świeże powietrze swym slicznym, malym noskiem! (FRA: 98)

Pingwiny te wdrapuja sie na brzeg aż do $50 \mathrm{~m}$ wysokosci i pod kepami trawy lub w dziurach skal buduja swoje gniazda. (GRZ: 17)

W londyńskim ogrodzie zoologicznym tasmański kangur szczurzy mial zwy- 
mit dem Schwanz zu umwickeln... (GRZ: 86)

Die übrigen Hilfsmittel für den Werkzeugkasten:...ein Ölkännchen mit dünnflüssigem Maschinenōl und schlieBlich einige Bogen Schleifpapier... (GÖK: 18) czaj owijac wiqzke slomy ogonem... (GRZ: 45)

W kompletnej skrzynce narzedziowej powinny jeszcze znaleźc sie:...oliwiarka z rzadkim olejem maszynowym i wreszcie pare arkuszy papieru sciernego... (GÖK: 15)

\subsubsection{Formal-semantische Restriktionen}

Wendet man sich innerhalb der Übersetzungsanalyse der oben (S.232) formulierten zweiten Fragestellung zu, so zeigt sich als zweites wesentliches Übertragungsproblem, diesmal auf die Zielsprache bezogen, die fehlende Möglichkeit, das dem der Vorlage entsprechende Lexem zu modifizieren, genauer zu suffigieren. Hier ist der Übersetzer durch ein restringiertes Angebot seines Sprachsystems dazu gezwungen, auf der Situation angemessene Ersatzlösungen auszuweichen. Dabei gilt es, zunächst auf jene Fälle, die strukturell-morphologischen Restriktionen unterliegen, zu verweisen:

Boleslaw był bardzo zdrów i mocny i na pewno dlugo by jeszcze musial blakać sie od brzeziny do polanki, gdzie chata Maryjki... (IWA: 131)

W ciszy mieszkanka potrzaskiwal sennie ogien. (NOW: 53)

Uklada Macius bajeczke dla Ali. (KOR: 74)

Józef skubnal palcami mech i przybliżywszy do oczu puszysta, zielona poduszeczke, przypatrywal sie jej 2 baczna uwaga. (FIN: 31)

Die durch die bereits suffigierte Form (Licht-ung, Wohn-ung, Mär-chen) bzw . den en Stamm vorliegenden Modifizierungsbeschränkungen haben den Übersetzer zur Verwendung des Grundwortes veranlaßt, was lediglich als approximative Äquivalenz gelten kann, da mit ihr doch ein Verlust an expressiver Wertung einhergeht. Lediglich die Wiedergabe von mieszkanko mit dem Kleinheit und Enge implizierenden Lexem Raum kann durch funktionelle Äquivalenz überzeugen. 
Bei den Beispielen für Adjektive resultiert die formale Beschränkung aus dem -I Stamm. Während kühl schon die Detensivierung mit sich führt, wäre die Wendung hatte lichtes Haar im zweiten Fall eher ein Äquivalent gewesen:

Pogoda byla nie ustalona i pelno chmur na niebie, chlodnawo. (IWA: 135)

"Kilo...", ucieszyl sie Kulas, "przyszedl Kilo."...'Kilo wariackich papierów' byl chudziutki i tysawy. (NOW: 118)

Restriktionen semantischer Art zeigen sich dagegen in folgenden Belegen:
Das Wetter war unbeständig und kühl, der Himmel hing voller Wolken. (IWA: 131)

"Kilo...", freute sich Kulas. "Kilo ist da."...'Das Kilo Jagdscheine' war mager und kahl. (NOW: 72)
A szczurek wdzieczny, że Macius go leczy: choć boli przymywanie rany, ale liże reke i tak slepkami mruga, jakby dziekowal. (KOR: 99)

Stach zdziwil sie: "Co to jest?" - "To mogilka", odpowiedziala Ola. "Czyja?" - "Jak to czyja? Mamusi." (IWA: 101f)

Mróz i slonce, rózgi krzaków na stromym brzegu po drugiej stronie wygladaly na bukiety ze zlota, powleczone gdzieniegdzie szara i sina farbka. (MIL: 21)

"Jutro dorsz", mlasnal wargami i powtórzyl: "dorsz z tranikiem." Co tydzień każda cela otrzymywala miske gotowanych dorszy. (NOW: 145f)
Und die Ratte ist dankbar, weil Hänschen sie pflegt. Obwohl das Auswaschen der Wunde sehr weh tun muß, leckt sie ihm die Hand und zwinkert mit den Augen, als wollte sie sich bedanken. (KOR: 134)

Stas wunderte sich: "Was ist das?" "Ein Grab", antwortete Ola. "Wessen Grab?" - "Was heiBt wessen? Meiner Mutti!" (IWA: 94)

Der Frost und die Sonne - die Ruten der Sträucher auf dem jenseitigen Ufer sahen wie goldene Sträuße aus, hie und da mit grauer und bläulicher Farbe überzogen. (MIL: 25)

"Morgen gibts Dorsch", schmatzte er und wiederholte: "Dorsch mit Tran." Alle Woche erhielt jede Zelle einen Topf mit gekochten Dorschen. (NOW: 98)

Zwei Aspekte sind zu unterscheiden: liegen mit Grab und Ratte Lexeme vor, deren negative Assoziationen im Deutschen generell nicht den verharmlosenden Effekt von Diminuierung dulden, zeigt sich in den übrigen Belegen die Verwendung von Stoffbezeichnungen als Behinderung der Zielsprache. Auch wenn im Deutschen mit Farbe nur das Grundwort gesetzt wurde, genügt doch schon die kompensatorische Bewegung, die Detensivierung mit bläulich, um funktionelle Äquivalenz zu erreichen; im letzten Beispiel hätte der Zusatz des ironisch gemeinten schmackhaft der Intention des Ausgangstextes besser entsprochen.

Im Gegensatz zu den bisherigen Belegen wäre eine Diminuierung der folgenden allenfalls als Okkasionalismus möglich, widerspricht aber in der Regel der Redenorm: 
To jeziorko posrodku buchty $w$ petli Issy, zasilała rzeka w czasie wiosennych roztopow przez bagnisty rów... (MIL: 63)

We wsi..., gdzie wówczas mieszkala moja matka, staruszka, byla szkolka czteroodzialowa. (FOR: 85)

Wszedl sinawy chlop...Siadl na brzezku krzesla, podwijajac nogi... (NOW: 34)

In zwei Fällen hat der Übersetzer Kompensationsmöglichkeiten nicht genutzt. Zum einen würde das Kompositum Dorfschule die Anzeige von Kleinheit und Begrenztheit unterstreichen, zum anderen könnte der Ausdruck von Intensität mit Hilfe des Zusatzes auf den äußersten (Stuhlrand) erzeugt werden.

Die unterschiedlichen Frequenzverhältnisse des Diminutivgebrauchs können zu der Annahme verleiten, das Problem formal-semantischer Restriktion gelte nur für die deutsche Sprache. Die hier aufgeführten Beispiele mōgen zeigen, daß es auch auf polnischer Seite unmöglich sein kann, bestimmte bereits suffigierte Wortformen zu modifizieren, was häufig zum Rückgriff auf analytische Bildungen führt:

"Natürlich hat er unser Feuerchen gesehen." (FRI: 51)

Manche hatten, wie das Mädchen Maria, Geschwisterchen dabei, die an der Hand geführt oder auf dem Arm getragen wurden... (END: 103)

Dieses Viertel ist typische Volksgegend,...vor allem die Kinder sind oft so schmutzig...So echte Kinder mit Rotznäschen... (FRA: 52)

..."das ist ja zum Auswachsen, wie hier heute getrödelt wird. MüBt ihr euer gemütliches Schwätzchen denn ausgerechnet jetzt abhalten?" (END: 197)
"Naturalnie tamten zuważyl nasze male ognisko." (FRI: 66)

Niektóre, jak na przyklad Maria, wziely ze soba mlodsze rodzeristwo i prowadzily je za reke albo niosly. (END: 84)

To jest typowa dzielnica robotnicza,.... już dzieci sa nieraz takie brudne ...Prawdziwe male zasmarkance... (FRA: 63)

"Szlag czlowieka może trafic od tego dzisiejszego guzdrania sie. Musicie wlaśnie teraz prowadzic sobie pogawędki?" (END: 161)

Eine Besonderheit der polnischen Sprache ist zu beobachten, wenn das der deutschen Ausgangseinheit entsprechende Derivat als eigenständiges Lexem auftritt und nicht als Modifikation zu nutzen ist:

So suche ich hastig auf dem Flughafen nach einem Kleinflugzeug, das ich chartern kann... Das rote Maschinchen ist gerade stark genug, um meinen
Szukam pospiesznie na lotnisku jekiegos malego samolotu do wynajecia... Czerwona maszyna jest maba, ale wastarczajaco silna, zeby uniesc w 
Mitarbeiter..., seine Frau, mich und unser ganzes Packzeug in die Luft zu ziehen. (GRZ: 13)

"Und der Alte hat gesagt, ich solle ihm abtelefonieren, weil er doch verreist...und da ist die Konferenz am Dienstag....ach du liebes Gottchen..." (TUC: 23f)

Sie stand gerade vor einer reich verzierten Spieluhr, auf der zwei winzige Figuren, ein Frauchen und ein Männchen, einander zum Tanz die Hand reichten. (END: 145) powietrze mnie, mojego wspólpracownika..., jego żone i nasz caly bagaż. (GRZ: 10f)

"I stary powiedzial, żebym do niego zadzwonila, bo on przecież wyjeżdża ...a we wtorek ma być konferencja...o dobry Boże..." (TUC: 27)

Stanela wlaśnie przed bogato zdobionym zegarem kurantowym, na którym dwie maleńkie figurki pani i pana podawaly sobie rece do tanca. (END: 119)

Maszynka bezeichnet u.a. Haushaltsgeräte (vgl. maszynka do mielenia migdalów) und kann daher nicht als Bezeichnung für ein kleines Flugzeug herangezogen werden. Die in den beiden nächsten Kontexten nötige Diminuierungsaussage wäre mit bożek ('Götze') bzw. panienka ('Fräulein') nicht zu erzielen.

Einen Sonderfall stellen die adjektivischen Intensiva dar. Anders als mit den Detensiva, die ihre formale Entsprechung im Deutschen haben und auch meist problemlos substituiert werden können, verhält es sich mit den Diminutiven auf -utki, eńki etc., denen kein analoges Derivationsmorphem zur Seite steht. Um dieser Lücke im morphologischen System der deutschen Sprache zu begegnen, muß der Übersetzer auf andere, zielsprachenkonforme Lösungen ausweichen. Will er funktionell äquivalent übertragen, so muß er, wie bei den Substantivdiminutiven, für jeden Fall aufs neue entscheiden, welche die optimalen Übersetzungsoptionen sind. Nicht selten haben die Intensiva ihren verstärkenden Charakter größtenteils eingebüßt und haben sich zu sprachüblichen Wendungen entwickelt, so daß die Wiedergabe durch das entsprechende Grundwort als gelungen anzusehen ist; der Versuch, hier in irgendeiner Form zu kompensieren, würde häufig als künstliche Überinterpretation erscheinen. Werden aber die diminutivischen Bildungen gezielt und mit Nachdruck eingesetzt, müssen lexikalische Mittel gefunden werden, die den Aussagewert explizieren; dazu zählen besonders adjektivische Zusammensetzungen und attributivische Fügungen. Vgl.:

To wszystko brzmialo jak $z$ bajki, $z$ czytanek dla grzecznych dzieci i czasami cichutko sie $w$ sobie smialam... (FIN: 73)

Dziwczyna położyla mu dlon na ramieniu. Pilegnowana, malutka dlon, kwitnaca rózowo malowanymi paznokciami. (FIN: 80)
Das klang alles wie Märchen aus einem Lesebuch für brave Kinder, und ich lachte hin und wieder leise in mich hinein... (FIN: 151)

Das Mädchen legte ihm die Hand auf die Schulter; eine gepflegte kleine Hand, an der die lackierten Fingernägel rosa blühten. (FIN: 139) 
...bo byl pulchniutki i okragly, malo ruchliwy, taki, którego od książki nie oderwiesz... (FIN: 10)

...wszystko to do pewnego stopnia bylo tej pogardy $i$ antypatii leciutkim wyrazem i pokrywka. (IWA: 49)

...zawstydzilam sie, ze jestem brudna $i$ predziutko jak mysz wsunelam sie do alkierza. (FOR: 53)

Jest wesola, mieszka w wesolym pokoju, sama go urzadzila, sama uszyla firanki, z muślinu, sama wyhaftowala obrus, sama, sama, sama, bo niby kto inny? Jest sa-miu-ten-ka. (FIN: 92)

Kokietowala go raczej Jola, mlodziutka wówczas, szesnastoletnia i przesticzna panna. (IWA: 36)

Zbudzilo ja cieniutkie drżenie szyb. (FIN: 7)

Stas poczul, ze mala placze, lzy cichutko spływały jej z oczu. (IWA: 130) ...er war mollig und rundlich und wenig regsam, so einer, den man von den Büchern nicht wegkriegt... (FIN: 14)

...all dies diente bis zu einem gewissen Grade als dezenter Ausdruck und leichter Deckmantel dieser Verachtung und Antipathie. (IWA: 36)

Ich schämte mich, daß ich so schmutzig war, und geschwind wie eine Maus schlüpfte ich in den Alkoven. (FOR: 50)

Sie ist fröhlich, wohnt in einem fröhlichen Zimmer, hat es selbst eingerichtet, selbst die Musselingardinen genäht, selbst das Tischtuch gestickt, selbst, selbst, denn wer auch sonst? Sie ist mut-ter-see-len-al-lein. (FIN: 136)

Schöne Augen machte ihm Jola, damals ein blutjunges, sechzehnjähriges und wunderhübsches Fräulein. (IWA: 21)

Ein ganz feines Beben der Fensterscheiben weckte sie. (FIN: 10)

Staś spürte, daß die Kleine weinte, ganz leise flossen ihr die Tränen aus den Augen. (IWA: 125)

In der Umkehrung des Übersetzungsvergleichs findet man die Beobachtungen bestätigt:

Viele Kinder hier laufen in einem dünnen Blüschen herum mit Holzschuhen an den bloßen Füßen... (FRA: 54)

So habe ich dann die grüne Stadt von 400000 Einwohnem und den sauberen Tiergarten...bewundert. (GRZ: 6)

Im schmalen Vorgarten wuchs eine winxige Edeltanne. (HEY: 112)

"Reden wir also offen miteinander. Du bist allein, armes Kind. Deine Freunde sind unerreichbar für dich." (END: 224)

Auch hier suchte sie sorgfältig alles ab, soweit es in der Dunkelheit möglich war. Sie hegte die zaghafte Hoffnung,
Wiele tutejszych dzieci biega w cieniutkich bluzach, w drewniakach na golych nóżkach... (FRA: 66)

W rezultacie...podziwialem czysciutki ogród zoologiczny i zielone miasto liczace 400 tys. mieszkariców. (GRZ: 5)

W waskim ogródku rosła maleńka jodelka. (HEY: 122)

"Porozmawiajmy wiec otwarcie. Jestes samiuteńka, biedne dziecko. Nie mozesz porozumiex sie z swoimi przyjaciólmi." (END: 180)

Także i tu starannie przeszukala wszystko mimo panujacej ciemnosci. Miala leciutkq nadzieje, ze jakims cudem 
daß die Schildkröte durch ein Wunder schon vor ihr nach Hause gekommen wäre. (END: 211)

Momo nickte unmerklich. (END: 85)

Mariefred ist eine klitzekleine Stadt am Mälarsee. (TUC: 30)

In seinem Wahn verfiel Marxentius Communus nun auf die Idee, die bestehende Welt hinfort sich selbst zu überlassen und lieber eine vollkommen nagelneue Welt zu bauen. (END: 47)

Hinter ihr zischelte es zwiefach: das waren die, die, ganz leise, sprechen wollten, und die andern, die die Flūsternden mit einem "Pst!" daran zu hindern suchten. (TUC: 37 )

Ein ganz kleines Durchgangszimmer soll Peter van Daans Reich werden. (FRA: 21) zólw doszedl do domu jeszcze przed nia. (END: 170)

Momo leciutko kiwnela glowa. (END: 69)

Mariefred jest maleńka miejscowoscia nad jeziorem Melar. (TUC: 37 )

Ale on wpadl na pomysl, aby odtad istniejacy świat pozostawic wlasnemu losowi, stworzyc zas inny, nowiuteńlki swiat. (END: 38)

Rozlegly sie dwojakiego rodzaju odglosy: tych, które chcialy po cichutku rozmawiac, $i$ innych uciszajacych tamte psykaniem. (TUC: 47)

Maleńki pokoik przejsciowy bedzie królestwem Piotra van Daana. (FRA: 22f)

\subsubsection{Pragmatisch-stilistische Inkompatibilitdten}

Zieht man das heuristische Modell des Nachvollzugs eines idealtypischen Übersetzungsvorgangs noch einmal heran, so läßt sich jetzt feststellen, daß es der Übersetzer bisher entweder mit "erstarten" Audrucksformen in der Ausgangssprache zu tun hatte oder ihm die notwendigen formalen Mittel auf Sprachsystemebene nicht zur Disposition standen. Kann er in einem gegebenen Textfall also eine Neutralisierung ausschließen und ist er auch nicht durch formal-semantische Restriktionen behindert, bereiten nun die "aktuellen", "lebendigen" Diminutive, die nämlich in ihrer eigentlichen Funktion, der subjektiv-wertenden Aussage, auftreten, das, wenn auch nicht quantitativ, so doch qualitativ gewichtigere Übersetzungsproblem.

In dem weiten Feld, das sich einer Übersetzung und deren Kritik jetzt öffnet, treten Diminutive nicht als Träger von sachlichen Aussagen, nicht als bloße Bezeichnungen oder Konstatierungen in den Blick, sondern vielmehr mit ihren affektiven Implikationen als Mittel stilistischer Sprachgestaltung. Die zumeist kodierte, d.h. erst vor dem Hintergrund eines textuellen Mikro- bzw. Makrobe- 
reichs in ihre Möglichkeit gesetzte Aussage muß vom Übersetzer in ihren vielfältigen Bezügen entschlüsselt werden. In dem dann stattfindenden Textverarbeitungs- und Textverbalisierungsproze $B$ bedarf es großer translatorischer Kompetenz, eines besonderen Feingefühls für stilistische Nuancierungen in der fremden und eigenen Sprachverwendung. Um dem übergeordneten Postulat zu entsprechen, mit der Erfassung und Umsetzung der Kommunikationsbedingungen, im näheren Kommunikationssituation und - ziel, ${ }^{35}$ die Invarianz des Sinns zu wahren, muß den innersprachlichen Instruktionen und außersprachlichen Determinanten sensibel Rechnung getragen und ein zielsprachengerechter Weg gefunden werden. Kenntnis und Vertrautheit der Gebrauchsdimensionen beider Sprachen ist besonders dann vonnöten, wenn sich, wie im vorliegenden Falle, pragmatisch-stilistische Inkompatibilitäten ergeben, mit der Folge, daß der unterschiedliche Einsatz morphologischer Elemente oft unmittelbar aufeinander trifft. Mit H.-M.Gauger gesprochen darf der Übersetzer nicht "das Bild seiner Sprache von den Diminutiven auf eine andere" übertragen, er darf nicht "mit den Augen seiner Kopräsenz die einer anderen" sehen, vielmehr muß er erkennen, daß "beide [Diminutive - B.K.] in verschiedener Weise vom Sprachbewußtsein ihrer jeweiligen Kopräsenzen ergriffen sind: Diminutiv ist nicht gleich Diminutiv". ${ }^{36}$

Will man im vorhinein eine Orientierung über die Angleichungsproblematik von Diminutiven auf Textebene geben, so ist es sinnvoll, Leitmotive und Konstanten herauszustellen, die die einzelnen, verwickelten Übertragungsrelationen wie die fallorientierte Analyse noch zeigen wird - durchsichtig machen. Man kann dabei bestimmte Spannungsfelder feststellen, in denen sich die beiden Sprachen bewegen und deren Pole wie folgt zu beschreiben sind:

- Die Diminutivverwendung im Polnischen zeigt sich häufig im Rahmen von Konventionen, d.h. in usueller Weise; das Deutsche ist eher durch individuellen, okkasionellen Gebrauch gekennzeichnet.

- Während im Polnischen das Gewicht der intendierten Aussage mehr durch Wortexpressivität getragen wird, verlagert es sich im Deutschen auf den umgebenden Kontext.

\footnotetext{
${ }^{3 s}$ Näheres dazu s. im theoretischen Teil der Arbeit S.81 und S.123.

36 GAUGER 1971. S.114; seine Beobachtung auf der Grundlage eines spanisch-französischen Diminutivvergleichs kommt dem polnisch-deutschen Verhälnis recht nahe.
} 
- Neigt der polnische Text zur Häufung von Diminutiven auch und besonders innerhalb einer schon markierten Passage, ist der deutsche Text vielmehr durch die Prinzipien der Ökonomie und Abwechslung geleitet.

Da diese Orientierungsbegriffe in der Praxis keine statischen Eckpunkte darstellen, vielmehr im Einzelfall meist aufeinander verwiesen und die Grenzen fließend sind, ist es angezeigt, einen von ihnen, den der Konvention, als methodischen Leitfaden, aus dem sich dann auch die anderen begreifen lassen, repräsentativ aufzunehmen und mit exemplarischen Textbesprechungen zu unterlegen.

\subsubsection{Konventionalisierte Kommunikationssituationen}

Die hier beabsichtigte Darstellung kommunikativer Standardsituationen kann nicht den Anspruch auf Vollständigkeit erheben, sie ist vielmehr der Versuch der Systematisierung von Textphänomenen, die bei der dieser Untersuchung voraufgehenden Lektüre in den Vordergrund rückten.

\section{Kind und Tier:}

Eine der gängigsten und bekanntesten Konventionen in beiden Sprachen ist der Diminutivgebrauch in der Rede von und mit Kindern bzw. Tieren, auch wenn einschränkend konstatiert werden muß, daß das Deutsche den allgemeinen Verwendungsnormen gemäß erheblich seltener die modifizierten Formen bemüht.

Besonders dieser Themenbereich gibt unabhängig von der sprachlichen Varietät dem Sprecher Anlaß, eine emotionale Haltung zu entwickeln und diese entsprechend anzuzeigen: ${ }^{37}$

"Prosze was, spojrzcie tylko na te dziewczynke....Spojrzcie na jej lagodna twarzyczke i rozumne oczeta." (KOR: 33)

...a jedna dziewczynka co dzien przechodzac tak przyjemnie usmiecha sie do niego i milym glosikiem mówi: "Dzień dobry!" (KOR: 141)
"Schauen Sie bitte nur einmal dieses kleine Mädchen an...Schauen Sie sich ihr heiteres Gesicht und ihre verständigen Augen an." (KOR: 45)

...ein kleines Mädchen, das jeden Tag an ihm vorbeikommt, lächelt ihn immer so nett an und sagt mit freundlicher Stimme "Guten Morgen". (KOR: 192)

\footnotetext{
"Im folgenden soll die bewāhrte Methode wieder aufgegriffen werden, Aussagen, die für den Übersetzungsvergleich PI. $\Rightarrow$ Dt. gelten, unmittelbar anschließend durch Exempel der gespiegelten Übertragungsrichtung $\mathrm{Dt}$. $\Rightarrow \mathrm{Pl}$. zu belegen.
} 
Chlopiec prowadzi za reke mala dziewczynke, która ladnie, predziutko przebiera nózkami i ma jasne wloski. (KOR: 72)

Dziecko ciażylo mu coraz bardziej w ramionach, ale nie byl to ciezzar wagi malego cialka. Odwrócil nieco glowe i wargami dotknal policzka dziewczynki. Miekki, cieply policzek. (FIN: 43)

Vgl. dazu:

"Das Fell ist so wunderschön, wahrscheinlich wird es eine wertvolle Ausfuhrware..." (GRZ: 61)

[Momo] dachte an die Stimmen der Sterne und an die Stunden-Blumen. Und dann begann sie mit klarer Stimme zu singen. (END: 266)
Der Junge hielt ein kleines Mädchen an der Hand, das niedlich und schnell seine Füßchen setzte und blondes Haar hatte. (KOR: 97)

Das Kind auf seinem Arm wurde immer schwerer, aber das war nicht die Last des kleinen Körpers. Er drehte den Kopf ein wenig, seine Lippen berührten die Backe des Mädchens. Die weiche, warme Backe. (FIN: 63f)

"Futerko jest przesliczne, prawdopodobnie bedzie ono stanowic cenny artykul eksportowy..." (GRZ: 35)

[Momo] myslala o glosach gwiazd i o Kwiatach Jednej Godziny. I dźwiecznym glosikiem zaczela spiewac. (END: 213)

Während die genannten Beispiele klassischen Funktionsfeldern des Diminutivs, Liebkosung, Mitleid etc., zuzuordnen und noch als individuell gekennzeichnet einzuschätzen sind, kehren die beliebig zu vermehrenden folgenden Belege erst den Gebrauchscharakter als Konvention hervor, insofern sich nämlich zeigt, daß völlig unabhängig von einem im Text lokalisierbaren Stimmungsgehalt Kinder und Tiere, ihre Attribute sowie vieles, was zu ihnen in Beziehung steht, gerade auch in einem sachlich gehaltenen informellen Kontext häufig diminutivisch angezeigt werden. Was also hier markiert wird, ist nicht das gerade durch das Diminutiv Bezeichnete, sondern die bestimmte Sprechweise ${ }^{38}$, die Kommunikationssituation, in die es gehört. ${ }^{39}$ Der Autor führt hier nicht mehr eine gezielt eingesetzte Gestaltung durch, vielmehr folgt er Sprachverwendungsnormen, die ihn, zwar nicht bezüglich des Einzelfalls, wohl aber des Kontexts, auf den Einsatz von Diminutiven festlegen: wo er seine Signale verteilt - davon kann man ausgehen -, bleibt ihm überlassen, obligatorisch ist dagegen die Kennzeichnung der Situation als solche:

Ulożyl Olenḱke w ramionach..., i, wolnym krokiem idac ku domowi, kotysal
Dann legte er Ola in seinen Armen... zurecht, ging mit langsamen Schritten

3* Vgl. dazu die Andeutungen von SEEBOLD 1983. Sp.1250.

30 Vgl. BRINKMANN 1971 (S.18), der dazu folgendes ăußert: "Bemerkenswert ist, daß die Diminuierung nicht dem Begriff zu gelten braucht, an dem sie erscheint; das Substantiv, das diminuiert wird, kann einfach die Stelle sein, an der ein Sprecher sein intimes Verhältnis zum Partner kennzeichnet." 
ja $w$ te i owa strone. To nótki, to glówka dziewczynki potracaly o liście, o krzaki... (IWA: 130)

Serduszko dziewczynki bilo pod jego palcami, czul je reka i obawial sie, ze jego bije tak samo. (IWA: 129)

Obok niej, w wózeczku przykrytym gaza, spal jej synek. (IWA: 48)

Przyniósl zwrotniczy od kumów stare, biedne ubranko. Przebral sie Macius, wzial kromke chleba... (KOR: 24f)

Ola usiadla w kaciku na malym stoleczku i cos gadala do lalki. (IWA: 100)

I Czing szczekal cichutko, ledwo, ledwo merdal ogonkiem, po czym lizal nas w policzki. (FIN: 35)

Albo [kanarek] wyfrunie na klatke, posiedzi troche - ale widac, że mu jakos dziwnie, nieswojo. Taki zdenerwowany, że nie wiem. Poprawia skrzydelka... (KOR: 63)

Nie, bylo to zwierzatko wieksze od myszy, z malym ogonkiem - rude, $z$ bialymi tapkami. (KOR: 90)

In der Umkehrung zeigt sich folgendes Bild:

Die Kinder blieben stumm. Ihre Gesichter waren plötzlich traurig und verschlossen. (END: 76)

Und plötzlich fing der Junge, der den ganzen Nachmittag der Spielverderber gewesen war, zu weinen an. Er versuchte es zu unterdrücken und wischte sich die Augen mit seinen schmutzigen Fäusten, aber die Tränen liefen in hellen Streifen durch die Schmutzflekken auf seinen Wangen. (END: 78)

...Erika war ungefähr so 16 , Lieselotte war ungefähr 12, und die Rita, die Kleine, nun jetzt ist sie 22 Jahre alt, die war da gut n Jahr. (RUN: 23) nach Hause und wiegte sie dabei hin und her. Köpfchen und Füße des Kindes stießen abwechselnd gegen Blätter und Büsche... (IWA: 125)

Das Herz des Kindes schlug unter seinen Fingern. Er spürte es mit der Hand und fürchtete, sein eigenes schlüge ebenso. (IWA: 124)

Neben ihr schlief ihr Söhnchen in einem mit Gaze zugedeckten Kinderwagen. (IWA: 34)

Der Weichensteller holte von seinen Verwandten einen alten, ärmlichen Anzug. Den zog Hänschen an, nahm ein Stück Brot mit... (KOR: 32f)

Ola setzte sich auf einen kleinen Hokker in der Ecke und redete mit ihrer Puppe. (IWA: 93)

Dann bellte Tsching leise, wedelte ganz sachte mit dem Schwanz und leckte uns die Backen. (FIN: 33)

Oder [der Vogel] fliegt oben auf das Bauer, sitzt da ein bißchen, aber man sieht, daß er sich nicht wohl fühlt. Er ist furchtbar aufgeregt, er zupft sich an den Flügeln... (KOR: 86)

Nein, das Tierchen war größer als Mäuse, es hatte einen kleinen Schwanz und war rothaarig mit weißen Pfoten. (KOR: 122)
Dzieci nie odzywaly sie. Na ich twarzyczkach zjawil sie nagle wyraz smutku i skrytosci. (END: 62)

Nagle chlopiec, który przez cale popoludnie przeszkadzal innym $w$ zabawie, zaczal plakac. Próbowal opanowac sie i brudnymi piastlcami tarl oczy, ale lzy nadal splywaly jasnymi strumyczkami po jego umorusanych policzkach. (END: 64)

...Erika miala już cos szesnascie lat, Liselotte okolo dwunastu, a Rita, najmlodsza, teraz ma dwadziescia dwa 
lata, miala wtedy cos ponad roczek. (RUN: 29)

"So? Ach! Das hast du nicht gewuBt! Das hat das gute Lieschen nicht gewuBt! Habe ich dir nicht schon tausendmal gesagt, daß man seinen Schrank nicht offenstehen läbt?" (TUC: 71)

Ursprünglich gab es keine Koalas auf der Känguruhinsel. Man hat diese Tiere...1924 eingeführt. (GRZ: 21)

Ein kleiner Vogel hüpfte heran, legte den Kopf schief und flog dann auf... (TUC: 90)

Auch ein kleiner Klāffer ist da, den wir durch sein Gebell kennen und dessen Schwanz man sieht, wenn er am Bootsrand entlangläuft. (FRA: 52)

Die Akrobatenfrau baut sich ein ziemlich großes Nest aus Gummiblättern und Rindenstücken in Astlöchern oder hohlen Bäumen... (GRZ: 55)
"Ach tak?! Nie wiedzialas o tym! Grzeczna Lizka o tym nie wiedziala! Czy nie mówilam ci już tysiace razy, ze swoja szanke zawsze trzeba zamykac?" (TUC: 88)

Pierwotnie nie bylo koali na Wyspie Kangura. Zwierzatka te...wprowadzono tu dopiero w 1924. (GRZ: 14)

Maly ptaszek podskakiwal w naszym kierunku, przekrzywil glowke, a potem odlecial... (TUC: 112)

Maja malego psiaka, którego poznajemy po szczekaniu i którego ogonek widac, kiedy biega po skraju lodzi. (FRA: 63f)

Samiczka buduje sobie z kawalków kory i liści eukaliptusów dośc duze gniazdko w dziupli na wielkiej wysokosci... (GRZ: 30)

Die Konvention ist eigentlich auch dem Deutschen vertraut, und es kann grundsätzlich ebenso diminutivisch reagieren (so ließen sich genügend Beispiele aus dem SD $\Rightarrow$ SD-Typ anführen, doch geben sie keinen Anlaß für eine Problembesprechung). Ein gravierender Unterschied aber zeigt sich sowohl hinsichtlich der Ausdehnung des zur Diminuierung disponierten Bereichs, als auch in der Frequenz dieses Mittels. So kann die Zielsprache einerseits im nichtexpressiven Sprachgebrauch lediglich auf Kinder geringen Alters und geringer Größe Diminutivformen anwenden:

Ola poszla spac, stara kucharka, Katarzyna, ulożyla ja do Ióieczka... (IWA: 107)

Boleslaw z werandy czy z lasu obserwowal z daleka wysoka, szczupla postac Stasia...byl prawie niebieski, a biale wloski Oli wygladaly niby sloneczna plama... (IWA: 108)

Onuté, tak jak jej matka...miala zólte wlosy, splatala je w warkoczyk. (MIL: 34)

"Ale ten, co do niej celowal, nie mógl strzelic. Widac oko mial czule na piek-
Ola ging schlafen, die alte Köchin Katarzyna brachte sie zu Bett... (IWA: 100)

Von der Veranda oder vom Wald aus beobachtete Boleslaw in der Ferne Stanislaws lange, schlanke Gestalt... Stas wirkte beinahe bläulich, und Olas Blondhaar sah aus wie ein Sonnenflecken... (IWA: 101)

Onuté hatte wie ihre Mutter gelbes Haar, sie flocht es zu einem Zöpfchen. (MIL: 43)

"Aber der, der zielte, konnte nicht abdrücken. Anscheinend hatte er ein für 
nosc...Potem podbiegl drugi,...odtracil tamtego $\mathrm{i}$ wystrzelil sam. Zamachala w powietrzu raczkami, upadla i tak już zostala." (FIN: 23f)
Schönheit empfindsames Auge...Dann lief ein anderer hin,... schob den ersten zur Seite und schoB selbst. Sie fuhr mit den Händen durch die Luft, fiel zu Boden und blieb so liegen." (FIN: 29)

In diesen Fällen handelt es sich um heranwachsende Mädchen bzw. eine junge Frau; hier erreicht das Deutsche Äquivalenz nur mit dem Verzicht auf das variierende Suffix, andernfalls, wie die letzte Textstelle demonstriert, würde gegen die Gebrauchsnorm verstoßen.

Andererseits muß das Deutsche gemäß seinen Normen mit der Markierung haushalten und sich auf den Einsatz an eher spezifisch angezeigten Stellen beschränken. Besonders deutlich wird die Neigung zur Modifikationsökonomie anhand von Textauszügen, in denen Diminutive der Ausgangssprache kumulativ aufteten und das Deutsche selektiv situationsgerechte Lösungen schafft:

Już po śmierci ptaszka przypomnial sobie Macius, ze ostatniego wieczora byl szczególnie smutny. Dziobek otwieral i oczki mrużyl, jakby sie dusil...A tu rano leży sztywny, nóżki wyprężone... (KOR: 83f)

Położyl na stole muszelki Alo i kamyczek Ali. I od razu zapomnial o wszystkim. Taka mila muszelka, taka jedyna na swiecie. Wie Macius, że muszelek jest na brzegu tysiac i wiecej, ale te muszelke dal Alo... (KOR: 92)

Zum Vergleich sei zitiert:

Viele Kinder hier laufen in einem dünnen Bliischen herum mit Holzschuhen an den bloßen Fußen, ohne Mantel, Mütze oder Handschwhe. (FRA: 54)

Sie holte eine Schachtel mit allerlei Schätzen unter dem Bett hervor und stellte sie vor Bibigirl hin...Und sie zeigte ihr eine hübsche bunte Vogelfeder, einen schön gemaserten Stein, einen goldenen Knopf, ein Stückchen buntes Glas..." Hier hab' ich zum Beispiel eine schöne rosa Muschel." (END: 88)
Erst nach dem Tode des Vogels fiel Hänschen ein, daß er am letzten Abend besonders traurig gewesen war. Er zwinkerte, öfnete den Schnabel und schloB inn wieder, als bekäme er keine Luft...Am Morgen lag er da, starr, die Beinchen ausgestreckt... (KOR: 113)

Alos Muschel und Alas Steinchen legte er auf den Tisch. Und gleich vergaß er alles um sich her. Wie lieb war inm diese Muschel, einzigartig auf der Welt. Hänschen wußte, es gab tausend $M u$ scheln am Strand und mehr, aber diese hatte ihm Alo gegeben... (KOR: 126)

Wiele tutejszych dzieci biega $w$ cieniutkich bluzach, w drewniakach na golych nożkach, bez plaszcza, bez czapki czy rekawiczek. (FRA: 66)

Wyciagnela spod lóżka pudelko z rozmaitymi skarbami i postawila je przed Bibi...I pokazala jej barwne piórko ptaka, kamyk o ladnym deseniu, zloty guzik, kawalek kolorowego szkla..."Patrz, mam tu na przyklad ladna różowa muszelke." (END: 71)

In der Gesprächskonstellation Kind-Erwachsener ist für das Polnische ergänzend zum Nomen auch die Verwendung von Intensiva - ihr wohl häufigstes Einsatzfeld - eine quasi unumgängliche Notwendigkeit. In der Mehrzahl sind sie 
usueller Art; dienen sie aber der Intensitätssteigerung, ist die übersetzende Sprache dazu veranlaßt, diesen Nachdruck ihren Möglichkeiten entsprechend anzuzeigen. So verhält sich das Deutsche, wie oben schon gezeigt, selektiv und variierend:

Mialo byc sloneczne popoludnie...Ola ciagnela pomalutku watek swych mysli. (IWA: 101)

Mialy jasne wlosy - sam jedwab! A nogi proste, thusciutkie jak serdelki. (FIN: 19)

Siedzial na brudnej, dawno nie pranej poscieli, dziecko leżalo cichutko i bylo okragle i duże... (FIN: 41)

Ola wstala bez slowa i wziela go za reke. Poczul raczke chudziutka i zimna. (IWA: 101)

Ein sonniger Nachmittag kündigte sich an...Ola spann langsam den Gedankenfaden fort. (IWA: 93f)

[Die Kinder] hatten blondes Haar, reine Seide, und gerade Beine, rund wie Würstchen. (FIN: 37)

Er saß auf dem schmutzigen, lange nicht gewaschenen Bettzeug. Das Kind lag ganz ruhig, es war rund und groß ... (FIN: 60)

Ola erhob sich wortlos und ergriff seine Hand. Er spürte ein sehr mageres und kaltes Händchen. (IWA: 93)

In die Diminuierungskonvention wird neben dem appellativischen auch der propriale Wortschatz einbezogen. Gängig, ja beinahe unabweislich ist es, Verwandtschaftsnamen in der Rede von und mit Kindern in hypokoristischer Form zu gebrauchen, was in expressiver Redeweise selbstverständlich ist, in der sachlichen Rede aber - aus der Perspektive des Deutschen - als Besonderheit gelten darf, vgl.:

"A oto jest fotografia twojej mamusi", powiedziala cichutko, cichutko Kampanella. (KOR:14)

[Dziewczynka] zawadzila o kamien czy korzen...i w krzyk...Braciszek sie zatrzymal, patrzy, co $z$ tego bedzie. (KOR: 72)

Nie widac bylo nikogo, ale $z$ izdebki dolatywaly dźwieki harmonijki. Ola scisnela mocniej Stasia za reke: "Wujaszku", powiedziala, "slyszy wujaszek? Tam graja." (IWA: 127f)

"Und hier hast du ein Foto deiner Mutter", sagte ganz, ganz leise Campanella. (KOR: 17)

[Das Mädchen] stolperte über einen Stein oder eine Wurzel...und heulte los...Der Bruder blieb stehen und schaute, was daraus würde. (KOR: 97)

Einen Menschen sah man nicht, doch klangen Harmonikatöne aus der Stube herüber. Ola drückte Stanislaws Hand fester. "Onkel Stas", sagte sie, "hörst du? Die spielen." (IWA: 122)

Im deutsch $\Rightarrow$ polnischen Vergleich ist anzuführen:

Vater hat die Dramen von Goethe und Schiller aus dem Schrank geholt und will nun jeden Abend vorlesen... Vaters gutes Vorbild hat Mutter angeregt, mir das Gebetbuch zu geben. (FRA: 40)
Tatus przyniósl z biblioteczki dramaty Goethego i Schillera i bedzie je nam teraz co wieczór czytac na glos... Dobry przyklad tatusia natchnal mame myśla, aby mi dać modlitewnik. (FRA: 46) 
Gestern abend war ich wieder recht traurig. Ich sah im Geiste Oma und Lies! Oma, liebe Oma! (FRA: 100)

Die meiste Zeit meiner Kindheit verbrachte ich eigentlich bei meinen Großeltern, bei meinem $\mathrm{Opa}$, bei meiner Oma, hier. (RUN: 105)
Wczoraj wieczorem bylam znow bardzo smutna. Widzialam w wyobrazni babunie i Lies! Babunia, kochana babunia! (FRA: 134)

Wieksza czesc mojego dzieciństwa spedzilam wlasciwie $u$ dziadków, u mojego dziadziusia i u mojej babci, tutaj. (RUN: 120)

Bezeichnend ist, daß die Konventionalisierung das Diminutiv zum Stilmittel einer ganzen Textsorte werden läßt, womit es über die standardisierte Sprechweise hinaus sogar die höhere Einheit "Kinderliteratur" markieren kann. Dies bedeutet im einzelnen, daB in die Modifizierung vermehrt auch Objekte einbezogen werden, die im Text in sehr losem oder gar keinem Verhältnis zu Kindern stehen; z.B.:

"Tu", pokazal paleczka na mapie nauczyciel geografii...Królowa Kampanella $z$ przestrachu malo nie zemdlala, nauczycielowi geografii laseczka aż wypadla $z$ reki... (KOR: 7)

Wybiegl jakis gruby pan, rekami macha, zlosci sie, krzyczy: "Dlaczego nie uprzedzono? Gdzie ja ich podzieje? Ani miseczek, ani kubków, nie mam dla nich." (KOR: 32f)

Vgl. ebenso:

Als er merkte, daß Momo mit dem Messer nicht gut zu Rande kam, strich er die Brötchen und legte sie ihr auf den Teller. (END: 149)

Zwischen dem Metallzaun und den Glaskasten schob sich langsam eine Schlange von Leuten weiter. Jeder nahm sich da und dort einen Teller oder eine Flasche und einen Pappbecher aus den Glaskästen. (END: 192)
"Hier", sagte der Erdkundelehrer und zeigte mit dem Stock auf die Landkarte...Königin Campanella wäre vor Schreck fast in Ohnmacht gefallen, dem Erdkundelehrer fiel der Stock aus der Hand... (KOR: 8f)

Aus dem Haus kam ein dicker Herr gelaufen, der fuchtelte mit den Händen, war sehr böse und rief: "Warum hat man mir vorher nichts gesagt? Wo soll ich sie denn unterbringen? Keine Schüsseln, keine Tassen habe ich für sie." (KOR: 44)

Kiedy zauważyl, ze Momo nie bardzo sobie radzi $z$ nożem, posmarowal buleczki i polożyl je na talerzyku dziewczynki. (END: 123f)

Miedzy metalowymi sztachetami i szklanymi szafkami powoli przesuwala sie kolejka osób. Każda z nich brala ze szklanych szafek jakis talerz albo butelke i papierowy kubeczek. (END: 157)

Auch hier gilt es für die deutsche Zielsprache, die angesprochene Zurückhaltung zu wahren.

Als Resümee läßt sich feststellen, daß das Deutsche zumeist zielsprachenkonforme Übersetzungsangebote geliefert hat; im Umkehrschluß aber, anhand weniger nicht ganz gelungener Übertragungsversuche, läßt sich noch einmal verdeutlichen, wie aufmerksam mit der Standardisierung umzugehen ist: 
"Jak mnie wtedy odkryli, to bylam jak malpka. Malp-ka. Niby czlowiek, niby zwierzatko." (FIN: 92)

...otwieral oczy a nad nim rozjarzone sloncem szpary $w$ gontach dachu, po tych gontach szuraja pazurki...i zgaduje sie kto tam chodzi - czy tylko wróble, czy jakies wieksze, może nawet leśne golebie. (MIL: 92)

[P:] To bylo najpiekniejsze lato...Sierpien 1980 roku...Tyle kwiatów...W brame stoczni Lenina ludzie wkladali czerwono-biale gozdziki...Matki, córeczki, synowie, cale rodziny czekaly pod brama... (RUM: 27)
"Als ich entdeckt wurde, war ich wie ein Äffchen. Äff-chen. Teils Mensch, teils kleines Tier." (FIN: 136)

...er öffnete die Augen, über ihm zwischen den Dachschindeln sonnenstrahlende Spalten, auf den Holzschindeln kratzten kleine Krallen,...und man möchte erraten, wer dort herumgeht vielleicht nur Spatzen, oder etwas größeres, sogar Waldtauben. (MIL: 125)

Das war der schönste Sommer...Dieser August 1980 ...Die vielen Blumen...Die Menschen steckten weiße und rote Nelken in das Tor der Leninwerft... Mütter, kleine Töchter, Söhne, ganze Familien warteten vor der Werft... (RUM: 33)

Die analytische Wiedergabeart mit ihrem spezifizierenden Epitheton klein wird dem allen Fällen eigenen standardisierten Gebrauch nicht gerecht. Eine Fehlinterpretation, die sogar einen Verstoß gegen die semantische Äquivalenz mit sich führt (gemeint ist die übertragene Bedeutung von pulpecik, vielleicht 'Knäuel', nicht die konkrete, 'Suppeneinlage'), liegt im folgenden vor:

Suka Murza oszczenila sie w tym czasie..., szczenieta piszczaly. Tomasz przytulal do twarzy te cieple pulpeciki i zagladal im w slepka... (MIL: 59)
Um diese Zeit warf die Hündin Murza..., die Kleinen [wimmerten] jämmerlich... Thomas schmiegte diese warmen Fleischklößchen an die Wangen und schaute in ihre...Äugelchen hinein. (MIL: 78)

\section{Idylle:}

Ein weiteres konventionalisiertes Einsatzfeld für Diminutive stellt, besonders in narrativem Textzusammenhang, die Schilderung einer idyllischen Naturszenerie im weitesten Sinne dar. Auch wenn es dem Deutschen nicht fremd ist, diese impressionistisch-beschauliche, Wohlgefühl erzeugende Beschreibung mit Diminutiven zu unterlegen, so begnügt es sich doch meist mit der expliziten oder auch impliziten Kontextaussage und vermeidet die Überstrapazierung dieses Mittels. Das folgende Material kann für die eine Seite die zwanglose und uneingeschränkte Nutzung dieses Stilmittels vorführen, für die andere Seite dessen kontrollierte Streuung:

Za dworem gaszcz drzew, to sad, a potem znowu pare sosen bardzo wy-
Hinter dem Gutshaus dichter Baumbestand: der Obstgarten, dann ein paar 
sokich, bo na wzgórzu stoja, a dalej lasek $i$ bezka $i$ znowu biala szosa, tamtedy sie idzie do wujostwa, a na przeciwnej stronie kotlinki, na horyzoncie powinien być las. (IWA: 29)

Wszystko to nieprawdopodobnie znudzilo Wiktora i zaraz po obiedzie, kiedy towarzystwo sie porozlazilo po pokojach i werandach, poszedl na duży spacer do ogrodu, do lasku, nad staw. (IWA: 63)

Jesienne dnie byly pogodne, slonce swiecilo uparcie, liśscie byly zólte i od samego tego koloru robilo sie cieplo i spokojnie. Jeziorko, czame w lecie, teraz zmienilo koloryt i stalo sie przyjemne...Teraz Boleslaw spotykal sie z Malwina nad jeziorkiem... (IWA: 168)

Czesto polegiwal na kilimku w malym ogrodku prza ganku. W tym ogrodku sloneczniki. Takie male wiejskie ogrodki, kolorowe od kwiatów, wiekszych i mniejszych, różnych. Kwiaty nie wiadomo jak sie nazywaja, ale ladne... (NOW: 103)

Widzialam go $w$ chlopskiej chacie..., stojacej...w ogrodzie, i tak niskiej, ze malwy $i$ inne czerwone kwiaty siegaly okien. Byla to malownicza chahupka pelna zapachu zielska i miety. Teraz dopiero spostrzegam jej malowniczosc ... (FIN: 55)
Kiefern, sehr hoch, weil auf einem Hügel stehend, ferner ein Wäldchen und eine Wiese und erneut die weiße Chaussee. Dort ging es zu seinem Onkel. Auf der anderen Seite des Kessels, am Horizont, müBte ein Wald sein. (IWA: 13)

Wiktor langweilte sich unwahrscheinlich, er begab sich gleich nach dem Essen, während alle sich auf die Zimmer und Veranden verteilten, zu einem langen Spaziergang in den Garten, zum Wald, an den See. (IWA: 51)

Die Herbsttage waren heiter, die Sonne schien ausdauernd, die Blätter vergilbten, und allein durch diese Farbe wurde es warm und ruhig. Der kleine schwarze See im Wald [sic!] veränderte jetzt sein Kolorit, er wurde angenehm und anmutig...Inzwischen traf sich Boleslaw mit Malwina an dem kleinen See. (IWA: 167)

Oft lag er auf einer Matte in dem kleinen Garten am Gehweg. In diesem Garten gab es Sonnenblumen. Solche kleinen Bauerngärten sind bunt von Blumen, größeren und kleineren, ganz verschiedenen. Wie die Blumen alle heißen, kann man nicht wissen, aber schön sind die... (NOW: 57)

Ich habe ihn in der Bauernkate gesehen...; die Kate stand... in einem Garten und war so niedrig, daß Malven und andere rote Blumen die Fenster überragten. Diese Kate war richtig malerisch, erfüllt mit Kräuter- und Pfefferminzgeruch. Wie malerisch sie war, merke ich erst jetzt... (FIN: 45)

Zu Recht verzichtet der Zieltext in dieser Situation auf die morphologisch markierten Formen, um optimale Äquivalenz zu erreichen. Einen möglichen Ausweg für den Fall, daß eine Hervorhebung unumgänglich erscheint, stellt dann die umschreibende, analytische Ausdrucksform dar.

Besonders diminutivreich sind die Naturschilderungen bei Cz.Milosz, die sich zudem noch der verklärten Erinnerung des Autors an Kindheit und Jugend verdanken. Können die direkt folgenden Belege noch als gelungene Übersetzungsoptionen angesehen werden, zeigt sich in der Mehrzahl der Beispiele die 
Gefahr, in die sich die Zielsprache begibt, wenn sie ohne Rücksicht auf die eigenen Gebrauchsnormen die modifizierte Variante übernimmt; vgl:

W malym brzozowym gaiku przy droice do strumienia drzewa rosly gesto i ziemie zaścielala warstwa suchych liści... Dróżka slużyła im za balkon do wygrzewania sie na sloncu... (MIL: 91)

Schodzili dróżka do rzeczki i przez kladke, na rzadowy las. (MIL: 95)

Piekny wieczór. Jeszcze jasnośc, ledwie podchodzaca różowoscia zza czarnej masy na widnokregu....A oto już ukazuja sie płaskie pastwiska i pola, na ich krawedzi kepa parku, za nia, w dziurze powietrza, w niebieskawej mgielce, majaczeja pagórki... (MIL: 85)
In einem kleinen Birkenwäldchen am Pfad, der zum Bache führte, wuchsen die Bäume dicht, und die Erde war mit einer Schicht dürrer Blätter bedeckt... Der Pfad diente ihnen als Sonnenterrasse... (MIL: 123)

Sie gingen einen $P f a d$ zu dem kleinen Fluß hinunter über den Steg zum Staatswald. (MIL: 130)

Ein schöner Abend. Noch Helligkeit, kaum von Rosa unterlaufen, hinter der dunklen Masse am Horizont... Und da tauchen schon flache Weiden und Felder auf, an deren Rand beginnt das Buschwerk vom Park, und dahinter in der Ferne im bläulichen Dunst ahnt man die Hügel... (MIL: 116)

Angenehme und ansprechende Stimmung - wie im letzten Beispiel - ist die Voraussetzung für eine Markierung, wechselt die Gemütslage, wird es zum Beispiel herbstlich-kalt und unwirtlich, so bleibt auch der Diminutivgebrauch aus:

Na dworze mgla, w niej tylko grube krople kapiace $z$ galęi pod gankiem...Zapachy jesieni... (MIL: 95)
Draußen Nebel und nichts als die schweren, von den Zweigen fallenden Tropfen nahe der Hausfront... Herbstgerüche... (MIL: 130)

Im folgenden wurde die pragmatisch-stilistische Äquivalenz verfehlt:

...zbliżala sie linia lasu i za pierwszymi jego drzewami otwieral sie zupelnie inny swiat: $z$ pagóreczka $w$ dolinke, tu borek, tu rojścik, krecace sie dróżki z jedna koleina miedzy bukietami zieleni. (MIL: 87)

Na skraju parku, na laweczce obrosnietej brodami siwego mchu, Helena Juchniewicz wpatrywala sie $w$ nabierajacy mocy ksieżyc. Wyszla żeby odpoczać $i$ odetchnać powietrzem letniego wieczoru...Kiedy Romuald... wspinal sie ku laweczce, krzyknela: "och", zdziwiona. (MIL: 85)
Die Linie des Waldes nahte, und hinter den ersten Bäumen öffnete sich eine ganz andere Welt: Hügelchen und Tälchen, hier ein Wäldchen, dort etwas Sumpf, gewundene kleine Pfade mit einzelner Wagenspur zwischen Buketts von Grün. (MIL: 119)

Am Parkrand, auf einem mit grauen Moosflechten bewachsenen Bänkchen, betrachtete Helena Juchniewicz den zunehmenden Mond. Sie ist ausgegangen, um auszuruhen und die sommerliche Luft zu atmen... Als Romuald...zum Bänklein emporstieg, schrie sie ein erstauntes: "Ach!". (MIL: 116!)

Während der polnische Text z.B. unbefangen auf engstem Raum fünf Diminutive versammeln kann, wirkt die deutsche Fassung nur noch wie die Parodie eines 
romantisch-märchenhaften Literaturversuchs. ${ }^{40}$ Eine deutsche Übersetzung muß ihrer Kopräsenz bewußt sein, in der Diminutive eher rare, weil mit stärkerem Aussagegewicht behaftete Erscheinungen sind, gelingt dies nicht, muß sie als stilistische Verzerrung und Verfremdung empfunden werden; man nimmt sie schlichtweg nicht ernst. Hier zeigt sich im Deutschen der von H.-M.Gauger herausgearbeitete "Widerspruch zwischen dem als Programm Angelegten, als pure Systemmöglichkeit an sich Lebendigen, und dem kümmerlich begrenzten Gebrauch, den die Normsprache faktisch von dieser Anlage macht". ${ }^{41}$

Wiederum findet man in der Spiegelung des Übersetzungsvergleichs die Gebrauchsgewohnheiten bestätigt:

Zürich könnte ein reizendes Städtchen sein. Es liegt am unteren Ende eines lieblichen Sees..., und da wir gestern auf unserm Bummel so freundliches Wetter hatten, September-Bläue mit leichtem Silberdunst, war ich wirklich entzückt... (FRI: 77)

Es wird Herbst. Da und dort, wie aus dem leeren Himmel, fallt ein gelbes Ahomblatt in den Sand. Man sieht es auch dem Himmel an; seine Bläue wird schon blasser, durchsichtiger. Die Luft ist frisch, vor allem am Vormittag. Eine versponnene Geräumigkeit. (FRI: 41)

Die Indianer, das Blumenvolk, flochten Flöße aus Rohr, luden Erde darauf und Tang, pflanzten sogar kleine Bäume und ruderten diese blühenden Inseln umher... (FRI: 28f)

Wir fuhren in die Umgebung Stockholms ... Wir sahen ... herrliche alte Laubbäume und einen blauen Sommerhimmel mit vielen weißen Wattewolken...Wir wollten ein ganz stilles, ein
Zurych móglby byc czarujacym miasteczkiem. Leży przy koncu uroczego jeziora...Podczas naszej wczorajszej wlóczegi mieliśmy piekna pogode. Bylem szczerze zachwycony niebieskoscia wrzesnia, przyslonieta lekka, srebrzysta mgielkq... (FRI: 101)

Nadchodzi jesien. Tu i ówdzie, jakby prosto $\mathrm{z}$ nieba, spada na piasek pożokly liš́ klonu. Jesień można poznać również po niebie; jego blekit staje sie już bledszy, bardziej przezroczysty. Powietrze bywa chlodnawe, zwlaszcza przed poludniem. Zasnuta mgielka przestrzen. (FRI: 51)

Indianie, lud kwiatów, wyplatali tratwy $z$ trzciny, pokrywali je ziemia i morszczynem, a nawet sadzili na nich male drzewka i plywali dookola na tych kwitnacych wyspach. (FRI: 35)

Pojechaliśmy...w okolice Sztokholmu ...Ogladalismy...wspaniale, stare lisciaste drzewa, i blekitne letnie niebo z bialymi chmurkami...Chcielismy bardzo cichego, malenkiego domku, na

\footnotetext{
* WANDRUSZKA 1969 (S.96) bezeichnet die durch "jahrhundertelange Abnützung unbrauchbar" gewordene "Poesie der Diminutive...Blümlein, Gräslein und Hälmchen" als "Gartenzwergdichtung". - Nicht zufällig kommen viele der hier zitierten Belege für Fehlübersetzungen aus einem bestimmten Werk, nămlich der Übersetzung von "Dolina Issy" durch Maryla Reifenberg. Die Hăufigkeit dieser fragwürdigen Wiedergaben ist vermutlich dadurch zu erklăren, daß die Übersetzerin den Usualitäten und Konventionen ihrer polnischen Muttersprache allzu sehr verhaftet blieb.
}

" GAUGER 1971. S.113. 
ganz kleines Häuschen, abgelegen, bequem, friedlich, mit einem kleinen Gärtchen... (TUC: 28f)

Mit dem letzten Beispiel wird erneut deutlich, daß das Deutsche auch bei Expressivitätsangabe auf ein Kompositum (Watte-wolken) zurūckgreift.

Eine weitere Domäne der Diminutivverwendung im Polnischen, der vom Übertragenden mit Sorgfalt zu begegnen ist, stellt der Bereich der vielfaltigen standardisierten Alltagssituationen dar. Auf der Grundlage des ausgewerteten Textkorpus kristallisieren sich drei Sphären heraus: vertrauter und höflicher Umgang miteinander (Abschn.3), Dienstleistungen (Abschn.4), im weiteren Verständnis auch Nahrungs- und Genußmittel (Abschn.5).

\section{Vertrautheit und Hoflichkeit:}

Im Polnischen besteht die Usance, im persönlichen Austausch, im alltäglichen Umgang, Vertrautheit und Höflichkeit durch Diminutive zu bekräftigen. Dabei ist eher freigestellt, was im einzelnen diminuiert wird, das Wertungsobjekt ist an sich zweitrangig, es dient allein als Instrument der Vermittlung von Verbindlichkeit und des Ausdrucks von wohlwollendem Affekt. Als Beispiel kann hier die Gesprächssituation zwischen Mann und Frau dienen, in der der Mann sich um die Gunst der Frau bemüht und sich (auch) über die Bezeichnungen gerade in Rede stehender Gegenstände ihrer Sympathie empfiehlt:

[S:] Najpierw to ja bym wolala zobaczyc [mieszkanie]. Potem bedziemy dalej rozmawiac. - [M:] Pani jest ostrożna. Boi sie pani wpuścic nas do mieszkania, jak jakich bandytów. A ja prosze pani, porzadny czlowiek jestem... - [S:] Nie lubie pospiechu. - [M:]...Ale tutaj nie ma co sie zastanawiac. Chetni sa. Pieniazki sa. Tylko sie zamieniać. (NUR: 7)

[S:] Zaraz pokaże panu mieszkanie. [M:] Nie mam pospiechu. Napijemy sie herbatki. Zjemy po ciasteczku. Zeby bylo po warszawsku. A pani, warszawianka? (NUR: 8)
[S:] Ich möchte erst einmal Ihre Wohnung sehen. Dann können wir weiter verhandeln. - [M:] Sie sind so vorsichtig. Wir sind doch keine Banditen, die man nicht über die Schwelle lassen darf. Ich bin ein anständiger Mann, meine Dame... - [S:] Ich mag nun mal nichts überstürzen. - [M:]...Aber was gibt's hier noch zu überlegen? Alles wie gehabt. Das Geld ist da, nichts wie tauschen. (NUR: 8)

[S:] Ich zeig Ihnen am besten gleich die Wohnung. - [M:] Damit hat's keine Eile. Trinken wir doch erst mal 'ne Tasse Tee. Essen wir die Torte dazu. Auf Warschauerische Art. Und Sie, sind sie eine echte Warschauerin? (NUR: 10) 
[M:] Ale ma pani mieszkanko, pierwsza klasa. Urzadzone. Czysciutko, cieplutko. Mebelki jak trzeba. (NUR: 8)

[S:/oschle/] O co chodzi? - [M:] Przynioslem kwiatek. Dla pani. Żeby nie bylo pusto na tym oknie w kuchni. (NUR: 13)

[M:] Tak sie pani przygladam, przygladam a pani nic, nawet okiem nie mrugnie... - [S:] Mial pan mie gazete. [M:] A jest gazetka, jest. Prosze bardzo. Polożylem na widoku... (NUR: 5)
[M:] Die Wohnung...ich muB schon sagen... alle Achtung. Und prima eingerichtet. Gemütlich, warm. Genau die richtigen Möbel. (NUR: 10)

[S:/kühl/] Worum geht es? - [M:] Ich möchte Ihnen diese Blume schenken. Damit das Fensterbrett in Ihrer Küche nicht so leer ist. (NUR: 16f)

[M:] Ich guck schon die ganze Zeit zu Ihnen herüber. Aber Sie lassen sich überhaupt nichts anmerken... - [S:] Sie sollten eine Zeitung bei sich haben. [M:] Da ist sie ja. Bitte. Ich habe sie ganz sichtbar hingelegt... (NUR: 6)

Ebenso betroffen sind Personen- und Verwandtschaftsnamen in der Anrede, die in ihrer hypokoristischen Form gebraucht werden:

...przypominam sobie jej slowa, gdy po pierwszej relacji o okrucieŕstwach, jakich dopuscili sie Niemcy w naszym miasteczku, zaprotestowalam cicho: "Agasiu, nie moge tego sluchać..." (FIN: 22)

Wstal od stolu w dobrym humorze i powiedzial: "Prosze cioci, ja na podwieczorku nie bede. Obiecalem już, że przyjde do Wilka." (IWA: 44)

/tańcza/[G:] Brawo, panie Wladziu. [M:] Widzi pani, Sabinko. Jeszcze niejednemu mlodemu dalbym rady.

(NUR: 12f)

Jej maż...,jeżeli przyjezdzal, rozpetywal jakis teatr...Wrzeszczal juz na wozie, wyrzucal w góre ramiona, wyskakiwal i biegl, a poly pudermantla albo burki rozwiewaly sie za nim i tak, gotow do uscisku, piszczal dyszkantem: "Mamusiu!" (MIL: 81)
Ich vergesse ihre Worte nicht mehr, mit denen sie mich zurechtwies, als ich nach ihrem ersten Bericht über die Grausamkeiten, die die Deutschen in unserem Städtchen verüben, leise protestierte: "Agaria, ich kann das nicht hören." (FIN: 27)

Er erhob sich guter Laune und sagte: "Liebe Tante, zum Vespern bin ich nicht da. Ich habe versprochen, nach Wilko zu kommen." (IWA: 31)

/sie tanzen/[St:] Bravo, Herr Ladislaus! - [M:] Sehen Sie, Sabinchen? Ich könnte es noch mit manchem Jüngeren aufnehmen. (NUR: 15)

Sobald ihr Mann...angefahren kam, entfesselte er irgendein Theater...Noch im Wagen schrie er, warf die Arme in die Luft, dann sprang er heraus und lief, daß die Schöße des Staubmantels oder des Wollumhangs auseinanderflatterten, und so, zur Umarmung bereit, kreischte er im Diskant: "Mutterchen..." (MIL: 110)

Bei den beiden ersten Beispielen kann man von funktional äquivalenten Übersetzungen sprechen, die vertraute Situation wird wiedergegeben. Während die Sprache des Originals wiederum unbefangen in der Wahl von markierten Bildungen ist, weil sie das Diminutiv "als sich selbst angemessen, als eine ihr normal 
verfügbare 'kongeniale' Möglichkeit des Sprechens ${ }^{\text {42 }}$ empfindet, unterliegt das Deutsche anderen Redenormen. Auch wenn die hypokoristische Verwendung ihm historisch gesehen nicht fremd ist, so stellt sie doch eine veraltende Konvention dar, die nicht mehr den Ausdruck echter Anteilnahme vermittelt. Ein Diminutivgebrauch im Deutschen würde hier das Konventionelle zum Vorschein kommen lassen, als übertriebene Galanterie aufgenommen werden müssen und damit seine Wohlwollen intendierende Expressivität ins Gegenteil verkehren; so geschehen im Fall Sabinchen. Zielsprachenkonform dagegen ist es, auf die Markierung zu verzichten, eventuell wie im Fall liebe Tante durch Adjektive u.ä. zu kompensieren, da diminutivische Kosung im Deutschen lediglich in der Anrede von Kindern, nicht von Erwachsenen - außer in engster Vertrautheit - üblich ist. Das letzte Beispiel kann als Gegenprobe verstanden werden: zur Entlarvung einer übertriebenen und gespielten Verbundenheit ist die Wahl von Mutterchen gerade geeignet.

Eine Bestätigung für die bemerkte Übertragungsrelation stellt sich ein, wenn das Deutsche zur Ausgangssprache wird:

"Na, da wollen wir ihm den Abschiedstrunk rüsten, was?" sagte Lydia. (TUC: 83)

Einmal sagte wieder jemand: "Ach Julika, habt ihr ein süßes Tier!" worauf Stiller antwortete: "Sehr süß, ja, demnächst werden wir Konfitūre daraus machen!" (FRI: 106)

"Na, ihr Affen", sagte Billie und lieB sich neben uns nieder. "Wie wars?" (TUC: 87f)
"No, to przygotujemy mu butelczyne na pożegnanie, co?" rzekla Lidia. (TUC: 103)

Pewnego razu ktos znowu powiedzial: "Ach, Juliko, ależ wy macie slodkiego pieska!" Na co Stiller: "Jest bardzo slodki, tak, wkrótce zrobimy z niego konfiture!" (FRI: 143)

"No, malpki", powiedziala Billie, lokujac sie obok nas, "jak tam poszlo?" (TUC: 109)

\section{Dienstleistungen:}

Konnte die gerade untersuchte Gebrauchskonvention als Erweiterung der meliorativen Emotionalisierung von der Sphäre des Kindes und Tieres auf die des enger vertrauten Erwachsenen verstanden werden, so setzt die nun zu besprechende Konstellation die angezeigte Richtung fort: vom Bekannten zum Fremden, mit dem man sich arrangieren muß, weil man seine Dienste in Anspruch

$\$ 2$ GAUGER 1971. S.113. 
nehmen will. Trotz breiter Materialgrundlage ließen sich nur zwei Bereiche, Friseur und Cafe, herausarbeiten:

[J:]Jaśnie wasy w sam raz, prosze jasnie pana... - [D:] Warto by je musnać $z$ boczku, $z$ lekka, nożyczkami. (TON: 7 )

[Kelnerka:] Prosze wode. - [M:] Zaraz. Co tak skromnie? Może kawke dla pani albo i cos mocniejszego? - [S:] Dziekuje. - [M:] To dla mnie prosze koniaczek. A dla ciebie, Rysio? - [Syn:] Mala kawa. - [M:] Mala kawa raz, koniaczek dwa...taki wie pani, wiekszy...- [Keln.:] Piecdziesiatka? - [M:] Może byc. I ciasteczko. Tylko jakies świeże, bo to dla tej pani. - [S:] Ja za ciastko dziekuje... - [Kein.:] To co mam jeszcze podać? - [M:] Mówilem. Koniak, kawa, ciastko. (NUR: 6)
[J:]Der hochverehrte Schnurrbart ist gerade richtig, allergnädigster Herr... [G:] Von der Seite, ganz leicht, sollte man ihn mit der Schere streifen. (TON: 11f)

[Kellnerin:] Bitte, Ihr Wasser. - [M:] Warum so bescheiden? Trinken Sie doch lieber einen Kaffee mit uns oder etwas stärkeres? - [S:] Nein, vielen Dank. - [M:] Dann eben nur für mich einen kleinen Kognak. Und du, Richard? - [Sohn:] Einen kleinen Schwarzen. - [M:] Einmal Kaffee und einen Kognak...einen großen. - [Kelln.:] Einen doppelten? - [M:] Meinetwegen. Und ein Stück Torte für die Dame, aber frisch. - [S:] Auf die Torte verzichte ich... - [Kelln.:] Was bestellen Sie nun also? - [M:] Wie gesagt: Kognak, Kaffee, Torte. (NUR: 7f)

Besonders die Kaffeehauspassage macht offensichtlich, wie der standardisierte Gebrauch doch im Gegensatz zur Neutralisierung Textfunktion übernehmen kann: sobald die Atmosphäre des guten Willens nicht mehr gegeben ist, ist der Diminuierung ihre Voraussetzung entzogen, so daß an ihre Stelle das Basislexem tritt. Die polnische Sprache verfügt somit über ein bequemes und wirkungsvolles Instrument, um Stimmungswechsel morphologisch anzuzeigen. Anders die deutsche Sprache: will sie funktionelle Äquivalenz erreichen, darf sie der Vorlage in formaler Sicht nicht folgen. Mit der nichtdiminutivischen Reaktion aber bleibt das spezifische emotionale Kolorit solcher Situationen und dessen Wechsel - und dies gilt wohlgemerkt für alle Konventionen! - im Deutschen unaussagbar. Die Eigenheit könnte allenfalls durch kompensatorische Strategien, sei es die Wahl von Höflichkeitspartikeln, wie bitte u.a., oder anderen verbindlichen Wendungen, in den deutschen Text verpflanzt werden.

\section{Nahrungs- und GenuBmittel:}

Die Gebrauchsnorm "Speisen und Getränke" ist, wie gesehen, auch innerhalb anderer Kommunikationssituationen anzutreffen, wo sie zur Kennzeichnung von Vertrautheit (vgl. herbatka) oder zur Respektbezeugung im Dienstleistungs- 
bereich (vgl. koniaczek) herangezogen wird. Doch darüberhinaus bildet sie in informellen Textzusammenhängen auch eine eigenständige Konvention, vgl.:

Katarzyna stukala nożem w kuchni, siekac cebulke do obiadu... (IWA: 1400)

Czul klujacy ból w zoladku. Bol ten odtad nie ustepowal. Karmili go kaszka i mlekiem. Naczelny lekarz przychodzil czesto. (NOW: 149)

im deutsch $\Rightarrow$ polnischen Vergleich:

Dahinter zogen sich...lange Glaskästen hin, in denen Schinken- und Käsebrote, Würstchen, Teller mit Salaten...und alles mögliche andere stand... (END: 192)

Fünf von sechs Sãcken waren bereits gut plaziert und Peter eben damit beschäftigt, Nr.6 zu bergen, als die Unternaht $\mathrm{riB}$ und sich ein Regen, nein, ein Hagel von braunen Bohnen über die Treppe ergoB. (FRA: 43)
Katarzyna hackte in der Küche mit dem Messer Zwiebeln für das Mittagessen... (IWA: 136)

Spürte stechenden Schmerz im Magen. Der Schmerz hatte seitdem nicht nachgelassen. Sie gaben ihm Brei und Milch. Der Oberarzt kam häufig. (NOW: 102)

Za nia ciagnely sie...szklane szafki, a w nich znajdowaly sie kanapki z szynka i z serem, kielbaski, talerze $z$ salatkami...i przeróżnymi innymi rzeczami... (END: 157)

Zdażyl już ulożyć tam piex worków i niósl wlaśnie szósty, kiedy dolny szew pekl i deszcz, nie, grad brazowych fasolek posypal sie na schody. (FRA: 51)

Besonders reiches Anschauungsmaterial bieten dafür Kochbücher:

...udusić na maśle $z$ drobno posiekana cebula oraz wloszczyzna (...z pietrusz$\mathrm{ka}$, selerem i 1 marchewka). (LEM: 255)

Oddzielnie podajemy wypieczona na sypko kaszkę krakowska (z koperkiem) i sezonowa jarzyne: w lecie zielona salatke, w zimie buraczki. (LEM: 102)

$11 / 2 \mathrm{~kg}$ chudej wieprzowiny na pieczeń zalewamy...wrzaca marynata przyrzadzona $z$ 1/2 szklanki bialego wytrawnego wina...i 1 listka laurowego. (LEM: 77)

Dodajemy 4 ziarnka ziela angielskiego i malutki kawaleczek listka laurowego. (LEM: 95)

Bezposrednio przed podaniem listki [salaty] (w calosci) ukladamy w salaterce... (LEM: 296)

Wątróbke, phucka i serce prosiecia... udusić na maśle... (LEM: 255) ...in Butter und feingehackter Zwiebel sowie Suppengrün (Petersilie, Sellerie und 1 Möhre) dünsten. (LEM: 261)

Dazu körnige Krakauer Grütze (mit Dill) und je nach Jahreszeit Salat oder Roterübengemüse reichen. (LEM: 104)

$11 / 2 \mathrm{~kg}$ mageres Schweinefleisch....mit kochendheißer Marinade aus $1 / 2$ Glas Weißwein... und einem Lorbeerblatt übergießen. (LEM: 81)

...mit 4 Körnern Piment und einem kleinen Stückchen Lorbeerblatt würzen. (LEM: 98)

Unmittelbar vor dem Servieren eine Salatschüssel mit ganzen Salatblättern auslegen... (LEM: 303)

Leber, Lunge und Herz des Spanferkels...in Butter...dünsten. (LEM: 261) 
Jeszcze jeden kulinarny slad wielowiekowego braterstwa polsko-litewskiego smakowite pierożkì miesne... (LEM: 54)

Teraz kolej na przyrzadzenie jasnej zasmažki z 5 dkg masla i $4 \mathrm{dkg}$ maki oraz drobniutko posiekanej cebuli. (LEM: 51)

...zagotowujemy ...z... 3 goździkami, kawaleczkiem cynamonu, kawaleczkiem cieniutko obranej skórki cytrynowej lub pomaraniczowej... (LEM: 31)
Ein weiterer kulinarischer Zeuge der jahrhundertelangen polnisch-litauischen Bindungen sind die wohlschmeckenden...Fleischpiroggen. (LEM: 60)

Jetzt ist eine helle Mehlschwitze aus $50 \mathrm{~g}$ Butter und $40 \mathrm{~g}$ Mehl sowie einer feingehackten Zwiebel herzustellen. (LEM: 57)

...mit ... 3 Nelken, einem Stückchen Zimt, einem Stückchen dünner Zitronen-oder Apfelsinenschale... aufkochen. (LEM: 31)

Wie allen Konventionen eigen, so ist auch hier der Einsatz von Diminutiven nicht obligatorisch festgelegt. Der in Rezeptangaben und -beschreibungen nur schwer in Regeln zu fassende Alternation zwischen Grundwort und Diminutiv kann dies veranschaulichen (vgl. z.B. marchew, LEM:79; koper, LEM:36; salata zielona, LEM:296; list laurowy, LEM:148; ziarna ziela angielskiego, LEM:77; liście kapusty, LEM:131; wątroba, płuca, LEM:63; pierogi, LEM: 306; drobno, LEM:74; cieńko, LEM:91).

Sowohl die diminutivischen Adjektive als auch Fälle wie z.B. plasterki, pasecaki (LEM:51) $\Rightarrow$ Streifen (LEM:57), koniuszki (LEM:50) $\Rightarrow$ Enden (LEM:56) zeigen, daß eine Parallele zum Konventionsbereich "Kind" zu beobachten ist: auch hier weitet sich der Diminutivgebrauch von den Produkten auf die Textumgebung aus, so daß er neben der Funktion der Markierung einer Standardisierung zum eigenen Stilmittel der Textsorte "Kochbuch" wird.

Alle Übersetzungslösungen (Wiedergabe durch das Basislexem) erfüllen die Forderung nach funktioneller Äquivalenz, so daß davon auszugehen ist, daß dem Deutschen eine vergleichbare Konventionshaltung, im Gegensatz zu den vorangegangenen typisierten Situationen, fremd ist.

Waren bisher für die Diminutivverwendung charakteristische Kommunikationssituationen herausgearbeitet worden, die nicht an einzelne diastratische Varietäten gebunden waren, so sollen zum Abschluß zwei Arten milieubedingten Diminutivgebrauchs vorgestellt werden, die nicht der Norm, sondern dem Substandard angehören. 


\section{Landliche Umgangsrede: ${ }^{.3}$}

Als ausgewählte Quelle für diesen weit gefächerten Bereich steht der Untersuchung der "Pamietnik matki" von M.Fornalska zur Verfügung. Diese Erinnerungen sind die von M.Rutkiewicz schriftlich fixierten mündlichen Berichte einer einfachen Bäuerin ohne jegliche Schulbildung. Ein Charakteristikum dieser in Abhängigkeit vom Bildungsgrad und von der schichtenspezifischen Zugehörigkeit stehenden Umgangsrede ist der vermehrte Einsatz diminutivischer Formen gerade auch in sachlich-informellem Zusammenhang für Objekte des täglichen Umgangs, überhaupt alles, was unmittelbar mit dem Dorfleben (Geräte, Gebäude u.a.) zusammenhängt:

"Jeżeli bede nosic jedna chusteczke, to czym sie bede różnić od dziewczat?" (FOR: 68)

...wlożylam swiateczne szatki, to jest piócienna, kolorowa spódniczke, bialy kaftanik i bialy fartuszek; wlosy... splotla mi jedna $z$ dziewczat w warkoczyk... (FOR: 56)

...wiec wtuliwszy sie do kacika cichutko rozmyślałam, co mnie czeka. (FOR: 22)

Ja z dwoma najetymi sasiadami przez ten czas sklecilam juz stodóke i oborke... (FOR: 78)

...kupujemy cala gospodarke $z$ ladna chata i rozwalona stodóka. (FOR: 71)

...chrust szedl na opal i ogrodzenie, a nierzadko i na oborke lub chlewik... (FOR: 45)

Idac po drabince, $z$ przerażeniem spostrzeglam, ie jest tam widno jak na dworze. (FOR: 83)
"Wenn ich nur ein Kopftuch tragen soll, wodurch unterscheide ich mich dann von den Mädchen?" (FOR: 64)

Ich...kleidete mich festlich, das heißt, ich zog einen roten Leinenrock und ein weißes Jäckchen an und band eine weiße Schürze um. Ein Mädchen flocht aus meinem...Haar einen Zopf... (FOR: 53)

So setzte ich mich still in eine Ecke und dachte darüber nach, was mich erwartete. (FOR: 28)

Während seiner Krankheit baute ich mit zwei Nachbarn Stall und Scheune. (FOR: 72)

...wir kauften die Wirtschaft samt dem hübschen Häuschen und einem Schuppen, der schon halb verfallen war. (FOR: 66)

Das Reisig wurde verbrannt oder für die Umzäunung verwendet, oftmals wurde auch ein Schweinestall daraus gebaut. (FOR: 45)

Als ich also eines Tages die Leiter hochstieg, sah ich mit Entsetzen, daß es oben taghell war. (FOR: 77)

* Dieser Begriff gilt hier nicht im Sinne einer eigenstăndigen Existenzform der polnischen Sprache (odmiana jezyka: jezyk potoczny), sondern lediglich eines Funktionalstils (styl potoczny ludowy). 
Po paru dniach raniutko poszlam na góre. "Góra" na wsi jest spichlerzem i skladem... (FOR: 82f)
Nach einigen Tagen kletterte ich morgens auf den Boden, der auf dem Lande meist als Speicher benutzt wird. (FOR: 77)

Darüberhinaus sind auch Verwandtschaftsnamen, die sich auch immer wieder mit dem Grundwort abwechseln können, von der Modifizierung betroffen:

Już tej jesieni myslalam, czego by nauczyc mego syna...Powiedzialam meżowi, ale on to wzial za dziwactwo, wiec zaczelam o tym rozmawiac $z$ moim małym synkiem. (FOR: 84)

...a że nic nie widzialam, po chacie prowadzila mnie córeczks. Starsza corka byla daleko, maż $z$ synem cale dnie spedzali w wiatraku... (FOR: 99)

...siostrzyczka pobiegla pedem naprzod, a ja szlam zadowolona $z$ przerwanej roboty. (FOR: 52)
In jenem Herbst machte ich mir Gedanken, was man meinen Sohn lernen lassen könnte...Ich sprach mit meinem Mann, doch er meinte, das wären Träume, und so beschloß ich, mit dem Jungen darüber zu sprechen. (FOR: 78ก)

Weil ich nichts sehen konnte, führte mich Malgosia durch das Haus. Meine große Tochter war weit weg. Der Sohn arbeitete mit dem Vater alle Tage in der Mühle. (FOR: 94)

Meine Schwester lief voraus, ich ging hinterdrein und war zufrieden, daB ich diese Schinderei für eine Weile unterbrechen konnte. (FOR: 49)

Alle korrekten Übersetzungsangebote zeigen, daß das Deutsche diese sprachliche Eigenart nicht nachvollziehen kann. Dies findet man in den ebenfalls auf deutsche ländliche und städtische Umgangssprache "einfacher Leute" rekurrierenden Aufzeichnungen von Erika Runge bestätigt: sie weisen keinen vergleichbaren Diminutivgebrauch auf.

Ein besonderes Problem stellen für die Zielsprache die Häufungen und Wiederholungen bei Kleinheitsangaben dar. Selbst die analytische bzw. analytisch-synthetische Bildeweise wird vom Deutschen vernachlässigt, wenn in der Textumgebung eine Verminderung bereits zum Ausdruck gekommen ist, vgl.:

Zaczelam meia namawiac, aby zdecydowal sie sprzedac nasze gospodarstwo; wszak ono takie male... Nabywca na nasze male gospodarst wo znalazl sie predko... (FOR: 90ff)

...i wydostala z dużej torby ksiązeczke. Byla to malenkka, zlożona z kilkunastu kartek ksiqizeczkn... (FOR: 17)

Byly równiez w gminie dwa mlyny, ktore należaly do dworu. Jeden maly, na malej rzece, z braku wody bardzo malo czynny... (FOR: 85 )
Ich überredete meinen Mann, unsere Wirtschaft zu verkaufen. Sie war so klein....Einen Käufer für unsere Wirtschant fanden wir schnell. (FOR: 86ก)

Dann holte sie aus der großen Tasche ein Buichlein hervor. Es war klein und hatte nur wenige Seiten... (FOR: 23)

Die Gemeinde besaß auch zwei Mühlen, die zum Gut gehörten. Die eine war klein, lag am wasserarmen Fluß und war daher selten in Betrieb. (FOR: 80) 
Eine weitere vom sozialen Milieu abhängige diminutivische Verwendung begegnet im Jargon gesellschaftlicher Randgruppen; dem Textkorpus entsprechend können Belege für den Funktionalstil des Jargons kleinkrimineller Kreise angeführt werden. Die jeweiligen Diminutive markieren dabei, wie schon in anderen Konventionsbereichen beobachtet, nicht die Einstellung des Sprechers zum einzelnen Objekt, vielmehr zeigen sie milieubedingtes Sprechen überhaupt an. Dabei liegt einer der seltenen Fälle vor, in denen das Polnische sein Suffixpotential auch produktiv nutzt (vgl. -ak, -yna):

"Do dzieciak6w przyzwyczaisz sie..." Mial czarniawa dziewczynke i szesciomiesiecznego brzdaca. (NOW: 108)

Chlopak znów spogladal na nia przez palce. W jej gebie wszystko bylo wielkie - miesiste usta i poteżna kwadratowa broda. (NOW: 52)

...[Kwadratowy] znalazl sie za brama wiezienia...Wpuszczajacy strażnik przymrużyl wyblakle oko i mruknal: "Wpadles, bratku." Każdemu to mówil. (NOW: 140)

Z uwaga obserwowal tego siódmego. Byl to chudy chlopina. Kuśtykal... (NOW: 40)

Kaczor pochylil sie nad wózkiem..." Udany", powiedzial Kaczor, "udany...", cmoknal jak na psiaka. (NOW: 109)

Dzieciak rozwrzeszczal sie nagle...Jego wrzask wibrowal przenikliwie $w$ mieszkaniu. Sczerwienial, pomarszczyl sie i grzebal pieluszki krzywymi nożynami. (NOW: 112)

Zegarek przeszedl $z$ reki do reki bez targu. Kaczor przeliczyl czerwone papierki. (NOW: 117)

[Kaczor] rozejrzal sie po izbie...Kobieta Trzyczwartaka... wygniatala w niecce kluski...[,] przestala, otarla o fartuch rece i mruknela: "Na wystawce?", Kaczor przytaknal. Trzyczwartak...przed-
"An die Gören gewöhnst du dich..." Er hatte ein schwarzhaariges Mädelchen und einen sechsmonatigen Knirps. (NOW: 62)

Der Bursche betrachtete sie wieder durch die Finger. An ihrer Visage war alles groß - die fleischigen Lippen und das kräftige Quadratkinn. (NOW: 29)

...[Quadratisch] war nun hinter dem Gefângnistor...Der Wachthabende zwinkerte mit ausgebleichtem Blick und brummte: "Geschnappt, Bursche." Das sagte er jedem. (NOW: 93)

Aufmerksam beobachtete er den Siebten. War ein mageres Kerichen. Hinkte... (NOW: 23)

Kaczor beugte sich über den Kinderwagen..." Der ist gut geraten", sagte Kaczor, "gut geraten..." Er schnalzte wie zu einem Hündchen. (NOW: 63)

Der Säugling fing plötzlich zu schreien an...Sein Quäken vibrierte durchdringend in der Behausung. Er wurde rot, bekam Runzeln und wühlte in den Decken mit seinen krummen Beinchen. (NOW: 66)

Die Uhr ging von Hand zu Hand, ohne Feilschen. Kaczor zählte die roten Papiere. (NOW: 71)

[Kaczor] sah sich in dem Raum um... Dreiviertels Frau...knetete in einem Trog gerade Klöße...[,] hörte auf, rieb sich die Hände an der Schürze $a b$ und brummte: "Gerade entlassen?", Kaczor 
stawil swojej babie, mówiac: "Moja... [,] a to kumpel $z$ odsiadki i wspólnik." (NOW: 108) nickte. Dreiviertel...stellte ihn der Frau vor: "Meine...[,] und das ist ein Kumpel aus dem Knast und mein Kamerad." (NOW: 62)

Während Gören, Bursche, mit Einschränkungen auch Kerkchen, die dem Stiltyp eigene derbe und leicht pejorative Färbung durch die Wahl eines synonymen Basislexems bzw. Derivats zielsprachenkonform wiedergeben konnten, bleiben die Übertragungen Hündchen und Beinchen hinter der ausgangssprachlichen Aussage zurück, da sie die Markierung des Jargons verfehlen. Beiden Diminutivformen haftet allzu sehr die meliorative Implikation der Kinder- bzw. Tierkonvention an. Die Übersetzung der zum spezifischen Vokabular der Gaunersprache zählenden Lexeme papierki und wystawka haben ebenso die pragmatisch-stilistische Äquivalenz verfehlt; Papiere muß sogar als Fehlübersetzung gedeutet werden, denn gemeint sind die roten 100-Zloty-Scheine.

Besonders auffallig ist die Diskrepanz zwischen polnischem und deutschem Diminutivgebrauch innerhalb dieser Konvention, wenn die deutsche Übersetzung versucht, dem häufigen Einsatz von Spitznamen durch Diminuierung formal zu entsprechen:

Ten w samodziale, zwany Ząbkiem, zapalil papierosa. (NOW: 49)

Może wychudl tylko i przygarbil sie troche. Siwy, ostrzyżony krótko, ulubieniec najcwanszych dziwek spod "Merkurego". "Wampirek", jak powiadala Zoska, ostatnia jego kochanica. (NOW: 127)
Der Grobleinerne, genannt Zähnchen, zündete sich eine Zigarette an. (NOW: 25)

Vielleicht war er nur ein wenig abgemagert und gebückt. Graues, kurz geschorenes Haar, der Schwarm der kessesten Nutten vom "Merkury". "Vampirchen", wie Zoska, seine letzte Geliebte sagte. (NOW: 80)

Die Übersetzungslösungen müssen als mißlungen gewertet werden, da auch sie durch ihre wohlwollende Akzentuierung, die dem Deutschen in der Regel eigen ist, die Sprachfärbung des Milieus nicht lebendig wiedergeben; sie hätten sich der auch im Deutschen gängigen Jargonwendungen kompensatorisch bedienen müssen.

Bezog sich die vorangegangene Problembesprechung in erster Linie auf die synthetische Bildeweise der Ausgangssprache, läßt sich nun eine Parallele zum analytisch-synthetischen Verfahren erkennen, dessen Syntagmen vom Deutschen meist in attributiven Fügungen entsprochen wird. Sie besteht darin, daß der 
Eigenart des Polnischen, bereits morphologisch gekennzeichnete Diminutive zur Verstärkung der Signalwirkung durch Adjektive (zuweilen auch noch diminuiert) zu begleiten, die deutsche Übersetzung lediglich im analytischen Bestandteil folgen kann und dabei das merkmalhafte Derivat durch das merkmallose Grundwort ersetzt. Auch hier können Belege für neutralisierte und konventionalisierte polnische Bildungen angeführt werden:

Stali wtedy na poludniu Rosji w malym miasteczku... (IWA: 117)

Nie, bylo to zwierzatko wieksze od myszy, z małym ogonkiem - rude z bialymi lapkami. (KOR: 90)

...wesola melodia wylatywala $z$ ust matki jak zwinny, mały ptaszek... (FIN: 61)

W niedużej kotlince, daleko, ale jak na dloni staly dwie wielkie stodoly, na krzyż do siebie zwrócone. (IWA: 29)

...wiec musiala to byc...pora przedwieczorna, slonce zachodzi, nad miastem zwisa cienka mgielka i cienie drzew leìa na ulicy. (FIN: 56)

Jeżeli barszcz podamy w filiżankach, odpowiedniejszym dodatkiem beda malutkie kruche pierożki $z$ farszem grzybowym... (LEM: 185)

...uczulam ogromna radosc, że w tej malenkkiej ksiazzeczce miesci sie taka wielka rzecz, że te maleńkie literki mówia... (FOR: 18)
Er war damals im Süden RuBlands in einer Kleinstadt stationiert... (IWA: 110)

Nein, das Tierchen war größer als Mäuse, es hatte einen kleinen Schwanz und war rothaarig mit weißen Pfoten. (KOR: 122)

...die fröhliche Melodie war dem Mund seiner Mutter entflogen wie ein kleiner, flinker Vogel... (FIN: 119)

In dem nachen Kessel standen fern, aber sehr gut sichtbar, über Eck zueinander zwei geräumige Scheunen. (IWA: 13)

...es muß also...die vorabendliche Stunde gewesen sein: die Sonne geht unter, über der Stadt hängt ein feiner Dunst, und die Schatten der Bäume liegen auf den Straßen. (FIN: 47)

Wird der Barschtsch in Tassen gereicht, sind kleine mürbe Piroggen mit Pilzfüllung...als Zugabe geeigneter. (LEM: 190)

Ich empfand eine ungeheure Freude darüber, daß in diesem kleinen Buch etwas so Großes verborgen war, daß diese kleinen Buchstaben...erzählten. (FOR: 24f)

Die analytische Komponente erweist sich somit im Deutschen als besser übertragbar, offensichtlich dem Sprachgebrauch angemessener und kompatibler. 


\subsubsection{Individuelle Kennzeichnung}

Die vorgeführte Dominierung von Übersetzungsproblemen durch konventionalisierte Bildungen im Polnischen darf nicht verdecken, daß der Einsatz diminutivischer Formen ebenso aktuell-intendiert sein kann und auch dann dem Übersetzer Schwierigkeiten zu bereiten vermag. Wie die Fallbeispiele nun zeigen sollen, hat der polnische Sprecher die Möglichkeit, Diminutive zum Instrument vielfäliger kommunikativer und nicht durch Konventionen vorgegebener Intentionen zu machen. Dieser Variabilität ist die zielsprachliche Version meist nicht gewachsen und zu Austarierungen gezwungen:

Karabinek spuscil w dziuple debu i nie tykal go wiecej. Przerobiony $z$ wojskowego, $z$ obcieta lufa, dawal sie schowac pod switka...I wtedy lufka na pochylonych plecach (siedzial do Baltazara bokiem), na szyi, na glowie z czapka bez kozyrka. (MIL: 32)
Den Karabiner hatte er in die Höhlung einer Eiche versenkt, er rührte ihn nicht mehr an. Umgearbeitet aus einem militärischen, den Lauf abgeschnitten, lieB er sich unterm Hemd verstecken... Und nun das Flintenläuflein auf den gebückten Rücken gerichtet (der Russe saß seitwärts zu Baltazar)...auf den Hals... auf den Kopf mit der schildlosen Mütze. (MIL: 40)

Die Vorlage will allein die geringe Größe der Waffe betonen.

Tomasz pedzil za Romualdem, z poczatku lekko, pózniej zadyszal sie, ledwo nadażal. $Z$ duktu skrecili $w$ bok, miedzy leszczyny, w parów, później dnem parowu i pod góre na wal. "Tu", pokazal mu Romuald na niski swierczek, gdzie ma stanaç... (MIL: 96)
... Thomas rannte hinter Romuald, erst leicht, später fing er an zu schnaufen, er konnte kaum nachkommen. Von der Schneise schlugen sie sich seitwärts, zwischen Haselsträucher, in die Schlucht, durch den Grund der Schlucht weiter und hinauf auf die Böschung. "Hier" - Romuald deutete auf das niedrige Tännlein, wo er stehen bleiben sollte... (MIL: 131)

Hier soll ein herausgehobener Punkt der Umgebung, der gerade nach Mühen erreicht worden ist, markiert werden. In beiden Belegen werden im Deutschen mit dem diminutivischen Derivat ganz andere Konnotationen hervorgerufen.

...czesto przychodzil i stawal na skraju lasu; patrzyl i myślal, ze można sobie wyobrazic, iz pola te ciagna sie bez konca, że nic już ich nie przerywa, że sie brzezina nie zacznie po tamtej stronie. Ale potem wracala myśl, iż to tylko polana, na ktorej chata Maryjki jest wysepka. (IWA: 115)

...za poletkami w gaszczu wisniowych drzew stala zapadnieta chatka starej
... [er kam] oft an diesen Ort, blieb am Waldrand stehen und dachte, man könne sich vorstellen, daß die Felder sich endlos dehnten, daß nichts sie mehr unterbreche, daß drüben nicht das Birkenwäldchen beginne. Doch dann kehrte der Gedanke zurück, es handle sich ja nur um eine Lichtung mit Maryjkas Kate als Insel in der Mitte. (IWA: 109)

...hinter den Feldern stand im Laubwerk der Kirschbaume die Kate der 
Maryjki, z której nie widać bylo nawet komina sponad drzewek. (IWA: 115) alten Maryjka; nicht einmal der Kamin überragte die Bäume. (IWA: 108)

In beiden Fällen soll Nachdruck auf die Kleinheit der Behausung gelegt werden, im ersten, indem sie in Beziehung zur räumlich beherrschenden Umgebung gesetzt wird; im zweiten wird die Kleinheit des Objekts (chatka) zusätzlich indirekt durch die Diminuierung von Bezeichnungen für die nächste Umgebung ausgedrückt.

In den folgenden Beispielen will der Sprecher mit der modifizierten Form jeweils bestimmte Eigenschaften, die er dem Objekt zuerkennt, unterstreichen, wie z.B.

Sanftheit:

...miedzy drzewami wilgoc poczela parowac. Mialo byc sloneczne popoludnie. "To tylko taki majowy deszczyk" ... (IWA: 101)

\section{Armseligkeit:}

Podwórko bylo male, czyste, ale smutne. Konie i krowy miescily sie w czerwonym budynku na parterze, na pietrze bylo mieszkanko... (IWA: 105)

\section{Einsamkeit, Verlassenheit:}

...przed czterema godzinami odlożylam sluchawke telefonu $w$ malej oberży wyoko w górach, trzeciorzedna miejscowosc $z$ trzeciorzednym lodowcem okrytym zazwyczaj mglami...;jest tu jedna tylko oberża, hotelik pusty... (FIN: 83)

[Dy:] Jakas stacyjka...Powygaszane perony, nic nie widać. Co to za stacja? - [I:] Ani jednego czlowieka... (RUM: 9)

\section{Bedeutungslosigkeit:}

[I:] Musze powiedziec, że nawet zaimponowala mi pani. - [Dz:] Oo! - [I:] Tak, odwaga $z$ jaka pani wypowiada swoja filozofijkę życiowa. Nie każdego na to stac. (RUM: 20) ...die Feuchtigkeit begann zwischen den Bäumen zu verdunsten. Ein sonniger Nachmittag kündigte sich an. "Es war nur so ein leichter Mairegen." (IWA: 93f)

Das Gehöft klein, sauber, aber traurig. Die Pferde und Kühe standen in einem roten Gebäude im Erdgeschoß, im Oberstock lag die kleine Wohnung. (IWA: 97)

Vor vier Stunden habe ich den Telefonhörer aufgelegt, oben in dem kleinen Berggasthof. Eine Ortschaft dritter Klasse, und ein Gletscher dritter Klasse, gewöhnlich von Nebelschwaden verdeckt...; es gibt hier nur einen Gasthof; das kleine Hotel ist leer... (FIN: 98)

[D:] Eine kleine Station...Keine Beleuchtung auf den Bahnsteigen, nichts zu sehen. Was mag das für eine Station sein? - [I:] Völlig menschenleer... (RUM: 10)

[I:] Ich muß gestehen, Ihre Haltung hat mich ziemlich beeindruckt. - [J:] Hört, hört! - [I:] Ja, der Mut, mit dem Sie Ihre bescheidene Lebensphilosophie verkünden. Das macht Ihnen so bald keiner nach. (RUM: 24) 
Darüberhinaus können Diminutive als Markierungspunkte innerhalb einer emotional gewichtigen Passage Indikatoren der darin ausgedrückten Stimmungen sein, z.B. als Bezeichnung für den Kulminationspunkt sehnsüchtiger Erwartung:

I poslał Maciuś list do Alo i Ali, że mu zabrali czólno, że musial ich opuscić. Juź wieczorem tego samego dnia mial odpowiedź... Ucalowal Macius ten liscik, schowal razem $z$ fotografia matki, ostatnim kawalkiem salaty, ktora jadl zmarly kanarek... (KOR: 99f)

oder der Betrübnis bzw. des Ärgernisses:

Uniosłam glowe i spostrzeglam, ze ktos sie zbliża. Rozloscilam sie, chcialam wstać i odejscc...: to szla kobieta...Szla wolnym krokiem, stapajac ostrożnie...A przecież tam, nad staw, prowadzi wygodna droga, miekka...Prowadzi ona nad brzeg, gdzie stoja dwa na plask sciete pniaki...Przyjdzie wiec tutaj, usiadzie na pniaczku...Tyle obiecywalam sobie po tym wieczorze! Sa mysli, które wiedna pod spojrzeniem obcych... (FIN: 97)
Und Hänschen schickte einen Brief an Alo und Ala, man habe ihm das Boot weggenommen, er müsse sie leider verlassen. Noch am Abend desselben Tages hatte er die Antwort da....Hänschen küBte den Brief, steckte ihn zur Fotografie seiner Mutter, zu dem letzten Stückchen Salat, an dem sein Kanarienvogel gepickt hatte... (KOR: 135)

Ich hob den Kopf und sah jemanden näher kommen. Ich wurde ärgerlich, wollte aufstehen und fortgehen...- ich sah eine Frau...Sie ging langsam und trat vorsichtig auf...Dabei ist der Weg dort am Teich bequem und weich...Er führt ans Ufer, wo ein Zwillingsbaumstumpf steht...Sie wird also herkommen und sich auf den Baumstumpf setzen... Was hatte ich mir alles von diesem Abend versprochen! Es gibt Gedanken, die unter den Blicken von Fremden welken... (FIN: 81)

Diminutivderivate stellen auch ein beliebtes Mittel zur Personencharakterisierung in literarischen Texten dar, vgl.:

$\mathrm{Z}$ drzwi $\mathrm{z}$ boku wyszedl rabin... Rabin: malurki, z twarza panienki - swietej Katarzyny tak jak na obrazie w kosciele w Giniu. Kolo policzków zwijaly mu sie puszyste blond wloski...Przed nim Baltazar czul swoj bezwladny ogrom. (MIL: 105f)

Aus einer Seitentür trat der Rabbi ein...Der Rabbiner: ganz klein, mit einem Madchengesicht, so wie das der heiligen Katharina auf dem Bild in der Kirche von Ginie. An den Wangen lockten sich flaumige, blonde Härchen ...Vor ihm spürte Baltasar seine eigene kraftlose Riesenhaftigkeit. (MIL: 144f)

Die mehrfach bemühten Diminutive auch in der Textumgebung dienen allein der Herausstellung des Kontrasts von kleiner äußerer Gestalt und großer innerer Kraft, die im weiteren Text expliziert wird.

Przychodzila z rodzicami, w różowej sukience, w blyszczacych lakierkach, rodzice pili herbate w salonie, dzieci szly do ogrodu i tam rozpoczynalo sie znecanie nad matym, strojnym gosciem. Poczatek byl raczej niewinny, zwykla gra w klasy polegajaca na rzucaniu pilki o sciane domu...Potem pytaliśmy: "Chcesz jeszcze raz od poczatku?" - a ona dobrze ulożona, oniesmielona cyrkowym pokazem, milczaco kiwala
Sie besuchte uns mit ihren Eltern, im rosa Kleid und mit glänzenden Lackschuhen. Die Eltern tranken im Salon Tee, wir Kinder gingen in den Garten, und dort fingen wir an, den kleinen, aufgeputzten Gast zu quälen. Der Beginn war ziemlich unschuldig; eine ganz gewöhnliche Ballschule, die darin bestand, daß man den Ball an eine Wand warf...Dann fragten wir: "Möchtest du noch einmal anfangen?", sie 
glowa, odbierala uslużnie podana pileczke... (FIN: 94) nickte gut erzogen, durch unsere Zirkusvorführung jedoch eingeschüchtert, schweigend mit dem Kopf und nahm gehorsam den Ball... (FIN: 131)

Hier beschränkt sich der Diminutivgebrauch nicht allein auf die Person selbst, sondern er begleitet sie gleichsam und besetzt auch das, wozu sie in Kontakt tritt. So spiegeln sich die spöttisch belachten Attribute des geschilderten Mädchens, "brav", "geziert", "zerbrechlich", in den anderen Diminutiven wider.

Im Überblick der zielsprachlichen Reaktionen lassen sich den drei möglichen Wiedergabeformen ebensoviele Äquivalenzgrade zuordnen. Die Beispiele, in denen sich der Übersetzer für eine formale Entsprechung entschieden hat, genügen nicht dem Postulat der funktionellen Äquivalenz. In ihnen äußert sich das grundlegende Übersetzungsproblem, daß die deutsche Suffixdiminution nur bedingt in der Lage ist, die Funktion der Herausstellung und des Nachdrucks in affektfreiem Zusammenhang nachzuvollziehen. Deutsche Diminutivmodifizierungen eröffnen wegen anderer Kopräsenzen unmittelbar und jederzeit einen zudem noch begrenzten Expressivitätsrahmen; was im Original z.B. noch reine Lokalisierung ist, wird in der Übertragung zur naiven, gekünstelten Beschreibung einer Märchenidylle (vgl. Tännlein). Ebenso wird durch die Suffixwahl bewirkt, daß die Schilderung des kleinen Wuchses und der dünnen Schläfenlocken (vgl. Härchen) eines Respekt fordernden Rabbiners unerlaubterweise ins rein Kindliche verkehrt wird.

Meint man nun aber, das Problem durch Ignorierung der formalen Vorgabe umgehen zu können, sieht man sich vor die Schwierigkeit gestellt, damit oft die bewußte, aktuelle Pointierung des Textes zu verfehlen. Die herausragende textliche Stellung, die zusätzliche Signalwirkung, die die zitierten Wörter Bäume, Insel, besonders aber Brief, Baumstumpf und Ball haben, geht verloren und findet allein noch im weiteren Kontext eine Stütze. In der konventionalisierten und standardisierten Redeweise fält der Markierungsverlust dagegen nicht so stark ins Gewicht, weil dort die Ausnahmeerscheinung Diminutiv zum Normalfall wird und ein pragmatisch-stilistischer Abnutzungseffekt eintritt.

Ein gangbarer Weg, um die Verluste so gering wie möglich zu halten, ist es, kompensatorische Mittel zu nutzen, d.h., wie die verbleibenden Belege zeigen, vor allem auf die analytische Bildeweise auszuweichen. Mit ihr ist es nämlich möglich, die Intention des Nachdrucks vermittels eines beigefügten Adjektivs zu benennen bzw. zu spezifizieren. Schöpft man dabei alle zur Verfügung stehenden 
sprachlichen Mittel aus, so müßte sich die Übersetzung immerhin zwischen optimaler und approximativer Äquivalenz bewegen.

Zieht man zum Vergleich Beispiele der umgekehrten Übersetzungsrichtung heran, wird der Charakter der intendierten Textmarkierung durch polnische Diminutive noch deutlicher. Der deutsche Text bietet, wie schon oben als Übersetzung, auch im Original ein lineares, nicht durch diminutive Suffixbildungen formal gleichsam reliefiertes Bild, in das nun die polnische Fassung - um in der Illustrierung zu bleiben - bei Übernahme aller lexikalischen Kontextangaben Markierungsspitzen in Form von Suffixdiminutiven hineinsetzt. Dies ist als Neigung zu verstehen, bereits in der engeren und weiteren Umgebung Ausgesagtes zusätzlich noch formal zu pointieren. Aus deutscher Sicht erscheint dies häufig redundant und der eigenen Textnorm unangemessen, im Polnischen macht aber gerade diese "Diminutivornamentik" den Textcharakter unabdingbar aus. Die oft aufgeworfene Frage jedoch nach einer möglichen "nationalpsychologischen" Deutung solcher Divergenzen ist auf dieser Grundlage, wenn überhaupt, nicht zu entscheiden.

Jene zusätzliche Akzentuierung mögen folgende Beispiele belegen, in denen der engere Kontext, d.h. Adjektive bzw. das erste Kompositumsglied, die Aussage spezifiziert:

Rip van Winkle, der Eichhömchenjäger, war ihnen wohlbekannt, und er hörte gar schnurrige Geschichten von dem Mann, der vor zwanzig Jahren, wie jedes Kind weiß, in eine Schlucht gestürzt oder den Indianern in die Hände gefallen war. (FRI: 75)

Zum Glück erkannten sie Julika meistens gar nicht; mit einer schulmadchenhafien Wollmuitze auf dem Kopf, so daß ihr immer schon rōtliches Haar versteckt war, huschte sie vorbei, ein sehr unscheinbares Mädchen... (FRI: 87)

Frau v.Daan will immer alles zugleich tun...und stöhnt zwischendurch über ihre gequetschte Rippe. Das kommt davon, wenn eitle, altere (!!) Damen die verrücktesten Tumübungen machen wollen, um den dicken Hintern wieder loszuwerden. (FRA: 50)
Znali dobrze Ripa van Winkle, który polowal na wiewiórki; uslyszal zabawne historyjki na temat czlowieka, który, jak każdemu dziecku wiadomo, dwadzieścia lat temu runal w wawóz albo wpadl w rece Indian. (FRI: 99)

Na szczescie przeważnie jej nie poznawano. W welnianej pensjonarskiej czapeczce na glowie, ukrywajacej jej ladne rude wlosy, przemykala sie nieśmialo...wygladala bardzo niepokaźnie. (FRI: 115)

Pani van Daan zawsze robi pare rzeczy na raz...a miedzy jednym i drugim zajeciem steka, ie ja boli stluczone zebro. Tak to jest, kiedy prozine, podstarzate (!) paniusie chca robic najbardziej zwariowane cwiczenia gimnastyczne, aby sie pozbyc grubego tylka. (FRA: 61) 
Heute wurde wieder furchtbar geschossen, besonders nachts. Ich habe meine wichtigsten Habseligkeiten zusammengerafft und am Tage einen "Fluchtkoffer" gepackt mit den nötigen Sachen. (FRA: 65)

"Warum erzählen Sie mir dann diesen ganzen Unsinn?" fragte der Polizist. "Halten Sie die Polizei denn für so blöd, daß sie auf solche Ammenmärchen hereinfallt?" (END: 180)

Nino war Pächter eines kleinen Lokals am Stadtrand, in dem meistens nur ein paar alte Männer saßen, die den ganzen Abend an einem einzigen Glas Wein tranken und von ihren Erinnerungen redeten. (END: 16)

Sich auf jemand verlassen können! Einmal mit jemand zusammen sein, der einen nicht miBtrauisch von der Seite ansieht, wenn irgendein Wort fallt, das vielleicht die als Berufsinteressen verkleidete Eitelkeit verletzen kōnnte... (TUC: 59)

...und dann sangen wir ein Reiterlied, das wir selber gedichtet hatten, und das ganz und gar blödsinnig war, von der ersten bis zur letzten Zeile... (TUC: 52)
Dzisiaj byla znowu straszliwa strzelanina, zwlaszcza noca. Jeszcze za dnia zebralam wszystkie moje najpotrzebniejsze rzeczy i spakowalam walizeczke. (FRA: 82)

"Wiec dlaczego opowiada mi pan te bzdury?" spytal policjant. "Myśli pan, ze policja jest taka glupia i da sie nabrać na pańskie bajeczki?" (END: 148)

Nino dzierżawil maly lokal na krancu miasta, zwykle siedzialo tam tylko paru starszych ludzi, którzy przez caly wieczor pili jedna jedyna szklaneczke wina i rozmawiali o swoich wspomnieniach. (END: 14)

Moc na kims polegac! Być razem z kims, kto nie obrzuci cie podejrzliwym spojrzeniem, gdy padnie jakies siowko mogace urazié zamaskowana zawodowymi interesami próżnosć... (TUC: 74)

...wreszcie [spiewaliśmy] pieśn rycerska, któraśsmy sami ulożyli, zupelnie idiotyczna od pierwszej do ostarniej linijki... (TUC: 64f)

Auch der weitere Kontext kann die Aussage spezifizieren:

Beppo Straßenkehrer wohnte in der Nähe des Amphitheaters in einer Hütte, die er sich aus Ziegelsteinen, Wellblechstücken und Dachpappe selbst zusammengebaut hatte. (END: 35 )

Und ja, kommt man nach Hause, dann wirds wieder Zeit für Abendbrotmachen und dann die Kinder in Bett bringen, die Sachen auswaschen, die sie vom Tag hatten, ja, und dann bin ich mal froh, wenn ich zwei Stunden sitzen kann. (RUN: 48)

...vor allem die Kinder sind oft so schmutzig, daß man sie nur mit einer Zange anfassen möchte...Gestern, als Margot und ich hier badeten, hatte ich eine Idee: Wenn man nun so ein paar von dieser kleinen Gesellschaft mit einer Angel hier heraufholen könnte, sie
Beppo Zamiatacz Ulic mieszkal w pobliżu amfiteatru w chatynce, ktora sam sobie zbudowal z cegiel, kawalków blachy falistej i papy. (END: 27)

No i tak, wraca sie do domu, to już jest czas na robienie kolacji, a potem trzeba dzieci polożyć spac, uprać rzeczy, które mialy na sobie przez caly dzien, tak, a potem to już jestem rada, gdy sobie moge choc ze dwie godzinki posiedziec. (RUN: 58)

...a już dzieci sa nieraz takie brudne, ze czlowiek balby sie ich dotknack gola reka...Wczoraj, kiedysmy sie tu obie $z$ Margot kapaly, przyszla mi do glowy taka myśl: schwytac kilku tych brudaskow na wedke, wciagnać na góre, wpakowac do wanny, czysciutko ubrac i znow wypuscic na ulice... (FRA: 63) 
ins Bad stopfte, sauber anzöge und

wieder laufen ließe... (FRA: 52)

Wurde in den vorangegangenen Fällen der Nachdruck auf ein bereits im Primärtext vorhandenes Lexem gelegt, zeigt sich nicht selten auch die Bereitschaft, darüberhinaus ein neues, diminuiertes Lexem in den zielsprachlichen Text einzubringen oder es stellvertretend für Pronomen/Adverbien zu setzen:

Niemand geht gerne zu einem Ehepaar in Krise..., es liegt in der Luft, selbst wenn man nichts davon weiß...; es ist gemūtlich wie auf einem Minenfeld, ein solcher Besuch bei einem Ehepaar in Krise... (FRI: 112)

Dann schimpfen und streiten die beiden, nur an den Armen hängend, und kümmern sich wenig um Zuschauer. (GRZ: 22)

Momo hockte sich neben sie und klopfte mit dem Fingerknöchel schūchtern auf den Rūckenpanzer. Die Schildkröte schob ihren Kopf hervor und blickte Momo an. (END: 187)

Übrigens: ein oder zwei Whisky sind erlaubt! (FRI: 77)

In die Beutelflughörnchen...kann man sich sehr leicht verlieben. Sie fühlen sich so weich an, sind hellgrau mit schwarzen Streifen, haben große Augen und werden leicht zahm. (GRZ: 55)

Die beiden aber schnackten unentwegt ...Jetzt waren sie bei den Geschichten angelangt. Die Prinzessin erzählte die vom Schuster Hagen... (TUC: 67)

Vor ihr saß die Schildkröte!...O Ohne sich zu besinnen[,] packte Momo sie und steckte sie unter ihre Jacke. (END: 229)

Ein anders Mal brachte ihr ein kleiner Junge seinen Kanarienvogel, der nicht singen wollte...Sie muBte ihm eine ganze Woche lang zuhören, bis er endlich wieder zu trillern und zu jubilieren begann. (END: 21)

Gleichzeitig fiel ihr ein, daß sie längst hätte Gerfried anrufen mūssen. Sie ging ...nikt nie odwiedza chetnie malżeństwa w okresie kryzysu, to wisi w powietrzu, nawet kiedy sie o tym nie wie... Taka wizyta u malżenstwa w okresie kryzysu jest jak spacerek po zaminowanym polu... (FRI: 151f)

Oba zwierzatka zawieszone tylko na ramionach krzycza i klóca sie, nie troszczac sie zupelnie o widzów. (GRZ: 14)

Przysiadla obok niego i nieśmialo zastukala palcem w pancerz zwierzatks. Zołw wysunal glowe i spojrzal na Momo. (END: 153)

Jeszcze cos: jedna lub dwie szklaneczki whisky sa dozwolone! (FRI: 101)

W lotopalankach...można sie naprawde zakochać. W dotyku sa miekkie jak puch, maja popielate futerko $w$ czarne paski i wielkje oczy; latwo sie oswajaja. (GRZ: 30)

Tamci oboje gawedzili bez przerwy... Teraz byli na etapie anegdot. Ksieżniczka opowiedziala hitoryjke o szewcu Hagenie... (TUC: 83)

Przed nia stal zólw!... Niewiele myslac Momo chwycila st worzonko i ukryla je pod swoim kubrakiem. (END: 185)

Innego dnia pewien chlopczyk przyniós! jej swojego kanarka, który nie chcial spiewać...Caly tydzien musiala sluchać ptaszka, zanim znów zaczal wyciagac swoje trele i radośnie spiewac. (END: 17)

Równocześnie pomyslala, że dawno już powinna zadzwonić do Gerfrieda. Po- 
also hinaber und drehte die Durchwahlnummer ihres Büros. (HEY: 73) szla wiec do budki i nakrecila numer swego pokoju. (HEY: 79)

Zum Abschluß sollte noch ein Blick auf die eher sekundäre Frage der Wiedergabeschwierigkeiten beim analytischen Verfahren geworfen werden. Manche Fälle der Problemdiskussion, die Erörterungen zum ASD $\Rightarrow$ AD-Typ sowie die eindeutige statistische Verteilung (vgl. oben Tab.l und Tab.10), der zu entnehmen ist, daß das Deutsche als Ausgangssprache analytische Diminutive wesentlich häufiger einsetzt als das Polnische, legen den Gedanken nahe, daß sich das Deutsche in den Ausdrucksformen der analytischen Bildeweise besser bewegt. Es ist daher zu vermuten, daß im polnisch $\Rightarrow$ deutschen Vergleich für die Zielsprache im $\mathrm{AD} \Rightarrow \mathrm{AD}$-Typ kein Übersetzungsproblem gegeben ist (ebenso wie im deutsch $\Rightarrow$ polnischen Kontrast $\operatorname{der} S D \Rightarrow$ SD-Typ unauffallig war) und dementsprechend häufig totale Äquivalenz, d.h. Deckungsgleichheit bis in die strukturelle Ebene hinein bestehen kann. Da die Domäne der polnischen Sprache in der synthetischen Diminution liegt, ergibt sich für sie eine Schwierigkeit: Trifft sie auf ein ausgangssprachliches analytisches Diminutiv, muß sie nämlich entscheiden, ob sie es in dieser Form beläßt oder doch lieber der ihr gemäßen Diminutionsart angleicht, d.h. mit einem Suffixdiminutiv bzw. seiner adjektivischen Erweiterung reagieren will.

Es finden sich doch viele Belege, in denen, für das Polnische ungewohnt, lediglich eine attributive Fügung mit Grundlexem begegnet, wo eher eine modifizierte Form zu erwarten wäre:

...ringsum die Gärten voll ewigem Frühling, Arkadien, aber indianisch. In einem schmalen Kanoe...paddelt sich eine alte Indianerin heran, ihren Säugling auf dem Rücken gebunden... (FRI: 28)

...die Figur [hat] etwas Ephebenhaftes, was bei einer Frau in ihren Jahren einen unerwarteten Reiz hat...Ihre sehr schmale Hand...ist keineswegs ohne Kraft... (FRI: 56)

Busse brachten sie vom Flugplatz der kleinen Stadt zu den verschiedenen Hotels. (HEY: 12) ...dookola ogrody pelne wiecznej wiosny, Arkadia, ale indianska. W waskiej lodzi...podplywa stara Indianka $z$ uwiazanym do plecow niemowleciem. (FRI: 33)

Wierzy sie..., ze jest $w$ niej cos $z$ efeba, co kobiecie $w$ jej latach dodaje nieoczekiwanego wdzieku...Jej bardzo waska reka...nie jest pozbawiona sily... (FRI: 72)

Autobusy rozwozily ich z lotniska malego miasta do rozmaitych hoteli. (HEY: 12) 
Unser Wohnzimmer und auch die anderen Räume sahen noch wie Rumpelkammern aus, unbeschreiblich!...Das kleine Zimmer war bis an die Decke mit Betten und Bettzeug vollgepfropft. (FRA: 21)

Die große schwere Tür aus grünem Metall öffnete sich. Momo stürzte hinein, rannte durch den Gang mit den steinernen Figuren, öfnete die ganz kleine Tür am anderen Ende... (END: 235)

Aber der Abgesandte des Königs...erklärte ihr, erst wenn der Fisch ausgewachsen sei, würde er sich in Gold verwandeln...Der junge Fisch wuchs nun von Tag zu Tag... (END: 44)

Ein leichter Wind hatte sich erhoben, dann wurden die Windstöße stärker, ein hauchzarter Regen fiel. (TUC: 98f)

Wenn die verhältnismäßig kleine Insel stärker bevölkert werden wird..., wird die Zahl dieser einzigartigen Tiere sich rasch verringern. (GRZ: 67)
Nasz pokoj i wszystkie inne wygladaly jeszcze jak graciarnia, balagan nie do opisania!...Mały pokój byl aż pod sufit zapchany lózkami i pościela. (FRA: 23)

Duże, cieżkie metalowe drzwi otworzyly sie przed nia. Momo wpadla przez nie, pobiegla przez galerie z kamiennymi figurami, otworzyla malenkkie drzwi na drugim koncu... (END: 189)

Jednak posel króla...wyjaśnil jej, że rybka zamieni sie $w$ zloto, dopiero kiedy wyrosnie...Mloda ryba rosla $z$ dnia na dzień... (END: 34)

Zerwal sie lekki wiatr. Potem jego podmuchy staly sie coraz silniejsze i zaczal padac delikatny deszcz. (TUC: 124)

Kiedy zwiekszy sie zaludnienie tej malej wyspy..., liczba tych osobliwych zwierzat szybko sie zmniejszy. (GRZ: 37)

Obwohl die Abwesenheit suffixaler Formen aus den Erfahrungen der Verwendungspraktik in der Ausgangssprache auffallig ist, kann dem polnischen Text doch nicht in derselben Entschiedenheit wie manchen deutschen Übersetzungen der Vorwurf verfehlter funktioneller Äquivalenz entgegengebracht werden. Zieht man Belege aus teilweise vergleichbaren Kontexten heran, die erwartungsgemäß suffixal (ASD bzw. SD) reagieren, verfestigt sich die These der unterschiedlichen pragmatisch-stilistischen Gewichtung des Diminutivs in beiden Sprachen:

Zuerst ging sie zu Nicola, dem Maurer.

Sie kannte das Haus gut, wo er oben unter dem Dach ein kleines Zimmer bewohnte. (END: 81)

Momo hockte sich nieder und sah direkt vor ihrer Nase auf der kleinen Tür ein Schildchen... (END: 134)

Die Kaiserin Strapazia hatte jedoch von einem Reisenden erfahren, jener König ...besitze einen kleinen Fisch, der sich, sobald er ausgewachsen sei, in pures Gold verwandeln würde. (END: 44)
Najpierw wybrala sie do murarza Nicoli. Dobrze znala dom, w którym mieszkal w malym pokoiku na poddaszu. (END: 65)

Momo przykucnela i zobaczyla tuż przed soba przytwierdzona na drzwiczkach tabliczke... (END: 110)

Cesarzowa Sztrapacja jednak dowiedziala sie od pewnego wedrowca, że $6 w$ król...ma nieduża rybke, która po wyrosnieciu zamieni sie w czyste zloto. (END: 33) 
Der Himmel war weiBgefleckt; wenn man von der Sonne recht schön angebraten war, kam eine Wolke, ein leichter Wind lief daher, und es wurde ein wenig kühl. (TUC: 32)

Diese kleinen Känguruhs sind auf dem Festland fast ausgerottet, sie hausen heute auf kleinen Inseln vor der Küste ... (GRZ: 97)
Niebo bylo upstrzone bialymi oblokami; gdy już slonce zbyt mocno przypiekalo, nadciagala chmura, wraz z nia pojawial sie wiaterek i robilo sie nieco chlodniej. (TUC: 40)

Te male kangury... wytepiono na stałym ladzie prawie zupelnie; dzisiaj zamieszkuja male wysepki przybrzeżne... (GRZ: 51)

Die Merkmalhaftigkeit im Polnischen hat nicht die Nachhaltigkeit und den Festlegungscharakter wie die im Deutschen; es ist nicht eine solch scharfe Trennung zwischen nichtmodifizierter und modifizierter Form zu registrieren. Die Grenze zwischen der Gebrauchsmöglichkeit von Grundwort und Derivat erscheint fließend, variabler setzbar. Mit der Diminuierung begibt man sich nicht in derselben Weise wie im Deutschen in ein ausgezeichnetes, für sprachliche Ausnahmeerscheinungen reserviertes Redefeld, man sucht damit nicht eine Nische auf, sondern verbleibt in der Usance.

Die offenbar weitgehend unproblematische Umsetzung analytischer Diminutive beider Sprachen im Gegensatz zur Reibungsvielfalt bei der Übertragung derivationeller Diminutive gibt Anlaß zu Rückschlüssen bezüglich des unterschiedlichen Charakters beider Verfahren. Indem die attributivische Diminution lexikalische Mittel nutzt, d.h. die Aussage durch Spezifizierung und Explikation eines Grundwortes mit einem weiteren Lexem - dem Attribut - zu Wege bringt, ist sie durch einen gleichsam definitorischen, begrifflich-informativen Charakter gekennzeichnet; sie ist eindeutiger und gibt dem Gemeinten eine deutlicher umrissene Kontur. Die synthetische Diminution dagegen gründet auf morphologischen Vorgängen, d.h. ihr Intendiertes ist in einer Bildung mit nichtexplikativem Morphemzusatz komprimiert, und deshalb ist sie eher chiffriert als offengelegt, elastischer, fließend, vieldeutiger. Weil sie in einer Sprache Implikationen auf eine individuelle Weise bündelt, wie es in gleicher Weise kaum in einer anderen Sprache geschehen kann, stellt im wesentlichen nur sie eine hermeneutische Herausforderung für den Übersetzer dar und muß daher von der analytischen Diminution im Hinblick auf ihre Leistungsfahigkeit unterschieden werden. 


\subsection{GEGENUBERSTELLUNG DER ÜBERSETZUNGSVERGLEICHE - INTERFERENZEN BEIDER SPRACHEN}

Der nun abgeschlossene Teil der Problembesprechung wurde angeregt durch die zwei statistischen Gesamtdarstellungen (Tab.1 und Tab.10), die die Frequenz der Wiedergabetypen im polnisch $\Rightarrow$ deutschen und deutsch $\Rightarrow$ polnischen Vergleich anschaulich machten, also sprachenpaarbezogen vorgingen. Dabei stellten sich häufig wiederkehrende Paarungen, Konstanten im Übersetzen, heraus, die in einer auf die Hauptmuster beschränkten Übersicht zusammengefaßt werden sollen: ${ }^{44}$

Tab.19: Frequenz der wichtigsten Wiedergabetypen

\begin{tabular}{|l|c|c|}
\hline \multirow{2}{*}{$\begin{array}{c}\text { WIEDERGABE- } \\
\text { TYP }\end{array}$} & \multicolumn{2}{|c|}{ BELEGE } \\
\cline { 2 - 3 } & PL.(AS) $\Rightarrow$ DT.(ZS) & PL.(ZS) $=$ DT.(AS) \\
\hline SD $\Leftrightarrow$ ND & $49,10 \%$ & $50,48 \%$ \\
\cline { 2 - 3 } AD $\Leftrightarrow A D$ & $10,67 \%$ & $19,37 \%$ \\
SD $\Leftrightarrow$ SD & $16,79 \%$ & $9,23 \%$ \\
\cline { 2 - 3 } ASD $\Leftrightarrow A D$ & $4,62 \%$ & $5,58 \%$ \\
\hline
\end{tabular}

Die hier schematisierte Möglichkeit, beide Übersetzungsvergleiche aufeinander zu beziehen, verdeutlicht, wie beide Sprachen nach bestimmten, beschreibbaren und charakteristischen Gesetzmäßigkeiten eine strukturelle Korrelation bilden. Der Übersetzungsaustausch zwischen beiden Sprachen ist somit kein diffuses Aufeinandertreffen, sondern kennt regelmäßig wiederkehrende Konstellationen.

Eine weitere wichtige Perspektive der Betrachtung des Diminutivgebrauchs ist die sprachenbezogene, im näheren die Frage, ob Veränderungen einer Sprache im Statuswechsel von Ausgangs- zu Zielsprache zu konstatieren, wenn ja, ob sie auch Regularitäten unterworfen sind. Im Verlauf der Problemdiskussion stie B man wiederholt auf das im weitesten Sinne als Interferenz zu bezeichnende Phänomen, daß eine Sprache, sieht sie sich vor eine ihr fremde Gebrauchsnorm gestellt, geneigt ist, dieser äußerlich-formal zu entsprechen und dabei Abweichungen von ihrem eigenen Standard zuzulassen. Ein solches

4 Aus methodischen Gründen werden lediglich Substantivdiminutive besprochen, adjektivische etc. Diminutive bleiben im weiteren Verlauf unberücksichtigt. 
Verfehlen, oft aber auch nur Verzerren funktioneller Äquivalenz konnte bisher lediglich exemplarische Darstellung finden. Mit Hilfe weiterer Statistiken sind diese Beobachtungen nun zu systematisieren, sie geben letztlich Auskunft über die Auswirkung der untersuchten Übersetzungsprobleme auf die Sprachen, sofern sie vor solche Schwierigkeiten gestellt werden.

Als Ausgangspunkt und Anlaß zu weiteren Fragestellungen sollen zunächst zwei Tabellen vorangestellt werden, die zeigen, welchen Anteil die drei Diminutionsarten an der Gesamtzahl der Diminutive der Texte einer Sprache haben, zum einen, wenn diese als Ausgangs-, zum anderen, wenn sie als Zielsprache eingesetzt wird:

Tab.20: Distribution der Diminutivformen in AS und ZS - im Polnischen ${ }^{4 S}$

\begin{tabular}{|c|c|c|}
\hline \multirow{2}{*}{$\begin{array}{c}\text { DMMINUTIONS- } \\
\text { ART }\end{array}$} & \multicolumn{2}{|c|}{ POLNISCH } \\
\hline & AUSGANGSSPRACHE & ZIELSPRACHE \\
\hline \multirow{3}{*}{$\begin{array}{l}\text { synthetisch } \\
\text { analytisch } \\
\text { analyt.-synth. }\end{array}$} & $79,29 \% \quad[1991]$ & $67,71 \% \quad[1099]$ \\
\hline & $13,10 \%$ & $23,66 \%$ \\
\hline & $7,61 \%$ & $8,63 \% \quad[140]$ \\
\hline
\end{tabular}

Tab.21: Distribution der Diminutivformen in AS und ZS - im Deutschen

\begin{tabular}{|c|c|c|}
\hline \multirow{2}{*}{$\begin{array}{l}\text { DIMINUTIONS- } \\
\text { ART }\end{array}$} & \multicolumn{2}{|c|}{ DEUTSCH } \\
\hline & AUSGANGSSPRACHE & ZIELSPRACHE \\
\hline \multirow{3}{*}{$\begin{array}{l}\text { synthetisch } \\
\text { analytisch } \\
\text { analyt.-synth. }\end{array}$} & $29,0 \% \quad[241]$ & $50,17 \% \quad[586]$ \\
\hline & $64,86 \% \quad[539]$ & $44,78 \% \quad[523]$ \\
\hline & $6,14 \%$ & $5,05 \%$ \\
\hline
\end{tabular}

Vorab soll ein Blick auf die Verteilung der Diminuierungsverfahren im Ausgangstext geworfen werden: Das schon dem bisherigen statistischen Material zu entnehmende Ungleichgewicht der Gebrauchsfrequenzen zwischen dem Polnischen und Deutschen wird hier noch einmal expliziert. Wurden im polnischen Original Worteinheiten diminuiert, dann bedeutete das in $79,29 \%$ aller Fälle Rückgriff auf die suffixale Methode, unter denselben Bedingungen belastete der deutsche Text sein Derivationspotential mit nur $29 \%$. Vorzug vor allen anderen

« Die Ziffern in eckigen Klammern geben hier und im folgenden die absolute Belegzahl an. 
Mitteln - wenn auch nicht in solch hohem Maße wie die Suffixe im Polnischen erhält dagegen im Deutschen mit $64,86 \%$ das in seiner Leistung nicht völlig deckungsgleiche analytische Verfahren; dem steht ein Anteil von nur 13,10\% im polnischen Ausgangstext gegenüber. Prozentual ausgedrückt kommen in der polnischen Vorlage $273,4 \%$ mehr Suffixdiminutive - fast dreimal so viel - zur Anwendung als in der deutschen, die wiederum gegenüber dem Polnischen ein Übergewicht von 495,1\% analytischer Bildungen - beinahe um das Fünffache aufweist. In Relation zu den anderen diminutivischen Verwendungsmöglichkeiten wird von dem kombinierten Verfahren in beiden Sprachen seltener Gebrauch gemacht.

Das eigentliche Interesse sollte aber dem Vergleich von Ausgangs- und Zielversion, also den Abweichungen des Diminutiveinsatzes gelten. Fünf Veränderungen beim Statuswechsel von Original zu Übersetzung sind dabei zu beobachten:

1. Die synthetische Diminution im Polnischen geht leicht zurück.

2. Die Nutzung analytischer Bildungen im Polnischen nimmt stark zu.

3. Im Polnischen werden vermehrt analytisch-synthetische Formen eingesetzt.

4. Im Deutschen erfahren suffixale Diminutive einen auffäligen Zuwachs.

5. Der Einsatz analytischer Diminutive nimmt im Deutschen beachtlich ab. Diese Tendenzen interlingualer Interferenz können zur Verdeutlichung prozentual angegeben werden:

ABWEICHUNGEN VON AS ZU ZS

Far das Polnische:

synthetisch: $\quad 79,29 \% \Rightarrow 67,71 \%=14,61 \%$ Abnahme analytisch: $\quad 13,10 \% \Rightarrow 23,66 \%=80,61 \%$ Zunahme analyt.-synth.: $7,61 \% \Rightarrow 8,63 \%=13,40 \%$ Zunahme

Far das Deutsche:

synthetisch: $\quad 29,0 \% \Rightarrow 50,17 \%=73,0 \%$ Zunahme analytisch: $\quad 64,86 \% \Rightarrow 44,78 \%=30,96 \%$ Abnahme analyt.-synth.: $6,14 \% \Rightarrow 5,05 \%=2,14 \%$ Abnahme

Diese auffalligen Mittelwertabweichungen sollen nun mit Hilfe von fünf Folgeauswertungen zu deuten versucht werden, die ausschließlich zielsprachenorientiert sind und als Mittel der Darstellung verschiedene Auswahlkriterien von ausgangs- und zielsprachlichen Formen nutzten. 
Tab.22: Wiedergabe ausgangssprachlicher Diminutive

\begin{tabular}{|c|c|c|}
\hline WIEDERGABEART & IM POLNISCHEN (ZS) & IM DEUTSCHEN (ZS) \\
\hline \multirow{5}{*}{$\begin{array}{l}\text { synthetisch (SD) } \\
\text { analytisch (AD) } \\
\text { analyt.-synth. (ASD) } \\
\text { nichtdiminut. (ND) } \\
\text { freie Wiedergb.(frW) }\end{array}$} & $25,63 \%$ [213] & $18,28 \%$ [459] \\
\hline & $42,24 \% \quad[351]$ & $19,91 \% \quad[500]$ \\
\hline & $16,25 \% \quad[135]$ & $2,31 \% \quad[58]$ \\
\hline & $10,95 \% \quad[91]$ & $53,01 \%[1331]$ \\
\hline & $4,93 \%$ & $6,5 \%$ \\
\hline
\end{tabular}

Die Tabelle zeigt an, mit welcher prozentualen Verteilung diminutivische Ausdrücke des Originals (ohne diese hier zunächst zu differenzieren) in der zielsprachlichen Version verwirklicht wurden. In einem ersten Überblick wird augenfallig, daß das Deutsche in mehr als der Hälfte aller Fälle, in denen es auf ein polnisches Diminutiv trifft, diesem keine diminutivische Form entgegensetzen kann bzw. will, während sich synthetische und analytische Reaktion die Waage halten. Fürs Polnische ist es dagegen frappierend, in welch geringem Ausmaß synthetische Formen als Übersetzung deutscher Diminutive eingesetzt werden, bei unerwartet hohem Anteil analytischer Bildungen. Beides soll noch weiter erläutert werden.

Schlüsselt man die für beide Sprachen jeweils wichtigsten Wiedergabearten weiter auf, im Hinblick darauf, in welchem Maße (genauer: zu welchem Anteil) sie jeweils auf die drei Diminutionsarten der Ausgangstexte reagiert haben, ergibt sich folgendes Bild:

Tab.23: Polnisch als Zielsprache

\begin{tabular}{|l|c|c|c|}
\hline \multirow{2}{*}{$\begin{array}{c}\text { WIEDERGABE- } \\
\text { (Polnisch) } \\
\text { PRT }\end{array}$} & \multicolumn{3}{|c|}{ AUSGANGSSPRACHE (Deutsch) } \\
\cline { 2 - 4 } & $\mathrm{SD}$ & $\mathrm{AD}$ & ASD \\
\hline $\begin{array}{l}\text { synthetisch (SD) } \\
\text { analytisch (AD) } \\
\text { analyt.-synth. (ASD) }\end{array}$ & $\mathbf{7 6 , 0 6 \%}$ & $18,78 \%$ & $5,16 \%$ \\
\cline { 2 - 4 } & $1,42 \%$ & $\mathbf{9 6 , 8 7 \%}$ & $1,71 \%$ \\
\cline { 2 - 4 } & $3,7 \%$ & $72,6 \%$ & $23,7 \%$ \\
\hline
\end{tabular}


Tab.24: Deutsch als Zielsprache

\begin{tabular}{|l|c|c|c|}
\hline \multirow{2}{*}{$\begin{array}{c}\text { WIEDERGABE- } \\
\text { ART } \\
\text { (Deutsch) }\end{array}$} & \multicolumn{3}{|c|}{ AUSGANGSSPRACHE (Polnisch) } \\
\cline { 2 - 4 } & SD & AD & ASD \\
\hline synthetisch (SD) & $97,39 \%$ & $0,65 \%$ & $1,96 \%$ \\
\cline { 2 - 4 } $\begin{array}{l}\text { analytisch (AD) } \\
\text { nichtdiminutiv. (ND) }\end{array}$ & $18,6 \%$ & $56,8 \%$ & $24,6 \%$ \\
\cline { 2 - 4 } & $98,2 \%$ & $1,05 \%$ & $0,75 \%$ \\
\hline
\end{tabular}

In ihrer Dominanz sind drei Werte besonders herauszustellen: Wenn der polnische Text analytisch antwortet, so tut er dies fast ausschließlich als Reaktion auf die strukturelle Entsprechung im Deutschen (=AD). Ebenso eindeutig verhält sich der deutsche Zieltext; die synthetische Wiedergabeart bezieht sich fast durchgehend auf polnische Suffixdiminutive. Wenn der deutsche Text dagegen keine Diminutive einsetzen kann, so sind es beinahe ausnahmslos suffixale Derivate der Vorlage, die ihn dazu zwingen.

Basierte Tab.22 nur auf der Erfassung von ausgangssprachlichen Diminutiven und umfaßte uneingeschränkt alle Wiedergabearten, soll umgekehrt die folgende Übersicht in der Zielsprache lediglich Diminutive (SD, AD, ASD) berücksichtigen, beim Primärtext aber keine Einschränkungen vornehmen (nichtdiminutivische Ausdrücke werden also eingeschlossen). Es wird zu ersehen sein, zu welchem Anteil sich die drei Diminutionsarten jeweils auf eine ausgangssprachliche Form beziehen.

Tab.25: Diminutivische Wiedergabe der Ausgangssprache (auch won ND)

\begin{tabular}{|c|c|c|c|}
\hline \multicolumn{2}{|c|}{ EINHEITEN DER } & POLNISCH (ZS) & DEUTSCH (ZS) \\
\hline \multirow{4}{*}{ SD } & \multirow{4}{*}{$\begin{array}{l}\text { SD } \\
\text { AD } \\
\text { ASD } \\
\text { ND } \\
\end{array}$} & $14,74 \% \quad[162]$ & $76,28 \% \quad[447]$ \\
\hline & & $3,64 \% \quad[40]$ & $0,51 \%$ \\
\hline & & $1,0 \% \quad[11]$ & $1,54 \%$ \\
\hline & & $80,62 \%$ [886] & $21,67 \% \quad[127]$ \\
\hline \multirow{4}{*}{ AD } & \multirow{4}{*}{$\begin{aligned} & \text { SD } \\
= & A D \\
& \text { ASD } \\
& \text { ND }\end{aligned}$} & $1,3 \%$ & $17,78 \%$ \\
\hline & & $88,54 \%$ [340] & $54,3 \% \quad[284]$ \\
\hline & & $1,56 \%$ & $23,52 \% \quad[123]$ \\
\hline & & $8,59 \% \quad[33]$ & $4,4 \%$ \\
\hline
\end{tabular}




\begin{tabular}{|c|c|c|c|}
\hline \multicolumn{2}{|c|}{ EINHEITEN DER } & \multirow[t]{2}{*}{ POLNISCH (ZS) } & \multirow[t]{2}{*}{ DEUTSCH (ZS) } \\
\hline ZS & AS & & \\
\hline \multirow{4}{*}{$\mathbf{A S D}=$} & \multirow{4}{*}{$\begin{array}{l}\text { SD } \\
\text { AD } \\
\text { ASD } \\
\text { ND }\end{array}$} & $3,57 \%$ & $15,25 \%$ \\
\hline & & $70,0 \%$ & $8,47 \%$ \\
\hline & & $22,86 \% \quad[32]$ & $74,58 \%$ \\
\hline & & $3,57 \%$ & $1,69 \%$ \\
\hline
\end{tabular}

Da hier auch Nichtdiminutive in der Quellensprache berücksichtigt wurden, kann ein auffalliger Wert hinzutreten, der statistisch bereits Geäußertes unterstreicht: mit $\mathbf{8 0 , 6 2 \%}$ bezieht sich der dominante Anteil polnischer Suffixdiminutive auf eine unmarkierte Einheit im Deutschen.

Zuletzt soll, um Vergleiche zu ermöglichen, demonstriert werden, welche statistischen Verhältnisse sich ergeben, wenn sowohl Ausgangs- als auch Zieltext nur im Hinblick auf Diminutivgebrauch besehen werden:

Tab.26: Diminutivische Wiedergabe von Diminutiven

\begin{tabular}{|l|l|c|}
\hline WIEDERGABEART & POLNISCH (ZS) & DEUTSCH (ZS) \\
\hline \multirow{2}{*}{$\begin{array}{l}\text { synthetisch } \\
\text { analytisch }\end{array}$} & $30,47 \%[213]$ & $45,13 \%$ \\
analyt.-synth. & $50,22 \%[359]$ \\
\cline { 2 - 3 } & $19,31 \%[135]$ & $49,16 \%[500]$ \\
\hline
\end{tabular}

Die bereits in Tab. 22 ermittelten Anteilswerte treten nun, auch in ihrem Widerspruch zu den für die Ausgangssprache angegebenen Zahlen (vgl. Tab.20 und Tab.21) deutlich zum Vorschein.

Diese verstreuten Informationen müssen nun gebündelt werden, im Versuch, die am Anfang konstatierten fünf auffalligen Abweichungen der Diminutivverwendung in Abhängigkeit vom Status des Textes zu erklären.

\section{Leichter Rückgang der polnischen Suffixdiminutive:}

Übersieht man die Verteilungsangaben zum synthetischen Diminutivgebrauch im polnischen Zieltext, so läßt sich eine starke Disproportion zwischen dem für alle Übersetzungen geltenden Wert (vgl. Tab.20: 67,71\%) und den für die Reak- 
tion nur auf Diminutive geltenden Angaben (vgl. Tab.22: 25,63\%; Tab.26: $30,47 \%$ ) feststellen. Zieht man jedoch zur Vermittlung die die synthetische Diminution betreffenden Ergebnisse von Tab.25 heran, so läßt sich eine eindeutige Schlußfolgerung ziehen: in letzterer wird offensichtlich, daß vier Fünftel der polnischen Suffixdiminutive auf der Übertragung nichtdiminutivischer Einheiten beruhen. Der Wiedergabetyp ND $\Rightarrow S D$ sorgt also für die noch recht hohe Zahl von $67,71 \%$. Sieht man dagegen von nichtdiminutivischen Formen im Ausgangstext ab, betrachte: somit nur, in welcher Weise sich das Polnische gegenüber der Diminutivstruktur des Deutschen verhält, wird ein krasser Rückgang des Gebrauchs morphologisch markierter Bildungen ersichtlich, der so stark ausfält, daß summa summarum der gesamtzielsprachliche Wert $(67,71 \%)$ knapp unter dem des Polnischen in der Quellenversion (79,29\%) bleibt. AufschluBreich ist der Vergleich der Angaben von Tab.22 und Tab.26 mit der in Tab.21 ablesbaren Distributionszahl zum synthetischen Diminutionsverfahren im deutschen Original: die Werte stimmen beinahe exakt überein, so daß angenommen werden kann, daß der starke Rückgang der Derivate im Kontakt mit dem frequentiell anders gelagerten Verfahren des deutschen Originals (vgl. in Tab.21 auch die starke Gewichtung analytischer Diminutive) auf einen Prozeß der Anpassung an diese Struktur zurückzuführen ist. Eine Ausgleichsbewegung dieser Verzerrung der eigenen Gebrauchsregularitäten bei der Auseinandersetzung mit vor allem auf lexikalischem Wege markierten Einheiten (AD) findet dann aber statt, wenn der polnische Übersetzer einer neutralen Grundform im Text (ND) gegenübertritt; dann ist ihm nichts vorgegeben, er ist an kein Muster gebunden und kann sich der Schwerpunkte bedienen, die seine Muttersprache setzt: er bringt seine Disposition in die Leerstelle ein.

\section{Starke Zunahme der polnischen analytischen Diminutive:}

Der Rückgang einer Verwendungsform - wie der gerade beobachtete - ist aber zwangsläufig mit dem Zuwachs einer anderen verbunden. Die schon angestiegene Frequenzzahl polnischer zielsprachlicher attributiver Fügungen von 23,66\% (vgl. Tab.20) fält noch größer aus, wenn nur die Erwiderung von vorgegebenen Diminutiven berücksichtigt wird; es kommt zur der überraschenden Beobachtung, daß die Hälfte aller polnischen Diminutive, die deutschen entgegnen, auf ein Syntagma zurückgreifen (vgl. Tab.26). Das Heranziehen zweier weiterer Werte kann diesen Vorgang durchsichtig machen. Zum einen kann der verstärkte Rückgriff auf analytische Diminutive genau lokalisiert 
werden: in Tab.25 bzw. Tab.23 läßt sich deutlich ersehen, in welcher RegelmäBigkeit $(88,54 \%$ bzw. 96,87\%) diese Diminutive als Reflex auf strukturell entsprechende und zudem dominierende Formen der deutschen Vorlage (AD) entstehen und sich darüberhinaus kaum einem anderen Übersetzungsmuster verdanken. Zum anderen kann ermessen werden, wie stark diese Anpassung an das fremde Schema sich durchgesetzt hat. Stellt man den für das Polnische geltenden Wert von 50,22\% (vgl. Tab.26) neben den des analytischen Diminutiveinsatzes im deutschen Quellentext von 64,86\% (vgl. Tab.21), so läßt sich feststellen, daß die Angleichung parallel zur synthetischen Diminution fast vollständig vollzogen worden ist.

Daß aber diese Störung aufs Ganze gesehen mit 23,66\% relativiert ist, kann man auf die Tatsache zurückführen, daß das $A D \Rightarrow A D-M u s t e r$ im deutsch $\Rightarrow$ polnischen Austausch absolut gesehen keine gravierende Rolle spielt, weil dieser durch den ND $\Rightarrow$ SD-Typ dominiert wird. In der obigen Ausschnittsbetrachtung dagegen (Reaktion auf gesetzte Diminutive) hat die Interferenz Gewicht.

\section{Geringer Zuwachs der polnischen analytisch-synthetischen Diminutive:}

Versucht man zu präzisieren, unter welchen Bedingungen diese insgesamt periphere Diminutionsart in ihrem Gebrauch verändert wird, so läßt der relativ hohe Wert von 19,31\% (vgl. Tab.26) erkennen, daß die Beeinflussung wiederum auf den Kontakt mit deutschen Diminutiven zurückzuführen ist. Sowohl Tab.25 als auch Tab.23 können belegen, daß dies in Dreiviertel aller Fälle im Gefolge eines analytischen Diminutivs der Ausgangsversion geschieht $(70,0 \%$ bzw. 72,6\%). Die in den beiden vorangegangenen Punkten beobachteten Tendenzen treffen hier zusammen. Die Entgegnung des kombinierten Verfahrens auf das epithetische kann einerseits als eine Anpassung an die vorgegebene Struktur des deutschen Diminutivs verstanden werden. Gleichzeitig ist sie aber auch Ausdruck der Neigung, die morphologisch merkmallose Einheit in eine merkmalhafte umzuwandeln. Die angesprochene Steigerung ist somit letztlich als Fortsetzung des oben untersuchten Angleichungsprozesses an die vorherrschende analytische Diminution im Deutschen zu sehen.

\section{Beachtlicher Anstieg der deutschen Suffixdiminutive:}

Obwohl sie nicht das Hauptanliegen der Untersuchung darstellen, seien auch die deutschen zielsprachlichen Veränderungen erläutert: Die erhebliche Zunahme 
synthetischer Formen kann hier ebenso als Assimilationsphänomen verstanden werden, wobei besonders die häufigen Suffixderivate der Vorlage den vermehrten Einsatz provoziert haben; Tab.25 zeigt, daß dreiviertel aller Belege, Tab.24, daß sogar fast alle synthetischen Bildungen, welche eine Übersetzung von Diminutiven darstellen (97,39\%), im Hinblick auf ein Modifikationsderivat gebildet sind. Der Anpassungsgrad an die Gebrauchsnorm der polnischen Ausgangssprache ist aber, verglichen mit dem des Polnischen in Zielsprachenfunktion, längst nicht so hoch ausgefallen. Hier scheinen die besprochenen Übersetzungsprobleme, d.h. die strengeren Gebrauchsrestriktionen im Deutschen, einer stärkeren Angleichung im Wege zu stehen.

\section{Deutliche Abnahme der deutschen analytischen Diminutive:}

Die Abweichung ist zunächst mit der Interdependenz der einzelnen Diminutionsverfahren im Zieltext zu erklären. Die Ausführungen zum vorangehenden Punkt und ein erneuter Blick auf Tab.26 verdeutlichen, daß die zielsprachliche analytische Diminution, im Gegensatz zur ausgeprägten Dominanz in der deutschen Originalfassung, gegenüber der synthetischen Diminution an Bedeutung eingebüßt hat. Die Aufwertung des einen Diminutionsverfahrens hat statistisch die Abwertung des anderen zur Folge. Gleichzeitig aber sorgt die Disposition des Polnischen als Vorlage (vgl. Tab.20: 13,10\% AD) dafü, daß auch absolut gesehen dem deutschen Übersetzer wenig Gelegenheit geboten wird, die analytischen Formen als strukturelle Entsprechung einzusetzen. Dies fallt umso mehr ins Gewicht, als das Deutsche ganz anders als das Polnische hierbei daran gehindert ist, in ähnlicher Weise ein neutrales Basislexem, eine Leerstelle, mit der ihm eigenen Diminutionsart zu besetzen (vgl. Tab.25: ND $\Rightarrow$ AD nur 4,4\%). Dies kann man wohl auf den bereits angesprochenen unterschiedlichen Charakter der morphologisch gebildeten Einheit gegenüber der lexikalisch gebildeten zurückführen. Letztere ist nicht so vielseitig nutzbar, nicht ein solch offenes Programm wie jene, sondern muß eher als ein Informationskonstrukt gedeutet werden. Daß jedoch die Interferenz mit 30,96\% Abweichung nicht so stark ausgefallen ist, liegt an der Neigung, auch synthetische bzw. analytisch-synthetische Formen (gemäß der Analogie von $\mathrm{ASD} \Rightarrow \mathrm{AD}$ zu $\mathrm{SD} \Rightarrow \mathrm{ND}$ ) in analytische umzuwandeln.

Faßt man obige Ausführungen zum Problem der zwischensprachlichen Interferenzen zu einem Resümee, so muß auf einen beachtlichen Unterschied im 
Grad der Abweichung bei den Diminutionsarten hingewiesen werden. Es fallt auf, daß auf der einen Seite die polnische analytische und die deutsche synthetische Bildeweise erheblich verzerrt, auf der anderen Seite das polnische synthetische und das deutsche analytische Verfahren geringfügiger in ihrem Gebrauch verändert werden. Diese Verteilung läßt eine Parallele zu den ausgangssprachlichen Frequenzverhältnissen erkennen: die im Ausgangstext schwach vertretenen Diminutionsarten (Polnisch: AD; Deutsch: SD) sind gleichzeitig die für eine Einwirkung am meisten anfalligen, die stark vertretenen dagegen (Polnisch: SD; Deutsch: AD) sind die, die sich im Modus der Übersetzung noch am ehesten durchsetzen können und am wenigsten einer assimilatorischen Überformung unterliegen. Gleichzeitig ist zu beobachten, daß die - der Kürze halber hier so zu nennenden - "starken" Verfahren im Zieltext nicht nur in Erwiderung ihrer formalen Entsprechung genutzt, sondern auch im Hinblick auf andere Muster tătig werden, während umgekehrt die sog. "schwachen" Verfahren durchgehend als Entgegnung ihres Pendant im Ausgangstext zu lokalisieren sind. Offensichtlich geht von der fremden Form des Originals eine prägende Signalkraft und Attraktion auf die Diminutionsarten aus, in denen sich die Zielsprache nicht selbstverständlich und eingeübt bewegen kann; der Möglichkeit, sie durch vertraute Bildungen zu übersetzen, wird nur bedingt entsprochen. Die enormen Zuwachsraten der polnischen analytischen und der deutschen synthetischen Diminution sind aus dieser quasi mechanischen Anpassung zu verstehen. Die überrepräsentierten Verfahren einer Sprache jedoch gleichen sich nicht so automatisch an die unterrepräsentierten im Quellentext an, sie finden immer noch in der Übersetzung anderer (Diminutiv-)Formen Gelegenheit, sich zum Einsatz zu bringen. 


\section{Resümee: Diminutive im Polnischen und Deutschen - "vergleichbar und unvergleichlich"}

Für den Versuch, Diminutive in beiden Sprachen in ihren Bildungs- und Verwendungsmöglichkeiten zu erfassen, hat sich die Methode des Übersetzungsvergleichs als fruchtbarer Forschungsansatz erwiesen. Seine Stärke liegt darin, den Charakter eines bestimmten Phänomens einer Sprache im Kontakt mit einer anderen Sprache, da, wo sie sich in gegebener Situation, angesichts einer konkreten Realisierung von Inhalten der Vorlage auf ihre eigensten sprachlichen Mittel besinnen muß, erkennen zu lassen. Anders als im system-bzw. verwendungsorientierten Sprachvergleich hat sich in dieser Studie immer wieder die "Feineinstellung" auf aktuelle Ereigniskonstellationen im Text ergeben und bewährt: sie zeigten sich als das allein mögliche tertium comparationis für die Entschlüsselung der in schematisierenden Konstruktionen nicht faßbaren jeweiligen Vielgestaltigkeit, des Reichtums sowie der Differenzierung und Andersartigkeit des Diminutivgebrauchs beider Sprachen.

Dabei hat es sich als aufschlußreich erwiesen, vorgefundene Übersetzungsangebote nicht nur konstatierend aufzunehmen, sondern sie auch einer Übersetzungskritik zu unterziehen. Denn erst in der Kritik, d.h. im Urteil über Gelingen und Versagen einer Wendung angesichts einer anderen, vom Quellentext vorgegebenen, kann das Verständnis für die sprachliche Erscheinung Diminutiv vertieft werden: die Erläuterung gescheiterter Übersetzungsversuche zeigte sich als genauso aussagekräftig wie die optimaler Übersetzungsoptionen.

Eine gängige These, die mit dem beschriebenen methodischen Rüstzeug überprüft wurde, statuiert für den Diminutiveinsatz der Vergleichssprachen die grundsätzliche und undifferenzierte Polarität von Reichtum und Armut. In dieser generalisierenden Form ließ sich die Gegenüberstellung nicht halten, denn sie gründet weitgehend nur auf dem formalen Kriterium des Vorhanden- bzw. Nichtvorhandenseins einer suffixalen Bildung. Führt man darüberhinaus jedoch den Gesichtspunkt der semantisch-pragmatischen Gewichtung in die Beurteilung ein, zeigen sich gravierende Unterschiede in der Wertigkeit von Diminutiv zu Diminutiv. Greift man auf die wichtigsten Beobachtungen zurück, so ist einerseits die umfangreiche Gruppe der polnischen Neutralisierungen abzusondern. Vornehmlich die Belege, denen ein deutsches Kompositum entgegengesetzt wurde, konnten demonstrieren, daß hier nicht eine stilistische Markierung, sondern vielmehr die Anzeige einer eigenen Gegenstandsklasse vorgenommen worden ist. An diesem Sprachausschnitt wird exemplarisch der typologisch- 
genetisch bedingte Unterschied in den Wortbildungsverfahren beider Sprachen offensichtlich. Während das Polnische den Aspekt des Größenunterschieds heranzieht (Derivate mit Diminutivsuffixen), verbalisiert das Deutsche die Funktion des neuen Objekts (Nominalkomposita).

Zum anderen zeigte sich in der verbleibenden Gruppe der aktuell-lebendigen Diminutive eine deutliche Unterscheidbarkeit zwischen den konventionalisiert genannten, innerhalb fester Kontexte schnell, unkompliziert und bequem handhabbaren, in ihrer Wirkung aber schablonisierten Formen und solchen, mit denen der Text seltenere, aber bewuBte, gezielte und damit individuelle Akzente setzt. Reduziert man das Phänomen des Diminutivs auf die Gegebenheiten der letztgenannten Bildungen und läßt Neutralisierungen und "leichtgewichtige" Konventionalisierungen dahinter zurückstehen, so rückt der polnische Diminutivgebrauch quantitativ in die Nähe des deutschen, dem zwar auch eine situativ-konventionalisierte Verwendung nicht unbekannt ist, dessen variierende Bildungen aber doch vornehmlich von einem nachdrücklichen, aktuell-legitimierten Wert geprägt sind.

Erscheinen somit die kontrastierten Sprachen im Lichte des Gewichtungsaspekts in einem quantitativ-statistischen Sinne vergleichbar, so sind sie es auch in einem qualitativ-äquivalenzbezogenen. Der im interlingualen Kontakt beherrschende Wiedergabetyp SD $\Rightarrow$ ND hat sich zwar als strukturelle, nicht aber als inhaltliche Opposition erwiesen; wegen der spezifischen unterschiedlichen Gewichtung des Diminutivs beider Sprachen konnte sich die zunächst auf gravierende Disproportion deutende Konfrontierung von markierter und unmarkierter Form als funktionell äquivalent herausstellen.

Doch im Vergleichbaren zeigt sich in einem auch das Unvergleichliche; ${ }^{46}$ die tarierende Sicht greift am Ende zu kurz und mit dem Erzielen funktioneller Äquivalenz ist zwar alles Mögliche getan, aber noch nicht alles Mögliche gesagt. Einer variierenden Bildung polnischer Texte - Neutralisierungen einmal ausgenommen - kann nämlich, wie es aus der Sicht der Zielsprache nahe liegen mag. nicht allein "Entbehrlichkeit", ein "Dreingabe-Charakter" und Redundanz nachgesagt werden, "ohne Zweifel (...) ist sie, von dem her gesehen, was gesagt werden möchte, durchaus notwendig" ${ }^{47}$ Richtet man sein Augenmerk nämlich in erster Linie auf die polnische Sprache, ohne sich von den Äquivalenzvorstellungen der zu kontrastierenden Sprachen beeinflussen zu lassen, so ist zunächst zu unterstellen, daß eine Wortform, die in der aktualisierten Rede leicht

\footnotetext{
“ Vgl. dazu GAUGER 1976. S.194.

${ }^{47}$ Zitate GAUGER 1971. S.124. 
verfügbar ist und auch häufig genutzt wird, für diese Sprache ein in bestimmter Weise signifikantes Instrument darstellt. Mag sie auch im Hinblick auf die Textfunktion für den deutschen Text zu vernachlässigen sein, so bildet sie doch die spezifische, individuelle Art und Weise von deren Verwirklichung im Polnischen. Diese Diminutive evozieren das der polnischen Sprache eigene Kolorit, sie suggerieren ein bestimmtes, letztlich unübersetzbares Assoziationsmilieu, in dem sich der polnische Sprecher/Leser selbstverständlich bewegt und das er auch vom Text erwartet. Zwar handelt es sich hier nicht um ein Gebot, im Range etwa eines grammatikalischen, sondern um Usancen, die wohl freier gehandhabt werden können, aber keineswegs ständig mißachtet werden. Darüberhinaus ist es aber die Form als Träger dieser Inhalte, die bei aller gültigen Orientierung an pragmatisch-stilistischen Aspekten nicht in Vergessenheit geraten sollte. Sie ist nicht einfach "variable Hülle unserer Gedanken", vielmehr "repräsentiert und fixiert [sie] Gehalte, Formen des Denkens, die in den Texten, einmal mehr und einmal weniger, Spuren oder Konturen markieren".$^{48}$ So ist die Suffixableitung im Polnischen eine solch zentrale Wortbildungskategorie, daß sie dem Text einen unverwechselbaren Charakter verleiht, auf dessen Vermittlung in einer sinn-, d.h. nicht formgemäßen Übersetzung notwendig verzichtet werden muß.

Diminutive stellen im Polnischen einen Bereich "besonders reiche[r] und feine[r] Wahlmöglichkeiten,(...) einen verwirrenden Überfluß an Ausdrucksmitteln ${ }^{\text {n99 }}$ dar, der auf einer bestimmten Mentalität der Zulassung von dem als "Programm Angelegten" beruht. Dieses Gepräge kann das Deutsche aufgrund seines eigenen. Charakters, der sich an dieser Stelle als Defizienz, an anderer aber als Stärke erweist, nicht nachvollziehen, es bedeutete andernfalls eine künstliche Überinterpretation, einen ungewollten Verfremdungseffekt. Man muß allerdings einschränkend hinzusetzen, daß, wie bei der Gegenüberstellung beider Übersetzungsvergleiche (PI. $\Rightarrow$ Dt. und Dt. $\Rightarrow \mathrm{PI}$.) gesehen, Beeinflussungen durch die Vorlage faktisch durchaus die Regel sind, daß darüberhinaus sie auch, wie es sich in der Analyse des Einzelfalls zeigte, bis zu einem gewissen Grade als Interferenz noch tragbar sind. Dies wiederum zeugt von der "erstaunliche[n] gegenseitige[n] Durchlässigkeit" der Sprachen, "ihre[r] instrumentale[n] Permeabilität", so die sowohl als Chance zur Bereicherung, aber auch als Gefahr der Befrachtung mit fremden, untypischen Ausdrucksformen gesehen werden muß.

\footnotetext{
“ NEUBERT 1988. S.51.

๑ WANDRUSZKA 1984. S.18.

so WANDRUSZKA 1973. S.12.
} 
Dies ändert nichts daran, daß polnische variierende Bildungen wie eine jede individuelle, nur einer Sprache eigene Gestaltung, in ihren ganzen Kopräsenzen und Bezugsfeldern unvergleichlich bleiben, mit dem scheinbaren Paradox, daß sie doch ständig verglichen werden können. Der übersetzungstheoretische Ansatz der funktionellen Äquivalenz schafft hier eine Vermittlung: Vergleichbar und zu übersetzen ist immer nur die interlinguale Konstante, die Situation, in deren Funktion die Sprachmittel stehen; unvergleichlich bleibt der jeweils einzigartige Zugang zu ihr. Anders formuliert: Der Übersetzungsvergleich zeigt, "daß keine Sprache immer alles sagen kann, daß aber auch keine Sprache immer alles sagen muß"; er führt die Sprachen "in ihrer ganzen Unzulänglichkeit", aber auch ihrer "unvergleichlichen Bildsamkeit und Beweglichkeit" vor. ${ }^{31}$

si Vgl. WANDRUSZKA 1971. S.136. 


\section{LITERATURVERZEICHNIS}

\section{Polnische Texte und ihre Übersetzungen}

FIN

FOR

IWA

KOR

LEM

MIL

NOW

NUR

RUM

Ida Fink: Skrawek czasu. Londyn 1987

Ida Fink: Eine Spanne Zeit. Erzählungen. Übers.v. Klaus Staemmler. Zürich 1983

Marcjanna Fornalska: Pamietnik matki. Warszawa 1960

Marcjanna Fornalska: Erinnerungen einer Mutter. Übers.v. Ludmilla und Kurt Kelm. Berlin 1982

Jarosław Iwaszkiewicz: "Panny z Wilka"; "Brzezina". J.I.: Dzieta. Opowiadania T.1. Warszawa 1958

Jarosław Iwaszkiewicz: "Die Fräulein von Wilko"; "Das. Birkenwäldchen". J.I.: Die Frăulein von Wilko. Drei Novellen. Übers.v. Klaus Staemmler. Frankfurt a.M. 1985

Janusz Korczak: Krobl Macius na wyspie bezludnej. Warszawa 1977

Janusz Korczak: Konig Hänschen auf der einsamen Insel. Übers. v. Katja Weintraub, überarb.v. Klaus Staemmler. München 5.Aufl. 1983

Maria Lemnis/Henryk Vitry: W staropolskiej kuchni i przy polskim stole. Warszawa 1986

Maria Lemnis/Henryk Vitry: Altpolnische Kache und polnische Tischsitten. Ubers.v. Dorota Matejak. Warszawa 1984

Czeslaw Milosz: Dolina Issy. (Dziela zbiorowe. T.5.) Paryż 1980 Czesław Milosz: Tal der Issa. Roman. Übers.v. Maryla Reifenberg. München 1983

Marek Nowakowski: Ten stary ztodziej. Opowiadania. Warszawa 1958

Marek Nowakowski: Die schrdgen Farsten. Ganovengeschichten. Übers.v. Rolf Fieguth. Berlin 1967

Maria Nurowska: Oferta. Słuchowisko (maschinenschriftl.) o.O. o.J.

Maria Nurowska: Der Wohnungstausch. Hörspiel (maschinenschriftl.). Übers.v. Peter Lachmann. o.O. o.J.

Witold Rumel: Oddychaj ponad czasem. Stuchowisko (maschinenschriftl.) 0.0 . $0 . J$.

Witold Rumel: Atme jenseits der Zeit. Hörspiel (maschinenschriftl.). Ubers.v. Peter Lachmann. 0.O. o.J. 
Michal Tonecki: Wasy pana dziedzica. Sluchowisko wedlug opowiadania Icyka Mangera (maschinenschriftl.) o.O. o.J.

Michal Tonecki: Der Schnurrbart des Herrn Gutsbesitzers. Hörspiel nach einer Erzählung von Itzak Manger (maschinenschriftl.). Ubers.v. Lieselotte Kaper. o.O. o.J.

\section{Deutsche Texte und ihre Übersetzungen}

END

FRA

FRI

GÖK

GRZ

HEY

RUN

TUC

Michael Ende: Momo oder Die seltsame Geschichte von den ZeitDieben und von dem Kind, das den Menschen die gestohlene Zeit zurackbrachte. Ein Märchen-Roman. Stuttgart 1973

Michael Ende: Momo czyli osobliwa historia o ztodziejach czasu i o dziecku, kaóre zwrocito ludziom skradziony czas. Przel. Teresa Jetkiewicz. Warszawa 1978

Das Tagebuch der Anne Frank. 12.Juni 1942 - 1.August 1944. Aus dem Holländischen übers.v. Anneliese Schütz. Frankfurt a.M. 1987

Dziennik Anny Frank. 12 czerwca 1942 - 1 sierpnia 1944. Przel. z j.niemieckiego Zofia Jaremko-Pytowska. Warszawa 1957

Max Frisch: Stiller. Roman. Frankfurt a.M. 1973

Max Frisch: Stiller. Przel. Jacek Frühling. Warszawa 1976

Roland Gööck: Selbermachen. Do it yourself. Gütersloh et al. 1970

Roland Gööck: Zrób to sam. Przel. Jan Guzera et al. Warszawa 1984

Bernhard Grzimek: Vierfußige Australier. Abenteuer mit Tieren und Menschen des 5.Kontinents. München 1966

Bernhard Grzimek: Z kamera przez Australie. Przel. Anna Czapik. Warszawa 1970

Richard Hey: Engelmacher \& Co. Roman. München 1985

Richard Hey: Fabrykant aniotkow $i$ spotka. Przel. Irena Naganowska. Warszawa 1979

Bottroper Protokolle. Aufgezeichnet von Erika Runge. Vorwort von Martin Walser. Frankfurt a.M. 1968

Erika Runge: Protokoty bottropskie. Przel. Zofia Rybicka. Poznań 1973

Kurt Tucholsky: Schloss Gripsholm. Eine Sommergeschichte. Hamburg 1988

Kurt Tucholsky: Zamek Gripsholm. Opowiesc wakacyjna. Przel. Zbigniew Fonferko. Warszawa 1985 
ADAMISZYN 1977 = Zbigniew Adamiszyn: " $Z$ badan nad zdrobnieniami $w$ malopolskiej pieśni ludowej". Zeszyty Naukowe Wyzszej Szkoty Pedagogicznej w Opolu. Seria A. Jezykoznawstwo. 6(1977) Opole. S.47-71

AGUD 1988 = Ana Agud: "Über Theorie und Praxis der Übersetzung". J.Albrecht/J.Lüdtke/H.Thun [Hgg.]: Energeia und Ergon. Sprachliche Variation - Sprachgeschichte - Sprachtypologie. Studia in honorem Eugenio Coseriu. 3.Bd.: Das sprachtheoretische Denken Eugenio Coserius in der Diskussion (2). Tübingen 1988 (= Tübinger Beiträge zur Linguistik 300). S.351-370

ALBRECHT 1973 = Jörn Albrecht: Linguistik und Übersetzung. Tübingen 1973 (= Romanistische Arbeitshefte 4)

ANDRJUSCHICHINA 1967 = Maria Andrjuschichina: "Bummelfritze-Bummelliese. Ein produktives Wortbildungsmodell der deutschen Gegenwartssprache". Sprachpflege. 16(1967) Leipzig. S.33-36

ANDRJUSCHICHINA 1968 = Maria Andrjuschichina: Vergleichende Untersuchung von Personenbezeichnungen im Russischen und Deutschen. Die Wiedergabe der suffixalen nomina agentis der russischen Sprache mit deutschen Sprachmitteln. Maschinenschriftl. Diss. Leipzig 1968

APEL 1983 = Friedmar Apel: Literarische Ubersetzung. Stuttgart 1983

APRESJAN 1963 = Ju.D.Apresjan: "Sovremennye metody izučenija značenij i nekotorye problemy strukturnoj lingvistiki". S.K.Saumjan [red.]: Problemy strukturnoj lingvistiki. Moskva 1963. S.102-150

BALCERZAN 1977 = Edward Balcerzan [red.]: Pisarze polscy o sztuce przekładu. 1440-1974. Antologia. Poznan 1977

BALCERZAN 1985 = Edward Balcerzan: "Przeklad literacki". Literatura polska. Przewodnik encyklopedyczny. T.2. Warszawa 1985. S.248

BALLY o.J. = Charles Bally: Traité de stylistique française. 2 vol. 2.ed. Heidelberg et al. o.J.

BALLY 1926 = Charles Bally: Le langage et la vie. Paris 1926

BALLY 1950 = Charles Bally: Linguistique générale et linguistique française. 3.éd. Berne 1950

BALLY 1966 = M.R.Mayenowa [red.]: Stylistyka Bally'ego. Wybór tekstów. Przel. U.Dambska-Prokop. Warszawa 1966

BARCHUDAROV 1975 = L.S.Barchudarov: Jazyk $i$ perevod. Voprosy obšej i Castnoj teorii perevoda. Moskva 1975

BARCHUDAROV $1977=$ L.S.Barchudarov: "Übersetzungstheorie als vergleichende Textlinguistik". O.Kade [Hg.]: Vermittelte Kommunikation, Sprachmittlung. Translation. Vorträge der Konferenz 'Ubersetzungstheorie und wiss. Grundlagen der Ausbildung von Sprachmittlern' am Moskauer Staatl. 
Pädag. Institut für Fremdsprachen vom 13.-16.5.1975. Leipzig 1977 (=Ubersetzungswissenschaftliche Beiträge 1). S.7-13

BARTMIŃSKI 1973 = Jerzy Bartmiński: $O$ jezyku folkloru. Wroclaw et al. 1973 (= Z dziejow form artystycznych w literaturze polskiej 32)

BASARA 1972 = Jan Basara: "Deminutywa formalne (na przykładzie gwar Slaska Cieszyńskiego w CSSR)". W.Doroszewski et al. [redd.]: Z polskich studiow slawistycznych. Seria 4. Jezykoznawstwo. Prace na VII Miedzynarodowy kongres slawistow w Warszawie 1973. Warszawa 1972. S.59-63

BAUSCH 1968 = K.-Richard Bausch: "Die Transposition. Versuch einer neuen Klassifikation". Linguistica Antverpiensia. 2(1968) Antwerpen. S.29-50

BELIĆ 1901/1904 = Alexander Belic: "Zur Entwicklungsgeschichte der slavischen Deminutiv- und Amplificativsuffixe". Archiv für slavische Philologie. 23(1901) Berlin. S.134-206; 26(1904). S.321-357

BENEŠ/VACHEK 1971 = E.Benes/J.Vachek [Hgg.]: Stilistik und Soziolinguistik. Beiträge der Prager Schule zur strukturellen Sprachbetrachtung und Spracherziehung. Übers.v. E.Beneš. Berlin 1971

BENNI et al. $1923=$ T.Benni/ J.Los/ K.Nitsch/ J.Rozwadowski/ H.Ulaszyn: Gramatyka jezyka polskiego. Krakow 1923

BICHEL 1973 = Ulf Bichel: Problem und Begriff der Umgangssprache in der germanistischen Forschung. Tübingen 1973 (= Hermae N.F.32)

BIEDERMANN 1981 = Johann Biedermann: Grammatiktheorie und grammatische Deskription in Rußland in der 2. Halfie des 18. und zu Anfang des 19.Jahrhunderts. Frankfurt a.M., Bern 1981

BIRKENMAJER 1924 = Józef Birkenmajer: "Oddrabnianie". Jezyk Polski. 9(1924) Krakow. S.143-146

BORST/MOTSCH 1986 = D.Borst/W.Motsch: "In welchem Maße sind Sprachen ineinander übersetzbar?". Zeitschrift für Sprachwissenschaft. 5(1986) Göttingen. S.167-186

BORYŚ 1974 = Wiesław Borys: "Deminutywa typu brzemiączki, ramiączki". Jezyk Polski. 54(1974) Kraków. S.119-124

BRACHIN 1977 = Pierre Brachin: "Le goût du diminutif". P.B.: La langue néerlandaise. Essai de présentation. Bruxelles 1977. S.81-84

BRANDSTETTER 1963/64 = Alois Brandstetter: "Semantische Studien zum Diminutiv im Mittelbairischen". Zeitschrifi für Mundartforschung. 30(1963/ 64) Wiesbaden. S.335-351

BRATUS $1969=$ Boms V.Bratus: The Formation and Expressive Use of Diminutives. Cambridge 1969 (= Studies in the Modern Russian Language 6)

BRINKMANN 1971 = Hennig Brinkmann: Die deutsche Sprache. Gestalt und Leistung. 2., neubearb. u. erw. Aufl. Düsseldorf 1971 
BRODOWSKA-HONOWSKA 1967 = Maria Brodowska-Honowska: Zarys klasyfikacji polskich derywatow. Wroclaw et al. 1967 (= Prace Komisji Jezykoznawstwa PAN 10)

BÜHLER 1934 = Karl Bühler: Sprachtheorie. Die Darstellungsfunktion der Sprache. Jena 1934

BURDZANOWSKA 1978 = Izabela M.Burdzanowska: "Tworzenie zdrobnien w jezyku polskim". Lubelskie Materiaty Neofilologiczne (1976). Lublin 1978. S.215-226

BUTTLER 1959 = Danuta Buttler: "Slownictwo Srodowiskowo-emocjonalne we wspólczesnej polszczyźnie" . Poradnik Jezykowy. (1959) Warszawa. S.68-78

BUTTLER 1982 = Danuta Buttler: "Miejsce jezyka potocznego wśród odmian wspołczesnej polszczyzny". S. Urbanczyk [red.]: Jezyk literacki $i$ jego warianfy. Wroclaw et al. 1982 (= Prace Komisji Slowianoznawstwa 43). S.17-28

BUTTLER et al. 1971 = D.Buttler/H.Kurkowska/H.Satkiewicz: Kultura jezyka polskiego. Zagadnienia poprawnosci gramatycznej. Warszawa 1971

BZDEGA 1976 = Andrzej Z.Bzdega: "Linguistische Übersetzungsanalyse". Kwartalnik Neofilologiczny. 23(1976) Warszawa, Poznan. S.283-293

BZDEGA 1986 = Andrzej Z. Bzdega: "Schwerpunkte und Perspektiven synchronen Sprachvergleichs". Kwartalnik Neofilologiczny. 33(1986) Warszawa, Poznañ. S.301-306

CATFORD 1965 = John C.Catford: A Linguistic Theory of Translation. An Essay in Applied Linguistics. London 1965

CHRISTENSEN 1976 = Gorm Christensen: "Das niederländische Diminutivsystem". Kopenhagener Beiträge zur germanistischen Linguistik. 7(1976) Kopenhagen. S.5-58

COSERIU $1970=$ Eugenio Coseriu: "Bedeutung und Bezeichnung im Lichte der strukturellen Semantik". P.Hartmann/H. Vernay [Hgg.]: Sprachwissenschaft und Ubersetzen. Symposion an der Univ. Heidelberg 24.2.-26.2.1969. München 1970 (= Commentationes Societatis Linguisticae Europaeae 3). S.104-121

COSERIU $1971 \mathrm{a}=$ Eugenio Coseriu: "System, Norm und 'Rede'" . E.C.: Sprache - Strukturen und Funktionen. 12 Aufsätze zur allgemeinen und romanischen Sprachwissenschaft. Hg.v. U.Petersen. 2.verb.Aufl. Tübingen 1971 (= Tübinger Beiträge zur Linguistik 2). S.53-72

COSERIU 1971b = Eugenio Coseriu: "Die lexematischen Strukturen". E.C.: Sprache - Strukturen und Funktionen. 12 Aufsätze zur allgemeinen und romanischen Sprachwissenschaft. Hg.v. U.Petersen. 2.verb. Aufl. Tübingen 1971 (=Tübinger Beiträge zur Linguistik 2). S.191-211

COSERIU 1973a = Eugenio Coseriu: Einfuhrung in die strukturelle Betrachtung des Wortschatzes. Hg.v. G.Narr. 2.Aufl. Tübingen 1973 (= Tübinger Beiträge zur Linguistik 14) 
COSERIU 1973b = Eugenio Coseriu: Probleme der strukturellen Semantik. Vorlesung gehalten im Wintersemester 1965/66 an der Universität Tübingen. Autor. und bearb. Nachschrift von D.Kastovsky. Tübingen 1973 (= Tübinger Beiträge zur Linguistik 40)

COSERIU 1978 = Eugenio Coseriu: "Falsche und richtige Fragestellungen in der Übersetzungstheorie" . L.Grähs/G.Korlén/B.Malmberg [Hgg.]: Theory and Practice of Translation. Nobel Symposium 39. Stockholm 6.-10.9.1976. Bern et al. 1978. S.17-32

COSERIU 1980 = Eugenio Coseriu: Textlinguistik. Eine Einführung. Hg.v. J.Albrecht. Tübingen 1980 (= Tübinger Beiträge zur Linguistik 109)

COSERIU 1981 = Eugenio Coseriu: "Kontrastive Linguistik und Übersetzung: ihr Verhältnis zueinander". W.Kühlwein/G.Thome/W.Wilss [Hgg.]: Kontrastive Linguistik und Übersetzungswissenschaft. Akten des Internationalen Kolloquiums Trier/Saarbrücken 25.-30.9.1978. München 1981. S.183-199

COSERIU 1988 = Eugenio Coseriu: Einfuhrung in die allgemeine Sprachwissenschafi. UUbers.v. M.Hübner. Tübingen 1988

CYRAN 1977 = Wladyslaw Cyran: Tendencje stowotworcze w gwarach polskich. Lodz 1977 (= Lodzkie Towarzystwo Naukowe. Wydzial II 78)

CZOCHRALSKI 1966 = Jan Czochralski: "Grundsätzliches zur Theorie der kontrastiven Grammatik". Linguistics. 24(1966) The Hague. S.17-28

DEUTSCHE WORTBILDUNG 1973-1984 = Deutsche Wortbildung. Typen und Tendenzen in der Gegenwartssprache. Eine Bestandsaufnahme des Instituts für deutsche Sprache. Forschungsstelle Innsbruck. 1.Bd.: I.Kühnhold/H. Wellmann: Das Verb. Düsseldorf 1973 (= Sprache der Gegenwart 29); 2.Bd.: H.Wellmann: Das Substantiv. Düsseldorf 1975 (= Sprache der Gegenwart 32); 3.Bd.: I.Kühnhold/O.Putzer/H.Wellmann: Das Adjektiv. Düsseldorf 1978 (= Sprache der Gegenwart 43); 4.Bd.: I.Kühnhold/H. P.Prell: Morphem- und Sachregister zu Bd. 1-3. Düsseldorf 1984 (= Sprache der Gegenwart 62)

DIECKMANN 1979 = Walther Dieckmann: "K.O.Erdmann und die Gebrauchsweisen des Ausdrucks 'Konnotationen' in der linguistischen Literatur" . Linguistische Arbeiten und Berichte. 13(1979) Berlin. S. 1-60

DLUSKA 1930 = Maria Dluska: "Przyczynek do zbierania polskich form hipokorystycznych". Jezyk Polski. 15(1930) Kraków. S.83-88

DOBRZYŃSKI $1974 / 1988$ = Walenty Dobrzyński: $Z$ badań nad rozwojem polskich deminutywów. Cz.1: Historyczny rozwoj rzeczownikow z formantem e na tle stowianiskim. Wroclaw 1974 (= Zeszyty Naukowe Wyższej Szkoły Pedagogicznej w Opolu. Seria B. Studia i Monografie 44); Cz.2: Apelatywne spieszczenia dezintegralne. Warszawa, Wroclaw 1988 (= Zeszyty Naukowe Wyższej Szkoły Pedagogicznej w Opolu. Seria B. Studia i Monografie 115)

DOBRZYŃSKI $1977=$ Walenty Dobrzyński: "Pochodniki dezintegralne w jezyku potocznym i w gwarach". Zeszyty Naukowe Wyzszej Szkoty Pedagogicznej w Opolu. Seria A. Jezykoznawstwo. 6(1977) Opole. S.33-46 
DOHERTY 1985 = Monika Doherty: "Wie begründet man eine Übersetzungsvariante?". Fremdsprachen. 29(1985) Leipzig. S.165-169

DOKULIL 1962 = Miloš Dokulil: Tvořent slov v cestinex. 1: Teorie odvozování slov. Praha 1962

DOKULIL 1964 = Milos Dokulil: "Zum wechselseitigen Verhältnis zwischen Wortbildung und Syntax". Travaux linguistiques de Prague. 1(1964) Prague. S.215-224

DOKULIL $1968=$ Milos Dokulil: "Zur Theorie der Wortbildung". Wissenschafiliche Zeitschrift der Karl-Marx-Universitat Leipzig. Gesellschafts- und sprachwissenschaftliche Reihe. 17(1968) Leipzig. S.203-211

DOKULIL 1979 = Milos Dokulil: Teoria derywacji. Przeł. A.Bluszcz, J.Stachowski. Wroclaw et al. 1979

DOROSZEWSKI 1928 = Witold Doroszewski: "Monografje słowotwórcze. Formacje $z$ podstawowem -k- w czesci sufiksalnej". Prace Filologiczne. 13(1928) Warszawa. S.1-261

DOROSZEWSKI 1957 = Witold Doroszewski: "O czasownikach deminutywnych". Poradnik Jezykowy. (1957) Warszawa. S.327-328

DOROSZEWSKI 1963 = Witold Doroszewski: Podstawy gramatyki polskiej. Cz.1. Wyd.2. Warszawa 1963

DRAŻYŃSKA 1974/75 = Maria Drażyńska: Diminutiv- und Augmentativbildungen im nominalen Bereich des Deutschen und Polnischen. Maschinenschrift. Diss. Poznań 1974/75

DRAŻYŃSKA-DEJA 1979 = Maria Drażyńska-Deja: "Strukturelle Konfrontation der Diminutivbildungen im nominalen Bereich des Deutschen und Polnischen". Studia Germanica Posnaniensia. 7(1979) Poznań. S.19-40

DUDEN-GRAMMATIK 1984 = G.Drosdowski [Hg.]: Duden-Grammatik der deutschen Gegenwartssprache. 4.neu bearb. und erw.Aufl. Mannheim et al. 1984 (= Duden 4)

EJDOWSKA 1972 = Krystyna Ejdowska: "Rzeczowniki zdrobniale rodzaju meskiego w jezyku polskim w porównaniu z rosyjskim". Lubelskie Materiaty Neofilologiczne. 1(1972) Lublin. S.157-172

ENCYKLOPEDIA 1978 = S.Urbańczyk [red.]: Encyklopedia wiedzy o jezyku polskim. Wroclaw et al. 1978

ERBEN 1980 = Johannes Erben: Deutsche Grammatik. Ein Abriß. 12.Aufl. München 1980

ERBEN 1983 = Johannes Erben: Einführung in die deutsche Wortbildungslehre. 2.durchges. und verm.Aufl. Berlin 1983 (= Grundlagen der Germanistik 17)

ERDMANN 1910 = Karl O.Erdmann: Die Bedeutung des Wortes. Aufsätze aus dem Grenzgebiet der Sprachpsychologie und Logik. 2.Aufl. Leipzig 1910 
ETTINGER 1974 = Stefan Ettinger: Diminutiv- und Augmentativbildung. Regeln und Restriktionen. Morphologische und semantische Probleme der Bistribution und der Restriktion bei der Substantivmodifikation im Italienischen, Portugiesischen, Spanischen und Rumänischen. Tübingen 1974 (= Tübinger Beiträge zur Linguistik 54)

ETTINGER 1980 = Stefan Ettinger: Form und Funktion in der Wortbildung. Die Diminutiv- und Augmentativmodifikation im Lateinischen, Deutschen und Romanischen (Portugiesisch, Spanisch, Italienisch und Rumänisch). Ein kritischer Forschungsbericht 1900-1975. 2.überarb. und erw. Aufl. Tübingen 1980 (= Tübinger Beiträge zur Linguistik 47)

ETTINGER 1981 = Stefan Ettinger: "Substantivmodifikation und Sprachgeographie". H.Geckeler et al. [Hgg.]: Logos semantikos. Studia linguistica in honorem Eugenio Coseriu 1921-1981. Vol.4: Grammatik. Berlin, New York 1981. S.29-41

ETTINGER 1984 = Stefan Ettinger: "Die Modifikation in der Lexikographie". D.Goetz/Th.Herbst [Hgg.]: Theoretische und praktische Probleme der Lexikographie. 1.Augsburger Kolloquium. München 1984. S.63-106

ETTINGER 1986 = Stefan Ettinger: Rez. zu "I.Polterauer: Die Deminutiva in der modernen nussischen Schriftsprache. Wien 1981". Zeitschrift für slavische Philologie. 45(1986) Heidelberg. S.438-444

ETTINGER 1988 = Stefan Ettinger: "Vom möglichen Nutzen der Übersetzung für die zweisprachige Lexikographie. Einige Anmerkungen zur Bedeutung und Bezeichnung anhand französischer Beispiele". J.Albrecht/J.Lüdtke/ H.Thun [Hgg.]: Energeia und Ergon. Sprachliche Variation - Sprachgeschichte - Sprachtypologie. Studia in honorem Eugenio Coseriu. 3.Bd.: Das sprachtheoretische Denken Eugenio Coserius in der Diskussion (2). Tübingen 1988 (= Tübinger Beiträge zur Linguistik 300). S.421-434

FEDOROV 1968 = Andrej V.Fedorov: Osnovy obšcej teorii perevoda. Izd.3-e, pererabot. i dop. Moskva 1968

FAULSEIT/KÜHN 1972 = D.Faulseit/G.Kühn: Stilistische Mittel und Moglichkeiten der deutschen Sprache. 5.überarb. Aufl. Leipzig 1972

FISCHER 1962 = Maja Fischer: Die Diminutive im Deutschen und im Franzosischen. Ein Vergleich von Gottfried Kellers Erzählungen 'Die Leute von Seldwyla' mit ihren französischen Übersetzungen. Diss. Zürich 1962

FISCHER 1963 = Maja Fischer: "Die Diminutive im Deutschen und im Französischen. Ein Vergleich von Gottfried Kellers Erzählungen 'Die Leute von Seldwyla' mit ihren französischen Ubersetzungen". Muttersprache. 73(1963) Berlin. S.129-138

FLEISCHER 1968 = Wolfgang Fleischer: Die deutschen Personennamen. Geschichte, Bildung und Bedeutung. 2.durchges. und erg.Aufl. Berlin 1968

FLEISCHER 1969 = Wolfgang Fleischer: "Stilistische Aspekte der Wortbildung". Deutsch als Fremdsprache. 6(1969) Leipzig. S.273-280

FLEISCHER 1974 = Wolfgang Fleischer: Wortbildung der deutschen Gegenwartssprache. 3.überarb. Aufl. Leipzig 1974 
FLEISCHER 1979 = "Kommunikativ-pragmatische Aspekte der Wortbildung". I.Rosengren [Hg.]: Sprache und Pragmatik. Lunder Symposium 1978. Lund 1979 (= Lunder grammatische Forschungen 48). S.317-329

FLEISCHER/MICHEL 1979 = W.Fleischer/G.Michel: Stilistik der deutschen Gegenwartssprache. 3.durchges. Aufl. Leipzig 1979

FÖRSTER 1982 = Uwe Förster: "Maskeraden im Gegenwartsdeutsch. Zur Stilfarbe im Sprachgebrauch". Muttersprache. 92(1982) Wiesbaden. S.316-336

FRIEDRICH 1916 = Johannes Friedrich: Deminutivbildungen mit nicht deminutiver Bedeutung (besonders im Griechischen und Lateinischen). Diss. Leipzig 1916

FURDAL 1973 = Antoni Furdal: Klasyfikacja odmian wspótczesnego jezyka polskiego. Wroclaw 1973

GAERTNER 1938 = Henryk Gaertner: Gramatyka wspotczesnego jezyka polskiego. Glosownia - semantyka - slowotworstwo. Lwow, Warszawa 1938

GARTNER 1898 = Theodor Gartner: "Die Nachsilben -chen und -lein". Wissenschafiliche Beihefie zur Zeitschrift des allgemeinen deutschen Sprachvereins. 14/15(1898) Berlin. S.167-176

GAUGER 1968 = Hans-Martin Gauger: "Determinatum und Determinans im abgeleiteten Wort?" H.E.Brekle/L.Lipka [Hgg.]: Wortbildung, Syntax und Morphologie. Festschrift zum 60.Geburtstag von Hans Marchand am 1.10. 1967. The Hague, Paris 1968. S.93-108

GAUGER 1971 = Hans-Martin Gauger: Durchsichtige Worter. Zur Theorie der Wortbildung. Heidelberg 1971

GAUGER 1976 = Hans-Martin Gauger: Sprachbewußtsein und Sprachwissenschäf. München 1976

GAWROŃSKI 1928 = Andrzej Gawronski: "Wartosć uczuciowa deminutywow". A.G.: Szkice jezykoznawcze. Warszawa et al. 1928. S.199-217

GEORGES 1951 = Karl E.Georges: Ausfuhrliches lateinisch-deutsches Handworterbuch. Bd.1. 9.Aufl. Basel 1951

GERSBACH/GRAF 1984/1985 = B.Gersbach/R.Graf: Wortbildung in gesprochener Sprache. Die Substantiv-, Verb- und Adjektiv-Zusammensetzungen und -Ableitungen im 'Häufigkeitswörterbuch gesprochener Sprache'. Bd.1. Tübingen 1984 (= Idiomatica 12); Bd.2. Tübingen 1985 (= Idiomatica 13)

GLADROW 1986 = Wolfgang Gladrow: "Probleme der Äguivalenz in der konfrontativen Linguistik". Kwartalnik Neofilologiczny. 33(1986) Warszawa, Poznan. S.325-332

GLADYSZ 1968 = Maria Gladysz: "Zdrabnianie imion osobowych we wspótczesnej polszczyźnie". Jezykoznawca. 18/19(1968) Lublin. S.79-85

GOOCH 1967 = Anthony Gooch: Diminutive, Augmentative and Pejorative Suffixes in Modern Spanish. A Guide to their Use and Meaning. Oxford et al. 1967 
GRABIAS 1978 = Stanisław Grabias: "Derywacja a ekspresja". T.Skubalanka [red.]: Studia nad sktadnia polszczyzny mówionej. Ksiega referatow konferencji poswieconej skladni i metodologii badań jezyka mówionego. Lublin 6.-9.10.1975. Wroclaw et al. 1978. S.89-102

GRABIAS 1980 = Stanisław Grabias: "Ekspresywnosć w strukturze znaczeniowej wypowiedzi, wyrazów i formantów". Poradnik Jezykowy. (1980) Warszawa. S.476-488

GRABIAS 1981 = Stanislaw Grabias: $O$ ekspresywnosci jezyka. Ekspresja a slowotwórstwo. Lublin 1981

GRAMATYKA 1984 = R.Grzegorczykowa/R.Laskowski/H.Wróbel [redd.]: Gramatyka wspótczesnego jezyka polskiego. Morfologia. Warszawa 1984

GREULE 1983/84 = Albrecht Greule: "Abi, Krimi, Sponti. Substantive auf -i im heutigen Deutsch". Muttersprache. 94(1983/84) Wiesbaden. S.207-217

GRIMM 1826/1831 = Jacob Grimm: Deutsche Grammatik. T.2. Drittes Buch: Von der Wortbildung. Göttingen 1826; T.3. Drittes Buch: Von der Wortbildung. Göttingen 1831

GROCHOWSKA 1975 = Alina Grochowska: "Przymiotniki z sufiksem -awy we wspólczesnym jezyku polskim". Jezyk Polski. 55(1975) Kraków. S.333-342

GRUCZA 1974 = Barbara Grucza: Die Konfrontation der substantivischen Ableitungen im Polnischen und Deutschen. Maschinenschriftl. Diss. Warszawa 1974

GRUITĂ 1984 = Mariana Gruił̧a: "Address in American English: Diminutives and Nicknames". Studia Universitatis Babes-Bolyai. Philologia. 29(1984) Cluj. S.47-51

GRZEGORCZYKOWA 1962 = Renata Majewska-Grzegorczykowa: "Ksztaltowanie sie funkcji znaczeniowych sufiksu -ina". Biuletyn Polskiego Towarzystwa Jezykoznawczego. 20(1961). Wroclaw et al. 1962. S.163-173

GRZEGORCZYKOWA 1971 = Renata Grzegorczykowa: Rez. zu "B.Kreja: Slowotworstwo rzeczowników ekspresywnych w jezyku polskim. Gdansk 1969". Poradnik Jezykowy. (1971) Warszawa. S.123-125

GRZEGORCZYKOWA 1978 = Renata Grzegorczykowa: "Struktura semantyczna wyrażeń ekspresywnych". M.Szymczak [red.]: Z zagadnien stownictwa wspótczesnego jezyka polskiego. Wroclaw et al. 1978 (= Prace Jezykoznawcze PAN 91). S. (17-123

GRZEGORCZYKOWA 1984 = Renata Grzegorczykowa: Zarys stowotwórstwa polskiego. Słowotwórstwo opisowe. Wyd.6. Warszawa 1984

GRZEGORCZYKOWA/PUZYNINA $1979=$ R.Grzegorczykowa/J.Puzynina: Stowotworstwo wspotczesnego jezyka polskiego. Rzeczowniki sufiksalne rodzime. Warszawa 1979

GÜRTLER 1909a = Hans Gürtler: Das Diminutivsuffix -chen im Frähneuhochdeutschen. Düsseldorf 1909 
GÜRTLER 1909b = Hans Gürtler: "Materialien zur Geschichte der Diminutiva auf -chen im Frühneuhochdeutschen". Zeitschrifi für deutsche Wortforschung. 11(1909) Straßburg. S.181-210

HARTMANN 1971 = Peter Hartmann: "Texte als linguistisches Objekt". W.-D.Stempel [Hg.]: Beiträge zur Textlinguistik. München 1971 (= Internationale Bibliothek für allgemeine Linguistik 1). S.9-29

HASSELROT 1957 = Bengt Hasselrot: Etudes sur la formation diminutive dans les langues romanes. Uppsala, Wiesbaden $1957(=$ Uppsala Universitets Arsskrift 11)

HASSELROT 1972 = Bengt Hasselrot: Etude sur la vitalité de la formation diminutive française au XXe siecle. Uppsala 1972 (= Acta Universitatis Upsaliensis. Studia Romanica Upsalensia 8)

HASSELROT 1976 = Bengt Hasselrot: "Quelques nouveaux diminutifs véritables". G.Colón/R.Kopp [edd.]: Mélanges de langues et de littératures romanes offerts d Carl Theodor Gossen. Vol.1. Bern, Liège 1976. S.317323

HASTENPFLUG 1914 = Fritz Hastenpflug: Das Diminutiv in der deutschen Originalliteratur des 12. und 13.Jahrhunderts. Diss. Marburg 1914

HELBIG 1986 = Gerhard Helbig: "Bemerkungen zu Zielen, Möglichkeiten und Grenzen des konfrontativen (kontrastiven) Sprachvergleichs". Kwartalnik Neofilologiczny. 33(1986) Warszawa, Poznan. S.271-289

HELTBERG 1964 = Kristine Heltberg: "O deminutywach i augmentatywach". Prace Filologiczne. 18(1964) Warszawa. S.93-102

HENSCHELMANN 1979 = Käthe Henschelmann: "Texttypologie und Übersetzen". W.Mair/E.Sallager [Hgg.]: Sprachtheorie und Sprachenpraxis. Festschrift für Henri Vernay zu seinem 60. Geburtstag. Tübingen 1979 (= Tübinger Beiträge zur Linguistik 112). S.53-70

HENZEN 1957 = Walter Henzen: Deutsche Wortbildung. 2.verb.Aufl. Tübingen 1957 (= Sammlung kurzer Grammatiken germanischer Dialekte. B.Ergänzungsreihe 5)

HERBERG $1979=$ Dieter Herberg: "Wortbildung und Expressivität". Sprachpflege. 28(1979) Leipzig. S.205-208

HÖHLE 1982 = Tilman N.Höhle: "Über Komposition und Derivation. Zur Konstituentenstruktur von Wortbildungsprodukten im Deutschen". Zeitschrift für Sprachwissenschaft. 1(1982) Göttingen. S.76-112

HÖSSELBARTH 1986 = Lutz Hößelbarth: "Zum Aspekt der Orientiertheit sprachvergleichender Untersuchungen". Kwartalnik Neofilologiczny. 33 (1986) Warszawa, Poznań. S.347-352

HOFMANN 1961 = Dietrich Hofmann: Die k-Diminutiva im Nordfriesischen und in verwandten Sprachen. Köln, Graz 1961 (= Niederdeutsche Studien 7) 
HOLZ-MÄNTTÄRI 1984 = Justa Holz-Mänttäri: Translatorisches Handeln. Theorie und Methode. Helsinki 1984

HOUSE 1973 = Juliane House: "Of the Limits of Translatability". Babel 19 (1973) Bonn. S.166-167

HOUSE 1977 = Juliane House: A Model for Translation Quality Assessment. Tübingen 1977 (= Tübinger Beiträge zur Linguistik 88)

INGARDEN 1955 = Roman Ingarden: "O thumaczeniach". M.Rusinek [red.]: $O$ szuce thumaczenia. Wroclaw 1955. S.127-190

IPPOLDT 1958 = Juliusz Ippoldt: "Rzeczowniki i przymiotniki zdrobniale w jezykach polskim i niemieckim". Jezyki Obce w Szkole. 2(1958) Warszawa. S.95-96

IWANOWA-PERCZYŃSKA 1978 = Nina Iwanowa-Perczyńska: "Wspólczesne imiona polskie oraz ich zdrobnienia $w$ porównaniu $z$ rosyjskimin". J.Wawrzyńczyk [red.]: Polsko-rosyjskie jezykoznawstwo konfrontatywne. Wybór materialów. Lódź 1978. S.22-25

JADACKA 1978 = Hanna Jadacka: "O interpretacji derywatów odprzymiotnikowych z sufiksem -awy". Poradnik Jezykowy. (1978) Warszawa. S.146-159

JÄGER 1968 = Gert Jäger: "Übersetzungswissenschaft und vergleichende Sprachwissenschaft" . R.Rủžicka [Hg.]: Probleme der strukturellen Grammatik und Semantik. Leipzig 1968. S.209-222

JÄGER 1972 = Gert Jäger: "Konfrontation und Translation". Deutsch als Fremdsprache. 9(1972) Leipzig. S.233-244

JÄGER 1975 = Gert Jäger: Translation und Translationslinguistik. Halle a.S. 1975

JÄGER 1980 = Gert Jäger: "Hypokoristika und Translation". Studia Onomastica. 1(1980) Leipzig (= Namenkundliche Informationen 26. Beiheft 2). S.60-68

JÄGER 1983 = Gert Jäger: "Theorie der sprachlichen Bedeutungen und Translation". G.Jäger/A.Neubert [Hgg.]: Semantik und Übersetzungswissenschaft. Materialien der 3.Internationalen Konferenz 'Grundfragen der Úbersetzungswissenschaft' . Leipzig 1983 ( = Ubersetzungswissenschaftliche Beiträge 6). \$.53-61

JÄGER 1986 = Gert Jäger: "Die sprachlichen Bedeutungen - das zentrale Problem bei der Translation und ihrer wissenschaftlichen Beschreibung". G.Jäger/A.Neubert [Hgg.]: Bedeutung und Translation. Leipzig 1986 (= Übersetzungswissenschaftliche Beiträge 9). S.5-66

JÄGER/MÜLLER 1982 = G.Jäger/D.Müller: "Kommunikațive und maximale Aquivalenz von Texten". G.Jäger/A.Neubert [Hgg.]: Aquivalenz bei der Translation. Leipzig 1982 (= Ubersetzungswissenschafuliche Beiträge 5). S.42-57

JAKOBSON 1960 = Roman Jakobson: "Linguistics and Poetics". Th.A.Sebeok [ed.]: Style in Language. New York et al. 1960. S.350-377 
JAKOBSON 1971 = Roman Jakobson: "On Linguistic Aspects of Translation". R.J.: Selected Writings. Vol.2: Word and Language. The Hague, Paris 1971. S.260-266

JANUS 1981 = Elżbieta Janus: Wyktadniki intensywnosci cechy (na materiale polskim $i$ rosyjskim). Wroclaw et al. 1981

JANUS 1986 = Elżbieta Janus: "Z zagadnień ekspresywów przymiotnikowych (na materiale polskim i rosyjskim)". T.Dobrzynska [red.]: Teoria tekstu. Zbiór studiów. Wroclaw et al. 1986. S.99-116

JARNATOVSKAJA 1984 = Valeria E.Jarnatovskaja: "Zu stilistischen Möglichkeiten der Morphologie in der deutschen Gegenwartssprache". Zeitschrifi fü Phonetik, Sprachwissenschafi und Kommunikationsforschung. 37(1984) Berlin. S.235-241

JEDLIČKA 1981 = Alois Jedliðka: "Probleme der Sprachsituation aus vergleichender Sicht" . Zeitschrift für Slawistik. 26(1981) Berlin. S.108-116

JEDLIČKA 1988 = Alois Jedliðka: "Wege zur Erarbeitung einer Systematik der Stile und Stilerscheinungen". Linguistische Studien. Reihe A. 186(1988) Berlin. S.9-26

JURKOWSKI 1978 = Marian Jurkowski: "Granice przekladu" . Przeglod Humanistyczny. 22(1978) Warszawa. S.81-86

KACZMAREK 1936 = Leon Kaczmarek: "Sposoby spieszczen w wielkopolskiej pieśni ludowej". Jezyk Polski. 21(1936) Kraków. S.141-147

KADE 1968 = Otto Kade: Zufall und Gesetzmaßigkeit in der Ubersetzung. Leipzig 1968 (= Beihefte zur Zeitschrift 'Fremdsprachen' 1)

KADE 1980 = Otto Kade: Die Sprachmittlung als gesellschaftliche Erscheinung und Gegenstand wissenschaftlicher Untersuchung. Leipzig 1980 (= Übersetzungswissenschaftliche Beiträge 3)

KANN 1973 = Hans-Joachim Kann: "Spielfreude in der Sprache: Super- und Mini-". Muttersprache. 83(1973) Wiesbaden. S.198-210

KARPLUK 1968 = Maria Karplukówna: "Ze slowotwórstwa rzeczowników polskich. Przyrostki -yszek, -yszka, -yszko". Symbolae philologicae in honorem Vitoldi Taszycki. Wroclaw et al. 1968 (= Prace Komisji Jezykoznawstwa PAN 17). S.147-154

KARPLUK 1982 = Maria Karpluk: "Ze slowotwórstwa rzeczowników polskich. Przyrostek -aszek". Zeszyty Naukowe Wydziatu Humanistycznego Uniwersytetu Gdaniskiego. Filologia Polska. Prace Jezykoznawcze. 8(1982) Gdansk. S.251-258

KEIPERT 1977 = Helmut Keipert: Die Adjektive auf -tebms. Studien zu einem kirchenslavischen Wortbildungstyp. T.1. Wiesbaden $1977(=$ Veröffentlichungen der Abteilung für Slavische Sprachen und Literaturen des Osteuropa-Instituts (Slavisches Seminar) an der Freien Universität Berlin 45)

KEIPERT 1981 = Helmut Keipert: "M.V. Lomonosov als Übersetzungstheoretiker". Wiener slavistisches Jahrbuch. 27(1981) Wien. S.27-48 
KEIPERT $1987=$ Helmut Keipert: "Kirchenslavisch und Latein. Über die Vergleichbarkeit zweier mittelalterlicher Kultursprachen". G.Birkfellner [Hg.]: Sprache und Literatur Altrußlands. Aufsatzsammlung. Münster 1987 (= Studia slavica et baltica 8). S.81-109

KEIPERT 1989a = Helmut Keipert: "Griechisch-slavische Übersetzungsprobleme bei einem fachsprachlichen Adjektivtyp. (Die Adjektive auf -Tıxó 'Bogoslovie' Johanns des Exarchen)". P.Dinekov et al. [redd.]: Studia slavico-byzantina et mediaevalia europensia. In memoriam Ivan Duj飞ev. Vol.1. Sofija 1989. S.201-216

KEIPERT 1989b = Helmut Keipert: "Dię Qual der Wahl. Die griechischen Verbaladjektive auf -TÉos und ihre Ubersetzungsäquivalente im älteren Kirchenslavisch". P.Dinekov et al.[redd.]: Vtori mezdunaroden kongres po bs lgaristika. Sofija, 23 maj - 3 juni 1986g. Dokladi (21). Kirilo- metodievistika simpozium. Sofija 1989. S.451-461

KLEMENSIEWICZ 1955 = Zenon Klemensiewicz: "Przeklad jako zagadnienie jezykoznawstwa". M.Rusinek [red.]: O sztuce ttumaczenia. Wroclaw 1955. S.85-97

KLIMASZEWSKA 1979 = Zofia Klimaszewska: Diminutive und augmentative Ausdrucksmoglichkeiten des Niederlandischen, Deutschen und Polnischen. Maschinenschriftl. Diss. Warszawa 1979

KLIMASZEWSKA 1983 = Zofia Klimaszewska: Diminutive und augmentative Ausdrucksmoglichkeiten des Niederlädischen, Deutschen und Polnischen. Eine konfrontative Darstellung. Wroclaw et al. 1983

KLOBUS 1985 = Alina Klobus: "Deminutywa, augmentatywa i ekspresywa w polszczyźnie mówionej mieszkańców Sieradza i okolicy . Rozprawy Komisji Jezykowej Lodzkiego Towarzystwa Naukowego. 31(1985) Wroclaw et al. S.81-95

KLOEPFER 1967 = Rolf Kloepfer: Die Theorie der literarischen Ubersetzung. Romanisch-deutscher Sprachbereich. München 1967 (= Freiburger Schriften zur romanischen Philologie 12)

KLUGE 1913 = Friedrich Kluge: $A b r i \beta$ der deutschen Wortbildungslehre. Halle a.S. 1913

KÖNIGS 1979 = Frank G.Königs: Übersetzung in Theorie und Praxis. Ansatzpunkte für die Konzeption einer Didaktik der Übersetzung. Bochum 1979 (= Manuskripte zur Sprachlehrforschung 14)

KÖNIGS 1986 = Frank G.Königs: "Übersetzungswissenschaftliche Forschung in Deutschland. Tendenzen und Perspektiven". Kwartalnik Neofilologiczny. 33(1986) Warszawa, Poznań. S.3-23

KOLLER 1978 = Werner Koller: "Äquivalenz in kontrastiver Linguistik und Ubersetzungswissenschaft". L.Grähs/G.Korlén/B.Malmberg [edd.]: Theory and Practice of Translation. Nobel Symposium 39. Stockholm, 6.-10.9. 1976. Bern et al. 1978. S.69-92

KOLLER 1981 = Werner Koller: "Textgattungen und Übersetzungsäquivalenz". W.Kühlwein/G.Thome/W.Wilss [Hgg.]: Kontrastive Linguistik und Ubber- 
setzungswissenschaft. Akten des Internationalen Kolloquiums Trier/Saarbrücken 25.-30.9.1978. München 1981. S.272-279

KOLLER 1987 = Werner Koller: Einfahrung in die Ubersetzungswissenschaft. 3.Aufl. Heidelberg, Wiesbaden 1987

KOMISSAROV $1973=$ V.N.Komissarov: Slovo o perevode. Moskva 1973

KOMISSAROV $1977=$ V.N.Komissarov: "Zur Theorie der linguistischen Ubersetzungsanalyse". O.Kade [Hg.]: Vermittelte Kommunikation, Sprachmittlung, Iranslation. Vorträge der Konferenz Ubersetzungstheorie und wiss. Grundlagen der Ausbildung von Sprachmittlern am Moskauer Staatl.Pädag. Institut für Fremdsprachen vom 13.-16.5.1975. Leipzig 1977 (= Ubersetzungswissenschaftliche Beiträge 1). S.44-51

KOMISSAROV $1980=$ V.N.Komissarov: Lingvistika perevoda. Moskva 1980

KOPEĆ 1966 = Jerzy Kopec: "Z problematyki deminutywów $w$ poezji dla dzieci". M.Adamus et al. [redd.]: Studia jezykoznawcze poswiecone profesorowi dokaorowi Stanistawowi Rospondowi. Wroclaw 1966. S.183-185

KOSTA 1986 = Peter Kosta: Probleme der Svejk-Übersetzungen in den westund sadslavischen Sprachen. Linguistische Studien zur Translation literarischer Texte. München 1986 (= Specimina Philologiae Slavicae. Suppl.Bd. 13)

KOSYL 1981 = Czeslaw Kosyl: "Slowotwórstwo i funkcjonowanie imion osobowych we współczesnej polszczyźnie". Acta Universitatis Lodziensis. Folia linguistica. 2 (1981) Lódź. S.67-78

KOWALIK 1977 = Krystyna Kowalik: Budowa morfologiczna przymiotników polskich. Wroclaw et al. 1977 (= Prace Instytutu Jezyka Polskiego 15)

KRAJCARZ 1981 a = Mariusz Krajcarz: "Zur Wiedergabe der niederländischen Diminutive im Polnischen". Institut voor Toegepaste Linguistik. 53(1981) Louvain. S.63-80

KRAJCARZ 1981b = Mariusz Krajcarz: "Die stilistischen Funktionen der niederländischen Diminutive". Roczniki Humanistyczne. Jezykoznawstwo. 29(1981) Lublin. S.65-74

KREJA 1957 = Boguslaw Kreja: "Geneza funkcji przymiotnikowego sufiksu -awy (typ bialawy, gorzkawy)". Jezyk Polski. 37(1957) Krakow. \$.351-357

KREJA 1963 = Boguslaw Kreja: "Pojecie derywacji wymiennej". Z polskich studiów slawistycznych. Seria 2. Jezykoznawstwo. Prace na V Miedzynarodowy kongres slawistów w Sofii. Warszawa 1963. S.133-140

KREJA 1969 = Boguslaw Kreja: Stowotworstwo rzeczowników ekspresywnych w jezyku polskim. Formacje na -ik, -k-, -isko i -ina. Gdańsk 1969 (= Gdańskie Towarzystwo Naukowe. Wydzial I 32)

KREJA 1970 = Boguslaw Kreja: "O tzw. derywacji wstecznej odprzymiotnikowej we wspótczesnym jezyku polskim". Studia z Filologii Polskiej $i$ Stowianskiej. 9(1970) Warszawa. S.61-94 
KREJA 1972 = Bogusław Kreja: "O syngulatywach na -inka typu śnieżynka we wspołczesnym jezyku polskim". Poradnik Jezykowy. (1972) Warszawa. S.61-66

KREJA 1974 = Boguslaw Kreja: "Nowe formy jezykowe jako skutek zmiany opozycji". Jezyk Polski. 54(1974) Kraków. S.171-184

KREJA 1976 = Boguslaw Kreja: "O pewnych typach derywacji słowotworczej". Biuletyn Polskiego Towarzystwa Jezykoznawczego. 34(1976) Wroclaw et al. S.41-53

KREJA 1978 = Bogusław Kreja: "Czasowniki zdrobniale na -k- w dialektach polskich oraz w innych jezykach slowianskich". M.Basaj et al. [redd.]: Z polskich studiow slawistycznych. Seria 5. Jezykoznawstwo. Prace na VIII Miedzynarodowy kongres slawistów w Zagrzebiu 1978. Warszawa 1978. S.61-71

KREJA 1986 = Bogusław Kreja: "Zagadnienia struktury funkcyjnej i formalnej niektórych derywatów na -ka". F. Sławski et al. [redd.]: Collectanea linguistica in honorem Adami Heinz. Wroclaw et al. 1986 (= Prace Komisji Jezykoznawstwa PAN 53). S.69-73

KRUISINGA 1942 = E.Kruisinga: Diminutieve en affektieve suffixen in de Germaanse talen. Amsterdam 1942 (= Mededeelingen d. Nederl. Ak. van Wetenschappen. Afd. Letterkunde. N.R. 5.9)

KÜPPER 1970 = Heinz Küpper: Worterbuch der deutschen Umgangssprache. Bd.6: Jugenddeutsch von A-Z. Hamburg, Düsseldorf 1970

KURKO 1975 = P.F.Kurko: "Smyslova struktura slova i semantyěna klasyfikacyja diminutyviv". Inozemna filolohija - Inostrannaja filologija. 38(1975) L'viv. S.64-69

KURKOWSKA 1954 = Halina Kurkowska: Budowa stowotworcza przymiotnikow polskich. Wroclaw 1954 (= Prace Jezykoznawcze PAN 1)

KURKOWSKA/SKORUPKA $1959=$ H.Kurkowska/S.Skorupka: Stylistyka polska. Zarys. Warszawa 1959

KURYLOWICZ 1956 = Jerzy Kuryłowicz: "La position linguistique du nom propre". Onomastica. 2(1956) Wroclaw. S.1-14

KURYLOWICZ 1968 = Jerzy Kurylowicz: "O niektórych wlaściwosciach imion skróconych". Symbolae philologicae in honorem Vitoldi Taszycki. Wroclaw et al. 1968 (= Prace Komisji Jezykoznawstwa PAN 17). S. 176-181

KURZOWA 1970a = Zofia Kurzowa: Polskie rzeczowniki meskie na -o na tle stowianskim. Wroclaw et al. 1970 (= Prace Komisji Jezykoznawstwa PAN 26)

KURZOWA 1970b = Zofia Kurzowa: "Meskie imiona zdrobniale i spieszczone w piesniach ludu polskiego (na podstawie 'Dziel wszystkich' Oskara Kolberga)". Onomastica. 15(1970) Wroclaw. S.234-281 
LALEWICZ 1973 = Janusz Lalewicz: "Krytyka teorii funkcji mowy BühleraJakobsona". Teksty. Teoria literatury. Krytyka. Interpretacja. 6(1973) Warszawa. S.16-33

LASKOWSKI 1966 = Roman Laskowski: Derywacja rzeczowników $w$ dialektach laskich. Cz.1: Abstracta, collectiva, deminutiva, augmentativa. Wroclaw et al. 1966 (= Prace Komisji Jezykoznawstwa PAN 11)

LAUSBERG 1973 = Heinrich Lausberg: Handbuch der literarischen Rhetorik. Eine Grundlegung der Literaturwissenschaft. 2 Bde. 2.verm.Aufl. München 1973

LEBIEDZIŃSKI 1981 = Henryk Lebiedziński: Elementy przekladoznawstwa ogolnego. Warszawa 1981

LETTENBAUER 1933 = Wilhelm Lettenbauer: Das Deminutivum im Russischen. Diss. München 1933

LEVÝ 1969 = Jił́ Levý: Die literarische Ubersetzung. Theorie einer Kunstgattung. Übers.v. W.Schamschula. Frankfurt a.M., Bonn 1969

LEWANDOWSKI 1979 = Theodor Lewandowski: Linguistisches Worterbuch. 3 Bde. 3.durchges. und erw.Aufl. Heidelberg 1979

LEYS $1970=$ O.Leys: "Einige allgemeine Betrachtungen zum Diminutiv- und Augmentativsystem in einer westfallischen Mundart". Orbis. 19(1970) Louvain. S.28-35

LIPKA 1981 = Leonhard Lipka: "Zur Lexikalisierung im Deutschen und Englischen". L.Lipka/H.Günther [Hgg.]: Wortbildung. Darmstadt 1981 (= Wege der Forschung 564). S.119-132

LOCKWOOD 1974/75 = W.B.Lockwood: "Thoughts on the Diminutive". German Life and Letters. N.S. 28(1974/75) Oxford. S.307-314

LUBAŚ 1979 = Wladyslaw Lubas: Spoleczne uwarunkowania wspótczesnej polszczyzny. Szkice socjolingwistyczne. Kraków 1979

LUDWIG 1974 = Klaus-Dieter Ludwig: "Zu einigen Fragen des Verhältnisses von Sprache, Wertung und Emotion". Sprachpflege. 23(1974) Leipzig. S.5154

MACZYŃSKI 1982 = Maciej Maczyński: "Derywacja czasowników ekspresywnych i onomatopeicznych. Zarys problematyki". Jezyk Polski. 62(1982) Kraków. S.255-259

MACZYŃSKI 1986 = Maciej Maczyński: "O tzw. deminutywach formalnych". Prace Filologiczne. 33(1986) Warszawa. S.279-284

MAŃCZAK 1980 = W.Mańczak: "Ze zjawisk współczesnego jezyka. Moda na zdrobnienia." Jezyk Polski. 60(1980) Kraków. S.69-71

MARKEY 1983 = Thomas L.Markey: "Die grammatikalische Multifunktionalität. L-Deminutiv im Bairischen". Annali Istituto Universitario Orientale. Filologia germanica. 26(1983) Napoli. S.53-66 
MASLOWSKA 1986 = Ewa Maslowska: "Problemy derywacji semantycznej rzeczowników ekspresywnych". Poradnik Jezykowy. (1986) Warszawa. S.479-485

MATERIALY 1958-1983 = W.Doroszewski/J.Puzynina [redd.]: Materiaty do bibliografii stowotworstwa jezyków stowianiskich. Z.1-6 (1874-1981). Warszawa 1958-1983

MATUSCHEK 1977 = Herbert Matuschek: Einwortexeme und Wortgruppenlexeme in der technischen Terminologie des Polnischen. München 1974 (= Slavistische Beiträge 114)

MAZURKIEWICZ 1987 = Anna Mazurkiewicz: "Derywacja deminutywów rzeczownikowych za pomoca formantow -ik, -ek, -ok w jezyku slowackim, polskim i rosyjskim. Cz.1: Zasób formantów". Studia z Filologii Polskiej $i$ Stowianskiej. 24(1987) Warszawa. S.207-214

MICHALEWSKI $1984={ }^{\circ}$ Kazimierz Michalewski: Dystrybucja polskich rzeczownikowych formantów przyrostkowych. Lodz 1984 (= Acta Universitatis Lodziensis. Folia linguistica 9)

MICHEL 1974 = Georg Michel: "Stil und Expressivität". Zeitschrift fur Phonetik, Sprachwissenschaft und Kommunikationsforschung. 27(1974) Berlin. S.132-140

MIEMIETZ 1981 = Bärbel Miemietz: Kontrastive Linguistik. Deutsch-Polnisch 1965-1980. Ein Literaturbericht. Gießen 1981 (= Vorträge und Abhandlungen zur Slavistik 2)

MINI-BETRACHTUNG 1967 = "Mini-Betrachtung". Sprachpflege. 16(1967) Leipzig. S.233

MOSER 1969 = Hans Moser: "Zum Deminutiv im Süd- und Südmittelbairischen". Zeitschrifi fur Dialeksologie und Linguistik. 36(1969) Wiesbaden. S. $190-200$

MOUNIN 1967 = Georges Mounin: Die Ubersetzung. Geschichte, Theorie, Anwendung. Übers.v. H.Stammerjohann. München 1967

MÜLLER 1953 = Alfons F.Müller: Die Pejoration von Personenbezeichnungen durch Suffixe im Neuhochdeutschen. Altdorf 1953

MÜLLER-OTT 1970a = Dorothea Müller-Ott: "Slawische Deminutiva mit Suffixen mit dem Element $-k-$. Produktivität und relative Häufigkeit der Suffixe". Actes du Xe Congrès International des Linguistes. Bucarest 28.8.2.9.1967. 4(1970) Bucarest. S.571-582

MÜLLER-OTT 1970b = Dorothea Müller-Ott: "Entropie des Deminutivsystems im Slawischen". Slavica Slovaca. 5(1970) Bratislava. S.3-16

MÜLLER-OTT 1972 = Dorothea Müller-Ott: "Zur Entstehung technischer Fachausdrücke aus Deminutivbildungen". Wiener slavistisches Jahrbuch. 17(1972) Wien. S.209-214 
MÜLLER-OTT 1975 = Dorothea Müller-Ott: "Besonderheiten der Deminutiva von Vornamen". F.V.Mareł [Hg.]: Bereiche der Slavistik. Festschrift zu Ehren von Josip Hamm. Wien 1975. S.203-213

MÜLLER-OTT 1983 = Dorothea Müller-Ott: "Die slawischen Deminutiva - ein Ubbersetzungsproblem". Festschrift zum 40jahrigen Bestehen des Instituts fur Ubersetzer- und Dolmetscherausbildung der Universitat Wien. Wien 1983. S.106-113

MÜLLER-OTT 1987 = Dorothea Müller-Ott: "Die sprachliche Lücke im morphologischen Bereich als Problem bei der Übersetzung Polnisch-Deutsch" Wiener slavistisches Jahrbuch. 33(1987) Wien. S.57-69

MUTHMANN 1988 = Gustav Muthmann: Räcklaufiges deutsches Worterbuch. Handbuch der Wortausgänge im Deutschen mit Beachtung der Wort- und Lautstruktur. Tübingen 1988 (= Germanistische Linguistik 78)

NABRINGS 1981 = Kirsten Nabrings: Sprachliche Varietaten. Tübingen 1981 (= Tübinger Beiträge zur Linguistik 147)

NEUBERT 1968 = Albrecht Neubert: "Pragmatische Aspekte der Übersetzung". A.N.[Hg.]: Grundfragen der Übersetzungswissenschaft. Leipzig 1968 (= Beihefte zur Zeitschrift 'Fremdsprachen' 2). S.21-33

NEUBERT 1981 = Albrecht Neubert: "Übersetzungswissenschaftlicher Sprachvergleich". Fremdsprachen. 25(1981) Leipzig. S.24-29

NEUBERT 1983 = Albrecht Neubert: "Translation und Texttheorie". G.Jäger/A.Neubert [Hgg.]: Semantik und Ubersetzungswissenschaft. Materialien der 3.Internationalen Konferenz 'Grundfragen der Übersetzungswissenschaft'. Leipzig 1983 (= Übersetzungswissenschaftliche Beiträge 6). S.100110

NEUBERT 1984 = Albrecht Neubert: "Text-bound Translation Teaching". W.Wilss/G.Thome [Hgg.]: Die Theorie des Ubersetzens und ihr Aufschlupwert für die Ubersetzungs- und Dolmetscherdidaktik. Akten des Internationalen Kolloquiums der AILA. Saarbrücken, 25.-30.7.1983. Tübingen 1984 (= Tübinger Beiträge zur Linguistik 247). S.61-70

NEUBERT 1986 = Albrecht Neubert: "Translatorische Relativität". M.SnellHornby [Hg.]: Ubersetzungswissenschafi - eine Neuorientierung. Tübingen 1986. $\$ .85-105$

NEUBERT 1988 = Albrecht Neubert: "Wortbildungsstrukturen in der Übersetzung". Zur Theorie der Wortbildung im Deutschen. Berlin 1988 (= Sitzungsberichte der AdW der DDR. Gesellschaftswissenschaften 4.1988). S.49-52

NEUMANN 1979 = Gerda Neumann: "Probleme beim Übersetzen von Kinderund Jugendliteratur". M.Gorschenek/A.Rucktäschel [Hgg.]: Kinder- und Jugendliteratur. München 1979. S.115-128

NIDA 1964 = Eugene A.Nida: Toward a Science of Translating with Special Reference to Principles and Procedures Involved in Bible Translating. Leiden 1964 
NIEDŹWIEDZKI 1919 = Wladysław Niedźwiedzki: "Potegowanie pojecc i stopniowanie bezwzgledne w jezyku polskim". Jezyk Polski. 4(1919) Kraków. S.44-48

NORD $1988=$ Christiane Nord: Textanalyse und Übersetzen. Theoretische Grundlagen, Methode und didaktische Anwendung einer übersetzungsrelevanten Textanalyse. Heidelberg 1988

NOWAKOWSKA-KEMPNA 1977 = Iwona Nowakowska-Kempna: "Możliwosci i ograniczenia przekladu nazw wlasnych nacechowanych stylistycznie". Zeszyty Naukowe Uniwersytetu Jagiellońskiego 457. Prace jezykoznawcze. 54(1977) Kraków. S.367-382

NOWOTNA-SZYBISTOWA 1969 = Magdalena Nowotna-Szybistowa: "Rzeczownikowe neologizmy deminutywne w jezyku Stanisława Ignacego Witkiewicza". Jezyk Polski. 49(1969) Kraków. S.362-369

OBREBSKA 1929 = A.Obrebska: "Technika spieszczeń $w$ dzisiejszej polszczyznie". Jezyk Polski. 14(1929) Kraków. S.65-71

ÖHMANN 1968 = Emil Öhmann: "Die Diminutiva im Mittelhochdeutschen". Neuphilologische Mitteilungen. 47(1946) Helsinki. Nachdruck Amsterdam 1968. S.115-125

ÖHMANN 1972 = Emil Öhmann: "Suffixstudien VIIl: Die deutschen Diminutivsuffixe -lein und -chen". Neuphilologische Mitteilungen. 73(1972) Helsinki. S.555-567

OTREBSKI 1968 = Jan Otrebski: "Die deminutiven Verben im Slavischen". Die Welt der Slaven. 13(1968) Wiesbaden. S.246-250

PAEPCKE 1968 = Fritz Paepcke: "Verstehen und Übersetzen". Linguistica Antverpiensia. 2(1968) Antwerpen. S.329-351

PAEPCKE 1971 = Fritz Paepcke: "Sprach-, text- und sachgemäßes Übersetzen" ..K.-R.Bausch/H.-M.Gauger [Hgg.]: Interlinguistica. Sprachvergleich und Übersetzung. Festschrift zum 60 .Geburtstag von Mario Wandruszka. Tübingen 1971. S.610-616

PAEPCKE 1979a = Fritz Paepcke: "Mitteilungsgeschehen - Textverstehen Ubersetzen". W.Mair/E.Sallager [Hgg.]: Sprachtheorie und Sprachenpraxis. Festschrift für Henry Vernay zu seinem 60. Geburtstag. Tübingen 1979 (= Tübinger Beiträge zur Linguistik 112). S.313-331

PAEPCKE 1979b = Fritz Paepcke: "Übersetzen als Hermeneutik". StefanGeorge-Seminar 1978. Heidelberg 1979. S.96-114

PAEPCKE 1986 = Fritz Paepcke: "Die Illusion der Äquivalenz. Übersetzen zwischen Unschärfe und Komplementarität" . E, Grözinger/A.Lawaty [Hgg.]: Suche die Meinung. Karl Dedecius, dem Ubersetzer und Mittler, zum 65.Geburtstag. Wiesbaden 1986. S.116-151

PAEPCKE/FORGET 1981 = F.Paepcke/Ph.Forget: Textverstehen und Übersetzen. Ouvertures sur la traduction. Heidelberg 1981 (= Studienbücher Deutsch als Fremdsprache 2) 
PAUL $1920=$ Hermann Paul: Deutsche Grammatik. Bd.5. Teil 4: Wortbildungslehre. Halle a.S. 1920

PELLEGRINI 1977 = Ines A.Pellegrini: Die Diminutive im Deutschen und im Italienischen. Zürich 1977

PFENNIG 1904 = Heinrich Pfennig: Das Deminutivum bei Schiller und seinen Zeitgenossen. Diss. Marburg 1904

PLEPELIĆ 1974 = Zvonko Plepelic: Die serbokroatischen Diminutiva auf -ca, -ce und -ac. Augsburg 1974

PLUTA 1967 = Feliks Pluta: "Deminutywa w Slaskiej pieśni ludowej". Zeszyty Naukowe Wyzszej Szkoty Pedagogicznej w Opolu. Seria A. Jezykoznawstwo. 3(1967) Opole. S.33-57

PLUTA 1980 = Feliks Pluta: " $Z$ badań nad deminutywami $w$ dialekcie sląskim". Studia Polonistyczne. 7(1979) Poznan 1980. S.125-140

PLUTA 1985 = Feliks Pluta: "Deminutywa w twórczosci Rafala Urbana". Zeszyty Naukowe Wyzszej Szkoty Pedagogicznej w Opolu. Filologia polska. 24(1985) Opole. S.129-140

PODRACKI 1977 = Jerzy Podracki: "Wiechowska gwara Warszawy" . Kronika Warszawy. 33(1977) Warszawa. S.19-32

PÖCKL 1978 = Wolfgang Pöckl: "Stil und Übersetzungsvergleich". Klagenfurter Beitrage zur Sprachwissenschaft. 4(1978) Klagenfurt. S.65-80

POHL 1980 = Alek Pohl: "Morphonologie des Diminutivs in der polnischen Gegenwartssprache". Scando-Slavica. 26(1980) Kopenhagen. S. 161-173

POHLING 1971 = Heide Pohling: "Zur Geschichte der Übersetzung". Studien zur Ubersetzungswissenschaft. Leipzig 1971 (= Beihefte zur Zeitschrift 'Fremdsprachen' 3/4). S.125-162

POLAŃSKA 1967 = Marta Polańska: "Deminutiva w polskiej literaturze dzieciecej". Jezykoznawca. 16/17(1967) Lublin. S.115-120

POLLAK 1975 = Seweryn Pollak [red.]: Przektad artystyczny. O sztuce tlumaczenia. T.2. Wroclaw et al. 1975

POLTERAUER 1981 = Ilona Polterauer: Die Deminutiva in der modernen russischen Schrifisprache. Wien 1981 (= Dissertationen der Universität Wien 148)

POLZIN 1901 = Albert Polzin: Studien zur Geschichte des Deminutivums im Deutschen. StraBburg 1901 (= Quellen und Forschungen zur Sprach- und Culturgeschichte der germanischen Völker 88)

POMIANOWSKA 1963 = Wanda Pomianowska: Klasyfikacja rzeczowników odrzeczownikowych. Studium ze slowotworstwa i geografii lingwistycznej. Wroclaw et al. 1963 (= Prace Jezykoznawcze PAN 35)

POPOVIĆ 1973 = Anton Popović: "Zum Status der Übersetzungskritik" . Babel. 19(1973) Bonn. S.161-165 
PREUSLER 1942/43 = Walther Preusler: "Diminutive und Koseformen im heutigen Hochdeutsch". Taal en Leven. 6(1942/43) 's-Gravenhage. S.145149

PREUSLER 1943/44 = Walther Preusler: Rez. zu "E.Kruisinga: Diminutieve en affektieve suffixen in de Germaanse talen. Amsterdam 1942". Anglia. Beiblätter. 54/55(1943/44) Halle a.S. S.16-18

PÜSCHEL 1983 = Ulrich Püschel: "Stilanalyse als Stilverstehen". B.Sandig [Hg.]: Stilistik. 1.Bd.: Probleme der Stilistik. Hildesheim et al. 1983 (= Germanistische Linguistik H.3-4/1981). S.97-126

PUZYNINA 1978 = Jadwiga Puzynina: "Transpozycja, mutacja, modyfikacja" . Poradnik Jezykowy. (1978) Warszawa. S.193-199

QUINTILIANUS $1975=$ Marcus F.Quintilianus: Ausbildung des Redners. Zwölf Bücher. 2.Teil: Buch VII-XII. Zweispr. Ausgabe hg. und übers.v. H. Rahn. Darmstadt 1975

RAMSTEIN 1969 = Martha Ramstein: Das Diminutiv als Stilmittel in neuerer deutscher Prosa. Untersuchungen zu den Ausdruckswerten des Diminutivs in Werken von Thomas Mann, Hermann Hesse, Robert Musil, E.T.A.Hoffmann, Joseph von Eichendorff und Eduard Mörike. Maschinenschriftl.Diss. Bern 1969

RECZEK 1968 = Stefan Reczek: "Deminutiva polskie. Charakterystyka, rozwój funkcji stylistycznej". Rocznik Naukowo-Dydakyczny Wyzszej Szkoty Pedagogicznej w Rzeszowie. Nauki humanistyczne. 5(1968) Rzeszów. S.373-386

RECZEK 1974 = Stefan Reczek: "Jak z małosci zrobić wielki problem" . Profile. 5(1974) Rzeszów. S.25 und 6(1974) S.22

REISS 1968 = Katharina Reiss: "Überlegungen zu einer Theorie der Übersetzungskritik". Linguistica Antverpiensia. 2(1968) Antwerpen. S.369-383

REISSS 1970 = Katharina Reiss: "Das Jahrhundert der Übersetzungen oder Die Ubersetzung in Bildern und Vergleichen". Linguistica Antverpiensia. 4(1970) Antwerpen. S.175-195

REISS 1976 = Katharina Reiss: Texttyp und Ubersetzungsmethode. Der operative Text. Kronberg i.Ts. 1976 (= Monographien Literatur, Sprache, Didaktik 11)

REISS 1981a = Katharina Reiss: "Der Übersetzungsvergleich. Formen - Funktionen - Anwendbarkeit". W.Kühlwein/G.Thome/W.Wilss [Hgg.]: Kontrastive Linguistik und Ubersetzungswissenschafi. Akten des Internationalen Kolloquiums Trier/Saarbrücken 25.-30.9.1978. München 1981. S.311-319

REISS 1981b = Katharina Reiss: "Elend und Glanz der Übersetzung oder Was hat das Übersetzen mit den kleinen grünen Männchen vom Mars zu tun?". W.Pöckl [Hg.]: Europdische Mehrsprachigkeit. Festschrift zum 70.Geburtstag von Mario Wandruszka. Tübingen 1981. S.409-419

REISSS 1983 = Katharina Reiss: "Quality in Translation oder Wann ist eine Ubersetzung gut?". Babel. 29(1983) Bonn. S.198-208 
REISS $1984=$ Katharina Reiss: "Adäquatheit und Äquivalenz". W.Wilss/G. Thome [Hgg.]: Die Theorie des Ubersetzens und ihr Aufschlußwert fur die Ubersetzungs- und Dolmetscherdidaktik. Akten des Internationalen Kolloquiums der AILA. Saarbrücken, 25.-30.7.1983. Tübingen 1984 (= Tübinger Beiträge zur Linguistik 247). S.80-89

REISS 1986 = Katharina Reiss: Moglichkeiten und Grenzen der Ubersetzungskritik. Kategorien und Kriterien für eine sachgerechte Beurteilung von Ubersetzungen. 3.Aufl. München 1986

REISS/VERMEER 1984 = K.Reiss/H.J.Vermeer: Grundlegung einer allgemeinen Translationstheorie. Tübingen 1984 (= Linguistische Arbeiten 147)

REITER 1988 = Norbert Reiter: "Die Regulierung der Perspektive durch Diminutivum und Augmentativum in bulgarischen Märchentexten". $\mathbf{R}$. Olesch/H.Rothe [Hgg.]: Slavistische Studien zum 10. Internationalen Slavistenkongre $\beta$ in Sofia 1988. Köln, Wien 1988 (= Slavistische Forschungen 54). S.659-693

REPA 1981 = Stanisława Repa: "Funkcje zdrobnień w jezyku Kazimierza Brodzińskiego". Rozprawy Komisji Jezykowej Wroctawskiego Towarzystwa Naukowego. 12(1981) Wroclaw. S.131-151

RIESEL 1959 = Elise Riesel: Stilistik der deutschen Sprache. Moskau 1959

RIESEL $1970=$ Elise Riesel: Der Stil der deutschen Alltagsrede. 2.Aufl. Leipzig 1970

RIESEL 1975 = Elise Riesel: "Grundsatzfragen der Funktionalstilistik" . Linguistische Probleme der Textanalyse. Jahrbuch 1973. Düsseldorf 1975 (= Sprache der Gegenwart 35). S.36-53

RIESEL/SCHENDELS 1975 = E.Riesel/E.Schendels: Deutsche Stilistik. Moskau 1975

RITTER 1976 = Eva Ritter: "Die Funktion des Diminutivs in der russischen Gegenwartssprache. Möglichkeiten und Grenzen des Bedeutungswandels". H.D.Pohl/N.Salnikov [Hgg.]: Opuscula slavica et linguistica. Festschrift für Alexander Issatschenko. Kagenfurt 1976. S.349-362

ROELANDTS 1956 = Karel Roelandts: "Augmentativ, Diminutiv und Zugehörigkeit im Lichte der Namengebung". Rheinische Vierteljahrs-Blatter. 21 (1956) Bonn. S.110-124

RÖSSLER 1979 = Gerda Rössler: Konnotationen. Untersuchungen zum Problem der Mit- und Nebenbedeutung. Wiesbaden 1979 (= Zeitschrift für Dialektologie und Linguistik. Beihefte N.F. 29 der 'Zeitschrift für Mundartforschung')

ROSSIPAL 1973 = Hans Rossipal: "Konnotationsbereiche, Stiloppositionen und die sogenannten 'Sprachen' in der Sprache". Germanistische Linguistik. H.4(1973) Marburg, Hildesheim. S.5-87

ROZWADOWSKI 1960 = Jan M.Rozwadowski: "Słowotworstwo i znaczenie wyrazow. Studium nad ich podstawowymi prawami" .J.R.: Wybor pism. T.3: Jezykoznawstwo ogólne. Warszawa 1960. S.21-95 
RUDOLPH 1983 = Jolanta Rudolph: Formen und Funktionen der substantivischen Deminutiva der polnischen Sprache der Gegenwart. Maschinenschriftl.Diss. Leipzig 1983

RUDOLPH 1984 = Jolanta Rudolph: "Funktionen der substantivischen Deminutiva in der polnischen Sprache der Gegenwart" . Linguistische Arbeitsberichte. Sektion: Theoretische und angewandte Sprachwissenschaft der KarlMarx-Universität. 46(1984) Leipzig. S.67-90 und 96

RŪĶE-DRAVIṆA 1959 = Velta Rūke-Dravip̧a: Diminutive im Lettischen. Lund 1959 ( = Acta Universitatis Stockholmiensis. Etudes de philologie slave 8)

RUSINEK 1955 = Michal Rusinek [red.]: O sztuce thumaczenia. Wroclaw 1955

SAFAREWICZOWA 1968 = Halina Safarewiczowa: "Deminutywne zwroty $z$ przyimkiem 2 w jezyku polskim". Slavia Occidentalis. 27(1968) Poznan. S. $227-230$

SANDERS 1893/1894 = D.Sanders: "Die Verkleinerungssilbe -chen". Zeitschrift fur deutsche Sprache. 6(1893) Paderborn. S.422-427 und 447-450; 7(1894) Paderborn. S.93-100 und 130-137

SANDIG 1978 = Barbara Sandig: Stilistik. Sprachpragmatische Grundlegung der Stilbeschreibung. Berlin, New York 1978

SARNOWSKI 1985 = Michal Sarnowski: "O możliwosci wykorzystania formacji deminutywnych $w$ tworzeniu systematycznej terminologii naukowej $w$ jezyku polskim i rosyjskim". Acta Universitatis Wratislaviensis. Studia linguistica. 9(1985) Wroclaw. S.33-41

SARNOWSKI 1987 = Michał Sarnowski: "Leksykalizacja deminutywów w jezyku rosyjskim i polskim". Acta Universitatis Wratislaviensis. Slavica Wratislaviensia. 44(1987) Wroclaw. S.59-70

SARNOWSKI 1991 = Michal Sarnowski: Quasi-deminutiwa $w$ jezyku rosyjskim i polskim. Wroclaw 1991 (= Slavica Wratislaviensia 58)

SATKIEWICZ 1969 = Halina Satkiewicz: Produktywne typy slowotworcze wspótczesnego jezyka ogolnopolskiego. Warszawa 1969

SCHARNHORST 1981 = Jürgen Scharnhorst: "Zum Wesen des Begriffs Funktionalstil". Zeitschrifi für Phonetik, Sprachwissenschaft und Rommunikationsforschung. 34(1981) Berlin. S.305-314

SCHARNHORST/ISING 1976/1982 = J.Scharnhorst/E.Ising [Hgg.]: Grundlagen der Sprachkultur. Beiträge der Prager Linguistik zur Sprachtheorie und Sprachpflege. 2 Bde. Berlin 1976 und 1982 (= Sprache und Gesellschaft 8)

SCHEIDWEILER 1984/85 = Gaston Scheidweiler: "Zur Konnotation der Diminutivsuffixe -chen und -lein - prosaisch oder poetisch?". Muttersprache. 95(1984/85) Wiesbaden. S.69-79

SCHIPPAN 1975 = Thea Schippan: Einführung in die Semasiologie. 2.überarb.Aufl. Leipzig 1975 
SCHIPPAN 1979 = Thea Schippan: "Zum Problem der Konnotationen". Zeitschrift für Phonetik, Sprachwissenschaft und Kommunikationsforschung. 32(1979) Berlin. S.679-684

SCHIPPAN 1984 = Thea Schippan: Lexikologie der deutschen Gegenwartssprache. Leipzig 1984

SCHIPPAN/SOMMERFELDT 1966 = Th.Schippan/K.-E.Sommerfeldt: "Wort und Kontext". Zeitschrifi für Phonetik, Sprachwissenschafi und Kommunikationsforschung. 19(1966) Berlin. S.533-552

SCHMIDT 1977 = Heide Schmidt: "Probleme der konfrontativen Beschreibung stilistischer Selektionsbeschränkungen". O.Kade [Hg.]: Vermittelte Kommunikation, Sprachmittlung, Translation. Leipzig 1977 (= Übersetzungswissenschaftliche Beiträge 1). S.111-121

SCHMIDT 1979 = Heide Schmidt: "Zur Bestimmung der stilistischen Information (als Voraussetzung für eine konfrontative Stilistik)" ${ }^{n}$ O.Kade [Hg.]: Sprachliches und Außersprachliches in der Kommunikation. Leipzig 1979 (= Ubersetzungswissenschaftliche Beiträge 2). S.46-89

SCHMIDT 1963 = Wilhelm Schmidt: Lexikalische und aktuelle Bedeutung. Ein Beitrag zur Theorie der Wortbedeutung. Berlin 1963 (= Schriften zur Phonetik, Sprachwissenschaft und Kommunikationsforschung 7)

SCHNEIDER 1959 = Wilhelm Schneider: Stilistische deutsche Grammatik. Die Stilwerte der Wortarten, der Wortstellung und des Satzes. 4.Aufl. Freiburg et al. 1959

SCHUHMACHER $1975=$ W.W.Schuhmacher: "Gedanken zum Diminutiv". Linguistics. 162(1975) The Hague, Paris. S.15-16

SCHWARZ 1905 = Hermann Schwarz: Das Suffix lich(t) bei Adjektiven im Neuhochdeutschen. Diss. Freiburg i.Br. 1905

ŚCIEBORA 1983 = Alina Ściebora: "Klasyfikacja slowotwórcza rzeczowników meskich utworzonych formantami zawierajacymi historyczne -k-". Poradnik Jezykowy. (1983) Warszawa. S.549-584

SEEBOLD 1983 = Elmar Seebold: "Diminutivformen in den deutschen Dialekten". W.Besch et al. [Hgg.]: Dialekologie. Ein Handbuch zur deutschen und allgemeinen Dialektforschung. 2.Halbbd. Berlin, New York 1983 (= Handbücher zur Sprach- und Kommunikationswissenschaft 1.2). S.1250-1255

SEIDEL-SLOTTY 1947 = Ingeborg Seidel-Slotty: "Über die Funktionen der Diminutiva". Bulletin Linguistique. 15(1947) Copenhague et al. S.23-54

SEIDELMANN 1979 = Erich Seidelmann: "Ausdruck- und inhaltbezogene Wortbildungslehre. Grundzüge eines funktionalen Modells". Zeitschrift für Dialekologie und Linguistik. 46(1979) Wiesbaden. S.149-186

SIATKOWSKA 1967 = Ewa Siatkowska: "Deminutywa rzeczownikowe we współczesnych literackich jezykach zachodnioslowianskich". Studia z Filologii Polskiej i Stowiańskiej. 6(1967) Warszawa. S.157-170 
SIEBERER 1966 = Anton Sieberer: "Das Wesen des Deminutivs". Die Sprache. 2(1950-52). Nachdruck Amsterdam 1966. S.85-121

SKUBALANKA 1973a = Teresa Skubalanka: "O ekspresywnosci jezyka" . Annales Universitatis Mariae Curie-Sktodowska. Sectio F. Nauki filizoficzne i humanistyczne. 27(1972) Lublin 1973. S.123-135

SKUBALANKA 1973b = Teresa Skubalanka: "Ekspresywnose jezyka a mowa potoczna". S.Skwarczyniska [red.]: Poetyka i stylistyka stowiańska. Materiały konferencji Komisji Poetyki i Stylistyki Slowianskiej Miedzynarodowego Komitetu Slawistów. Warszawa 18.-20.4.1972. Wroclaw et al. 1973. S.177183

SKUBALANKA 1976 = Teresa Skubalanka: "Założenia analizy stylistycznej". H.Markiewicz/J.Sławiński [redd.]: Problemy metodologiczne wspótczesnego literaturoznawstwa. Kraków 1976. S.250-273

SLOWNIK TERM.LIT. 1976 = M.Glowiński et al.: Stownik terminów literackich. Wroclaw et al. 1976

SMOCZYŃSKI $1962=$ P.Smoczyński: "Nomina appellativa i propria we wzajemnym oddzialywaniu slowotworczym". Biuletyn Polskiego Towarzystwa Jezykoznawczego. 21(1962) Wroclaw. S.61-82

SNELL-HORNBY 1986 = Mary Snell-Hornby [Hg.]: Ubersetzungswissenschaft - eine Neuorientierung. Zur Integrierung von Theorie und Praxis. Tübingen 1986

SPYCHER 1955-1957 = Peter C.Spycher: "Die Struktur der Adjektive auf -ig und -lich in der deutschen Schriftsprache der Gegenwart. Ein Beitrag zur Darstellung der Ableitung vom Standpunkt der synchronischen Sprachwissenschaft. Erster Teil: Allgemeines". Orbis. 4(1955) Louvain. S.74-90; "Zweiter Teil: Die Bedeutungs- und Wortbildungskategorien der Suffixtypen -ig und -lich". 5(1956). S.435-452 und 6(1957). S.410-426

STANKIEWICZ 1954 = Edward Stankiewicz: "Expressive Derivation of Substantives in Contemporary Russian and Polish". Word. 10(1954) New York. S.457-468

STANKIEWICZ 1961 = Edward Stankiewicz: "Grammatical Neutralization in Slavic Expressive Forms". Word. 17(1961) New York. S.128-145

STANKIEWICZ 1964 = Edward Stankiewicz: "Problems of Emotive Language". Th.A.Sebeok et al. [edd.]: Approaches to Semiotics. Cultural Anthropology. Education. Linguistics. Psychiatry. Psychology. Transactions of the Indiana University Conference on Paralinguistics and Kinesics. The Hague 1964. S.239-264

STASZEWSKA 1985 = Zdzisława Staszewska: "Formacje deminutywno-ekspresywne w gwarze okolic Radomska". Poradnik Jezykowy. (1985) Warszawa. S.226-247

STEIN 1980 = Dieter Stein: Theoretische Grundlagen der Ubersetzungswissenschaft. Tübingen 1980 (= Tübinger Beiträge zur Linguistik 140) 
STEPANOWA/FLEISCHER $1985=$ M.D.Stepanowa/W.Fleischer: Grundzage der deutschen Wortbildung. Leipzig 1985

STÖRIG 1963 = Hans J.Störig [Hg.]: Das Problem des Ubbersetzens. Darmstadt 1963 (= Wege der Forschung 8)

STOLZE 1982 = Radegundis Stolze: Grundlagen der Textäbersetzung. Heidelberg 1982

SZOBER 1953 = Stanisław Szober: Gramatyka jezyka polskiego. Oprac. W. Doroszewski. Wyd.3. Warszawa 1953

SZYMCZAK 1966 = Mieczysław Szymczak: Nazwy stopni pokrewienistwa $i$ powinowactwa rodzinnego w historii $i$ dialektach jezyka polskiego. Warszawa 1966

SZYMCZAK 1969 = Mieczyslaw Szymczak: "O analogii semantyczno-slowotwórczej w polskiej terminologii rodzinnej". Prace Filologiczne. 19(1969) Warszawa. \$.119-126

SZYMCZAK 1973 = Mieczyslaw Szymczak: "O tzw. nieprzekladalnych kategoriach jezykowych". S.Skwarczynska [red.]: Poetyka i stylistyka stowianiska. Materiały konferencji Komisji Poetyki i Stylistyki Słowianskiej Miedzynarodowego Komitetu Slawistow. Warszawa 18.-20.4.1972. Wroclaw et al. 1973. S.20-26

TASZYCKI 1961 = Witold Taszycki: "Powstanie i rozwoj rzeczownikow typu cielak. Ustep $z$ historii narzecza mazowieckiego". Rozprawy $i$ studia polonistyczne. T.2: Dialektologia i problem pochodzenia polskiego jezyka literackiego. Wroclaw et al. 1961. S.236-249

TENNERT 1982 = Renate Tennert: Die Darstellung der Diminutiva (Substantive mit Suffixen der subjektiven Wertung) im Russischen unter besonderer Beracksichtigung der Problematik ihrer Ubersetzung ins Deutsche. Maschinenschriftl. Diss. Jena 1982

TENNERT 1984 = Renate Tennert: "Äquivalenzbetrachtung russischer und deutscher Diminutiva". Wissenschafiliche Zeitschrift der Friedrich-SchillerUniversitdt Jena/Tharingen. Gesellschaftswissenschaftliche Reihe. 33(1984) Jena. S.673-678

THESAURUS 1909-34 = Thesaurus Linguae Latinae. Vol.5. Pars prior D. Leipzig 1909-34

THIEL 1978 = Rudolf Thiel: "Die Diminuierung im Deutschen". Sprachpflege. 27(1978) Leipzig. S.116-117

TIEFENBACH 1987 = Heinrich Tiefenbach: "-chen und -lein. Überlegungen zu Problemen des sprachgeographischen Befundes und seiner sprachhistorischen Deutung. Mit fünf Karten". Zeitschrift fur Dialekologie und Linguistik. 54(1987) Stuttgart. S.2-27

TOMICZEK 1979 = Eugeniusz Tomiczek: "Die direkte Anrede im Polnischen kontrastiert mit dem Deutschen". Kwartalnik Neofilologiczny. 26(1979) Warszawa, Poznañ. S.81-99 
TOMICZEK 1983 = Eugeniusz Tomiczek: System adresatywny wspótczesnego jezyka polskiego i niemieckiego. Socjolingwistyczne studium konfrontatywne. Wroclaw 1983

ULLMANN 1972a = Stephen Ullmann: Grundzage der Semantik. Die Bedeutung in sprachwissenschaftlicher Sicht. Ubers.v. S.Koopmann. 2.Aufl. Berlin, New York 1972

ULLMANN 1972b = Stephen Ullmann: Sprache und Stil. Aufsätze zur Semantik und Stilistik. Übers.v. S.Koopmann. Tübingen 1972

ULLMANN 1973 = Stephen Ullmann: Semantik. Eine Einführung in die Bedeutungslehre. Ubers.v. S.Koopmann. Frankfurt a.M. 1973

URBAŃCZYK 1945 = Stanisław Urbańczyk: "Dwa przyczynki do znajomości polskich deminutywow: 1.Zdrobnienia typu mateńka, serdeńko, maleńki (Drobiazg ze stosunkow jezykowych polsko-ruskich); 2. Geneza miekkich spólglosek s, z, 6 w spieszczeniach typu Stas, Kasia, nózia, malusi." Sprawozdania z czynnosci $i$ posiedzen Polskiej Akademii Umiejetnosci $w$ Rrakowie. 45(1939-44) Kraków 1945. S.15-18

VERMEER 1986 = Hans J.Vermeer: "Übersetzen als kultureller Transfer". M.Snell-Hornby [Hg.]: Ubersetzungswissenschaft - eine Neuorientierung. Tübingen 1986. S.30-53

VLACHOV/FLORIN $1980=$ S. Vlachov/S. Florin: Neperevodimoe $v$ perevode. Moskva 1980

WANDRUSZKA 1967 = Mario Wandruszka: "Romanische und germanische quantifizierend-qualifizierende Suffixe" . Archiv für das Studium der neueren Sprachen und Literaturen. 203(1967) Braunschweig et al. S.161-175

WANDRUSZKA 1969 = Mario Wandruszka: Sprachen - vergleichbar und unvergleichlich. München 1969

WANDRUSZKA 1971 = Mario Wandruszka: Interlinguistik. Umrisse einer neuen Sprachwissenschaft. München 1971

WANDRUSZKA 1973 = Mario Wandruszka: "Kontrastive Linguistik in Österreich". Anzeiger der Osterreichischen Akademie der Wissenschafien. Philosophisch-historische Klasse. 110(1973) Wien. S.1-21

WANDRUSZKA 1974 = Mario Wandruszka: "Deutsch im Übersetzungsvergleich". K.Engel/P.Grebe [Hgg.]: Sprachsystem und Sprachgebrauch. Festschrift für Hugo Moser zum 65.Geburtstag. T.1. Düsseldorf 1974 (= Sprache der Gegenwart 33). S.308-327

WANDRUSZKA 1975 = Mario Wandruszka: "Über die Natur natürlicher Sprachen". B.Schlieben-Lange [Hg.]: Sprachtheorie. Hamburg 1975. S.319-342

WANDRUSZKA 1979 = Mario Wandruszka: "Über den Zufall in unseren Sprachen". W.Mair/E.Sallager [Hgg.]: Sprachtheorie und Sprachenpraxis. Festschrift für Henri Vernay zu seinem 60.Geburtstag. Fübingen 1979 (= Tübinger Beiträge zur Linguistik 112). S.427-441 
'WANDRUSZKA 1984 = Mario Wandruszka: Das Leben der Sprachen. Vom menschlichen Sprechen und Gespräch. Stuttgart 1984

'WARCHOL 1970 = Stefan Warchol: Rez. zu "J.Zima: Expresivita slova v současne zeštinè. Praha 1961". Rocznik Slawistyczny. 31(1970) Wroclaw et al. S.124-136

WARCHOL 1972 = Stefan Warchol: "Ze slowotworstwa czasownikow ekspresywnych w jezykach slowiańskich". W.Doroszewski et al. [redd.]: Z polskich studiow slawistycznych. Seria 4. Jezykoznawstwo. Prace na VII Miedzynarodowy kongres slawistow w Warszawie 1973. Warszawa 1972. S.121-127

WARCHOL 1974a = Stefan Warchol: "Formacje ekspresywne a system jezyka". Biuletyn Polskiego Towarzystwa Jezykoznawczego. 32(1974) Wroclaw et al. S.71-86

'WARCHOL 1974b = Stefan Warchol: "Morfologiczno-semantyczne wlasciwosci sufiksu *- i *-et-ko w jezyku polskim i rosyjskim". Slavisticky Sbornik Olomoucko-Lublinský. (1974) Praha. S.149-166

WARCHOL 1979 = Stefan Warchol: "O ekspresywach z formantem -ik typu awansik, pawilonik w jezyku polskim". Annales Universitatis Manae Curie-Sktodowska. Sectio F. Nauki filozoficzne i humanistyczne. 32(1977) Lublin 1979. S.259-262

'WARCHOL 1982 = Stefan Warchol: "Charakterystyka ekspresywow z -ik w jezyku polskim w porównaniu ze slowackim". Jezyk Polski. 62(1982) Krakow. S.12-21

WARCHOL 1984 = Stefan Warchol: Geneza $i$ rozwoj stowiariskich formacji ekspresywnych z sufiksalnym -k-i c-. Warszawa, Lodz 1984 (= Lubelskie Towarzystwo Naukowe. Prace Wydziahu Humanistycznego 19)

WEDKIEWICZ 1929 = Stanislaw Wedkiewicz: "Kilka uwag o technice spieszczen". Jezyk Polski. 14(1929) Kraków. S.110-120

WEGERA 1982 = Klaus-Peter Wegera: "Möglichkeiten und Grenzen der philologischen Auswertung einer elektronisch gespeicherten Datei zum Frühneuhochdeutschen. Aufgezeigt am Beispiel der Diminutivsuffixe". H.Fix et al. [Hgg.]: Sprachen und Computer. Festschrift zum 75.Geburtstag von Hans Eggers, 9.Juli 1982. Dudweiler 1982 (= Sprachwissenschaft - Computer Linguistik 9). S.207-222

WELLMANN 1969 = Hans Wellmann: "Die Substantivbildung mit er und -ling im heutigen Deutsch". Germanistische Studien. Innsbruck 1969 (= Innsbrucker Beiträge zur Kulturwissenschaft 15). S.337-354

WIERZBICKA 1969 = Anna Wierzbicka: Dociekania semantyczne. Wroclaw 1969

WIERZBICKA 1984 = Anna Wierzbicka: "Diminutives and Depreciatives. Semantic Representation for Derivational Categories". Quaderni di semantica. 5(1984) Bologna. S.123-130 
WILKOŃ 1987 = Aleksander Wilkon: Typologia odmian jezykowych wspótczesnej polszczyzny. Katowice 1987 (= Prace Naukowe Uniwersytetu Slaskiego w Katowicach 865)

WILMANNS 1899 = Wilhelm Wilmanns: Deutsche Grammatik. Gotisch, Alt-, Mittel- und Neuhochdeutsch. 2.Abt.: Wortbildung. 2.Aufl. Straßburg 1899

WILSS 1974 = Wolfram Wilss: "Probleme und Perspektiven der Übersetzungskritik". International Review of Applied Linguistics in Language Teaching. 12(1974) Heidelberg. S.23-41

WILSS $1975=$ Wolfram Wilss: "Übersetzen". H.Stammerjohann [Hg.]: Handbuch der Linguistik. Darmstadt 1975. S.515-537

WILSS $1977=$ Wolfram Wilss: Übersetzungswissenschaft. Probleme und Methoden. Stuttgart 1977

WILSS $1981=$ Wolfram Wilss [Hg.]: Übersetzungswissenschaft. Darmstadt 1981 (= Wege der Forschung 535)

WÖLFFLIN $1876=$ E.Wölfflin: "Zuneigung oder Abneigung durch Diminutive". Philologus. 34(1876) Göttingen. S.153-156

WOJTASIEWICZ 1957 = Olgierd Wojtasiewicz: Wstep do teorii thumaczenia. Wroclaw, Warszawa 1957

WOJTASIEWICZ 1975 = Olgierd Wojtasiewicz: "Wybrane zagadnienia z teorii przekładu". S.Pollak [red.]: Przektad artystyczny. Wroclaw et al. 1975. \$.347-352

WREDE 1908 = Ferdinand Wrede: "Die Diminutiva im Deutschen". Deutsche Dialektgeographie. 1(1908) Marburg. S.73-144

WROBBL 1969 = Henryk Wróbel: "Uwagi o wzajemnym oddziaływaniu na siebie pni i formantow slowotworczych". Prace Naukowe Uniwersytetu Slaskiego w Katowicach. Prace Jezykoznawcze. 1(1969) Katowice. S.105117

WROBEL 1973 = Henryk Wróbel: "Schematy slowotwórcze polskich hipokorystykow". Prace Naukowe Uniwersytetu Sloskiego w Ratowicach. Prace Jezykoznawcze. 2(1973) Katowice. S.27-48

WUNDERLICH 1972 = Dieter Wunderlich: "Zur Konventionalität von Sprechhandlungen". D.W. [Hg.]: Linguistische Pragmatik. Frankfurt a.M. 1972 (= Schwerpunkte Linguistik und Kommunikationswissenschaft 12). S.11-58

ZABROCKI 1970 = Ludwik Zabrocki: "Grundfragen der konfrontativen Grammatik". Probleme der kontrastiven Grammatik. Jahrbuch 1969. Düsseldorf 1970 (= Sprache der Gegenwart 8). S.31-52

ZALESKI 1958 = Jan Zaleski: "Wyrazy zdrobniałe $w$ polszczyźnie XVI wie$\mathrm{ku}^{\prime}$. Zeszyty Naukowe Uniwersytetu Jagiellońskiego 17. Filologia. Prace Jezykoznawcze. 4(1958) Kraków. S.93-184 
ZALESKI 1959 = Jan Zaleski: "Wolacz $w$ funkcji mianownika $w$ imionach meskich i rzeczownikach pospolitych". Jezyk Polski. 39(1959) Kraków. S.32-50

ZAREBINA 1954 = Maria Zarebina: "O niektórych sposobach spieszczen" . Jezyk Polski. 34(1954) Krakow. S.180-197

ZIETARSKA 1973 = Jadwiga Zietarska: "Glówne kierunki polskich studiów nad przekladami w latach 1950-1973". Poradnik Jezykowy. (1973) Warszawa. S.309-319

ZIMA 1961 = Jaroslav Zima: Expresivita slova $v$ současné Cestinè. Studie lexikologická a stylistická. Praha 1961 (= Rozpravy CSAV. Rada spolezenských ved 71.16)

ZWOLIŃSKI 1968 = Przemyslaw Zwoliński: "O chronologii kilku nie dostrzezonych funkcji niektórych sufiksow w jezyku polskim". Biuletyn Polskiego Towarzystwa Jezykoznawczego. 26(1968) Wroclaw et al. S.121-126

ZYCH 1981 = Anna Zych: "Nazwy mlodych zwierzat $w$ jezyku polskim i rosyjskim". Prace Naukowe Uniwersytetu Slaskiego w Katowicach. Prace Jezykoznawcze. 8(1981) Katowice. S.51-59 